\title{
The Parameterisation of Electrochemical Machining
}

By

Rachel N Muir

Ph.D Thesis

The University of Edinburgh

2006

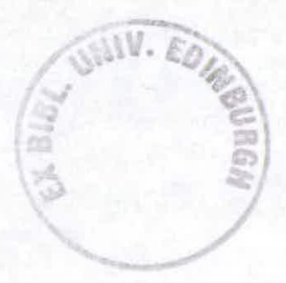




\begin{abstract}
Electrochemical Machining (ECM) is a non-conventional, non-contact technique, popular in the aerospace and automotive industries, used to machine ultra hard metal alloys. This work details a novel system, using ultrasound to dynamically measure the interelectrode gap. For greater accuracy, thermocouples have also been incorporated into the system to allow for the often significant temperature variation due to resistive heating. This gives time-resolved data for the dissolution valency and overpotential during ECM. The time averaged dissolution valencies found using this technique are the same, to within experimental error, as those found in previous literature demonstrating the accuracy of this approach. These parameters determine the ECM process and are used in computer modelling; generally these have been assumed to be time invariant however, significant variation is found in this work. This variation has also been used to give insight into high current dissolution processes.
\end{abstract}

Iron machined in sodium chloride $(\mathrm{NaCl})$ electrolyte was initially studied to eliminate any possible effects of alloy composition which might be seen when machining more complex materials. Data collected for the iron based stainless steels Jethete and SS410 machined in $\mathrm{NaCl}$ electrolyte showed common current-voltage transients for differing electrolyte concentration. A number of trends were observed for the iron and stainless steel data which can be discussed in relation to changes in the structure and permeability of the surface oxide to metal ions during ECM. More pertinent to industrial ECM, the machining parameters of the hard metal alloys Inconel 718 (In718) and Titanium $6 / 4$ (Ti6/4) in both $\mathrm{NaCl}$ and $\mathrm{NaNO}_{3}$ were also investigated. The current-voltage characteristics were found to have some similarities to those of iron and stainless steels. Similar results were also found for In718 and Ti6/4 using an ECM cell of a longer electrolyte flow path length.

Together these data begin to provide a parameter base for ECM under industrial conditions. They also allow insight into ECM parameter alterations, dissolution valency and current-voltage characteristics, at different electrolyte concentrations, electrolyte flow rates, applied voltages and cell path length. This data has been further employed to advance the understanding if the chemical processes established at the metal electrode/surface oxide/electrolyte interfaces during machining. 


\section{Declaration}

I hereby confirm that the work of this thesis has been performed exclusively from my own efforts except where referred to as being otherwise in the text. 


\section{Acknowledgements}

I would like to thank the people who have helped me complete the work in this thesis. I would like to extend my thanks to my industrial sponsors DutchAero BV for funding to complete this thesis. I also wish to thank Andy Mount (my supervisor) for all his help during the course of my work. I would like to thank Derek Jardine and George Duff who were especially helpful on the technical side of the project and contributed many ideas and suggestions to this work as well as designing and constructing equipment. Also, thanks to Dave Curry (Engineering postgraduate student) for help in carrying out some of the experiments in this work and for the use of his simulation work in this thesis, and Craig McGowan for his invaluable assistance in the technicalities of using Microsoft Word to produce a thesis document. Finally, I would like to thank my family, friends and my partner for endless encouragement and kind words throughout the course of the last 3 years. 


\section{List of Symbols}

A area of electrode, both tool and workpiece $\left(\mathrm{cm}^{2}\right)$

a activity of species

C specific heat capacity

c concentration of a given ion

D diffusion co-efficient

$\mathrm{D}_{\mathrm{T}} \quad$ thermal diffusivity

E electric field

e efficiency of machining process

F Faradays constant $\left(96,485 \mathrm{C} \mathrm{mol}^{-1}\right)$

f workpiece feed rate

$\Delta \mathrm{G} \quad$ Gibbs free energy

$\mathrm{h}$ height of workpiece, measured with the ultrasound probe

I current (amps)

IRC interface reflection coefficient

J current density

K equilibrium constant

k electrochemical machining constant

$\mathrm{M}$ molar mass of workpiece $\left(\mathrm{g} \mathrm{mol}^{-1}\right)$

$\mathrm{n}$ dissolution valency

$\mathrm{O}_{(\mathrm{aq})} \quad$ aqueous oxidised species

$\mathrm{P}$ power

$\mathrm{P}_{\mathrm{r}} \quad$ Prandlt number

$\mathrm{p}$ number of data points contained in a data-set

$\mathrm{R} \quad$ universal gas constant

$R_{(a q)} \quad$ aqueous reduced species

$\mathrm{R}_{\mathrm{n}} \quad$ Reynolds number

$\mathrm{r} \quad$ solvated ionic radius

$\mathrm{T}$ temperature

TOF time of flight

TS transition state

$\mathrm{t}$ time (secs)

U electrolyte flow velocity $\left(\mathrm{L} \mathrm{min}^{-1}\right)$

$\mathrm{u} \quad$ mobility of a given ion

V applied voltage (Volts)

$\mathrm{V}_{\mathrm{m}} \quad$ volume of material

$\mathrm{V}_{0} \quad$ overpotential, potential required to drive the chemical reactions at both the ECM electrode surfaces (Volts) 


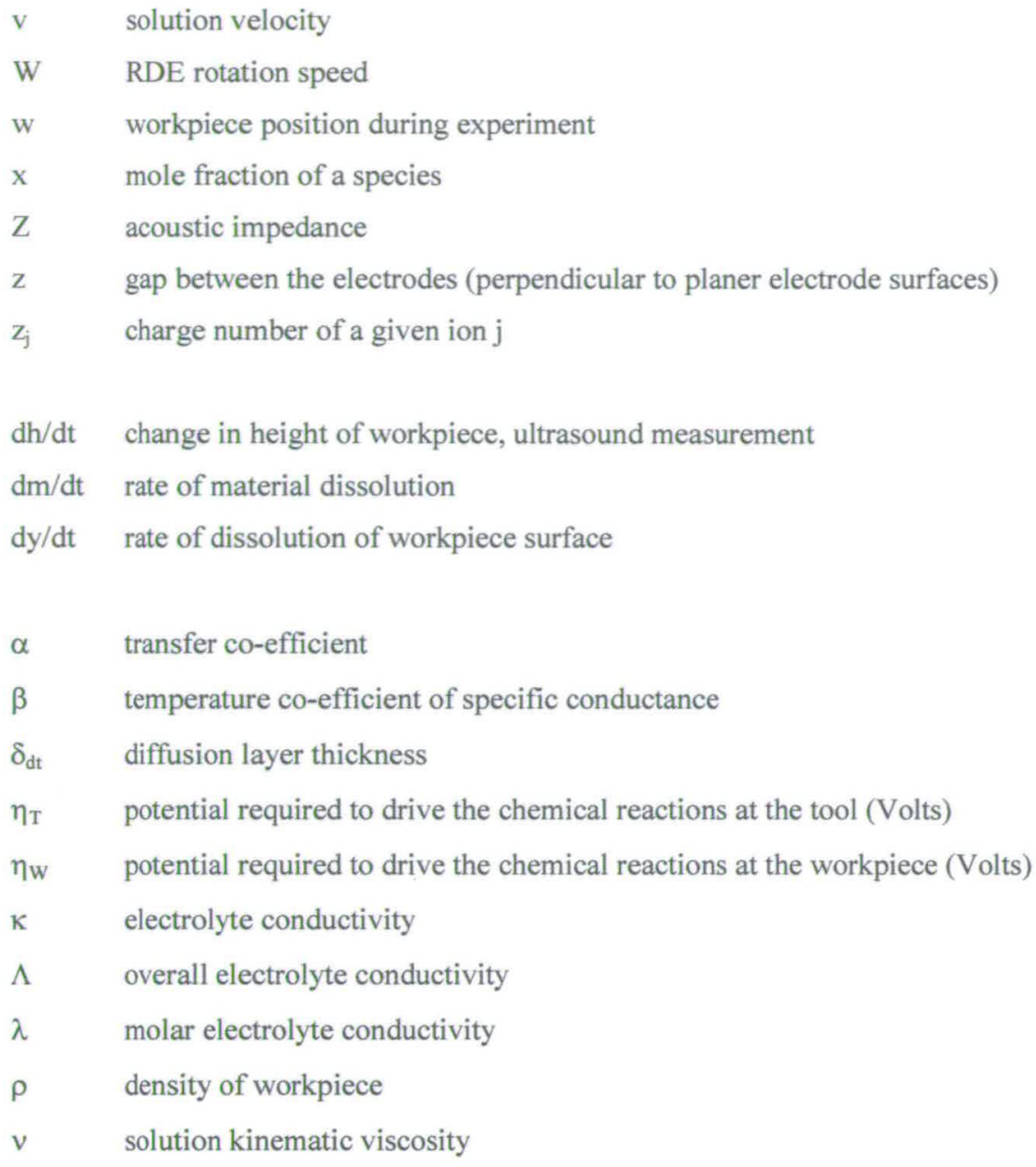

\section{Subscripts}

i initial value (value at time designated as initial time for fitting purposes)

j a given ion type

$\mathrm{T}$ parameter value at the tool

W parameter value at the workpiece

obs observed value

true true value

$\infty \quad$ value as time approaches infinity

$0 \quad$ value at time zero 


\section{Contents}

The Parameterisation of Electrochemical Machining 1

Chapter 1

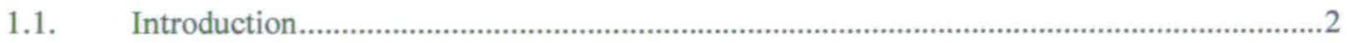

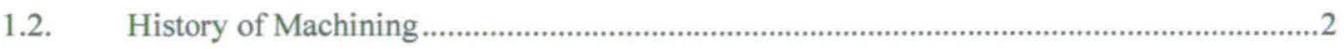

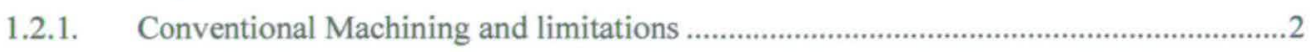

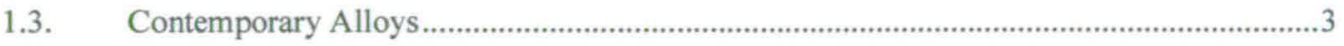

1.3.1. Machinability of Contemporary Alloys and Tool Materials. ..........................................4

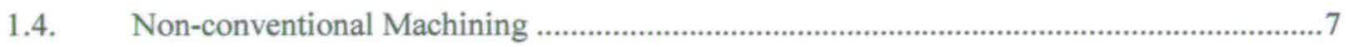

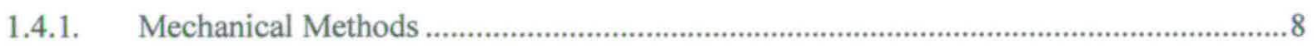

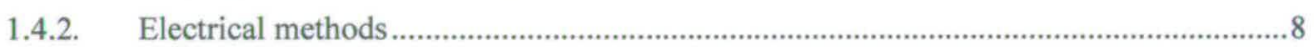

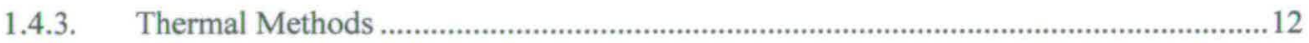

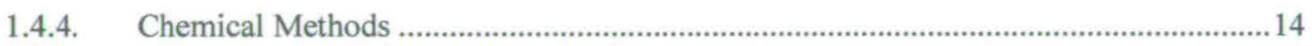

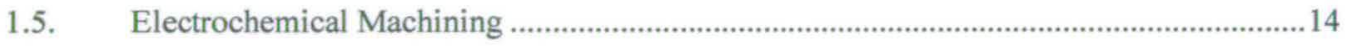

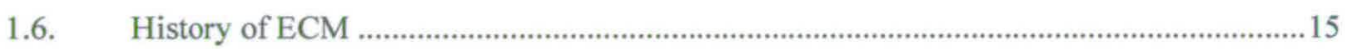

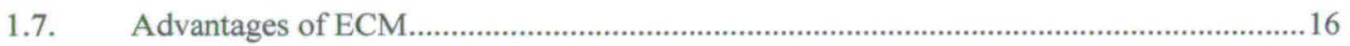

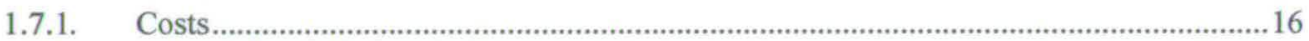

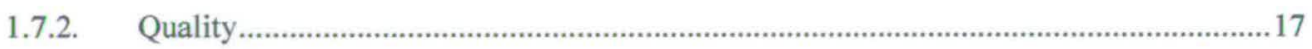

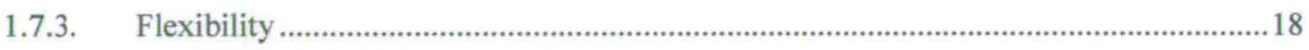

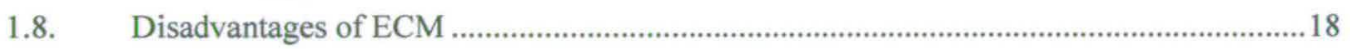

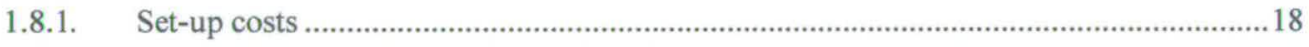

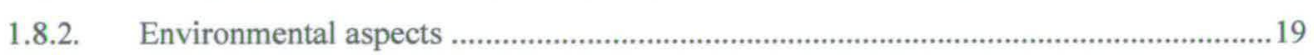

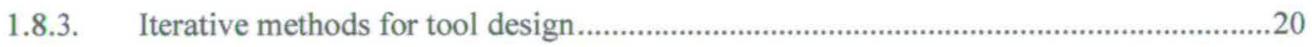

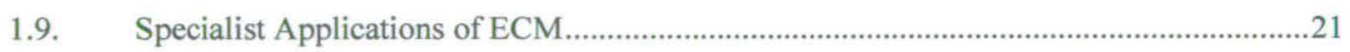

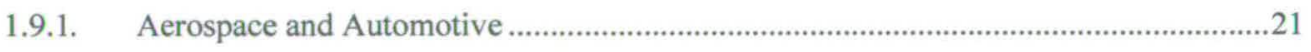

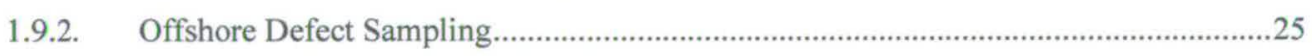

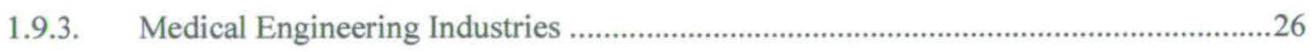

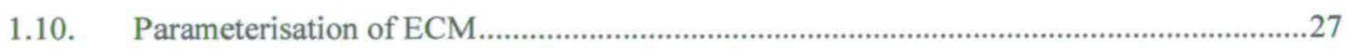

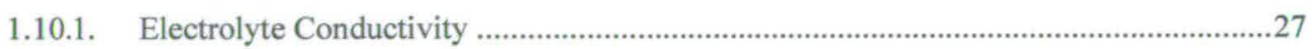

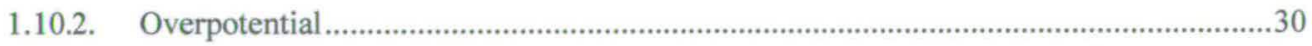

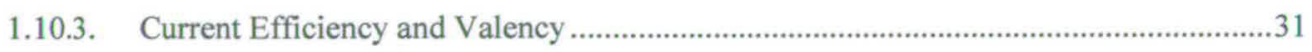

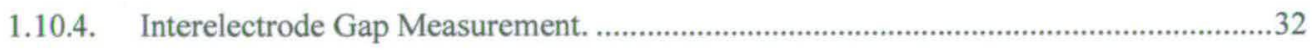

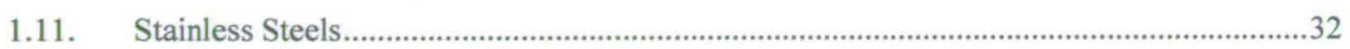

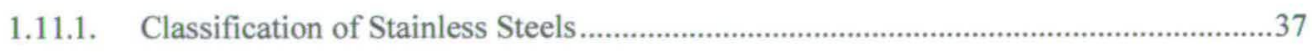

1.11.2. Function of Alloying Elements ......................................................................

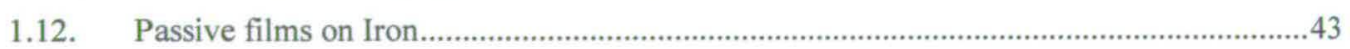

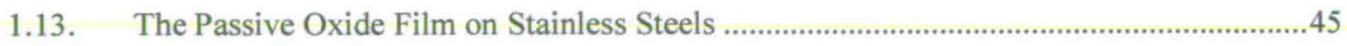




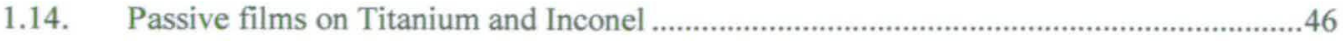

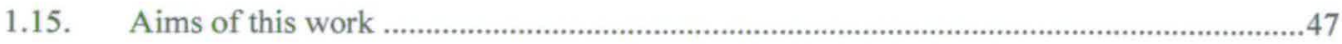

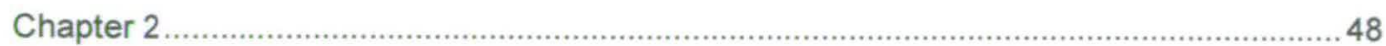

2.1. Metal Removal and Removal Rates.........................................................................

2.2. ECM Electrochemistry, a two electrode system ........................................................50

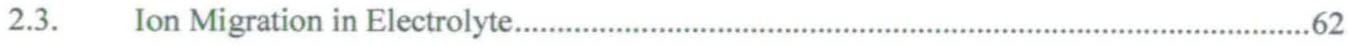

2.3.1. Electrolyte Conductivity ..........................................................................................63

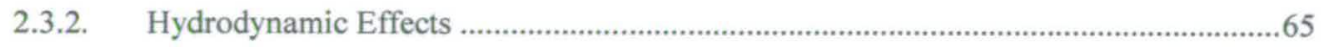

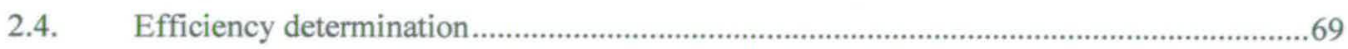

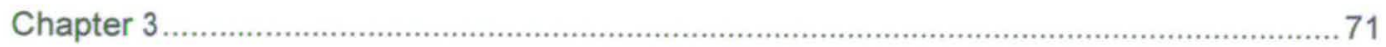

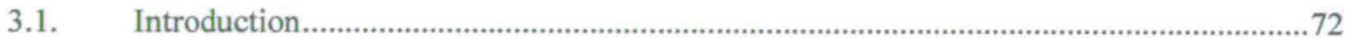

3.2. Chronoamperometric analysis of Machining Parameters ..............................................72

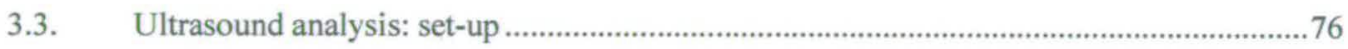

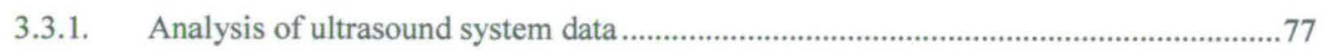

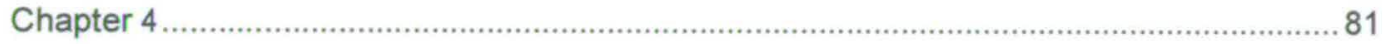

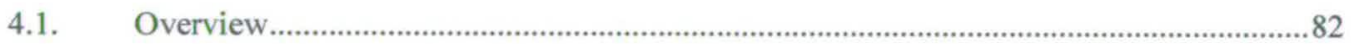

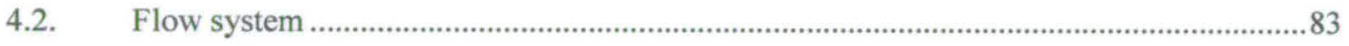

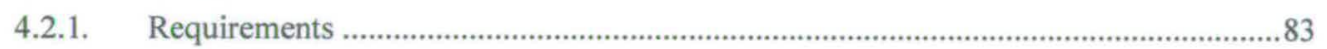

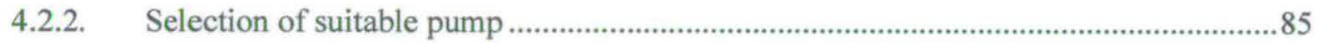

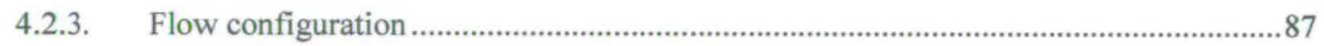

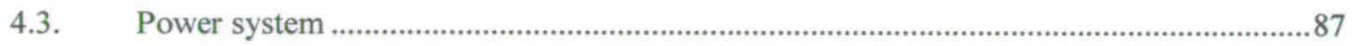

4.4. Feed and control system....................................................................................

4.5. Parameter control and monitoring / data acquisition system ..........................................90

4.5.1. Segment and total dissolution currents....................................................................90

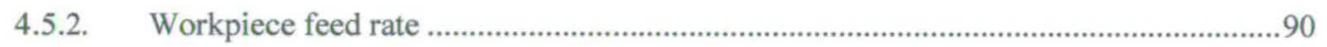

4.5.3. Machining voltage .................................................................................................

4.5.4. Inlet and outlet electrolyte temperatures ..............................................................91

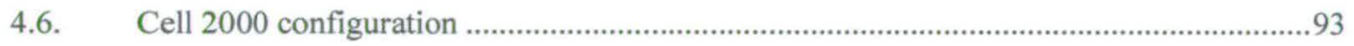

4.6.1. Electrode configuration …………............................................................................95

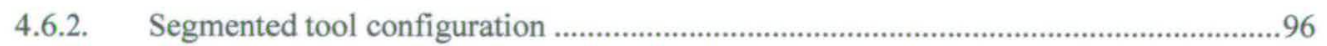

4.6.3. Workpiece drive pin assembly ……………............................................................98

4.6.4. Electrolyte sampling tubes ........................................................................................102

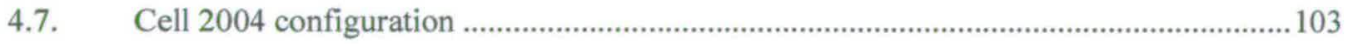

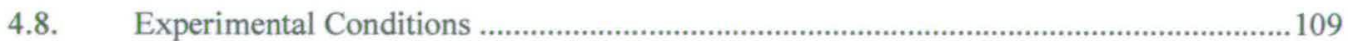

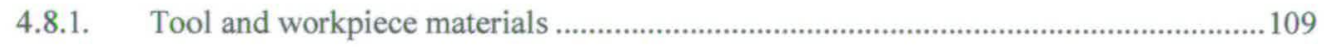

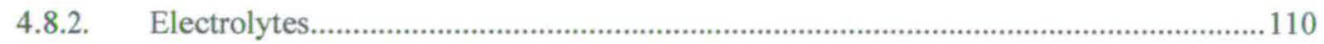

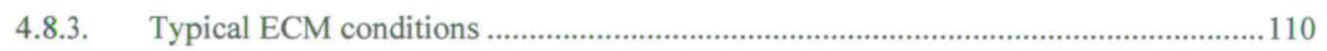

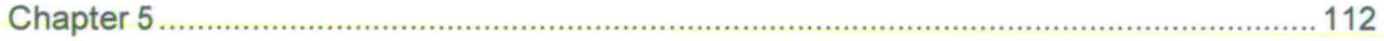




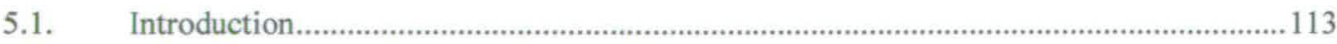

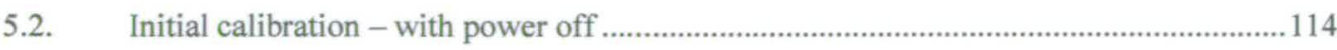

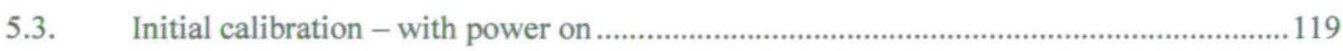

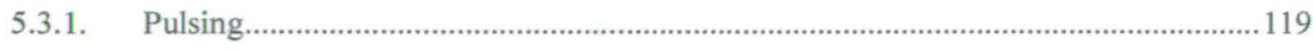

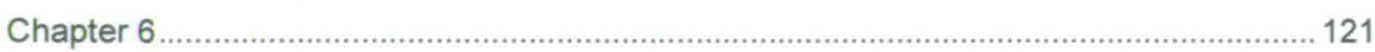

6.1. Critical comparison of analysis Methods.......................................................................122

6.1.1. Dissolution valency $(\mathrm{n})$ measurement. ...................................................................... 122

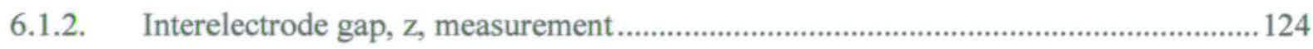

6.1.3. Comparison of measured ECM machining parameter, $\mathrm{k}$. ........................................ 125

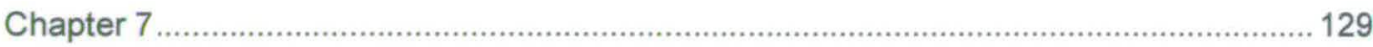

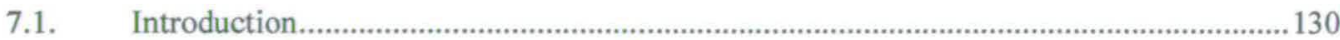

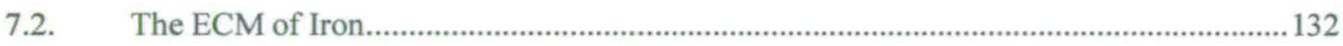

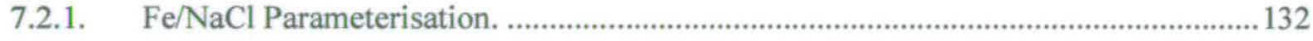

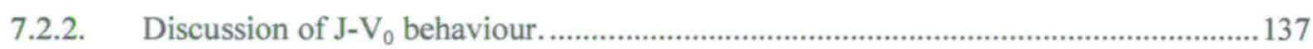

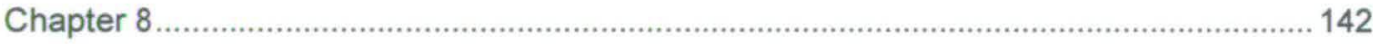

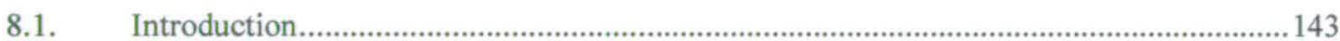

8.2. Thermocouple measurements. ........................................................................................ 145

8.3. Variations in Segment Current During ECM.............................................................. 150

8.4. Alloy composition effects. .............................................................................................. 153

8.5. Basic Parameter Measurements for SS410 and Jethete in $\mathrm{NaCl}$...................................155

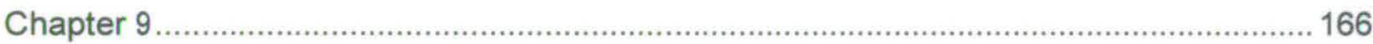

9.1. Introduction and previous literature ....................................................................... 167

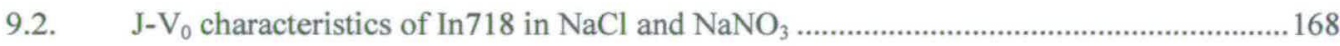

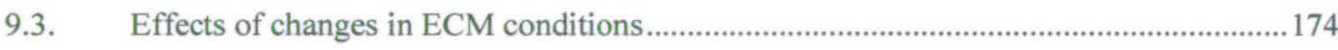

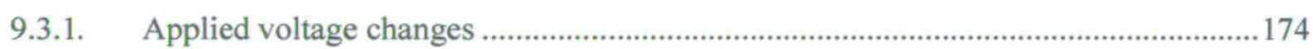

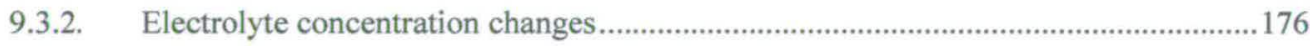

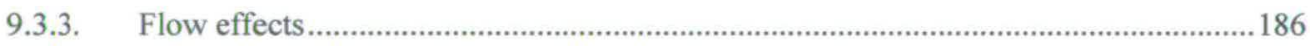

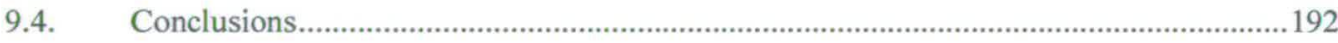

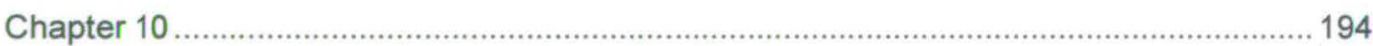

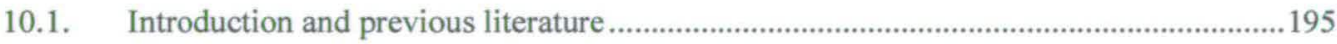

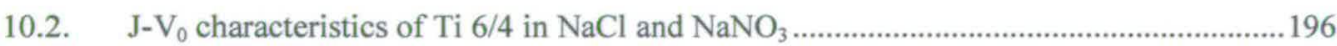

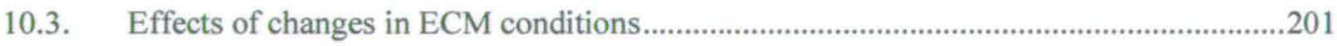

10.3.1. Electrolyte concentration changes..............................................................................201

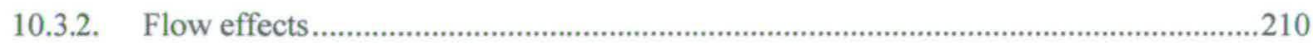

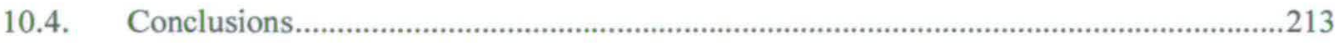

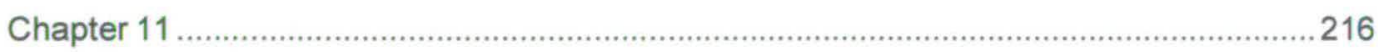

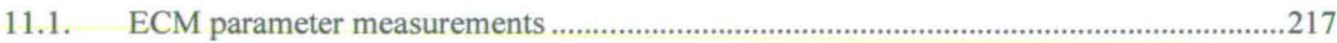


11.1.1. Dissolution valency measurements .........................................................................217

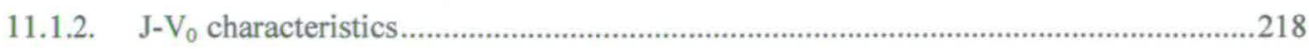

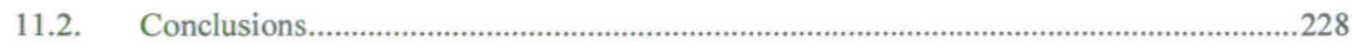

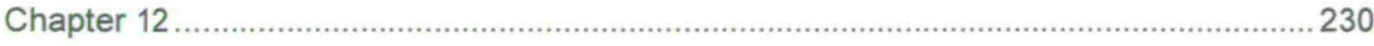

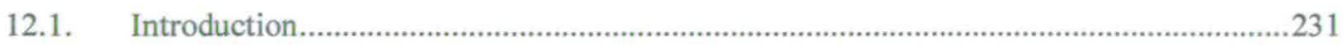

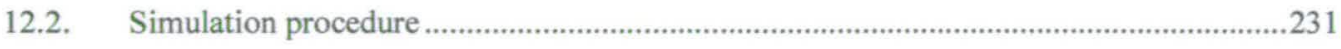

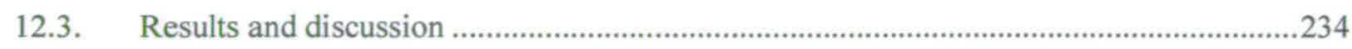

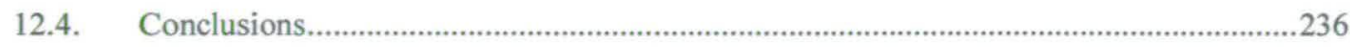

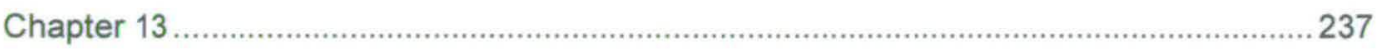

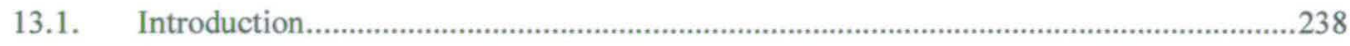

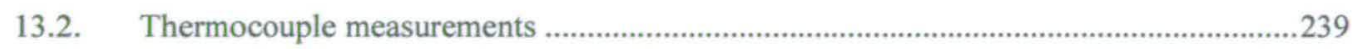

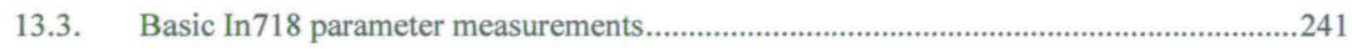

13.4. Basic Ti6/4 parameter measurements …........................................................................247

13.5. Cell 2004 parameterisation conclusions...........................................................................255

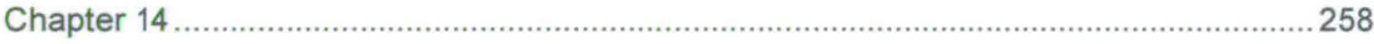

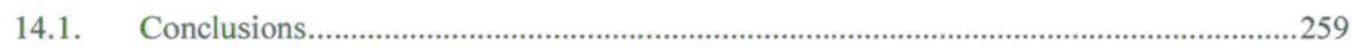




\section{Chapter 1}

Introduction 


\subsection{Introduction}

The subject of this thesis is the parameterisation of the Electrochemical Machining (ECM) process, which is a non-conventional machining method particularly applicable to contemporary alloys. In this chapter, therefore, contemporary alloys used in the aerospace and automotive industries are described. Conventional machining methods, their limitations and the advent of non-conventional machining methods to combat these limitations are then discussed. Of these techniques, a critical comparison is presented between ECM and its main rival, Electrical Discharge Machining (EDM). For various reasons, EDM is currently the method of choice for manufacturers, however, with further research, ECM has the potential to surpass EDM in accuracy, machining time and economically. The ECM process is then discussed, including advantages, disadvantages, specific applications and the need for parameterisation. Most of the ECM parameterisation in the literature has been carried out in Stainless steels. Thus, these are examined, in particular looking at their classification and the explicit functions of the alloying elements.

\subsection{History of Machining}

"All down the ages the rate of man's material progress has been
determined by his tools, because all tools represent synthetic
extensions of the human hand, being designed to magnify its
cunning or its power." [1]

Power driven tools have evolved and developed over the past 200 years or so. After about 1800 , a standard practice of metal cutting emerged whereby a hardened, tempered tool steel was used to cut an annealed (or softened) workpiece material which, if required, could be hardened and tempered after machining [2].

\subsubsection{Conventional Machining and limitations}

All forms of conventional machining rely on the same process, the movement of a sharp tool of hard material through a soft workpiece and the removal of a chip (Figure 1.1). A sharper harder tool requires a smaller force to cut the material. However, the tool point must be 
strong enough to withstand the force which is applied to it and must be resistant to the abrasion caused as the tool rubs across the workpiece. In addition the increased temperature at the tool-workpiece contact facilitates plastic deformation of the tool and has a tendency to increase the wear of the tool [3].

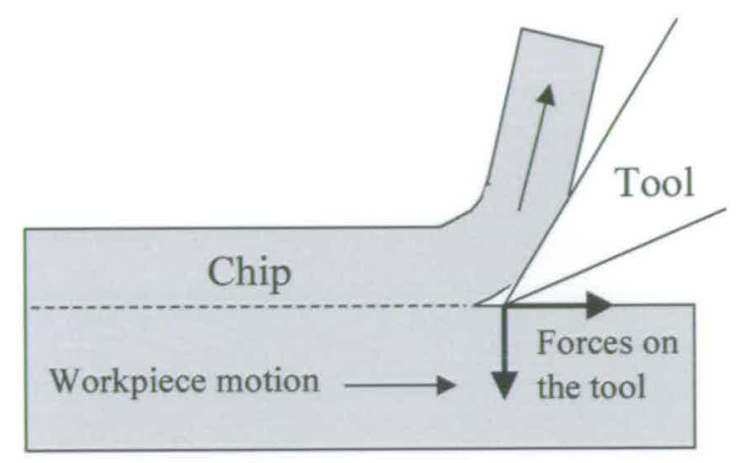

Figure 1.1: Conventional metal cutting, the chip production technique [4].

For conventional machining to succeed, the tool material must be harder than the workpiece material. Materials commonly used are cemented carbides, coated carbides and ceramics, with the tool material chosen depending on the workpiece material, see section 1.3.1 [5]. Even the newest tool materials have difficulty cutting materials of similar strength; clearly new machining techniques are required which are not restricted by the relative strengths of the tool and the workpiece. These techniques come under the category of non-conventional machining techniques and are typically used to machine contemporary alloys.

\subsection{Contemporary Alloys}

The development of the aerospace and automotive industries requires the production of harder, lighter and more temperature resistant alloys. Common alloys used for aeroengine manufacture are steel, and alloys of nickel, titanium and aluminium. Other materials used in the industry are carbon, ceramic and metal-matrix composites [5]. These materials are used because they offer valuable properties sought after by these industries e.g. corrosion resistance and high strength-to-weight ratio over a range of temperatures [6]. Due to their superior properties these alloys are often termed "super alloys". The most widely used super alloys are nickel-based and are commonly used in the manufacture of turbine blades, which are required to operate at high temperatures and pressures [7]. Nickel-based alloys are also 
used in marine equipment [8], nuclear reactors [9], petrochemical plants and the food processing industry [10]. The major alloying elements in nickel-based alloys are chromium, cobalt and molybdenum, however, other trace alloying elements can be added such as tungsten, tantalum and molybdenum to increase corrosion resistance and strength at elevated temperatures [11].

Titanium alloys are also widely used in aeroengine manufacture. Titanium alloys, like nickel-based alloys, have a high strength-to-weight ratio. They maintain their high strength at increased operating temperatures and display exceptional corrosion resistance [12]. Titanium alloys account for $30 \%$ of the total engine mass in commercial and $40 \%$ in military aircraft [13]. Gamma titanium aluminides (Ti-Al) are becoming increasingly popular in the aerospace industry for use in high temperature components, due to their high strength-toweight ratios over a range of temperatures and good corrosion and oxidation resistance [14]. Work has been done on the effects of various alloying elements on the oxidation behaviour of Ti-Al. The results report niobium additions to be effective in improving the oxidation resistance of $\mathrm{Ti}-\mathrm{Al}$ in air while chromium is detrimental in this area $[15,16,17]$. Work has also been conducted into the effects of high speed milling and grinding on the fatigue strength of Ti-Al. The results show that high speed milling increased the fatigue strength of the alloy, while finish grinding reduced the fatigue strength when compared to rough grinding and polished samples. With high speed milling, the surface integrity of the alloy was seen to deteriorate as the material was milled [18], as well as producing increasingly worn tools [19].

\subsubsection{Machinability of Contemporary Alloys and Tool Materials.}

Advanced materials such as those described in section 1.2 provide a severe challenge for cutting tool materials due to their unique combination of properties such as high temperature strength, hardness and chemical wear resistance. The low thermal conductivity of these alloys also results in significantly increased temperatures at the tool-workpiece interface, thereby accelerating tool wear [5]. While these properties are desirable design characteristics, they result in poor conventional machinability of these alloys.

Tool materials with improved hardness such as cemented carbides, ceramics, coated ceramics and cubic boron nitride ( $\mathrm{CBN})$ are the most frequently used for machining these 
aerospace alloys. The development of carbide tools allowed metals to be machined at twice the rate previously achieved with high speed cutting tools [5]. Tool fracture can also be reduced through the incorporation of a wear resistant coating onto the tool producing coated tools. A good deal of research has been done in this area investigating coating materials including TiC [20], TiN [21], $\mathrm{Al}_{2} \mathrm{O}_{3}$ [22], TiCN [23], TiAlN [24] and TiZrN [25]. More recently diamond coatings have been investigated. The diamond coating is applied to the tool using Chemical Vapour Deposition (CVD) [26] resulting in a tool which is highly wear resistant $[27,28]$. One of the main causes of tool failure with diamond coated tools is the poor adhesion of the diamond film to the substrate [29]. Investigations have been carried out into substrate pre-treatment in an attempt to enhance the adhesive strength of the film. The usual pre-treatment carried out involves scratching and acid etching of the substrate surface to remove the surface cobalt. This had been found to inhibit the cobalt-catalysed formation of non-diamond carbon at the diamond-substrate interface which reduces the adhesive properties of the film $[30,31]$.

Besides fracture resistance the tool material must have sufficient high temperature strength in order to maintain its shape at elevated cutting temperatures, resisting plastic deformation of the tool [6]. Table 1.1 shows the typical softening temperatures of some tool materials.

\begin{tabular}{cc}
\hline Tool material & Softening temperature $\left({ }^{0} \mathrm{C}\right)$ \\
\hline High speed steel $^{\mathrm{a}}$ & 600 \\
$\mathrm{WC}$ & 1100 \\
$\mathrm{Al}_{2} \mathrm{O}_{3}$ & 1400 \\
$\mathrm{CBN}$ & 1500 \\
Diamond & 1500
\end{tabular}

Table 1.1: Typical softening temperatures of some tool materials [5] ${ }^{\mathrm{a}}$ when a tool steel contains more than 7 wt. \% tungsten, molybdenum and vanadium, along with more than 0.6 wt. \% carbon they are referred to as high speed steels (HSS). The term is descriptive of their ability to cut metals at the relatively high speeds in use in the 1940's [32].

Cooling and lubrication techniques such as cryogenic systems, flood cooling and high pressure cooling can be used to lower the temperature at the tool/work material contact and so improve tool resistance to plastic deformation [33, 34]. Notwithstanding the obvious advantages of using cooling and lubrication fluids, there are some significant drawbacks. The fluids lead to pollution of the atmosphere and/or water system and produce health 
hazards, mainly to the skin and respiratory system [35]. Consequently, cleaning of the workpiece is required after machining to remove any residual fluid from the machined components and any residual fluid on the evacuated chips must be removed prior to disposal to reduce environmental damage.

Tool fracture and plastic deformation are sudden and catastrophic tool failures. Gradual wear in the form of Chemical Dissolution Wear (for high speed machining) and Mechanically Activated Wear (for low speed machining) must also be considered when selecting a tool material [36]. At high cutting speeds, favoured by industry for economic reasons, the chemical dissolution of the tool material into the workpiece is an important contributor to tool wear. In this case the tool material dissolves into the chip at elevated temperatures; the tool materials most resistant to dissolution wear the least. In low speed machining chemical dissolution of the tool into the workpiece material can be considered to be insignificant due to the relatively low temperatures. In this region wear is caused by micro-fracturing of the tool $[36,5]$.

Nickel based alloys are commonly used in the aerospace industry. They are known to be among the most difficult alloys to machine due to their high strength and thermal resistance [10]. Inconel 718 (In718) is a nickel based alloy, it microstructure contains highly abrasive carbide particles decreasing its machinability [37]. In addition, the austenitic matrix of In718 causes rapid work hardening (the increase in hardness and strength produced by cold plastic deformation or mechanical working [38]) during machining contributing to tool wear [39]. Machining of $\operatorname{In} 718$ is carried out industrially with either a coated carbide tool or a ceramic tool [10]. Various studies detail the relative merits of different tool coatings for machining In718 $[6,37,40]$. The main wear mechanism when machining In718 is abrasion [37]. Cemented carbide tools are largely used for machining $\operatorname{In} 718$ at very low cutting speeds, $20-30 \mathrm{~m} / \mathrm{min}$ [37]. At cutting speeds up to $100 \mathrm{~m} / \mathrm{min}$ under dry conditions, coated carbide tools are used [37]. A Physical Vapour Deposition (PVD) [41] TiAlN or TiCN coatings were found to outperform TiN coatings at cutting speeds of $76 \mathrm{~m} \mathrm{~min}^{-1}$, while at the lower cutting speed of $46 \mathrm{~m} \mathrm{~min}^{-1}$ TiAlN has been observed to show an excellent performance [42]. A further increase in cutting speeds, up to $200-700 \mathrm{~m} / \mathrm{min}$, can be achieved with the use of ceramic tools [37]. $\mathrm{Al}_{2} \mathrm{O}_{3}-\mathrm{TiC}$ was found to be the most stable for machining In718 as it has the most thermal resistance when high speed machining [37]. However, ceramics are poor thermal conductors and vulnerable to thermal cracks [37]. 
Ceramic tool materials are not generally used to machine titanium alloys due to high chemical wear rates resulting from high reactivity of the titanium alloy with the ceramic material [43]. In the machining of titanium, CVD coated carbides and ceramics have struggled to replace cemented carbides due to their reactivity with titanium at the high machining temperatures used in industry [44]. At these temperatures the titanium atoms diffuse into the carbide tool and react with the carbon forming a layer of titanium carbide which bonds to both the tool and the chip to form a seizure zone [3].

\subsection{Non-conventional Machining}

The term non-conventional machining applies to a range of mechanical, electrical, thermal and chemical material removal techniques. These techniques began to emerge following the Second World War in reaction to changing machining desires [45]. Design engineers began creating complex parts that were meant to be stressed close to their ultimate material capabilities. Metallurgists were creating space-age alloys with improved corrosion, erosion and temperature resistance. These alloys, however, proved much more difficult to machine than conventional materials.

Non-conventional techniques are classified according to the type or energy used to remove material, the classical terms: mechanical, electrical, thermal and chemical, are somewhat oversimplified because, for example, both chemical and electrical energies are important in ECM. However, for the sake of conciseness these 4 classes will be used. Non-conventional processes are usually characterised by high power consumption and lower material removal rates than conventional methods. Since these are disadvantages, non-conventional techniques are usually preferred when machining complex parts or exotic materials. Nonconventional processes also differ in their effects on surface integrity. They often compare favourably in terms of reducing surface roughness and less heat-affected zones, cracks and residual stress [45]. 


\subsubsection{Mechanical Methods}

\subsubsection{Abrasive Jet Machining (AJM)}

In AJM a high pressure gas drives a stream of abrasive particles through a nozzle at high speeds. This technique can be used to cut a wide range of materials. It differs from conventional sand blasting in that the abrasive is much finer. AJM has a number of main advantages: the carrier gas acts as a coolant; there are no vibration problems because the tool is not in contact with the workpiece; precise control of the needle-like, high speed stream of particles can be obtained and, finally, the beam can be focussed onto small areas [46].

The most frequently used abrasive in AJM is aluminium oxide although silicon carbide is also common. It is important that the abrasive particles have sharp edges rather than rounded surfaces. Particle size is also important; the best cutting results have been obtained with the bulk of the particles varying between 15 and 40 microns. In addition, specific powders for cleaning, etching, abrading and fine polishing are available [45].

This method can be used to make fine cuts or a wide spray to "frost" large areas. It is commonly used for frosting, cleaning and operations on semi-conductors, including cutting, drilling, cleaning and thinning [45]. AJM is also used for various metalworking applications: drilling and cutting thin sections of hardened metal, applying numbers or trade names to parts and removing chrome, corrosion and contaminants from small areas. AJM is a valuable process for special problem areas on those small parts with sufficient value to justify handling individually. This process is not practical for removal of large amounts of material and it is uneconomical to recycle the abrasive [47].

\subsubsection{Electrical methods}

The electrical methods are the main competitors of ECM, they are detailed as follows: 
ECG was the precursor of ECM. A combination of electrochemical dissolution and the abrasive action of diamond particles is used to remove material from electrically conducting workpiece materials. The majority of the material is removed by electrolysis but some is removed by the abrasive, which is in contact with the workpiece. One of the functions of the abrasive is to act as a spacer between the grinding wheel tool electrode and the workpiece, allowing electrolyte to flow between the two electrodes and facilitating electrolysis. ECG is primarily electrochemical dissolution in contrast to conventional grinding in which material is removed by the grit cleaving a small chip from the workpiece. ECG's ability to grind hard materials makes it a useful technique in the aerospace, automotive and medical manufacturing industries. Wheel wear is considerably less than observed in conventional machining and the low forces involved make ECG a desirable process for the manufacture of fragile parts such as hypodermic needles. A disadvantage of ECG is the expensive set-up cost [48].

\subsubsection{2. \\ Electric Discharge Machining (EDM)}

The first scientist to observe a spark discharge causing metal erosion was Sir Joseph Priestley, an English chemist, around 1770 [49]. Over 150 years later, in 1943, two Russians, B. R. and N. I. Lazarenko, while investigating the wear of switch contacts, had the idea that spark discharges could be used for machining metals [45]. Since then EDM has expanded into one of the most extensively used non-conventional material removal processes [2]. It machines irrespective of workpiece hardness and there is no direct contact between the electrodes, thus eliminating mechanical stress and vibration problems [45]. This technique is based on an arc caused by a dielectric breakdown in the gap between the workpiece and the tool leading to pitting, or material removal, on the workpiece. In the material erosion mechanism electrical energy is transformed into thermal energy via a series of discrete electrical discharges taking place in a dielectric fluid. The ionisation channel between the surface of the anode and the cathode creates thermal energy and a temperature in the range of $8000-12,000^{\circ} \mathrm{C}$. As a result of this ionisation channel heating, melting of material at the surface of the workpiece occurs. A pulsing direct current is used and removal of the field results in destruction of the ionisation channel. A reduction in temperature 
follows and the circulating dielectric fluid removes the molten material from the surface of the workpiece (Figure 1.2) [45].

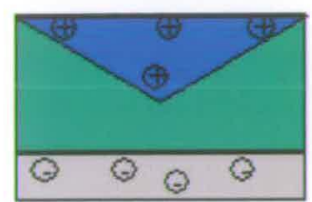

(a)

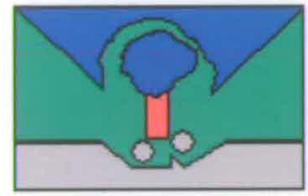

(d)

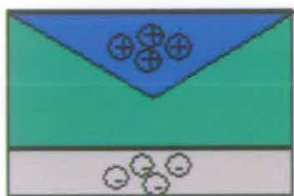

(b)

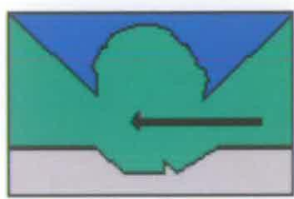

(e)

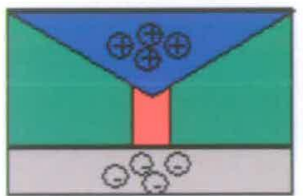

(c)

Tool

Dielectric fluid

Workpiece

Figure 1.2: Typical series of steps for EDM. a) The energised electrodes approach one another. b) Concentration of the electrical field. c) Creation of an ionisation channel between the electrode and workpiece. d) Spark and heat production at the workpiece and the tool. The workpiece material melts locally and disintegrates; a small portion of the tool material is also removed. e) Current halted and evacuation of the metallic particles by flushing with dielectric.

The main qualities for the dielectric fluid are that it should:

- Possess sufficiently high dielectric strength to remain electrically non-conducting until the breakdown voltage is reached

- Be able to de-ionize rapidly after the discharge

Liquid hydrocarbon products such as paraffin or light transformer oils, and more recently deionized water, are mainly used as di-electric fluids [47].

Electrically conductive materials with high melting points are chosen as tool materials for EDM. In early EDM machines the tool material used was brass, however it is a relatively high wear material. Nowadays, graphite is widely used in pulse EDM. Copper is also a stable material under sparking conditions as its wear is comparable with graphite. Other tool materials have been investigated such as aluminium, which displays very high wear, and 
copper-boron and silver-tungsten, which both exhibit extremely low wear and are therefore occasionally used [47].

The volume of material removed per discharge is generally in the range of $10^{-6}-10^{-4} \mathrm{~mm}^{3}$, and the metal removal rate is usually between $2-400 \mathrm{~mm}^{3} / \mathrm{min}$. EDM is a reproductive shaping process in which the form of the tool electrode is mirrored in the workpiece due to the ionisation efficiency decreasing markedly with distance [2].

The disadvantages of EDM compared to ECM are the tool wear during machining; the heat produced at the workpiece in the region of machining and also at the tool which lead to electrode damage and also the low material removal rate (compared to ECM) [4].

A number of EDM variations have been developed over the years. Wire-cut EDM (WEDM) is common due to its ability to machine high strength, temperature resistive metals into elaborate shapes and profiles. A thin wire acts as the tool electrode and is wound continuously between two spools at rates of up to $3 \mathrm{~m} \mathrm{~min}^{-1}$ [47]; this continuous feeding of the tool ensures a fresh portion of the tool electrode is always presented to the workpiece. Di-electric fluid is injected into the machining zone and the tool wire is then moved through the workpiece eliminating the need for the elaborately shaped tools required in EDM [50] (Figure 1.3).

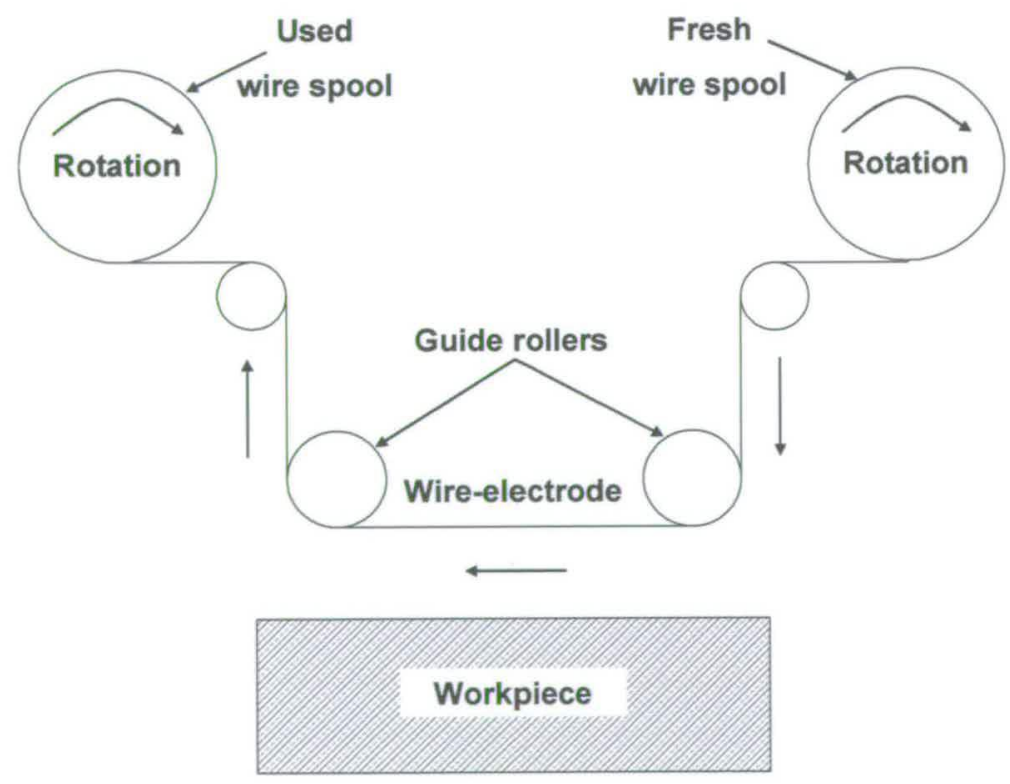

Figure 1.3: Principles of wire-EDM [47]. 
Drilling is a common task performed by EDM. Hollow, tubular electrodes are generally used with the di-electric fluid flushed down the centre of the tube. Because rotation of the tool is unnecessary, irregularly shaped holes which are tapered or even curved can be produced [47].

An advantage EDM has over ECM is that the initial outlay costs of buying an EDM machine are significantly less than buying an ECM machine [2]. However the disadvantages of EDM are that EDM tools are subject to wear and need to be replaced far more often than ECM tools; EDM is a slow process and so cavities are generally roughed out prior to heat treatment and then finishing by EDM. The material removal rate for an ECM machine is higher than for an EDM machine. In EDM a large temperature rise is produced at the site of machining leading to possible workpiece damage [47].

\subsubsection{Thermal Methods}

\subsubsection{1. \\ Laser Beam Machining (LBM)}

LASER is an acronym for Light Amplification by Stimulated Emission of Radiation [51]. The light energy emitted by a laser has several characteristics which distinguish it from other light sources:

- The light emitted is monochromatic; the beam can be focused using simple optics.

- The light beam is highly collimated, with typical divergence angles of $10^{-2}-10^{-4}$ radians

- Because of the small beam divergence, the laser has a highly focused power density. The laser beam energy can be collected with simple optics and focussed onto a small area

The power density of a laser is sufficient to cut any material, even diamond [52]. Material is typically removed in the gaseous or liquid states. When the laser is concentrated on an area of the workpiece, the material is heated and melts. However, some of the underlying material is heated so dramatically that it evaporates forcing the liquid above it to be removed from the surface of the workpiece at high velocity. In some cases cutting can be enhanced 
through the introduction of a gas at the point at which the laser beam is working. An advantage of laser beam machining is the possibility of machining a surface which is not perpendicular to the beam, a requirement with mechanical drilling. The laser can be used to drill, cut, mark, heat-treat or even weld. Although the heat-affected zone is shallow, very high thermal stress may be applied which can result in material charring and bubbling. Laser techniques are the most versatile of the non-traditional techniques as they can be used to cut and drill virtually any material, hard, soft, brittle, or ductile, insulator or conductor. However the costs of machining with laser techniques are high and it is a dangerous technique requiring skilled operators [45].

\subsubsection{Electron Beam Machining (EBM)}

Electrons can be accelerated and formed into a narrow beam by an electric field. This beam can then be focused and bent by electrostatic and electromagnetic fields. Electron beam cutting machines use relatively high power beams with electron velocities exceeding onehalf the speed of light. At the point where the beam impacts the workpiece surface, the kinetic energy of the electrons is transformed into thermal energy, vaporising or melting the material in the vicinity. The process is carried out in a vacuum to prevent collisions between the electrons and gas molecules which would scatter the beam. This adds to the expense of the process [45].

Electron beams are used for a wide variety of applications: welding, cutting, heat-treating, drilling and glazing [47]. The power density focussed on a specific area can be altered according to the application. Hole cutting is a typical application of EBM. The electron beam is focussed on one spot until either the beam has penetrated the workpiece or until a specific hole depth has been achieved. This technique can be used to drill holes in ceramics, an example of high power density EBM. EBM is an extremely precise cutting tool able to cut holes of a very small size, as small as $0.05 \mathrm{~mm}$ in diameter; therefore it is excellent for micromachining [53]. EBM can cut any known material that can exist in a vacuum with no cutting tool pressure or wear [45]. The unavoidable use of a vacuum for machining adds to the already expensive set-up and operating costs of this technique. In addition, EBM requires high operator skill further adding to the running costs. The material removal rate is low, $\approx 1.6 \mathrm{~mm}^{3} / \mathrm{min}$, consequently, only relatively small cuts are economically viable [45]. Finally, heat is produced at the site of machining leading to possible workpiece damage [54]. 


\subsubsection{Chemical Methods}

\subsubsection{Photochemical Milling (PCM)}

This technique is mainly used for machining extremely thin materials that would be difficult to machine with normal mechanical applications [45]. Thorough cleaning of the workpiece is required to insure uniform adhesion of the photographic material and uniform chemical dissolution of the metal. The cleaning process varies depending on the workpiece material. After cleaning the parts are dried in air and coated with a photoresist, photographic material that will polymerise when exposed to light of a particular wavelength. The photoresist remains on the metal when it goes through the developing stage and acts as a barrier to the etching solution applied to the metal. The required patterns are produced on a photographic film or glass plate called the phototool. The phototool image is printed onto the photoresist using light as the exposure source. The photoresist is developed creating durable stencils on the metal. The material is then etched through the stencil apertures generally using aqueous ferric or cupric solutions mixed with hydrochloric acid [55]. PCM is used for machining thin materials, such as washers, and in some decorative applications [47].

\subsection{Electrochemical Machining}

Although the application of electrolytic machining for metal-working is relatively new, the fundamental principles are not. Michael Faraday (1791-1867) observed that if two electrically conducting metals (electrodes) are placed in a conductive electrolyte and subject to a direct current, metal may be deplated from the positive electrode (the anode) and plated onto the negative electrode (the cathode) [56]. Faraday's work initiated research into Electroplating and Electropolishing, both well established processes in our manufacturing industries. The controlled electrolytic removal of material from an electrically conducting workpiece appears to have been initially applied to Electropolishing. Electropolishing was developed by Jacquet in 1935 and nowadays is widely used for the preparation of samples for metallographic examination [2,57]. Electropolishing is ideal for this application because there is no surface damage to the workpiece in comparison to traditional polishing techniques. In the same way ECM can be used to remove an electrically conductive 
workpiece material through anodic dissolution [58]. For many years, the metalworking industry concentrated on the cathodic side of the electrochemical cell, e.g. Electroplating. More recently, in the 1960's and 70's, focus on the anodic reaction lead to an increase in research into ECM as a metal-working process [45]. ECM is the process of metal removal via anodic dissolution of a workpiece alloy. The workpiece is biased as the anode and the tool the cathode in an electrochemical cell with an aqueous salt electrolyte, typically $\mathrm{NaCl}$, $\mathrm{NaNO}_{3}$ or $\mathrm{KCl}$, flowing through the interelectrode gap to remove the soluble and insoluble products of machining. The electrodes are moved toward each other resulting in workpiece material dissolution governed by ion migration.

\subsection{History of ECM}

The first ECM-like process was patented by W. Gusseff, a Russian, in 1929 [47] (British Patent No. 335,003), and was later published by C. F. Burgess in a paper for the Electrochemical Society (United States) as a process for anodic metal removal in 1941. In his paper Burgess drew attention to the remarkable differences between mechanical and electrolytic methods for metal removal, this former being a method of "brute force and violence" while the latter "cool, steady, non-deforming magic". Consequently, while it may be pleasing to trace the progress of ECM back to Electroplating and Electropolishing, it does not seem to have originated that way. Indeed, the ECM process was invented many years before it became an established metal-working technique; it seems likely that the timing of its début onto the commercial stage was determined by the escalating need to shape difficult to machine alloys [2].

It was not until the 1950 's that the first significant developments took place and ECM machines began to be produced for industrial purposes. In 1954 the Battelle Memorial Institue, sponsored by Cleveland Twist Drill Company, developed an electrochemical method for sharpening carbide-tipped drills (British Patent No. 854,541). This patent was bought by the Steel Improvement and Forge Company (SIFCO) which supported further work at the Battelle Memorial Institute and also conducted work in its own laboratories. SIFCO adapted the new method to machine the curved surfaces of gas-turbine blades. As a result the first specialised blade machining equipment was created which inspired widespread interest. One of these machines was imported to Britain by Metachemical Machines Ltd and made available to Rolls-Royce Ltd. Rolls-Royce pioneered its own 
improvements and became the first British company to use the technique for mass production of gas-turbine blades [2].

Meanwhile, in 1959 a commercial ECM machine was produced by Anocut Engineering Company in Chicago. Anocut Engineering played a major role in pioneering the ECM process [59]. Anocut developed ECM for a wide range of applications and the company manufactured a large number of specialised and general machines. Also, during this era (1956-1966), other organisations were developing electrochemically assisted grinding machines, combining electrolytic and grinding action. Early reports suggested wheel wear to be $1 / 3$ of that observed in conventional grinding. From this point electrochemical metal removal founded many metal shaping techniques. The main commercial use of ECM in the current era is in the aerospace industry but it also finds uses in the automotive, offshore and medical engineering industries [45].

\subsection{Advantages of ECM}

\subsubsection{Costs}

ECM has the ability to produce a large, complex-contoured surface in a single pass of the ECM tool, resulting in the obvious economic advantages of a direct saving in machining time. Additional economic advantages arise from the possibility of unskilled or even automated machine operation and the absence of tool wear. A single ECM machine can perform machining tasks more rapidly and can replace several mechanical machining operations; therefore the capital investment in both the machines and the buildings they occupy is less. ECM is a technique which can be used to manufacture modern, technologically advanced components without increasing the cost of the final product. For example, enhanced jet-engine performance necessitates the use of complex-shaped components of "super-alloy" material; the time frame for conventional machining of these parts has increased several fold over the past 10 years and labour costs have soared. Component complexity and material hardness pose no obstacles to ECM and the rapid production of parts minimises the burden of increased labour costs [59]. However, these cost advantages must be weighed against increased costs of tool design. 


\subsubsection{Quality}

ECM is a controlled process in which metal is dissolved producing surfaces that are consistently free from imperfections or stress [2]. Metals can be machined regardless of their strength and there is no necessity for the tool to be harder than the workpiece. The process does not produce burrs and complex-shaped components can be produced with a high quality surface finish [59]. The untreated ECM surface, however, does not have the fatigue strength of surfaces produced by mechanical machining. Mechanical finishing methods impart compressive stresses to the surface layers improving the fatigue strength of the part. When the fatigue strength is critical, post-electrochemical mechanical finishing procedures can be used to re-establish or impart compressive stresses to the surface raising the fatigue properties of the component. These post-electrochemical mechanical finishing techniques include glass-bead or vapour blasting the surface and regularly result in electrochemically machined components exhibiting comparable or greater fatigue strengths than mechanically finished parts [2].

\begin{tabular}{l|c|c|c}
\hline Machining technique & $\begin{array}{c}\text { Maximum rate of } \\
\text { metal removal }\end{array}$ & Attainable & $\begin{array}{c}\text { At maximum rate of } \\
\text { metal removal }\end{array}$ \\
\cline { 3 - 4 } & $\mathrm{m}^{3} \times 10^{-6} / \mathrm{min}$ & $\mu \mathrm{m}$ & $\mu \mathrm{m}$ \\
\cline { 3 - 4 } & 1640 & 2.54 & 127 \\
\hline Turning & 819 & 2.54 & 127 \\
Grinding & 4.92 & 12.7 & 127 \\
EDM & 16.4 & 50.8 & 127 \\
ECM & 0.00819 & 5.08 & 25.4 \\
EBM & 0.00492 & 12.7 & 127 \\
Ruby laser & & &
\end{tabular}

Table 1.2: Comparison of metal removal rates and accuracy for varying conventional and non-convention machining techniques [2].

Table 1.2 gives a comparison of different conventional and non-conventional machining techniques. The comparison of the accuracy and tolerance measurements are quoted from literature published in 1968, current accuracies attainable with Electrochemical Micromachining are of the order of hundreds of nanometres, however, Table 1.2 can still be used for comparison of machining techniques. The accuracy, or tolerances, which can be 
machined with the use of ECM compares favourably to other available machining techniques (Table 1.2), the accuracy in this instance is the \pm error in machining a given dimension. The definitive limits of dimensional quality are defined by the precision of the machines and tooling and the electrolyte selection. While the metal removal rate of ECM compares unfavourably to the conventional methods of Turning and Grinding, it has the highest rate of metal removal of the non-conventional techniques listed. The accuracy of EBM outstrips that of the other non-conventional techniques however ECM accuracy compares favourably to EDM and LBM at maximum metal removal rate.

\subsubsection{Flexibility}

A general purpose electrochemical machine can produce a variety of component shapes, large or small in area. ECM replicates machining produced by almost all mechanical cutting techniques: hard or tough materials, complex contours, and thin sections are all easily machinable [2].

\subsection{Disadvantages of ECM}

ECM is more challenging as a machining technique than as a finishing technique. Consequently the early success of ECM in finish machining was a disadvantage in that it obscured many problems associated with using ECM as a machining technique [2]. This is largely due to complications involving the flow geometry, tool design and obtaining chemical parameters for the process.

\subsubsection{Set-up costs}

The set-up costs for ECM are significantly greater than other non-conventional and conventional machining techniques, particularly EDM, its main competitor. 
Nowadays ECM machines are generally purpose built, making exact costs difficult to estimate, however an ECM machine generally costs twice as much as an EDM machine, although it is worth noting that an ECM machine was capable of at least 4 times the metal removal rate of an EDM machine in 1968 (Table 1.2). These measurements were taken when ECM was a fledgling machining technique and the interim years of improvement to the process has lead to the current ECM metal removal rate being more like $5-10$ times that of EDM. These figures strongly indicate that in the long term ECM may prove to be of better value than EDM [60].

\subsubsection{Environmental aspects}

Hydrogen is the main gaseous product produced during ECM, it must be removed from the machining area due to the explosion hazard it poses. Other noxious gases can also be produced, such as chlorine when machining with chloride electrolyte, which requires removal via an adequate ventilation system and disposal.

The waste electrolyte also requires disposal. It contains the solid products of machining such as metal hydroxides and oxides. The current British method of recycling the electrolyte through many machining processes means the solid products contained in the electrolyte are unclassified. This makes disposal problematic. There are also soluble products in the electrolyte to consider. Current policy is to dispose of these products down the drain. Previous work has shown these soluble products may contain the chromium VI ion $(\mathrm{Cr}(\mathrm{VI}))$ [60]. The U.S. Environmental Protection Agency (EPA) and the International Agency for Research on Cancer have classified $\mathrm{Cr}(\mathrm{VI})$ as a human carcinogen as research found it to be carcinogenic in animals. The EPA recommends a maximum exposure for inhalation of hexavalent chromium of $8.7 \times 10^{-3} \mu \mathrm{g} \mathrm{m}^{-3}$ [61], [62]. It is also known to be a respiratory tract irritant and can initiate an asthmatic response. It is a powerful skin and eye irritant and can be corrosive [63]. For these reasons the European Union issued legislation controlling management of End-of-Life Vehicles, aimed to reduce the number of heavy metals dumped in landfill sites in the form of old vehicles [64], [65]. This legislation aims to increase the amount of re-use and recovery to $85 \%$ per vehicle by $1^{\text {st }}$ January 2006 . The British Parliament issued a Standard Note detailing that in order to meet the EU regulations vehicles must be designed taking waste management considerations into account; however they argue 
that small car manufacturers should be exempt from the legislation for economic reasons [66]. This sort of legislation is set to make dumping electrolyte a thing of the past and thorough classification of ECM products will be of increasing importance.

\subsubsection{Iterative methods for tool design}

As the ECM dissolution rate is governed by migration of ions across the electrolyte, the dissolution rate is governed by tool-workpiece separation distance. Therefore, the required tool shape is often considered simply to be the inverse of the required workpiece shape [2, 67]. Variations in electrolyte conductivity along the flow path mean this is not the case [ 68 , 69]. It is often impossible to predict the exact path of the turbulent electrolyte over the long flow paths often employed in ECM [59]. Correct, reproducible electrolyte flow across the tool is fundamental to the success of the technique and is an important feature of tool design. Decisions as to whether the electrolyte should flow from side to side or outwards from portings in the tool must be made. Attention must be given to areas of the tool shape where cavitation of the electrolyte may occur. Natural features of the workpiece surface, such as ridges can be used as electrolyte supply points. Although tools with portings are simple to manufacture and operate, they leave a small ridge in the workpiece surface [59]. Flow cavitation, resulting in process failure, can be a problem when sharp changes in flow direction or channel width occur. One critical section is entry into the machining area cavitation in this area can be overcome by rounding the edge of the tool [59]. The variables discussed in section 1.10 also influence the final shape of the workpiece. The resulting complexity in tool design can be achieved by using empirical methods for determining tool corrections. Empirical methods, however, are time consuming and expensive and make the overall ECM process uneconomical. This project aims to measure the relevant ECM parameters discussed in section 1.10 to enable full computer aided design of the tool without the need for some empirical methods. This will also further electrochemical knowledge of the ECM dissolution process.

\subsection{Specialist Applications of ECM}

Due to the advantages of ECM (section 1.7) the technique has been applied successfully in the aerospace industry, where the advantages of using the process often outweigh the 
Parameterisation of Electrochemical Machining economic disadvantages. Nevertheless ECM is also finding niche applications in other industries such as the automotive, offshore and medical engineering industries.

\subsubsection{Aerospace and Automotive}

\subsubsection{Drilling}

Electrochemical drilling is useful for producing deep $(600 \mathrm{~mm})$, small $(0.1 \mathrm{~mm})$ diameters holes in hard components. A conventional drill bit of $0.1 \mathrm{~mm}$ diameter would have a relatively short length to avoid breakage and therefore would be unsuitable for this application. ECM can be used to avoid stress to the component being machined, for this reason it is an ideal technique for drilling cooling holes in turbine blades (Figure 1.4).

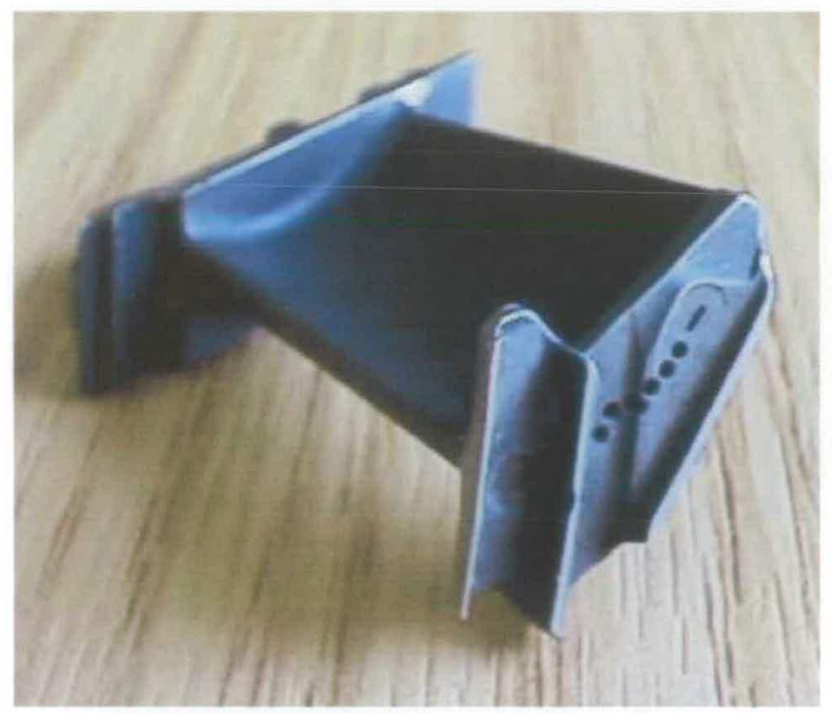

Figure 1.4: Pegasus turbine blade with axial cooling holes produced by ECM.

The drilling process is illustrated in Figure 1.5. The cathode is generally a tube with insulated sides, with electrolyte supplied down the centre to the working gap. Reverse flow tools can also be used. Overcut can be a problem during Electrochemical Drilling resulting from the sideways current flow to the end surface of the tool. This can result in a hole diameter $0.01-0.02 \mathrm{~mm}$ greater than the tool diameter [2]. Overcut is generally minimised by insulation of the tool. 


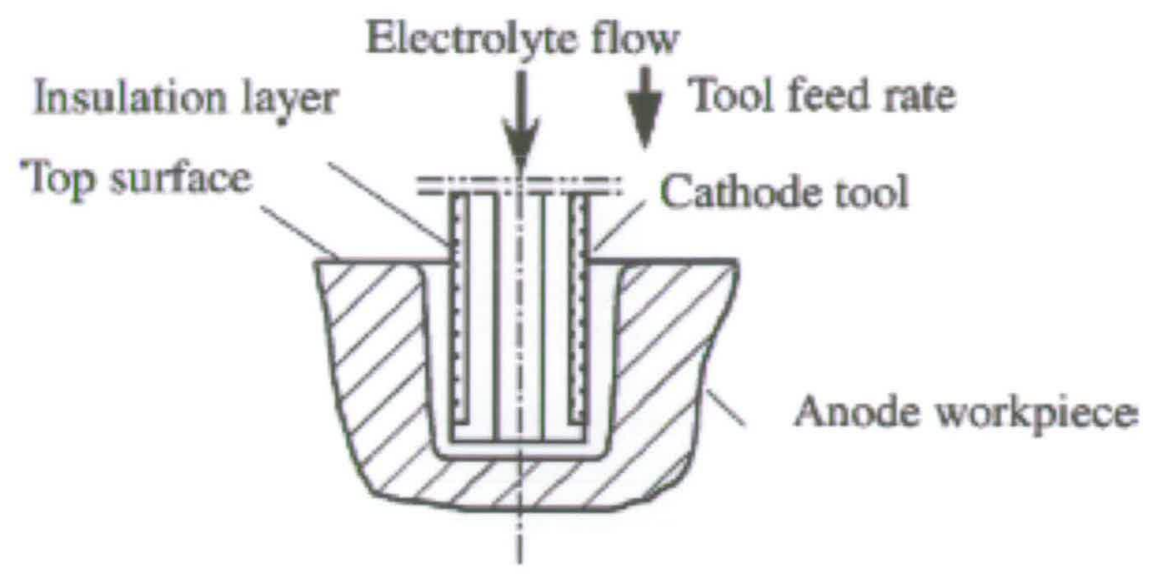

Figure 1.5: Schematic of ECM drilling showing traditional insulation of drilling tool [70].

Zhu and $\mathrm{Xu}$ developed a dual pole tool to increase the accuracy of Electrochemical Drilling, by reducing the overcut observed [70]. The dual pole tool was created with a metallic brush outside the insulating coating of the cathode. This brush was connected to the anode, substantially reducing the electric field at the side gap between the tool and the workpiece (Figure 1.6).

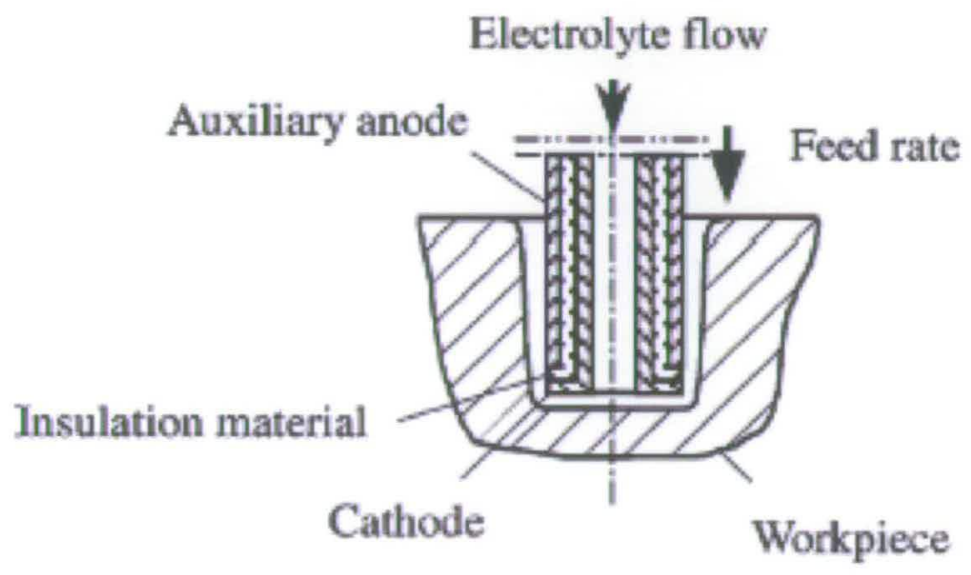

Figure 1.6: Diagram of dual pole tool for Electrochemical Drilling [70].

This research shows that the standard deviation of the machined holes with the dual pole tool is approximately half that of the insulated tool at hole depths ranging from $0-20 \mathrm{~mm}$. The lower standard deviation measured indicates increased repetitive machining accuracy. The dual pole tool showed a significant decrease in overcut for hole depths up to $16 \mathrm{~mm}$. 
ECM can be viewed as a cavity sinking process, with other applications being special cases of this technique. The main application of Electrochemical Cavity Sinking, and indeed of ECM, is for shaping blades and other components for gas-turbine engines (Figure 1.7).

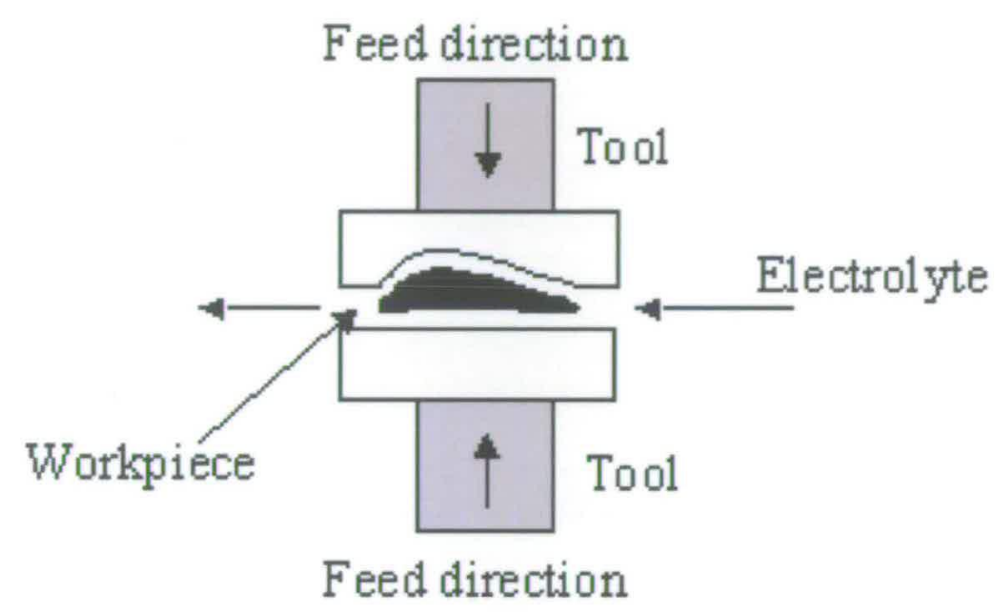

Figure 1.7: ECM cavity sinking of a turbine blade [60].

Due to the reduction of fatigue life (discussed in section 1.7.2), blades produced using ECM are typically finished using abrasive polishing [2].

The automotive industry is subject to the same quest for harder materials and the need to machine intricate and complex shapes as the aerospace industry. Electrochemical shaping and cavity sinking can be used with the same benefits and advantages in this industry to produce complex engine parts.

\subsubsection{Thinning of trailing edges}

A convenient method of producing certain aerofoil blades is to fold a sheet of metal into the desired form and seam-weld the trailing edges. Following this process the trailing edges require thinning down and radiusing (rounding at the trailing edge) [71]. ECM can be used in this application to avoid imparting stress to the aerofoil section; however limiting the machining to the section requiring thinning is problematic. Areas not requiring thinning are 
Parameterisation of Electrochemical Machining exposed to low current densities and undergo an etching effect resulting in poor surface finish. The tool can be designed to reduce this effect but not eliminate it [2].

\subsubsection{Offshore Defect Sampling}

ECM can be advantageous in defect sampling. Defect sampling is carried out on welded structures when a defect is detected. There are two benefits to defect sampling: firstly to prevent further growth of the defect, and secondly to acquire a sample of the defect for analysis, possibly providing an estimate of the residual life of a structure.

Offshore structures are continuously exposed to the cumulative effects of both cyclic and random loading due to surface waves and deep sea currents. Consequently offshore structures are subject to stringent inspection codes and practices [72]. Crack growth data can be collected using non-destructive testing (NDT) techniques such as ultrasonics, eddy current measurements or magnetic particle inspection [67]. In general NDT has been successful in determining the defect position and size. The sampling of defects is regularly carried out onshore by grinding two converging slots that intersect at a point beneath the defect. These grinding operations impart stress to the structure and often damage or destroy the defect sample rendering subsequent analysis difficult. Grinding is also limited by the accessibility of the defect. For example subsea defect removal is not viable using a grinding technique [73].

A subsea ECM weld defect sampling system has been developed by Edinburgh University combining a NDT method to detect the defect and ECM for removal, retaining the defect sample for any further analysis required [67], [74]. The ECM sampling tool consisted of a section of "U" shaped tubing (Figure 1.8, right). The tube was traversed into the workpiece below and around the defect, removing a "scoop" sample containing the weld defect (Figure 1.8 , left). 
Parameterisation of Electrochemical Machining
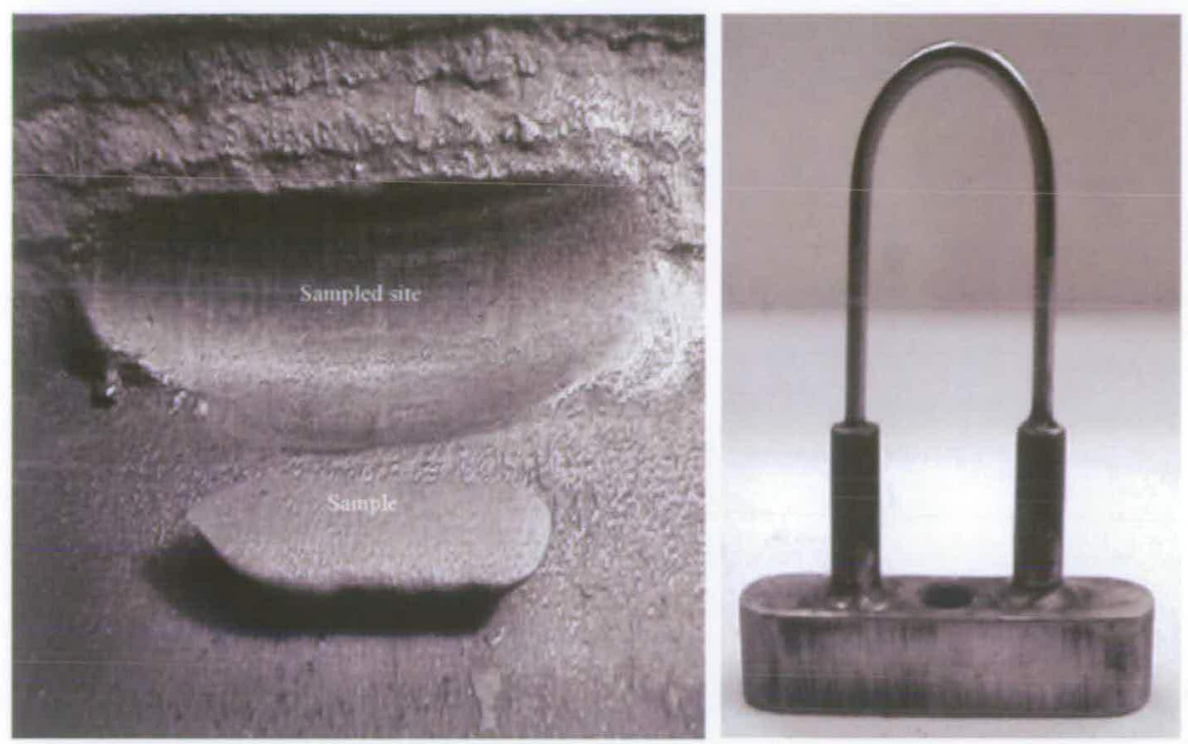

Figure 1.8: Sampled site in welded section and removed sample (left) and "U-shaped" ECM sampling tool (right).

Results have shown this technique to be successful in the removal of defects from complex component shapes and in the removal of weld defects from structures situated underwater $[67,74]$

\subsubsection{Medical Engineering Industries}

Titanium alloys offer an excellent combination of biocompatibility, corrosion resistance and mechanical properties making them ideal for surgical implants including fracture fixation implants. However, the very mechanical properties which make them attractive for this application: stress corrosion cracking resistance, fatigue strength and wear characteristics, impose the standard problems with shaping the implants. Also favouring ECM as a machining technique is the possibility of complex-shaped implants with curved surfaces that are difficult to machine using other methods [75]. 


\subsection{Parameterisation of ECM}

The fundamental equations describing the inter dependence of the main ECM parameters are:

$$
I=\frac{\kappa\left(V-V_{0}\right) A}{z}=\frac{n F A \rho \frac{d y}{d t}}{M}
$$

\section{Equation 1.1}

With the electrode feed rate (f) governing the workpiece dissolution rate (dy/dt). The relationship shown in Equation 1.1 contains a number of fixed physical values relating to the ECM system, namely the electrode area (A), Faradays constant (F), the workpiece molecular mass (M) and the workpiece density ( $\rho$ ). The inter dependency of the ECM characteristic parameters of dissolution current (I), the electrode feed rate (f), the applied voltage (V) and the interelectrode gap (z) are therefore governed by the basic ECM parameters of the dissolution valency $(n)$, the overpotential $\left(V_{0}\right)$ and the electrolyte conductivity $(\kappa)$. ECM is generally characterised and modelled assuming ideal conditions, namely the basic parameters above remaining constant throughout machining. During machining changes can occur in these parameters with time and flow path position, leading to variations in the characteristic ECM parameters. This limits the current accuracy of modelling the ECM process. The level of knowledge of the changes undergone by these parameters during machining is limited, as is the understanding of the relationship between the parameters [60].

\subsubsection{Electrolyte Conductivity}

The electrolyte conductivity is given by Equation 1.2, taking each type of ion, $\mathrm{j}$, into account 


$$
\kappa=\sum_{j} c_{j} u_{j} b_{j} F
$$

\section{Equation 1.2}

Where $c$ is the concentration of each ion, $j, u$ is its mobility, $b$ is its charge number and $F$ is Faradays constant. One of the factors contributing to $\kappa$ changes is the production of soluble and insoluble products during machining and the consumption of electrolyte ions. $\kappa$ may increase or decrease due to whether the replacement ions are more or less mobile or more or less charged and will decrease according to electrolyte ion consumption (Equation 1.2).

An increase in electrolyte temperature will lead to increased electrolyte conductivity, due to increase ion mobility at higher temperatures. The conductivity of most electrolytes often varies linearly with temperature according to Equation 1.3

$$
\kappa=\kappa_{0}\left[1+\beta\left(T-T_{0}\right)\right]
$$

\section{Equation 1.3}

Where $\beta$ is the temperature coefficient of the conductivity and $\kappa_{0}$ is the initial conductivity at temperature $T_{0}[2]$. The temperature along the interelectrode gap, in the direction of the flow, can be expected to increase linearly due to Joule heating during the process [76, 77]. A fast electrolyte flow rate will disperse the power produced across a larger volume of electrolyte more effectively than a slow flow rate, making temperature effects more prominent at slow electrolyte flow velocities [78]. While Joule heating is often taken into account for modelling purposes [77], any heat produced due to viscous friction of the electrolyte is generally ignored, as is heat lost through conduction by the electrode materials [79]. A study carried out by Jain et al. studied the comparison between the experimental effect of the tool material on the temperature distribution during ECM. The study concluded that the cathode material had a significant effect on the parameters when compared to computed values of temperature distribution and other machining parameters, however, there were some discrepancies between the experimental and computational data for the measurements of temperature distribution along the electrolyte flow path [79]. Temperature effects have been studied in this work (Chapter 8, Chapter 9 and Chapter 10); however the 
cathode material remained the same throughout, precluding cathode material variation from these studies.

Hydrogen gas is produced at the tool through Reaction 1.1 and can be present in the electrolyte as hydrogen bubbles or, at elevated pressures, as dissolved hydrogen.

$$
2 \mathrm{H}_{2} \mathrm{O}_{(l)}+2 e_{(s)} \rightarrow \mathrm{H}_{2(g)}+2 \mathrm{OH}_{(a q)}^{-}
$$

\section{Reaction 1.1}

Dissolved hydrogen has no charge and hence $\kappa$ would be expected to be essentially unaffected, however, hydrogen bubbles will result in voids in the electrolyte in the interelectrode gap and lead to a decrease in the overall value of $\kappa$. Hopenfield and Cole attempted to model the production of hydrogen gas during ECM by assuming a linear $\mathrm{H}_{2}$ boundary layer, $\delta$, increasing along the flow path length of the interelectrode gap (Figure 1.9) $[80]$.

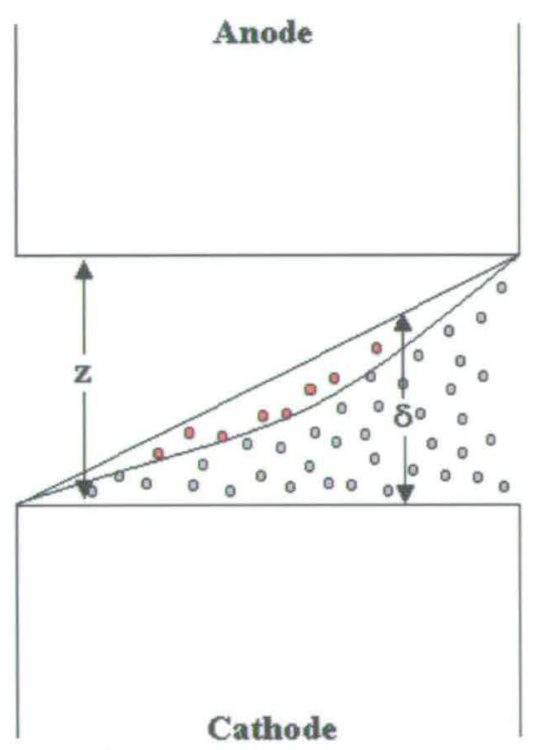

Figure 1.9: Hopenfield and Cole model of hydrogen gas production during ECM (grey and red bubbles). $\mathrm{z}$ is the interelectrode gap and $\delta$ is the progression of the hydrogen gas front into the interelectrode gap. Also shown is the model proposed by Pramanik (grey bubbles only) 
The linear release of hydrogen along the length of the interelectrode gap can also be distorted by the pressure in the gap. At the inlet and the outlet passages the pressure is lower than in the centre of the gap meaning a more realistic hydrogen gas production profile is shown by the grey bubbles in Figure 1.9. An experimental study by Pramanik et al. [81] reported on pressure variations within the interelectrode gap for planar parallel electrodes of dimensions $100 \mathrm{~mm}$ long by $40 \mathrm{~mm}$ width and for gap values in the range of $0.1 \mathrm{~mm}$ to $0.75 \mathrm{~mm}$ and electrolyte flow volumes in the range of $12 \mathrm{~L} \mathrm{~min}^{-1}$ to $35 \mathrm{~L} \mathrm{~min}^{-1}$. The results of this study showed a general trend of an increase in pressure towards the centre of the flow path (Figure 1.9) for the full range of gaps and flow volumes studied. Pramaniks experimental set-up, however, included electrolyte introduction into the cell via an aperture situated at the centre of the electrolyte flow path. This system induced a two directional flow, possibly producing atypical flow effects which may have effected the pressure measurements. Back pressure can be applied to the cell to re-establish a linear hydrogen gas production and simplify modelling of the procedure, or to force $\mathrm{H}_{2}$ to dissolve and hence produce no effects on $\kappa$.

To minimise $\kappa$ changes in this work, high flow rates are used and any taper in the interelectrode gap present after machining was analysed to assess its significance (section 4.6.2).

\subsubsection{Overpotential}

$\mathrm{V}_{0}$ is generally assumed to be invariant during ECM and consequently variations are rarely accounted for in modelling $[82,83] . \mathrm{V}_{0}$ is the potential required to drive the reactions at the electrodes, which is not available to drive ion migration in the electrolyte. The reaction at the tool is given by Reaction 1.1, the reaction at the workpiece is Reaction 1.2.

$$
M_{(s)} \rightarrow M_{(a q)}^{n+}+n \bar{e}_{(s)}
$$

\section{Reaction 1.2}


The assumption of a constant $\mathrm{V}_{0}$ infers little change in $\mathrm{V}_{0}$, c.f. $\left(\mathrm{V}-\mathrm{V}_{0}\right)$ (Equation 1.1), when $\mathrm{V}$ is changed. Consequently measurement of changes in $\mathrm{V}_{0}$ can be used to probe changes in surface electrochemical reactions.

\subsubsection{Current Efficiency and Valency}

The production of current in the ECM system through any process other than anodic dissolution of the workpiece alloy constitutes an inefficiency in the system. These processes include gas production at the anode and reactions between the ECM products in the electrolyte allowing further redox reactions to occur following the initial dissolution. Typically, current efficiencies of 75-90\% are common for ECM [2]. The observed workpiece dissolution valency is given by Equation 1.4.

$$
n=\frac{n_{\text {true }}}{e}
$$

\section{Equation 1.4}

Where the observed valency ( $n$ ) is given by the true valency $\left(n_{\text {true }}\right)$ divided by the efficiency of the process (e). Clearly this causes some confusion with the measurement of the valency and the efficiency whereby an increase in the observed valency could result from an actual increase in workpiece dissolution valency or a decrease in efficiency of the process. Two methods have been used practically. Firstly, a theoretical $\mathrm{n}$ can be applied to a system and values derived for experimental $\mathrm{n}$ values. Secondly, $100 \%$ efficiency $(\mathrm{e}=1)$ can be assumed by choosing appropriate ECM conditions (typically fast electrolyte flow rate and relatively low I) in which case $n=n_{\text {true }}$.

The literature presents many reports of current efficiency dependencies. Landolt showed that when machining Nickel in $1 \mathrm{M} \mathrm{NaClO}_{3}$, the current efficiency falls rapidly from close to $100 \%$ for current densities of $30 \mathrm{~A} \mathrm{~cm}^{-2}$ or more, to below $20 \%$ as the current density drops below $10 \mathrm{~A} \mathrm{~cm}^{-2}$ [84]. It is re-assuring that this work also produces evidence for current efficiency drops at current densities below $10 \mathrm{~A} \mathrm{~cm}^{-2}$, observed in this work (section 8.5), however, the study reports a constant metal dissolution efficiency in $\mathrm{NaCl}$, contradictory to the work in this thesis (section 8.5). 
Datta and Landolt reported current efficiency dependencies on flow velocity during the dissolution of $\mathrm{Fe}$ in $\mathrm{NaNO}_{3}$ electrolyte. For low flow velocities, in the region of $1 \mathrm{~ms}^{-1}$, current efficiencies of $70 \%$ were observed, whereas efficiencies close to $100 \%$ were observed at flow velocities higher than $10 \mathrm{~ms}^{-1}[85]$.

A study carried out be Béjar et al. investigated the effects of flow velocity, current density, electrolyte concentration and temperature on dissolution efficiency when machining a HSS anode in $\mathrm{NaNO}_{3}$ [76]. The current efficiency was observed to decrease with decreasing concentration of electrolyte. An electrolyte temperature of $20{ }^{\circ} \mathrm{C}$ produced a high stable current efficiency (of the order of $70 \%$ ) over a large range of current densities while the current efficiency was observed to increase with current density for temperatures of $35{ }^{\circ} \mathrm{C}$ and $50{ }^{\circ} \mathrm{C}$. It is interesting to note that for this study, Béjar et al. assumed a constant dissolution valency of $\mathrm{n}=3$, the measured efficiency of $\mathrm{e} \approx 2 / 3$ suggests another possible explanation for the results is a dissolution valency of $\mathrm{n}=2$ and an efficiency of $\mathrm{e}=1$.

\subsubsection{Interelectrode Gap Measurement.}

In most cases presented in the literature, methods of measurement of the interelectrode gap required interruption or termination of the machining process. Hopenfield and Cole used a travelling microscope to optically observe the interelectrode cross-section through a glass plate [86]. The maximum cell inlet pressure measured in the micro system used for this study was $34.8 \mathrm{psi}$, which corresponds to approximately 2.4 atmospheres. The higher pressures established during ECM studies pertinent to industry, approximately 8 atmospheres for this work, would necessitate a significantly thicker glass plate in order for this technique to be a useful gap measurement technique, this may lead to possible problems with transmission of the light required for optical techniques. Another technique involved a mechanical probe introduced to the interelectrode gap through a hole in the tool surface to make contact with the workpiece producing a manual gap measurement. This process was used in a study by Bejar et al. to produce gap-time data at pre-determined time intervals after interruption of the machining process [76]. 


\subsection{Stainless Steels}

Stainless steels are a family of iron-based alloys containing a minimum of $10.5 \% \mathrm{wt}$ chromium. This percentage corresponds to the minimum generally required to obtain satisfactory resistance to atmospheric corrosion or spontaneous rusting [87]. Generally, metals, including alloys, can be characterised with band theory as having highly delocalised electrons with delocalisation extending throughout the solid [88]. These electrons form a delocalised band with a maximum energy given by the Fermi Energy (Figure 1.10). To form a chemical bond between a metal and an oxygen molecule, the basic reaction required in the formation of a surface oxide film, electrons are transferred from the delocalised band in the metal to the oxygen molecule. The electrons transferred are the highest energy electrons in the delocalised band, i.e. those with an energy corresponding to the Fermi Energy. For favourable electron transfer the Fermi Energy of the metal must be greater than the energy of the Lowest Unoccupied Molecular Orbital (LUMO) of the oxygen molecule into which the electrons are being transferred. Through the addition of elements with higher redox potentials, such as chromium, to form an alloy, it is possible to increase the energy of the Fermi Level making the electron transfer shown in Figure 1.10 more favourable and consequently promoting the formation of a surface oxide film.
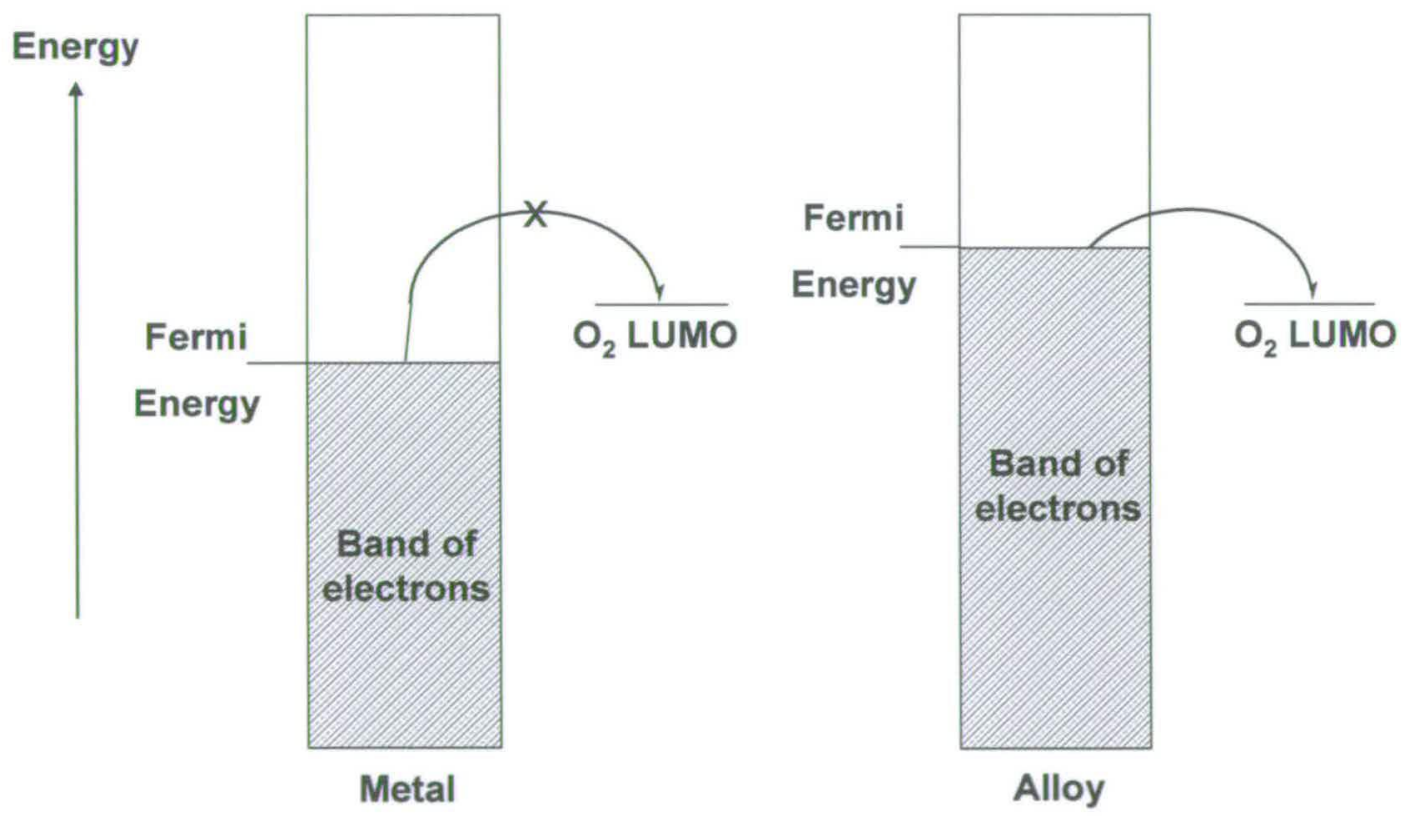

Figure 1.10: Schematic depicting the changing Fermi energy of alloys compared to a simple metal. 
The ability of the oxide layer to heal itself means the steel is corrosion resistant no matter how much of the surface is removed. This is not the case when carbon or low alloy steels are protected from corrosion by metallic coatings such as zinc or cadmium, or organic coatings such as paint [89].

Stainless steel can be selected for use compared to other materials for a number of different reasons besides resistance to corrosion. These include:

- Aesthetic qualities: it can be polished to a satin or mirror finish.

- Stainless steel is far more resistant to dry corrosion than carbon steels. Dry corrosion occurs at high temperatures and causes the steel to oxidise or scale up.

- Because there is no coating to break down there is no contamination of any liquids brought into contact with stainless steel, making it ideal for food storage.

- Many anti corrosion coatings are fire hazards or the materials themselves have low melting points.

The American Iron and Steel Institute (AISI) has a standard that is widely accepted in the United States and other countries, however it does not cover all metals and, in many instances, does not convey enough information to speak about all the attributes of some metals [90]. Consequently, two United States organisations, the American Society for Testing and Metals (ASTM) and the Society of Automotive Engineers (SAE), have developed an independent standard of metal classification [91]. The Unified Numbering System (UNS) for metals and alloys provides means of correlating many internationally used metal and alloy numbering systems. It avoids the confusion caused by having more than one numbering system. A few examples of worldwide standards are [90]:

From Canada, C.S.A. standards

From Germany, D.I.N. standards

From France, A.F.N.O.R. standards

From the UK, B.S. standards

From Italy, U.N.I. standards

From Japan, J.I.S. standards 
The UNS establishes 18 series of designations for metals and alloys each consisting of a single letter prefix followed by five digits. The single letter prefix is designated as follows [92]:

(A) Aluminium and Aluminium Alloys

(B) Copper and Copper Alloys

(D) Specified Mechanical Properties Steels

(E) Rare Earths and Similar Metals and Alloys

(F) Cast Irons

(G) AISI and SAE Carbon and Alloy Steels

(H) AISI and SAE H-Steels

(J) Cast Steels

(K) Miscellaneous Steels and Ferrous Alloys

(L) Low Melting Metals and Alloys

(M) Miscellaneous Nonferrous Metals and Alloys

(N) Nickel and Nickel Alloys

(P) Precious Metals and Alloys

(R) Reactive and Refractory Metals and Alloys

(S) Heat and Corrosion Resistant Steels (including Stainless), Valve Steels and Iron-based Super Alloys

(T) Tool Steels, Wrought and Cast

(W) Welding Filler Metals

(Z) Zinc and Zinc Alloys

The UNS system for classification of stainless steels follows the AISI standard. By prefixing the AISI number with $\mathrm{S}$ and adding an extra two digits it becomes a UNS number, e.g. AISI 304 stainless steel is converted to S30400 UNS. The key to the AISI stainless steel numbering system is [90]:

Austenitic Stainless Steels (section 1.11.1)

- The first digit is " 3 ", as in $3 \mathrm{xx}$

- The second and third numbers do not correlate to any particular chemical combination or process. However, in general, chromium content increases with the last two-digit number. 
Martensitic and Ferritic Stainless Steels (section 1.11.1)

- The first digit is " 4 ", as in $4 \mathrm{xx}$

- The second and third numbers do not correlate to any particular chemical combination or process. However, in general, chromium content increases with the last two-digit number.

The most everyday application of stainless steel is in cutlery [93]. Cheap cutlery is made from grades 409 and 430 while more expensive cutlery from specially produced 410 and 420 for knives and 304 for spoons and forks. The different grades are used as 410/420 can be hardened and tempered to give the knife blades a sharp edge whereas 304 is more ductile and can be moulded into the shaped required for the forks and spoons [94].

Large amounts of stainless steel are used in food production and storage. The most commonly used grades are 304 and 316 [95]. Typical uses are in dairies, milk storage, ham curing, frozen and salted fish storage. The lack of contamination from stainless steel containers can be a disadvantage in winemaking. Newer winemaking techniques use large vats and stainless steel storage containers where traditional methods use barrels of oak. The new system gives greater economies of scale; however, in conventional winemaking the acid of the wine dissolves some of the wood to give an oak body taste to the wine. Using stainless steel vats the winemaking companies must deliberately add oak chips to give the flavour in order to satisfy traditional wine drinkers [94].

The pumping and containment of oils, gases and acids creates a large market for stainless steels. Special grades of stainless steel have been developed with greater corrosion resistance and are used in a wide variety of industries: de-salination plants, sewage plants, offshore oil rigs, harbour supports and ships propellers [96].

Architecture is a growing market with many modern buildings using stainless steel for cladding [97]. Clean melted stainless steel is often used for medical implants and artificial hips. A great deal of medical equipment such as orthopaedic beds, cabinets and examination machines are made from stainless steel because of its hygienic and easy to clean properties [98]. Also cars are increasingly made from stainless steel, primarily the exhaust systems and catalytic converters [99]. 


\subsubsection{Classification of Stainless Steels}

Although stainless steels depend on the presence of chromium, other alloying elements are often added to enhance their properties. The categorisation of stainless steels is unusual among metals as it is based on the nature of their microstructure, denoting the arrangement of the atoms which make up the grains of the steel [100]: the predominant classes are ferritic, martensitic and austenitic.

Below its melting point $\left(1536{ }^{\circ} \mathrm{C}\right)$ iron has three allotropes, see Table 1.3.

\begin{tabular}{ccc}
\hline Temperature range $\left({ }^{\circ} \mathrm{C}\right)$ & Symbol & Crystal structure \\
\hline $1536-1392$ & $\delta-\mathrm{Fe}$ & Body centred cubic (bcc) \\
$1392-911$ & $\gamma-\mathrm{Fe}$ & Face centred cubic (fcc) \\
911 and below & $\alpha-\mathrm{Fe}$ & Body centred cubic (bcc)
\end{tabular}

Table 1.3: Different allotropes of iron below the melting point [101].

These allotropes of iron are the basis for the classification of stainless steels. Figure 1.11 shows the phase diagram of iron-chromium alloys.

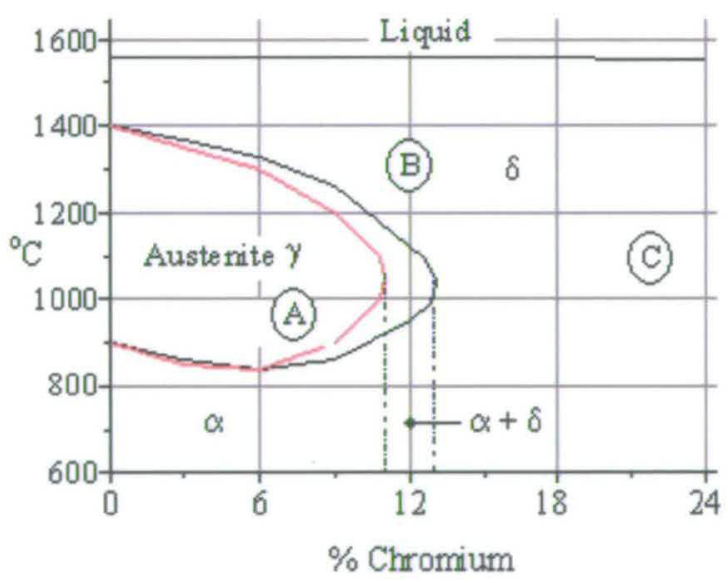

Figure 1.11: Phase diagram of iron-chromium alloys: A, martensitic alloys; B, semi-ferritic alloys; C, ferritic alloys [102].

In the solid state area of Figure 1.11 three regions are evident: 
- An area limited by a loop (red) known as the " $\gamma$ - loop", inside this loop the austenite phase is stable, also known as the $\gamma$ - phase, with a fcc crystalline structure.

- An area located beyond a second loop (black), where the bcc ferrite phase ( $\alpha$ - or $\delta$ phase) is stable from room temperature up to the melting point of the alloy. By analogy with $\mathrm{Fe}$, ferrite produced on solidification is referred to as $\delta$ - ferrite while ferrite produced from the transformation of austenite on cooling is termed $\alpha$ - ferrite.

- An area between the two loops, where both ferritic and austenitic phases coexist, their respective portions being dependent on the usual laws of phase diagrams.

Using Figure 1.11 two classes of stainless steel can be defined. The first is Martensitic stainless steel, which has properties similar to iron-chromium alloys containing less than $12 \%$ chromium. The carbon in martensitic steels is the element responsible for the considerable variation in mechanical characteristics of the steels. Martensitic steels solidify as $\delta$ - ferrite, but are completely transformed to $\gamma$ - ferrite, austenite, during cooling. When cooling is slow the austenite decomposes into $\alpha$ - ferrite and carbides, however when rapid cooling is used this does not occur and the austenite transforms to martensite. The second is Ferritic stainless steel, which behaves like iron-chromium alloys with adequate chromium to remain beyond the external " $\gamma$ - loop". They are essentially ferritic over the whole temperature range corresponding to the solid state and consequently do not harden on quenching like martensitic steels [102].

\subsubsection{Austenitic Stainless Steels}

Austenitic steels usually contain between 18 and $30 \mathrm{wt} \%$ chromium, 8 to $20 \mathrm{wt} \%$ nickel and between 0.03 and $0.1 \mathrm{wt} \%$ carbon [103]. Austenite is usually formed with a mixture of carbon and iron, when it is only stable above $720{ }^{\circ} \mathrm{C}$. However the nickel and chromium content of stainless steels are sufficient to retain the austenitic phase at room temperature [103], as the nickel and chromium widen the temperature range over which the austenite exists $(\gamma$ - loop in Figure 1.11), increasing its stability at low temperatures. The austenite phase has an fcc microstructure like that of $\gamma$ - iron (Table 1.3). However, the majority of austenitic stainless steels display $\gamma$ - and $\delta$ - ferrite phases at temperatures greater than 1000 ${ }^{\circ} \mathrm{C}$, whilst at $650{ }^{\circ} \mathrm{C}$ the composition of the steels has been shown to consist of $\gamma+\alpha$ which transforms to $\gamma+\sigma$ on aging [101]. The $\sigma-$ phase is specific to an $\mathrm{Fe}-\mathrm{Cr}$ alloy system, it displays a tetragonal microstructure based on the $\mathrm{FeCr}$ compound [101]. The austenite 
structure increases the hardness of the stainless steel. In practice, austenitic stainless steels generally contain more than $15 \%$ chromium and a nickel content determined by the necessity to maintain a stable austenitic structure over the temperature range from $1100{ }^{\circ} \mathrm{C}$ to room temperature without the formation of martensite.

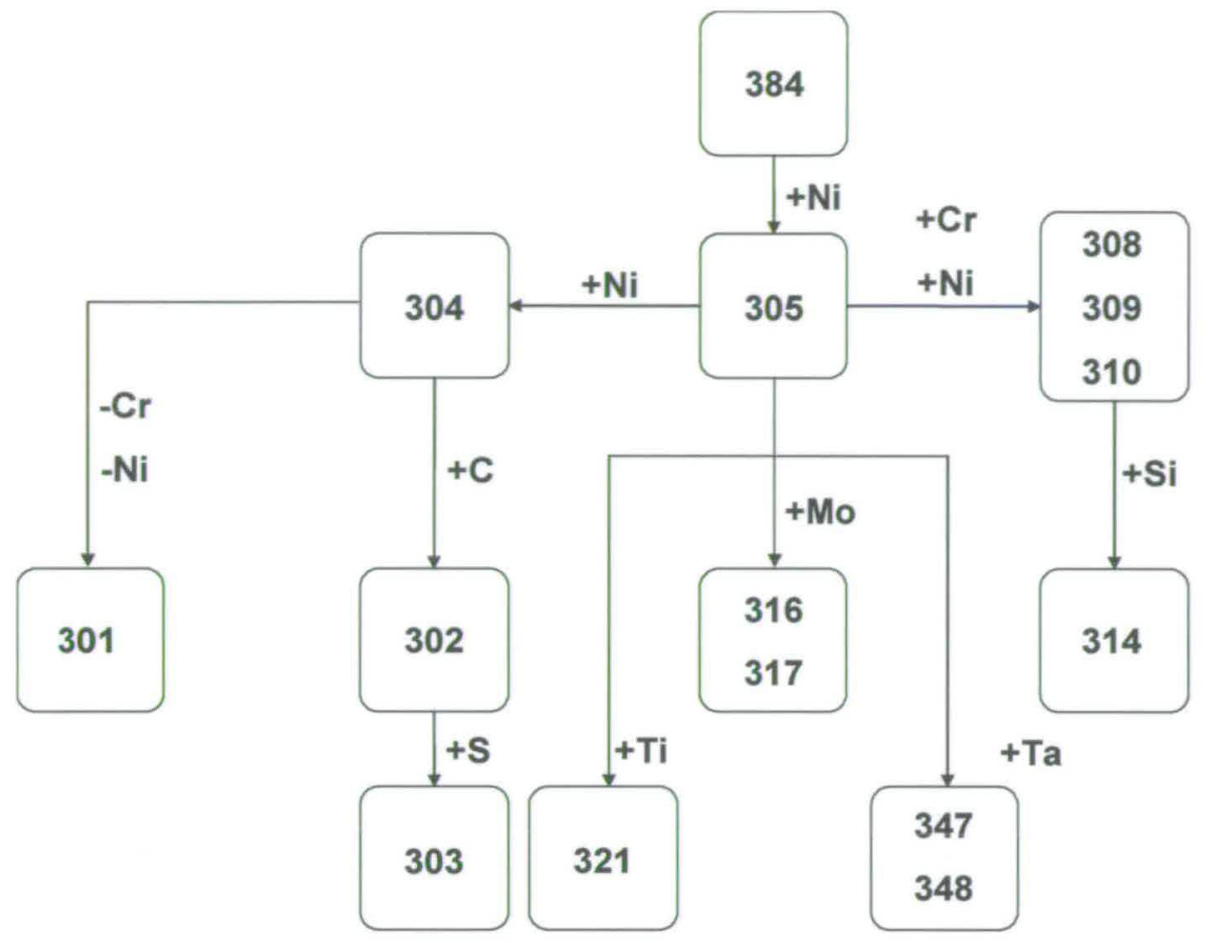

Figure 1.12: Schematic of Austenitic Stainless Steel grades, also known as the 300 series [87].

\subsubsection{Ferritic Stainless Steels}

Ferritic stainless steels contain approximately half the 400 series. They contain from $10.5-$ $30 \mathrm{wt} \%$ chromium. Ferritic stainless steels have a bcc crystal structure and are known to be ferritic at all temperatures below their melting points. The ferritic stainless steels have higher yield strengths and lower ductilities than austenitic stainless steels. 


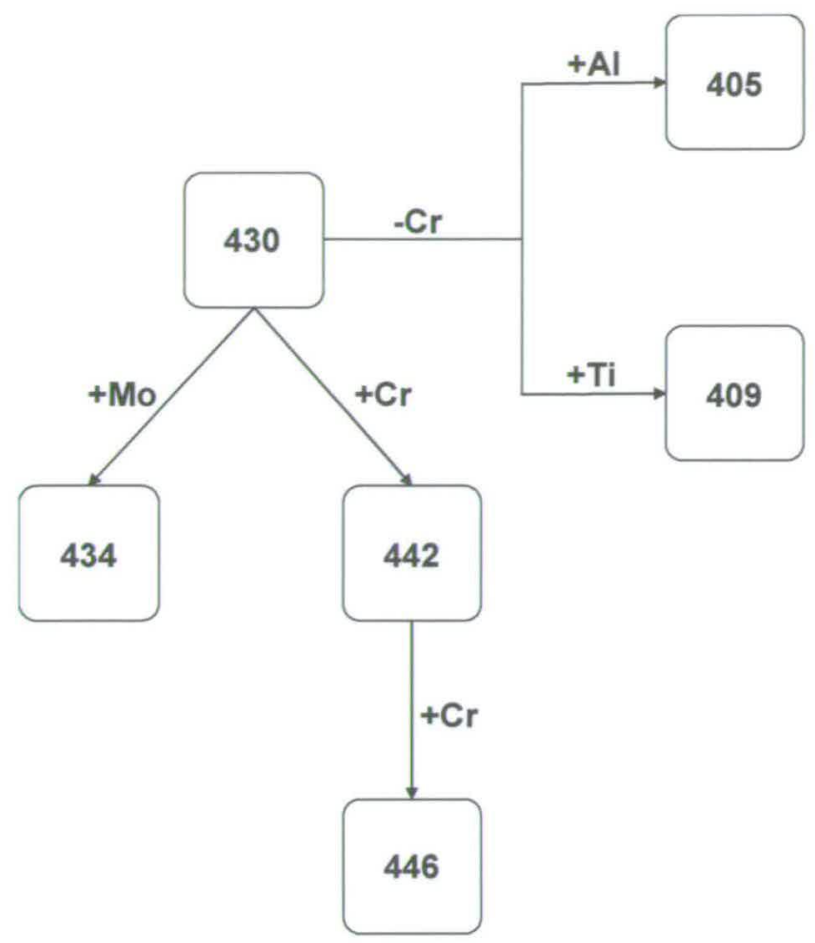

Figure 1.13: Schematic of Ferritic Stainless Steel grades [87].

\subsubsection{Martensitic Stainless Steels}

Martensitic steels are essentially alloys of iron, chromium and carbon that posses a bcc or body centred tetragonal crystal structure in the hardened condition. They are ferromagnetic and hardenable by heat treatments. The chromium content of these steels generally ranges from 11.5 to $18 \mathrm{wt} \%$ and their carbon content can be as high as $1.2 \mathrm{wt} \%$. The chromium and carbon amounts are chosen to ensure a martensitic structure after hardening. The most commonly used martensitic stainless steel is SS410 [104]. Molybdenum and nickel can be added to improve mechanical or corrosion properties. 
Parameterisation of Electrochemical Machining

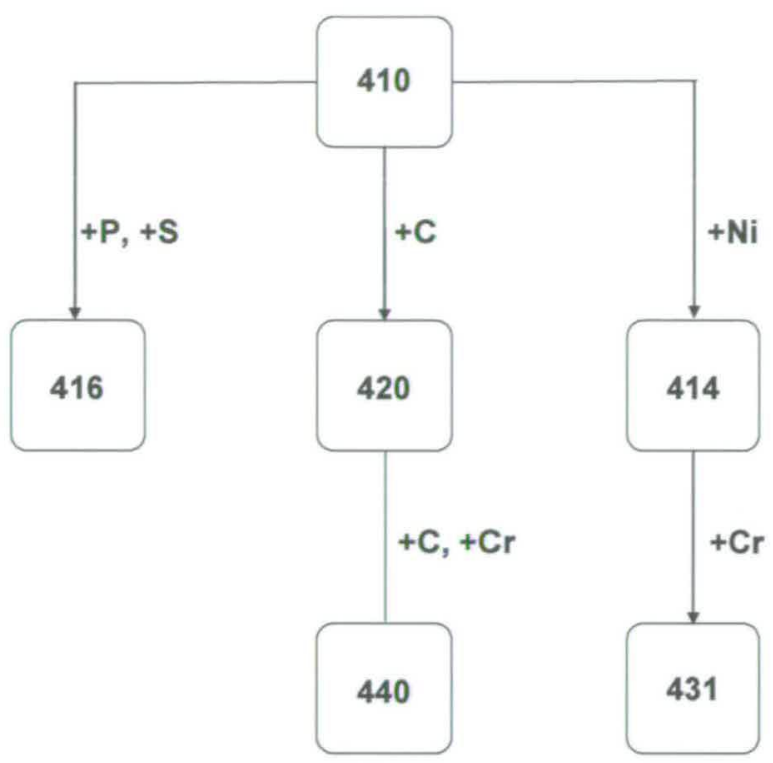

Figure 1.14: Schematic of Martensitic Stainless Steel grades [87].

\subsubsection{Function of Alloying Elements}

The corrosion protection of Stainless Steels is dependent on the $\mathrm{Cr}$ content of the steel. Other alloying elements are considered to enhance the properties of stainless steel in the following ways [105]:

\subsubsection{Nickel.}

$\mathrm{Ni}$ is useful in the re-passivation of the protective oxide layer, re-passivation is the re-growth of the protective oxide film after it has sustained damage of some kind, for example mechanical damage or electrochemical damage. Ni additions are especially useful for repassivating in reducing environments. Using the band theory of metals and alloys described in section 1.11.1, Ni additions can be viewed as further fine tuning of the energy of the Fermi level to that of the oxygen molecule LUMO resulting in more favourable surface oxide formation. Ni is also used to stabilise the austenitic form of iron and so is useful in the production of austenitic stainless steels. 
Mo in moderate quantities combined with chromium is used to stabilise the passive oxide film in the presence of chlorides, which are known to attack the oxide films. Chlorides are known to cause pitting corrosion of the passive oxide layer.

\subsubsection{Manganese.}

$\mathrm{Mn}$ is also an austenite stabiliser. In a stainless steel with lower amounts of $\mathrm{Ni}, \mathrm{Mn}$ will perform the same job as Ni. It is unknown what effect $\mathrm{Mn}$ has on the corrosion resistance of stainless steel.

\subsubsection{Copper.}

$\mathrm{Cu}$ can be added to stainless steels to increase hardening.

\subsubsection{Carbon.}

$\mathrm{C}$ is another element used to increase the strength of stainless steels.

\subsubsection{Sulfur.}

$\mathrm{S}$ is added to the stainless steel to promote mechanical properties such as ductility, formability and weldability. This helps in the machining of the stainless steels.

\subsubsection{Nitrogen.}

$\mathrm{N}$ is used to stabilise the austenite structure of the stainless steel, which strengthens the steel. The addition of nitrogen makes it possible to increase the content of Mo, which in turn increases the corrosion resistance of the stainless steel in chloride environments. 


\subsection{Passive films on Iron}

The growth and breakdown of passive films on metal and alloy surfaces has been a subject of intense interest over the past 160 years. The passivity of metals and alloys in contact with reactive environments depends wholly on these films. During the 18 th century the surprising lack of reactivity observed when iron was immersed in a concentrated solution of nitric acid was remarked upon by various authors [106]. A metal is described as passive if it is only slightly, or not at all, attacked either when acting as an anode in an electric circuit, or when treated with chemical reagents. It is described as active when, under the same conditions, it is vigorously attacked. Special interest is shown when a metal, such as $\mathrm{Fe}$, can be shown to be either active or passive under these conditions [107]. The passive nature of these metals was shown to be a result of the formation of a passive oxide film on the metal surface.

Iron oxides are common compounds which are wide spread in nature. The crystal structure of the iron oxides of interest to work on passive oxide films, Magnetite $\left(\mathrm{Fe}_{3} \mathrm{O}_{4}\right)$ and Maghemite $\left(\gamma-\mathrm{Fe}_{2} \mathrm{O}_{3}\right)$ are very similar. The structures are based on the spinel structure, also sometimes called garnet structure, named after the mineral spinel $\mathrm{MgAl}_{2} \mathrm{O}_{4}$ [108]. The spinel structure has a general formula of $\mathrm{AB}_{2} \mathrm{O}_{4}$ where $\mathrm{A}$ is a divalent ion $\left(\mathrm{A}^{2+}\right)$ and $\mathrm{B}$ is a trivalent ion $\left(\mathrm{B}^{3+}\right)$. The structure is based on a cubic close packed array of oxide ions with $\mathrm{A}^{2+}$ occupying tetrahedral holes and $\mathrm{B}^{3+}$ occupying octahedral holes [109]. 


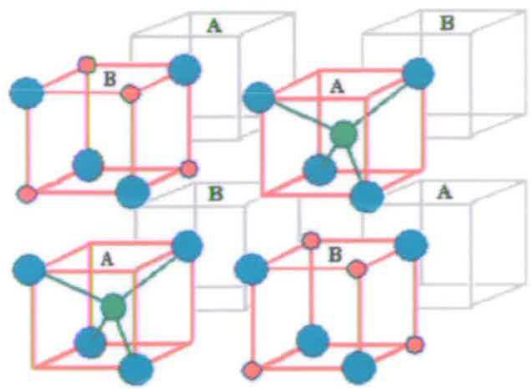

(a)

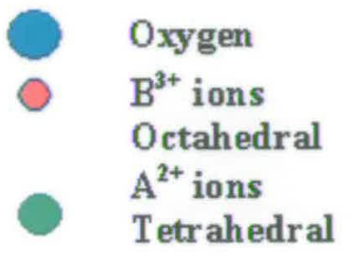

Figure 1.15: The spinel structure, the red cubes denote the A- and B-type octants (a) the arrangement of the octants in the unit cell and (b) positioning of 2 B-type octants in the spinel unit cell, also shown are the $\mathrm{A}^{2+}$ tetrahedral ion positions [adapted from 108 and 109].

Figure 1.15 shows that the $\mathrm{A}^{2+}$ ions occupy tetrahedral positions in the A-type octants and the corners and face-centres of the unit cell while the $\mathrm{B}^{3+}$ ions occupy octahedral sites comprising half the corners of the B-type octants. When compounds of the same general spinel formula $\left(\mathrm{AB}_{2} \mathrm{O}_{4}\right)$ adopt the inverse-spinel structure, half the $\mathrm{B}^{3+}$ ions now occupy tetrahedral sites while the remaining half along with the $\mathrm{A}^{2+}$ ions occupy octahedral site (which are double in number to the tetrahedrally occupied positions) [109].

Magnetite is considered to have an inverse spinel structure (Figure 1.15) with a fcc unit cell based on $32 \mathrm{O}^{2-}$ ions. It contains both divalent and trivalent iron ions with a formula $\mathrm{B}(\mathrm{AB}) \mathrm{O}_{4}$ where $\mathrm{A}$, in spinel terminology, is the divalent $\mathrm{Fe}^{2+}$ and $\mathrm{B}$ is the trivalent $\mathrm{Fe}^{3+}$ [110]. Maghemite is considered to have a very similar structure to Magnetite. It differs in the fact than all or most of the iron is present in the trivalent state. This increase charge compared to the Magnetite structure is compensated for through cation vacancies randomly distributed amongst the octahedral sites [109]. In this way the Maghemite structure can be considered to be a Magnetite structure containing vacancy defects in the octahedral sites. The conversion of Magnetite to Maghemite has been postulated to occur through oxidation of $\mathrm{Fe}^{2+}$ and migration of the iron cations to the surface, with oxygen layers added to the 
surface as the ions migrate [111]. This conversion is facile and occurs under air at around $200{ }^{\circ} \mathrm{C}$. Although these iron oxide structures are well characterised, indeed the main details of the structure of Hematite were established in 1915 [109], the history of the study of the iron oxide films formed on the surface of iron has been far from straight forward. A large number of in situ and ex situ surface techniques have been used to study these films including Auger Electron Spectroscopy (AES) [112], X-ray Photoelectron Spectroscopy (XPS) [113], Secondary Ion Mass Spectroscopy (SIMS) [114] and Surface Enhanced Raman Spectroscopy [115]. It is generally agreed that the passive oxide film on iron comprises a spinel type structure of an inner layer of $\mathrm{Fe}_{3} \mathrm{O}_{4}$ and an outer layer of either $\gamma-\mathrm{Fe}_{2} \mathrm{O}_{3}$ or a hydrated oxide $[116,117,118,119]$.

\subsection{The Passive Oxide Film on Stainless Steels}

The oxides formed on stainless steel are thicker than those formed on Fe [120,121]. Oxide films on stainless steels are generally considered to have a duplex character with an inner $\mathrm{Cr}$ rich region and an outer Fe rich region [120, 122]. The preferential dissolution and reprecipitation of $\mathrm{Fe}$ compared to $\mathrm{Cr}$ leads to the outer $\mathrm{Fe}$ rich region. $\mathrm{The} \mathrm{Cr}$ rich inner region maintains the passivity of stainless steels. Passivity refers to the lack of reaction of the underlying metal with the atmosphere. If the oxide film sustains damage, the $\mathrm{Cr}$ in the underlying metal of the damaged area will repassivate the metal.

However, contradictions in the literature results addressing the structure of the passive films of stainless steels can be explained by the effects of the type of electrolyte used for growth, potential changes applied, temperature, $\mathrm{pH}$ and the time allowed for film growth on the oxide film. All these factors will contribute to the differing structures observed [123]. There are also the differences in the techniques used. Ex-situ surface techniques require removal of the film from its growth environment and exposure to extremely harsh conditions such as vacuum, these conditions will dehydrate and affect the film structure [124]. All these differences will result in structural changes in the film before the initial stripping techniques used is even considered. The underlying metal will inevitably already have an oxide film, which must be removed before growth of the film is studied, via the individual's experimental method. If the film is not entirely removed by the stripping procedure and a monolayer of oxide is present at the start of film growth, this will possibly affect the structure and certainly affect the initial growth mechanism of the film [124]. 


\subsection{Passive films on Titanium and Inconel}

Other than stainless steels, this thesis examines the ECM properties of an alloy of Titanium (Ti), Ti 6/4, and a Ni super alloy, In718 (for alloy compositions see Table 4.1). Ti is a strong and light metal, it is not easily corroded by sea water and hence is used in propeller shafts, rigging and other parts of boats exposed to sea water. Since Ti does not react inside the human body, it is ideal for use as artificial hip replacements, pins for setting bones and for other biological implants. Also, $\mathrm{Ti}$ is used extensively in the aerospace and automotive industries (section 1.2) [125]. Crystalline titanium dioxide occurs in three polymorphs: rutile (tetragonal), anatase (tetragonal) and brukite (orthorhombic). Of the three phases, rutile is known to be the most stable and has been the subject of many experimental and theoretical studies, a lesser amount of literature is present for the other two, less stable, phases [126]. It is generally accepted that Ti metal in air is spontaneously covered by a surface oxide layer of 1.5-10 nm thickness. The oxide consists of $\mathrm{TiO}_{2}$ and is amorphous in crystal structure and morphologically homogeneous. Ti is a typical valve metal for which oxide growth involves field-assisted migration of ions through the oxide film. Furthermore, the structure of the anodic oxide film has been observed to change to a crystalline phase (e.g. rutile, anatase or brukite) above a critical oxide thickness of about $8 \mathrm{~nm}$ [127]. A Raman study of anodic oxide films of thickness greater than $10 \mathrm{~nm}$ showed the films to be primarily composed of an anatase type $\mathrm{TiO}_{2}$ [128]. In a study into the Electrochemical Micromachining properties of $\mathrm{Ti}$, the passive film thickness was seen to increase linearly from approx. $8-50 \mathrm{~nm}$ during a voltage increase of approx. $0-12$ Volts. At 12 Volts, after the initial passive region, the film was suddenly dramatically reduced in thickness to about $6 \mathrm{~nm}$. These data were recorded at current densities of the order of $0.001-1.0 \mathrm{~A} \mathrm{~cm}^{2}$, far lower than those used for the work in this thesis [58]. These films have been considered to be highly doped n-type semiconductors, with $\mathrm{Ti}^{3+}$ as the donor ions [129].

In718 is a nickel-based alloy, the further major constituents are $\mathrm{Cr}$ (approximately $21 \%$ ) and Fe (approximately 12\%). The oxide layer present on nickel-based alloys is described as having a duplex structure similar to that found on stainless steel, formed by an inner Cr-rich layer and either an outer Fe-rich layer for stainless steels, or an Ni-rich layer for $\mathrm{Ni}$ based alloys such as In718 [130]. 


\subsection{Aims of this work}

Generally previous studies have been aimed at investigation of the growth of oxide films, at very low applied voltages and currents. Where dissolution has been investigated, it has been studied at low currents, of the order of $\mu \mathrm{A} \mathrm{cm} ~^{-2}$, which produces relatively low fields. The characterisation of electrochemical dissolution current-voltage characteristics at high current densities is experimentally challenging. One of the aims of this thesis is the study of dissolution at high voltages and current densities to extend electrochemical understanding in this area. From an engineering point of view, this thesis aims to increase the parameter base for ECM in the hope of finding standard systems for the eventual prediction of ECM characteristics for differing workpiece materials. The approach detailed in this work can be widely applied to the ECM of other workpiece materials. Chapter 2 presents a theoretical background for ECM. Chapter 3 presents the theoretical development of the chronoamperometric and ultrasound analysis methods. Chapter 4 presents details of the experimental set-up and experimental conditions used during this work and Chapter 5 presents a critical comparison of the analysis used in this thesis and previous analysis methods. Chapter 6 details preliminary studies into direct ECM interelectrode gap measurements. The remaining chapters detail the results of this work with Chapters 7-12 dealing with results collected using cell 2000 (Chapter 4), Chapter 7 deals with the ECM of pure iron and Chapter 8 looks at the stainless steels Jethete and SS410; Chapters 9 and 10 deal with the ECM of In718 and Ti $6 / 4$ respectively while the overall conclusions drawn from the cell 2000 studies are discussed in Chapter 11 and the benefits of these parameterisation studies to ECM computer simulation are demonstrated in Chapter 12 . Finally Chapter 13 presents preliminary results for Ti $6 / 4$ and In718 using a longer path length cell. 


\section{Chapter 2}

Theoretical background of ECM 


\subsection{Metal Removal and Removal Rates}

The passage of current through the ECM cell results in dissolution of the workpiece at a rate given by Faraday's laws of electrolysis [130], which state:

1. The amount of chemical change produced by an electric current, that is, the amount of any substance dissolved, is proportional to the quantity of electricity passed, the charge, Q, where Q=It

2. The amount of different substances dissolved by the same quantity of electricity is proportional to $\mathrm{M} / \mathrm{n}$, the molecular mass of the substance over the dissolution valency.

These laws give rise to an equation for rate of material dissolution $(\mathrm{dm} / \mathrm{dt})$ for a given current I as Equation 2.1:

$$
\frac{d m}{d t}=\frac{I M}{n F}
$$

\section{Equation 2.1}

where $\mathrm{F}$ is Faradays constant. This can be related to the feed rate as follows:

The volume of material removed $\left(\mathrm{V}_{\mathrm{m}}\right)$ is given by Equation 2.2

$$
\frac{d V_{m}}{d t}=\frac{1}{\rho} \frac{d m}{d t}=\frac{I M}{n F \rho}
$$

\section{Equation 2.2}

This can be related to the rate at which the workpiece surface is receding from the tool (dy/dt): 


$$
\frac{d V_{m}}{d t}=A \frac{d y}{d t}
$$

\section{Equation 2.3}

where $\mathrm{A}$ is the electrode surface area. Substitution of Equation 2.2 and re-arrangement gives Equation 2.4:

$$
\frac{d y}{d t}=\frac{I M}{A n F \rho}
$$

\section{Equation 2.4}

At equilibrium machining the workpiece surface is receding from the tool, dy/dt, at the same rate as the workpiece is being fed towards the tool, the feed rate, $f$ (Figure 2.2). Consequently at equilibrium machining conditions Equation 2.5 gives the feed rate of the workpiece:

$$
\frac{d y}{d t}=f=\frac{I_{\infty} M}{A n F \rho}
$$

\section{Equation 2.5}

\subsection{ECM Electrochemistry, a two electrode system}

During the ECM process material is removed from a workpiece through anodic dissolution. To achieve this, an external electrical source is used to bias the workpiece material as the anode and the tool as the cathode. Electrolyte flows through the interelectrode gap (z), between the workpiece and the tool, at a high flow velocity (U). The workpiece material, of thickness $\mathrm{y}$, is moved towards the tool until the current across the cell is high enough to facilitate dissolution of the workpiece material. As dissolution occurs the workpiece surface recedes from the tool at a rate of dy/dt. (Figure 2.1) 


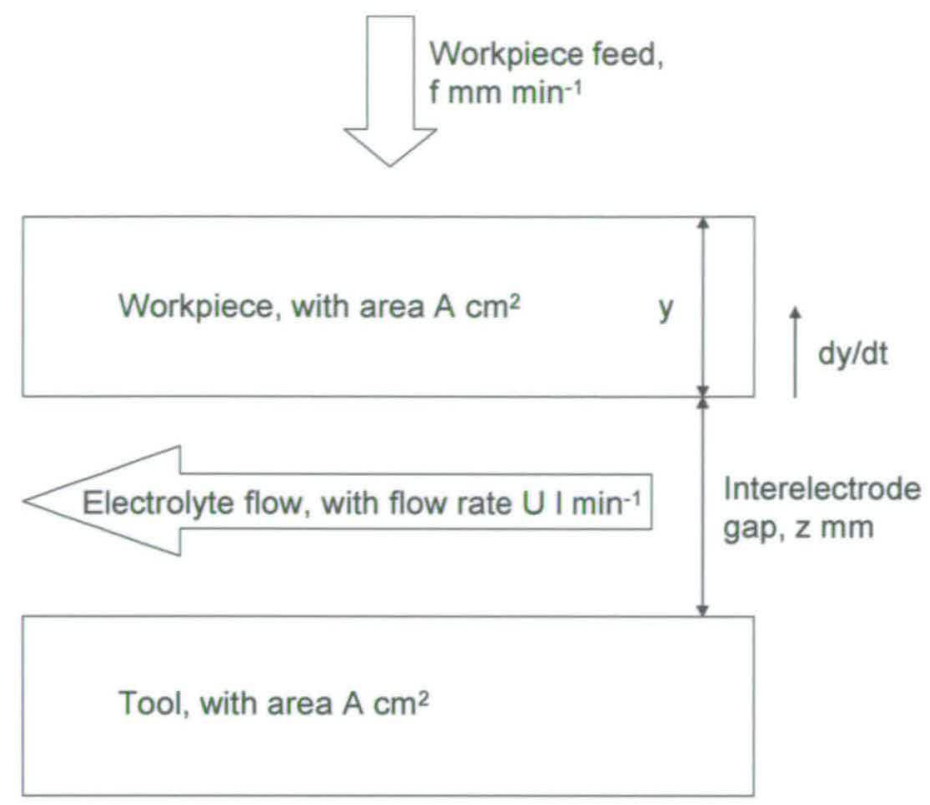

Figure 2.1: Set-up of planar ECM system

Current is governed by the migration of ions across the gap. This type of electrochemical cell is an electrolytic cell and converts electrical energy to chemical energy. This is the reverse of a galvanic cell in which a spontaneous chemical reaction can be used to produce electrical energy. As the workpiece and tool are brought closer together the field increases and hence the current across the cell increases (the rate of anodic dissolution increases). At some time, the current will be such that the rate of anodic dissolution of the workpiece is equal to the feed rate $(\mathrm{dy} / \mathrm{dt}=\mathrm{f})$ and the change in the interelectrode gap $(\delta z)$ becomes zero. At this time machining is said to have reached equilibrium. (Figure 2.2) 


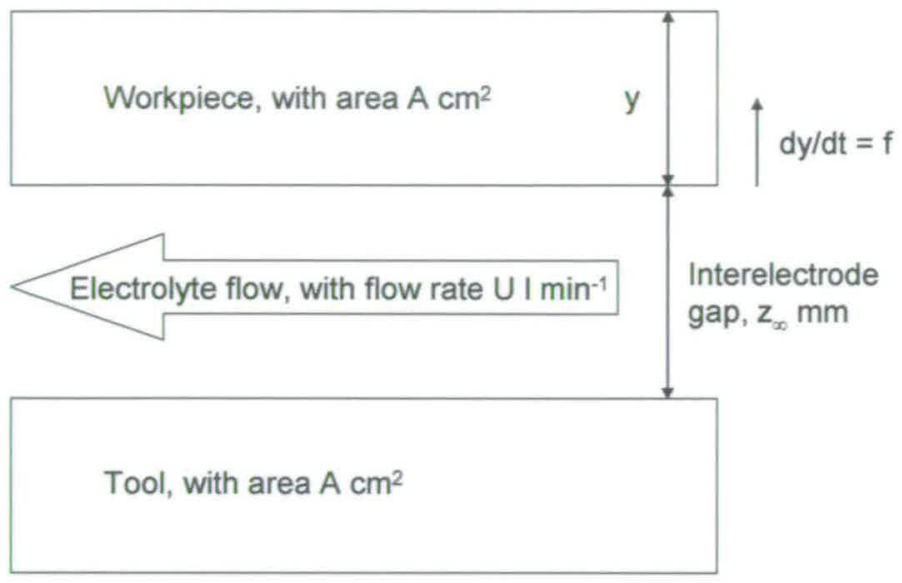

Figure 2.2: Planar ECM system under equilibrium machining conditions.

The chemistry during ECM can be described using electrochemical reactions for the processes occurring at the surface of the two electrodes (the workpiece and tool). At the workpiece, the material is oxidised and the reaction can be described in terms of a general metal $\mathrm{M}$ loosing $\mathrm{n}$ electrons and producing an $\mathrm{M}$ species of valency $\mathrm{n}$, by Reaction 2.1:

$$
M \rightarrow M(n)+n e^{-}
$$

\section{Reaction 2.1}

The corresponding reaction at the tool is the reduction of water (from the electrolyte) by Reaction 2.2 producing hydrogen gas and hydroxide ions:

$$
2 \mathrm{H}_{2} \mathrm{O}+2 e^{-} \rightarrow \mathrm{H}_{2(g)}+2 \mathrm{OH}_{(a q)}^{-}
$$

\section{Reaction 2.2}

In the simplest case, the two redox products of Reaction 2.1 and Reaction 2.2, $\mathrm{M}(\mathrm{n})$ and $\mathrm{OH}^{-}$ ions, diffuse, convect and migrate through the electrolyte towards each other; they may meet 
and combine to form insoluble metal hydroxide particles by Reaction 2.3, either in the gap or in the electrolyte tank:

$$
M(n)+n O H^{-} \rightarrow M(O H)_{n}
$$

\section{Reaction 2.3}

The overall cell reaction during the ECM of iron is therefore Reaction 2.4:

$$
\mathrm{M}+n \mathrm{H}_{2} \mathrm{O} \rightarrow \mathrm{M}(\mathrm{OH})_{n}+\frac{n}{2} \mathrm{H}_{2}
$$

\section{Reaction 2.4}

The potential drop across the two electrode ECM system is shown schematically in Figure 2.3 .

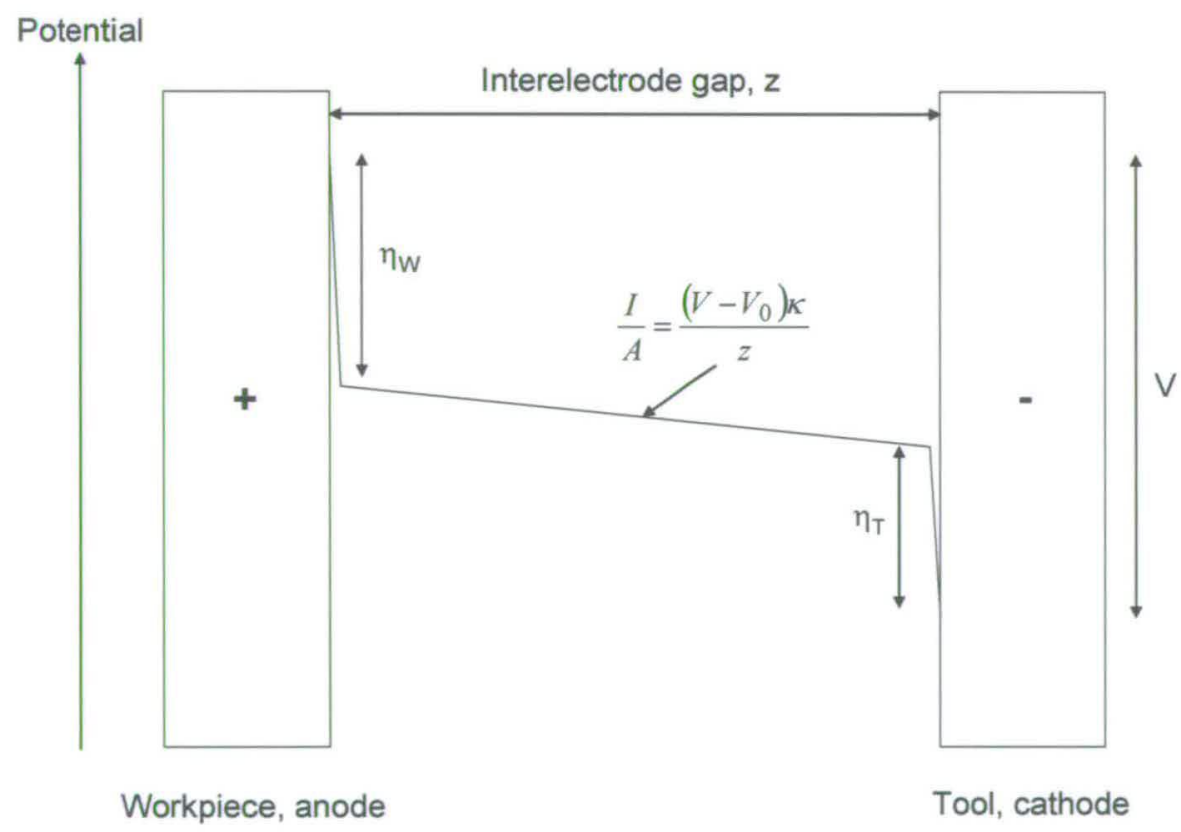

Figure 2.3: Potential distribution across an ECM cell during machining.

In the ECM system used there is no reference electrode. The second electrode used in the cell to complete the circuit is the tool and the workpiece is effectively the working electrode. 
The potential used to drive the electrochemical reactions at the workpiece and the tool (in the simplest case Reaction 2.1 and Reaction 2.2) is called the overpotential, $\mathrm{V}_{0}$. The components of $V_{0}$ are $\eta_{W}$ and $\eta_{T}$, the separate overpotentials of the electrochemical reactions at the workpiece and the tool respectively, which in turn encompass a thermodynamic and kinetic term (Figure 2.4). In other words $\mathrm{V}_{0}$ can be found by:

$$
V_{0}=\eta_{W}+\eta_{T}
$$

\section{Equation 2.6}

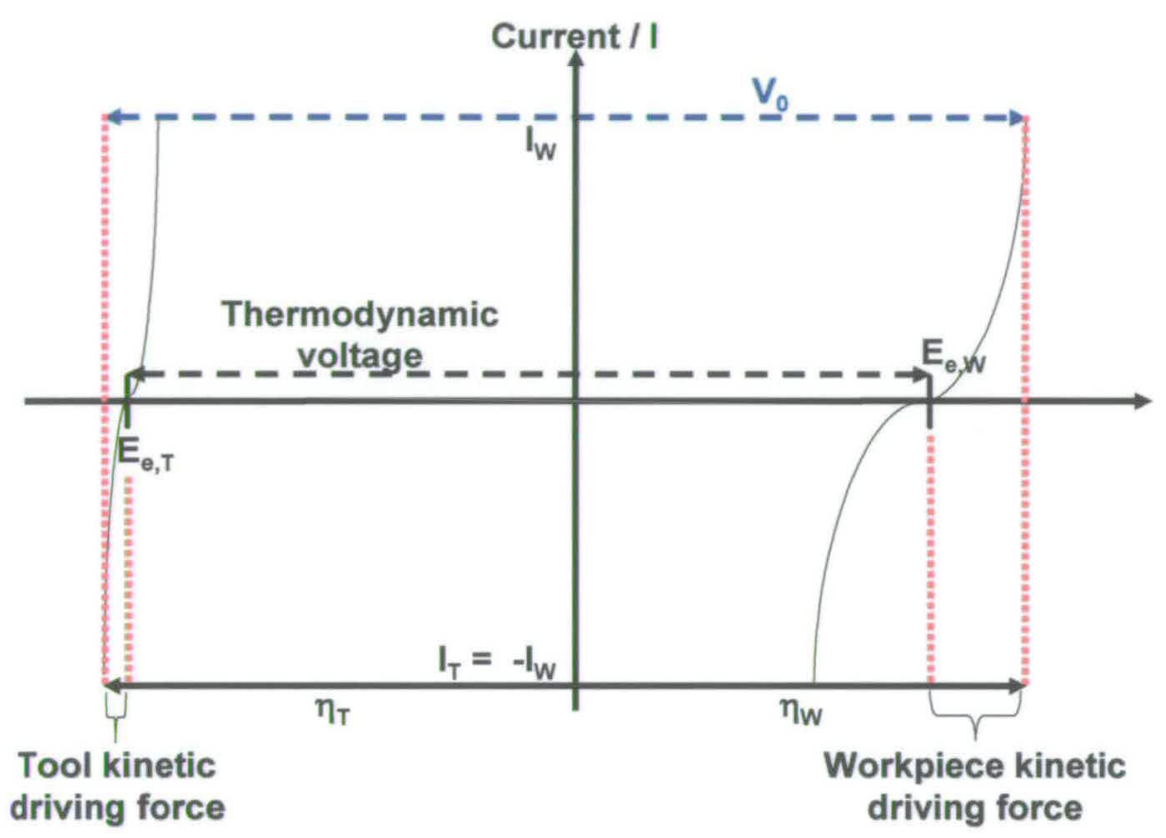

Figure 2.4: Schematic of the electrode components of $\mathrm{V}_{0}$.

The gradient of the I- $\eta_{\mathrm{T}}$ data for the reduction of water (Figure 2.4) is far steeper than that for the reaction at the workpiece; consequently, for the changes in current produced through movement of the electrodes, $\eta_{\mathrm{T}}$ tends to remain essentially constant. Therefore, variations in $\mathrm{V}_{0}$ can be further narrowed down to variations in $\eta_{\mathrm{w}}$ and hence the reaction at the workpiece.

In the simplest case, transition state theory can be used to quantitatively discuss the reaction kinetics of the reaction [131]: 


$$
A+B \rightarrow C
$$

\section{Reaction 2.5}

This reaction can be described using the free energy plot shown in Figure 2.5.

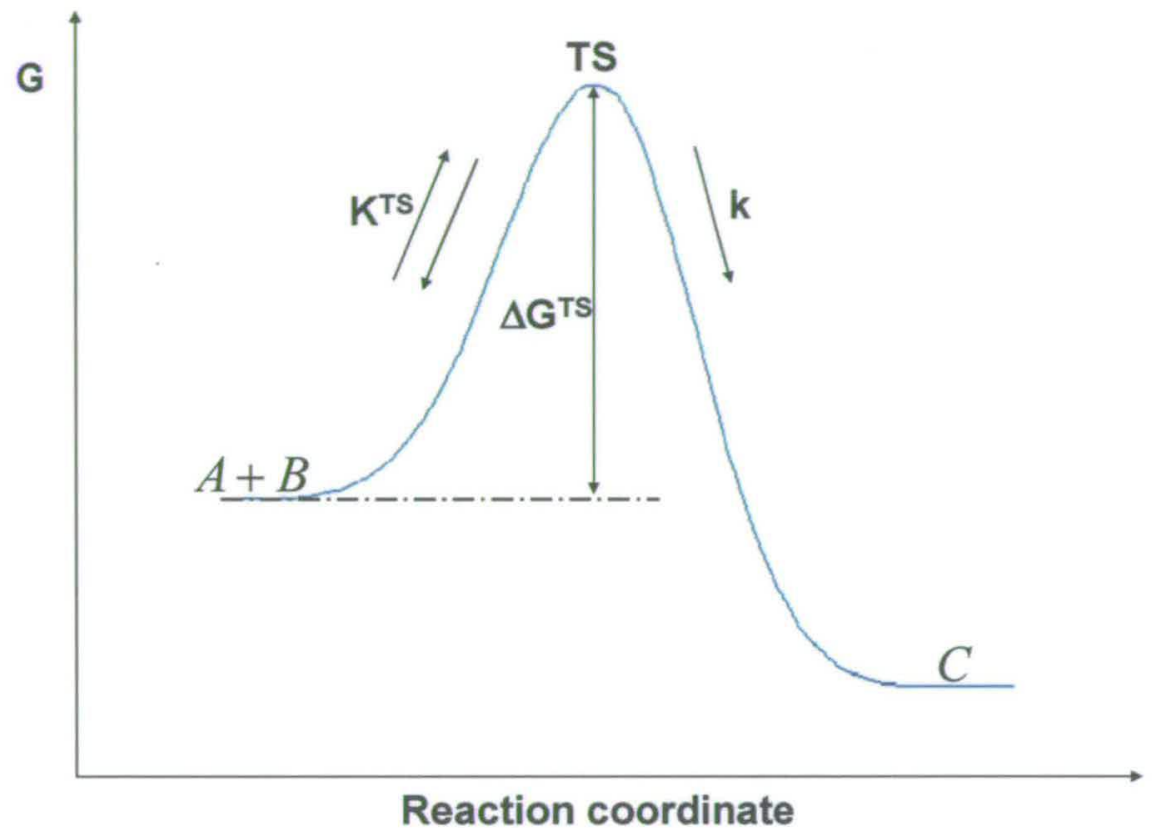

Figure 2.5: Free energy plot for the simple reaction shown in Reaction 2.5.

Where TS is the reaction transition state, $\mathrm{K}^{\mathrm{TS}}$ a rate constant between the reactants and the transition state, $\mathrm{k}$ is the reaction rate constant and $\Delta \mathrm{G}^{\mathrm{TS}}$ is the difference in free energy of the transition state and the reactants. The reactants can be thought of as being in equilibrium with the transition state, hence the rate of production of $\mathrm{C}$ can be found through the rate equation:

$$
\frac{d C}{d t}=k[T S]
$$

\section{Equation 2.7}


where [TS] is the concentration of the transition state species. The reactants/transition state equilibrium can be shown using the activities of the transition state and the reactants as Equation 2.8:

$$
K^{T S}=\frac{a_{T S}}{a_{A} a_{B}} \approx \frac{[T S]}{[A][B]}
$$

\section{Equation 2.8}

which, assuming dilute concentrations, can be approximated to the species concentrations.

Substituting [TS] in Equation 2.7 using Equation 2.8 gives the following rate equation:

$$
\frac{d C}{d t}=k K^{T S}[A][B]
$$

\section{Equation 2.9}

The relationship between the difference in free energy and the equilibrium constant is defined as:

$$
K^{T S}=\exp \left(-\frac{\Delta G^{T S}}{R T}\right)
$$

\section{Equation 2.10}

where $\mathrm{R}$ is the gas constant and $\mathrm{T}$ is the temperature in Kelvin. The overall change in free energy of a chemical reaction can be found through the difference in free energies of the reactants (j) and the products (i) using the chemical potentials (also called molar free energies) of these species ( $\mu$ ): 


$$
\Delta G=\sum_{i, \text { products }} n_{i} \mu_{i}-\sum_{j, \text { reac tan ts }} n_{j} \mu_{j}
$$

\section{Equation 2.11}

Using this equation $\Delta \mathrm{G}^{\mathrm{TS}}$ for Reaction 2.5 can be defined as:

$$
\Delta G^{T S}=\mu_{T S}-\mu_{A}+\mu_{B}
$$

\section{Equation 2.12}

Substitution of Equation 2.10 into Equation 2.9 gives:

$$
\frac{d C}{d t}=k[A][B] \exp \left(-\frac{\Delta G^{T S}}{R T}\right)
$$

\section{Equation 2.13}

The observed rate constant $\left(\mathrm{k}_{\mathrm{obs}}\right)$ for Equation 2.13 is therefore:

$$
k_{o b s}=k \exp \left(-\frac{\Delta G^{T S}}{R T}\right)
$$

\section{Equation 2.14}

This transition state theory can also be used to describe electrochemical reaction kinetics, for reactions including charges species, such as the expulsion of a metal ion from the workpiece and evolution of hydrogen at the tool. For electrochemical reactions, the free energies of the charged species can be altered through variation of the electric field. For the general electrochemical reduction of an oxidised species $\mathrm{O}^{\mathrm{z}+}$ with $\mathrm{n}$ electrons to the reduced species $\mathrm{R}^{(\mathrm{z}-\mathrm{n})+}$ (Reaction 2.6): 


$$
O^{z+}+n e^{-} \stackrel{\left.T S^{(z-\alpha}{ }^{n}\right)^{n+}}{\longrightarrow} R^{(z-n)+}
$$

\section{Reaction 2.6}

the free energy plot can be seen in Figure 2.6.

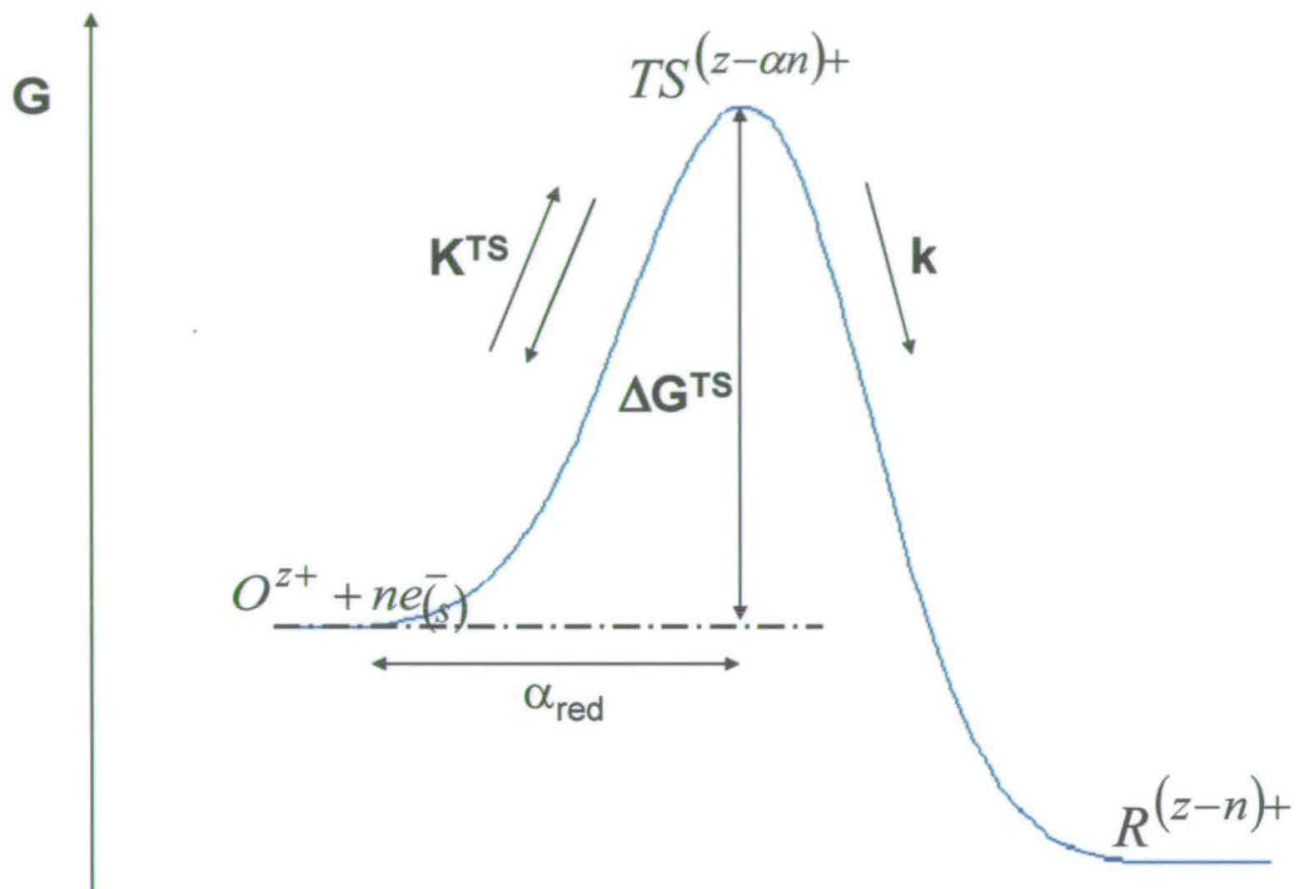

\section{Reaction coordinate}

Figure 2.6: Free energy plot for the simple electrochemical reduction shown in Reaction 2.6.

The value of $\alpha$ (the transfer co-efficient, in this case for the reduction) reflects the sensitivity of the transition state to the drop in electric potential present. The oxidation and reduction transfer coefficients for an electrochemical reaction are related through the equation: 
Parameterisation of Electrochemical Machining

$$
\alpha_{\text {red }}+\alpha_{o x}=1
$$

\section{Equation 2.15}

Consequently the value of the transfer coefficient for an electrochemical reduction or oxidation varies between $0<\alpha<1$ where a value of $\alpha$ approaching 0 represents a transition state which resembles the reactants in its potential dependence and a value of $\alpha$ approaching 1 represents a transition state which resembles the products in it's potential dependence. For many reactions the transfer coefficient is found to have a value of 0.5 depicting intermediate behaviour of the transition state, this value of the transfer coefficient can then be used to represent the effective charge of the TS. The transition state is strictly depicted as the intersection of the potential surfaces of the reactants and the products, different values of $\alpha$ and corresponding positions of the TS are depicted in this manner in Figure 2.7.

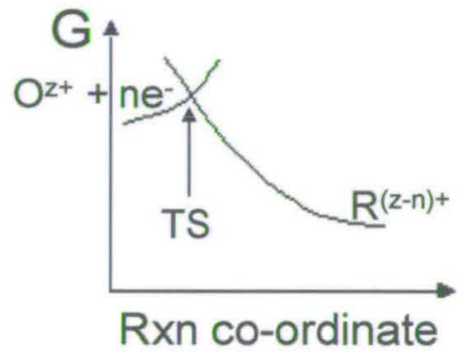

(a)

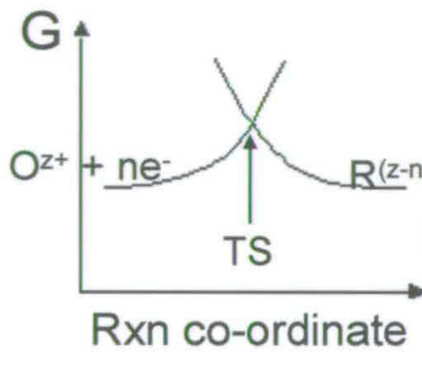

(b)

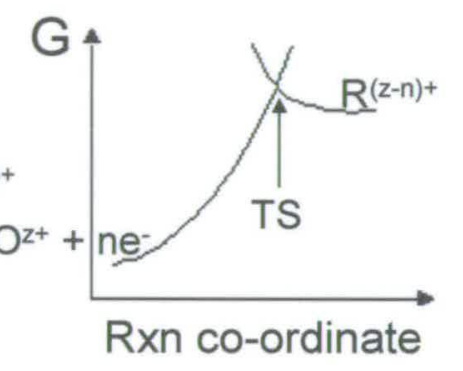

(c)

Figure 2.7: Free energy potential surfaces for (a) $\alpha \rightarrow 0$, (b) $\alpha=0.5$ and (c) $\alpha \rightarrow 1$.

For an electrochemical reaction the electrochemical potential of the reacting species must be taken into account, for a general species $i$ this is found as:

$$
\mu_{i}^{\prime}=\mu_{i}+z F \phi_{i}
$$

\section{Equation 2.16}


Where $\mu_{\mathrm{i}}$ is the chemical potential of the species, $\mathrm{z}$ is the charge on the ion and $\phi_{\mathrm{i}}$ is the potential experienced by ion $i$, consequently $z F \phi_{i}$ can be thought of as the extra energy imparted to the ion through the presence of the electric field.

Adapting Equation 2.11 for the electrochemical situation gives the free energy change as:

$$
\Delta G^{\prime}=\sum_{i, \text { products }} n_{i} \mu_{i}^{\prime}-\sum_{j, \text { reac tan } t s} n_{j} \mu_{j}^{\prime}
$$

\section{Equation 2.17}

Substituting with Equation 2.16 gives the free energy change:

$$
\Delta G^{T S^{\prime}}=\Delta G^{T S}+\sum_{i, \text { products }} n_{i} z_{i} F \phi_{i}-\sum_{j, \text { reac tan ts }} n_{j} z_{j} F \phi_{j}
$$

\section{Equation 2.18}

For Reaction 2.6 this can be written as:

$$
\Delta G^{T S^{\prime}}=\Delta G^{T S}+\alpha_{r e d} n F E
$$

\section{Equation 2.19}

where $\mathrm{E}$ is the potential drop. Substituting $\Delta \mathrm{G}^{\mathrm{TS}}$ for $\Delta \mathrm{G}^{\mathrm{TS}}$ in Equation 2.14 gives the observed reaction rate constant as:

$$
k_{o b s}=k \exp \left(-\frac{\Delta G^{T S}}{R T}\right) \exp \left(\frac{-\alpha_{r e d} n F E}{R T}\right)
$$

Equation 2.20 
Through the relationship for current (I)

$$
I=n F A k c
$$

\section{Equation 2.21}

Where A is the electrode area and $\mathrm{c}$ is the species concentration, the rate constant is observed to be directly proportional to the current; consequently the current is exponentially dependent on the potential during this general electrochemical reaction. Conversely theoretical analysis of the oxidation process:

$$
R^{(z-n)+} \stackrel{\left.T S^{(z-\alpha}{ }_{o x}^{n}\right)+}{\longrightarrow} O^{z+}+n e^{-}
$$

Reaction 2.7

yields the rate constant

$$
k_{o b s}=k \exp \left(-\frac{\Delta G^{T S}}{R T}\right) \exp \left(\frac{\alpha_{o x} n F E}{R T}\right)
$$

\section{Equation 2.22}

in which the current is again exponentially dependent of the potential. This is known as Tafel behaviour (Figure 2.8). 


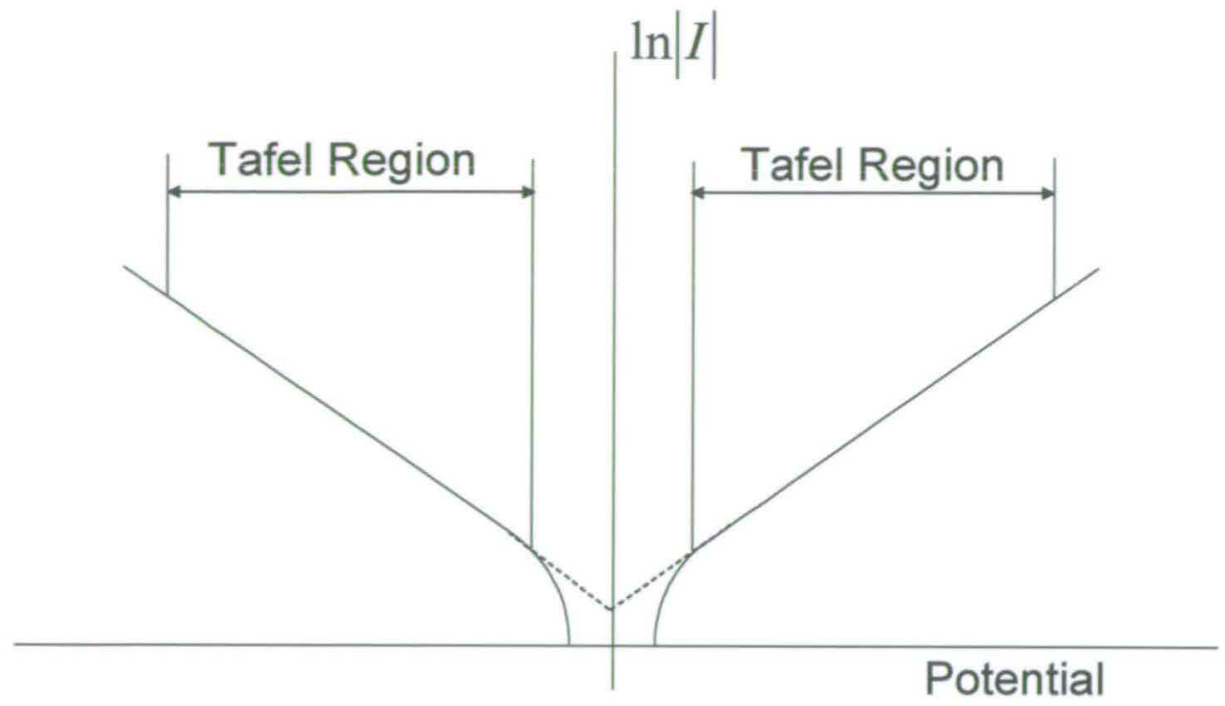

Figure 2.8: A schematic showing the Tafel region.

\subsection{Ion Migration in Electrolyte}

In order for current to flow in the ECM circuit there must be an ohmic current drop across the electrolyte to drive ion migration. This differs from typical electrochemical studies in which an inert electrolyte is deliberately added to the solution to remove the field across most of the solution between the electrodes. Consequently in these electrochemical studies the movement of the electrodes towards each other has no effect on the current passed in the cell. In ECM, however, the ohmic drop across the electrolyte solution results in the establishment of an electric field which is dependent on the distance between the two electrodes ( $\mathrm{z}$ ). Decreasing $\mathrm{z}$ causes an increase in the electric field between the electrodes giving higher currents. The current density across the electrolyte can be calculated using the potential dropped across the electrolyte $\left(\mathrm{V}-\mathrm{V}_{0}\right)$, the electrolyte conductivity $(\kappa)$ and the distance between the electrodes ( $\mathrm{z}$ ) through the simple ohmic law:

$$
\frac{I}{A}=\frac{\left(V-V_{0}\right) \kappa}{z}
$$

\section{Equation 2.23}


In order to achieve current flow the electrolyte must be conducting, i.e. it must contain ions of some description. The equilibrium reaction

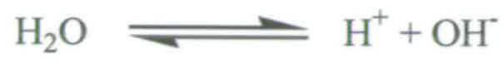

Reaction 2.8

shows that pure water contains ions, however a large potential would be required across a water solution to give a significant current, this would leave less overpotential at the electrode surfaces leading to smaller dissolution rates and an uneconomically slow machining process. A solution to this is to add a salt such as $\mathrm{NaCl}$ to the electrolyte; this increases the ion concentration in the electrolyte giving a greater current flow.

\subsubsection{Electrolyte Conductivity}

The migrational field in the equilibrium gap is determined by the electrolyte conductivity, which is a measure of the movement of all the ions in a solution under the influence of an electric field. It follows that, using Equation 2.23, at a constant electric field and interelectrode gap, the current is governed by the electrolyte conductivity.

For a solution, containing various ions, the overall conductivity is given by the sum of the conductivities of all the ions present [132]:

$$
\kappa=\sum_{j} \kappa_{j}
$$

Equation 2.24

which in turn can be found as: 


\section{Equation 2.25}

Where $z_{j}$ is the charge number of the ion and $c_{j}$ is the concentration of each ion type. $u_{j}$ is the mobility of each ion type, which is the proportionality co-efficient between the ion velocity and the electric field strength; it is given by:

$$
u_{j}=\frac{z_{j} e}{6 \pi \eta r_{j}}
$$

\section{Equation 2.26}

Where $z_{j}$ is the charge on the ion, $\eta$ is the solution viscosity and $r_{j}$ is the solvated radius of the ion. From Equation 2.26 it can be seen that smaller, highly charged ions will have a greater mobility. A value for the molar conductivity of a particular ion $\mathrm{j}$ in the electrolyte $\left(\lambda_{\mathrm{j}}\right)$ can be calculated using:

$$
\lambda_{j}=\frac{\kappa_{j}}{c_{j}}=z_{j} u_{j} F
$$

\section{Equation 2.27}

And the overall electrolyte molar conductivity $(\Lambda)$ can be found using:

$$
\Lambda=\sum_{j} n_{j} \lambda_{j}=\frac{\kappa}{c}
$$

\section{Equation 2.28}


where $\mathrm{c}$ is the overall concentration of the solution.

Most conductivity modelling is carried out using solutions of infinite dilution. In concentrated solutions with high ionic strengths, such as is used in ECM, the interactions between various ions in the solutions make conductivity modelling using infinite dilution analysis problematic. For this reason experimental measurement of conductivity is required for the purposes of this thesis.

\subsubsection{Hydrodynamic Effects}

In ECM the flow rate is high compared to the electrode feed rate, consequently for the purposes of hydrodynamic modelling the electrodes can be considered stationary. Therefore, the most appropriate system for modelling the ECM hydrodynamic conditions is the channel electrode cell [133].

A channel electrode cell contains two electrodes facing each other on opposite sides of an electrolyte channel (Figure 2.9), such as is seen in the ECM set-up.

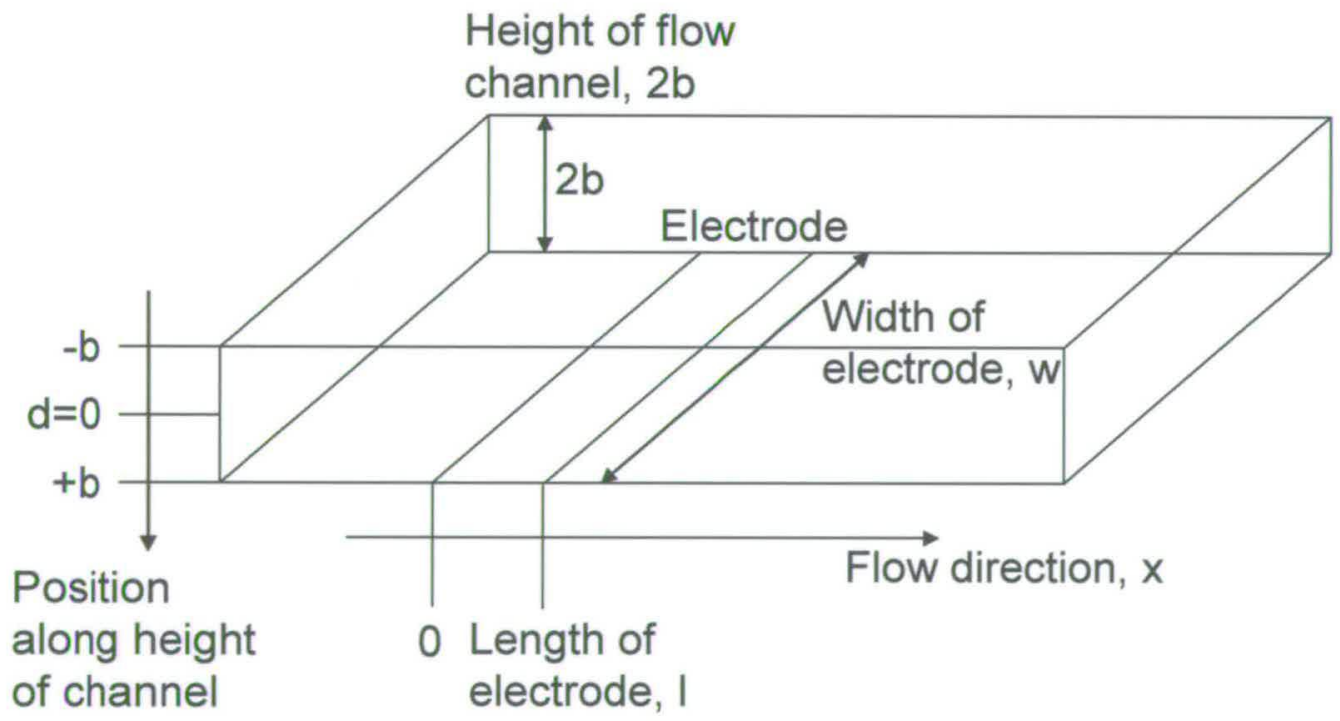

Figure 2.9: Schematic of a channel electrode system showing dimensions of the cell. 
In the ECM system studied, mass transport to the electrodes is under turbulent hydrodynamic conditions. Turbulent conditions are related to the value of the Reynolds number which is given by Equation 2.29:

$$
R_{n}=\frac{\mathrm{v}_{0} b}{v}
$$

\section{Equation 2.29}

Where $v_{0}$ is the solution velocity at the centre of the channel, $b$ is shown in Figure 2.9 and $v$ is the kinematic viscosity of the electrolyte solution. When $R_{n}<2000$ the flow is smooth and laminar, however once $R_{n}$ exceeds 2000 the flow becomes turbulent. $R_{n}$ for the ECM system can be calculated using Equation 2.29: using the cell width, $1.5 \mathrm{~cm}$, and a typical equilibrium interelectrode gap, $0.4 \mathrm{~mm}$, the interelectrode channel area is $1.5 \mathrm{~cm} \times 0.040 \mathrm{~cm}$ $=0.060 \mathrm{~cm}^{2}$, for a flow rate of $161 \mathrm{~min}^{-1}\left(267 \mathrm{~cm}^{3} \mathrm{~s}^{-1}\right) \mathrm{v}_{0}=267 \mathrm{~cm}^{3} \mathrm{~s}^{-1} / 0.060 \mathrm{~cm}^{2}=4450$ $\mathrm{cm} \mathrm{s}^{-1}$. Substitution into Equation 2.29 and using the kinematic viscosity of water as $v=$ $0.0114 \mathrm{~cm}^{2} \mathrm{~s}^{-1}, \mathrm{R}_{\mathrm{n}}$ is given by [134]:

$$
R_{n}=\frac{4450 \mathrm{~cm} \mathrm{~s}^{-1} x(0.040 / 2 \mathrm{~cm})}{0.01 \mathrm{~cm}^{2} \mathrm{~s}^{-1}}=8900
$$

The Reynolds number is of the order of 9000 , therefore under these ECM conditions the flow in the cell is turbulent.

The flow in channel electrode systems is generally chosen to be laminar to assist analysis and hydrodynamic modelling; however some turbulent systems have been studied [133]. Provided that $4 \times 10^{3}<\mathrm{R}_{\mathrm{n}}<10^{5}$, as is the case in ECM experiments, the turbulent velocity profile can be described by the empirical equation:

$$
\frac{\mathrm{v}_{x}}{\mathrm{v}_{0}}=\left(\frac{b-d}{b}\right)^{\frac{1}{7}}
$$

\section{Equation 2.30}


Where $\mathrm{b}$ and $\mathrm{d}$ are shown in Figure 2.9 and $\mathrm{v}_{\mathrm{x}}$ represents the flow velocity in the $\mathrm{x}$-direction at some value of $\mathrm{d}$. This velocity profile is compared to the laminar velocity profile, given by Equation 2.31 in Figure 2.10

$$
\mathrm{v}_{0}=\mathrm{v}_{x}\left(1-\frac{d^{2}}{b^{2}}\right)
$$

\section{Equation 2.31}

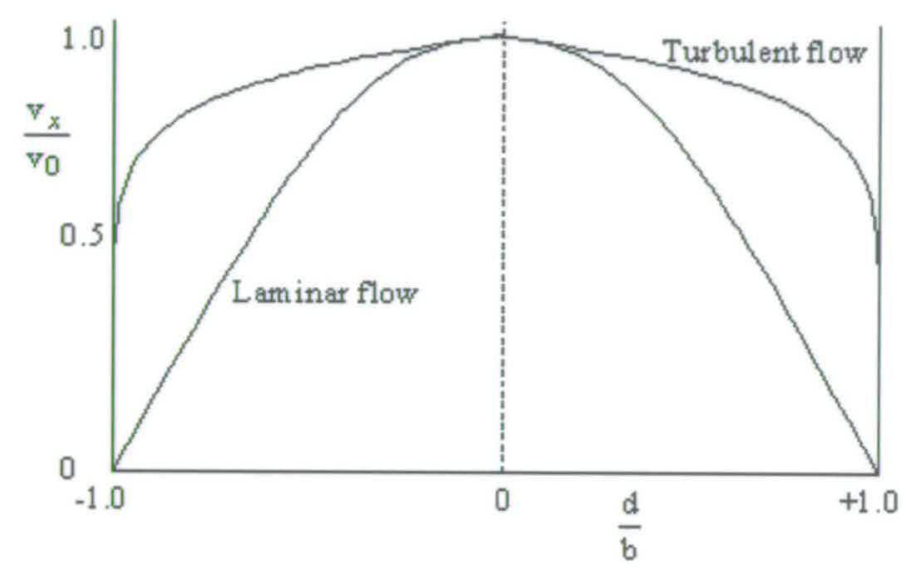

Figure 2.10: Comparison of laminar $\left(R_{n}<2000\right)$ and turbulent $\left(4 \times 10^{3}<R_{n}<10^{5}\right)$ flow velocity distributions [133].

It can be seen from Figure 2.10 that for a turbulent flow region the electrolyte flow is faster in the region of the cell walls. The electrolyte in the region of the cell walls is slowed by friction at the walls, however the effect of friction on the speed of the electrolyte is less pronounced for turbulent flows. This leads to a thinner diffusion layer next to the electrode for turbulent flow systems.

Von Kárman [135] established three zones within the boundary layer found at the cell walls in a tubular channel electrode under turbulent conditions. The Laminar Sublayer was identified as the layer closest to the tube walls. The turbulent eddies present in the flow were damped out due to friction at the cell wall; consequently the flow in this region can be 
Parameterisation of Electrochemical Machining considered to be laminar. In this region the flow velocity varies linearly with the distance from the cell wall, as observed with a laminar flow channel electrode. The Boundary Region is a region in which the transition between laminar and turbulent flow is observed and the Turbulent Core, in which the velocity profile is defined by Equation 2.30.

These three regions are depicted schematically in Figure 2.11 for an electrode which is generating material:

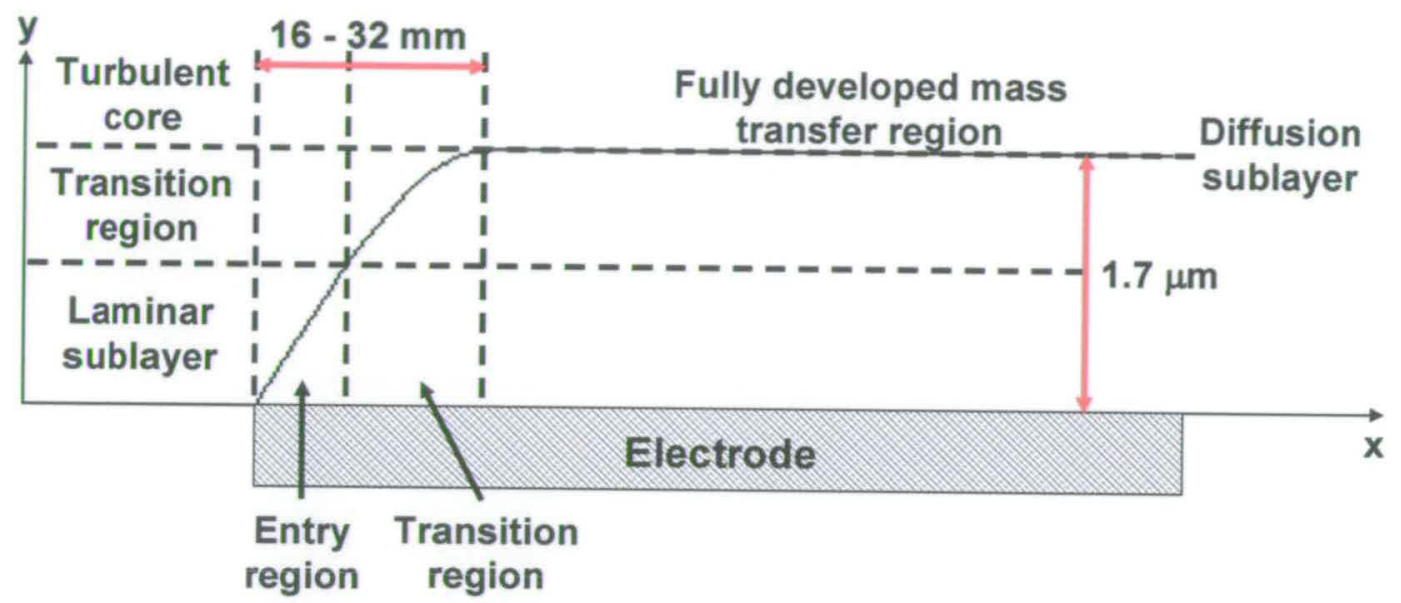

Figure 2.11: Schematic depicting the 3 regions in the vicinity of the cell wall for turbulent flow, shown as a function of distance along the flow path length of the electrode, $\mathrm{x}$ (not to scale) [133].

Equation 2.32 [133] was used to calculate the dimension of the diffusion sublayer:

$$
\delta_{d t}=\frac{50 b}{R_{n}^{7 / 8} \operatorname{Pr}^{1 / 3}}
$$

Equation 2.32

where $b$ is half the interelectrode gap distance (in this case b was chosen to be $0.2 \mathrm{~mm}$ ), $R_{n}$ is the Reynolds number (calculated previously as 8900 ) and Pr is given by Equation 2.33: 


$$
\operatorname{Pr}=\frac{v}{D_{\mathrm{T}}}
$$

\section{Equation 2.33}

Using the kinematic viscosity of $5 \mathrm{M} \mathrm{NaCl}, v=1.6 \times 10^{-6} \mathrm{~m}^{2} \mathrm{~s}^{-1}$ [136] and the thermal diffusivity of water $\mathrm{D}_{\mathrm{T}}=0.14 \times 10^{-6} \mathrm{~m}^{2} \mathrm{~s}^{-1}$ (the value for $\mathrm{NaCl}$ was not available), $\delta_{\mathrm{dt}}$ was found to be $1.7 \mu \mathrm{m}$ (Equation 2.32), approximately $0.4 \%$ of an interelectrode gap distance of $0.4 \mathrm{~mm}$, consequently the approximation of turbulent conditions across the entire interelectrode gap is valid.

A lead-in length is required to establish the fully developed mass transfer region in the cell (Figure 2.11). This length is typically $40-80$ cell diameters, which for the ECM system is found by multiplying the interelectrode gap by between 40 and 80 giving a lead-in length of $16-32 \mathrm{~mm}$ when an equilibrium machining gap of $0.4 \mathrm{~mm}$ is established. A consequence of this is that the diffusion sublayer will be thinner than the calculated $1.7 \mu \mathrm{m}$ across the majority of the $40 \mathrm{~mm}$ cell length giving more efficient mixing in the turbulent core of the electrolyte.

\subsection{Efficiency determination}

The theoretical treatment in this work assumes a machining efficiency of $100 \%$, i.e. all the anode current can be attributed to dissolution products. However, there are possible side reactions which could produce extra current and lead to an efficiency of less than $100 \%$. For example, the anode potential may be raised sufficiently to result in the evolution of oxygen, reaction of products and cycling of reagents in the interelectrode gap. In this instance the anode current is not entirely produced by dissolution and the current efficiency drops from $100 \%$. In practice, industrial current efficiencies for ECM are $75-90 \%[2]$. The efficiency of ECM can be expressed as: 


$$
\mathrm{e}=\frac{\text { actual metal removal rate }}{\text { theoretical metal removal rate }} \times 100
$$

\section{Equation 2.34}

This can also be expressed in terms of dissolution valency as Equation 2.35:

$$
\mathrm{e}=\frac{\mathrm{n}_{\text {true }}}{\mathrm{n}}
$$

\section{Equation 2.35}

Under ideal conditions the observed valency ( $n$ ) is equal to the true valency $\left(n_{\text {true }}\right)$ and the efficiency is $100 \%$, where $\mathrm{n}$ is the number of electrons produced per mole of dissolving material. If $\mathrm{n}$ is greater than $\mathrm{n}_{\text {true }}$ then electrons are being produced by processes other than dissolution which constitutes an inefficiency in the ECM process and results in a lowering of the efficiency. However, if the efficiency of the ECM process $\neq 1, n_{\text {true }}$ can only be determined from the ECM current if the dissolution efficiency if known. 


\section{Chapter 3}

Theoretical Development 


\subsection{Introduction}

This chapter provides the theoretical background to the analysis methods used throughout this work. It defines and compares two analysis methods, the Chronoamperometric method and the Ultrasound method, and provides a critical comparison of the two methods.

\subsection{Chronoamperometric analysis of Machining Parameters}

The system analysed is a planar tool and workpiece with a constant voltage, V, applied between the electrodes. The workpiece is moved towards a tool, each of the same area, A, at a constant feed rate, $f$. The assumptions for the theory are [137]:

1. The current across the cell is governed by migration of the ions in the electrolyte, necessary for stable ECM.

2. The electrolyte is being pumped through the interelectrode gap, $z$, at a high enough flow rate that the build up of machining products or the decrease in ions from the electrolyte does not significantly affect the electrolyte conductivity, $\kappa$.

3. All the current, I, leads to electrode dissolution, i.e. the process is $100 \%$ efficient.

From 1) and 2) the current across the electrolyte is then given by Equation 3.1

$$
I=\frac{\kappa\left(V-V_{0}\right) A}{z}
$$

\section{Equation 3.1}

where $\mathrm{V}_{0}$ is the ECM overpotential, which is defined as the voltages required at both the tool and the workpiece electrodes to drive the electrochemical reactions. This means that $\left(\mathrm{V}-\mathrm{V}_{0}\right)$ is the component of the applied voltage available to drive ion migration in the interelectrode gap.

From 3) I is also the dissolution current of the workpiece given by Equation 2.4: 
The Parameterisation of Electrochemical Machining

$$
I=\frac{n F A \rho}{M} \frac{d y}{d t}
$$

where $\mathrm{y}$ is the erosion thickness of the workpiece, $\mathrm{n}$ is the workpiece dissolution valency, $\mathrm{F}$ is Faraday's constant and $\rho$ and $\mathrm{M}$ are the density and the relative molecular mass of the workpiece respectively.

The change in interelectrode distance $(\mathrm{dz} / \mathrm{dt})$ is given by Equation 3.2:

$$
\frac{d z}{d t}=\frac{d y}{d t}-f
$$

\section{Equation 3.2}

combining Equation 2.4 with Equation 3.1 and Equation 3.2 gives:

$$
\frac{d z}{d t}=\frac{k}{z}-f
$$

\section{Equation 3.3}

where $\mathrm{k}$, which is a combined machining constant (also often called the $\mathrm{C}$-function when it varies [67]), is given by Equation 3.4

$$
k=\frac{\kappa\left(V-V_{0}\right) M}{n F \rho}
$$

\section{Equation 3.4}

At the equilibrium current, $\mathrm{I}_{\infty}$, (I when $\mathrm{t} \rightarrow \infty$ ), when a steady state current has been reached, $\mathrm{dy} / \mathrm{dt}=\mathrm{f}, \mathrm{dz} / \mathrm{dt}=0$ and hence $\mathrm{z}$ remains constant at $\mathrm{z}_{\infty}$, from Equation $3.3, \mathrm{f}=\mathrm{k} / \mathrm{z}_{\infty}$ and so 
The Parameterisation of Electrochemical Machining

$$
\frac{d z}{d t}=k\left[\frac{1}{z}-\frac{1}{z_{\infty}}\right]
$$

\section{Equation 3.5}

If a constant value of $k$ can be assumed (which assumes constant values of $\kappa,\left(\mathrm{V}-\mathrm{V}_{0}\right)$ and $n$ from Equation 3.4), this equation can be integrated from $\mathrm{t}=\mathrm{t}_{0}, \mathrm{z}=\mathrm{z}_{0}$ to $\mathrm{t}=\mathrm{t}$ and $\mathrm{z}=\mathrm{z}$ to give Equation 3.6 (appendix 1).

$$
\ln \left[\frac{z_{\infty}-z_{0}}{z_{\infty}-z}\right]+\frac{z_{0}-z}{z_{\infty}}=\frac{k\left(t-t_{0}\right)}{z_{\infty}^{2}}
$$

\section{Equation 3.6}

Equation 3.1 can be used to interrelate $I_{0}$ and $z_{0}, I_{\infty}$ and $z_{\infty}$ and $I$ and $z$. Therefore, substituting the appropriate values of $\mathrm{I}_{0}, \mathrm{I}$ and $\mathrm{I}_{\infty}$ for $\mathrm{z}_{0}, \mathrm{z}$ and $\mathrm{z}_{\infty}$ gives Equation 3.7.

$$
\ln \left(\frac{1-\frac{I_{\infty}}{I_{0}}}{1-\frac{I_{\infty}}{I}}\right)+\left(\frac{I_{\infty}}{I_{0}}-\frac{I_{\infty}}{I}\right)=\frac{f^{2}\left(t-t_{0}\right)}{k}
$$

\section{Equation 3.7}

This equation enables analysis of current-time data (chronoamperometric) to give the combined ECM machining parameter $\mathrm{k}$. The 4 parameters in this equation are $\mathrm{t}_{0}, \mathrm{I}_{0}, \mathrm{k} / \mathrm{f}^{2}$ and $I_{\infty}$. $t_{0}$ and $I_{0}$ can be assigned fixed values dependent on the initial time and initial currents chosen for the experimental data. $I_{\infty}$ can be chosen or determined as $I$ at long $t$, under equilibrium machining conditions. A non-linear regression with iterative fitting of the $\mathrm{I}$ and $\mathrm{t}$ data is typically then performed to calculate $\mathrm{f}^{2} / \mathrm{k}$ (and hence $\mathrm{k}$ ). Once $\mathrm{k}$ has been determined in this fashion values Equation 3.4 can be used with known values of two of the ECM 
parameters $\mathrm{n}, \mathrm{\kappa}$ or $\mathrm{V}_{0}$ to calculate the third, unknown, parameter, this is usually carried out to calculate $\mathrm{V}_{0}[137]$.

A similar iterative fitting analysis can also be carried out using gap measurement data. Substitution of Equation 3.1 into Equation 3.7 gives Equation 3.8. Iterative fitting is then carried out in the same fashion as for Equation 3.7, fixing $z_{0}$ and $t_{0}$ as the initial gap and time respectively and iteratively fitting the data to obtain values of $\mathrm{f}^{2} / \mathrm{k}$ (and hence $\mathrm{k}$ ) and $\mathrm{z}_{\infty}$ as before.

$$
\left[\ln \left(\frac{1-\frac{z_{0}}{z_{\infty}}}{1-\frac{z}{z_{\infty}}}\right)+\left(\frac{z_{0}}{z_{\infty}}-\frac{z}{z_{\infty}}\right)\right]=\frac{f^{2}\left(t-t_{0}\right)}{k}
$$

\section{Equation 3.8}

This theoretical analysis can only be applied for constant $\mathrm{k}$. Often $\mathrm{n}, \mathrm{\kappa}$ and $\mathrm{V}_{0}$ are not always constant during the entire ECM transient leading to a breakdown in the assumption of a constant value of $\mathrm{k}$. However, under the high flow conditions typically employed in ECM the assumption of constant $\kappa, \mathrm{V}$ and typically $\mathrm{n}$ can be assumed to be valid, although $\mathrm{V}_{0}$ is unlikely to be constant over the entire ECM transient (Chapter 5) as $\mathrm{V}_{0}$ varies with I. In this case iterative fitting using Equation 3.7 or Equation 3.8 to the entire ECM transient is likely to be unsuitable. However, iterative fitting to small time portions of the transient may be appropriate, if the variation in $\mathrm{I}$ is small enough that the assumption of a constant $\mathrm{V}_{0}$ is valid.

For a planar tool only one value of $\mathrm{k}$ can be calculated for the entire path length. It is apparent that this value of $\mathrm{k}$ is often not constant along the whole flow path; this lead to the development of the segmented tool. The segmented tool allows values of $k$ to be determined for each segment along the flow path length during the experiment. These variations in $\mathrm{k}$ along the flow path are thought to be due to two factors [138]:

1. Electrolyte conductivity sensitivities to the position along the flow length and the geometric form of the gap.

2. Valency and overpotential sensitivities to the applied voltage, the current density, the electrolyte concentration, the flow velocity and machining products. 
The errors in the chronoamperometric analysis caused by the assumption if a constant $\mathrm{k}$ led to the development of a method for real-time analysis of $\mathrm{k}$ to probe the ECM parameter more accurately variations: the ultrasound analysis.

\subsection{Ultrasound analysis: set-up}

In the ultrasound system set-up, an ultrasound transmit/receive transducer is spring loaded against the upper face of the workpiece above segment 4 (section 4.6) [139]. The lower face of the workpiece is machined perpendicular to the probe and orthogonal to the acoustic axis of the ultrasound beam. The ultrasound device takes thickness readings of the workpiece throughout the ECM process. The experimental set-up for the Ultrasound analysis can be seen in Figure 3.1 and is discussed in more detail in the Experimental Equipment (Chapter 4).

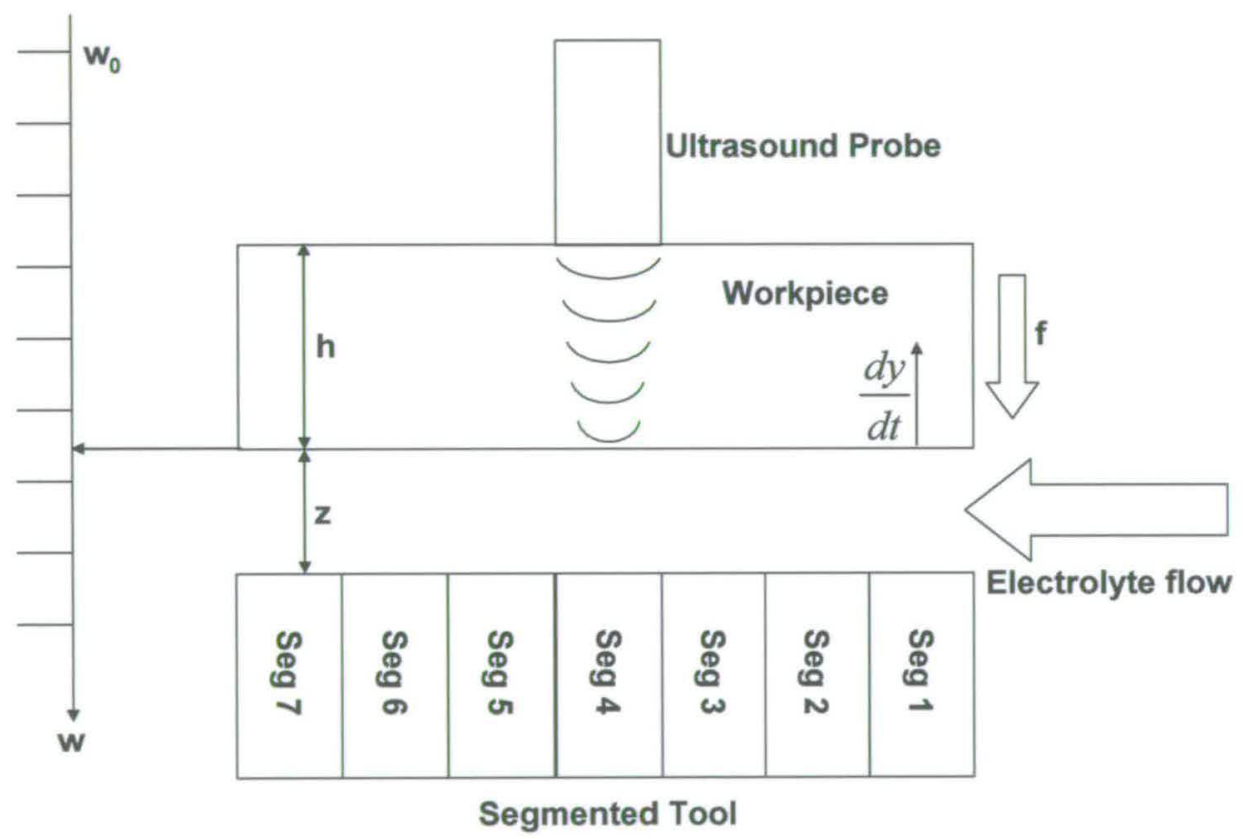

Figure 3.1: Schematic diagram of the ultrasound system.

The ultrasound probe transmits a pulse which reflects from the workpiece face (Figure 3.1). Knowing the velocity of sound in the workpiece material $\left(\mathrm{v} \mathrm{ms}^{-1}\right)$, the workpiece thickness, $\mathrm{h}$, can be determined, at the position of the ultrasound probe, from Equation 3.9. 


$$
h=\frac{t \mathrm{v}}{2}
$$

\section{Equation 3.9}

\subsubsection{Analysis of ultrasound system data}

Analysis of ultrasound system data is carried out in a number of stages, each producing realtime data for a defined ECM parameter.

\subsubsection{Interelectrode gap measurement}

The workpiece position (w) during the experiment can be found using Equation 3.10.

$$
\mathrm{w}-\mathrm{w}_{0}=\mathrm{f}\left(\mathrm{t}-\mathrm{t}_{0}\right)
$$

\section{Equation 3.10}

Where $\mathrm{w}$ and $\mathrm{w}_{0}$ denote the workpiece positions on the feed axis at times $t$ and $\mathrm{t}_{0}$ respectively.

The interelectrode gap is then calculated by comparing the distance the workpiece has moved with the amount machined, given by the ultrasound measurements (Figure 3.1) and Equation 3.11. 
The Parameterisation of Electrochemical Machining

$$
z=\left(h_{o}-h\right)-\left(w-w_{o}\right)+z_{o}
$$

\section{Equation 3.11}

The ultrasound probe continuously determines $\mathrm{h}$ and therefore $\mathrm{z}$ throughout the experiment. This gives real-time z-t data in addition to the I-t data normally collected during ECM.

Equation 3.3 can then be used to calculate the machining constant $\mathrm{k}$ at any time during the experiment provided $\mathrm{dz} / \mathrm{dt}$ can be accurately determined from the gradient of the $\mathrm{z}$ vs. $\mathrm{t}$ data at time $\mathrm{t}$.

\subsubsection{Overpotential measurement}

The overpotential throughout machining can be calculated at any time using Equation 3.1.

$$
V_{0}=V-\frac{I z}{\kappa A}
$$

when $\mathrm{k}$ varies along the flow path length this equation only applies at the position where $\mathrm{h}$, and therefore $\mathrm{z}$, is measured. Consequently the value of I used must also be measured at this point, this data is achieved using the segmented tool.

\subsubsection{Dissolution Valency measurement}

Measurements of real-time valency can also be made using Equation 3.12.

$$
\frac{d y}{d t}=\frac{I M}{A n F \rho}
$$

Equation 3.12

As erosion leads to workpiece thinning 


$$
\frac{d y}{d t}=-\frac{d h}{d t}
$$

\section{Equation 3.13}

By combining Equation 3.12 and Equation 3.13, the dissolution valency is given by Equation 3.14:

$$
\frac{d h}{d t}=-\frac{I M}{A n F \rho}
$$

\section{Equation 3.14}

Hence the dissolution valency can be determined from dh/dt and I. Equation 3.12 was derived assuming a $100 \%$ dissolution efficiency. In this case $n$ is the true valency of the dissolving workpiece atoms and $\mathrm{n}=\mathrm{n}_{\text {true }}$. However, this analysis still applies when $100 \%$ dissolution efficiency is not found. In this case $\mathrm{n}$ is the total number of electrons required to dissolve each workpiece atom and is related to $\mathrm{n}_{\text {true }}$ by Equation 2.35

$$
n=\frac{n_{\text {true }}}{e}
$$

where $\mathrm{e}$ is the dissolution efficiency.

As the charge, $\mathrm{Q}$, passed during ECM is given by

$$
I=\frac{d Q}{d t}
$$

\section{Equation 3.15}


Substituting for Equation 3.14 gives the relationship

$$
\frac{d Q}{d h}=-\frac{A \rho n F}{M}
$$

\section{Equation 3.16}

It is evident from Equation 3.16 that a plot of $\mathrm{Q}$ vs. $\mathrm{h}$ will yield changes in dissolution valency as changes in gradient. Therefore, $n_{\text {true }}$ cannot be measured directly but can be inferred from the knowledge of likely chemical dissolution valencies and workpiece composition. 


\section{Chapter 4}

ECM Experimental Configuration 
The Parameterisation of Electrochemical Machining

\subsection{Overview}

When the ECM project was started at Edinburgh University, off-the-shelf experimental ECM equipment with the desired flexibility was not available. Consequently, all the ECM equipment used for this work, tooling and ECM flow cells, was developed and purpose built. The purpose built machine, which has been further developed during this work, has allowed for more accurate and in depth data collection than would have been possible on a standard industrial set-up.

The experimental ECM set-up is shown in Figure 4.1.

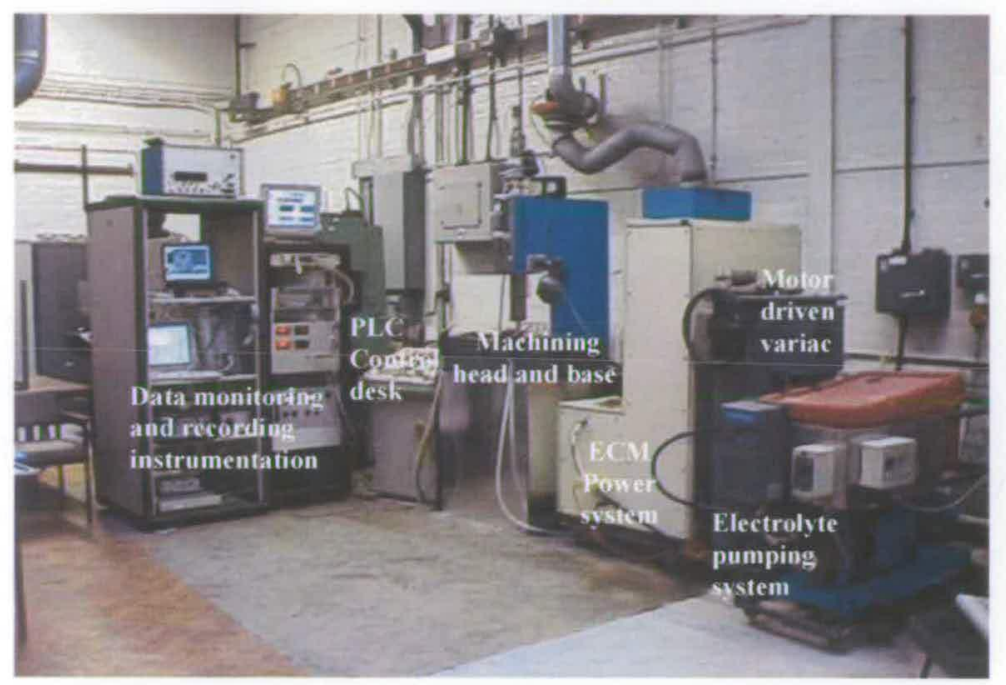

Figure 4.1: View of the entire ECM set-up.

The equipment and set-up depicted in Figure 4.1 was assembled by Clifton [67]. The equipment was designed and built specifically to allow the user to monitor and control the machining parameters and variables with minimum adjustment during machining, but also to allow small changes in experimental parameters between, and in some cases during, machining runs. The system can be divided into modules which will be discussed separately in this chapter with attention paid to their design, development, set-up, calibration and operation. 


\subsection{Flow system}

\subsubsection{Requirements}

Solid ECM products must be removed at a sufficient rate in order to maintain stable dissolution and hence the required machining rate during an experiment. Also ion depletion and electrochemical reaction in the electrolyte must be kept to a minimum to avoid changes in electrolyte conductivity. Furthermore the power input into the electrolyte in the interelectrode gap leads to Joule heating of the electrolyte which also produces changes in the electrolyte conductivity. These effects can be minimised by flowing the electrolyte through the interelectrode gap at a sufficiently high velocity. At high flow velocities the changes in electrolyte conductivity through ion depletion can be assumed to be negligible and the solid dissolution products are removed at a sufficient rate to enable stable dissolution. The Joule heating of the electrolyte is minimised and in some cases can be neglected (section 8.2), in other cases it can be measured and allowed for in calculations using thermocouples placed at the inlet and outlet of the ECM cell (section 4.5.4).

The flow rate required may differ between experiments owing to differences in other parameters such as the applied voltage and feed rate which determine the interelectrode gap; for example, flow velocity, $v$, changes during machining with the interelectrode gap, $z$, when the volume flow rate, $\mathrm{U}$, is kept constant (Equation 4.1).

$$
v=\frac{U}{z d}
$$

\section{Equation 4.1}

where $d$ is the depth of the workpiece and the tool (Figure 4.2). 
The Parameterisation of Electrochemical Machining

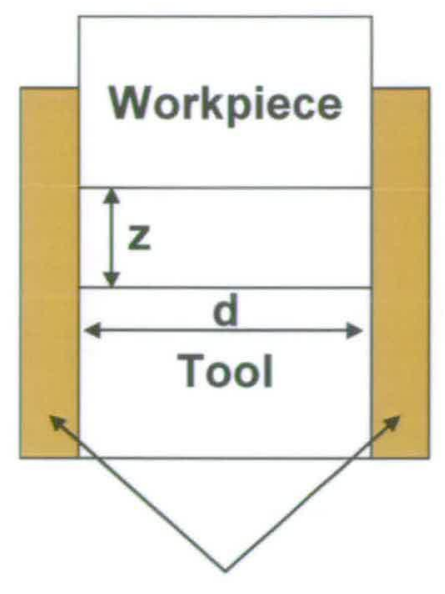

\section{Side cheeks}

Figure 4.2: Schematic showing parameters $\mathrm{z}$ and $\mathrm{d}$

There is no clear agreement in the literature of a sufficient flow velocity to maintain stable dissolution. Previously flow velocities in the range $30-50 \mathrm{~m} \mathrm{~s}^{-1}$ have been shown to be sufficient [2]; in this work stable dissolution has been observed for the flow velocity range $15-80 \mathrm{~m} \mathrm{~s}^{-1}$. A physical limitation of the flow rate is often the cell since the inlet pressure of the cell, $P_{\text {inlet }}$ is often very large. The maximum value of $P_{\text {inlet }}$ for this system is $10-20$ bar. The possibility of back pressure application to the ECM cell and the use of an electrolyte filter were also considered when selecting the electrolyte pump for the system, consequently a pump able to sustain pressures of up to 40 bar was considered necessary.

Using Equation 4.1 the volume flow rate required to achieve the correct flow velocities can be calculated from the cross sectional area $(\mathrm{z} \times \mathrm{d})$ and the required flow velocity $(\mathrm{v})$. For an interelectrode gap of $\mathrm{z}=0.4 \mathrm{~mm}$ with $\mathrm{d}=15 \mathrm{~mm}$ (interelectrode area $=6 \times 10^{-6} \mathrm{~m}^{2}$ ), a flow volume of $\mathrm{U}=9 \times 10^{-5} \mathrm{~m}^{3} \mathrm{~s}^{-1}$ is required to achieve a flow velocity of $\mathrm{v}=15 \mathrm{~m} \mathrm{~s}^{1}$, this equates to a flow rate of $5.4 \mathrm{~L} \mathrm{~min}^{-1}$. Consequently a flow rate of $5.4 \mathrm{~L} \mathrm{~min}^{-1}$ can be considered to be the minimum required for stable dissolution.

The electrolytes used in this study were $\mathrm{NaCl}$ and $\mathrm{NaNO}_{3}$; however more corrosive acid based electrolytes can be used, these corrosive electrolytes are known to attack a wide range of metal parts. A pump which would resist the corrosive action of these electrolytes was required so as not to limit the range of electrolytes which could be employed by the system. 
The Parameterisation of Electrochemical Machining Consequently the requirements for the flow system can be summarised in a number of points:

- Continuous flow velocities of up to $30 \mathrm{~L} \mathrm{~min}^{-1}$

- Pressures of up to 40 bar

- Able to withstand corrosive liquids

- Able to deal with liquids containing particulate matter (i.e. metal oxides and hydroxides)

\subsubsection{Selection of suitable pump}

Bennett [2] discusses the absence of suitable ECM pumping systems commercially available in 1968. However, since this report there have been a number of significant advances in pumping technology providing a wide range of commercially available pumps to choose from. Considering the system requirements addressed in section 4.2.1 and the need for maximum flexibility and control of the flow velocity, the advantages and disadvantages of a range of pumping systems are discussed.

The pumping systems commercially available for use with low viscosity liquids can be grouped into two categories: those operating through rotodynamic principles and those through positive displacement principles [140][141].

Rotodynamic pumps are so called because the output flow volumes and pressures achieved are a fundamental characteristic of rotational velocity for both systems. The rotodynamic systems can be further split into the categories axial and centrifugal. Axial rotodynamic pumping systems display a characteristic steep decline of operating efficiency beyond the specified optimum flow capacity and consequently are better suited to systems in which the required flow volume can be fixed. Consequently this pump was thought to be unsuitable for parameterisation measurements which required the alteration of flow volume.

Centrifugal systems produce a far broader spectrum of operating outputs (flow volumes and pressures) and the efficiency has been observed to gradually decrease outside the specified optimum conditions [141]. This system has been used to produce stable dissolution over the broad range of flow volumes and pressures encountered as the interelectrode gap closes 
The Parameterisation of Electrochemical Machining towards equilibrium, and is commonly used in commercially available ECM systems. An examination of centrifugal system parameters with reference to the work at Edinburgh University eliminated them as a possibility due to their maximum operating pressures of 25 bar. This maximum pressure would limit the investigation of stable ECM at very small, but still commercially realistic, $\mathrm{z}$ values $(0.2 \mathrm{~mm}$ and below). Another substantial limitation of the centrifugal pumping systems is the difficulty of accurately varying the flow rate. Control of the flow rate is vital in carrying out a full parameterisation of the ECM process. Finally the centrifugal pumps do not lend themselves to pumping corrosive liquids as the substance being pumped usually comes into contact with moving corroding parts within the pump itself.

Positive displacement pumps, which use a mechanical force to push liquid through the pump either through gears, pistons or diaphragms [142], were also considered for use in the ECM system. Of the positive displacement pumps commercially available, gear pumps and hydrostatic pumps were found to be incapable of providing the required flow volumes. Reciprocating piston pumps were found to be capable of producing the required volumes, however the rate of liquid delivery from this type of pump is variable. The piston action of this pump produces pulses of pressure which would be incapable of producing stable dissolution. These pulses can be smoothed out using a pulsation damper, however this damper must be pre-set to a specific operating pulse rate and hence a specific flow rate. This would not allow variation of the flow rate during machining and therefore precludes the use of a reciprocating piston pump for the ECM system under consideration.

After careful consideration of the merits of different pumping systems, a diaphragm pump was chosen for the set-up at Edinburgh University. This system combines the advantages of the high flow rates achieved using the centrifugal pump and the high pressure capability of positive displacement systems without pumping. The system selected was a Hydracell model D-25XL (Wanner Engineering Inc. USA), this pump is a diaphragm pump in which all the metallic parts are isolated from the liquid being pumped. Consequently the system can be used to pump corrosive liquids without sustaining corrosion damage to the working parts of the pump. The system provides high pressure, up to $70 \mathrm{bar}$, with flow volumes of up to $45 \mathrm{~L} \mathrm{~min}^{-1}$ possible. This more than satisfies the specifications mentioned in section 4.2.1; furthermore the system allows the flow velocity, for any specified interelectrode gap, to be varied to a high resolution while still maintaining negligible pulsing. 


\subsubsection{Flow configuration}

Figure 4.3 shows the integrated flow system, which incorporates a $3.3 \mathrm{kV}$ motor driven by an S3 frequency inverter (PIV electronics, Netherlands) to enable flow volume management through pump speed control. The electrolyte is contained in a $60 \mathrm{~L}$ capacity reservoir above the pump assembly and the electrolyte temperature is maintained at a constant temperature $\left( \pm 1^{\circ} \mathrm{C}\right.$ ) using a TN2-1 kV heater/temperature sensor (Therm-o-level Ltd, UK) working in parallel with a water driven heat exchanger. A PDH13 high pressure filter (Pall process filtration $\mathrm{Ltd}, U K$ ) is incorporated at the outlet of the pump to remove precipitated solids of diameter $>10 \mu \mathrm{m}$, however this was not used for the work in this thesis owing to the pressure drop resulting from the liquid passing through the filter.

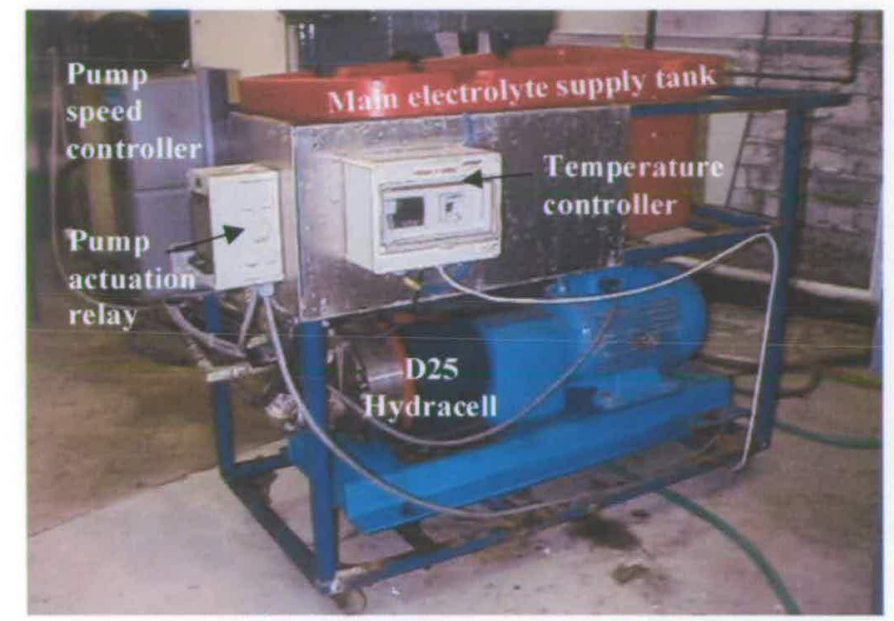

Figure 4.3: Flow system assembly including pump speed controller and electrolyte tank with temperature incorporation.

\subsection{Power system}

The main requirement for a power supply system used for constant voltage ECM is the ability to supply a potential across the ECM gap and maintain that potential regardless of the dissolution current being passed in the system. Therefore a RDOA 100/240/415 3 phase variac power supply (Bonar Brentford Electric Ltd, UK), and an isolating transformer/rectifier (Goodyear Ltd) with an output of $600 \mathrm{~A}$ at $45 \mathrm{~V}$ was chosen. In the case of low currents $(<100 \mathrm{~A})$ the voltage can be controlled through the use of regulating 
transistors and associated control electronics situated in the supply line. However, inefficiencies in the system cause the build up of heat within these devices which was considered to be too great at the high currents envisaged for the proposed ECM studies, making automated control of the voltage impractical in this situation. Accordingly a mechanical motor controlled precision arrangement was developed which was capable of adjusting the relative displacement between the primary and secondary stages of the system's variac transformer. The system under manual control was found to achieve voltage regulation to an accuracy of $\pm 1 \%$.

\subsection{Feed and control system}

A central console system was designed to control the feed, flow and power systems in the ECM set-up. The console system incorporated an S5-100U programmable logic controller (PLC) (Siemens plc) which had been programmed (using a S5-ladder programme assembly) to provide sequencing and control of ECM operations, which include:

1. Timed sequencing of flow, ECM power and tool feed systems. The objective of this sequencing is to protect the tooling assembly by ensuring the flow rate through the cell is adequate to maintain stable dissolution before the subsequent concurrent initiation of the ECM power and workpiece feed rate. The delay between these operations is at least one order of magnitude less than the sample interval used by the data logger.

2. Selection and adjustment of voltage and flow rate parameters. Control signals are generated by the PLC to enable variation in the voltage or flow rate during experiments. This input parameter control allows the precise measurement of the effect of voltage and flow rate on fundamental ECM parameters, shown in this thesis.

3. Workpiece feed control and positioning. Feed reference signals are generated at pre-defined values by the PLC. These are delivered to a DC D110 servo controller which powers a 33D-series servo/tacho motor (Unimatic plc). The stop and start positions of the feed are controlled by limiting switches connected to the PLC. 
The Parameterisation of Electrochemical Machining

To provide the workpiece drive system with stability as well as positional accuracy, a speed compensated pulsed width modulated MSM series servo driver (Infranor Ltd, Switzerland) is used. This drives the DC motor/tacho system enabling highly accurate motor velocities to be achieved. High positional resolution is achieved by using a precision ground anti-backlash ballscrew ( $6 \mathrm{~mm}$ pitch) system to drive the main feed axis. The ballscrew is connected to the motor via a high ratio (100:1) anti-backlash gearbox, ensuring a high motor velocity, thereby producing a high torque whilst maintaining the feed rate at the appropriate, relatively low values for this work $\left(\leq 1 \mathrm{~mm} \mathrm{~min}^{-1}\right)$.

The base and head of the ECM machine were donated to Edinburgh University by AmTech Ltd. The system provides the required rigidity through an I-beam construction configuration, with machining head rigidity provided using box-section construction and using four symmetrically positioned linear bearings situated down the length of travel (Figure 4.4).

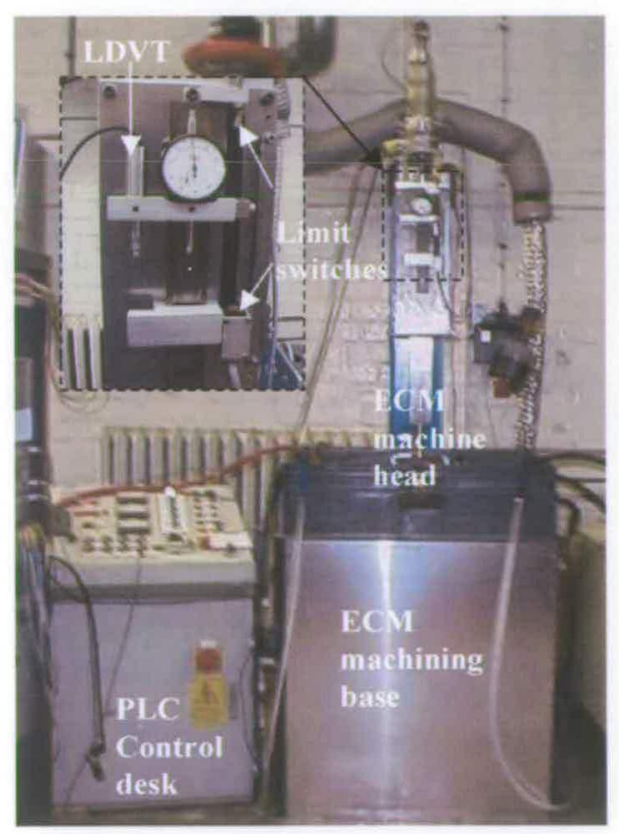

Figure 4.4: PLC control desk and machining base (insert shows LDVT workpiece position sensor and limit switches). 


\subsection{Parameter control and monitoring / data acquisition system}

The process parameters were measured and logged as follows:

\subsubsection{Segment and total dissolution currents}

These are monitored using split core, type 200 SID Hall effect transducers (LEM-HEME, Switzerland), providing a RMS accuracy of $\pm 1 \%$. The segment current Hall effect transducer monitoring system is shown in Figure 4.5.

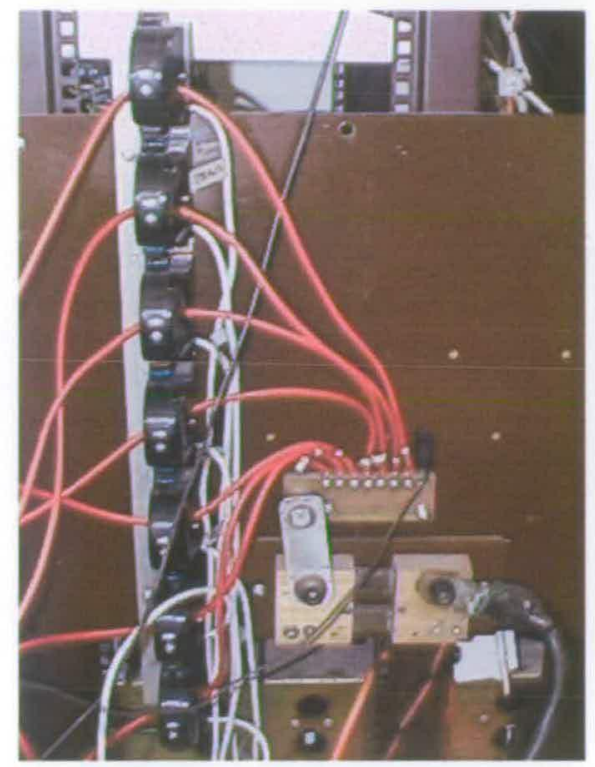

Figure 4.5: Segment current Hall effect transducer monitoring array.

\subsubsection{Workpiece feed rate}

This is monitored using a digital to analogue (DAC) module. This module processes signals from an EC6-CW25C incremental optical encoder (Omron Ltd, UK) located on the main drive shaft. The EC6-CW25C produces 1000 pulses per revolution, consequently, with the 6 mm pitch ball screw and 100:1 gear ratio, this translates to 16,000 pulses per mm of travel, giving an estimated resolution of workpiece feed rate data of $\pm 0.01 \mathrm{~mm} \mathrm{~min}^{-1}$. For 
calibration, the overall average feed rate was compared to the total feed measured over a large measured time interval according to Equation 4.2.

$$
\mathrm{w}-\mathrm{w}_{0}=\mathrm{f}\left(\mathrm{t}-\mathrm{t}_{0}\right)
$$

\section{Equation 4.2}

where the total feed is was measured by a mechanical gauge positioned on the main drive shaft.

\subsubsection{Machining voltage}

This is measured using a Type-3192 Hall effect voltage transducer (LEM-HEME, Switzerland) with an accuracy of $\pm 1.0 \%$.

\subsubsection{Inlet and outlet electrolyte temperatures}

These are measured using two class B3 wire connection resistance thermometer assemblies (Roxspur Measurement and Control, $U K)$ positioned in the electrolyte flow at the inlet $\left(\mathrm{T}_{\text {in }}\right)$ and outlet $\left(\mathrm{T}_{\text {out }}\right)$ of the ECM cell. Mixing due to placement of both inlet and outlet thermocouples is negligible (confirmed by experiments, see section 8.2). For calibration purposes water at $40{ }^{\circ} \mathrm{C}$ was introduced into the system in place of electrolyte and circulated, with $T_{\text {in }}$ and $T_{\text {out }}$ being measured with time during slow cooling of the bulk. The thermocouple temperature reading was cross referenced at regular intervals with a thermometer in the bulk solution; all three were found to agree to within $\pm 0.5^{\circ} \mathrm{C}$ at all times. The inlet and outlet thermocouple readings were observed to agree to within $\pm 0.1{ }^{\circ} \mathrm{C}$, showing that the main difference in temperature was due to thermal gradients in the bulk and that the thermocouple system can be accurately used to determine cell temperature over the range applicable to these investigations.

The programmable parameter monitoring and logging system developed can be seen in Figure 4.6 and Figure 4.7. All sensor and transducer signals were routed through a central board (Figure 4.8) allowing the system to be reconfigured easily and also allowing the use of 
The Parameterisation of Electrochemical Machining

additional monitoring equipment such as oscilloscopes and multimeters without disruption to the primary data monitoring system.

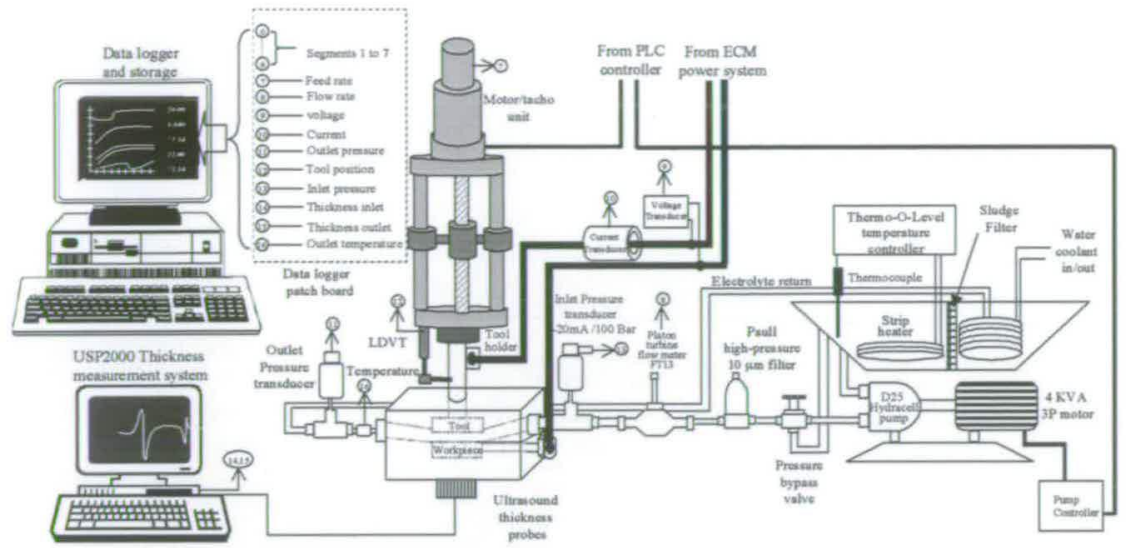

Figure 4.6: Detailed schematic of parameter monitoring system and transducer arrangement.

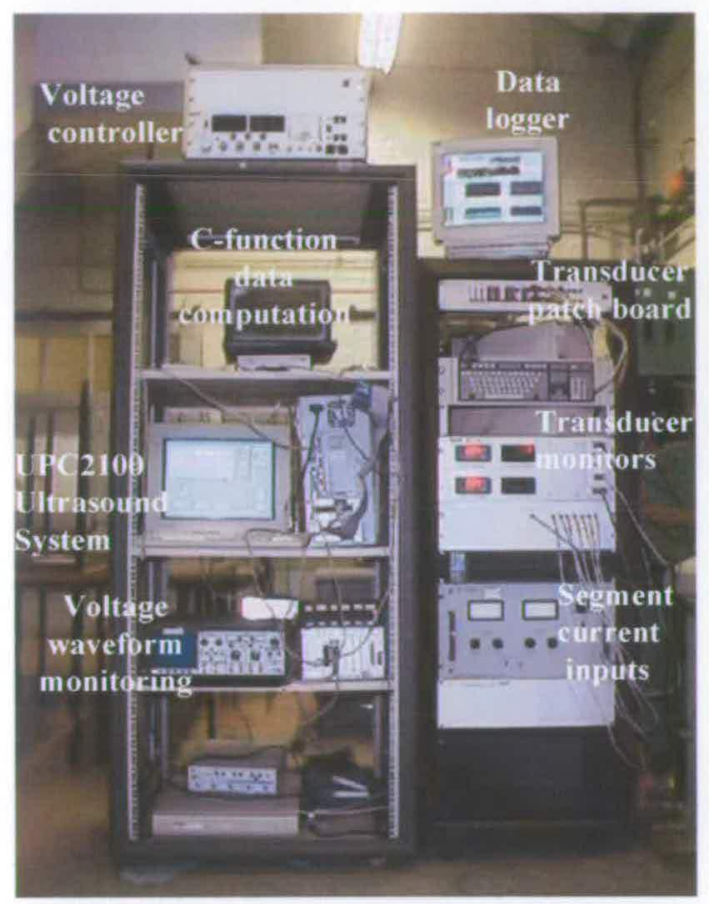

Figure 4.7: System monitoring and data logging. 


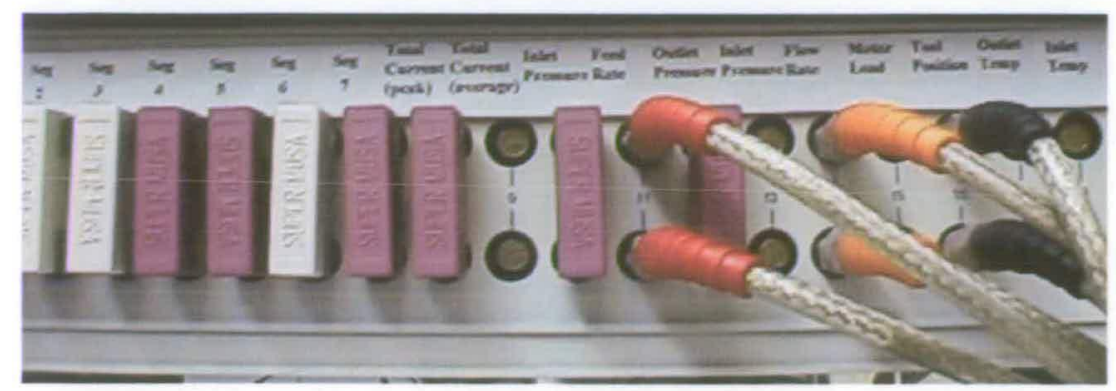

Figure 4.8: Patchboard for routing of parameter signals to data logger and/or diverting to monitors.

The data was logged using a PCI - $20377 \mathrm{~W}$ multifunction data acquisition board (Burr Brown inc. USA) located inside an Optiplex GX100 PC (Dell inc. USA). Visual Designer (Burr Brown inc. USA) was used to write in house virtual instrument control programmes to $\log$ all the system parameters in real-time. This enabled rapid software reconfiguration easily incorporating additional ECM parameters and manipulation of the data. It is also possible to incorporate into the data logging programme real-time data analysis. However, for the purpose of this work all data analysis was completed after the experiment.

\subsection{Cell 2000 configuration}

The ECM celli configuration used for the majority of this work will be referred to as cell 2000 , this is an enhanced version of the cell 2000, cited in previous works [60][67], with the addition of ultrasound measurement. Cell 2000 consists of three main elements which enclose the electrode configuration ${ }^{\mathrm{ii}}$; these are a tooling plate, a workpiece holder/feed pin assembly and the main cell enclosure (or central block and top plate)

\footnotetext{
${ }^{\mathrm{i}}$ The ECM cell is the enclosure assembly and any auxiliary components related to guiding and confining the electrolyte flow in a predetermined way.

ii The electrode configuration is the term used to describe the tool and workpiece block array and any components directly attached to these, such as the tool side cheeks and the workpiece drive pin assembly.
} 
The Parameterisation of Electrochemical Machining

Figure 4.9 shows the main cell split into the tooling plate, central block and top plate. The tooling plate is used to locate the tooling assembly precisely at the bottom of the cell (vide infra). The central block is used, along with the side cheeks, to align the electrodes and the flow feed ports and uses eight toggle clamps symmetrically around the sides to clamp the cell together. The workpiece feed port locates and seals the workpiece in alignment with the tooling face, via the central guide channel.

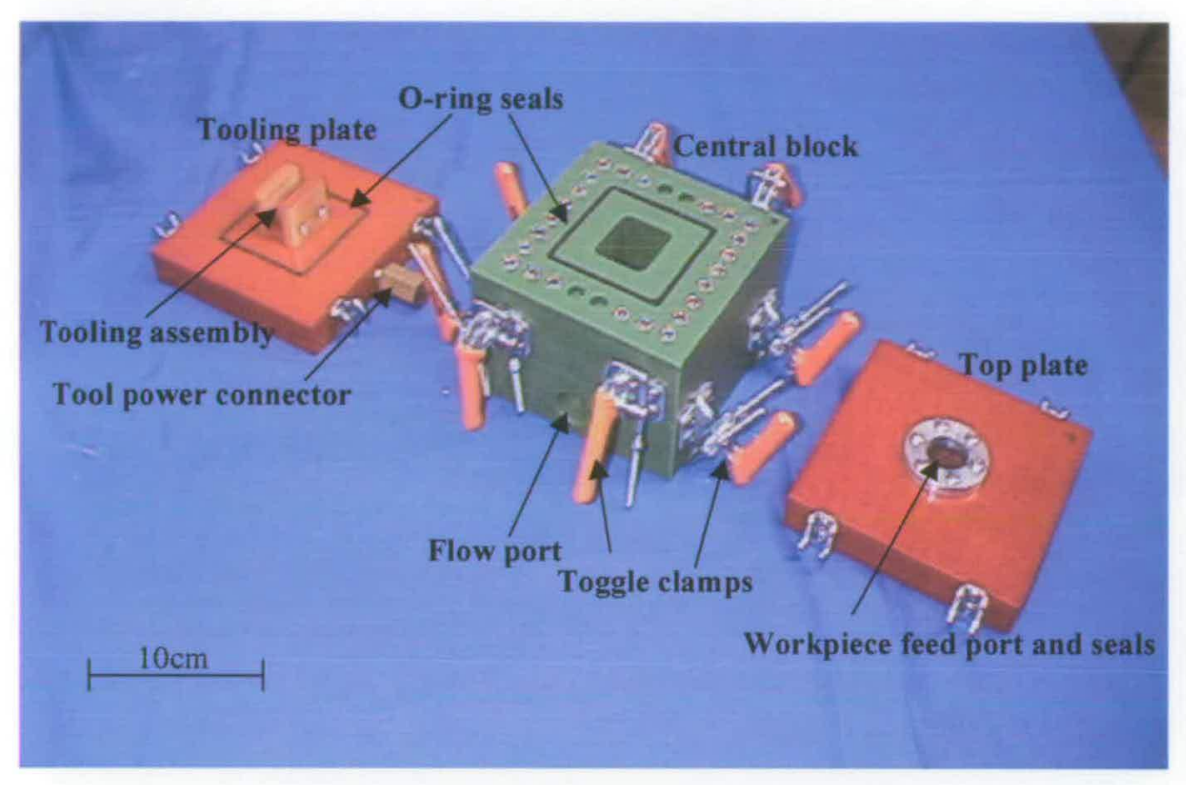

Figure 4.9: Component parts of cell 2000.

Cell 2000 was therefore designed to feed the workpiece towards the tool maintaining a uniform electrolyte flow path allowing the flow volume, and hence flow velocity, to be controlled. Feeding of the workpiece towards the tool (as opposed to vice versa) was chosen to allow precise alignment of the flow inlet and the interelectrode gap channel. In previous set-ups used at Edinburgh University the tool had been fed towards the workpiece which resulted in considerable distortion of the flow path after a period of machining (Figure 4.10). 


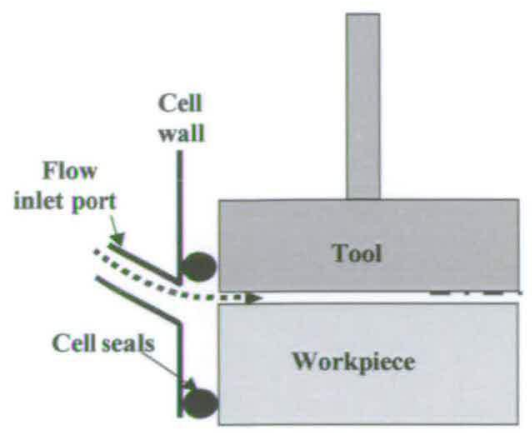

(a) Initial conditions for fixed workpiece

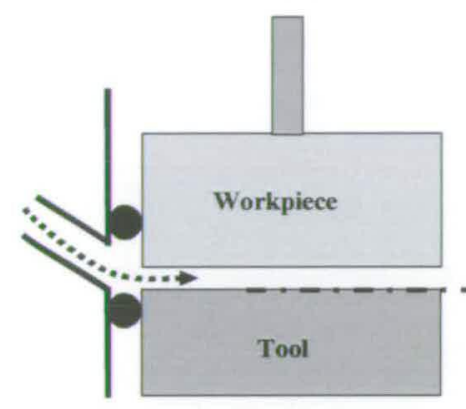

(c) Initial conditions for fixed tool

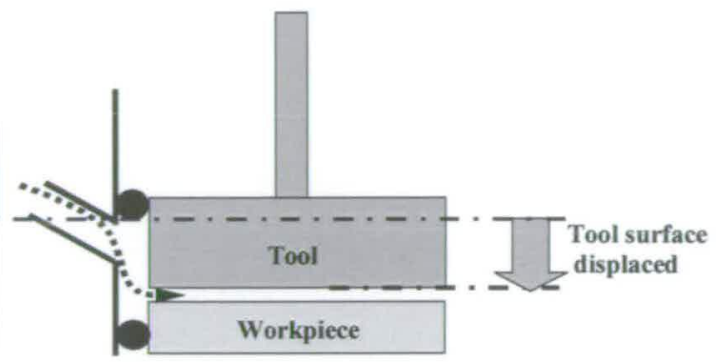

(b) End conditions for fixed workpiece

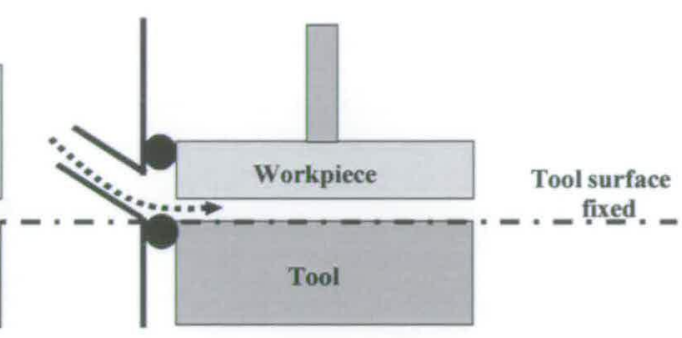

(d) Final conditions for fixed tool

Figure 4.10: Schematic showing flow variation for fixed workpiece or tool configurations.

\subsubsection{Electrode configuration}

In order to complete accurate parameterisation of the ECM system under investigation, the electrolyte flow velocity, and therefore volume, is an important variable, consequently the ability to accurately control and determine them is essential. It was vital for this work that the tool and workpiece were fully enclosed within a flow confinement system. The flow channel was encapsulated through the inclusion of insulating side cheeks attached to either side of the tool and extending beyond the tool face. These side cheeks also enclosed the workpiece and were used as a guide in positioning the electrodes. As the workpiece feeds towards the tool the side cheeks are flush with the wall at the cell inlet ensuring that the electrolyte is delivered exclusively to the interelectrode gap and is not lost around the edges of the electrode configuration. Electrolyte flow effects, such as low velocity boundary layers, are known to produce instability in dissolution systems. In cell 2000 the side cheeks were modified to include recesses to minimise these effects. These recesses move the boundary layer effects away from the active dissolution area of the tool face, ensuring that the flow velocity, in a direction perpendicular to the direction of flow, is relatively constant 
The Parameterisation of Electrochemical Machining across the entire workpiece surface, producing efficient product removal over the entire active dissolution area of the electrodes. The volume of these recesses was less than $1 \%$ of the total flow volume and hence have a negligible effect on the overall flow velocity. The general electrode configuration can be seen in Figure 4.11.

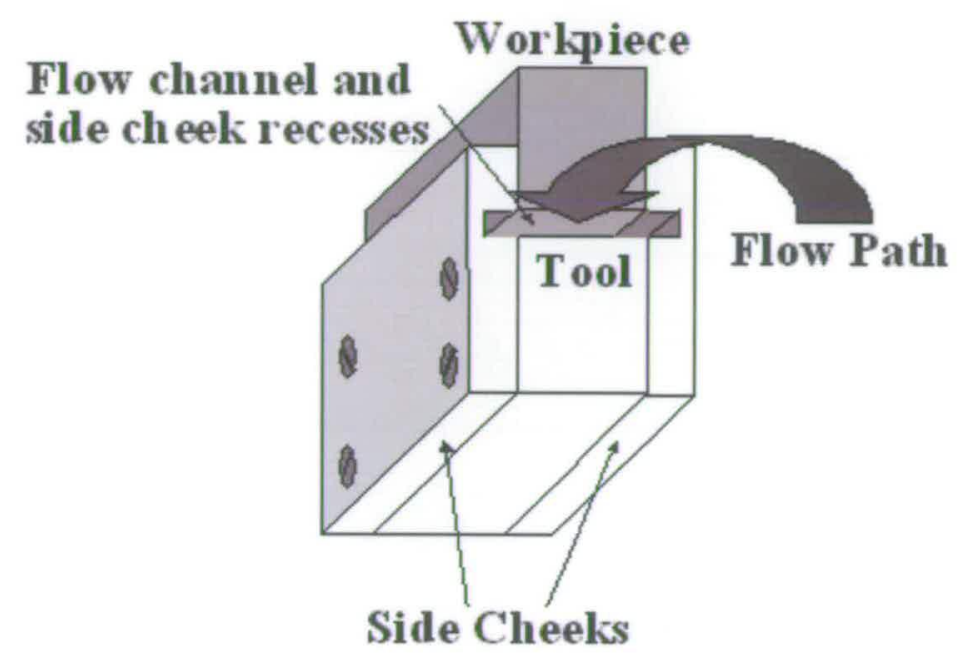

Figure 4.11: Electrode configuration for cell 2000, recesses are exaggerated for clarity.

\subsubsection{Segmented tool configuration}

An extension of the planar electrode system is the planar segmented tool. It has been shown previously that both planar and segmented tools can be used to collect current-time (chronoamperometric) data which can be used to analyse the ECM parameters for a workpiece material [60]. The segmented tool is split into seven segments and is shown in Figure 4.12 and Figure 4.13. 
The Parameterisation of Electrochemical Machining

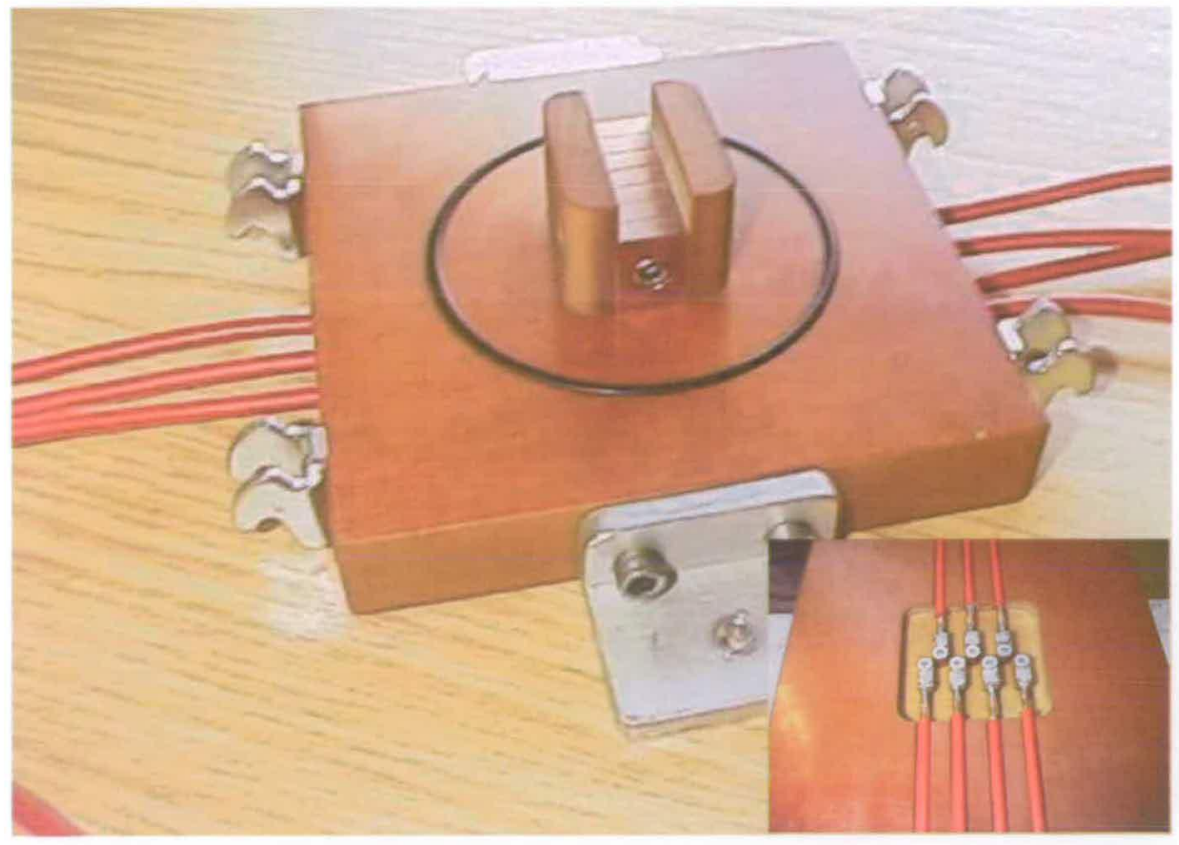

Figure 4.12: Segmented tooling plate for cell 2000 with underside view (insert) showing individual segment connections.

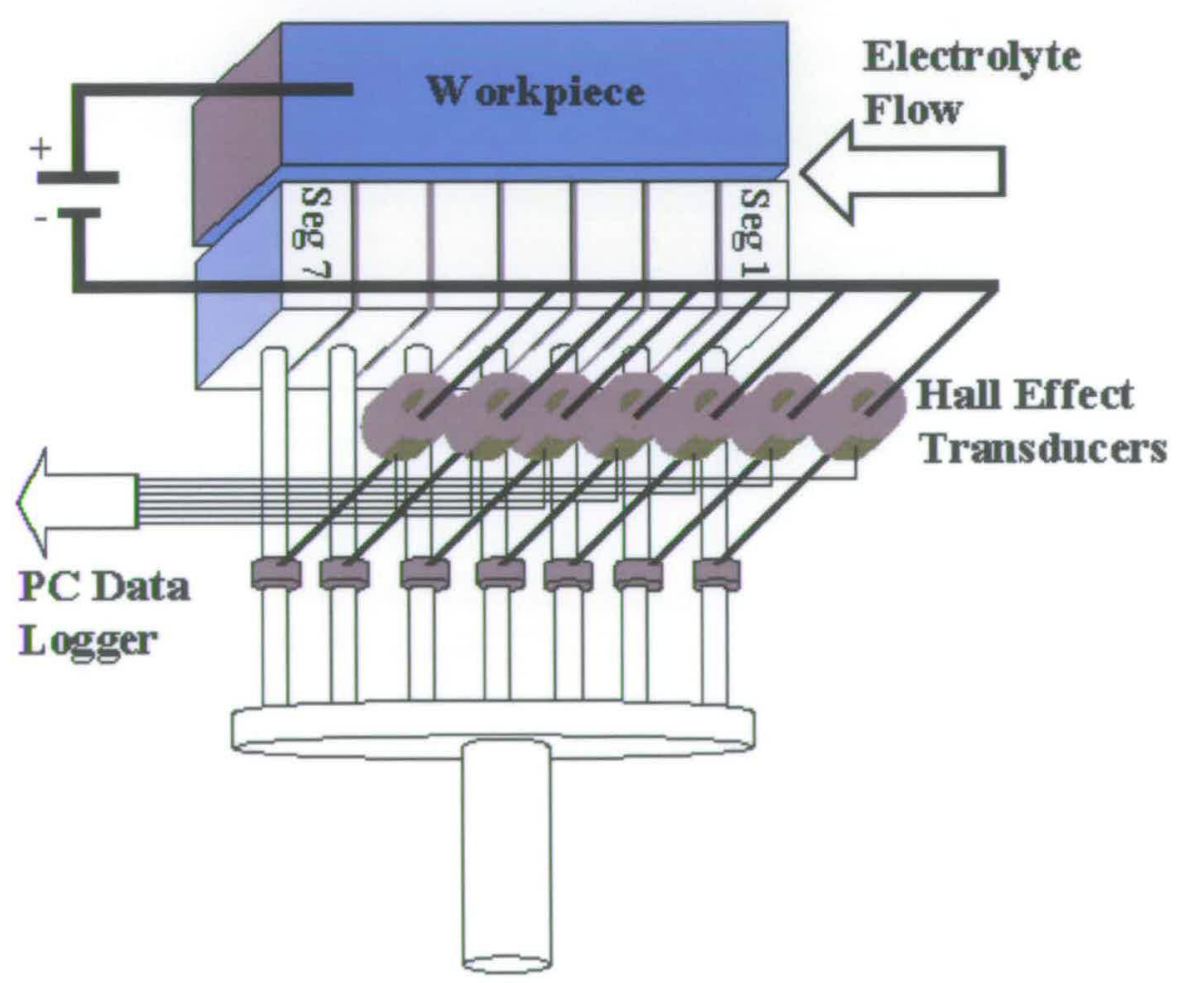

Figure 4.13: Segmented tool arrangement showing power distribution and monitoring setup. 
The segments of the tool are numbered sequentially with segment 1 at the flow inlet and segment 7 at the flow outlet. This tool arrangement was designed to allow the collection of chronoamperometric data correlating to positions along the flow path. The tool segments are insulated from one another using $0.05 \mathrm{~mm}$ mica spacers and are held together by two insulated brass bolts. The tool length is $40 \mathrm{~mm}$ and the width $15 \mathrm{~mm}$ giving a total tool area of $600 \mathrm{~mm}^{2}$. The six $0.05 \mathrm{~mm}$ mica spacers account for $(6 \times 0.05 \times 15)=4.5 \mathrm{~mm}^{2}$ of the total area giving segment areas of $85.1 \mathrm{~mm}^{2}$. Each segment is powered separately using one of an array of seven power connection pins (Figure 4.12 and Figure 4.13). An array of seven Hall effect transducers are employed to measure the individual currents flowing through each segment.

\subsubsection{Workpiece drive pin assembly}

The complete workpiece drive pin assembly can be seen in Figure 4.14(a).

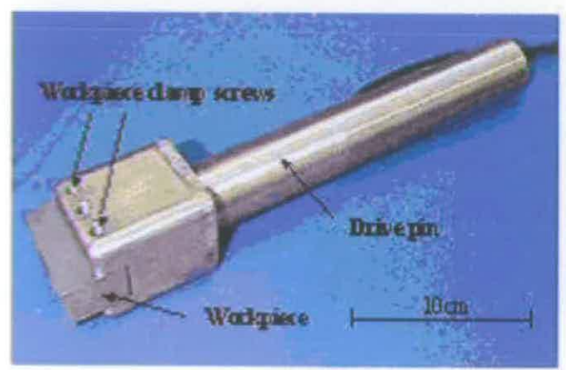

(a)

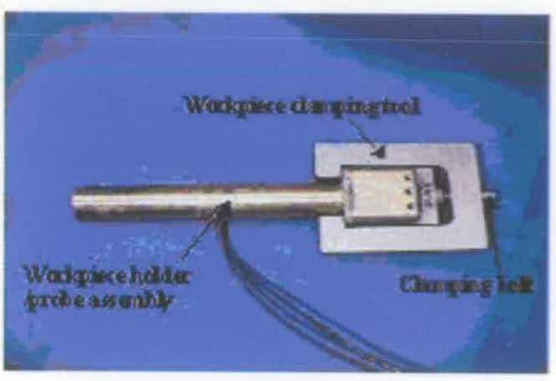

(b)

Figure 4.14: The workpiece drive pin assembly showing (a) the procedure for workpiece clamping and (b) the complete assembly.

In this enhanced cell system an ultrasound probe is also incorporated into the workpiece assembly. The ultrasound transmit/receive transducer (Buechler Krautkramer Ltd) is positioned against the upper face of the workpiece opposite segment 4 of the tool [139]. The lower face of the workpiece is perpendicular to the probe and orthogonal to the acoustic axis of the ultrasound beam. In order to achieve the maximum power for received ultrasound pulses a spring is secured to the drive pin end plate to ensure a parallel contact between the ultrasound probe and the workpiece. The spring also served to guarantee an adequate 
contact pressure between the probe and the workpiece surface. The ultrasound device collected thickness data of the workpiece throughout the ECM process. The PC based ultrasound software (USPC 2100) allows a thickness measurement to be measured and recorded in the following manner: the software instigates an initial pulse, IP, from the ultrasound probe at a frequency of $5 \mathrm{MHz}$ and measures the time of flight, TOF, of the reflection of that pulse from a surface (Figure 3.1). The USPC 2100 software then displays a scan of the initial pulse and the reflection (Figure 4.15)

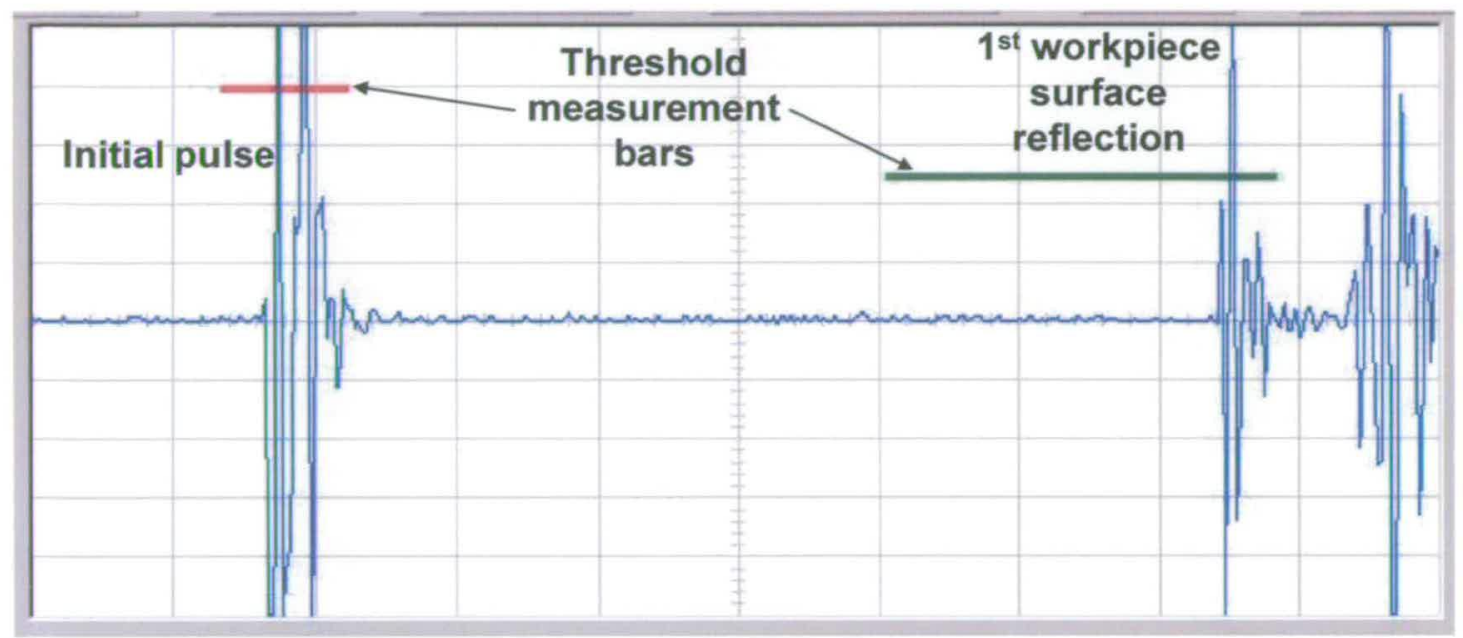

Figure 4.15: Typical USPC 2100 scan of the initial pulse, reflection and threshold bars.

The initial pulse and reflection thresholds (red and green bars respectively in Figure 4.15) are set on the PC and the USPC 2100 software calculates the thickness measurement, h, of the sample using the equation:

$$
h=\frac{\mathrm{v} \times \mathrm{TOF}}{2}
$$

\section{Equation 4.3}

where $\mathrm{v}$ is the speed of sound in the sample, set through calibration of the system with a sample of the same material with a known thickness. The pulse repetition frequency, PRF, used for this work was $500 \mathrm{~Hz}$, producing thickness measurements every $2 \mathrm{~ms}$. The USPC 2100 software allows for the alteration of the PRF up to a maximum value of $10,000 \mathrm{~Hz}$ 
producing a subsequent thickness measurement every $0.1 \mathrm{~ms}$ this will, however, considerably reduce the measurable thickness per cycle.

The ultrasound contact points are sealed so that electrolyte could not reach the probe and the workpiece located firmly against the seal by the clamping arrangement shown in Figure 4.14(b). Figure 4.16 illustrates the workpiece assembly fitted into the top plate, showing the position of the ultrasound probe.

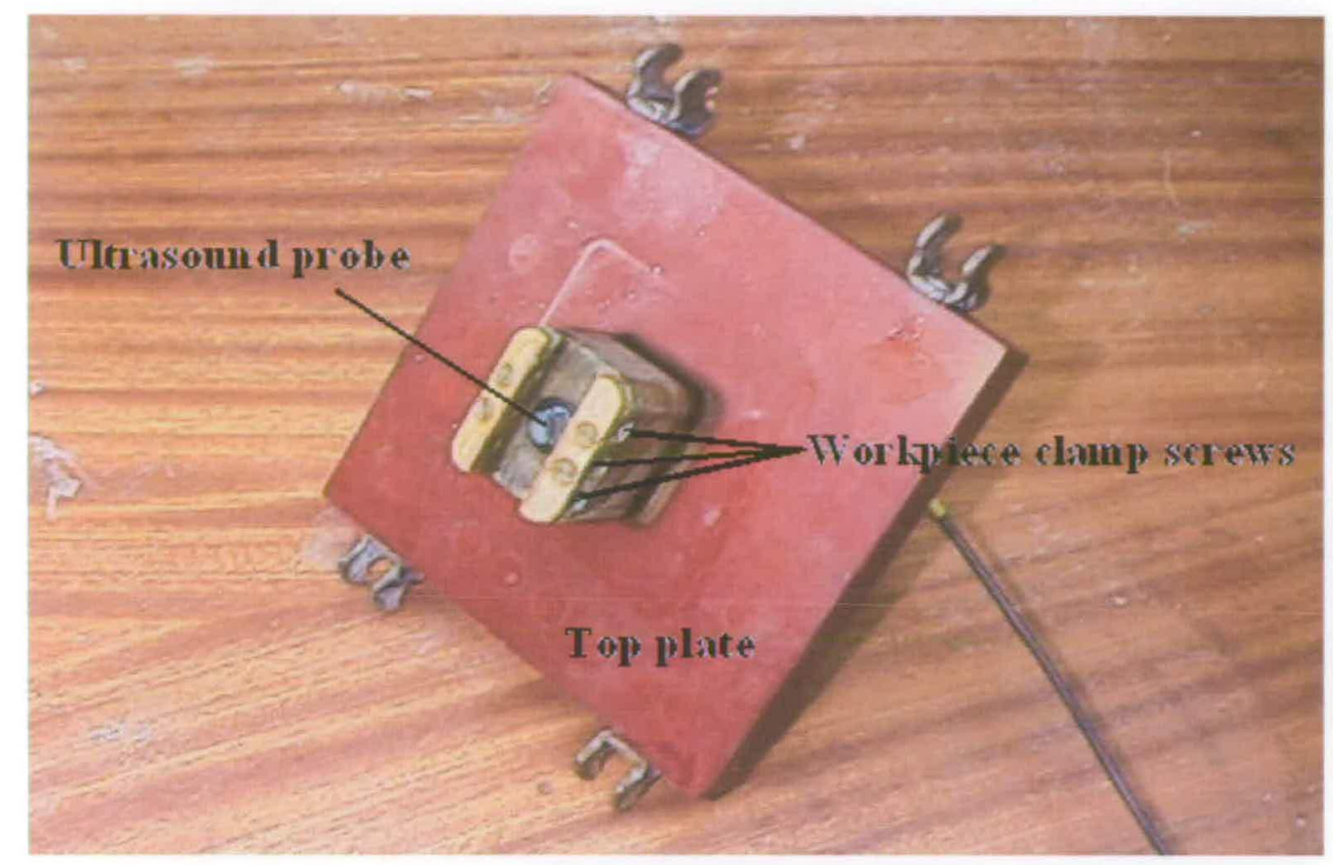

Figure 4.16: Workpiece assembly fitted into top plate showing position of ultrasound probe in cell 2000.

Accurate measurement of the interelectrode gap was carried out before and after each experiment. This measurement procedure (Figure 4.17) comprised of aligning the tool and the workpiece in the absence of the central block, inserting a feeler gauge to set the initial interelectrode gap distance required and noting the position of the drive shaft using the mechanical gauge (section 4.5.2). A polyether impression material (Permadyne, EPSE) was injected into the interelectrode gap and allowed to solidify (for at least 5 minutes until hard) to accurately determine the size of the gap (Figure 4.17, insert). After solidification the gap cast was removed and the thickness measured using a micrometer. Gap casting provided accurate initial gap measurements along with confirmation that the interelectrode gap was 
The Parameterisation of Electrochemical Machining

parallel before machining was begun. It was also carried out after machining to determine the final interelectrode gap accurately and assess any tapering present.

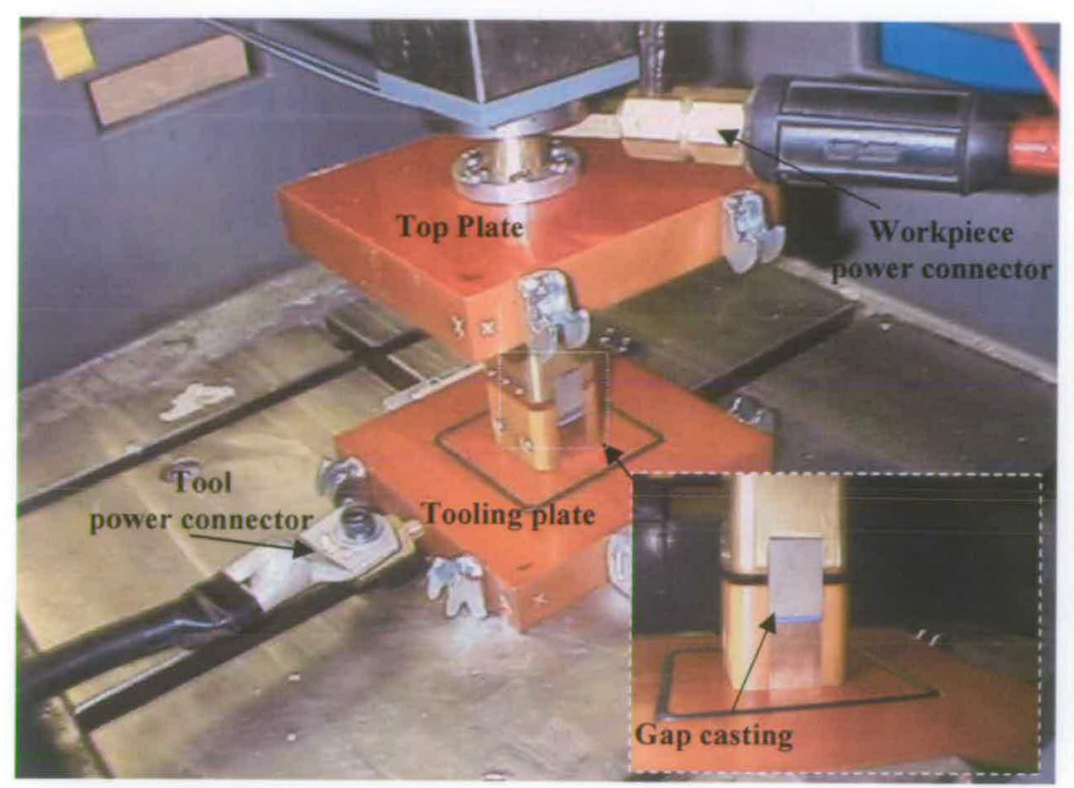

Figure 4.17: Cell 2000 interelectrode gap measurement technique.

After the initial set-up procedures the central section of the cell was positioned onto the tool plate and sealed using the four lower toggle clamps, the workpiece was fed back to the appropriate initial position, using the mechanical gauge measurement taken during casting, and the top four toggle clamps fastened to ensure sealing of the cell against electrolyte loss into the machining chamber. Finally, before machining can commence, the flow inlet and outlet and gap voltage monitoring clips were attached as shown in Figure 4.18. 
The Parameterisation of Electrochemical Machining

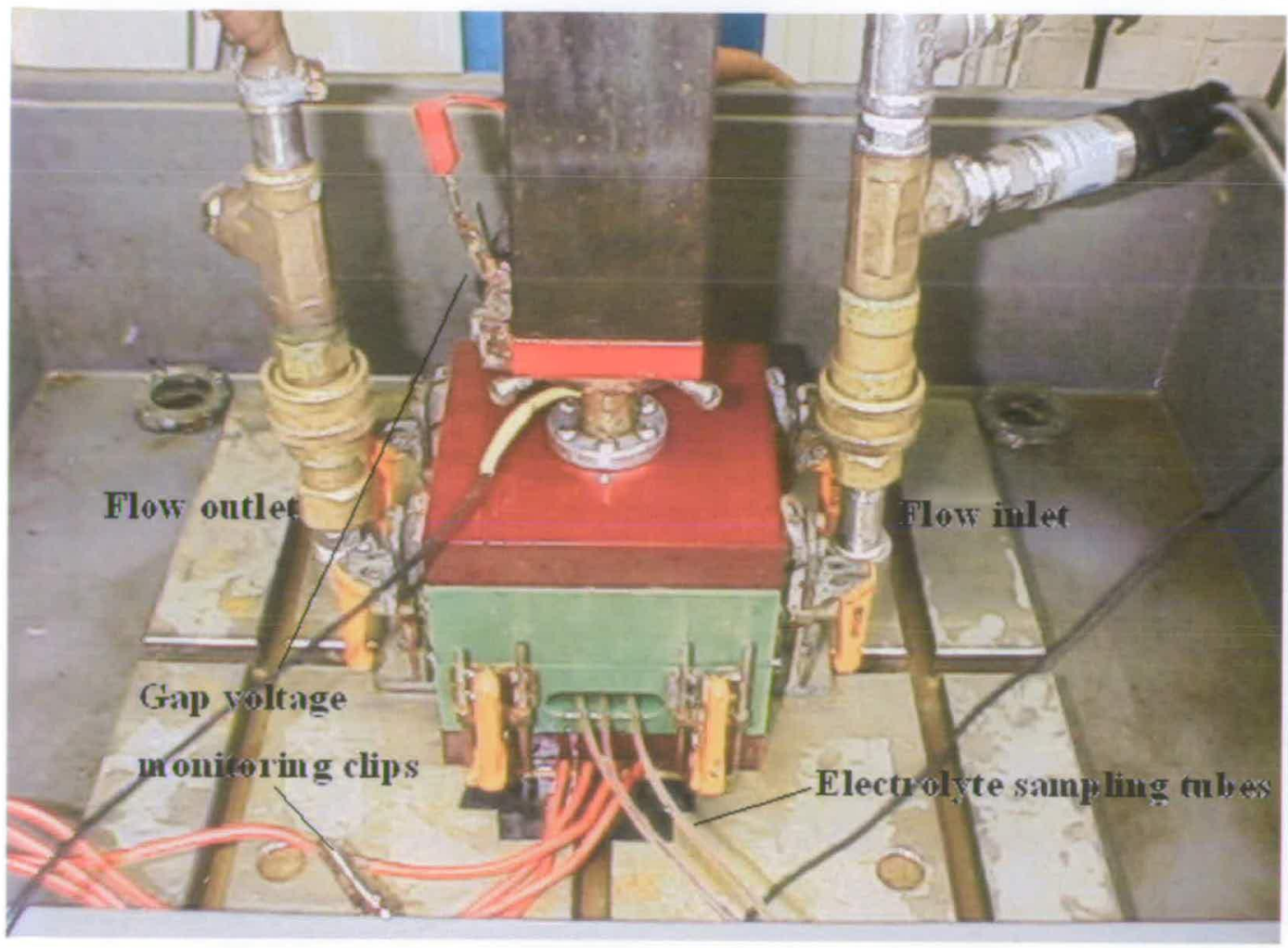

Figure 4.18: Cell 2000 assembled in machining chamber.

\subsubsection{Electrolyte sampling tubes}

Tubes ( $0.4 \mathrm{~mm}$ in diameter) have also been used to allow electrolyte sampling to take place in three positions along the flow path. The inlet sampling tube (IT) is placed above segment 2, the middle sampling tube (MT) above segment 4 and the outlet sampling tube (OT) above segment 6. These tubes can be seen in Figure 4.18 and a schematic of their positioning is shown in Figure 4.19. 


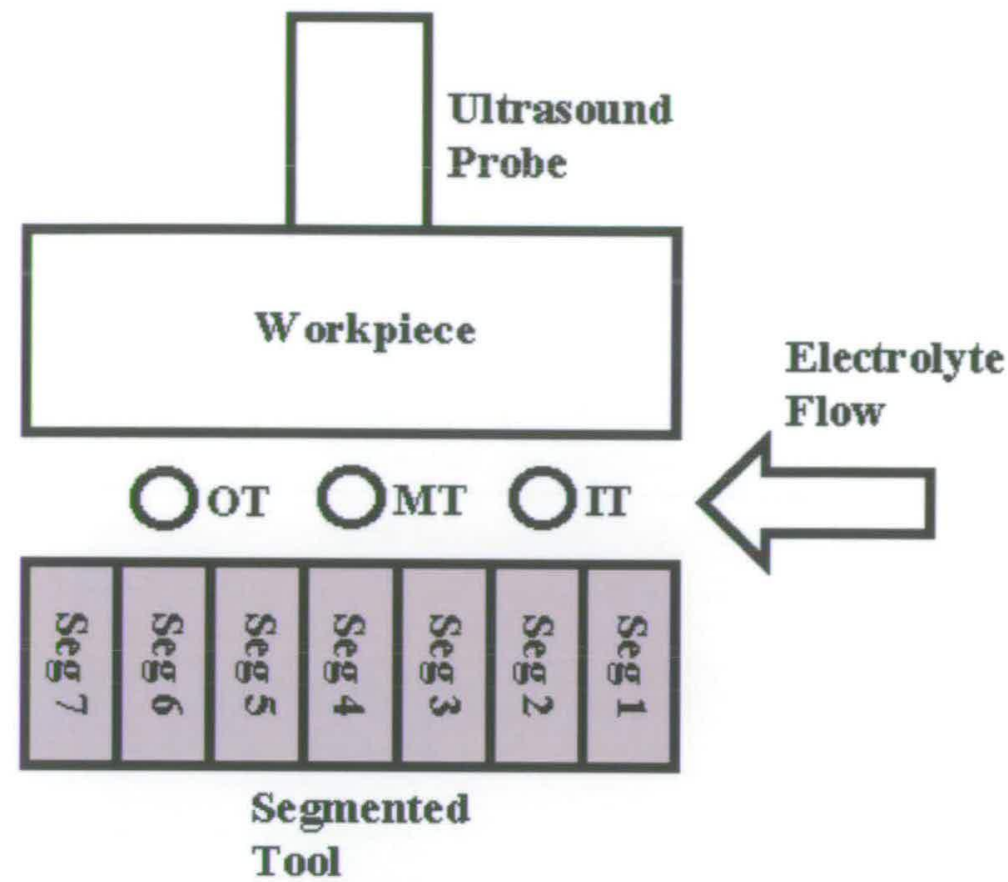

Figure 4.19: Schematic showing positioning of electrolyte sampling tubes along flow path.

\subsection{Cell 2004 configuration}

The purpose of the construction of cell 2004 was to position an ultrasound probe into the tool assembly with the aim of assessing the feasibility of direct real-time gap measurements in this configuration. In many ways the configuration of this cell 2004 is similar to cell 2000 , section 4.6. This includes the three main elements which enclose the electrode configuration of a tooling plate, a workpiece holder/feed pin assembly and the main cell enclosure (or central block and top plate)

However, in the cell 2004 the dimensions of these elements are different as is the ultrasound set-up configuration. Figure 4.20 shows the main cell split into the tooling plate, the central block and a top plate with the workpiece holder/feed pin assembly positioned through the top plate. The workpiece is again fed downwards towards the tool for the same reasons as those discussed for cell 2000 in section 4.6. 


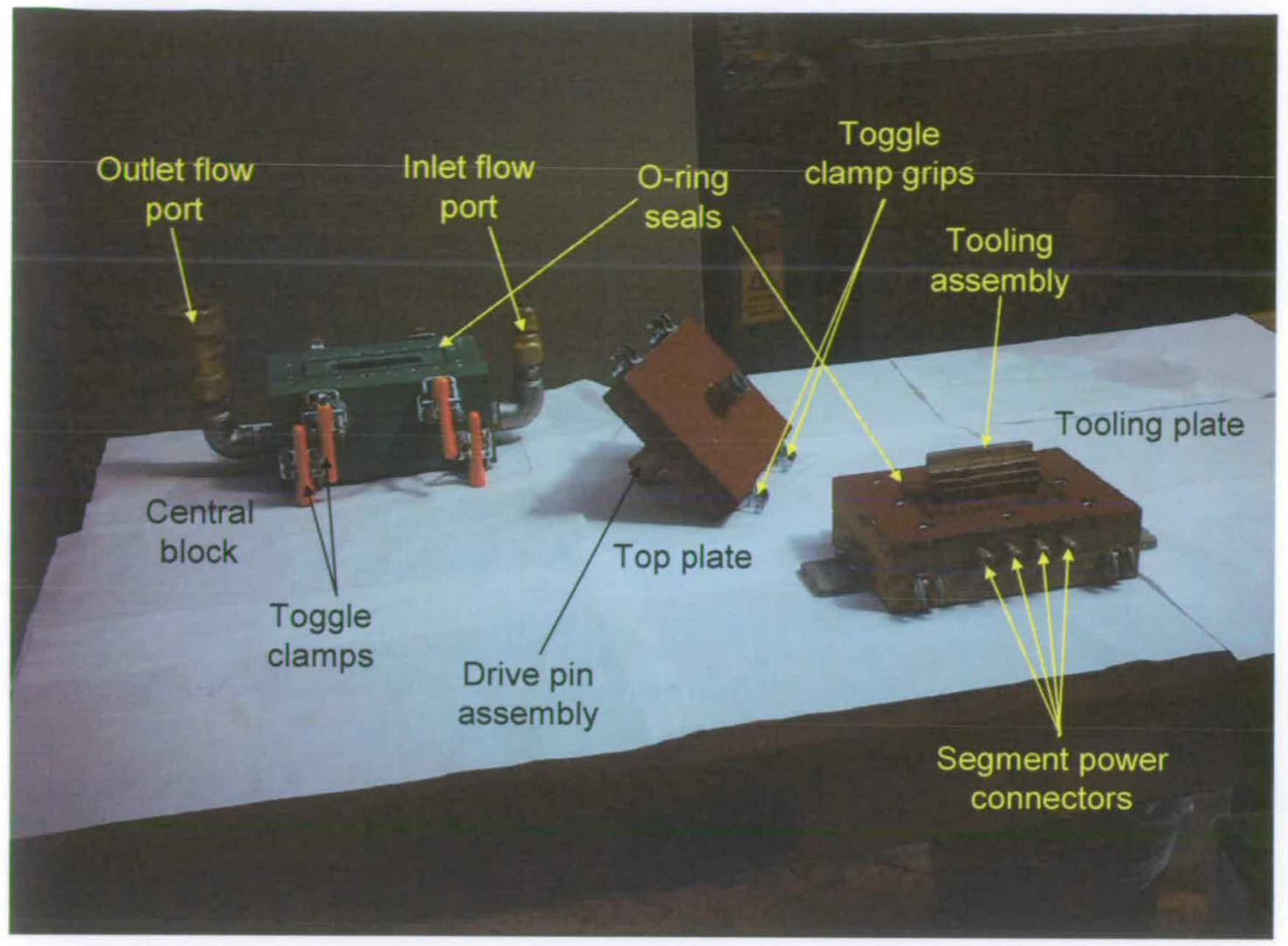

Figure 4.20: Component parts of cell 2004.

The flow channel is encapsulated through the inclusion of insulating side cheeks attached to either side of the tool and extending beyond the tool face. These side cheeks also enclose the workpiece and are used as a guide in positioning the electrodes (Figure 4.21). 


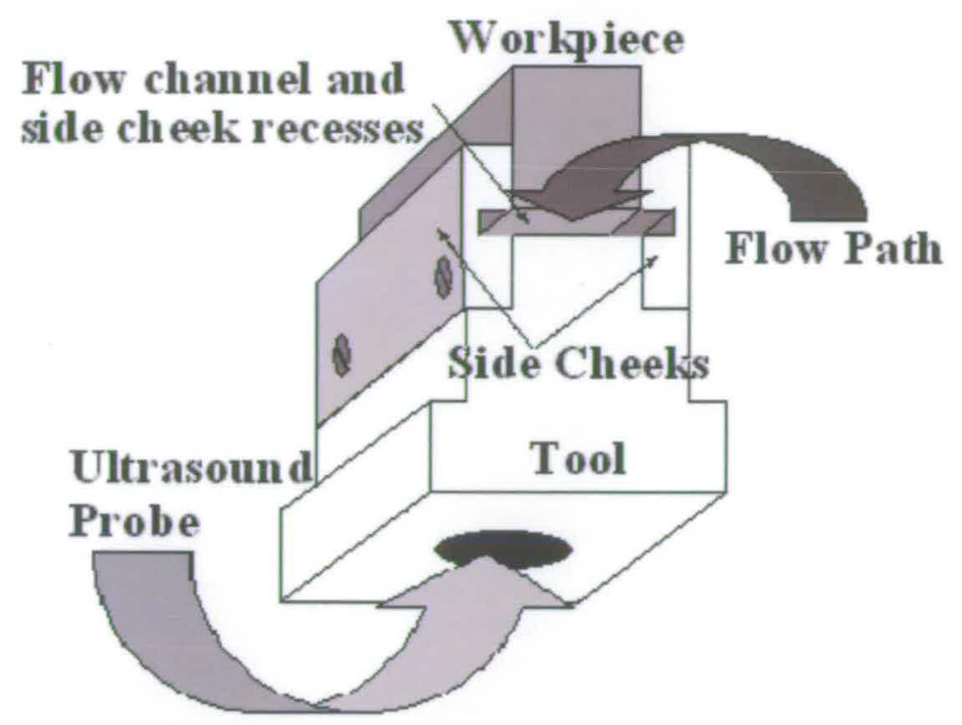

Figure 4.21: Electrode configuration for the segment containing the ultrasound probe for cell 2004, recesses are exaggerated for clarity.

The tool is split into four segments and is shown in Figure 4.22. The segmented tool arrangement, power distribution and monitoring set-up is similar to that depicted in Figure 4.13 , but in this case for only 4 segments. 
The Parameterisation of Electrochemical Machining

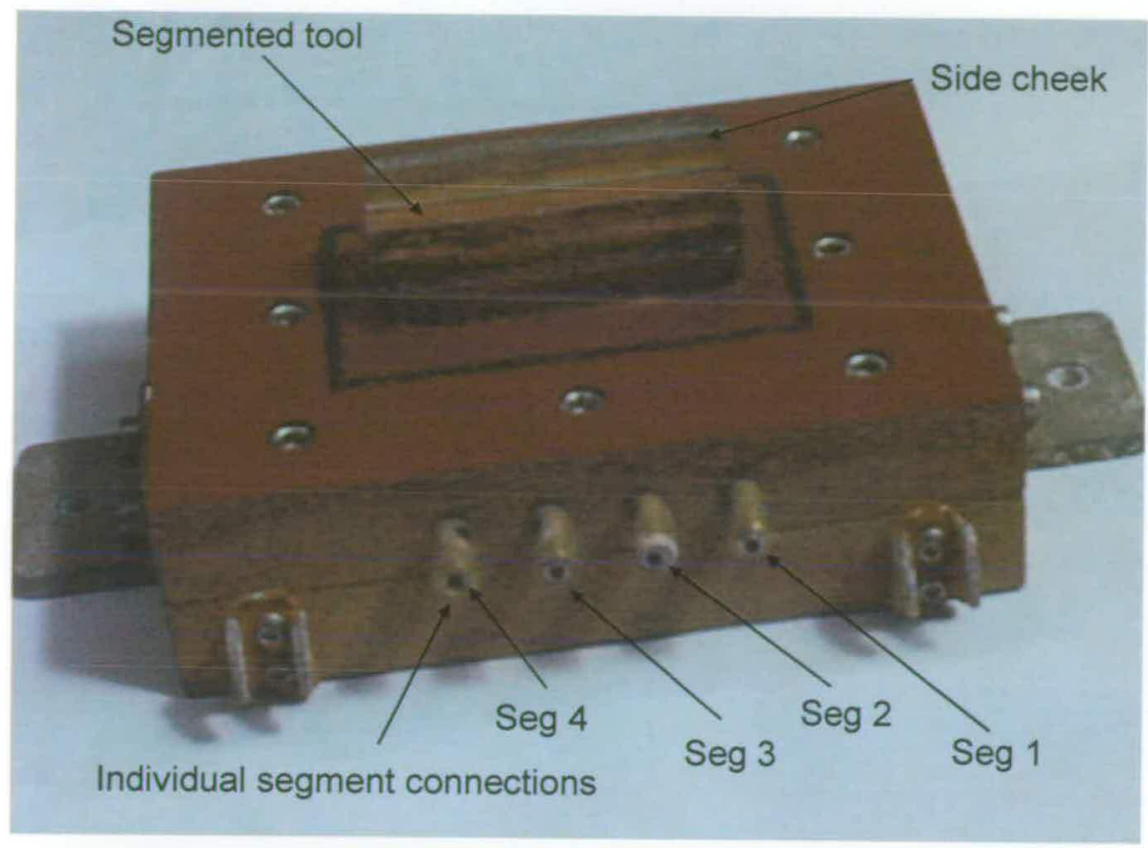

Figure 4.22: Segmented tooling plate for cell 2004 with front side cheek removed to show individual segment connections.

The segments of the tool are numbered sequentially with segment 1 at the flow inlet and segment 4 at the flow outlet. The tool segments were insulated from one another using 0.05 $\mathrm{mm}$ mica spacers. The tool length was $80 \mathrm{~mm}$ and the width $7.5 \mathrm{~mm}$ giving a total tool area of $600 \mathrm{~mm}^{2}$. The three $0.05 \mathrm{~mm}$ mica spacers accounted for $(3 \times 0.05 \times 7.5)=1.125 \mathrm{~mm}^{2}$ of the total area giving segment areas of $149.7 \mathrm{~mm}^{2}$. Each segment was powered separately using one of an array of four power connection pins (Figure 4.22 and Figure 4.13). An array of four Hall effect transducers were employed to measure the individual currents flowing through each segment.

The complete workpiece drive pin assembly can be seen in Figure 4.23. 
The Parameterisation of Electrochemical Machining

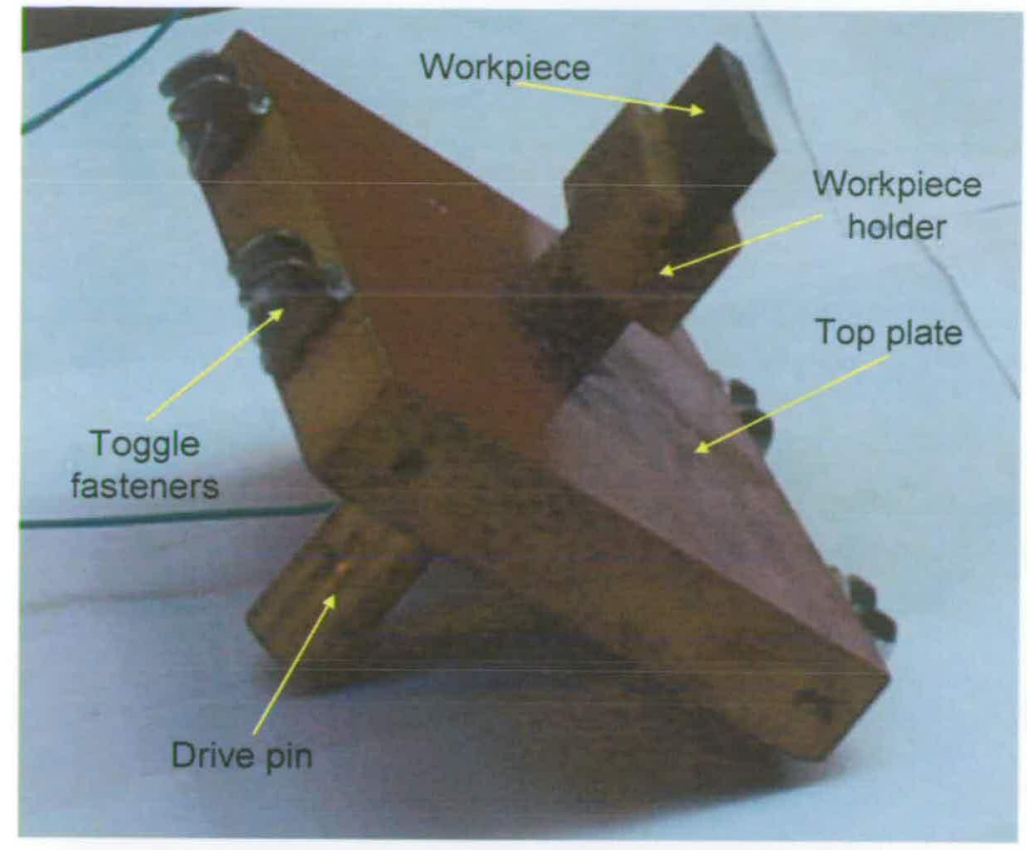

Figure 4.23: The workpiece drive pin assembly and top plate.

The ultrasound transmit/receive transducer (Buechler Krautkramer Ltd) is positioned inside segment 2, $6 \mathrm{~mm}$ away from the face of the tool (Figure 4.24).

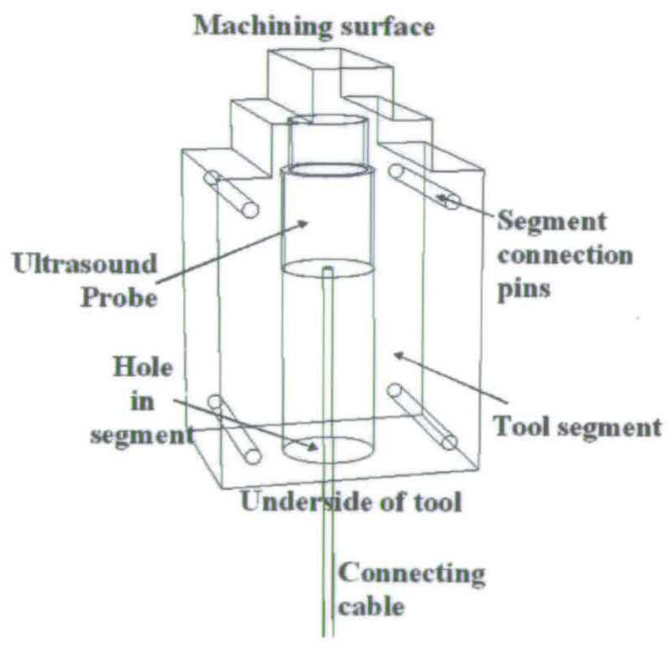

Figure 4.24: Schematic of segment 2 showing ultrasound probe positioning.

The flexible design of the tool, with equal sized segments being held together by insulated brass screws, made it possible to exchange the positions of segments allowing ultrasound 
The Parameterisation of Electrochemical Machining measurements to be collected from behind any segment. The upper face of the tool was perpendicular to the probe and orthogonal to the acoustic axis of the ultrasound beam, as for cell 2000. In order to achieve the maximum power for received ultrasound pulses a spring was placed behind the ultrasound probe to ensure a parallel contact between the ultrasound probe and the underside of the tool. The spring also served to guarantee an adequate contact pressure between the probe and the tool. The ultrasound device collects interelectrode gap data. The ultrasound contact points are sealed so that electrolyte could not reach the probe.

Accurate measurement of the interelectrode gap before and after each experiment and alignment of the cell was carried out as detailed for cell 2000 (section 4.6.2). Cell 2004 is assembled in a similar fashion to cell 2000 with the central block being used along with the side cheeks to align the electrodes and eight supporting toggle clamps placed symmetrically around the sides used to clamp the cell together (Figure 4.25)

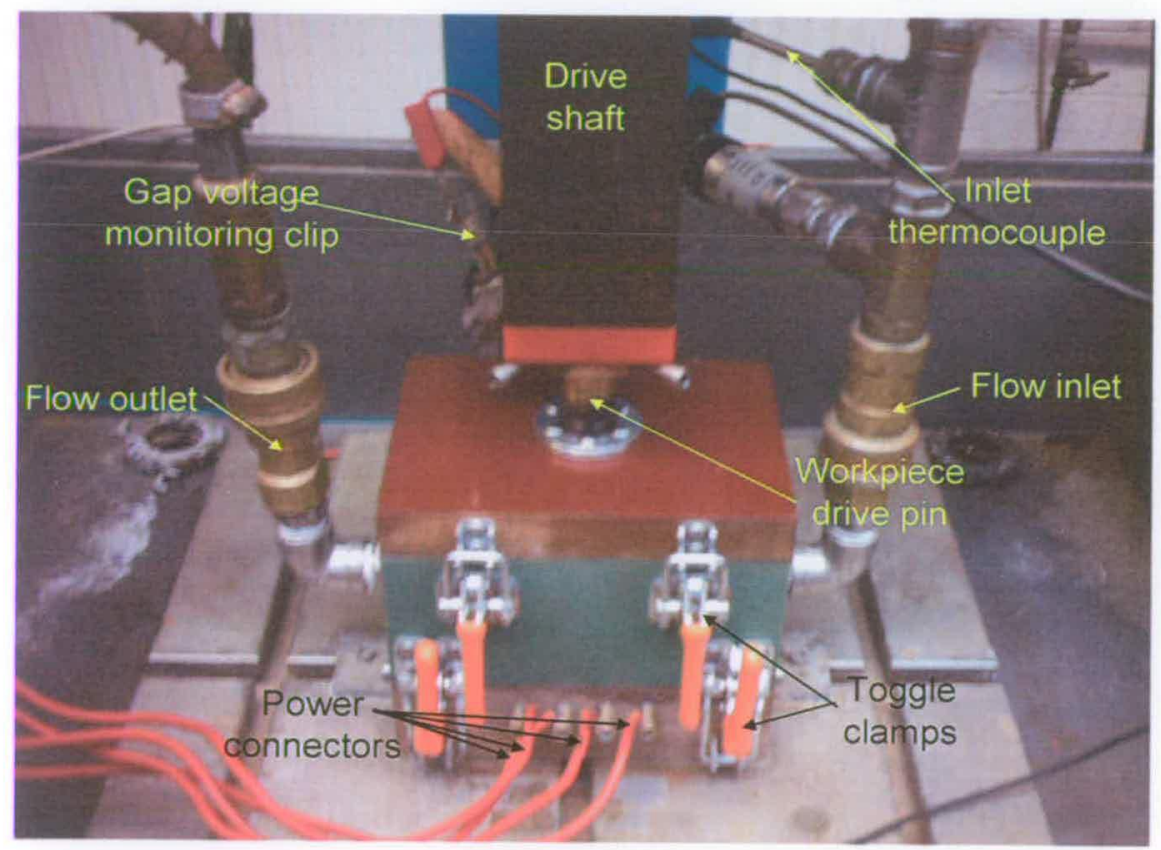

Figure 4.25: Cell 2004 assembled in machining chamber.

In order to directly compare cell 2000 and cell 2004, cell 2004 was directly adapted by incorporating the ultrasound probe behind the workpiece (Figure 4.26), an equivalent configuration to cell 2000 (section 4.6) 
The Parameterisation of Electrochemical Machining

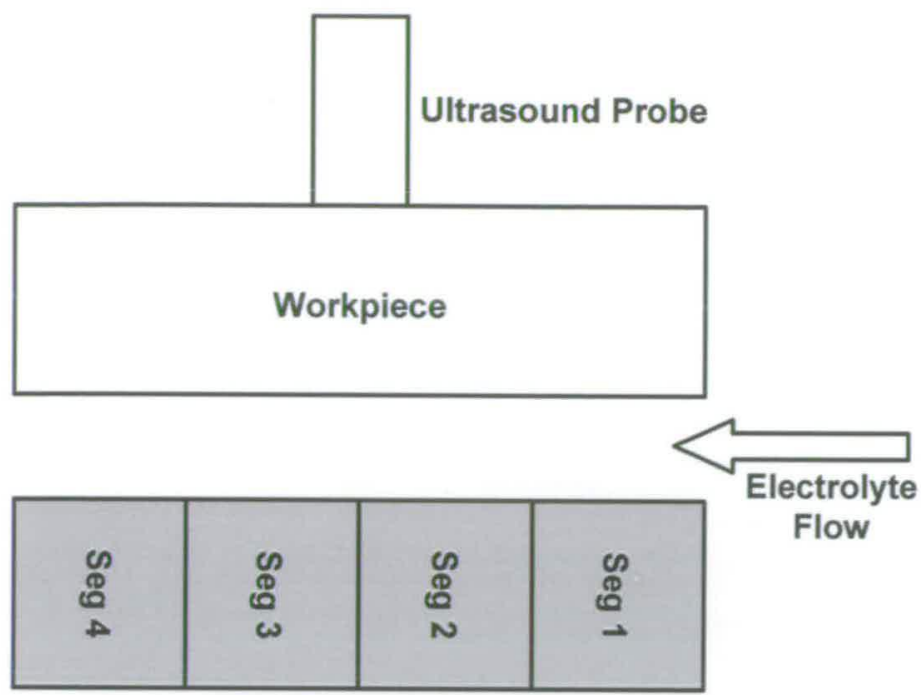

Segmented Tool

Figure 4.26: Schematic showing alternative configuration of cell 2004, used for comparison with cell 2000 data.

Unlike cell 2000, cell 2004 does not include electrolyte sampling tubes along the flow path.

\subsection{Experimental Conditions}

\subsubsection{Tool and workpiece materials}

The metals used in this thesis were the stainless steels SS410 and Jethete, and Ti 6/4 and In718, the atomic compositions of these materials is shown in Table 4.1.

\begin{tabular}{|c|c|c|c|c|c|c|c|c|c|c|c|c|c|c|c|c|c|c|}
\hline Alloy & Fe & Cr & Ni & Mo & Mn & Cu & C & S & P & Si & N & V & Co & Al & Ti & B & Ta & O \\
\hline J & $80.63^{\mathrm{b}}$ & $12.50^{\mathrm{a}}$ & $3.00^{\mathrm{a}}$ & $2.00^{\mathrm{a}}$ & $0.90^{\mathrm{a}}$ & - & $0.13^{\mathrm{a}}$ & 0.02 & 0.03 & 0.35 & $0.04^{\mathrm{a}}$ & $0.40^{\mathrm{a}}$ & - & - & - & - & - & - \\
\hline SS410 & 85.50 & 12.75 & 0.23 & 0.01 & 0.72 & 0.08 & 0.14 & 0.02 & 0.04 & 0.49 & 0.02 & - & - & - & - & - & - & - \\
\hline In718 & 18.23 & 18.37 & 53.75 & 3.02 & 0.19 & 0.03 & 0.06 & 0.002 & 0.005 & 0.09 & - & - & 0.23 & 0.5 & 1.00 & 0.004 & 5.22 & - \\
\hline Ti 6/4 & 0.17 & - & - & - & - & - & 0.01 & - & - & - & 0.02 & 3.76 & - & 5.89 & 89.91 & - & - & 0.15 \\
\hline
\end{tabular}

Table 4.1: Composition in atomic weight percent of the stainless steels Jethete (J), SS410, ${ }^{a}$ maximum amount, ${ }^{b}$ minimum amount, and $\operatorname{In} 718$ and Ti 6/4. 
The Parameterisation of Electrochemical Machining

The workpieces were heat treated prior to use to remove any compositional variation according to the manufacturer's (Bodycote International Plc.) annealing process; heating in air at $990^{\circ} \mathrm{C}$ for 18 mins, then $670{ }^{\circ} \mathrm{C}$ for 36 mins and at $655^{\circ} \mathrm{C}$ for 36 mins.

\subsubsection{Electrolytes}

A reservoir of $60 \mathrm{~L}$ of electrolyte was used with an initial temperature of $30^{\circ} \mathrm{C} .5 .0 \%, 12.0$ $\%, 15.0 \%$ and $25.0 \% \mathrm{w} / \mathrm{w}$ solutions $\left(0.85 \mathrm{~mol} \mathrm{dm}^{-3}, 2.05 \mathrm{~mol} \mathrm{dm}^{-3}, 2.56 \mathrm{~mol} \mathrm{dm}{ }^{-3}\right.$ and 4.27 mol dm${ }^{-3}$ respectively) of sodium chloride and $5.0 \%, 15.0 \%, 21.7 \%$ and $25.0 \% \mathrm{w} / \mathrm{w}$ solution of sodium nitrate were investigated $\left(0.59 \mathrm{~mol} \mathrm{dm}^{-3}, 1.77 \mathrm{~mol} \mathrm{dm}^{-3}, 2.56 \mathrm{~mol} \mathrm{dm}^{-3}\right.$ and $2.95 \mathrm{~mol} \mathrm{dm}^{-3}$ respectively) (Anderson, Gibb and Wilson). The electrolyte conductivity, $\kappa$, was measured for the $5.0 \%$ chloride and nitrate electrolytes and the $12.0 \%$ chloride and $15.0 \%$ nitrate electrolytes. Previous studies have shown $\kappa=0.20 \pm 0.1 \mathrm{~S} \mathrm{~cm}^{-1}$ for $15.0 \%$ chloride and $21.7 \%$ nitrate solutions [137][143]. The $25.0 \%$ electrolyte solutions were normalised to the $\mathrm{I}-\mathrm{V}_{0}$ plots for $15.0 \%$ electrolyte concentration for the chloride data and $21.7 \%$ electrolyte concentration for the nitrate data. For data taken with thermocouples fitted to the system (section 4.5.4) $\kappa$ was measured and calibrated for temperature dependence where possible. Unless otherwise stated, the electrolyte flow rates used were 16.0 L min $\mathrm{mor}^{-1}$ the cell 2000 and $8.0 \mathrm{~L} \mathrm{~min}^{-1}$ for cell 2004. Any electrolyte conductivity measurements were made with a 4010 Conductivity Meter (Jenway).

\subsubsection{Typical ECM conditions}

Unless otherwise stated, the experimental conditions used throughout this thesis, for both cell 2000 and cell 2004, were as follows.

$\mathrm{z}_{0}=1.00 \pm 0.1 \mathrm{~mm}$; both $\mathrm{z}_{0}$ and $\mathrm{z}_{\infty}$ values were confirmed by casting using the polyether impression material. The experiments were conducted by first machining to the steady state machining current ("equilibrium machining conditions") at $\mathrm{f}=0.5 \mathrm{~mm} \mathrm{~min}^{-1}$ (the approach phase), when a rising current transient was recorded. The feed was then halted, ( $f=0 \mathrm{~mm}$ $\min ^{-1}$ ), when dissolution of the workpiece resulted in the recession of the workpiece surface away from the tool (the retract phase) producing a falling current transient (approach/retract experiments). In some cases the electrodes were then allowed to approach steady state 
The Parameterisation of Electrochemical Machining

machining again by re-establishing $\mathrm{f}=0.5 \mathrm{~mm} \mathrm{~min}^{-1}$, and producing a rising current transient (the re-approach phase, approach/retract/re-approach experiments). 


\section{Chapter 5}

Feasibility of direct gap measurement using ultrasound behind the tool. 


\subsection{Introduction}

The purpose of cell 2004 was to position an ultrasound probe into the tool assembly with the aim of assessing the feasibility of direct real-time gap measurements in this configuration (Figure 5.1).

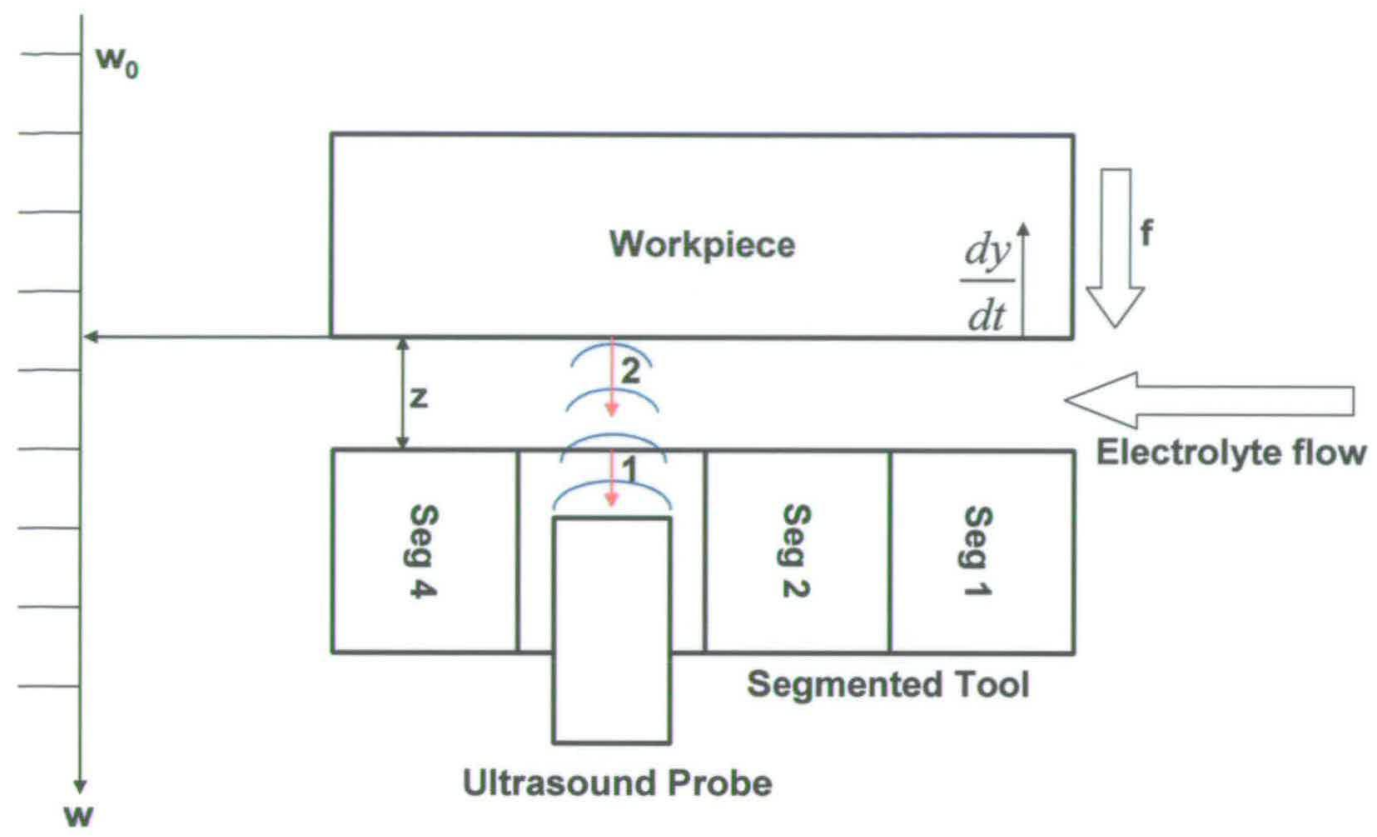

Figure 5.1: Schematic diagram of ultrasound system for direct gap measurement.

Figure 5.1 shows the positioning of the ultrasound probe in segment 3 of the tool. The reflections 1 and 2 indicate the reflection of the ultrasound pulse from the tool surface $\left(1^{\text {st }}\right.$ tool surface reflection, Figure 5.2) and the reflection of the ultrasound pulse from the workpiece surface $\left(1^{\text {st }}\right.$ gap reflection, Figure 5.2). Depending on the attenuation of the signal, on reflection at each interface, multiple gap reflections may be observed between each ultrasound pulse. If the speed of sound in the electrolyte medium, $v$, is known the interelectrode gap, $\mathrm{z}$, can be calculated directly using the difference in the time of flight of reflections 1 and 2, TOF, by: 
The Parameterisation of Electrochemical Machining $z=\frac{\mathrm{v} \times \mathrm{TOF}}{2}$

\section{Equation 5.1}

This would enable direct measurement of $\mathrm{z}$ during the experiment for the measured TOF.

\subsection{Initial calibration - with power off}

The cell 2004 set-up was initially calibrated with no power supplied to the system. The ultrasound signal through $\mathrm{H}_{2} \mathrm{O}$ at a flow rate of $\mathrm{U}=8.0 \mathrm{~L} \mathrm{~min}{ }^{-1}$, was found to be significant and measurable providing a direct measurement of $\mathrm{z}$ for the system (Figure 5.2).

Detection

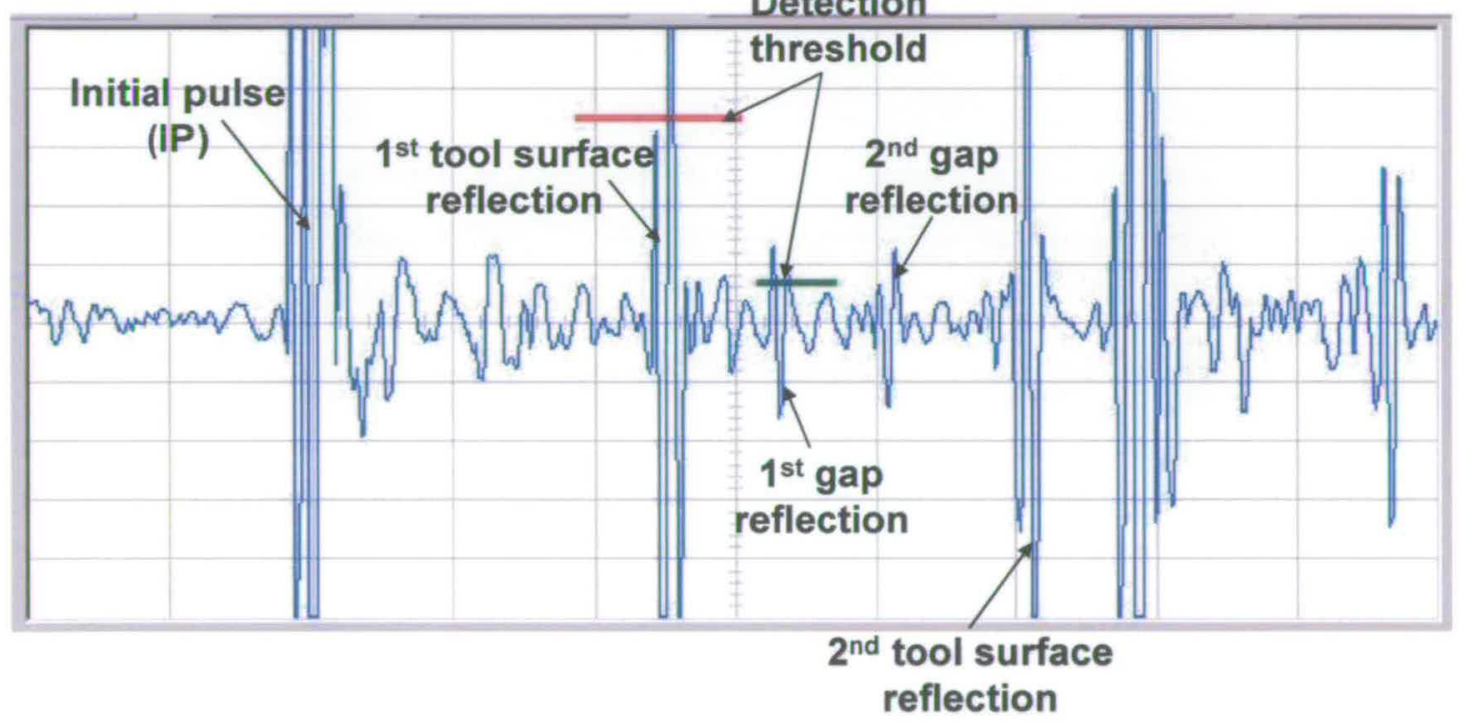

Figure 5.2: Plot showing the ultrasound measurement of $\mathrm{z}=1 \mathrm{~mm}$ in $\mathrm{H}_{2} \mathrm{O}$ electrolyte.

Figure 5.2 clearly shows that, for this system (section 4.6.3), the initial ultrasound pulse is followed by two tool reflections and 2 gap reflections. The threshold lines were set up to enable the TOF to be measured between the first tool surface reflection and the first gap reflection. As $\mathrm{z}$ was reduced the number of signal echoes relating to the interelectrode gap 

were observed to increase, within a given pulse, as expected, as the time of flight of the ultrasound signal in the interelectrode gap decreased (Figure 5.3).

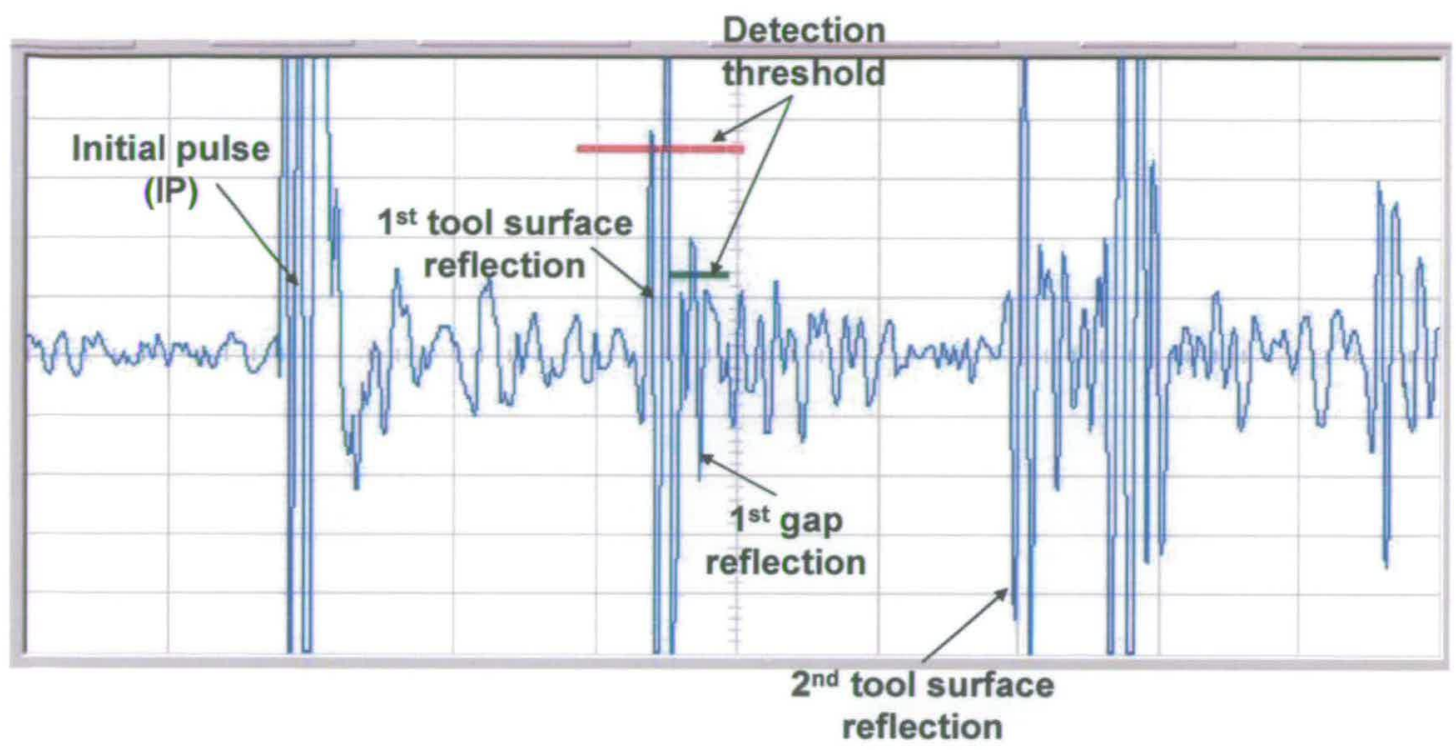

Figure 5.3: Plot showing the ultrasound measurement of $\mathrm{z}=0.2 \mathrm{~mm}$ in $\mathrm{H}_{2} \mathrm{O}$.

Using Equation 5.1, v can be determined, through measurement of the TOF for known $\mathrm{z}$ values, for all the relevant $\mathrm{z}$ values for ECM (Figure 5.4)

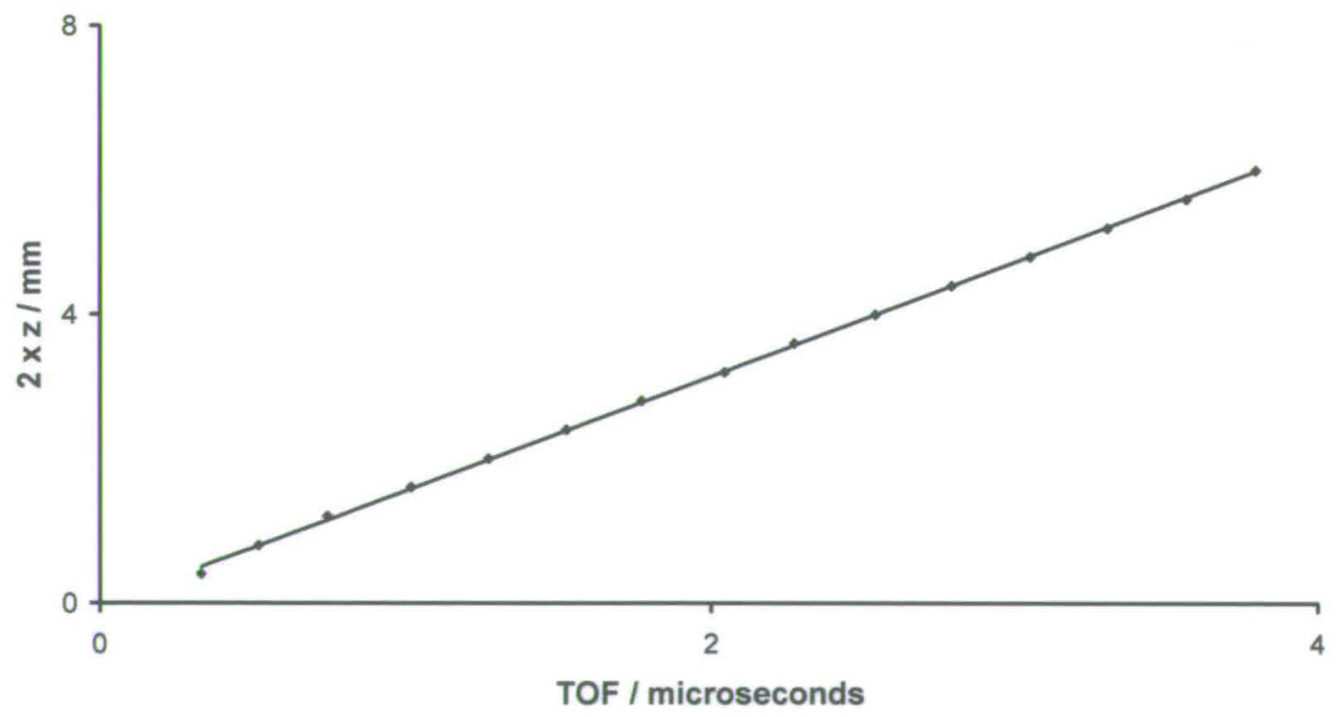

Figure 5.4: Plot of TOF vs. values of know $\mathrm{z}$ in $5.0 \%$ chloride solution at $\mathrm{T}=30^{\circ} \mathrm{C}$. 
$\mathrm{v}$ can be calculated, from the gradient of Figure 5.4, in $5.0 \%$ chloride solution at a temperature of $\mathrm{T}=30^{\circ} \mathrm{C}$ as $\mathrm{v}=1587 \mathrm{~ms}^{-1}$.

The temperature dependent speed of sound in the electrolyte was calculated from the gradient of Figure 5.4 and Equation 5.1. This experiment was carried out with pure water and nitrate and chloride electrolytes of $5.0 \%, 15.0 \%$ and $25.0 \%$ concentrations. Figure 5.5 and Figure 5.6 show these data, with literature values for water and seawater. The close correspondence of the measured data for water with the literature data confirms the accuracy of this technique. It is also reassuring that the data for seawater is comparable to $3-5 \%$ chloride as expected. In both chloride and nitrate electrolytes with increasing electrolyte concentrations, a corresponding increase in the speed of propagation of sound was observed. This is due to the increased electrolyte density, resulting from increased electrolyte concentrations, allowing faster pressure changes and hence faster propagation of pressure or sound waves [144]. However, the increase in speed measured for chloride was observed to be more significant (Figure 5.5). It is possible that this is due to larger amounts of water captured in the solvation shell of the smaller $\mathrm{Cl}^{-}$ion solvation shell compared to the similarly sized solvation shell of the larger $\mathrm{NO}_{3}^{-}$ion. This leads to an apparent increase in concentration for $\mathrm{NaCl}$ solutions compared to $\mathrm{NaNO}_{3}$ solutions of the same conductivity leading to increased $v$ values for these $\mathrm{NaCl}$ solutions. This theory is supported by the greater discrepancy between the $\mathrm{v}$ values for similar $\mathrm{NaCl}$ and $\mathrm{NaNO}_{3}$ solutions at high concentrations [145]. 


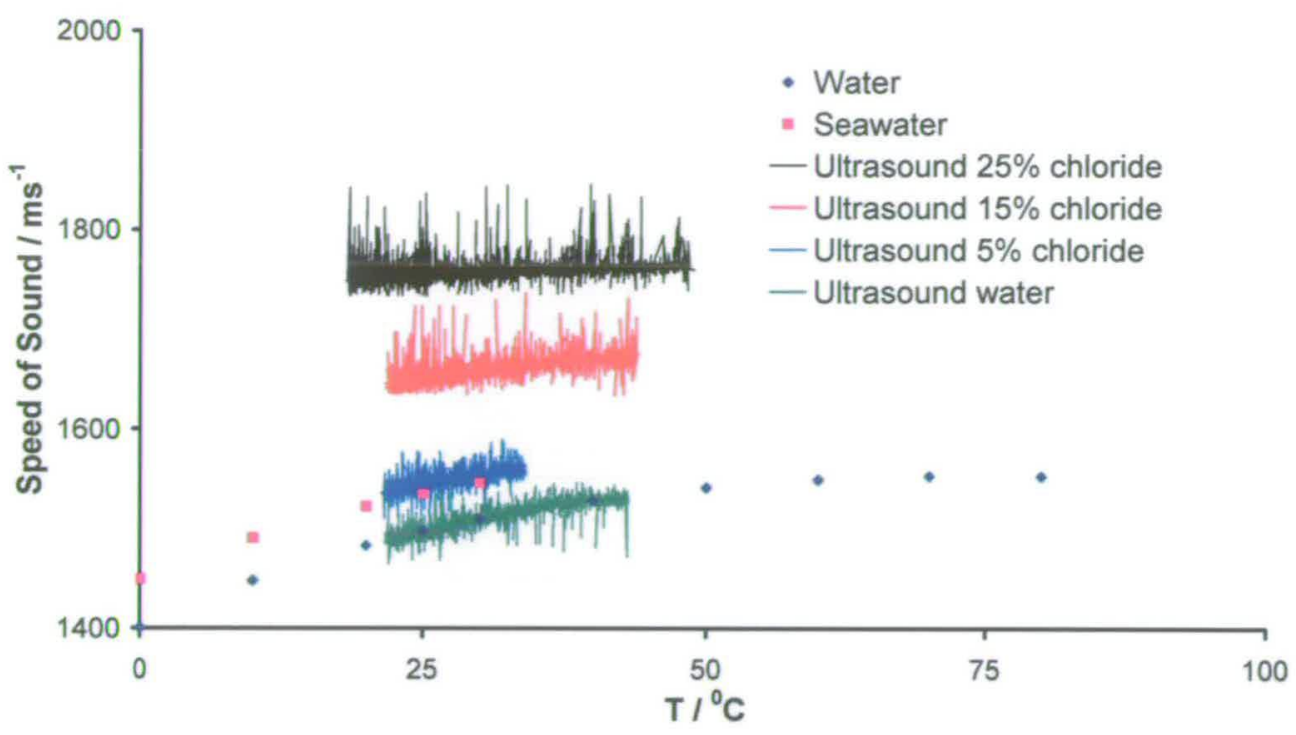

Figure 5.5: Plot to show temperature dependence of speed of sound measurements made with cell 2004 in difference concentrations of chloride electrolyte. Also shown are the literature values of the temperature dependent speed of sound in water and seawater [146].

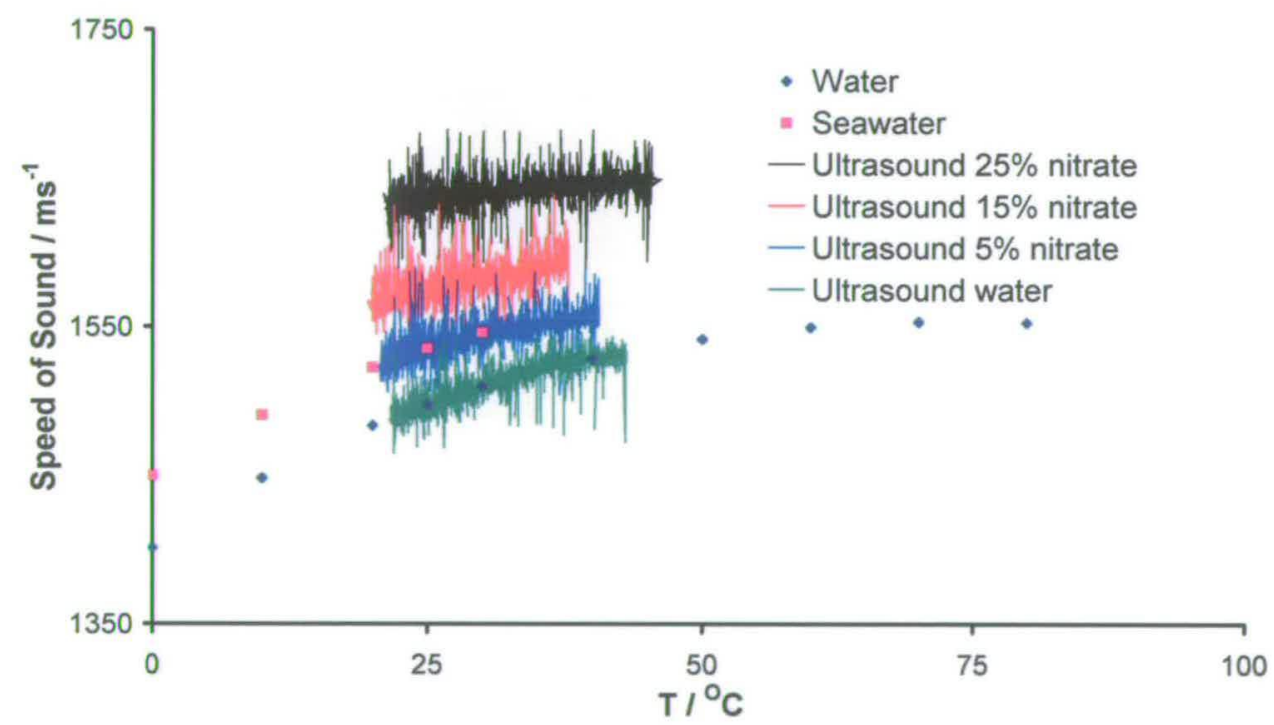

Figure 5.6: Plot to show temperature dependence of speed of sound measurements made with cell 2004 in difference concentrations of nitrate electrolyte. Also shown are the literature values of the temperature dependent speed of sound in water and seawater [146]. 
The Parameterisation of Electrochemical Machining

There was no electrolyte temperature or conductivity, in the ranges measured, at which the ultrasound signal could not be determined while the ECM power was off.

Further tests were then carried out with a second ultrasound probe due to mechanical failure of the first probe. Unfortunately the strong, measurable, ultrasound signal achieved for the previous results could not be re-produced. The inability to recreate the signal produced with the first probe was possibly due to slight misalignments in the reflective surfaces in the system (the tool and the workpiece). This shows the importance of exact alignment of the ultrasound probe in order to enable the maximum signal to pass through the tool/electrolyte interface into the interelectrode gap. Non-parallel surfaces would potentially not reflect the gap reflection ultrasound signal back to the probe for detection. This is especially problematic in this system due to the large reflectance of the ultrasound signal at the tool interface and the subsequently small signal strength passing into the electrolyte medium. This effect can be quantified by calculating the interface reflection co-efficient, IRC, of the ultrasound signal. The IRC between a copper and seawater boundary, literature data similar to the system studied in this work, can be calculated through the acoustic impedances, Z, of both media using Equation 5.2.

$$
I R C=\frac{Z_{\text {copper }}-Z_{\text {seawater }}}{Z_{\text {copper }}+Z_{\text {seawater }}}
$$

\section{Equation 5.2}

Where the acoustic impedance of a media can be calculated using the density of the medium, $\rho$, and the speed of sound in the medium (Equation 5.3) [147].

$$
Z=\rho \mathbf{v}
$$

\section{Equation 5.3}

Using Equation 5.2 and Equation 5.3 for the tool/electrolyte interface, assumed to be copper/sea water (salinity $=35$ ) with $\rho_{\text {copper }}=8960 \mathrm{~kg} \mathrm{~m}^{3}, \mathrm{v}_{\text {sound } / \text { copper }}=4760 \mathrm{~m} \mathrm{sec}^{-1}, \rho_{\text {seawater }}$ $=1023 \mathrm{~kg} \mathrm{~m}^{3}$ and $\mathrm{v}_{\text {sound/seawater }}=1535 \mathrm{~m} \mathrm{sec}^{-1}$ [146], the system IRC $=0.929$, in other words 
$92.9 \%$ of the ultrasound signal is reflected at a copper/sea water interface and $7.1 \%$ of the signal is transmitted into the electrolyte, assuming there are no energy losses at the interface. From these data it is predictable that no signal through the electrolyte was measured.

In order to decrease the IRC the acoustic impedance of the tool material must be similar to that of the electrolyte, or at least significantly lower that that for copper to allow a greater signal transmission into the electrolyte. Changing the tool material to a lower acoustic impedance material would be inherently difficult due to the requirement for an electrically conducting tool material which, by definition, will have high acoustic impedances. A more realistic approach may be to increase the surface roughness of the tool thus reducing the IRC and allowing greater transmission through the interface [148], however, care must be taken to maintain the electric field conditions in the gap necessary for stable machining.

\subsection{Initial calibration - with power on}

The ECM system at Edinburgh University is set-up to machine with a continuous DC current. In order for the cell 2004 to be most immediately useful, gap measurements were required to be made during machining with the power on. However, it was observed that, when machining, although the $1^{\text {st }}$ tool surface ultrasound reflection (Figure 5.2) remained unchanged, the $1^{\text {st }}$ gap ultrasound reflection (Figure 5.2) was drastically attenuated to the extent that no recordable signal was observed. This was due to the ECM products (and hydrogen gas) causing significant refractive index changes in the electrolyte and consequently scattering the ultrasound signal. It was found that there was no gap size, flow rate, electrolyte temperature or composition at which the ultrasound signal could be observed. The ultrasound direct gap measurement technique was therefore proved to be incompatible with a continuous DC current.

\subsubsection{Pulsing}

Pulsed current ECM is a technique in industry. It was therefore thought possible that in a system in which a measurable ultrasound signal was observed with the ECM power off, this signal could be used during the current off pulses to measure $\mathrm{z}$ directly during ECM. Successful gap measurement using this technique would require the pulse off time to be greater than the ultrasound software send, receive and thickness calculation time ( $2 \mathrm{~ms}$ for a 
The Parameterisation of Electrochemical Machining pulse repetition frequency of $500 \mathrm{~Hz}$, section 4.6.3) and ideally the interelectrode gap flushing time (so that the electrolyte composition is known and no scattering due to ECM products could occur). Table 5.1 shows the finite time required to flush any remaining ECM products from a gap of $\mathrm{z}=1 \mathrm{~mm}$ at the flow rates applicable to the Edinburgh ECM set-up.

\begin{tabular}{|c|c|c|c|}
\hline Flow Rate $\left(\mathrm{L} \mathrm{min}^{-1}\right)$ & Flow Rate $\left(\mathrm{m}^{3} \mathrm{~s}^{-1}\right)$ & Flow velocity $\left(\mathrm{ms}^{-1}\right)$ & $\begin{array}{c}\text { Time required to } \\
\text { flush gap (millisecs) }\end{array}$ \\
\hline 8 & $1.33 \times 10^{-4}$ & 17.78 & 4.50 \\
\hline 10 & $1.67 \times 10^{-4}$ & 22.22 & 3.60 \\
\hline 12 & $2.00 \times 10^{-4}$ & 26.67 & 3.00 \\
\hline 14 & $2.33 \times 10^{-4}$ & 31.11 & 2.57 \\
\hline 16 & $2.67 \times 10^{-4}$ & 35.56 & 2.25 \\
\hline 18 & $3.00 \times 10^{-4}$ & 40.00 & 2.00 \\
\hline 20 & $3.33 \times 10^{-4}$ & 44.44 & 1.80 \\
\hline
\end{tabular}

Table 5.1: Table to show the gap flushing time calculated for a gap of $\mathrm{z}=1 \mathrm{~mm}$ for the flow rates accessible for cell 2004.

These data show that the minimum pulse off time for successful $\mathrm{z}$ measurement to be of the order of milliseconds, however, the exact limit of the pulsing speed would be easily found using a pulsed ECM system and increasing the pulsing speed until the ultrasound signal could no longer be detected. Ideally a system of latching would be used in which the PC logging the ECM system power would instigate the USPC 2100 software to send out the initial ultrasound pulse when the ECM power was off resulting in $\mathrm{z}$ measurements only being recorded during power off times. Continuous, fast power pulsing would allow nearcontinuous $\mathrm{z}$ data to be collected during the off pulse time.

Once a method has been found to achieve a stronger ultrasound entry signal to the electrolyte the author is confident that direct ultrasound measurement of $\mathrm{z}$ with pulsed voltage will be possible. However, for this remainder of this work, cell 2004 was utilised with continuous machining and the ultrasound probe positioned in the same way as cell 2000 (Chapter 4). 


\section{Chapter 6}

Critical comparison of analysis methods 


\subsection{Critical comparison of analysis Methods.}

An experiment was carried out using the stainless steel SS410 (see chapter 4 for experimental set-up), the workpiece was machined in an approach/retract experiment using $15.0 \% \mathrm{NaCl}$ electrolyte, $\mathrm{f}=0.5 \mathrm{~mm} \mathrm{~min}^{-1}$ during the approach phase and $\mathrm{z}_{0}=1.00 \mathrm{~mm}$. The experimental data was analysed using the chronoamperometric and the ultrasound analysis techniques and the results compared.

\subsubsection{Dissolution valency $(n)$ measurement.}

The chronoamperometric analysis method (section 3.2) yields universal values of $\mathrm{n}$ at equilibrium machining through Equation 6.1, found using Equation 2.4 under equilibrium machining conditions where $\mathrm{dy} / \mathrm{dt}=\mathrm{f}$.

$$
I_{\infty}=\frac{n A F f \rho}{M}
$$

\section{Equation 6.1}

where $\rho=7.75 \mathrm{~g} \mathrm{~cm}^{-3}[146], \mathrm{f}$ is the feed rate, in this instance $0.5 \mathrm{~mm} \mathrm{~min}^{-1}$ and $\mathrm{M}=55.15 \mathrm{~g}$ $\mathrm{mol}^{-1}$ found using Equation 6.2

$$
M=\sum_{j} x_{j} M_{j}
$$

\section{Equation 6.2}

where $\mathrm{x}$ is the mole fraction of each element, $\mathrm{j}$, present in the alloy and $\mathrm{M}$ is the molecular mass of that element (Table 4.1). Using Equation 6.1 and the observed value of $\mathrm{I}_{\infty}(135 \mathrm{~A})$ for the experiment conducted with SS410, in agreement with [143] the universal $n$ values was found to be $2.1 \pm 0.1$. 
It is reassuring that these data are consistent with the dissolution valency of $n=2.1$, calculated using the subdivision of the stainless steel into individual valencies, $\mathrm{n}_{\mathrm{j}}$, and mole fractions, $\mathrm{x}_{\mathrm{j}}$ (Table 4.1), for each element, $\mathrm{j}$, assuming a dissolution efficiency of $100 \%$ and using a dissolution valency of $\mathrm{Cr}=\mathrm{Cr}$ (III) and a dissolution valency of 2 for all other constituents.

$$
n=\sum_{j} n_{j} x_{j}
$$

\section{Equation 6.3}

However, this universal measurement of $\mathrm{n}$ at equilibrium gives no indication as to whether $\mathrm{n}$ alters during machining. The ultrasound analysis method uses the ultrasound thickness data to produce direct, time-resolved $\mathrm{n}$ data throughout the machining run (Chapter 3 ). The change in workpiece thickness with time, $\mathrm{dh} / \mathrm{dt}$, can be calculated using a number of data points, $\mathrm{p}$, centred on time t, through Equation 6.4.

$$
\left(\frac{d h}{d t}\right)_{t}=\frac{h_{\left(t-\frac{(p-1)}{2} \Delta t\right)}-h_{\left(t+\frac{(p-1)}{2} \Delta t\right)}}{(p-1) \Delta t}
$$

\section{Equation 6.4}

From Figure 6.1, measuring $\mathrm{dh} / \mathrm{dt}$ over larger values of $\mathrm{p}$ yields significant noise reduction in the data and more consistent values of n. However, care is necessary when using larger values of $\mathrm{p}$ to assess data in which short-time changes in $\mathrm{n}$ are expected, because larger values of $\mathrm{p}$ will prohibit the detection of these changes. From Figure 6.1 reasonable smoothing is found with $\mathrm{p}=100$, therefore, for all further $\mathrm{n}$ measurements in this work $\mathrm{p}$ is chosen as 100 . It is reassuring that these data are consistent throughout the machining run with the overall time averaged valency of $n=2.0 \pm 0.1$ calculated using Equation 6.1 and Equation 6.3 which indicates for the first time that $n$ is invariant throughout the ECM experiment. 


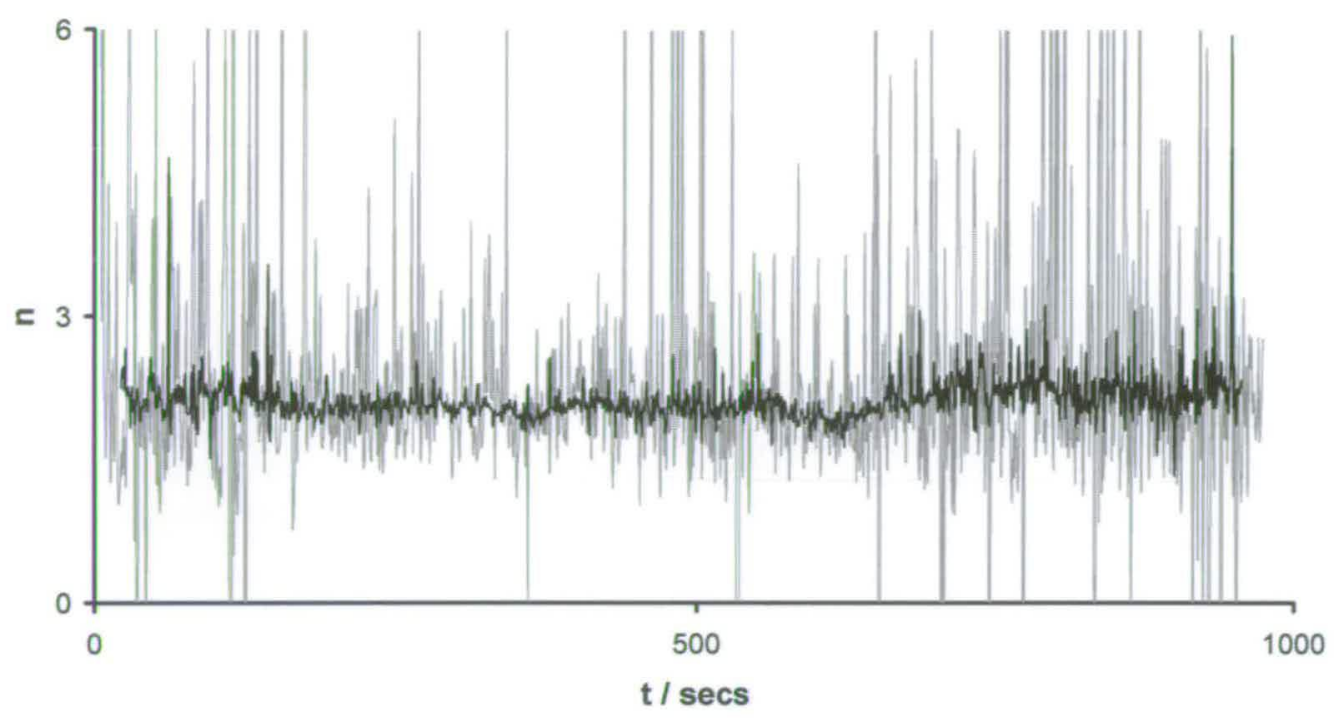

Figure 6.1: Time-resolved plot of the variation in $\mathrm{n}$ against $\mathrm{t}, \mathrm{p}=11$ shown in grey and $\mathrm{p}=$ 100 shown in black, using the ultrasound method of analysis.

\subsubsection{Interelectrode gap, $\mathrm{z}$, measurement}

Time-resolved parameterisation of ECM requires time-resolved measurement of $\mathrm{z}$ during the machining process. Previous gap measuring techniques have included optical observation of $\mathrm{z}$ using a travelling microscope [149] and the introduction of a mechanical probe through a hole in the tool in order to make contact with the workpiece surface and mechanically measure $\mathrm{z}$ [150]. Both these techniques require disruption of the machining process in order to take a gap measurement. Furthermore, unlike the ultrasound analysis technique used in this thesis, the chronoamperometric analysis method provides no direct measurement of $\mathrm{z}$ during machining. For ultrasound measurements Equation 3.11 can be used to produce a time-resolved plot of $\mathrm{z}$ for this experiment (Figure 6.2). As expected, Figure 6.2 shows $\mathrm{z}$ decreasing until equilibrium machining is established, during the approach phase, followed by a steadily increasing $z$ during the retract phase. 


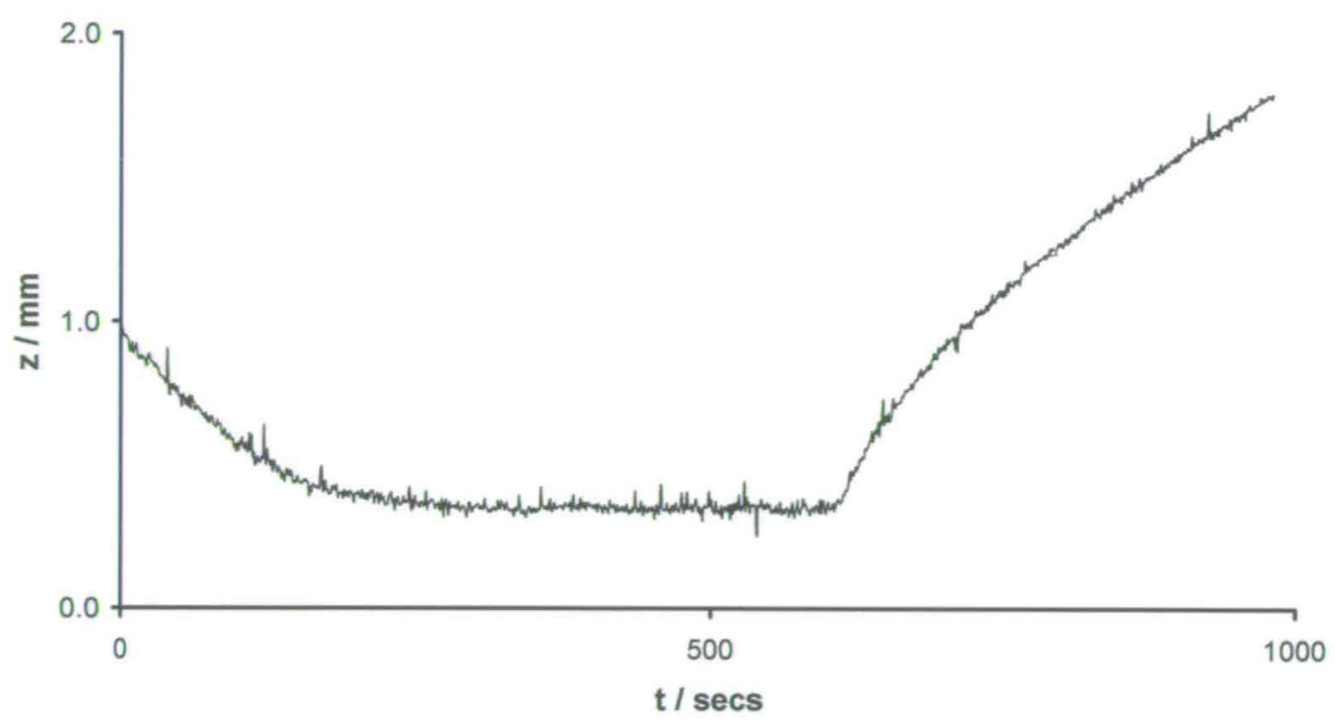

Figure 6.2: Time-resolved plot of the variation in $z$ vs. $t$ using the ultrasound method of analysis.

\subsubsection{Comparison of measured ECM machining parameter, $\mathbf{k}$.}

Both I- and z- iterative fitting analysis was used (section 3.2) to fit Equation 3.7 and Equation 3.6 to I-t and z-t data respectively, collected using the ECM ultrasound experimental set-up, to give values of $\mathrm{f}^{2} / \mathrm{k}$ and hence $\mathrm{k}$. This was then compared to ultrasound analysis of the same data set. For these data, $\left(\mathrm{V}-\mathrm{V}_{0}\right) \mathrm{k}$ is found using the ultrasound $\mathrm{z}$ data and the collected I data. $\mathrm{k}$ is then calculated from Equation 3.4 with the average $\mathrm{n}$ found independently through the $\mathrm{dh} / \mathrm{dt}$ and the I data (section 3.3.1). The fixed parameter for the iterative fitting of the data for the I-t analysis was the observed value of $\mathrm{I}_{\infty}$ $=135 \mathrm{~A}$ and for the $\mathrm{z}$-t analysis was $\mathrm{z}_{\infty}=0.32 \mathrm{~mm}$ (found from the ultrasound data at equilibrium machining). Both I- and $z$ - iterations were carried out using the entire approach phase producing an average, global I-analysis and $\mathrm{z}$-analysis value of $\mathrm{f}^{2} / \mathrm{k}$ (and hence $\mathrm{k}$ ) for these data. This I- and z- iterative analysis was also performed over a number of smaller data sets where the assumption of constant $k$ is more likely to be appropriate. 40 point (38 second) data sets were used during the approach phase producing a number of local $\mathrm{k}$ values during the approach phase. For the I-analysis it was possible to fit 14 sections, while for the z-analysis it was only possible to fit 9 ; no data could be collected using iterative fitting during equilibrium machining conditions. These local $\mathrm{k}$ values are plotted in Figure 6.3 at 
the central $t$ value of each 40 point data section $(t=38$ secs $)$. For both local and global iterative fitting, larger errors were recorded for the I-analysis compared to the z-analysis. Also, both local and global I-analysis gave systematically higher $\mathrm{k}$ data compared to $\mathrm{z}$ analysis. Also shown in Figure 6.3 are the real-time ultrasound $\mathrm{k}$ data for comparison.

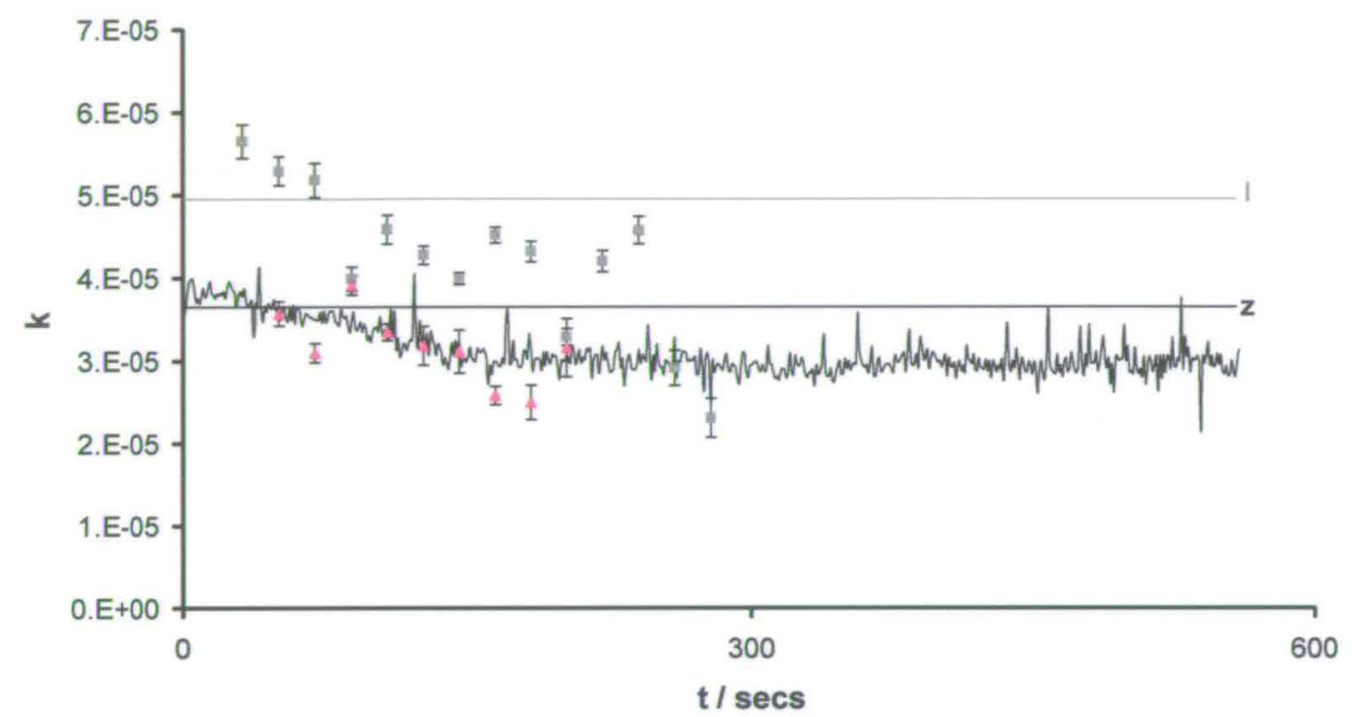

Figure 6.3: Comparison of the electrochemical machining constant determined using the ultrasound analysis method with both global iterative fitting I-analysis, z-analysis data (lines shown with errors of $\pm 4.48 \times 10^{-7}$ and $\pm 2.97 \times 10^{-7}$ for the I- and $z$ - fitting respectively) and 40 point data set iterative fitting I analysis $(\mathbf{m})$ and z-analysis $(\mathbf{\Delta}) \mathrm{k}$ data.

It is reassuring that the ultrasound analysis and the global z-analysis give comparable values of $\mathrm{k}$. It is also reassuring that the local $\mathrm{z}$-analysis typically gives the same value of $\mathrm{k}$ as the corresponding ultrasound analysis within experimental error. However, the errors on the global z-analysis are larger than the ultrasound analysis and the observed ultrasound analysis shows the value of $\mathrm{k}$ to change by $24 \%$ during machining. This suggests that the assumption of a constant $\mathrm{k}$ is certainly in error for the global I-analysis and $\mathrm{z}$-analysis. However, between successive local z-analysis measurements the change is typically within experimental error, which explains why local $\mathrm{z}$-analysis, assuming a constant $\mathrm{k}$ in the given period, is relatively successful.

In contrast, both local and global I-analysis show systematic differences in $\mathrm{k}$ compared to these measurements. Also, typically successive local I-analysis values are not the same 
within experimental error. This suggests that $\mathrm{k}$ is varying significantly both locally and globally. This violates the assumption of a constant $\mathrm{k}$ and may explain the errors in the calculated values of $\mathrm{k}$. Local analysis would be expected to be more accurate at higher I values when $V_{0}$ is approximately constant and the same, within experimental error, as $V_{0}$ for $I_{\infty}$ and $z_{\infty}$. However, large errors in the local analysis at these higher I values would be expected due to the iterative fitting to small changes in I and $\mathrm{z}$ data.

A further advantage of the ultrasound analysis is the direct production of $\left(\mathrm{V}-\mathrm{V}_{0}\right) \kappa$ from Equation 3.1 which, if a constant $\kappa$ is assumed, produces values for $\left(\mathrm{V}-\mathrm{V}_{0}\right)$ and hence $\mathrm{V}_{0}$. However, $\mathrm{z}$ - and $\mathrm{I}$ - iterative fitting analysis yields values for $\mathrm{k}$, a value of $\mathrm{n}$ must be assumed in this case in order to yield values of $\left(\mathrm{V}-\mathrm{V}_{0}\right) \kappa$ from which $\mathrm{V}_{0}$ can be found through the assumption of $\kappa$. This assumption of $\mathrm{n}$ provides additional error in the calculation of $\mathrm{V}_{0}$ from $\mathrm{k}$ in the chronoamperometric analysis especially if $\mathrm{n}$ is changing. Furthermore, a changing $\mathrm{n}$ will also invalidate the theoretical approach through the integration of Equation 3.5 assuming a constant value of $n$.

Therefore, Equation 3.4 was then used to calculate average values of $\mathrm{V}_{0}$ for the global I- and $\mathrm{z}$ - analysis using values of $\mathrm{n}$ found from the ultrasound analysis and assuming a constant $\mathrm{\kappa}=$ $0.2 \mathrm{Scm}^{-1}$. The global $\mathrm{V}_{0}$ values from the I- and $\mathrm{z}$ - analysis were found to be $\mathrm{V}_{0}=0.86 \pm$ $0.42 \mathrm{~V}$ and $\mathrm{V}_{0}=2.74 \pm 1.31 \mathrm{~V}$. $\mathrm{V}_{0}$ data was also calculated from the local $\mathrm{k}$ data seen in Figure 6.3. A comparison between the global and local I- and z- analysis $\mathrm{V}_{0}$ data and the ultrasound $\mathrm{V}_{0}$ data can be seen in Figure 6.4 


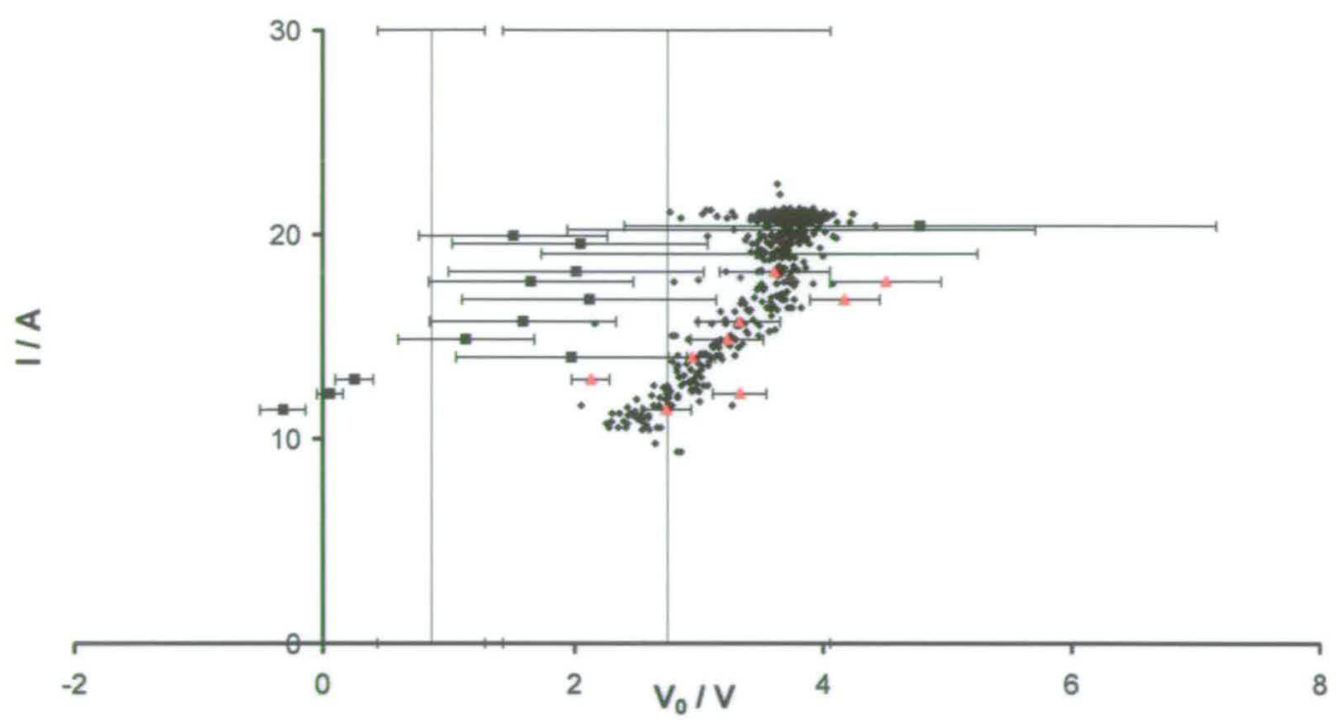

Figure 6.4: Comparison of time-resolved ultrasound $\mathrm{I}-\mathrm{V}_{0}$ data and both global I-analysis, $\mathrm{z}$ analysis data and local I analysis ( $\mathbf{m})$ and z-analysis $(\boldsymbol{\Delta}) \mathrm{I}^{-} \mathrm{V}_{0}$ data.

The chronoamperometric z-analysis was observed to be the more accurate, in line with the more accurate $\mathrm{k}$ values produced for this technique (Figure 6.3), with the I-analysis producing a systematically lower $\mathrm{V}_{0}$ value compared to the entire ultrasound data set, in line with the systematic error observed in the I-analysis $\mathrm{k}$ values (Figure 6.3). The error bars calculated for both I- and z- analysis were observed to span the entire ultrasound data set (Figure 6.4). These large errors in the chronoamperometric analysis can be attributed to the breakdown of the assumptions of constant $\mathrm{V}_{0}$ and constant $\mathrm{n}$ and the inherent inaccuracy of the iterative curve fitting analysis compared to the simple linear relationship given by Equation 3.1.

The continuous nature of the $\mathrm{I}-\mathrm{V}_{0}$ data produced using the ultrasound analysis therefore facilitates detailed investigation of trends in $\mathrm{I}-\mathrm{V}_{0}$ data under ECM conditions. It allows direct time-resolved measurement of $\mathrm{n}$ and $\mathrm{V}_{0}$ throughout machining compared to discrete or single values obtained with the chronoamperometric analysis which are less accurate due to the errors in the measurement and the assumptions used in obtaining the values. It also provides accurate time-resolved $\mathrm{z}$ data without interruption of machining. These are powerful grounds for the use of the ultrasound technique for determining ECM dissolution characteristics. Consequently, this ultrasound analysis technique is used to characterise ECM throughout the remaining chapters of this thesis. 


\section{Chapter 7}

The ECM of Iron 


\subsection{Introduction}

This chapter concerns the parameterisation of the ECM of iron. There have only been limited previous studies of the ECM of iron by Lohrengel et al. [151, 152]. The lack of study is mostly due to the infrequent use of pure iron in industry, which means there is no great need to machine iron. However, if machining were required the material properties of iron make conventional machining techniques more economically viable than ECM. Nonetheless, $\mathrm{Fe}$ is a simple system which can be considered as the first step to understanding the ECM of Fe based alloys such as steels.

The literature shows no examples of detailed ECM macro machining parameterisation studies of Fe under industrially relevant conditions. However, there is one macro ECM experiment as a calibration experiment for steel ECM, detailed by Haisch et al. [153], which uses gravimetric measurements (correlating charge to weight loss) at flow rates up to $7 \mathrm{~ms}^{-1}$ to measure the machining efficiency of iron. These measurements were conducted in a flow channel cell at current densities between $0-60 \mathrm{Acm}^{-2}$ in $20 \% \mathrm{NaCl}$ electrolyte. Further low current density (up to $3.5 \mathrm{~A} \mathrm{~cm}^{-2}$ ) Rotating Cylinder Electrode (RCE) studies on the iron rich steel $100 \mathrm{Cr} 6$ (96.92\% Fe, $1.43 \% \mathrm{Cr}, 0.97 \% \mathrm{C}$ and traces of other elements) were also detailed in this work.

A further study by Lohrengel et al., in which relatively high current densities have been applied when micro machining [151], offers information regarding iron dissolution characteristics. In this study the machining of $\mathrm{Fe}$ in $2.94 \mathrm{~mol} \mathrm{dm}^{-3} \mathrm{NaNO}_{3}$ electrolyte was investigated. The studies used a novel three electrode flow-through micro-cell with a double-barrelled capillary (Figure 7.1). 


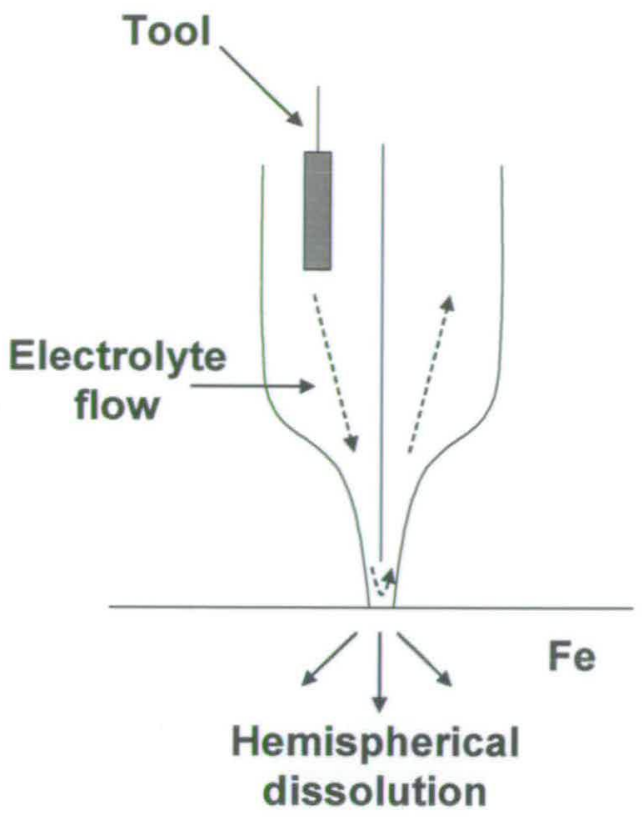

Figure 7.1: Schematic of three electrode double barrelled capillary cell used by Lohrengel et al. $[151]$

Lohrengel's system also includes a reference electrode in the cell, although it is not clear precisely where. This allows measurements of absolute voltages for the anode to be investigated; this is a significant advantage over other systems in which voltage measurements are only available with reference to the counter electrode (the tool).

From Figure 7.1 a number of disadvantages are obvious in Lohrengel's microsystem when relating the results to industrial ECM. The first is the relatively complex flow path. In the micro system set-up the flow will impact the anode essentially perpendicular to the surface and result in a non-homogeneous electrolyte flow path. The second drawback is the stated positioning of the counter electrode in the micro system, in the inlet of the micro-cell; as a result the machining products produced at the tool, e.g. hydrogen, are carried through the cell by the electrolyte and across the workpiece surface. Chemical reaction of the products with the workpiece could significantly affect the dissolution process and, depending on it's position, the reference electrode. A further disadvantage is that this microsystem set-up comprises a stationary tool and workpiece system which prohibits movement of the electrodes towards each other and consequently precludes the study of prolonged dissolution at high current densities or with steady state currents often found in industrial ECM. During the dissolution experiments the machining area was observed to increase [153]. This is 
Parameterisation of Electrochemical Machining

consistent with the expected hemispherical dissolution (Figure 7.1), which would result in a continuously increasing machining area. Such an increase would make current density calculation prone to error. Finally, significant $i R$ drops were observed in these measurements. The electric field between the tool and the workpiece during these micro electrode experiments is constrained by the confined geometry of the tip of the capillary. Consequently the $\mathrm{iR}$ drop in this capillary cell would be expected to be greater than that generally expected in a micro electrode system resulting in problems measuring the absolute anode potential.

As a result there is a lack of systematic studies of the effects of changes in machining conditions (such as feed rate and electrolyte concentration) on the industrial ECM characteristics of the Fe system. This chapter therefore presents such measurements made using experimental conditions relevant to industrial.

\subsection{The ECM of Iron}

\subsection{1. $\mathrm{Fe} / \mathrm{NaCl} \mathrm{Parameterisation.}$}

Figure 7.2 shows chronoamperometric data for a retract/approach/re-retract experiment (section 4.8) for $\mathrm{Fe}$ in $15.0 \% \mathrm{NaCl}$. The initial retract phase lasted $60 \mathrm{~s}$. Throughout this experiment it is clear that segments 1 and 2 give significantly different currents to the other segments. It is also clear that the changes in the currents on segments 1 and 2 are correlated, and the average current of segments 1 and 2 is the same at all times as the other segments within experimental error. This is consistent with a short circuit between segments 1 and 2 . This is a highly unusual effect attributable to conducting material becoming trapped between the segments. The difference in the segment 1 and 2 currents is an experimental measurement artefact diagnostic of shorting but does not affect the machining since the average current of segments 1 and 2 remain the same as the other segments. Also, as the ultrasound probe is positioned at segment 4 and the ultrasound analysis is carried out using measurements taken for segment 4 , this effect does not influence the remainder of the analysis. 


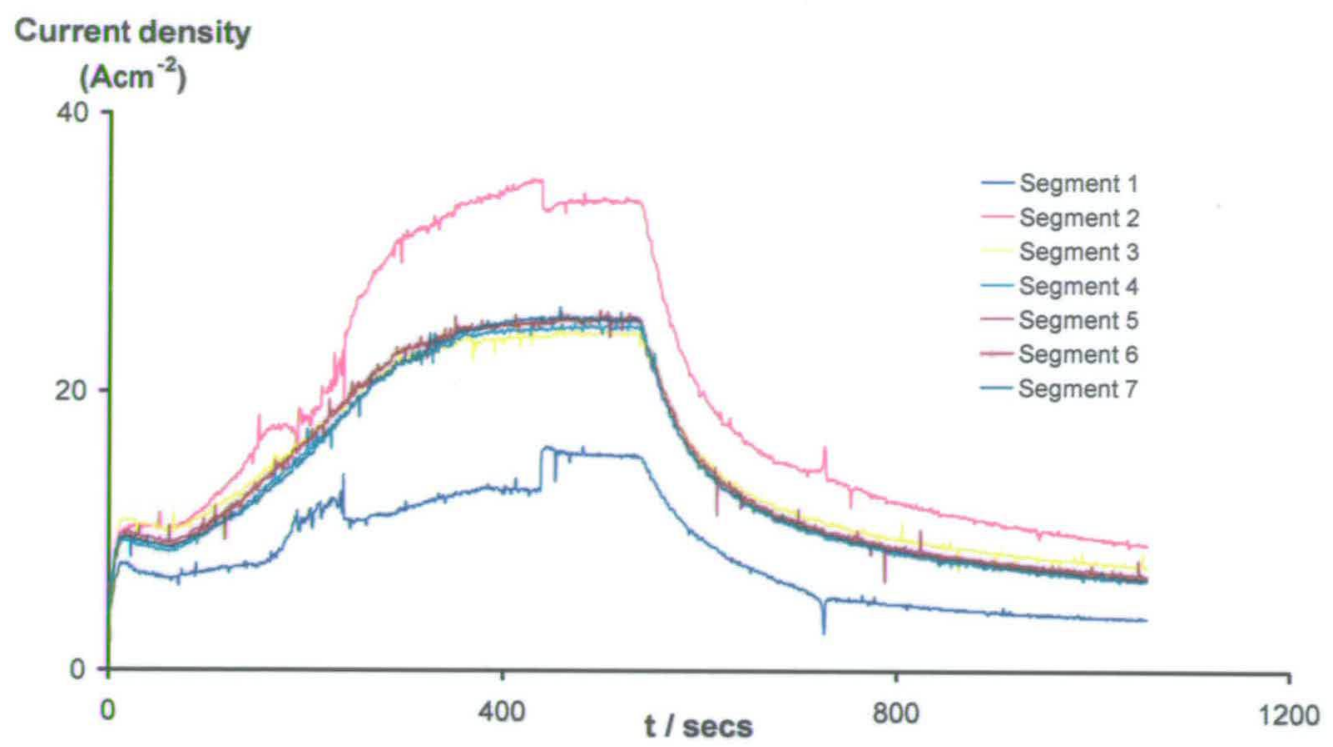

Figure 7.2: Experimental current density transients for segments 1-7 for ECM of $\mathrm{Fe}$ at $\mathrm{U}=$ $16.0 \mathrm{~L} \mathrm{~min}^{-1}$ with $15.0 \% \mathrm{NaCl}$ electrolyte with an applied voltage of $\mathrm{V}=6.0$ Volts for this data $\mathrm{z}_{0}=0.990 \mathrm{~mm}$.

During the approach phase $\mathrm{Fe}$, machined with an equilibrium current, $\mathrm{I}_{\infty}=21.5 \pm 0.5 \mathrm{~A}$ for each segment 3-7 and for the average of segments 1 and 2 (Figure 7.2). Using Equation 6.1, with the segment area, $A=0.857 \mathrm{~cm}^{2}$, the feed rate of the workpiece, $f=0.51 \mathrm{~mm} \mathrm{~min}^{-1}$ and the density and molecular mass of $\mathrm{Fe}, \rho=7.87 \mathrm{~g} \mathrm{~cm}^{-3}$ and $\mathrm{M}=55.847 \mathrm{~g} \mathrm{~mol}^{-1}$ respectively [146], the average dissolution valency of the workpiece under these conditions was calculated to be $\mathrm{n}=2.0 \pm 0.1$ across segments $1-7$, which indicates low valency $\mathrm{Fe}$ (II) dissolution of the Fe workpiece (Figure 7.2). This is consistent with previous measurements for iron [153].

Figure 7.3 shows the corresponding workpiece thickness measured using the ultrasound probe. The scatter of the data shows the error of the thickness measurements to be of the order of $\pm 0.02 \mathrm{~mm}$. 


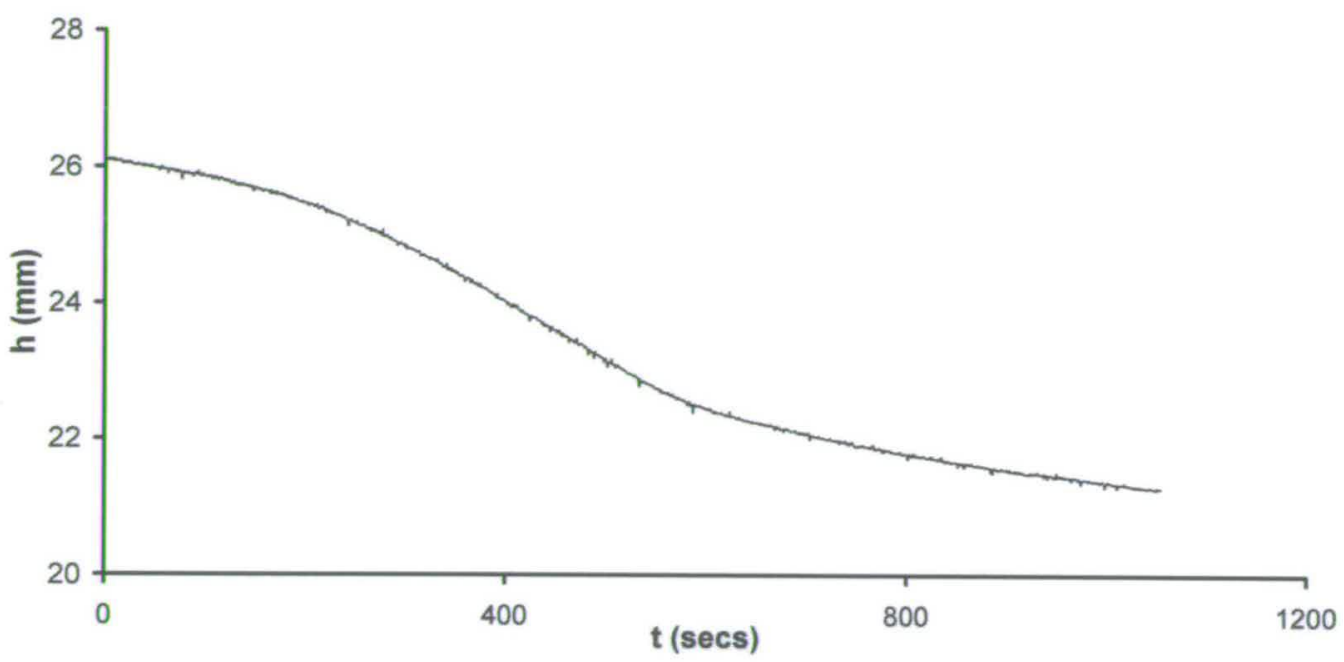

Figure 7.3: Experimental workpiece thickness during machining measured opposite to segment 4 during ECM of Fe.

From these data, $\mathrm{dh} / \mathrm{dt}$ was calculated with $\mathrm{p}=100$, using Equation 6.4. Then, using Equation 3.14, the corresponding time-resolved values of $\mathrm{n}$ were calculated (Figure 7.4).

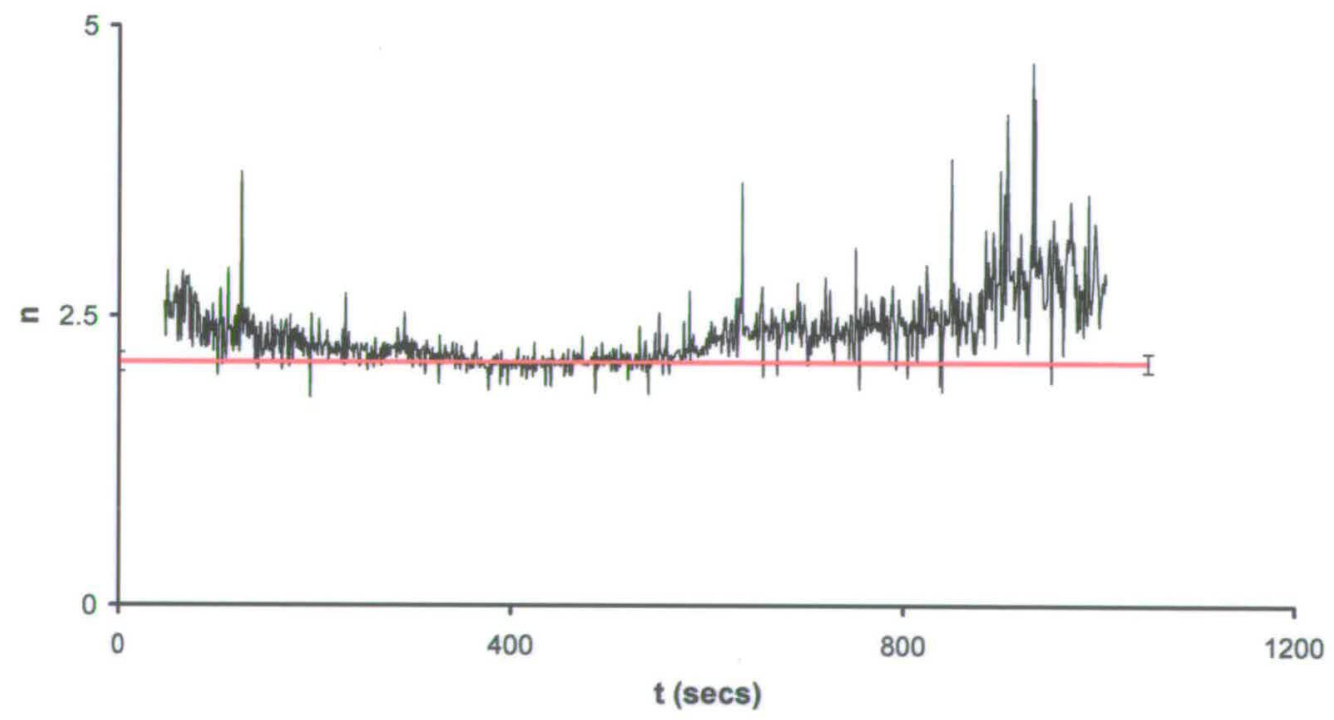

Figure 7.4: The calculated time-resolved $n$ data (Equation 3.14) during ECM of Fe, with $p=$ 100 from Equation 6.4, also shown is the average $n$ value (red line) of $n=2.1 \pm 0.08$ at equilibrium with the error on this value. 
From these data the time-average dissolution valency during equilibrium machining $\left(\mathrm{I}_{\infty}=\right.$ $21.5 \pm 0.5 \mathrm{~A}$ ) was found to be $\mathrm{n}=2.1 \pm 0.1$. It is gratifying that this is the same, to within experimental error, as the result from $\mathrm{I}_{\infty}$ and Equation 6.1, and the results detailed previously by Haisch et al. [153]. This confirms low valency $\mathrm{Fe}$ (II) dissolution during equilibrium machining. It is re-assuring that the chronoamperometric, gravimetric and ultrasound methods give the same value of $\mathrm{n}$ during equilibrium machining, however it is interesting to note the slight increase in the time-resolved $\mathrm{n}$ shown in Figure 7.4 during lower current periods of machining. This observation contradicts the work of Haisch et al. who found $\mathrm{n}=$ 2 at all current densities between 0 and $60 \mathrm{~A} \mathrm{~cm}^{-2}$ [153]. The increase in $\mathrm{n}$ observed in this work is consistent with either an increase in dissolution valency or a decrease in efficiency of the dissolution process (Equation 2.35). An increase in valency, through mixed $\mathrm{Fe}$ (II)/Fe(III) dissolution is unlikely at low currents since higher valency machining occurs at higher currents. Reductions in current efficiency at higher current density when machining iron have been observed in a previous study by Hoare et al. [154]. In these studies the authors attribute the efficiency decrease to the formation of a soluble salt film on the anode surface at high current densities [154]. However the high current densities in this study (of the order of $100 \mathrm{~mA} \mathrm{~cm}^{-2}$ ) are significantly lower than those used in this work (of the order of $30 \mathrm{~A} \mathrm{~cm}^{-2}$ ) and also used in industrial ECM. Furthermore, Hoare et al. make no mention of electrolyte flow rate in the study which infers a stationary electrolyte, a condition conducive to surface film formation unlike the high electrolyte flow velocities used in this work. This explanation describes inefficiency at higher current density; this is inconsistent with this work which shows decreased inefficiency at low current density. The results in this work are consistent with another competing electro-oxidation reaction which does not result in ion dissolution.

Figure 7.5 shows a time-resolved plot of the interelectrode machining gap, z, from Equation 3.11 . 


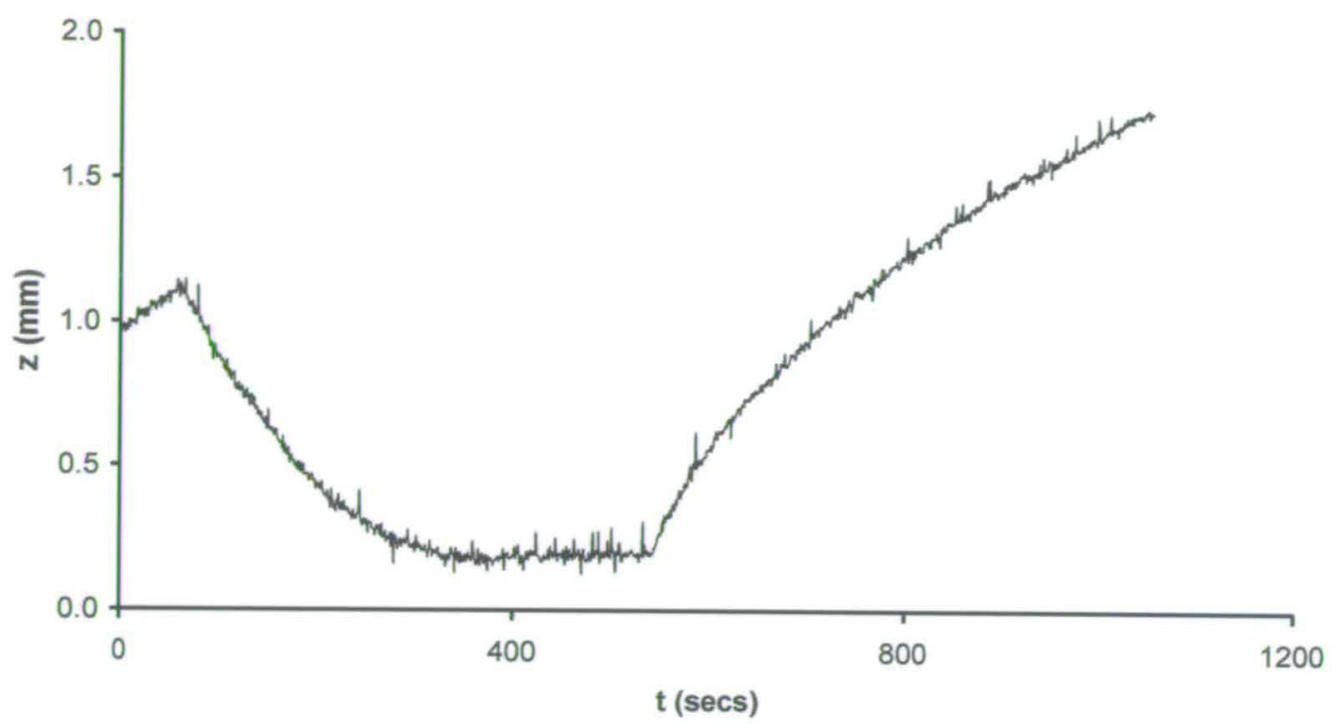

Figure 7.5: Time-resolved plot of the variation in interelectrode machining gap against time during ECM of $\mathrm{Fe}$.

As expected $\mathrm{z}$ increases initially in the retract phase as machining occurs while the workpiece is stationary, it then decreases during the approach phase until a steady state machining gap is established (equilibrium machining), followed by a steadily increasing $\mathrm{z}$ during the re-retract phase. A polyether impression material was used to cast the initial and final interelectrode gap distances $z_{0}$ and $z$ respectively (section 4.6.2) giving measurements of $0.990 \pm 0.005 \mathrm{~mm}$ and $1.725 \pm 0.005 \mathrm{~mm}$ respectively. It is encouraging that the corresponding ultrasound $\mathrm{z}_{0}$ and $\mathrm{z}$ measurements of $0.97 \pm 0.02 \mathrm{~mm}$ and $1.73 \pm 0.02 \mathrm{~mm}$ are the same to within experimental error.

Figure 7.6 shows $\mathrm{J}-\mathrm{V}_{0}$ data during ECM, calculated using the $\mathrm{z}$ values shown in Figure 7.5, the measured values of current and applied voltage (Equation 3.1) and assuming a constant value of $\kappa=0.20 \mathrm{Scm}^{-1}$. The assumption of a constant $\kappa$ can be justified for this data as thermocouple measurements carried out in Chapter 8 show little temperature variation when machining at these low applied voltages in $\mathrm{NaCl}$ electrolyte. Also previous studies have shown $15.0 \% \mathrm{NaCl}$ electrolyte to have a constant conductivity, measured as $\kappa=0.20 \mathrm{Scm}^{-1}$, under the high flow rate conditions used in this work [137]. In this case the current density (J) is most appropriately the current density measured at the point on the workpiece surface where $\mathrm{z}$ is measured (segment 4). In contrast to other established gap measurement 
techniques, this allows $\mathrm{V}_{0}$ to be calculated at any time during ECM without interruption of the process.

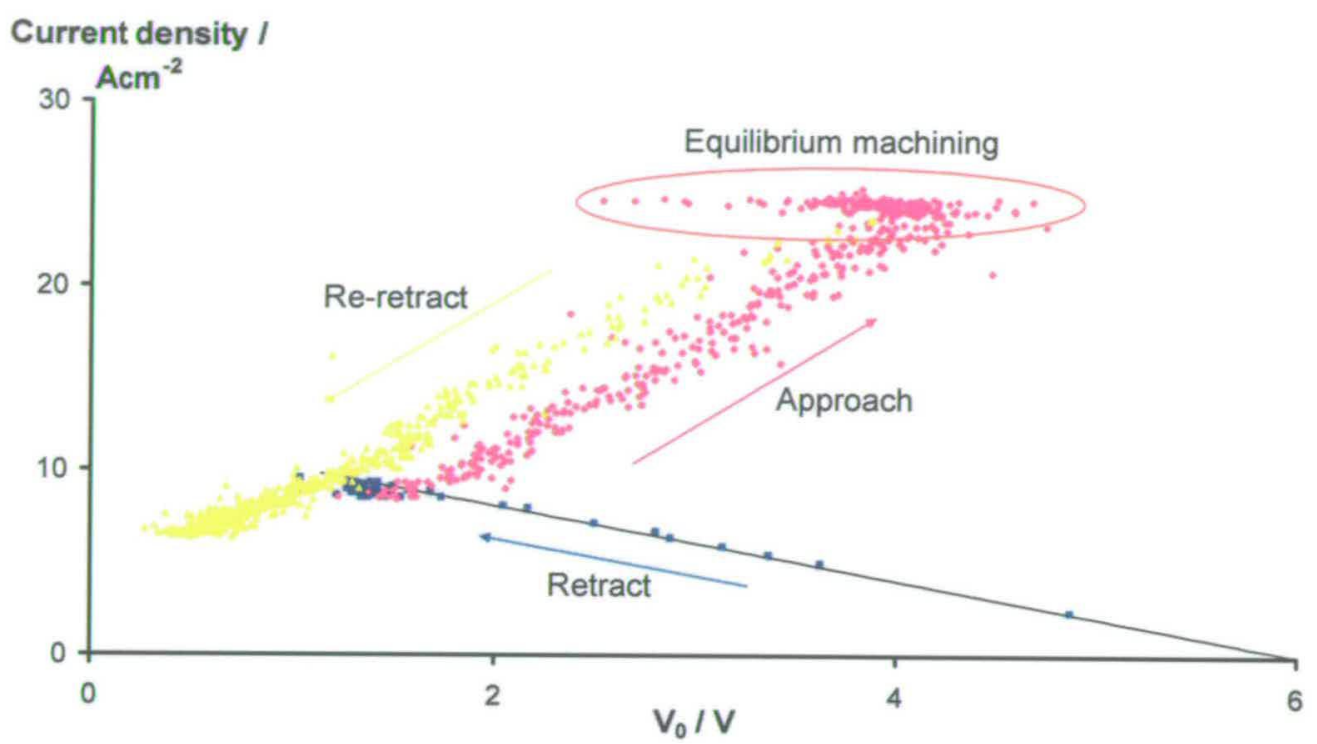

Figure 7.6: J vs. $\mathrm{V}_{0}$ data for the electrochemical dissolution of Fe. For this plot, and all subsequent $\mathrm{J}-\mathrm{V}_{0}$ plots for cell 2000 , $\mathrm{J}$ is $\mathrm{I}_{4}$, the segment current at the position along the flow path where the ultrasound probe is positioned and consequently where $\mathrm{z}$ and $\mathrm{V}_{0}$ are measured. Also shown is the theoretical line calculated from Equation 7.2 with the appropriate experimental electrolyte conductivity of $\kappa=0.20 \mathrm{~S} \mathrm{~cm}^{-1}$.

It is clear that, in contrast to the simplifying previous assumption (section 1.10), a dependence of $\mathrm{J}$ on $\mathrm{V}_{0}$ is observed during ECM, as expected.

\subsubsection{Discussion of $\mathrm{J}-\mathrm{V}_{0}$ behaviour.}

The results in this chapter have demonstrated the ability to measure $\mathrm{J}-\mathrm{V}_{0}$ characteristics under ECM conditions. The data collected can now be compared to previous literature regarding the dissolution of $\mathrm{Fe}$ and $\mathrm{Fe}$-based alloys such as steel.

Initially, at the start of the retract phase, $\mathrm{V}_{0}$ is found to decrease linearly with increasing current density (Figure 7.6). Quantitatively, this phenomenon can be modelled through differentiation of Equation 3.1 with respect to the two variables during machining, $\mathrm{V}_{0}$ and $\mathrm{z}$, to give Equation 7.1: 


$$
d I=-\frac{\kappa A}{z} d V_{0}-\frac{\kappa A\left(V-V_{0}\right)}{z^{2}} d z
$$

\section{Equation 7.1}

Initially $\left(\mathrm{V}-\mathrm{V}_{0}\right)$ is small, $\mathrm{z}$ is large, and $\mathrm{dz}$ is small (as there is no feed and little dissolution, see Figure 7.3), consequently the second term of Equation 7.1 can be assumed to be negligible and Equation 7.1 then simplifies to:

$$
d I=-\frac{\kappa A}{z} d V_{0}
$$

\section{Equation 7.2}

In this case an initial linear dependence of $\mathrm{V}_{0}$ on I with a gradient of $-\mathrm{kA} / \mathrm{z}$ should be observed. In addition, from Equation 3.1 when $\mathrm{J}=0.0 \mathrm{~A}, \mathrm{~V}-\mathrm{V}_{0}=0.0 \mathrm{~V}$, consequently the $\mathrm{V}_{0}$ axis intercept of this line should lie at $\mathrm{V}_{0}=\mathrm{V}=6.0 \mathrm{~V}$ for this data. Figure 7.6 also shows this theoretical line. The close correspondence of this line with the experimental data confirms the validity of Equation 7.2 in the retract phase. Figure 7.6 shows the data on this line starts at low $\mathrm{J}$ and high $\mathrm{V}_{0}$ and moves to high $\mathrm{J}$ and low $\mathrm{V}_{0}$. This is consistent with breakdown of a passivating layer on the workpiece. It is likely that this passivating layer is the passive oxide film present on the Fe workpiece prior to machining which is broken down in order to establish the required surface conditions for workpiece electrochemical dissolution (often termed active dissolution).

Figure 7.6 then shows a small increase in $\mathrm{V}_{0}$ with a negligible change in $\mathrm{J}$ at the start of the approach phase, i.e. when the feed is applied. Following this a positive linear $\mathrm{J}-\mathrm{V}_{0}$ dependence is observed during this phase. During the re-retract phase a positive linear $\mathrm{J}-\mathrm{V}_{0}$ dependence is again observed, however, the $\mathrm{V}_{0}$ values are systematically lower during this phase compared to the approach phase. The initial $\mathrm{V}_{0}$ increase with a negligible change in $\mathrm{J}$ when the feed is applied is consistent with an extra voltage requirement as the system is "driven" forwards during the approach phase. During the re-retract phase the system is allowed to relax at its own rate resulting in a slightly lower surface potential measurement. This results in hysteresis in the approach/re-retract behaviour. 
It is interesting to note that the $\mathrm{V}_{0}$ values at the end of the retract phase are consistent with the $\mathrm{V}_{0}$ values measured for the system at the same $\mathrm{I}$, and the same $\mathrm{z}$, during the re-retract phase $\left(\mathrm{V}_{0}=1.3 \mathrm{~V}, \mathrm{I}=7.7 \mathrm{~A}\right.$ and $\mathrm{z}=1.1 \mathrm{~mm}$ respectively). These observations infer that there is no long term change in $V_{0}$ during the retract phases of repeated approach/retract experiments.

Finally both approach and re-retract phases show a positive, essentially linear dependence of the $\mathrm{J}-\mathrm{V}_{0}$ data. This data can be compared to low current data collected by Haisch et al. using a RCE with an iron rich $100 \mathrm{Cr} 6$ steel anode in $20 \% \mathrm{NaCl}$ electrolyte [153] (Figure 7.7).

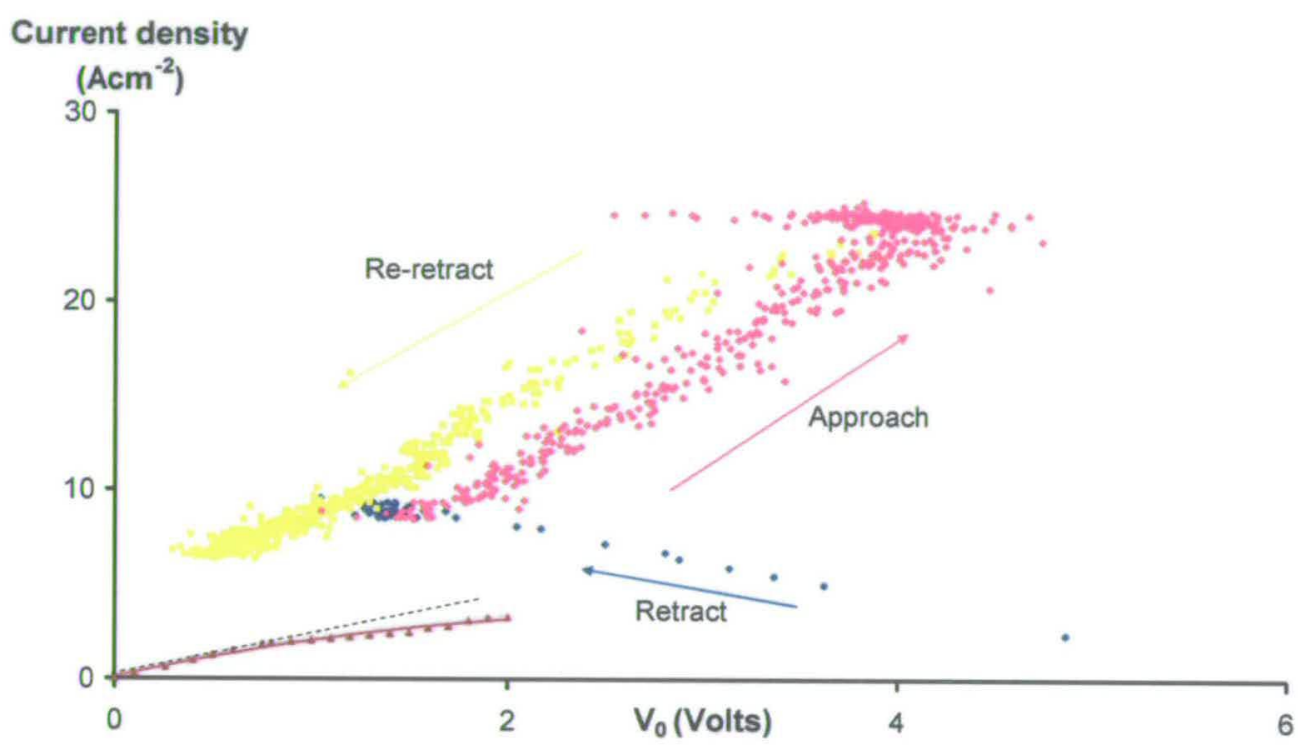

Figure 7.7: Comparison of $\mathrm{J}-\mathrm{V}_{0}$ data from Figure 7.6 with low current density literature data (brown) [153].

At current densities below $1.75 \mathrm{~A} \mathrm{~cm}^{-2}$ Haisch et al. observe a linear increase in anode potential vs. NHE [153], indicated by the dashed line in Figure 7.7. This initial linear section was observed to intersect the anode potential axis at a potential of $0.0 \mathrm{~V}$. Haisch et $a l$. postulate that this line is consistent with active dissolution of iron at low current densities. During the ECM dissolution process detailed in this work, the mean $\mathrm{V}_{0}$ value when $\mathrm{J}=0$ $\mathrm{Acm}^{-2}$, calculated through extrapolation of the positive linear data for the approach and reretract phases (Figure 7.6), was found to be $0.0 \pm 0.1 \mathrm{~V}$, which under these conditions is also likely to be active dissolution. The consistency of this result with that observed by Haisch et 
al. implies that the potential of the tool at $\mathrm{J}=0.0 \mathrm{~A} \mathrm{~cm}^{-2}$ is similar to the NHE reference electrode potential. It is likely that the reaction at the NHE reference electrode [153] and the tool are similar:

$$
\mathrm{H}^{+}+\mathrm{e}^{-} \rightleftharpoons 1 / 2 \mathrm{H}_{2}
$$

\section{Reaction 7.1}

However, the tool is made of copper chrome not $\mathrm{Pt}$, and $\left[\mathrm{H}^{+}\right]$in the ECM system is not equal to 1 , as for NHE. This result therefore suggests that the change in $\left[\mathrm{H}^{+}\right]$and metal has not had a significant effect on the electrode potential of Reaction 7.1. This is in part explicable by the fact that the potential of the reaction is only logarithmically related to $\left[\mathrm{H}^{+}\right]$through the Nernst equation. Thus, as long as this is the tool reaction, direct comparison of $\mathrm{V}_{0}$ values from extrapolation to $\mathrm{J}=0.0 \mathrm{~A} \mathrm{~cm}^{-2}$ with workpiece potentials w.r.t NHE should be possible.

The gradient of the linear phase found by Haisch et al. at current densities below $1.75 \mathrm{~A} \mathrm{~cm}^{-2}$ was found to give a resistance of $0.4 \Omega \mathrm{cm}^{-2}$ which was attributed to an uncompensated ohmic resistance of the electrolyte [153]. This residual resistance of the electrolyte therefore prohibits surface resistance measurements below $0.4 \Omega \mathrm{cm}^{-2}$. The increased gradient of the high current density dissolution data in this work indicate an observed resistance of $0.16 \pm$ $0.1 \Omega \mathrm{cm}^{-2}$. The analysis technique used in this work eliminates all the ohmic resistance attributable to solution electrolyte migration in the gap. Consequently the ohmic dependence of the high current density data in this work infers a resistive, or ohmic, process at the electrode surface with a resistance of $0.16 \pm 0.1 \Omega \mathrm{cm}^{-2}$. This is inconsistent with a Tafel process being the rate determining step at the electrode, which would result in an exponential dependence of $\mathrm{J}$ on $\mathrm{V}_{0}$ in these data (section 2.2).

Haisch et al. also observed that at current densities greater than $3.5 \mathrm{~A} \mathrm{~cm}^{-2}$ the $\mathrm{J}-\mathrm{V}$ characteristics approach a limiting current (Figure 7.7) [153]. This limiting current was seen to be lower at lower rotation speeds [153]. Haisch et al. attribute this current limitation to the formation of a precipitated iron oxychloride salt film. The absence of breaks or gradient changes during the approach and re-retract data in this work suggest no analogous film formation in this work at higher current density. This is possibly due to the increased flow velocities $\left(15-50 \mathrm{~ms}^{-1}\right)$ used for this study compared to Haisch et al. $\left(7 \mathrm{~ms}^{-1}\right)$, which may 
Parameterisation of Electrochemical Machining

decrease local ion concentration and inhibit film formation [155] or the immediate formation of a salt film on application of the ECM voltage. 


\section{Chapter 8}

The ECM of Stainless Steels in NaCl 


\subsection{Introduction}

Stainless steels are probably one of the most common classes of metal material used around the world [156]. For this reason the machining of stainless steels is of great interest in industry. While stainless steels are far more widely used than iron, similar machining criteria apply (section 7.1); the material properties of stainless steels, as with iron, typically make conventional machining techniques more economically viable than ECM for simple machining of single components (however, there are niche markets for which macro ECM stainless steel parameter bases may be useful, e.g. mass produced complex shaped components such as razor and turbine blades [151]). For this reason, much of the current literature concerning ECM of stainless steels concentrates on Electrochemical Micromachining (ECMM) $[157,158]$, which is an area in which the machining of stainless steels using ECM still has general advantages over other machining techniques. Compared to micro EDM, ECMM has the advantage of high material removal rates with no tool wear during the process. Also ECMM is more cost effective than techniques such as laser beam machining [157]. Although micromachining studies are typically carried out at relatively low currents, less than 1 A [157], given the size of the workpiece this can correspond to similarly high current densities as ECM. However, relatively low, if not stationary, electrolyte flow velocities are typically used $[157,158]$. As discussed previously (Chapter 7) this parameter difference may result in significant differences in machining characteristics for ECMM compared to macro ECM. Consequently it is unwise to simply utilise ECMM characterisation studies as a basis for macro ECM characteristics.

There are limited literature studies concerning the macro ECM characteristics of steels machined in $\mathrm{NaCl}$. A study, by Haisch et al., into the machining of the iron rich steel $100 \mathrm{Cr} 6$ in $\mathrm{NaCl}$ was detailed in section 7.2.2 [153]. In addition, a number of current efficiency studies have been carried out on steels using $\mathrm{NaCl}$ electrolyte $[159,160,161]$, under a variety of conditions with current densities relevant to industrial ECM (ranging from $1-100 \mathrm{~A} \mathrm{~cm}^{-2}$ ). Mao used a closed cell system with a mild steel anode being continuously fed into the cell to maintain an interelectrode gap of $0.8 \mathrm{~mm}$ [159]. 2.0 or $4.5 \mathrm{M} \mathrm{NaCl}$ electrolyte was pumped into the system at a flow rate of $2 \mathrm{~L} \mathrm{~min}{ }^{-1}$, giving electrolyte flow velocities of the order of $0.5 \mathrm{~ms}^{-1}$ for this cell (which uses an electrode disc diameter of $0.953 \mathrm{~cm}$ ). These flow velocities are low compared to those used for industrial ECM and this work. 
Studies by Chin et al. were carried out using a mild steel anode [160]. The anode dimensions $\left(A=0.0792 \mathrm{~cm}^{2}\right)$ were small compared to those used in this work while the cathode was $0.40 \mathrm{~cm}^{2}$, which would be expected to produce a non linear field at the anode edges. A 4.0 M solution of $\mathrm{NaCl}$ was introduced into the linear flow path with an initial interelectrode gap of $0.2 \mathrm{~mm}$ and with Reynolds numbers (see section 2.3.2) of the same order as those used in industry and this work $\left(\mathrm{R}_{e}=8000\right)$ resulting from comparable electrolyte flow velocities. The electrolyte temperature was maintained at approximately 21 ${ }^{0} \mathrm{C}$ during machining. Current densities between 1 and $100 \mathrm{~A} \mathrm{~cm}^{-2}$ were investigated. The machining times were short compared to the machining times in this study. Although the authors do not specify exact values, dissolution varied from 120 secs at low current densities to 5 secs at $100 \mathrm{~A} \mathrm{~cm}^{-2}$.

Current efficiency studies by Mileham were carried out on low to medium carbon steel in a radial flow cell using $10 \% \mathrm{w} / \mathrm{w} \mathrm{NaCl}$ with the electrolyte temperature ranging from $15-20$ ${ }^{0} \mathrm{C}$ [161]. In this study, the electrolyte was introduced into the interelectrode gap via a 6.35 $\mathrm{mm}$ diameter hole in the cathode resulting in essentially perpendicular electrolyte impact with the anode and non-uniform electrolyte flow. The gap inlet flow velocities were measured as $30 \mathrm{~ms}^{-1}$ however this velocity undergoes rapid reduction (by a factor of four) due to gap volume expansion. These conditions are consistent with industrial ECM processes in which the electrolyte is introduced through a porting in the tool (section 1.8.3), however, as with the Lohrengel system (section 7.1), tool products are likely to impinge on the anode surface which could produce differences in these observations compared to those collected in a system with a laminar electrolyte flow across the workpiece, such as in this work.

Previous initial studies concerning the macro ECM of stainless steels have been carried out at Edinburgh University. The segmented tool assembly, detailed in Chapter 4, was used to study dissolution valency changes during the ECM of the stainless steel SS316 [162]. The stainless steels SS316, SS410, Jethete, Duplex (D) and Super Duplex (SD) were studied in both $\mathrm{NaCl}$ and $\mathrm{NaNO}_{3}$ electrolytes using the segmented tool assembly [143]. In this study the chronoamperometric analysis technique detailed in section 3.2 was used to determine time-averaged dissolution valencies $(n)$ and overpotentials $\left(V_{0}\right)$ for machining in these systems. For the high $\mathrm{Cr}$ steels $\mathrm{SD}(25.58 \% \mathrm{Cr})$ and $\mathrm{D}(23.00 \% \mathrm{Cr})$ high valency dissolution was observed for both electrolytes, consistent with iron dissolving as $\mathrm{Fe}(\mathrm{III})$ and 
Parameterisation of Electrochemical Machining

chromium as $\mathrm{Cr}(\mathrm{VI})$. Average $\mathrm{V}_{0}$ values for these stainless steels were found to be $9.6 \pm 0.8$ $\mathrm{V}$ and $9.2 \pm 0.8 \mathrm{~V}$ for SD and D respectively. For the low $\mathrm{Cr}$ steels Jethete $(12.50 \% \mathrm{Cr})$ and SS410 $(12.75 \% \mathrm{Cr})$ similar high valency dissolution was observed when machining in $\mathrm{NaNO}_{3}$, while low valency machining was observed in $\mathrm{NaCl}$ consistent with iron dissolving as $\mathrm{Fe}(\mathrm{II})$ and chromium as $\mathrm{Cr}(\mathrm{III})$. Average $\mathrm{V}_{0}$ values for these two steels were found to be $7.5 \pm 0.9 \mathrm{~V}$ and $5.6 \pm 0.9 \mathrm{~V}$ for Jethete in $\mathrm{NaNO}_{3}$ and $\mathrm{NaCl}$ respectively. For SS410 average $\mathrm{V}_{0}$ values were found to be $9.5 \pm 0.8 \mathrm{~V}$ and $4.0 \pm 1.0 \mathrm{~V}$ for machining in $\mathrm{NaNO}_{3}$ and $\mathrm{NaCl}$ respectively. For the intermediate $\mathrm{Cr}$ steel SS316 $(17.00 \% \mathrm{Cr})$ high valency dissolution was observed when machining in $\mathrm{NaNO}_{3}$, while machining in $\mathrm{NaCl}$ initially produced high valency machining followed by a valency change to low valency machining [162]. Average $\mathrm{V}_{0}$ values for this steel were found to be $9.7 \pm 0.8 \mathrm{~V}$ and $5.7 \pm 0.9 \mathrm{~V}$ for $\mathrm{NaNO}_{3}$ and $\mathrm{NaCl}$ respectively.

The work in this chapter uses the ultrasound system to produce time-resolved $\mathrm{n}, \mathrm{J}$ and $\mathrm{V}_{0}$ data for Jethete and SS410 under different ECM conditions. In this way, variations in these parameters during machining can be investigated. As these stainless steels contain over 80 $\%$ atomic weight of iron, they could be expected to display dissolution trends related to those observed for iron (Chapter 7). All the work in this Chapter was carried out using cell 2000 (section 4.6).

\subsection{Thermocouple measurements.}

Any significant temperature effects during machining would be expected to lead to a breakdown in the assumption of constant $\kappa$ during machining (Chapter 3). Temperature measurements were therefore made to test the validity of this assumption. Figure 8.1 shows a typical plot of the inlet and outlet temperature during the machining of SS410 in $12.0 \%$ $\mathrm{NaCl}$. 


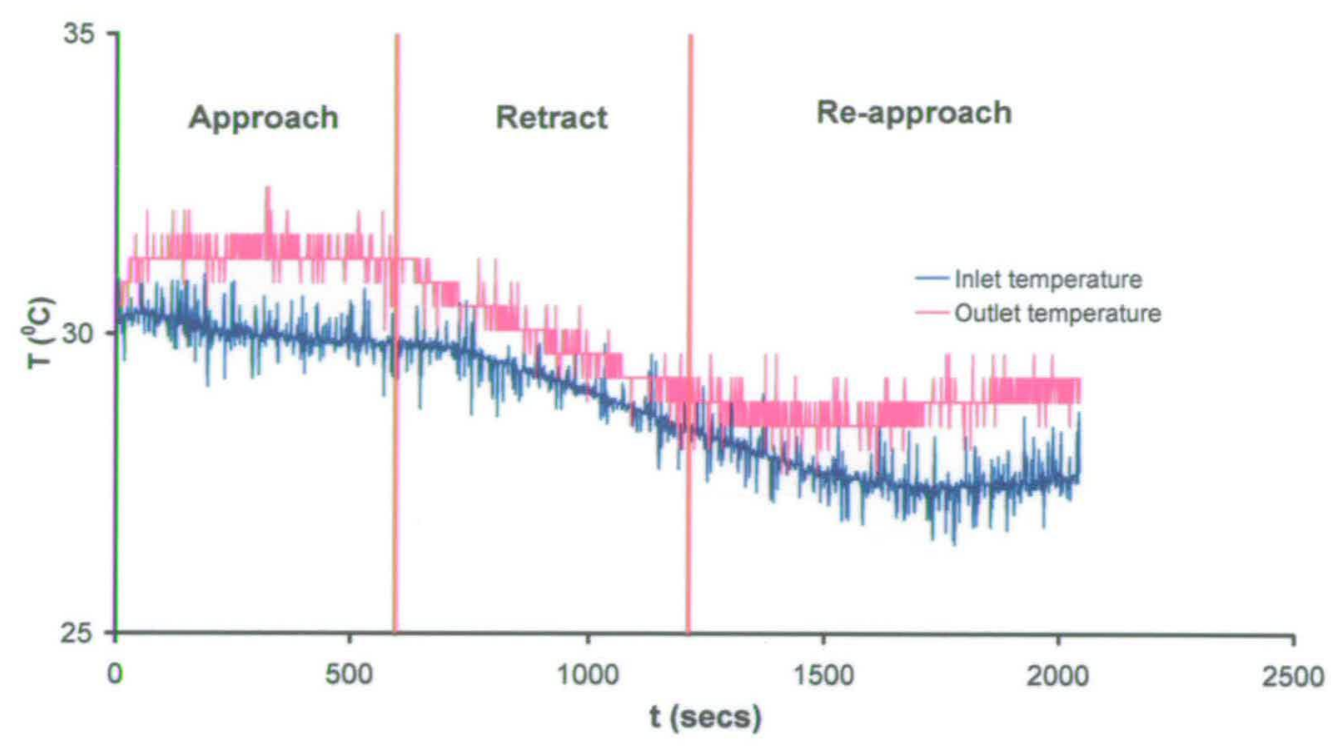

Figure 8.1: Inlet and outlet thermocouple data for machining of SS410 in $12.0 \% \mathrm{NaCl}$ with an applied voltage of $\mathrm{V}=10.0 \mathrm{~V}$.

This clearly shows an increase in electrolyte temperature of the order of $0.4-1.0{ }^{\circ} \mathrm{C}$ across the interelectrode gap during ECM. Thermocouple data collected at the same flow rate $(\mathrm{U}=$ $16.0 \mathrm{~L} \mathrm{~min}^{-1}$ ) with no ECM power at comparable $\mathrm{z}$ values (Figure 8.2) showed negligible temperature differences between the inlet and the outlet temperatures at all $\mathrm{z}$ and a variety of T. This demonstrates that the temperature differences seen in Figure 8.1 are not due to fluid compression or friction as a result of electrolyte flow and that the increase seen is a consequence of the application of ECM power. 


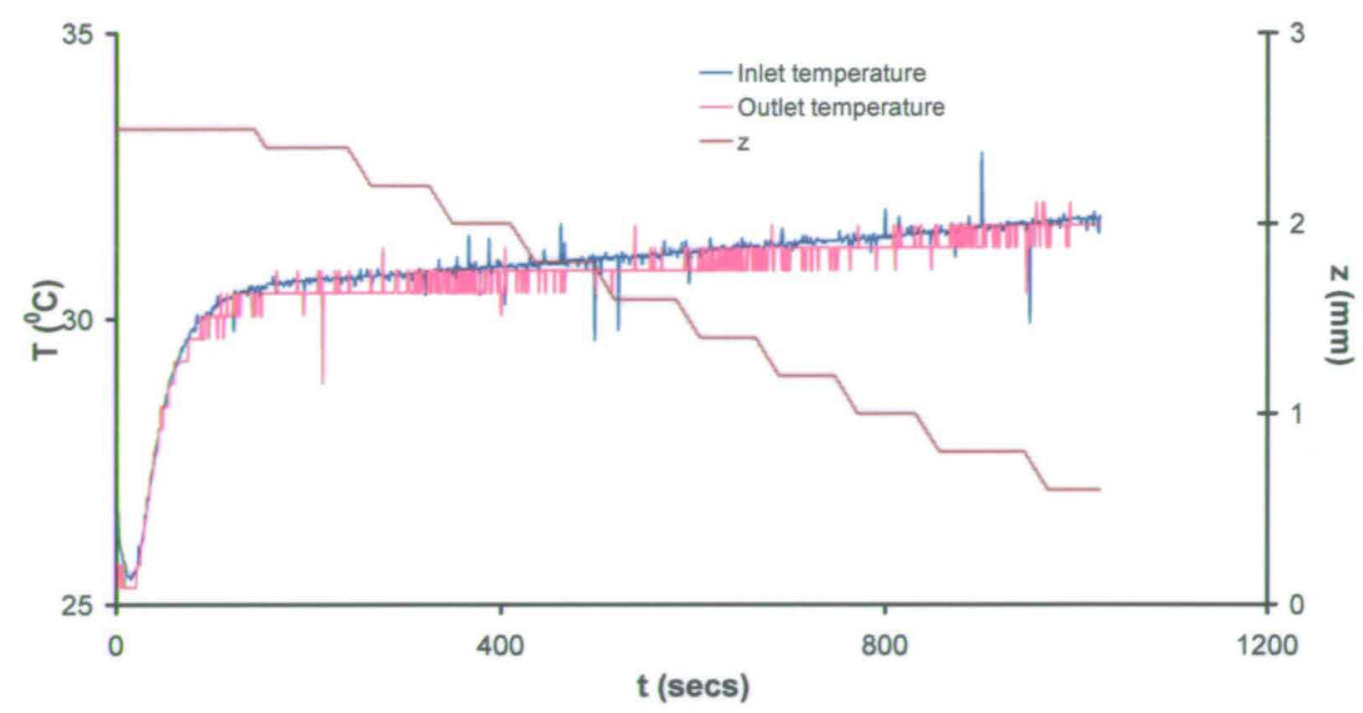

Figure 8.2: Inlet and outlet calibration thermocouple data for $\mathrm{U}=16.0 \mathrm{~L} \mathrm{~min}^{-1}$ and no power. Also shown is the $\mathrm{z}$ value at which the data were collected.

In this case Joule heating is the simplest explanation of the temperature increase seen in Figure 8.1 between the inlet and the outlet thermocouples. The total power, P, dissipated in this experiment can be calculated using the simple relationship:

$$
P=I V
$$

\section{Equation 8.1}

Assuming insignificant thermal dissipation within the cell, this would lead to the following relationship for the theoretical outlet temperature:

$$
T_{\text {out }}=T_{\text {in }}+\frac{P}{U C}
$$

\section{Equation 8.2}


Where $U$ is the electrolyte flow rate $\left(\mathrm{cm}^{3} \mathrm{~s}^{-1}\right), \mathrm{C}$ is the specific heat capacity of the electrolyte (assumed to be that of water, $\mathrm{C}=4.186 \mathrm{~J} \mathrm{~cm}^{-3} \mathrm{~K}^{-1}$ ) [163] and $\mathrm{P}$ is the total power dissipated $\left(\mathrm{J} \mathrm{s}^{-1}\right)$ found using Equation 8.1.

Figure 8.3 shows the comparison of the experimental data with the theoretical values calculated from Equation 8.1 and Equation 8.2.

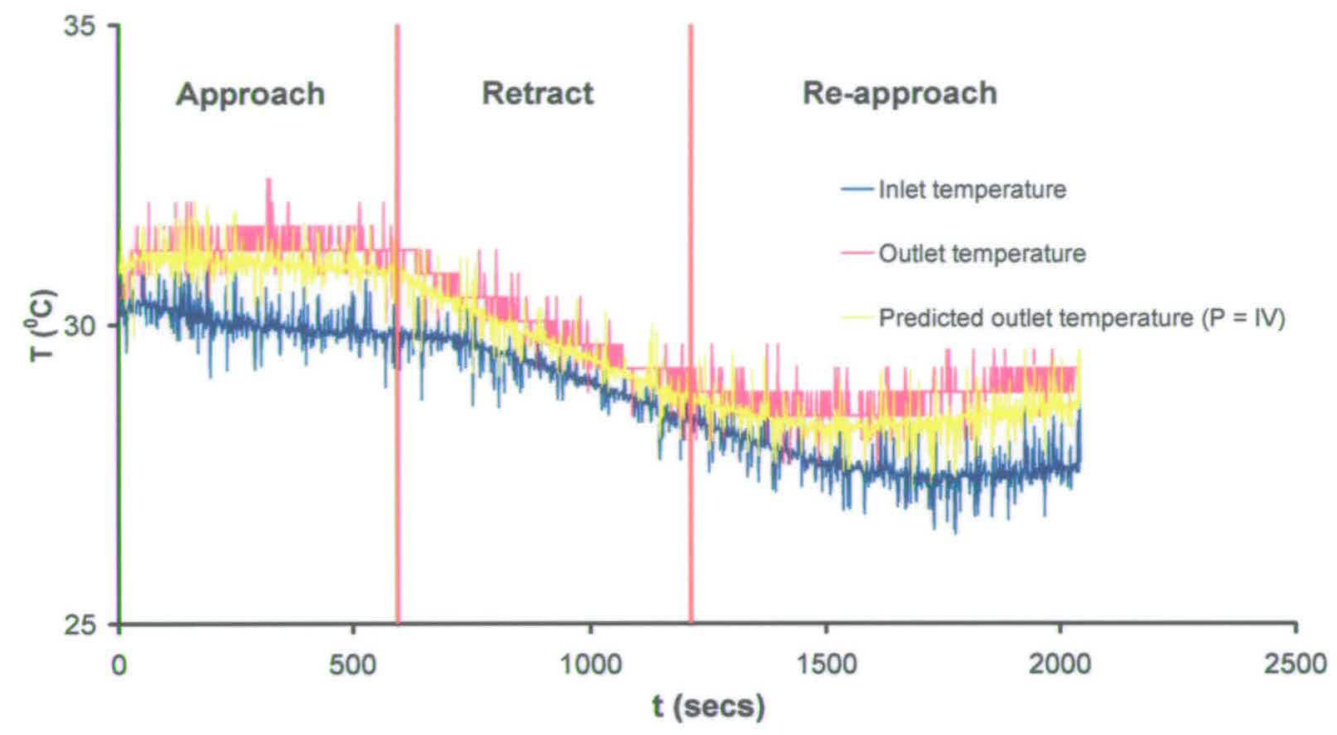

Figure 8.3: Plot to show the comparison of the temperature profiles in Figure 8.1 with theoretical outlet temperatures calculated using Equation 8.1.

It is satisfying that the predicted temperature rise shown in Figure 8.3 is of the same order of magnitude as that observed experimentally, implying that Joule heating is responsible for this temperature increase. The close comparison of the predicted outlet temperature using Equation 8.1 to calculate the dissipated power infers that all the IV energy put into the system results in a temperature rise of the electrolyte. A Joule heating effect would be expected to give a temperature increase which would vary linearly along the flow path length. Consequently, in this case, the temperature at segment $4\left(\mathrm{~T}_{4}\right)$, the position where the ultrasound probe is located, could be predicted using Equation 8.3. 


$$
T_{4}=\frac{\left(T_{o u t}-T_{i n}\right)}{2}
$$

\section{Equation $\mathbf{8 . 3}$}

It is interesting that although the predicted outlet temperature data fits well during the retract phase, Figure 8.3 does still show the measured outlet temperature to be marginally greater during the approach and re-approach phases than the theoretical outlet temperature calculated using Equation 8.1 (with a difference of $0.4{ }^{\circ} \mathrm{C}$ ). This could suggest a small contribution from additional exothermic reactions in these phases in the gap (Chapter 13). Even allowing for these effects, the temperature changes during machining are still very small.

In addition, a cooling coil placed in the bulk electrolyte during machining removes the effects of accumulated Joule heating during electrolyte recycling in order to maintain a constant bulk electrolyte temperature. Figure 8.1 shows that over the duration of the experiment, the bulk electrolyte temperature has decreased by $1.0{ }^{\circ} \mathrm{C}$ implying that the heat accumulated through Joule heating during machining is approximately balanced by the heat removed by the cooling coil.

Significant, uncorrected, temperature variations would lead to errors in the assumption of a constant $\kappa$ used in the ultrasound analysis method when calculating $\mathrm{J}-\mathrm{V}_{0}$ data. Conductivity calibration measurements carried out for $12.0 \% \mathrm{NaCl}$ show that a $1.0{ }^{\circ} \mathrm{C}$ electrolyte temperature change will lead to a decrease in conductivity of $\Delta \kappa=2.23 \mathrm{mS} \mathrm{cm}^{-1}$. For this experiment, at an equilibrium machining current of $\mathrm{I}_{\infty}=19.0 \mathrm{~A}$ with an interelectrode gap of $\mathrm{Z}_{\infty}=0.61 \mathrm{~mm}$, an electrode area of $\mathrm{A}=0.857 \mathrm{~cm}^{2}$ and an applied voltage of $\mathrm{V}=10 \mathrm{~V}$, this conductivity change will give rise to a $0.18 \mathrm{~V}$ error in an equilibrium $\mathrm{V}_{0}$ value of $\mathrm{V}_{0, \infty}=3.2$ V. This small error is present in data sets collected prior to the fitting of the thermocouples into the ECM system; however, the error is of the order of the scatter on the $\mathrm{J}-\mathrm{V}_{0}$ data, consequently, the analysis was not greatly affected. Unless otherwise stated, all subsequent data presented in this thesis have been corrected for temperature variations.

Indeed the absence of either segmented current separation during machining for the two stainless steels (vide infra Figure 8.4 and Figure 8.5) or tapering of the interelectrode gap, 
measured by gap casting after machining, is also an indication that there are no significant changes in $\kappa$ during the experiment.

\subsection{Variations in Segment Current During ECM}

Figure 8.4 and Figure 8.5 show typical chronoamperometric transients for Jethete and SS410.

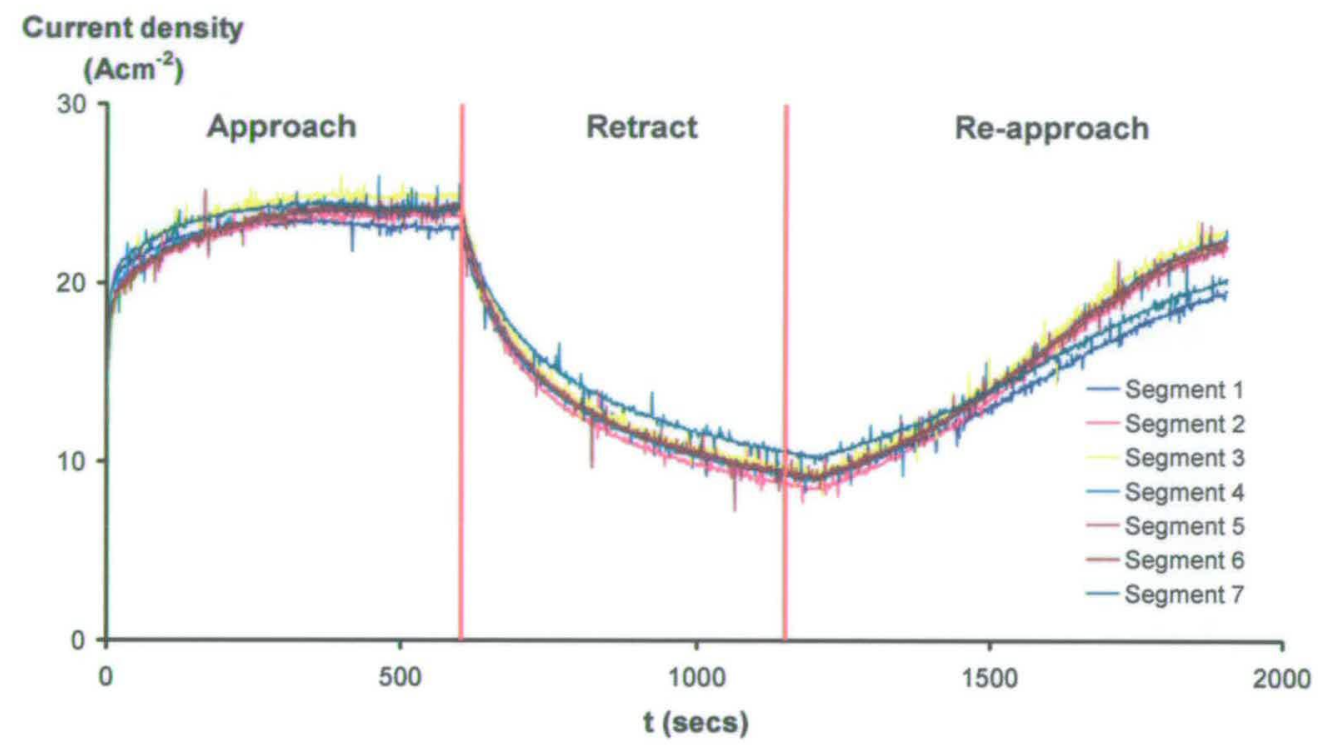

Figure 8.4: Experimental current density transients for Jethete machined in $12.0 \% \mathrm{NaCl}$ with $\mathrm{V}=15.0 \mathrm{~V}$. 


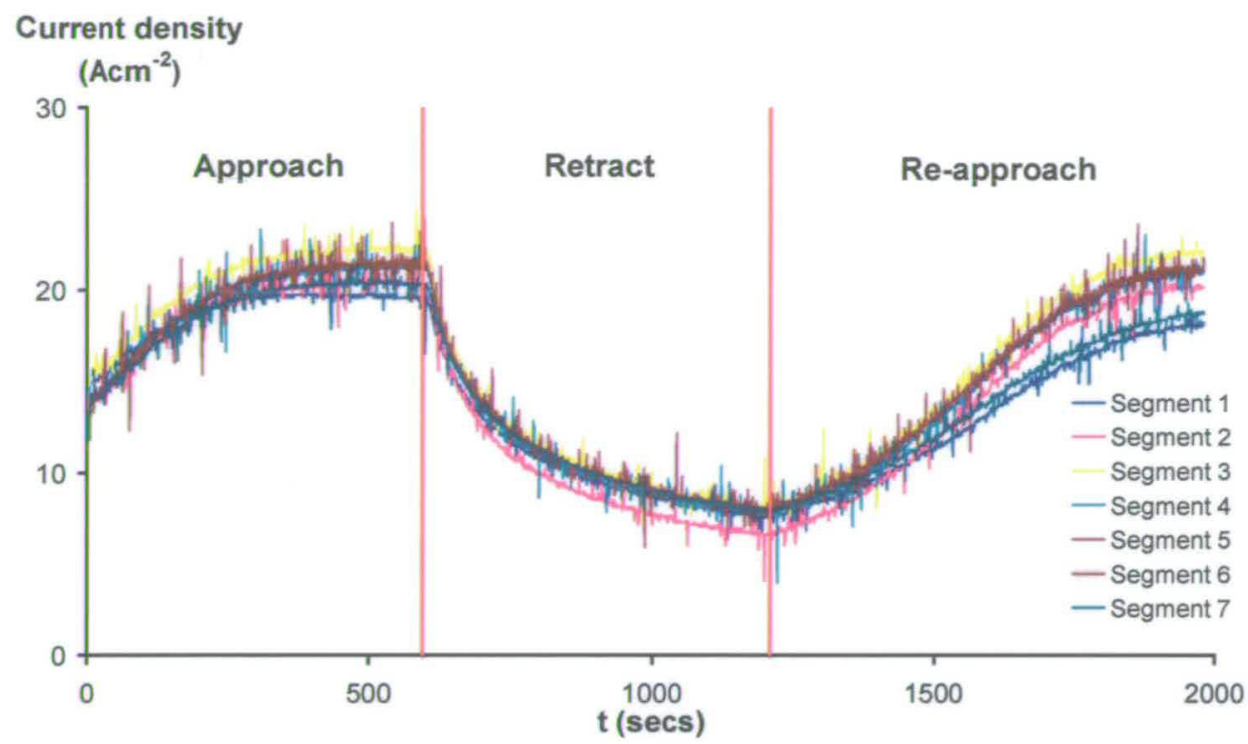

Figure 8.5: Experimental current density transients for SS410 machined in $12.0 \% \mathrm{NaCl}$

$$
\text { with } \mathrm{V}=10.0 \mathrm{~V} \text {. }
$$

These data show a systematic variation in the segment currents for segments 1 and 7 ( $J_{1}$ and $J_{7}$ ). Typically during the retract phase $J_{1}$ and $J_{7}$ were observed to be higher than the remaining segment currents $\left(\mathrm{J}_{2}-\mathrm{J}_{6}\right)$ while during the re-approach phase they were observed to be systematically lower than $J_{2}-J_{6}$. This trend can be attributed to a rounding effect. Figure 8.6 shows a schematic of the field present in the interelectrode gap illustrating the difference between the field in the gap, $\mathrm{E}_{\mathrm{G}}$, and the field at the edge of the tool which curls around the edge of the workpiece, $\mathrm{E}_{\mathrm{E}}$, at large and small $\mathrm{z}$ values. 


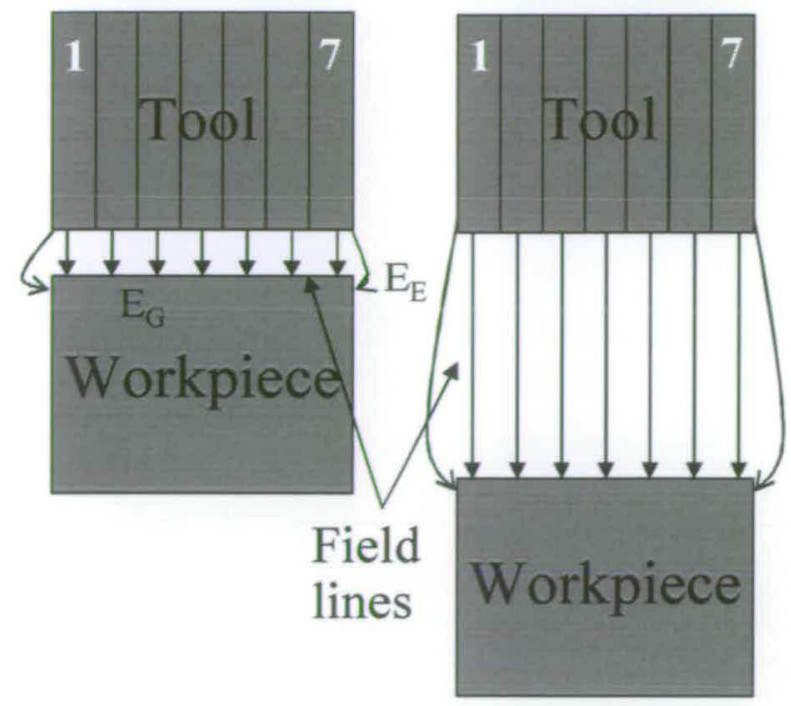

Figure 8.6: Schematic diagram of the effect of the ratios of $E_{E} / E_{G}$ on $E C M$.

At small $\mathrm{z}$ values (i.e. equilibrium machining), the distance of the field lines is appreciably different, therefore, $E_{E}$ is expected to be much smaller than $E_{G}$. However, at large $z$ values (i.e. during the retract phase) the two fields are expected to be roughly equal. Consequently the machining resulting from $\mathrm{E}_{\mathrm{G}}$ and $\mathrm{E}_{\mathrm{E}}$ at large $\mathrm{z}$ values is approximately equal and the periphery of the workpiece is eroded at both ends (segments 1 and 7) during the retract phase. Figure 8.7 shows an exaggerated schematic of the rounding of the workpiece during this phase. It is clear from this figure if $\mathrm{z}$ at segments 1 and 7 were increased producing larger $\mathrm{z}$ values at these segments during the re-approach phase, this would lead to the drops in $\mathrm{J}_{1}$ and $\mathrm{J}_{7}$ observed during this phase and illustrated in Figure 8.4 and Figure 8.5. 
Parameterisation of Electrochemical Machining

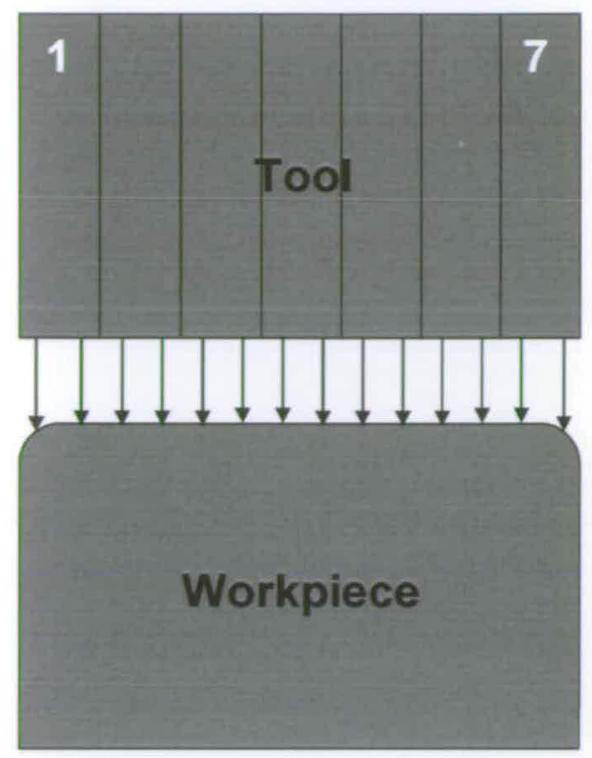

Figure 8.7: Schematic diagram of the exaggerated rounding of the workpiece during the reapproach phase.

These edge effects are only observed for segments 1 and 7 and consequently do not influence the ultrasound analysis which is carried out using segment 4 data.

\subsection{Alloy composition effects.}

Figure 8.8 shows typical approach/retract $\mathrm{J}-\mathrm{V}_{0}$ data for two different SS410 workpieces in $15.0 \% \mathrm{NaCl}$ (section 4.8). 


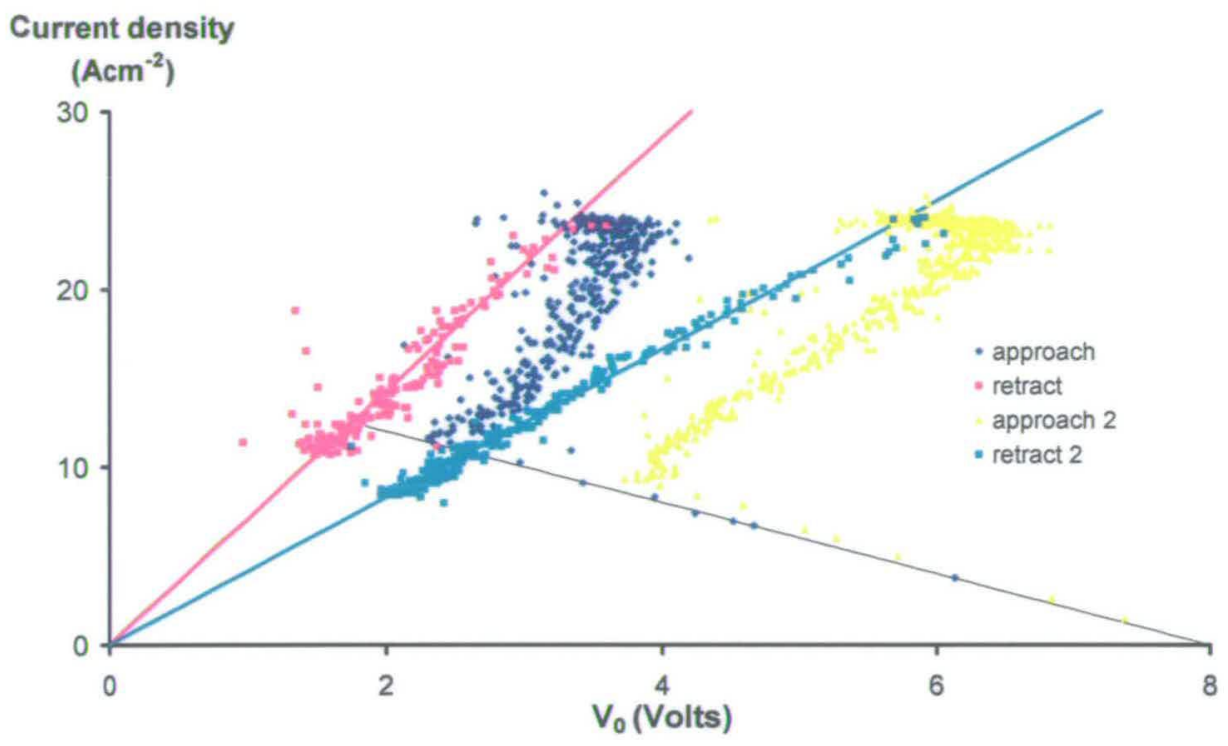

Figure 8.8: Comparison of two non-heat treated SS410 workpiece (workpiece 1 and 2) machined in $15.0 \% \mathrm{NaCl}$ at an applied voltage of $8.0 \mathrm{~V}$. Also shown is the theoretical line calculated from Equation 7.2 with the appropriate experimental electrolyte conductivity of $\kappa$ $=0.20 \mathrm{~S} \mathrm{~cm}^{-1}$ and also theoretical $\mathrm{J}-\mathrm{V}_{0}$ linear transient with ohmic resistances of $0.140 \Omega \mathrm{cm}$ ${ }^{2}$ and $0.240 \Omega \mathrm{cm}^{-2}$ (pink and blue respectively).

Figure 8.8 shows some clear similarities to the $\mathrm{J}-\mathrm{V}_{0}$ data collected for $\mathrm{Fe}$ (Chapter 7). Initially, as with $\mathrm{Fe}$, the data lie on the theoretical line calculated from Equation 7.2 (section 7.2.2). As with $F e$ the early time low $J$, high $V_{0}$ data change to higher $J$, lower $V_{0}$ data, consistent with the breakdown of passivity. Following the initial linear decrease of $\mathrm{V}_{0}$, both workpieces show positive, essentially linear $\mathrm{J}-\mathrm{V}_{0}$ characteristics for the approach phase consistent with an ohmic process with resistance measurements of $0.140 \pm 0.01 \Omega \mathrm{cm}^{-2}$ and $0.240 \pm 0.01 \Omega \mathrm{cm}^{-2}$ for workpieces 1 and 2 respectively (measured for the retract phase transient to eliminate errors in the calculation through driving the system forward in the approach phase, section 7.2.2). The $\mathrm{V}_{0}$ axis intercept at $\mathrm{J}=0 \mathrm{Acm}^{-2}$ for both retract phases is at $\mathrm{V}_{0}=0.0 \mathrm{~V}$, previously attributed to active dissolution for $\mathrm{Fe}$ (section 7.2.2). However, unlike for $\mathrm{Fe}$, the final point of the initial linear decrease of $\mathrm{V}_{0}$ with increasing $\mathrm{J}$ does not correspond to $\mathrm{V}_{0}$ values at the same current on the retract phase inferring a long term change in $\mathrm{V}_{0}$ during these two phases. Furthermore, the $\mathrm{J}-\mathrm{V}_{0}$ characteristics for workpieces 1 and 2 do not show the same values of $V_{0}$ and large hysteresis is observed between the approach and retract phases for both workpieces. It is likely that the tool reaction is the same for both experiments, since the machining conditions for both workpieces were identical; these 
observations are therefore consistent with the effects of workpiece surface compositional variations between workpieces 1 and 2. Typically, workpiece samples were sectioned by sawing, milling and finally grinding the samples to sizes from a larger heat-treated sample. It is possible that sectioning these samples may have introduced compositional variation. To test this hypothesis SS410 workpieces were heat-treated in-house to the manufacturers specifications (section 4.8) prior to machining. Figure 8.9 shows typical $\mathrm{J}-\mathrm{V}_{0}$ data for an approach/retract/re-approach experiment (section 4.8) on a heat-treated SS410 workpiece.

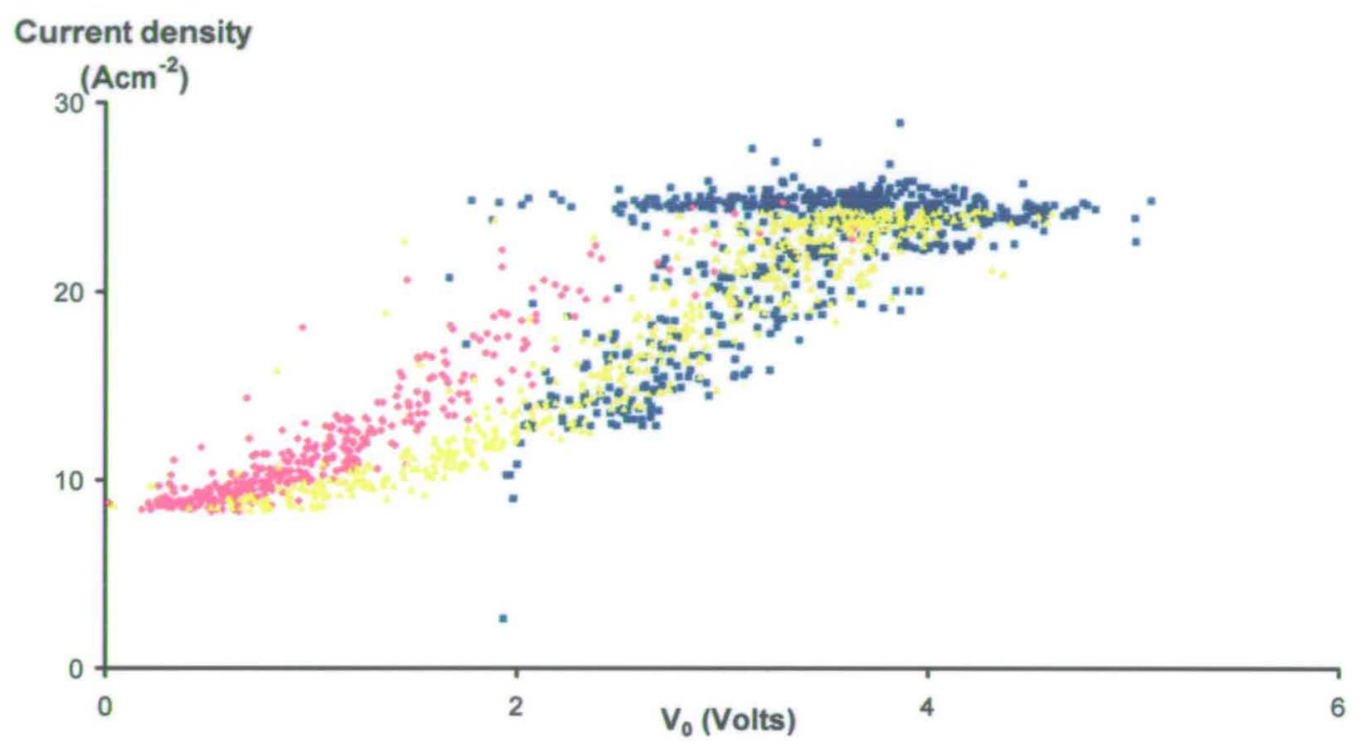

Figure 8.9: $\mathrm{J}-\mathrm{V}_{0}$ plot for heat treated SS410 workpiece machined in $15.0 \% \mathrm{NaCl}$ at an applied voltage of $8.0 \mathrm{~V}$. Approach phase is shown in blue, retract in pink and re-approach in yellow.

The $\mathrm{J}-\mathrm{V}_{0}$ plot showed the re-approach $\mathrm{V}_{0}$ values to be similar to the approach $\mathrm{V}_{0}$ values indicating the removal of any previous compositional changes in the workpiece. Repeat $\mathrm{J}-\mathrm{V}_{0}$ data were also found to be superimposable. Consequently all future workpieces were heat treated to these specifications in house after cutting to size and prior to machining. 


\subsection{Basic Parameter Measurements for SS410 and Jethete in $\mathrm{NaCl}$.}

Figure 8.10 and Figure 8.11 shows $\mathrm{J}-\mathrm{V}_{0}$ characteristics for approach/retract/re-approach experiments carried out for Jethete and SS410 in $12.0 \% \mathrm{NaCl}$ electrolyte (with the conductivities measured as $\kappa=0.1897 \mathrm{~S} \mathrm{~cm}^{-1}$ and $\kappa=0.1843 \mathrm{~S} \mathrm{~cm}^{-1}$ respectively). Ultrasound gap measurements gave equilibrium machining gaps for Jethete and SS410 of $z_{\infty}$ $=0.80 \pm 0.01 \mathrm{~mm}$ and $\mathrm{z}_{\infty}=0.60 \pm 0.05 \mathrm{~mm}$ respectively. The larger machining gap observed for Jethete is a consequence of the higher applied voltage for this system compared to $\mathrm{SS} 410(\mathrm{~V}=15.0 \mathrm{~V}$ and $10.0 \mathrm{~V}$ respectively $)$.

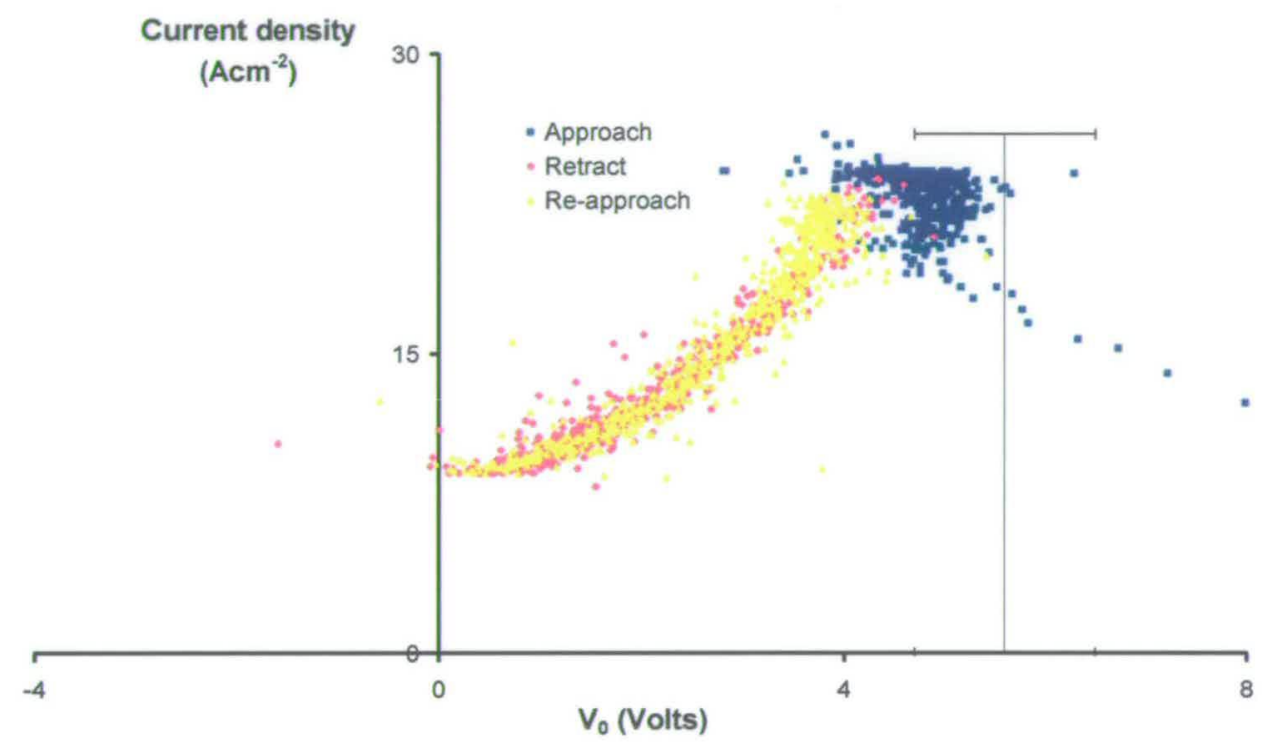

Figure 8.10: $\mathrm{J}$ vs. $\mathrm{V}_{0}$ data for the electrochemical dissolution of Jethete machined in $12.0 \%$

$\mathrm{NaCl}$ at an applied voltage of $\mathrm{V}=15.0 \mathrm{~V}$. Also shown is the $\mathrm{V}_{0}$ value found previously using chronoamperometric analysis of $\mathrm{V}_{0}=5.6 \pm 0.9$ [143]. 


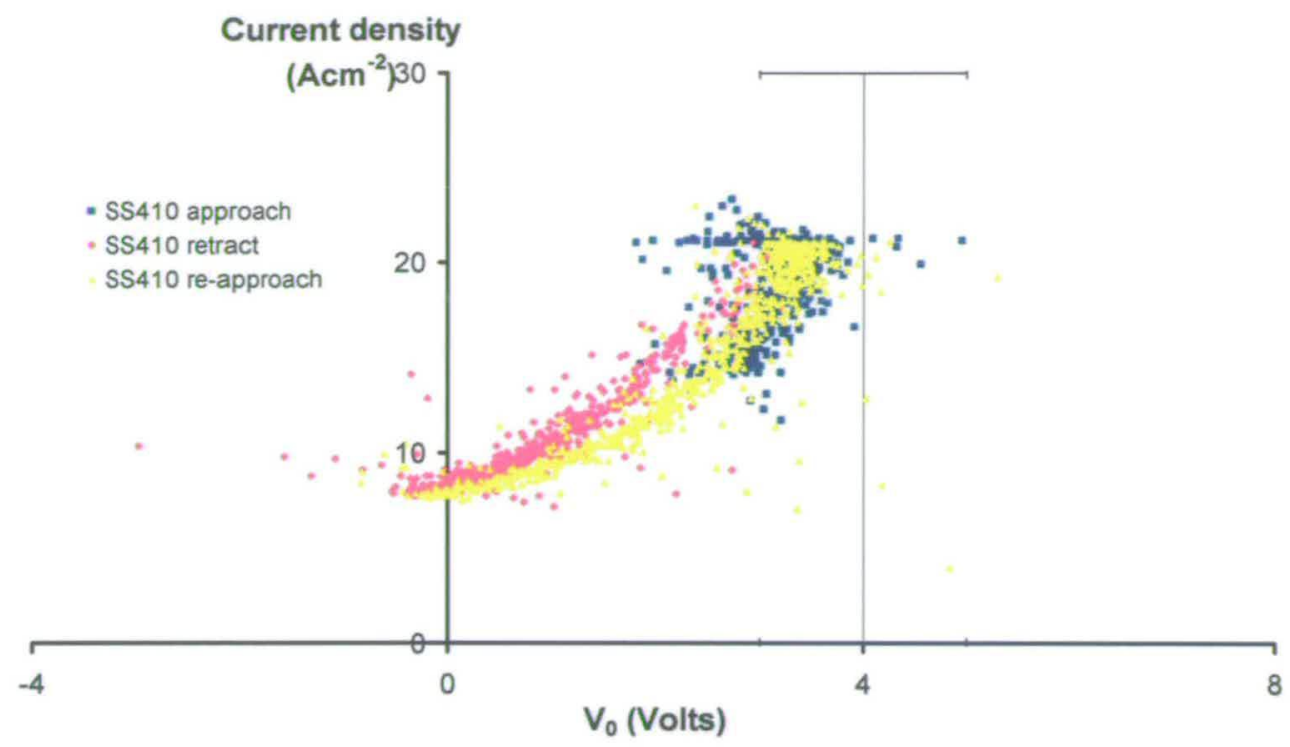

Figure 8.11: J vs. $\mathrm{V}_{0}$ data for the electrochemical dissolution of SS410 machined in $12.0 \%$ $\mathrm{NaCl}$ at an applied voltage of $\mathrm{V}=10.0 \mathrm{~V}$. Also shown is the $\mathrm{V}_{0}$ value found previously using chronoamperometric analysis of $\mathrm{V}_{0}=4.0 \pm 1.0$ [143].

It is re-assuring that the data in Figure 8.10 and Figure 8.11 show the approach phase $\mathrm{V}_{0}$ data to be broadly consistent, to within experimental error, with the previously calculated, timeaveraged $\mathrm{V}_{0}$ values found using chronoamperometric analysis for the approach phase data with current densities up to $90 \mathrm{~A} \mathrm{~cm}^{-2}$ [143]. The ultrasound method used in this work also shows the approach phase $V_{0}$ values to vary within the error bounds of the chronoamperometric method. Therefore the ultrasound system provides much more information concerning the temporal variation in $\mathrm{V}_{0}$ data during the approach, retract and reapproach phases.

The initial linear decrease of $\mathrm{V}_{0}$ with increasing $\mathrm{J}$ observed when machining $\mathrm{Fe}$ (section 7.2.2) is not obvious for these SS410 data (Figure 8.11). However, this phenomenon has been observed when machining Jethete, which is consistent with the theoretical analysis detailed in section 7.2.2. Figure 8.12 shows $\mathrm{J}-\mathrm{V}_{0}$ data for the approach phases of approach/retract/re-approach experiments on Jethete carried out using different initial interelectrode gaps $\left(\mathrm{z}_{0}\right)$. 


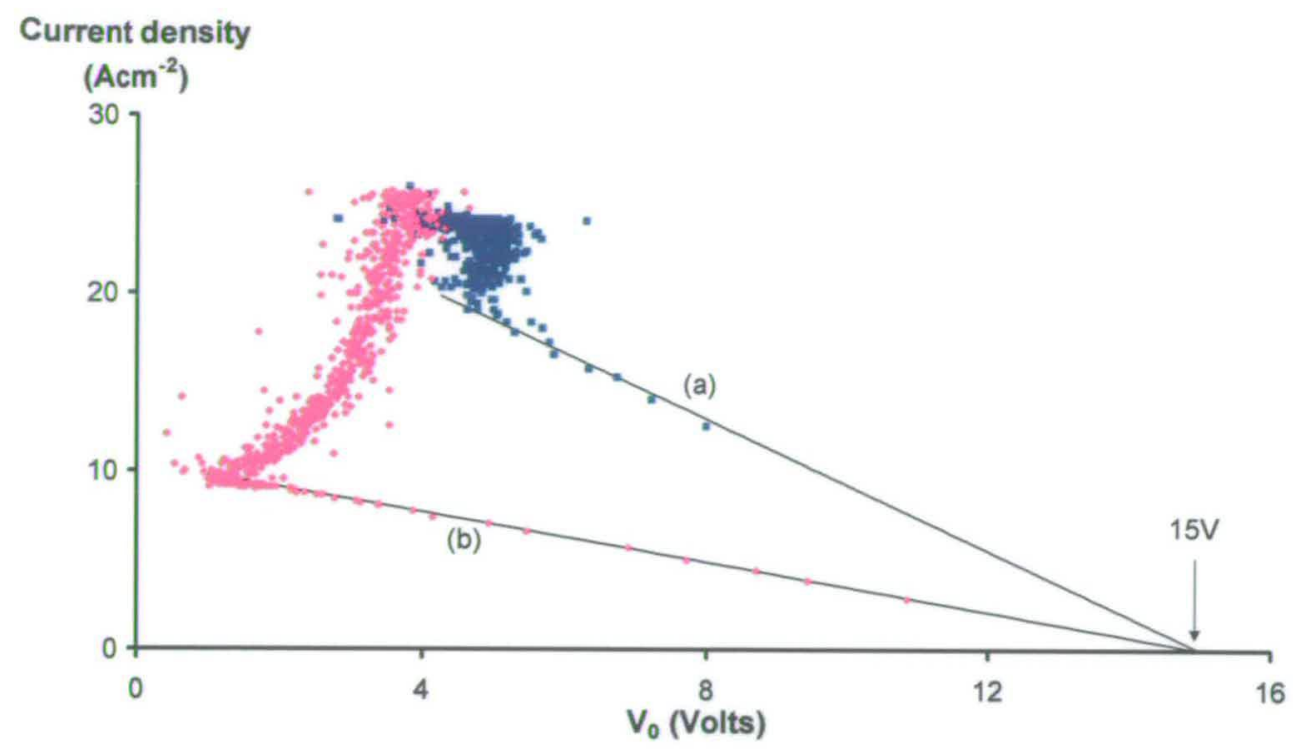

Figure 8.12: Approach $\mathrm{J}-\mathrm{V}_{0}$ data for the electrochemical dissolution of Jethete with (a) $\mathrm{z}_{0}=$ $1.055 \mathrm{~mm}$ and an electrolyte concentration of $12.0 \%$ and (b) $\mathrm{z}_{0}=2.960 \mathrm{~mm}$ and an electrolyte concentration of $15.0 \%$ both machined at $\mathrm{V}=15.0 \mathrm{~V}$. Also shown are theoretical lines calculated from Equation 7.2 with the appropriate electrolyte conductivities of $\kappa=0.184 \mathrm{~S} \mathrm{~cm}^{-1}$ and $\kappa=0.210 \mathrm{~S} \mathrm{~cm}^{-1}$ respectively.

Figure 8.12 shows the close correspondence of the initial linear decrease to the theoretically calculated lines found using Equation 7.2. In these data, as with $\mathrm{Fe}$ (section 7.2.2), the data on this line start at low $\mathrm{J}$ and high $\mathrm{V}_{0}$ and move to high $\mathrm{J}$ and low $\mathrm{V}_{0}$ consistent with breakdown of a passivating layer on the workpiece. Following this initial linear decrease of $\mathrm{V}_{0}$ with increasing $\mathrm{J}$, Figure 8.12 shows systematically higher values of $\mathrm{V}_{0}$ during the approach phase for the $\mathrm{z}_{0}=1.055 \mathrm{~mm}$ data. This is consistent with the temporal mixing of the two terms in Equation 7.1.

Furthermore Figure 8.13 shows the approach and retract data for the Jethete experiment carried out with $\mathrm{z}_{0}=2.960 \mathrm{~mm}$. From these data it is clear that the $\mathrm{V}_{0}$ values at the end of the initial linear decrease are consistent with the $\mathrm{V}_{0}$ values measured for the system at the same $\mathrm{I}$, and the same $\mathrm{z}$, during the retract phase $\left(\mathrm{V}_{0}=1.03 \mathrm{~V}, \mathrm{I}=8.4 \mathrm{~A}\right.$ and $\mathrm{z}=2.9 \mathrm{~mm}$ respectively). These observations infer that, as with $\mathrm{Fe}$ (section 7.2.2), there is no long term change in $\mathrm{V}_{0}$ during the approach and retract phases in spite of a small hysteresis. 


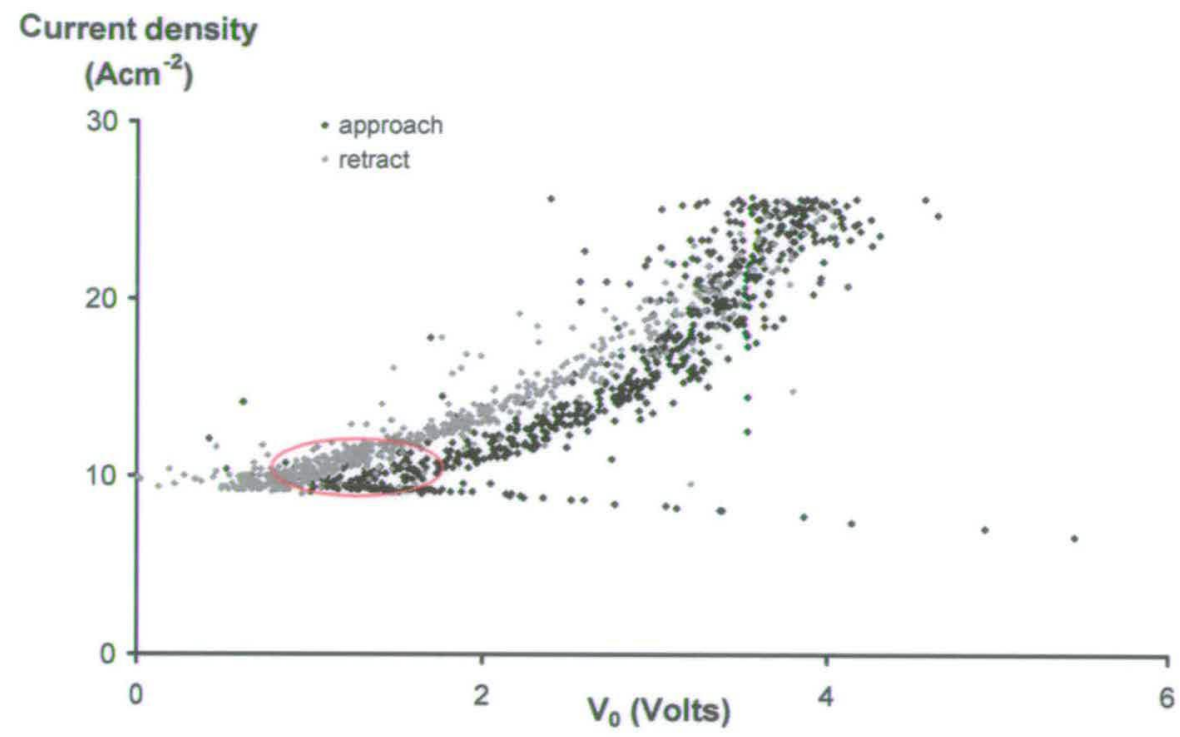

Figure 8.13: Approach and retract $J$ vs. $V_{0}$ data for the electrochemical dissolution of Jethete with $\mathrm{z}_{0}=2.960 \mathrm{~mm}$ and an electrolyte concentration of $15.0 \%$ machined at $\mathrm{V}=15.0 \mathrm{~V}$.

Figure 8.14 and Figure 8.15 show comparisons of the $\mathrm{J}-\mathrm{V}_{0}$ data for SS410 and Jethete and SS410 with those observed for Fe (section 7.2.1) respectively.

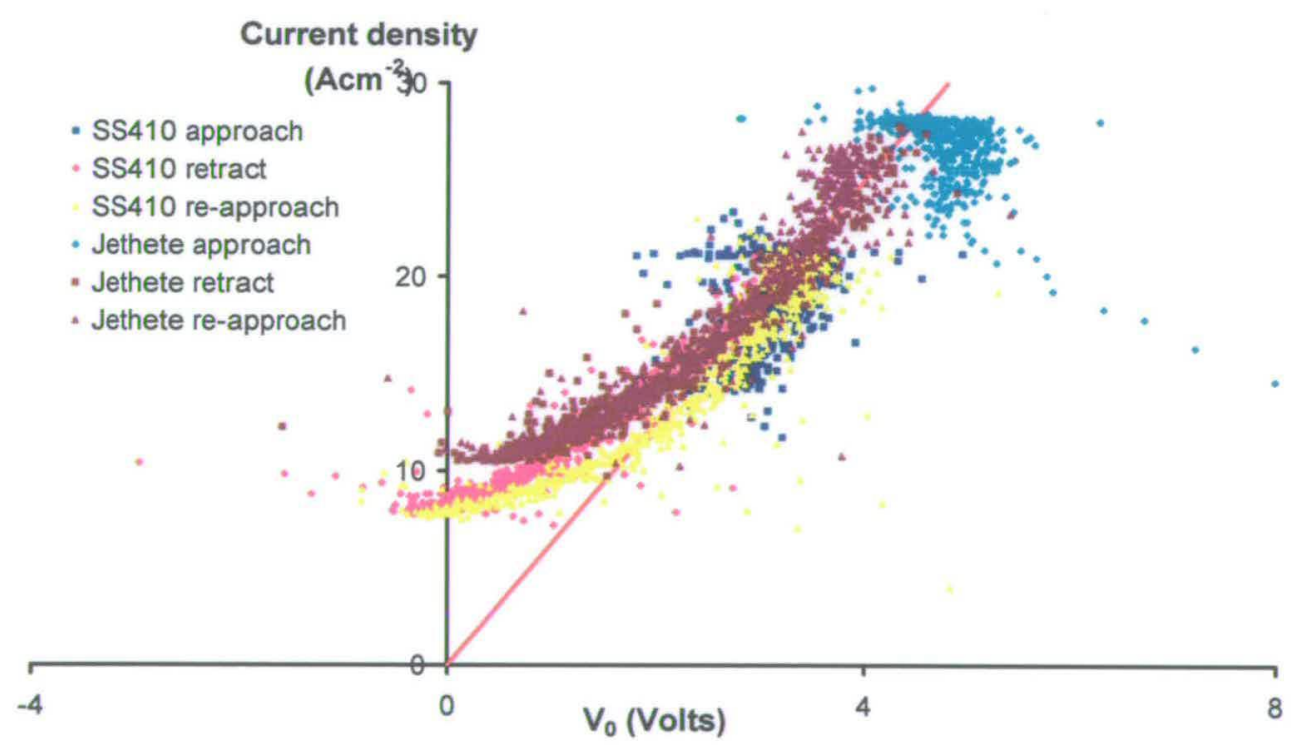

Figure 8.14: Comparison of the $\mathrm{J}-\mathrm{V}_{0}$ data for the electrochemical dissolution of Jethete and SS410. Also shown is the theoretical $\mathrm{J}-\mathrm{V}_{0}$ linear transient with an ohmic resistance of $0.16 \pm$ $0.01 \Omega \mathrm{cm}^{-2}$. 


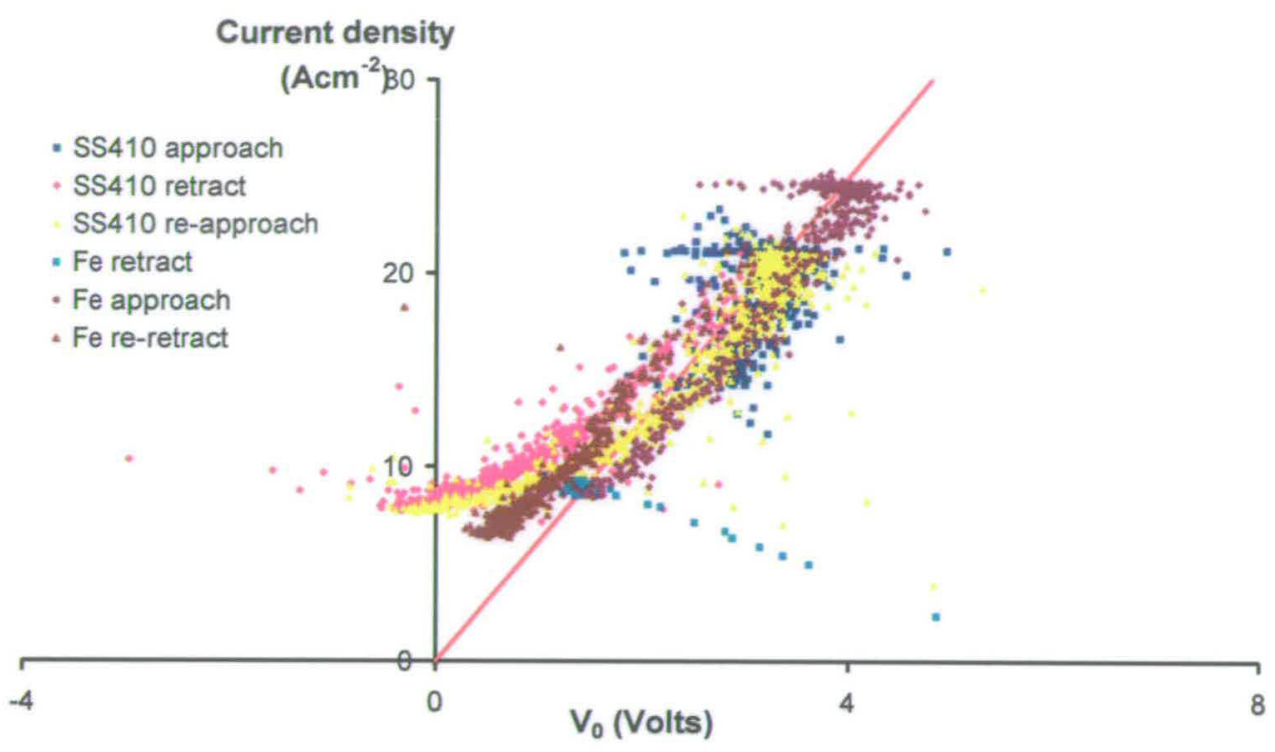

Figure 8.15: Comparison of the $\mathrm{J}-\mathrm{V}_{0}$ data for the electrochemical dissolution of $\mathrm{Fe}$ and

SS410. Also shown is the theoretical $\mathrm{J}-\mathrm{V}_{0}$ linear transient with an ohmic resistance of $0.16 \pm$ $0.01 \Omega \mathrm{cm}^{-2}$.

It is highly satisfactory that the $\mathrm{J}-\mathrm{V}_{0}$ data for both stainless steels follow an essentially common curve in approach, retract and re-approach phases (Figure 8.14) despite the differences in interelectrode gap and applied voltage. This implies that for these data the J$\mathrm{V}_{0}$ characteristics are independent of ECM conditions such as the interelectrode gap and applied voltage and give an insight into the surface dissolution properties of the workpiece.

Figure 8.15 also shows the high current dissolution $\mathrm{J}-\mathrm{V}_{0}$ characteristics to be the same, within experimental error, as those measured for Fe. The high current SS410 data follows the same positive linear $\mathrm{J}-\mathrm{V}_{0}$ line as the $\mathrm{Fe} J-\mathrm{V}_{0}$ data with a similar extrapolated $\mathrm{V}_{0}$ axis intercept of $0.0 \pm 0.1 \mathrm{~V}$. Consequently this data is also consistent with the literature RCE data for an iron rich steel in $\mathrm{NaCl}$ given by Haisch et al. in which an anode potential of $0 \mathrm{~V}$ was observed at $\mathrm{J}=0.0 \mathrm{Acm}^{-2}$ and attributed to an active dissolution process [153] (section 7.2.2). A further literature study by Chin et al. showed the formation of oxide films on a steel anode during ECM with $\mathrm{NaNO}_{3}$ electrolytes [160]. However, in this study oxide films were not observed to form on the steel anodes in chloride media due to the destructive nature of the chloride anion towards the oxide films. This is consistent with the active dissolution process postulated by Haisch et al. [153]. The similarity in the $\mathrm{V}_{0}$ axis intercept implies the 
same thermodynamic dissolution mechanisms at high current density for these stainless steels and Fe. The gradient of the high current density stainless steel data is also the same as that measured for Fe indicating an ohmic surface resistance of $0.16 \pm 0.01 \Omega \mathrm{cm}^{-2}$.

However, Figure 8.15 also shows a trend towards lower $\mathrm{V}_{0}$ values for the low current density $\mathrm{J}-\mathrm{V}_{0}$ data for SS410 compared to Fe. Electrochemically this is indicative of two different electro-oxidations, one occurring at low current density and voltage and reaching a limiting current of approximately $\mathrm{J} \approx 8 \mathrm{~A} \mathrm{~cm}^{-2}$ and a different higher voltage electro-oxidation at high currents which does not appear to reach a limiting current over the current range studied. It is interesting that in this low current, low voltage electro-oxidation regime, there is a corresponding increase in observed dissolution valency (Figure 8.16 and Figure 8.17)

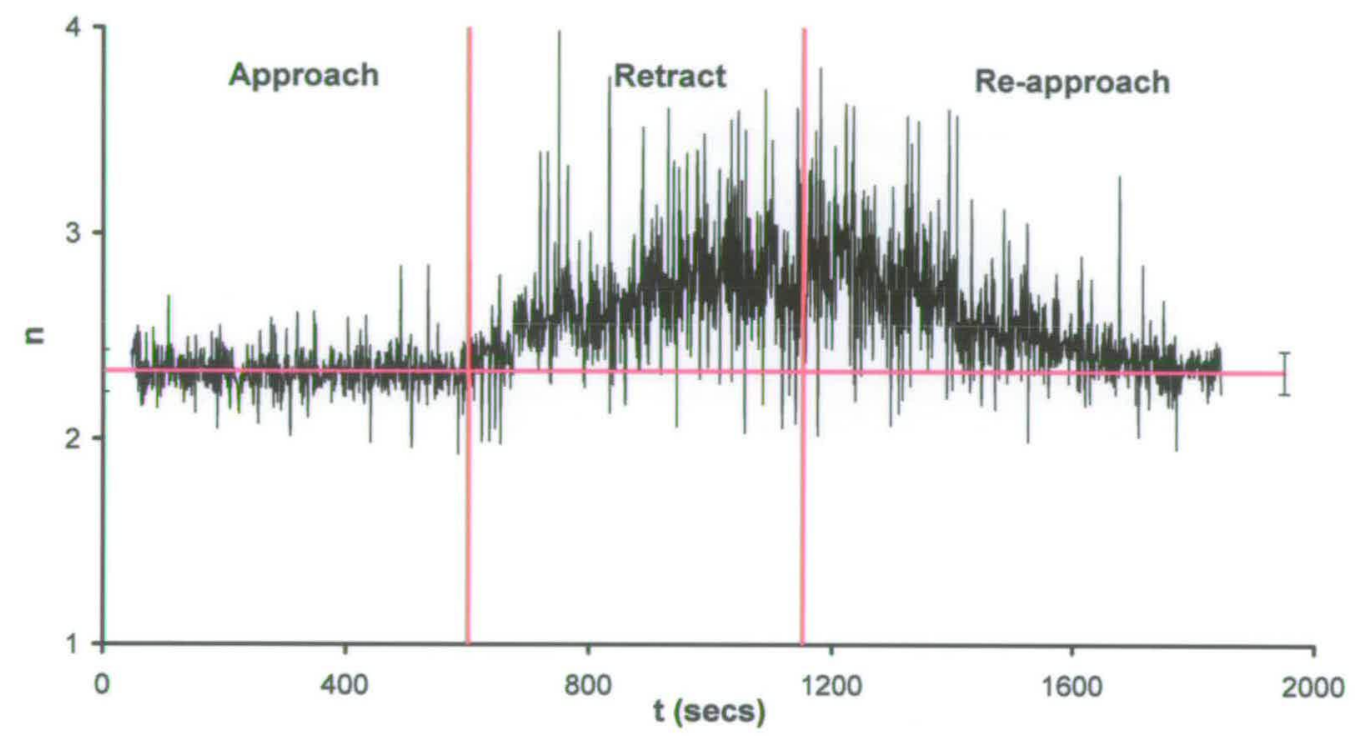

Figure 8.16: Experimental time-resolved n, with $\mathrm{p}=100$ from Equation 6.4, for the stainless steel Jethete, the red line depicts the average dissolution valency at equilibrium with an error shown. 


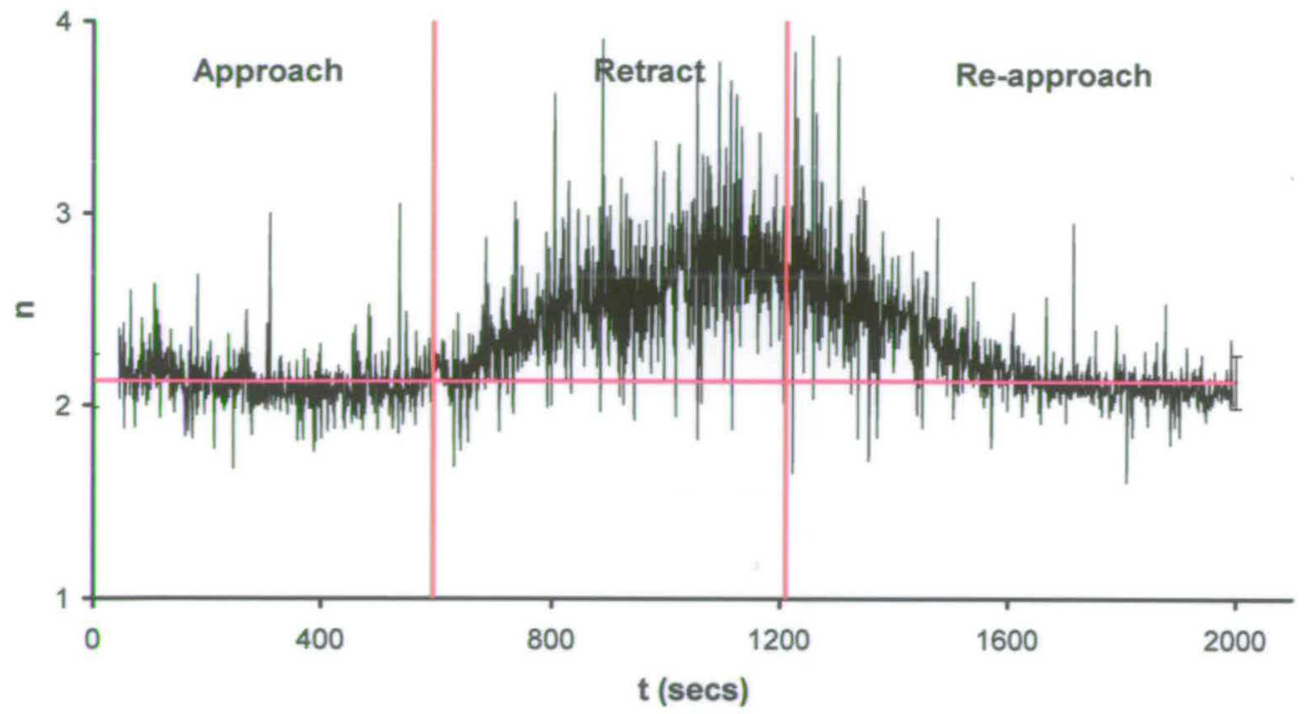

Figure 8.17: Experimental time-resolved n, with $\mathrm{p}=100$ from Equation 6.4, for the stainless steel SS410, the red line depicts the average dissolution valency at equilibrium with an error shown.

From Figure 8.16 and Figure 8.17 the equilibrium dissolution valencies were found to be $\mathrm{n}=$ $2.3 \pm 0.1$ and $n=2.1 \pm 0.2$ for Jethete and SS410 respectively which is the same to within experimental error. These $\mathrm{n}$ values are consistent with the values obtained previously and suggest that, during equilibrium $\mathrm{Fe}$ and $\mathrm{Cr}$ are both undergoing low valency dissolution as $\mathrm{Fe}(\mathrm{II})$ and $\mathrm{Cr}$ (III) respectively [143]. It is reassuring that the time-averaged experimentally measured valency is consistent with that calculated through the subdivision of individual dissolution valencies for the steels, inferring a machining efficiency of $100 \%$ at equilibrium machining conditions. This is consistent with previous literature suggesting that under these conditions $\mathrm{Cr}$ dissolves with a dissolution valency of 3 [161].

However, during the low current/low voltage regime $n$ was observed to increase to $n=2.9 \pm$ 0.2 and $n=2.8 \pm 0.2$ for Jethete and SS410 respectively. This increase in $n$ is consistent with either an increase in dissolution valency or a decrease in efficiency of the dissolution process (Equation 2.35) at low current densities. It is interesting that while an increase in $n$ at low current densities was also observed in the dissolution valency for Fe (Figure 7.4), low current $\mathrm{V}_{0}$ deviation was not observed (Figure 8.15). Figure 8.16 and Figure 8.17 show this valency change occurring between approximately $t=700$ and $1500 \mathrm{~s}$, corresponding to current densities below approximately $12 \mathrm{~A} \mathrm{~cm}^{-2}$ (Figure 8.4 and Figure 8.5) 
In contrast, Figure 8.18 shows the time-resolved valency for an experiment in which the retract phase was terminated to ensure that the current density did not drop below $12 \mathrm{~A} \mathrm{~cm}^{-2}$. These data show a constant dissolution valency throughout machining of $2.1 \pm 0.1$ confirming that this higher valency is present only at current densities below $12 \mathrm{~A} \mathrm{~cm}^{-2}$.

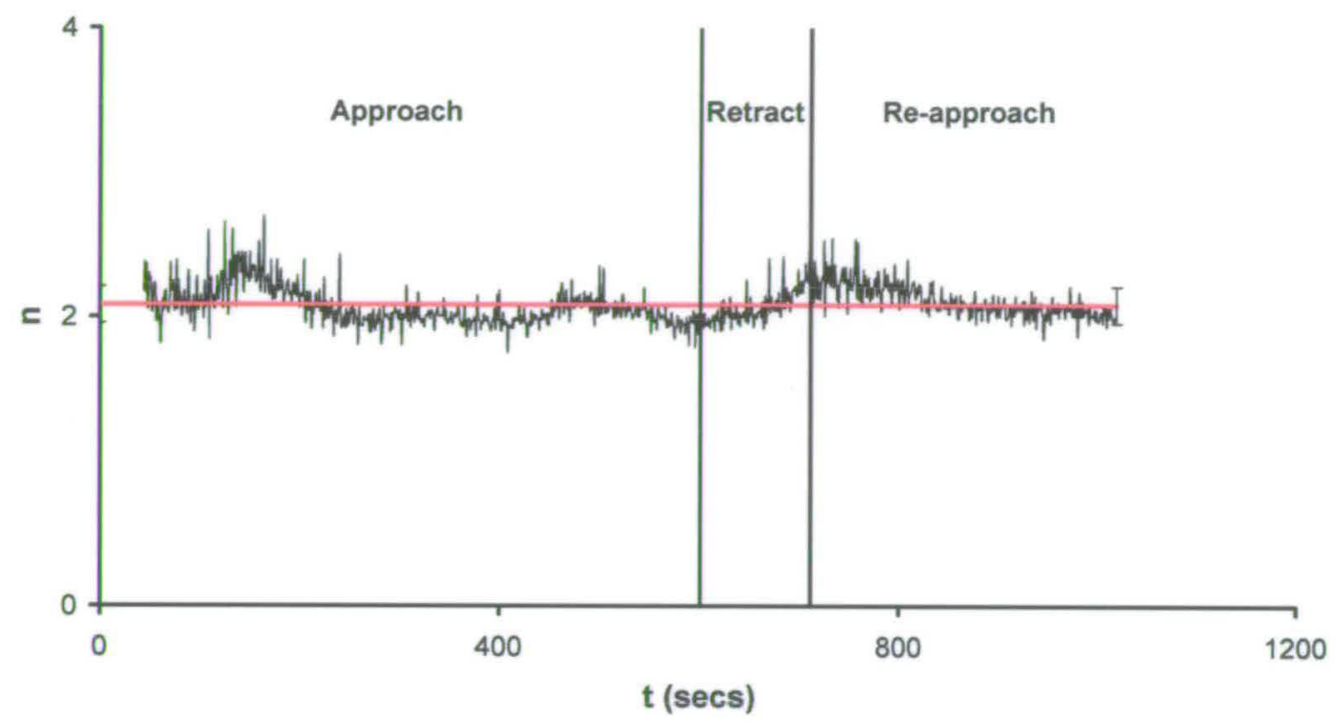

Figure 8.18: Time-resolved valency, with $\mathrm{p}=100$ from Equation 6.4, for SS410 machined with a $\mathrm{J}>12 \mathrm{~A} \mathrm{~cm}^{-2}$ in $15.0 \% \mathrm{NaCl}$ with an applied voltage of $\mathrm{V}=8.0 \mathrm{~V}$, the red line depicts the average dissolution valency throughout machining with an error shown.

In contrast, reductions in current efficiency at higher rather than lower current density when machining low or medium carbon steels have been observed in previous studies by Mao [159], Chin et al. [160] and Mileham [161]. Mao observed the current efficiency to decrease from $98.0 \%$ to $93.9 \%$ with current density increases from $26 \mathrm{~A} \mathrm{~cm}^{-2}$ to $62 \mathrm{~A} \mathrm{~cm}^{-2}$. In this study the high current density inefficiency was attributed to the evolution of oxygen at the steel anode [159]. The study, however, failed to detect oxygen gas during the process, which was attributed to the consumption of the oxygen produced in oxidising some of the ferrous products. Using a flow channel cell with a Reynolds number measured as $\mathrm{R}_{\mathrm{e}}=8000$, Chin et al. observed a decrease in current efficiency for mild steel from $100 \%$ to $80 \%$ as the current density was increased from $7 \mathrm{~A} \mathrm{~cm}^{-2}$ to $50 \mathrm{~A} \mathrm{~cm}^{-2}$ [160]. Decreasing the Reynolds number was found to decrease the current density at which the transition from high to low current efficiency occurred. Chin et al. attribute the decrease in current efficiency to an increase in dissolution valency from $\mathrm{Fe}$ (II) at lower current density to $\mathrm{Fe}$ (III) at higher current density 
(also observed by Mileham [161]). A decrease in efficiency at high current densities from approximately $100 \%$ to approximately $72 \%$ as the current density increased from $5.8 \mathrm{~A} \mathrm{~cm}^{-}$ ${ }^{2}$ to $35.6 \mathrm{~A} \mathrm{~cm}^{-2}$ for a steel anode was observed by Mileham [161]. Through examination of the surface profiles after machining Mileham et al. attribute this to a change from low valency machining to high valency machining.

The results in this thesis, however, show inefficiencies at low current density. It is unlikely that such increasing current densities would lead to a high to low valency transition. Valency changes have also been observed by Mount et al. when machining the stainless steel SS316 [164]. However, these changes were not related to the current density but instead attributed to product accumulation through electrolyte recycling. Landolt presents a model of rate limiting water diffusion to the anode surface [165]. In this model, as the cations form aquo complexes and at high salt concentrations, the activity of free water in the vicinity of the anode surface is reduced. This reduction in the activity of water would be expected to become more pronounced with increasing current density and result in a decrease in oxygen evolution. This model predicts valency increase through inefficiency by increased oxygen evolution at low current densities. This is consistent with the observations in this work.

An alternative explanation is possible, as it is interesting that the observed dissolution valencies seen in Figure 8.16 and Figure 8.17 appear to have a limiting value at approximately 3 , the theoretical dissolution valency for $\mathrm{Cr}$ under these conditions [161]. The increase in the low current/low voltage dissolution valency could also therefore be explained with preferential dissolution of $\mathrm{Cr}(\mathrm{III})$ at low current and $\mathrm{Fe}(\mathrm{II})$ at higher currents. Furthermore, the Pourbaix diagram for an alloyed steel [166] and the standard electrode potentials for $\mathrm{Cr}^{3+}{ }_{\text {(aq) }} \mid \mathrm{Cr}_{(\mathrm{s})}$ and $\mathrm{Fe}^{2+}{ }_{\text {(aq) }} \mid \mathrm{Fe}_{(\mathrm{s})}, \mathrm{E}^{\theta}=-0.74$ and $\mathrm{E}^{\theta}=-0.44 \mathrm{~V}$ respectively [146], show that at $\mathrm{pH}=7.0 \mathrm{Cr}$ is more easily oxidised to $\mathrm{Cr}^{3+}$ than $\mathrm{Fe}$ is to $\mathrm{Fe}^{2+}$. Consequently preferential dissolution of $\mathrm{Cr}(\mathrm{III})$ at low current densities could also explain the lower anode surface potentials observed at these current densities in Figure 8.14 and Figure 8.15.

Together the data in this chapter and Chapter 7 show the J-V $\mathrm{V}_{0}$ ECM characteristics of low chromium stainless steels of similar composition to be closely related to the ECM transient of their major constituent, Fe. The initial linear decrease for both $\mathrm{Fe}$ and stainless steels, consistent with the initial breakdown of a passive layer is observed, and corresponds closely to the theoretical gradient and intercept from Equation 7.2. The positive, essentially linear, high current stainless steel $\mathrm{J}-\mathrm{V}_{0}$ data corresponds closely to the $\mathrm{J}-\mathrm{V}_{0}$ data for Fe with a $\mathrm{V}_{0}$ 
Parameterisation of Electrochemical Machining

axis intercept of $\mathrm{V}_{0}=0.0 \mathrm{~V}$ and a gradient corresponding to an ohmic surface resistance the same as that for $\mathrm{Fe}\left(0.16 \pm 0.01 \Omega \mathrm{cm}^{-2}\right)$ previously attributed to active dissolution of $\mathrm{Fe}$ and steels, consistent with no oxide surface film. A low current increased valency has been observed during ECM for both Fe (Chapter 7) and stainless steels. For stainless steels this valency increase was also observable in the $\mathrm{J}-\mathrm{V}_{0}$ characteristics as a decrease in $\mathrm{V}_{0}$. This is consistent with an alternative electro-oxidation reaction, either oxygen evolution suppressed at high current densities by dehydration of the electrolyte in the vicinity of the anode [165], or preferential dissolution of $\mathrm{Cr}(\mathrm{III})$ at low current densities. There are two possible explanations for these observations; firstly, the effect can be entirely explained through low current density oxygen evolution in Fe, SS410 and Jethete. Secondly, low current density oxygen evolution exists in both systems, however the SS410 and Jethete systems have an extra high valency component through preferential dissolution of $\mathrm{Cr}(\mathrm{III})$ at low current densities. Interestingly the $\mathrm{J}-\mathrm{V}_{0}$ characteristics for Fe do not show a decrease of $\mathrm{V}_{0}$ at low currents consistent with this alternative electro-oxidation reaction. The role of $\mathrm{Cr}$ in this low current behaviour could be probed through the investigation of the ECM J- $\mathrm{V}_{0}$ characteristics of stainless steels with differing \% Cr contents, such as Duplex $(23.00 \% \mathrm{Cr})$, Super Duplex $(25.58 \% \mathrm{Cr})$ or SS316 $(17.00 \% \mathrm{Cr})$, under industrial ECM conditions.

In addition to providing insight into the $\mathrm{J}-\mathrm{V}_{0}$ characteristics of Fe, SS410 and Jethete, the data in this chapter and Chapter 7 provide useful results for an ECM parameter base of these materials. The data presented have shown similar ECM characteristics for two stainless steels of similar composition and also for ECM of Fe, the major constituent of these steels. The common $\mathrm{J}-\mathrm{V}_{0}$ curves observed for Fe, SS410 and Jethete (Figure 8.14 and Figure 8.15) show independence of the $J-V_{0}$ characteristics of $z$ and $V$, greatly simplifying the ECM parameter base. Furthermore, the reproducible approach and retract $\mathrm{J}-\mathrm{V}_{0}$ data at high flow rate show independence of the $\mathrm{J}-\mathrm{V}_{0}$ characteristics on the initial interelectrode gap. 


\section{Chapter 9}

The ECM of In 718 


\subsection{Introduction and previous literature}

Nickel based super alloys are widely used in industries, such as the aerospace and automotive, which require ultra hard metal alloys. The characteristics which make ultra hard metal alloys so desirable, namely high temperature strength, hardness and chemical wear resistance, also make machining of these alloys by conventional methods problematic. Complex shaped components manufactured from super alloys with high surface integrity requirements are found in engines both in the aerospace and automotive industries. It is often economical to machine large numbers of these components using ECM [151]. Chapter 7 and Chapter 8 have shown the similarities in ECM characteristics of Fe and Fe base alloys. It is therefore possible that characterisations carried out for a nickel based alloy may be relevant to other alloys of similar composition, and indeed nickel itself, and may provide a parameter base for the ECM of these materials.

A number of studies into the high current dissolution characteristics of nickel have been carried out by Datta and Landolt [136, 167, 168]. Surface brightening during high rate nickel dissolution in nitrate electrolytes was studied by Datta and Landolt [167]. A flow channel cell with linear electrolyte velocities of between 1.0 and $17.6 \mathrm{~ms}^{-1}$ was used with current densities between 0.5 and $30.0 \mathrm{~A} \mathrm{~cm}^{-2}$. Current-voltage behaviour, current efficiency for metal dissolution and surface appearance were evaluated as a function of applied current density, flow rate and electrolyte composition. In a further study, Datta and Landolt used a channel cell to study high rate anodic dissolution of nickel in $5.0 \mathrm{M} \mathrm{NaCl}$ [136]. The current densities and electrolyte flow velocities in this study were below $33.0 \mathrm{~A} \mathrm{~cm}^{-2}$ and $17.6 \mathrm{~ms}^{-1}$ respectively. Gravimetric measurements were used to calculate mean current efficiencies and the average surface roughness of the samples after dissolution was measured using surface profilometry (also known as the Talysurf method). A further study by Landolt et al. used coulometry and AES to study the thickness and composition of anodic films formed in nickel in alkaline electrolytes consisting of $\mathrm{NaNO}_{3}$ and $\mathrm{NaOH}$ under high rate transpassive dissolution conditions [168]. A channel cell with current densities up to $30.0 \mathrm{~A} \mathrm{~cm}^{-2}$ and an electrolyte flow velocity of $10.0 \mathrm{~ms}^{-1}$ was used with varying concentrations of alkaline aqueous $\mathrm{NaNO}_{3}$.

Previous macro ECM characterisation of In718 has been carried out at Edinburgh University using the chronoamperometric analysis detailed in section 3.2 [137]. In this time-averaged 
dissolution valencies and $\mathrm{V}_{0}$ values were found for In718 in $\mathrm{NaCl}$ and $\mathrm{NaNO}_{3}$ electrolytes. The time-averaged $\mathrm{V}_{0}$ values were found to be $\mathrm{V}_{0}=3.0 \pm 0.4$ and $\mathrm{V}_{0}=5.4 \pm 0.4 \mathrm{~V}$ for $\mathrm{NaCl}$ and $\mathrm{NaNO}_{3}$ electrolytes respectively. The time-averaged dissolution valencies were found in this study to be $\mathrm{n}=3.0 \pm 0.2$ for both electrolyte types consistent with Fe dissolving as $\mathrm{Fe}(\mathrm{III}), \mathrm{Cr}$ as $\mathrm{Cr}(\mathrm{III})$ and $\mathrm{Ni}$ as $\mathrm{Ni}(\mathrm{III})$.

Studies into the time-resolved changes in $\mathrm{n}, \mathrm{J}$ and $\mathrm{V}_{0}$ would provide a valuable contribution to the literature data already present for the dissolution of nickel and nickel alloys, as well as giving an insight into the surface processes during ECM. Furthermore the increased parameter base for these industrially useful ECM alloys would be useful in the area of ECM computer modelling. All the work in this Chapter has been carried out on cell 2000 (section 4.6).

\subsection{J- $\mathrm{V}_{0}$ characteristics of $\ln 718$ in $\mathrm{NaCl}$ and $\mathrm{NaNO}_{3}$}

Figure 9.1 shows typical chronoamperometric data for segments 1-7, and typical z-t data, for an approach/retract and re-approach (section 4.8) experiment using In718 with $15.0 \% \mathrm{NaCl}$ electrolyte and an applied voltage of $\mathrm{V}=13.0 \mathrm{~V}$.

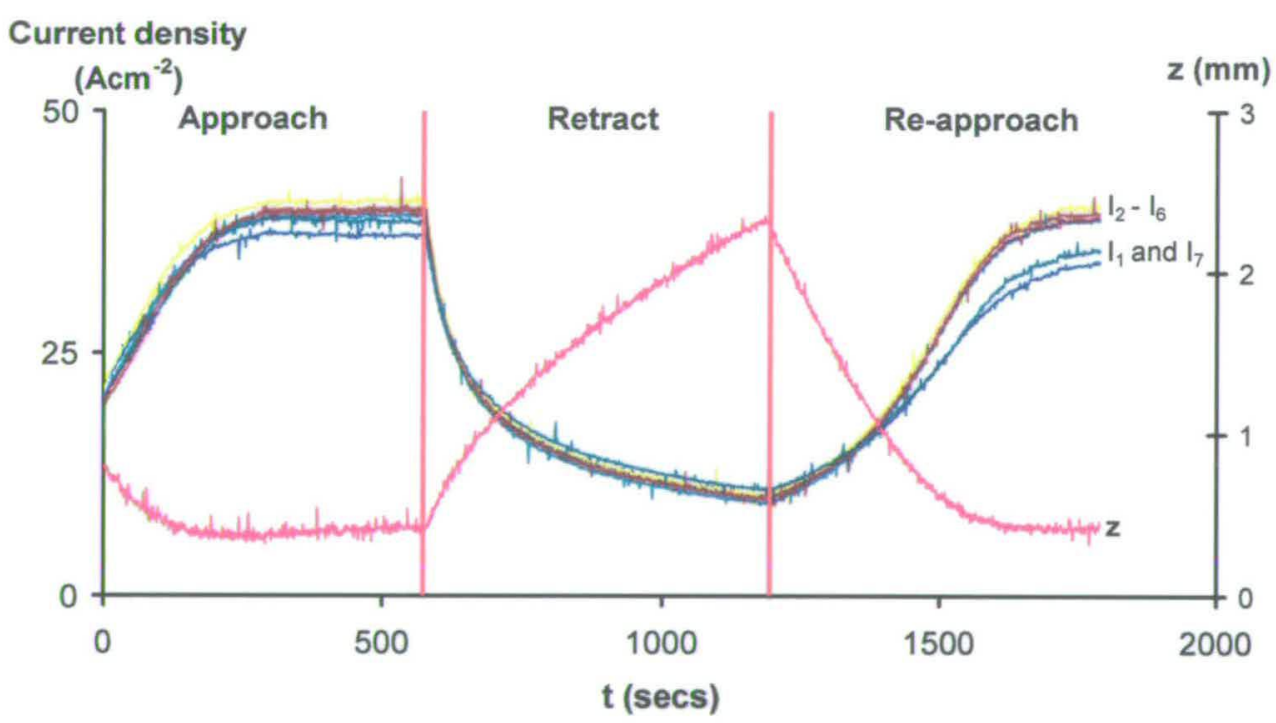

Figure 9.1: Experimental chronoamperometric transients for In718 machined in $15.0 \%$ $\mathrm{NaCl}$ with $\mathrm{V}=13.0 \mathrm{~V}$. Also shown is z-t data for the same experiment. 
As with steels (Chapter 8), decreased values of $\mathrm{J}_{1}$ and $\mathrm{J}_{7}$ were seen during approach and reapproach phases with increased current densities seen for these segments during the retract phase. This can be attributed to the rounding effect, which has previously been observed for steels (section 8.3). From these data equilibrium values for segment 4 were found to be $J_{\infty}=$ $39 \pm 0.7 \mathrm{Acm}^{-2}$ and $\mathrm{z}_{\infty}=0.43 \pm 0.03 \mathrm{~mm}$. Figure 9.2 shows typical chronoamperometric and z-t data for the same material machined with $21.7 \% \mathrm{NaNO}_{3}$ electrolyte and an applied voltage of $\mathrm{V}=16.0 \mathrm{~V}$.

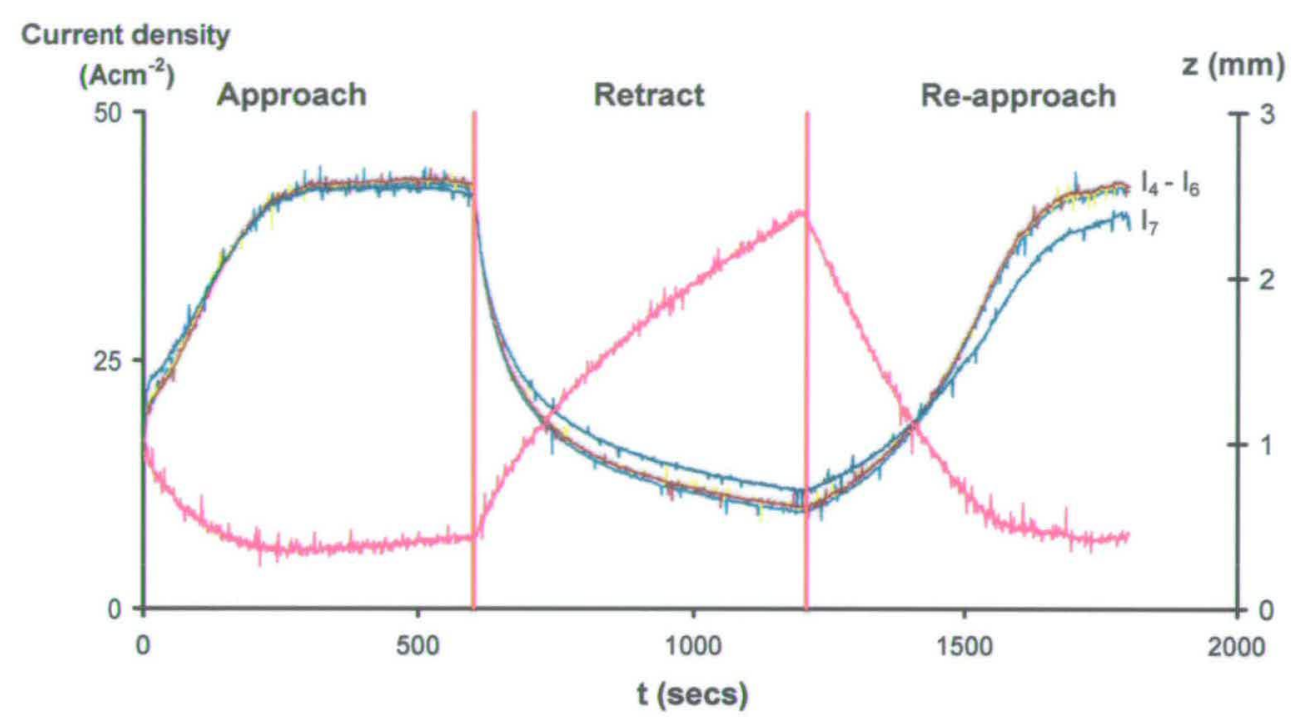

Figure 9.2: Experimental chronoamperometric transients for In 718 machined in $21.7 \%$ $\mathrm{NaNO}_{3}$ with $\mathrm{V}=16.0 \mathrm{~V}$. Also shown is z-t data for the same experiment.

Current data for segments 1-3 were unavailable for this experiment due to loose connections in the measuring system. However, since the $V_{0}$ and $n$ calculations require the use of the segment 4 current measurements, the subsequent analysis was unaffected. In the absence of these data, the segment 7 current density transient shows similar rounding effects observed for steels (section 8.3) and for $\mathrm{NaCl}$ electrolyte (Figure 9.1). From these data equilibrium values for segment 4 were found to be $\mathrm{J}_{\infty}=43 \pm 0.7 \mathrm{Acm}^{-2}$ and $\mathrm{z}_{\infty}=0.38 \pm 0.03 \mathrm{~mm}$. As previous chronoamperometric measurements had produced a higher mean value of $\mathrm{V}_{0}$ for $\mathrm{NaNO}_{3}$ compared to $\mathrm{NaCl}$ electrolyte [137], the applied voltage was increased when machining in $\mathrm{NaNO}_{3}$ in an attempt to maintain a constant $\mathrm{J}_{\infty}$ and $\mathrm{z}_{\infty}$ between experiments. The percentage composition of the electrolytes was calculated to maintain a constant molarity and hence a similar electrolyte conductivity of $\kappa=0.20 \mathrm{~S} \mathrm{~cm}^{-1}$ [137]. This 
increased applied voltage could lead to increased temperature effects through Equation 8.1 (section 8.2). It is therefore possible that some temperature effects were present during this experiment (which could explain the curvature of the $\mathrm{NaNO}_{3}$ re-approach transient in Figure 9.3, section 9.3.2.2 vide infra); however as these data were obtained prior to thermocouple measurement, thermocouple data were not available for $21.7 \% \mathrm{NaNO}_{3}$, which prohibited quantitative temperature correction.

Figure 9.3 shows the corresponding $\mathrm{J}-\mathrm{V}_{0}$ transients for In718 machined in $\mathrm{NaCl}$ and $\mathrm{NaNO}_{3}$.

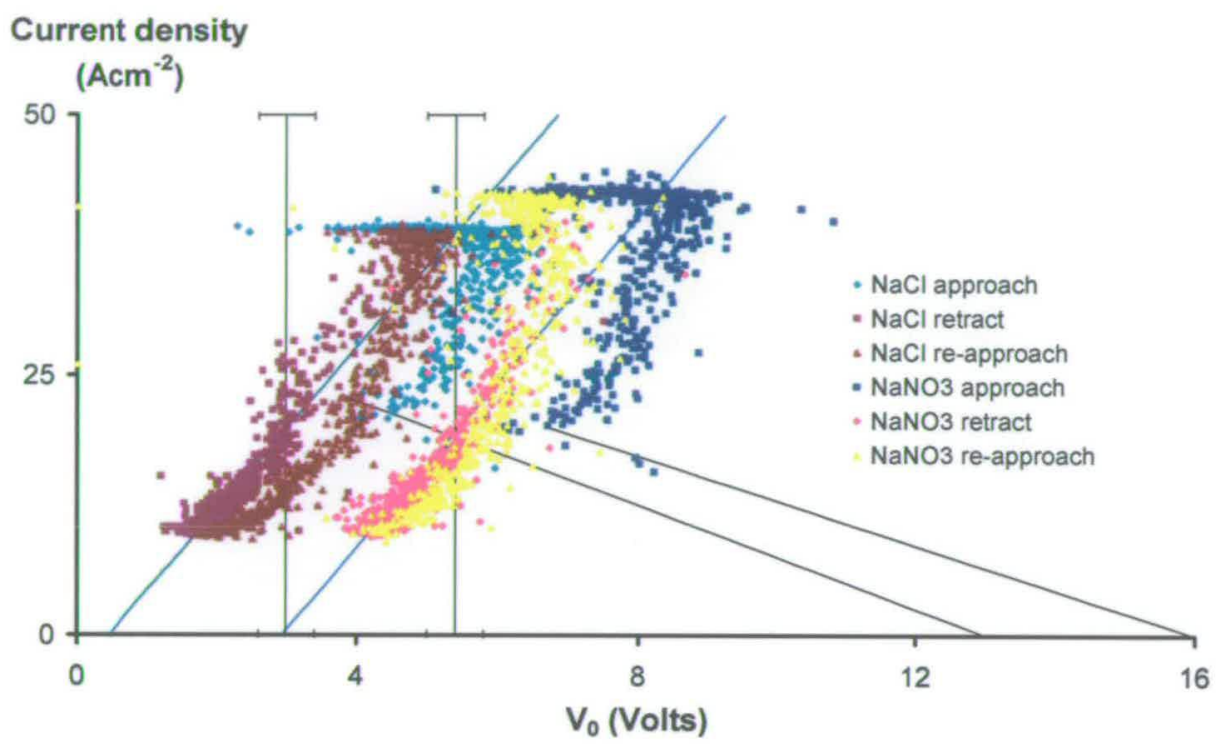

Figure 9.3: $\mathrm{J}-\mathrm{V}_{0}$ data for $\operatorname{In} 718$ machined in $15.0 \% \mathrm{NaCl}$ and $21.7 \% \mathrm{NaNO}_{3}$ at $\mathrm{V}=13.0 \mathrm{~V}$ and $\mathrm{V}=16.0 \mathrm{~V}$ respectively. Time-averaged $\mathrm{V}_{0}$ values for $\operatorname{In} 718$ found through the chronoamperometric analysis in section 3.2 as $\mathrm{V}_{0}=3.0 \pm 0.4$ and $\mathrm{V}_{0}=5.4 \pm 0.4 \mathrm{~V}$ for $\mathrm{NaCl}$ and $\mathrm{NaNO}_{3}$ electrolytes respectively are given [137]. Also shown are the theoretical lines calculated from Equation 7.2 with the appropriate experimental electrolyte conductivity of $\kappa$ $=0.20 \mathrm{~S} \mathrm{~cm}^{-1}$ and initial interelectrode gaps $\mathrm{z}_{0}=0.80 \mathrm{~mm}$ and $\mathrm{z}_{0}=0.93 \mathrm{~mm}$ for $\mathrm{NaCl}$ and $\mathrm{NaNO}_{3}$ respectively. Finally, linear least square fits of the retract $\mathrm{J}-\mathrm{V}_{0}$ data are shown. These both give ohmic resistances of $0.13 \pm 0.01 \Omega \mathrm{cm}^{-2}$ and $\mathrm{V}_{0}$ axis intercepts of $0.5 \pm 0.2$ $\mathrm{V}$ and $2.9 \pm 0.2 \mathrm{~V}$ for $\mathrm{NaCl}$ and $\mathrm{NaNO}_{3}$ (green and blue) respectively.

The close comparison of the mean value of these ultrasound $J-V_{0}$ data with the mean $V_{0}$ values previously obtained using a chronoamperometric method is re-assuring and indicates the accuracy of this method. Figure 9.3 also shows that for machining in $\mathrm{NaCl}$ and $\mathrm{NaNO}_{3}$, 
the $\mathrm{J}-\mathrm{V}_{0}$ characteristics for $\mathrm{In} 718$ show some similarities with those observed for $\mathrm{Fe}$ (section 7.2.2) and the stainless steels investigated (section 8.5). The initial linear decrease of $\mathrm{V}_{0}$ with increasing $\mathrm{J}$ for $\mathrm{In} 718$ shows the same close correspondence to the theoretically calculated lines found using Equation 7.2. As with Fe (section 7.2.2) and Jethete (section 8.5), the data on this line start at low $\mathrm{J}$ and high $\mathrm{V}_{0}$ and move to high $\mathrm{J}$ and low $\mathrm{V}_{0}$ consistent with initial breakdown of a passivating layer on the workpiece. Following the establishment of the required surface conditions for workpiece electrochemical dissolution, the $\mathrm{J}-\mathrm{V}_{0}$ transients again follow positive, essentially linear, ohmic characteristics as seen for $\mathrm{Fe}$ (section 7.2.2) and the stainless steels (section 8.5). Figure 9.3 shows linear least square fits of the retract data in these electrolytes. The retract data were analysed as these are likely to have minimal hysteresis effects, which have previously been attributed to the driven feed regime of the approach and re-approach phase (section 7.2.2). It is interesting that these ohmic gradients are equal, to within experimental error for the two electrolytes, with an ohmic resistance of $0.13 \pm 0.01 \Omega \mathrm{cm}^{-2}$, and are of the same order as those measured for Fe and stainless steel (section 8.5).

It is re-assuring that both the chronoamperometric and the ultrasound method produce greater mean $\mathrm{V}_{0}$ values when machining with $\mathrm{NaNO}_{3}$ compared to machining with $\mathrm{NaCl}$. This is consistent with literature evidence from Datta et al. showing increased surface potentials when machining nickel in $\mathrm{NaNO}_{3}$ [167] compared to $\mathrm{NaCl}$ [136]. In these studies Datta et al. observed anode potentials of $-0.25 \pm 0.1$ vs. SCE and $1.75 \pm 0.1 \mathrm{~V}$ vs. MSE, equivalent to $0.0 \pm 0.1 \mathrm{~V}$ and $1.1 \pm 0.1 \mathrm{~V}$ vs. SHE, at $\mathrm{J}=0.0 \mathrm{~A} \mathrm{~cm}^{-2}$ when machining nickel in $5 \mathrm{M} \mathrm{NaCl}$ and $5 \mathrm{M} \mathrm{NaNO}_{3}+1 \mathrm{M} \mathrm{HNO}_{3}$ respectively. Datta et al. attribute these values to an active dissolution mechanism in $\mathrm{NaCl}$ and a passive dissolution mechanism in $\mathrm{NaNO}_{3}$. The value of $\mathrm{V}_{0}$ at $\mathrm{J}=0.0 \mathrm{~A} \mathrm{~cm}^{-2}$ for $\mathrm{NaCl}, 0.5 \pm 0.2 \mathrm{~V}$, shown in Figure 9.3 is broadly consistent to that found by Datta et al., and consequently consistent with an active dissolution mechanism. While the positive potential at $\mathrm{J}=0.0 \mathrm{~A} \mathrm{~cm}^{-2}$ found for $\mathrm{NaNO}_{3}$ in this work can be attributed to passive dissolution, in the same way as the results found by Datta et al., the absolute potential shown in Figure 9.3, $2.9 \pm 0.2 \mathrm{~V}$, is larger than the corresponding literature value found by Datta $e t$ al. There are two possible explanations for this discrepancy; firstly the use of acidified $\mathrm{NaNO}_{3}$ by Datta et al. could be expected to result in increased breakdown of the passive film compared to neutral $\mathrm{NaNO}_{3}$, and a corresponding lowering of the anode potential at $\mathrm{J}=0.0 \mathrm{~A} \mathrm{~cm}^{-2}$. Secondly, the use of In718 in this work compared to nickel, used by Datta et al., may also induce changes in $\mathrm{V}_{0}$. In718 is a nickel based alloy to which $\mathrm{Cr}$ has been added $(21.0 \% \mathrm{Cr}$ in this instance) in order to increase the 
material's passivating ability. It is possible that a passive film of increased resistance and/or thickness is present when machining In718 in $\mathrm{NaNO}_{3}$ compared to pure nickel, which could be expected to result in higher $\mathrm{V}_{0}$ at $\mathrm{J}=0.0 \mathrm{~A} \mathrm{~cm}^{-2}$ for this work.

Figure 9.4 and Figure 9.5 show the time-resolved dissolution valency data for In718 machined in $\mathrm{NaCl}$ and $\mathrm{NaNO}_{3}$ respectively.

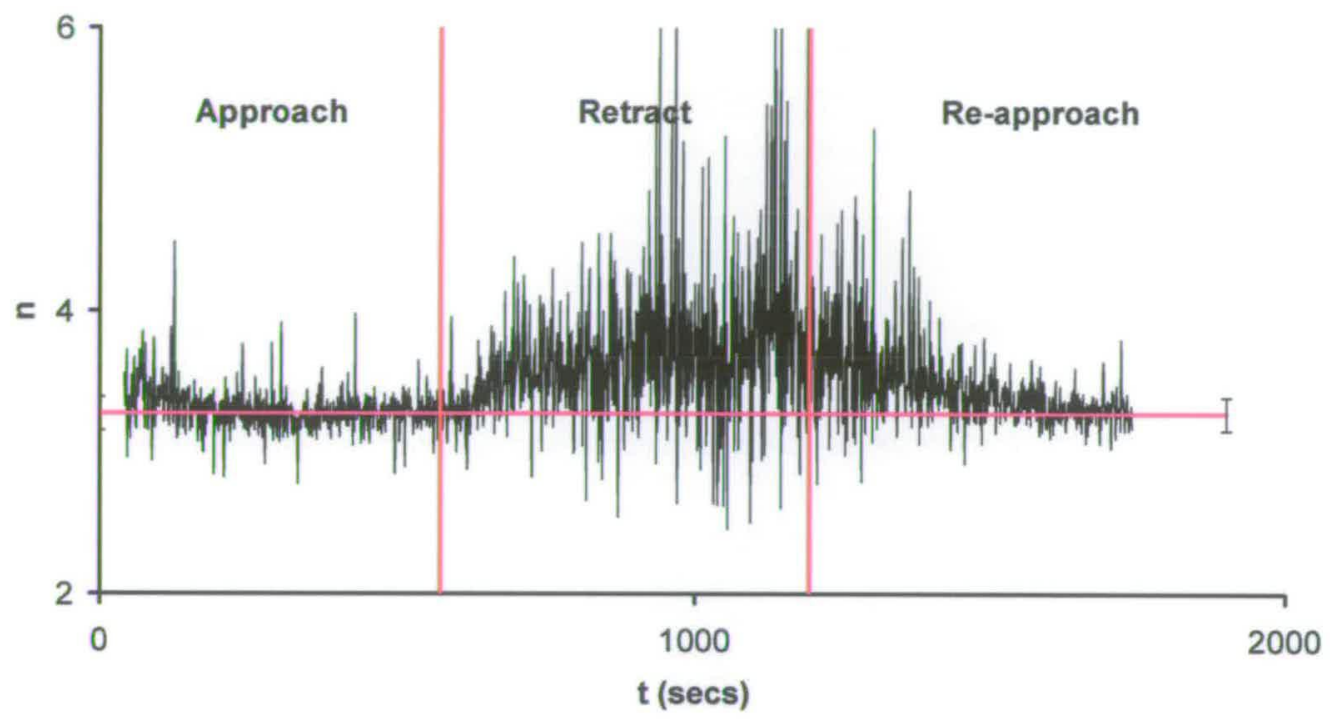

Figure 9.4: Experimental time-resolved n, with $\mathrm{p}=100$ from Equation 6.4, for $\operatorname{In} 718$ machined in $15.0 \% \mathrm{NaCl}$, the pink line depicts the average dissolution valency at equilibrium with an error shown. 


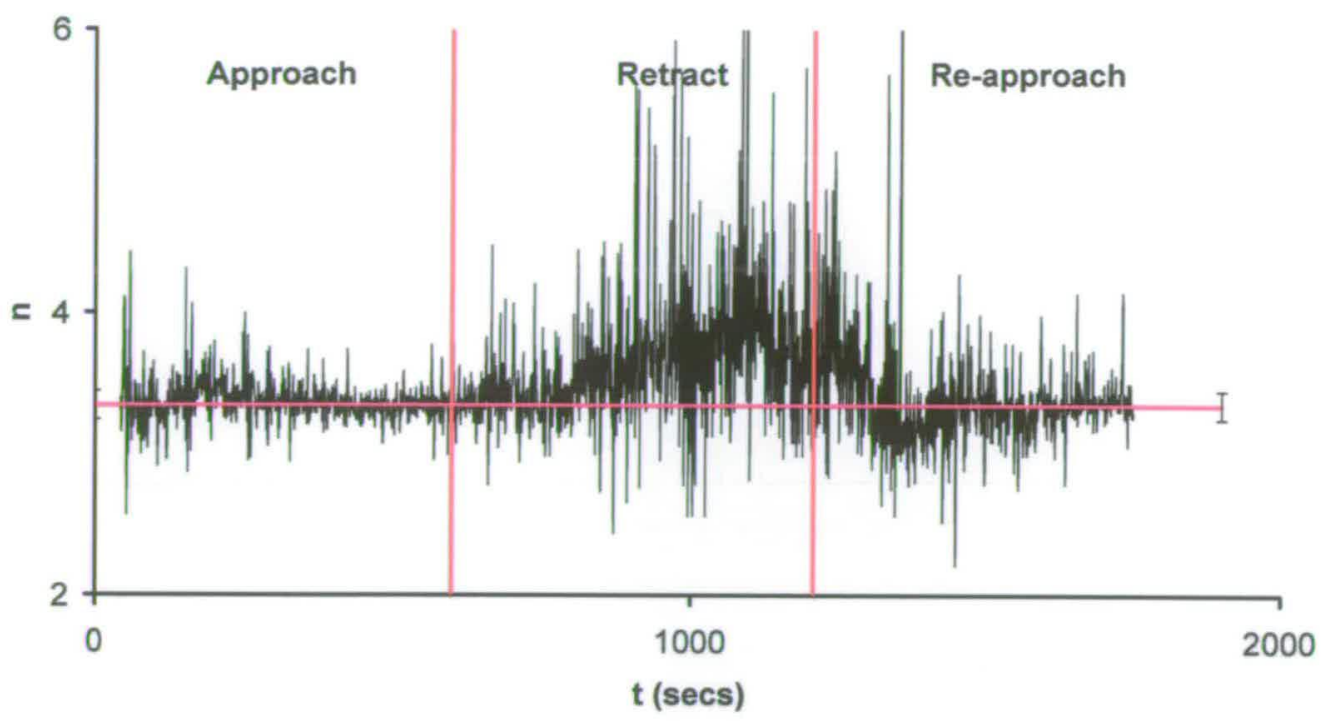

Figure 9.5: Experimental time-resolved n, with $\mathrm{p}=100$ from Equation 6.4, for $\operatorname{In} 718$ machined in $21.7 \% \mathrm{NaNO}_{3}$, the pink line depicts the average dissolution valency at equilibrium with an error shown.

These figures show the equilibrium dissolution valencies for In718 in both electrolytes to be $\mathrm{n}=3.3 \pm 0.1$. These $\mathrm{n}$ values are consistent with the values obtained previously [137] and suggest that, during equilibrium, the majority of the $\mathrm{Ni}, \mathrm{Fe}$ and $\mathrm{Cr}$ present in the alloy are dissolving with the chemically reasonable valencies of $\mathrm{Ni}(\mathrm{III}), \mathrm{Fe}(\mathrm{III})$ and $\mathrm{Cr}$ (III) or $\mathrm{Ni}(\mathrm{II})$, $\mathrm{Fe}$ (III) and $\mathrm{Cr}$ (IV). However, care must be taken when assigning individual valencies since it is possible that the same mean valency could be achieved by balancing a lower valency for one element (e.g. Ni) with a higher valency of another (e.g. Cr). Careful measurements under a variety of elemental compositions close to the alloy composition would be necessary to establish these elemental valencies, which is beyond the scope of this thesis. As with stainless steels and $\mathrm{Fe}$ (section 8.5 and section 7.2.2 respectively) higher dissolution valencies were observed during the low current density machining. Figure 9.4 and Figure 9.5 show $n$ to increase to $n=3.8 \pm 0.2$ for both electrolytes. This increase in $n$ is consistent with either an increase in dissolution valency or a decrease in efficiency of the dissolution process (Equation 2.35) at low current densities (section 7.2.2 and section 8.5), and for In718 is found to be of the same magnitude in both electrolytes. A study by Datta et al. [168] into the dissolution efficiency of nickel in $\mathrm{NaNO}_{3}$, with a flow velocity of $10 \mathrm{~ms}^{-1}$, found $\mathrm{n}=2$ dissolution at current densities of approximately $20 \mathrm{~A} \mathrm{~cm}^{-2}$ reducing to $\mathrm{n}=2.66$ at around 8 A cm$~^{-2}$ (the lowest current density measured in this work). Datta et al. attribute this 
inefficiency to low current density oxygen evolution. It should be noted that while the low current density inefficiency for nickel in $\mathrm{NaNO}_{3}$ observed by Datta et al. is consistent with the work in this thesis, the dissolution valency measurements of $n=2$ and $n=2.66$ are significantly different. This difference may be explicable, as previously discussed, by the use of In718 in this work compared to pure nickel used by Datta et al. It is interesting that, as with $\mathrm{Fe}$, no low current density trend to lower $\mathrm{V}_{0}$ values is observed for $\operatorname{In} 718$ as seen for stainless steels (section 8.5).

\subsection{Effects of changes in ECM conditions}

\subsubsection{Applied voltage changes}

Figure 9.6 shows a comparison of the $\mathrm{J}-\mathrm{V}_{0}$ characteristics for $\mathrm{V}=11.0 \mathrm{~V}$ applied voltage in $15.0 \% \mathrm{NaCl}$ with those collected at $\mathrm{V}=13.0 \mathrm{~V}$ (Figure 9.3, section 9.2). During the lower voltage experiment the equilibrium interelectrode gap was observed to have decreased to $\mathrm{z}_{\infty}$ $=0.22 \pm 0.03 \mathrm{~mm}$, as expected due to the lower applied voltage. The equilibrium dissolution valency was also found to be $\mathrm{n}=3.3 \pm 0.2$ increasing to $\mathrm{n}=3.8 \pm 0.2$ at low current densities, consistent with measurements made at $\mathrm{V}=13.0 \mathrm{~V}$ (Figure 9.4). 


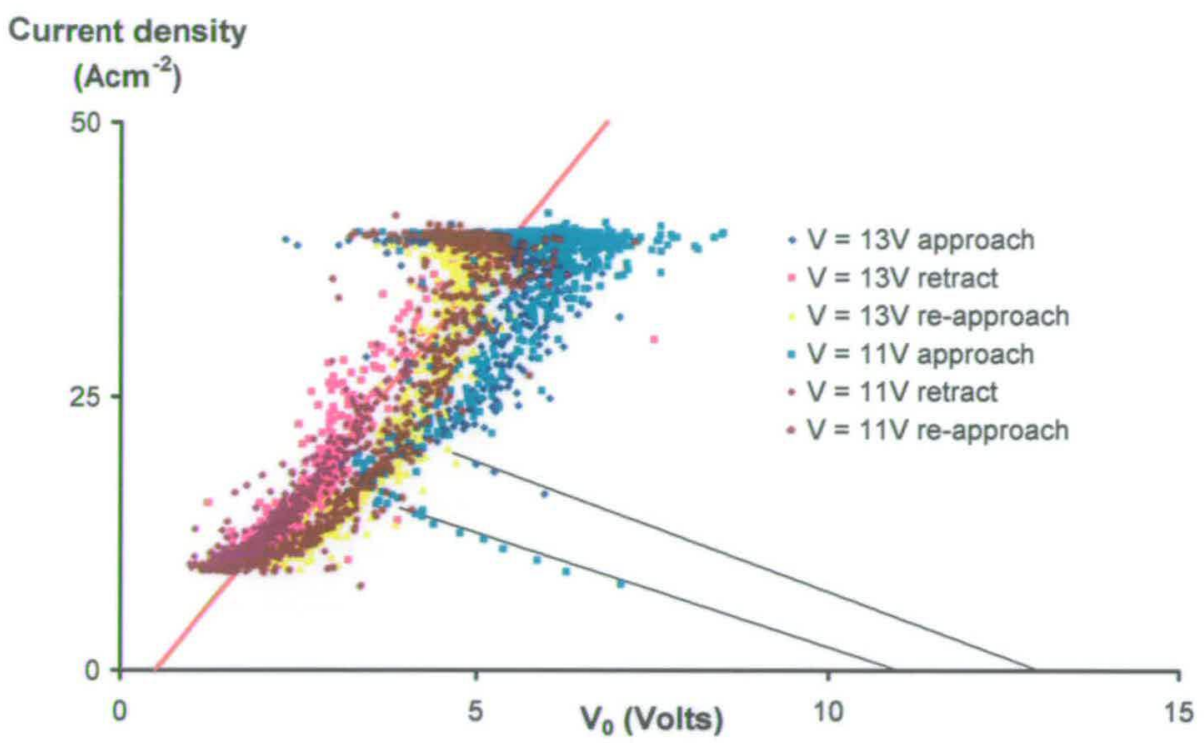

Figure 9.6: Plot showing the comparison of the $\mathrm{J}-\mathrm{V}_{0}$ characteristics for In718 machined at applied voltages of $\mathrm{V}=11.0 \mathrm{~V}$ and $\mathrm{V}=13.0 \mathrm{~V}$ in $15.0 \% \mathrm{NaCl}$. Also shown are the theoretical lines calculated from Equation 7.2 and the linear least squares fit of the retract J$\mathrm{V}_{0}$ data with an ohmic resistance of $0.13 \pm 0.01 \Omega \mathrm{cm}^{-2}$ and $\mathrm{V}_{0}$ axis intercepts of $0.5 \pm 0.2 \mathrm{~V}$.

It is clear from these data when machining at an applied voltage of $\mathrm{V}=11.0 \mathrm{~V}$, that as expected from Equation 7.2 the initial linear decrease of $\mathrm{V}_{0}$ with increasing $\mathrm{J}$ is consistent with a $\mathrm{V}_{0}$ axis intercept, during this region, of $11.0 \mathrm{~V}$. Figure 9.6 also shows the gradients of the initial linear decreases when machining at applied voltage of $\mathrm{V}=11.0 \mathrm{~V}$ and $\mathrm{V}=13.0 \mathrm{~V}$ to be the same consistent with machining in electrolytes of the same conductivity with the same initial interelectrode gap. As with Fe, Jethete and $\ln 718$ at $\mathrm{V}=13.0 \mathrm{~V}$ (section 7.2.2, section 8.5 and section 9.2) the data in this region start at low $\mathrm{J}$ and high $\mathrm{V}_{0}$ and move to higher $\mathrm{J}$ and lower $\mathrm{V}_{0}$ consistent with the breakdown of passivity. It is interesting that these data show lower currents and longer breakdown times than the data at $\mathrm{V}=13.0 \mathrm{~V}$. This indicates that the rate of film breakdown depends upon the applied voltage. The subsequent positive, essentially linear, retract $\mathrm{J}-\mathrm{V}_{0}$ characteristics follow the same line as the $\mathrm{V}=13.0 \mathrm{~V}$ $\mathrm{J}-\mathrm{V}_{0}$ data with a similar extrapolated $\mathrm{V}_{0}$ axis intercept of $0.5 \pm 0.2 \mathrm{~V}$ and a gradient consistent with an ohmic resistance of $0.13 \pm 0.01 \Omega \mathrm{cm}^{-2}$. These data show that the $\mathrm{J}-\mathrm{V}_{0}$ characteristics are essentially independent of the applied voltage and the interelectrode gap under the conditions investigated, which simplifies parameterisation. 


\subsubsection{Electrolyte concentration changes}

\subsubsection{1. $\quad 25.0 \%$ electrolyte concentration}

In718 was machined in $25.0 \% \mathrm{NaCl}$ and $25.0 \% \mathrm{NaNO}_{3}$ with applied voltages of $\mathrm{V}=13.0 \mathrm{~V}$ and $\mathrm{V}=19.0 \mathrm{~V}$ respectively. During these experiments the equilibrium interelectrode gap was observed to have increased to $\mathrm{z}_{\infty}=0.64 \pm 0.03 \mathrm{~mm}$ for both electrolytes compared to the $\mathrm{z}_{\infty}=0.43 \pm 0.03 \mathrm{~mm}$ and $\mathrm{z}_{\infty}=0.38 \pm 0.03 \mathrm{~mm}$ at $15.0 \% \mathrm{NaCl}$ and $21.7 \% \mathrm{NaNO}_{3}$ respectively (section 9.2). This is expected due to the increased conductivities of $25.0 \%$ $\mathrm{NaCl}$ and $25.0 \% \mathrm{NaNO}_{3}$ over $15.0 \% \mathrm{NaCl}$ and $21.7 \% \mathrm{NaNO}_{3}$ respectively. Consequently the gap required to establish the same migrational field in the gap and support the same current is larger for these larger electrolyte concentrations. The equilibrium dissolution valency was found to be $\mathrm{n}=3.1 \pm 0.1$ and $\mathrm{n}=3.3 \pm 0.1$ for $25.0 \% \mathrm{NaCl}$ and $25.0 \% \mathrm{NaNO}_{3}$ respectively increasing to $\mathrm{n}=3.8 \pm 0.2$ at low current densities for both electrolytes. This is consistent, to within experimental error, with measurements made at lower electrolyte concentrations (Figure 9.4 and Figure 9.5).

Figure 9.7 and Figure 9.8 show comparisons of the $\mathrm{J}-\mathrm{V}_{0}$ characteristics for $15.0 \%$ (Figure 9.3) and $25.0 \% \mathrm{NaCl}$ and $21.7 \%$ (Figure 9.3) and $25.0 \% \mathrm{NaNO}_{3}$ respectively. 


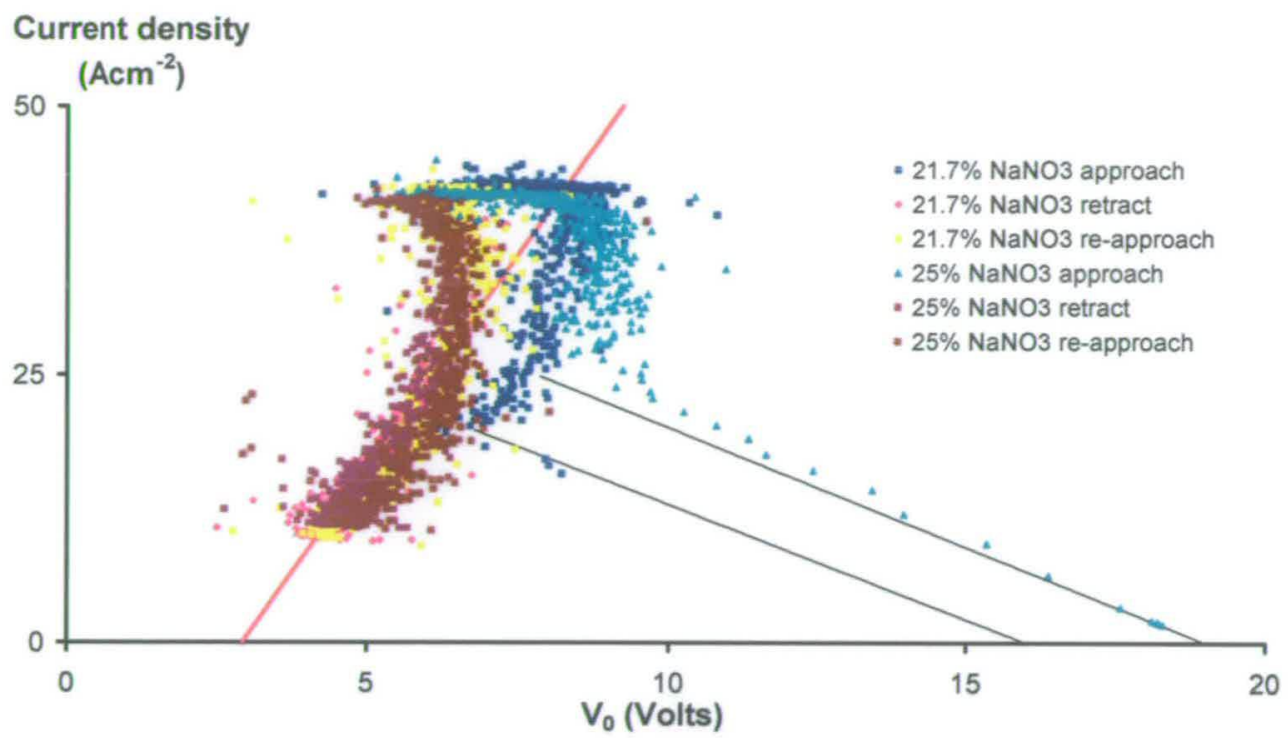

Figure 9.7: Comparison of the $\mathrm{J}-\mathrm{V}_{0}$ characteristics for In718 machined at $\mathrm{V}=13.0 \mathrm{~V}$ in 15.0 $\%$ and $25.0 \% \mathrm{NaCl}$ electrolytes. Also shown are the theoretical lines calculated from Equation 7.2, with conductivities of $\kappa=0.20 \mathrm{~S} \mathrm{~cm}^{-1}$ and $0.27 \mathrm{~S} \mathrm{~cm}^{-1}$ for $15.0 \%$ and $25.0 \%$ respectively and the linear least squares fit of the retract $\mathrm{J}-\mathrm{V}_{0}$ data with an ohmic resistance of $0.13 \pm 0.01 \Omega \mathrm{cm}^{-2}$ and $\mathrm{V}_{0}$ axis intercepts of $0.5 \pm 0.2 \mathrm{~V}$. 


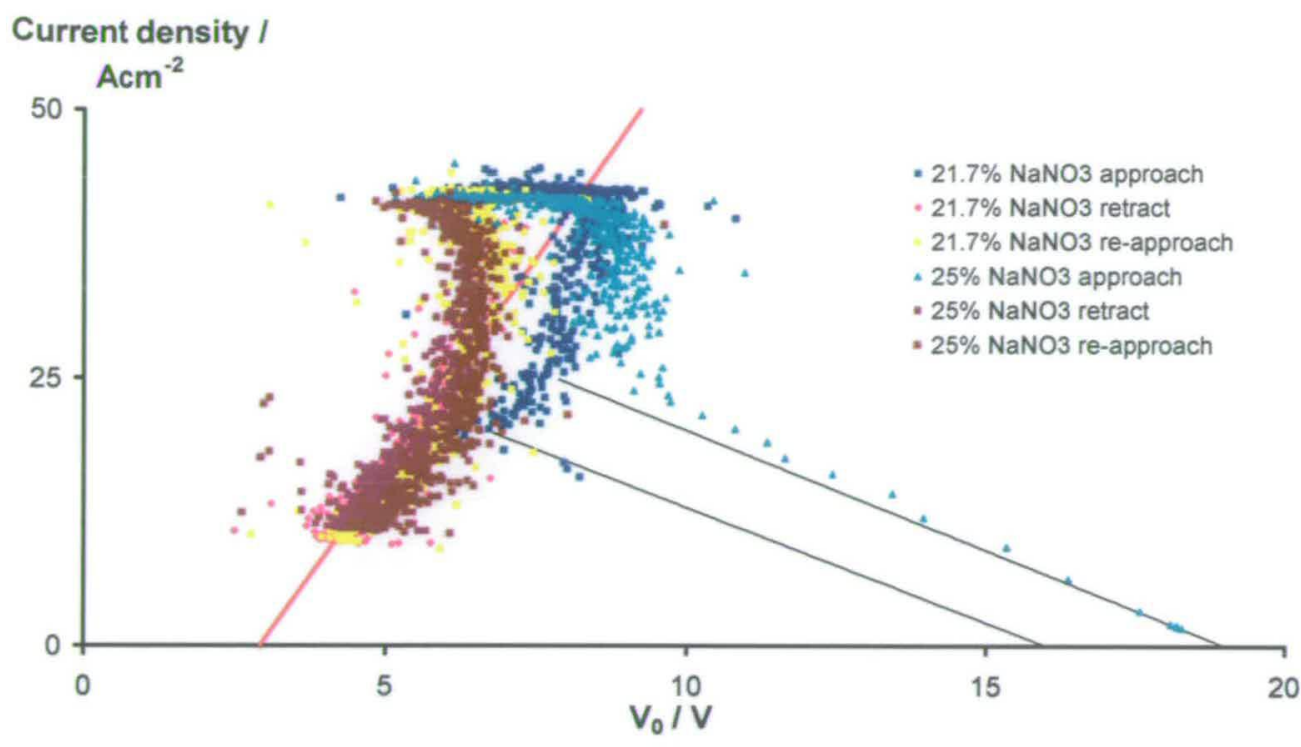

Figure 9.8: Comparison of the $\mathrm{J}-\mathrm{V}_{0}$ characteristics for In 718 machined $21.7 \%$ and $25.0 \%$ $\mathrm{NaNO}_{3}$ electrolytes at $\mathrm{V}=16.0 \mathrm{~V}$ and $\mathrm{V}=19.0 \mathrm{~V}$ respectively. Also shown are the theoretical lines calculated from Equation 7.2, with conductivities of $\kappa=0.20 \mathrm{~S} \mathrm{~cm}^{-1}$ and $0.21 \mathrm{~S} \mathrm{~cm}^{-1}$ for $21.7 \%$ and $25.0 \%$ respectively and the linear least squares fit of the retract $\mathrm{J}-\mathrm{V}_{0}$ data with an ohmic resistance of $0.13 \pm 0.01 \Omega \mathrm{cm}^{-2}$ and $\mathrm{V}_{0}$ axis intercepts of $2.9 \pm 0.2$

V.

It is clear from these data that the initial linear decrease of $\mathrm{V}_{0}$ with increasing $\mathrm{J}$ occurs with gradients and $\mathrm{V}_{0}$ axis intercepts broadly consistent with the theoretical lines calculated using Equation 7.2 with the electrolyte conductivity and applied potential parameters appropriate for each experiment. The data on this line start at low $\mathrm{J}$ and high $\mathrm{V}_{0}$ and move to higher $\mathrm{J}$ and lower $\mathrm{V}_{0}$ consistent with the breakdown of passivity as seen for $\mathrm{Fe}$, Jethete and In718 at $\mathrm{V}=13.0 \mathrm{~V}$ (section 7.2.2, section 8.5 and section 9.2). It is interesting to note that the $\mathrm{J}-\mathrm{V}_{0}$ data in this region for $25.0 \% \mathrm{NaCl}$ and $25.0 \% \mathrm{NaNO}_{3}$ initially follow the theoretical lines calculated from Equation 7.2 but trend to higher current densities at lower $\mathrm{V}_{0}$ values, this is consistent with the temporal mixing of the two terms in Equation 7.1.

Figure 9.7 and Figure 9.8 also show the subsequent positive, essentially linear, $\mathrm{J}-\mathrm{V}_{0}$ characteristics for $25.0 \% \mathrm{NaCl}$ and $25.0 \% \mathrm{NaNO}_{3}$ to have an ohmic resistance of $0.13 \pm$ $0.01 \Omega \mathrm{cm}^{-2}$, consistent with the $\mathrm{J}-\mathrm{V}_{0}$ data collected for $15.0 \% \mathrm{NaCl}$ and $21.7 \% \mathrm{NaNO}_{3}$. It is interesting that all these experiments show the same positive $\mathrm{J}-\mathrm{V}_{0}$ gradient despite changes in applied voltage, electrolyte concentration and type, and interelectrode gap. However, it 
should be noted that the conductivities of the electrolytes at a concentration of $25.0 \%$ could not be measured directly. Consequently a conductivity of $0.27 \mathrm{~S} \mathrm{~cm}^{-1}$ was assumed for 25.0 $\% \mathrm{NaCl}$ in order to normalise this transient to the $15.0 \% \mathrm{NaCl}$ transient, while a conductivity of $0.21 \mathrm{~S} \mathrm{~cm}^{-1}$ was assumed for $25.0 \% \mathrm{NaNO}_{3}$ to normalise this transient to the $21.7 \% \mathrm{NaNO}_{3}$ transient. Once normalised, all the data are the same to within experimental error. Such close correspondence of all the data with one common normalisation parameter further justifies the normalisation process, although it would be more accurate to independently measure the $25.0 \%$ concentration electrolyte conductivity. However, a conductivity meter able to measure conductivities greater than $0.20 \mathrm{~S} \mathrm{~cm}^{-1}$ was not available for these studies. As a result it can be concluded that the $\mathrm{J}-\mathrm{V}_{0}$ characteristics are essentially independent of electrolyte concentration.

\subsubsection{2. $\quad 5.0 \% \mathrm{NO}_{3}^{-}$electrolyte}

Figure 9.9 shows the time-resolved dissolution valency when machining In718 in $5.0 \%$ $\mathrm{NaNO}_{3}$ at an applied voltage of $\mathrm{V}=45.0 \mathrm{~V}$. This increased applied voltage is necessary to maintain reasonable values of $z_{\infty}$ in these relatively low conductivity electrolytes.

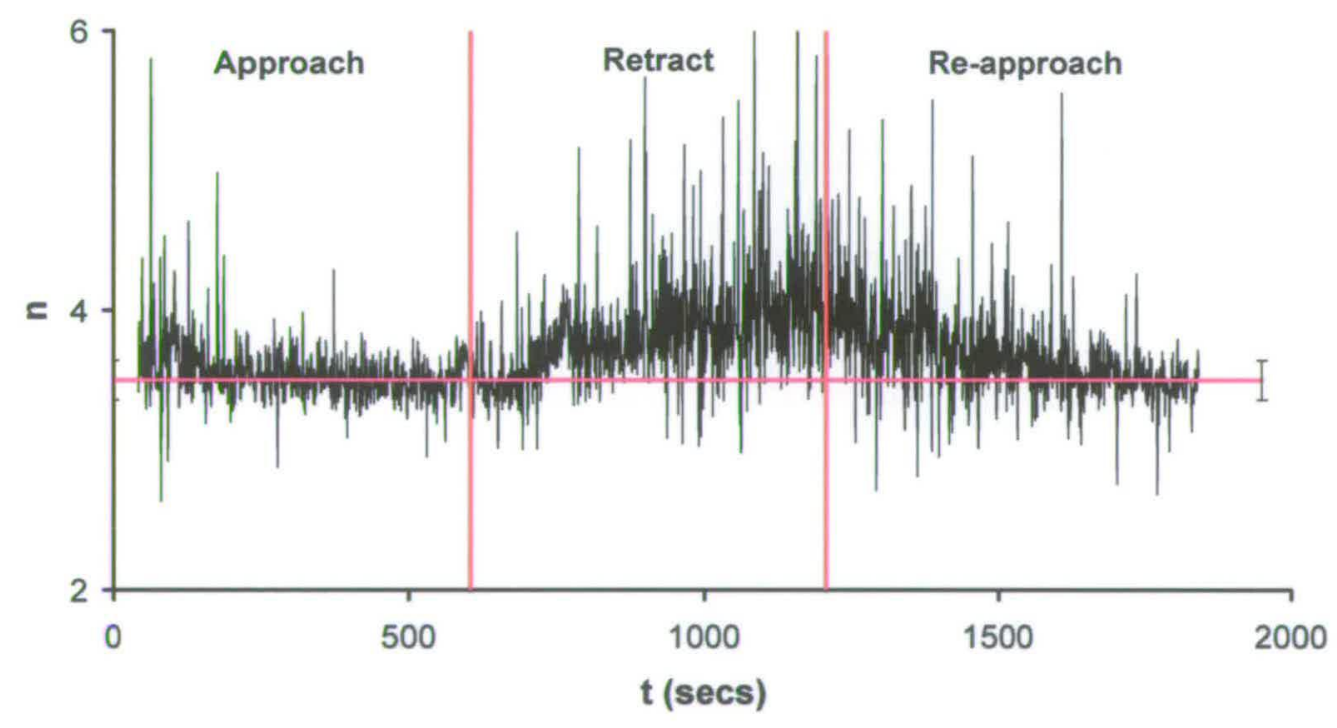

Figure 9.9: Experimental time-resolved n, with $\mathrm{p}=100$ from Equation 6.4, for $\operatorname{In} 718$ machined in $5.0 \% \mathrm{NaNO}_{3}$ at an applied voltage of $\mathrm{V}=45.0 \mathrm{~V}$, the pink line depicts the average dissolution valency at equilibrium with an error shown. 
This figure shows the equilibrium dissolution valencies for $\operatorname{In} 718$ in this electrolyte to be $\mathrm{n}=$ $3.5 \pm 0.2$ increasing to $n=4.0 \pm 0.3$ at low current density. These values are again consistent, to within experimental error, with previous measurements for In718 in higher concentration electrolytes.

Figure 9.10 shows the measured inlet and outlet temperature profiles when machining $\operatorname{In} 718$ in $5.0 \% \mathrm{NaNO}_{3}$ with an applied potential of $\mathrm{V}=45.0 \mathrm{~V}$.

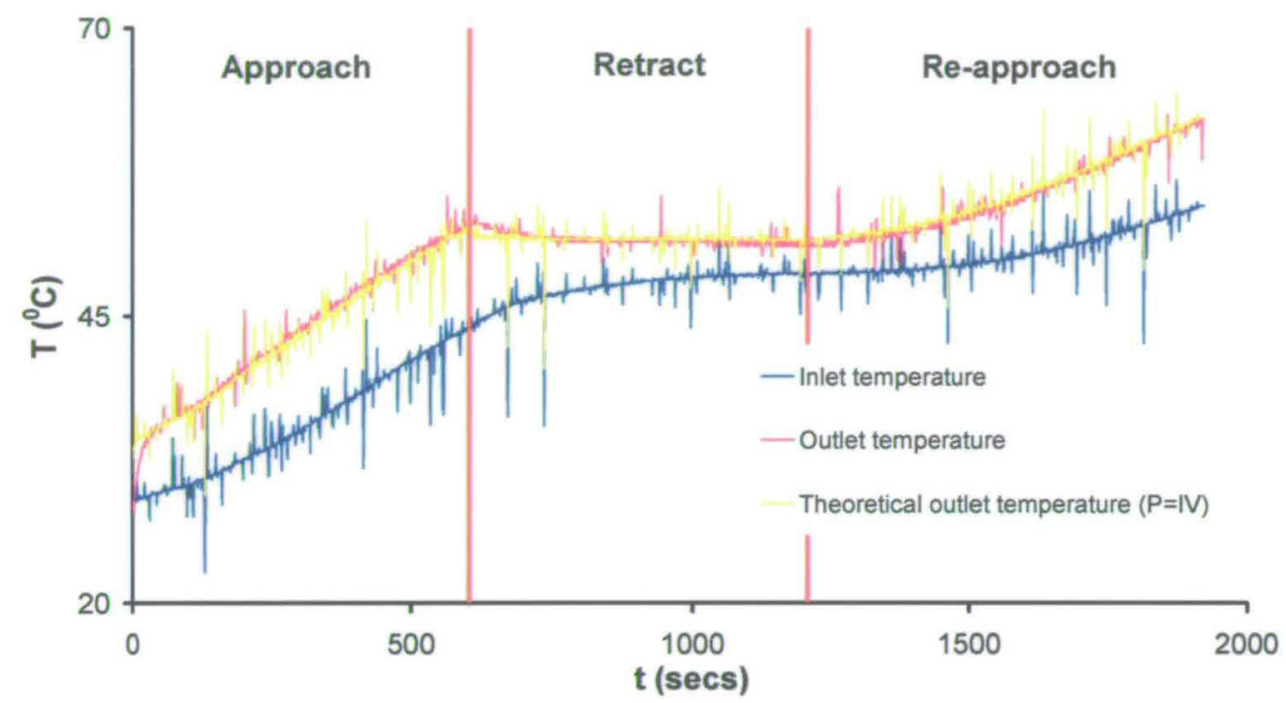

Figure 9.10: Plot to show the inlet and outlet temperature profiles during machining In718 in $5.0 \% \mathrm{NaNO}_{3}$ electrolyte at $\mathrm{V}=45.0$ Volts. Also show is the theoretical outlet temperature calculated using Equation 8.1.

These data show a temperature difference between the inlet and the outlet thermocouple of the order of $4-10^{\circ} \mathrm{C}$ with an overall temperature increase during machining of $25^{\circ} \mathrm{C}$ for the inlet thermocouple and $32{ }^{\circ} \mathrm{C}$ for the outlet thermocouple. It is satisfying that the outlet temperature can be accurately predicted using the simple Joule heating theory discussed in section 8.2 (Equation 8.1 and Equation 8.2). This infers that, as with stainless steels, all the electrical energy put into the system results in a temperature rise of the electrolyte. This would be expected to give a temperature increase which would vary linearly along the flow path length (section 8.2). Consequently the temperature at the ultrasound probe can be calculated using Equation 8.3. The consistency between the predicted and measured outlet 
temperatures also implies negligible thermal losses through the metal electrodes in the system studied; this simplifies thermal analysis and can, at least in part, be explained by the short residence time of electrolyte in the cell due to the high electrolyte flow rate used. The large electrolyte temperature increases shown in Figure 9.10 would be expected to affect the electrolyte conductivity during machining. Figure 9.11 shows the measured temperature dependence of $\kappa$ for the temperature range seen in Figure 9.10.

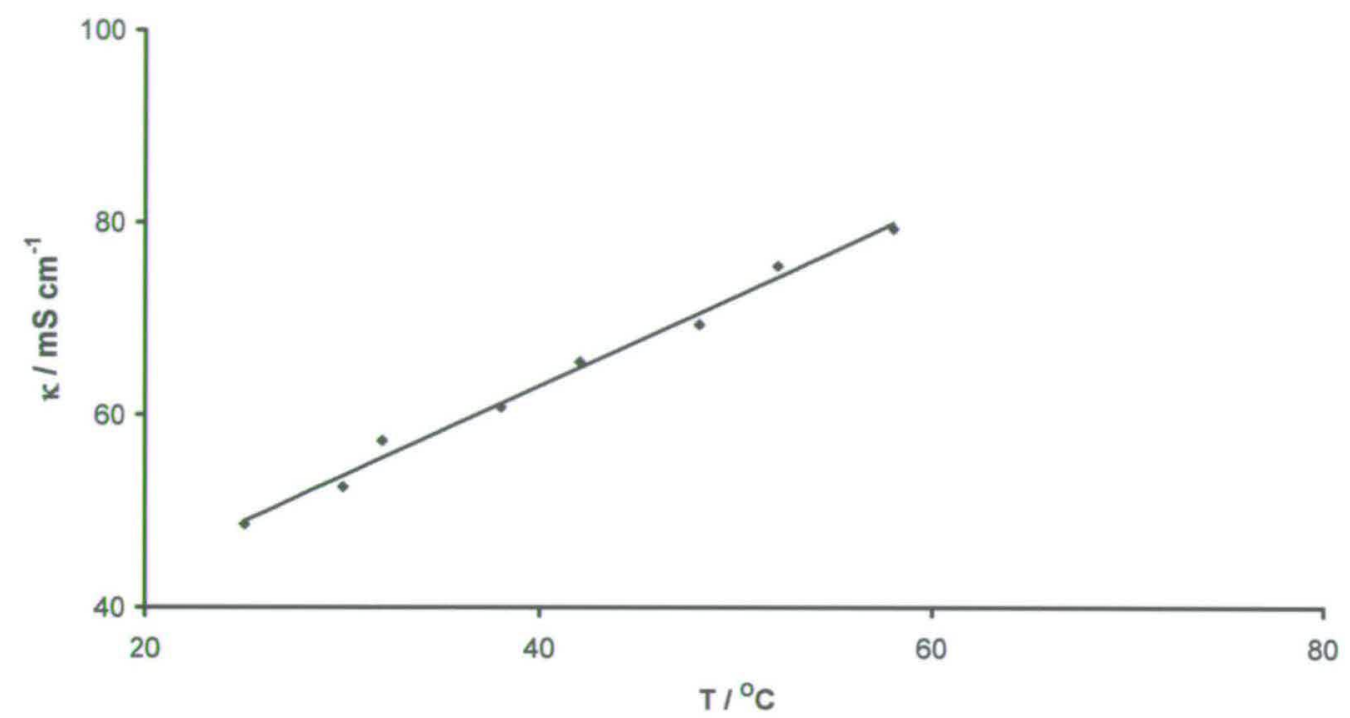

Figure 9.11: Calibration plot showing $\kappa$ vs. $\mathrm{T}$ for $5.0 \% \mathrm{NaNO}_{3}$. These data fit the empirical line $\kappa=B T+D$ shown. Linear regression gives $r=0.995, B=(0.94 \pm 0.04) \mathrm{mS}^{0} \mathrm{C}^{-1}$ and $\mathrm{D}$ $=(25.48 \pm 1.60) \mathrm{mS}$. This equation was used to calculate values of $\kappa$ as a function of temperature.

Figure 9.12 shows the time-resolved $\mathrm{z}$ data collected when machining $\ln 718$ in $5.0 \%$ $\mathrm{NaNO}_{3}$. 


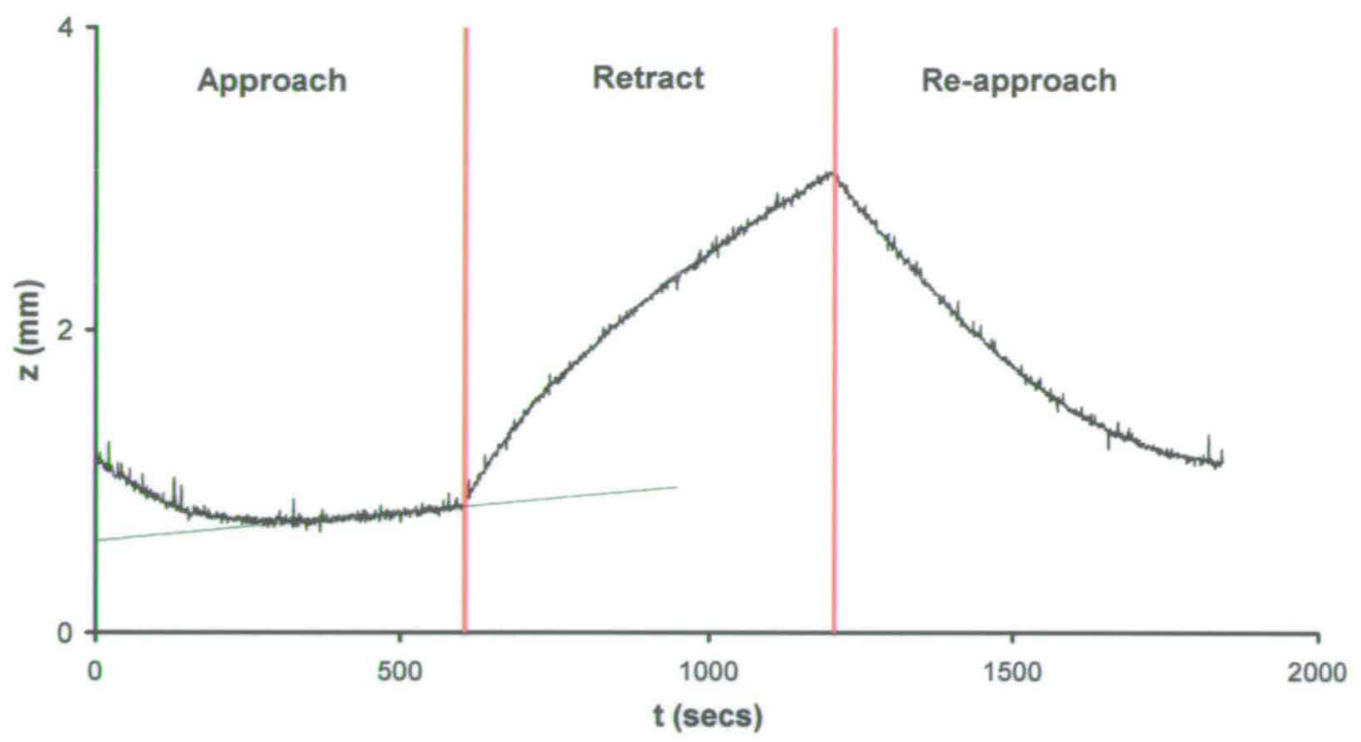

Figure 9.12: Plot of the time-resolved $\mathrm{z}$ data collected when machining In718 in $5.0 \%$ $\mathrm{NaNO}_{3}$. Also shown is the least squares fit through the approach phase $\mathrm{z}_{\infty}$ data (green line).

Figure 9.12 clearly shows that $z_{\infty}$ increases with time; this increase was found to occur at a rate of $3.7 \times 10^{-4} \mathrm{~mm} \mathrm{~s}^{-1}$. At the start of the approach phase $\mathrm{z}_{\infty}=0.72 \pm 0.03 \mathrm{~mm}$ which was found to have increased by $15 \%$ to $\mathrm{z}_{\infty}=0.83 \pm 0.03 \mathrm{~mm}$ at the end of the approach phase. This is in line with the increasing electrolyte temperature (Figure 9.10) and hence increasing electrolyte conductivity (Figure 9.11). Figure 9.13 shows the effects on the $\mathrm{J}-\mathrm{V}_{0}$ characteristics of an (incorrect) assumption of constant $\kappa$ when machining at $5.0 \% \mathrm{NaNO}_{3}$ with the temperature increases shown in Figure 9.10. 


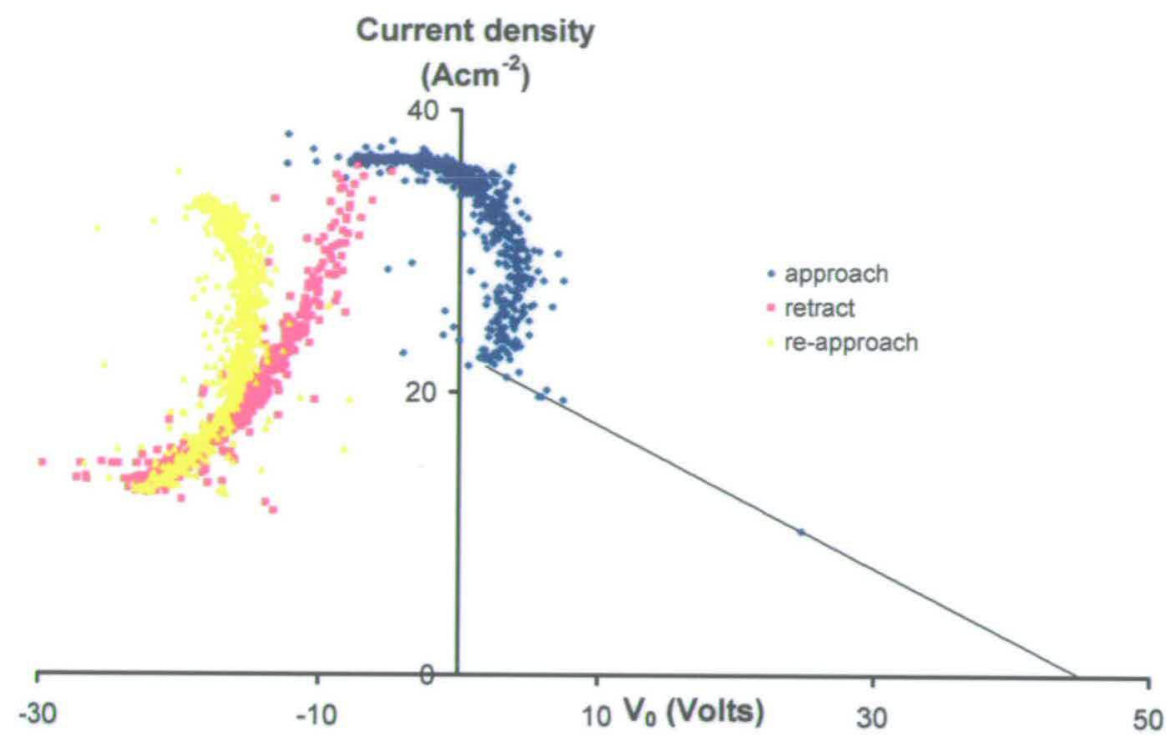

Figure 9.13: $\mathrm{J}-\mathrm{V}_{0}$ data for machining of In718 in $5.0 \% \mathrm{NaNO}_{3}$ with an assumed constant $\mathrm{\kappa}$ equal to the initial bulk value of $\kappa=58.0 \mathrm{mS} \mathrm{cm}^{-1}$. Also shown is the theoretical line calculated from Equation 7.2.

The initial linear decrease of $\mathrm{V}_{0}$ with increasing $\mathrm{J}$, starting from higher $\mathrm{V}_{0}$ and lower $\mathrm{J}$ and moving towards lower $\mathrm{V}_{0}$ and higher $\mathrm{J}$, consistent with Equation 7.2 shows initial breakdown of the passive surface layer on the anode. Subsequent to this initial trend the data in Figure 9.13 clearly show large, negative and chemically unrealistic $V_{0}$ values, a large hysteresis and curvature of the re-approach phase towards smaller $V_{0}$ values. Figure 9.14 shows the $J-V_{0}$ data for the same experiment with $\kappa$ calculated at all times using the temperature at segment 4, the position at which the $\mathrm{J}-\mathrm{V}_{0}$ data is calculated (Equation 8.3), and the calibration data in Figure 9.11 . 


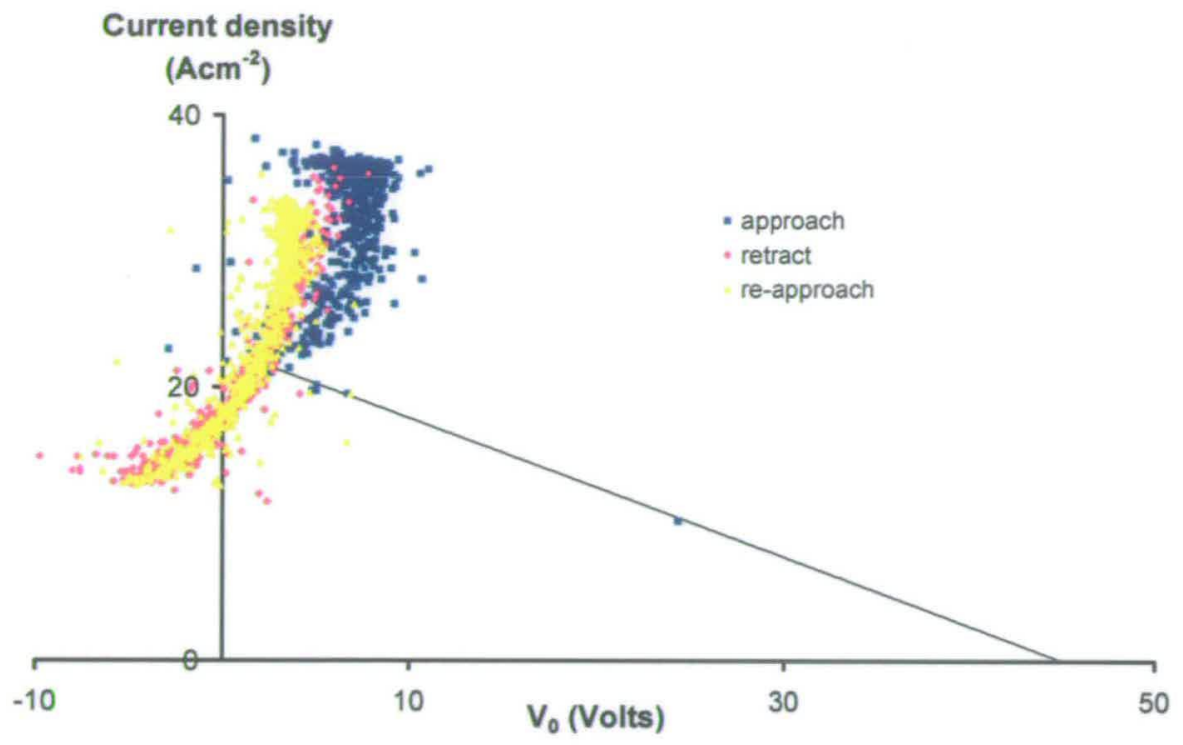

Figure 9.14: Corresponding $J-V_{0}$ data to Figure 9.13 with $V_{0}$ calculated using the time dependent conductivity values of $\kappa$ adjusted for the measured temperature. Also shown is the theoretical line calculated from Equation 7.2, with a conductivity of $\kappa=58.0 \mathrm{mS} \mathrm{cm}^{-1}$.

Figure 9.14 shows a reduction of the unrealistic negative $V_{0}$ values, the hysteresis and the reapproach curvature shown in Figure 9.13 showing that these observations were the result of the incorrect assumption of a constant $\kappa$ value.

Figure 9.15 shows a comparison of the $\mathrm{J}-\mathrm{V}_{0}$ data in Figure 9.14 with $\mathrm{J}-\mathrm{V}_{0}$ data collected at $21.7 \% \mathrm{NaNO}_{3}$ (Figure 9.3). 


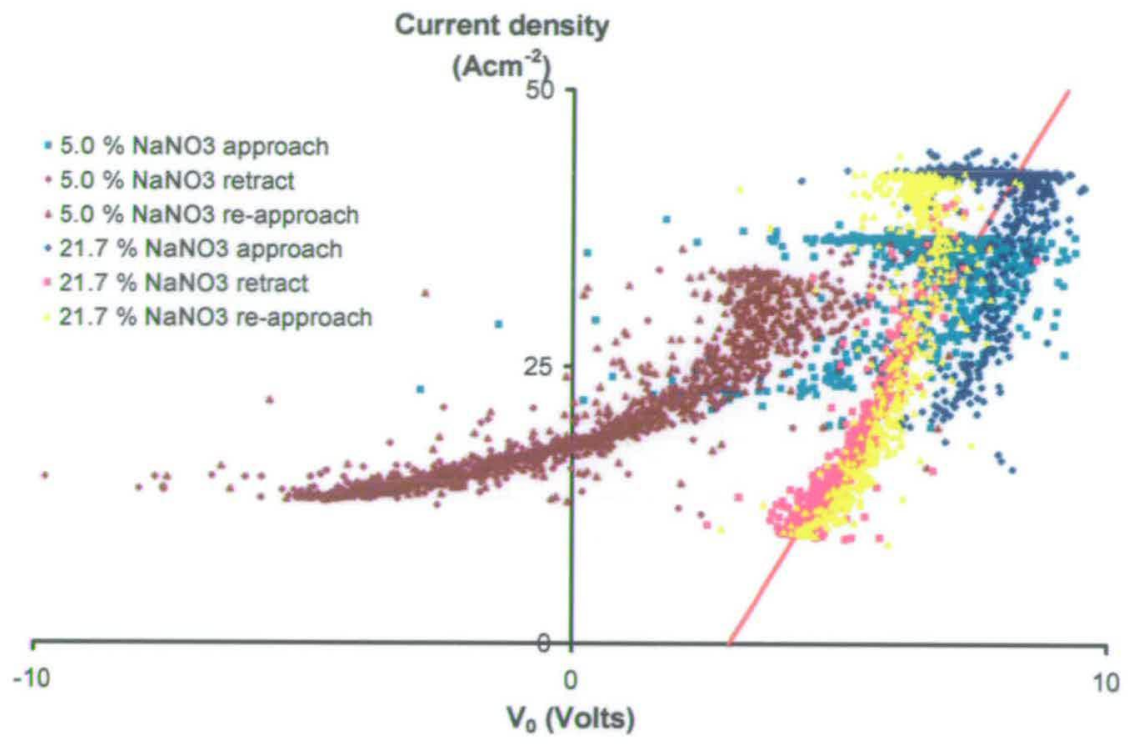

Figure 9.15: Comparison of the $\mathrm{J}-\mathrm{V}_{0}$ characteristics for In 718 machined $5.0 \%$ and $21.7 \%$ $\mathrm{NaNO}_{3}$ electrolytes at $\mathrm{V}=45.0 \mathrm{~V}$ and $\mathrm{V}=16.0 \mathrm{~V}$ respectively. Also shown is the linear least squares fit of the $21.7 \% \mathrm{NaNO}_{3}$ retract $\mathrm{J}-\mathrm{V}_{0}$ data with an ohmic resistance of $0.13 \pm$ $0.01 \Omega \mathrm{cm}^{-2}$ and $\mathrm{V}_{0}$ axis intercepts of $2.9 \pm 0.2 \mathrm{~V}$.

Figure 9.13 and Figure 9.14 show that electrolyte temperature corrections remove a number of the unrealistic, temperature related $\mathrm{J}-\mathrm{V}_{0}$ features. However, Figure 9.15 clearly shows that there are still some significant differences in the $\mathrm{J}-\mathrm{V}_{0}$ transients of $5.0 \% \mathrm{NaNO}_{3}$ and $21.7 \% \mathrm{NaNO}_{3}$ after temperature correction. While it is satisfying that the approach phase data for $5.0 \% \mathrm{NaNO}_{3}$ are broadly consistent with the approach phase data for $21.7 \%$ $\mathrm{NaNO}_{3}$, Figure 9.15 shows the retract and re-approach transients trending to smaller $\mathrm{V}_{0}$ values than expected with a small, but significant residual re-approach curvature towards smaller $\mathrm{V}_{0}$ values. It has previously been shown that $\mathrm{NaNO}_{3}$ induces oxide formation on nickel [169]. Therefore, one explanation could be that lower concentrations of $\mathrm{NaNO}_{3}$ produces active dissolution and markedly lower $\mathrm{V}_{0}$ values. However, these $\mathrm{V}_{0}$ values appear significantly lower than those found for active dissolution in $15.0 \% \mathrm{NaCl}$ (Figure 9.3). While the temperature corrections detailed in this section correct for the effects of electrolyte temperature, the effects of increasing temperature on other parts of the system are not addressed. As the temperature increases observed in this experiment are significant, this may be expected to increase the rate of the dissolution processes during machining, hence decreasing the magnitude of $\mathrm{V}_{0}$. This effect could be difficult to quantify since for large 
rates of temperature increase, the temperature distribution in the cell also may not be homogeneous.

These results demonstrate the importance of electrolyte temperature measurements and corrections when experiments are carried out at the relatively large values of $\mathrm{V}$ and $\mathrm{J}$ obtained at relatively high $\mathrm{f}$ and for relatively low electrolyte concentrations $(5.0 \%)$. However, care must be taken since the $\mathrm{J}-\mathrm{V}_{0}$ characteristics at high cell temperatures may be significantly different from those observed at low cell temperatures despite electrolyte conductivity corrections. This will also effect ECM parameterisation.

\subsubsection{Flow effects}

Constant $\mathrm{U}$ and $\mathrm{L}$ parameters (electrolyte flow rate and width of workpiece respectively) combined with significant changes in $\mathrm{z}$ during the approach retract and re-approach phases of ECM experiments give rise to changes in $\mathrm{v}$ (the electrolyte flow velocity) throughout the experiment. These can be calculated using Equation 9.1.

$$
\mathrm{v}=\frac{\mathrm{U}}{\mathrm{zL}}
$$

\section{Equation 9.1}

Figure 9.16 shows typical time-resolved flow velocity data for an approach/retract/reapproach experiment. 


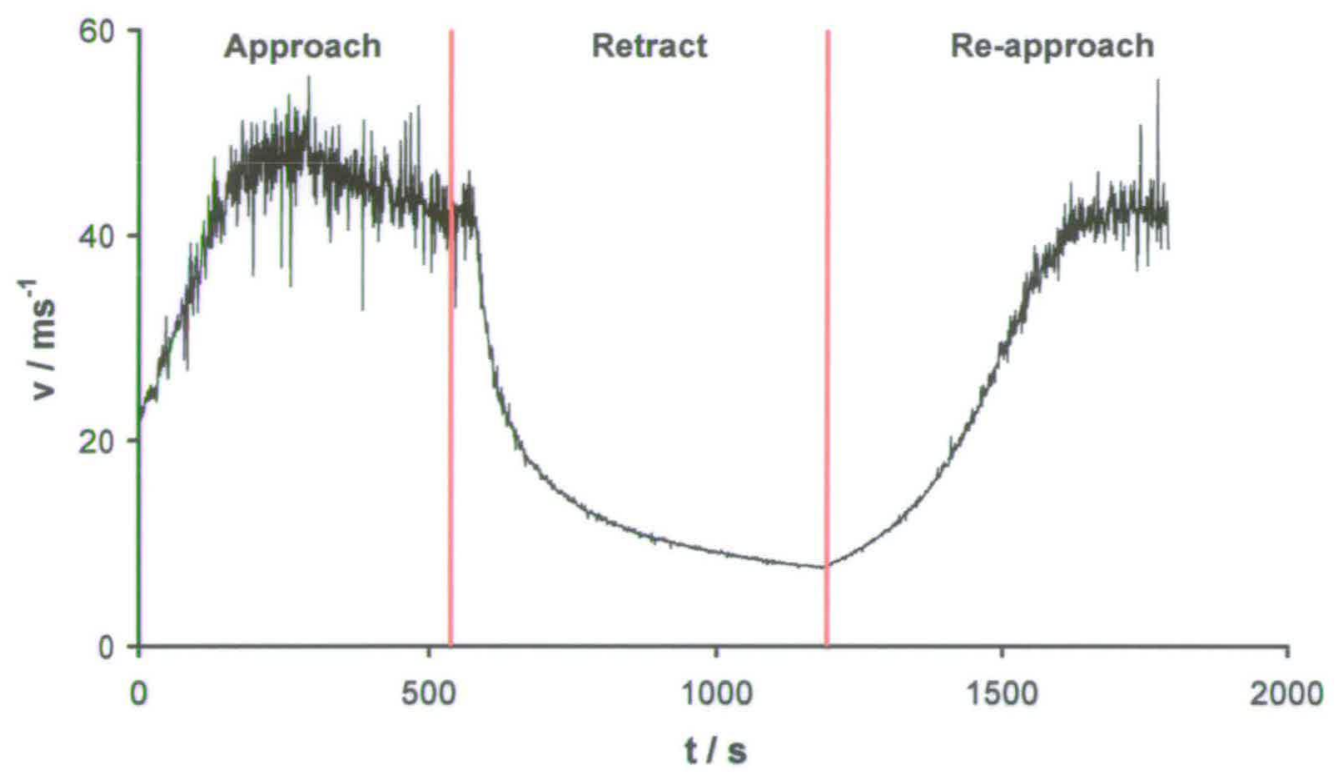

Figure 9.16: Time-resolved flow velocity data for $\operatorname{In} 718$ machined in $15.0 \% \mathrm{NaCl}$ with $\mathrm{V}=$ $13.0 \mathrm{~V}$ and $\mathrm{U}=16.0 \mathrm{~L} \mathrm{~min}^{-1}$.

Consequently flow velocity effects on ECM parameters can be investigated by altering the flow rate parameter of the experiment resulting in the flow velocity changes observed in Figure 9.17. For the purposes of this work $\mathrm{U}=16.0 \mathrm{~L} \mathrm{~min}^{-1}$ and $20.0 \mathrm{~L} \mathrm{~min}^{-1}$ were used. 


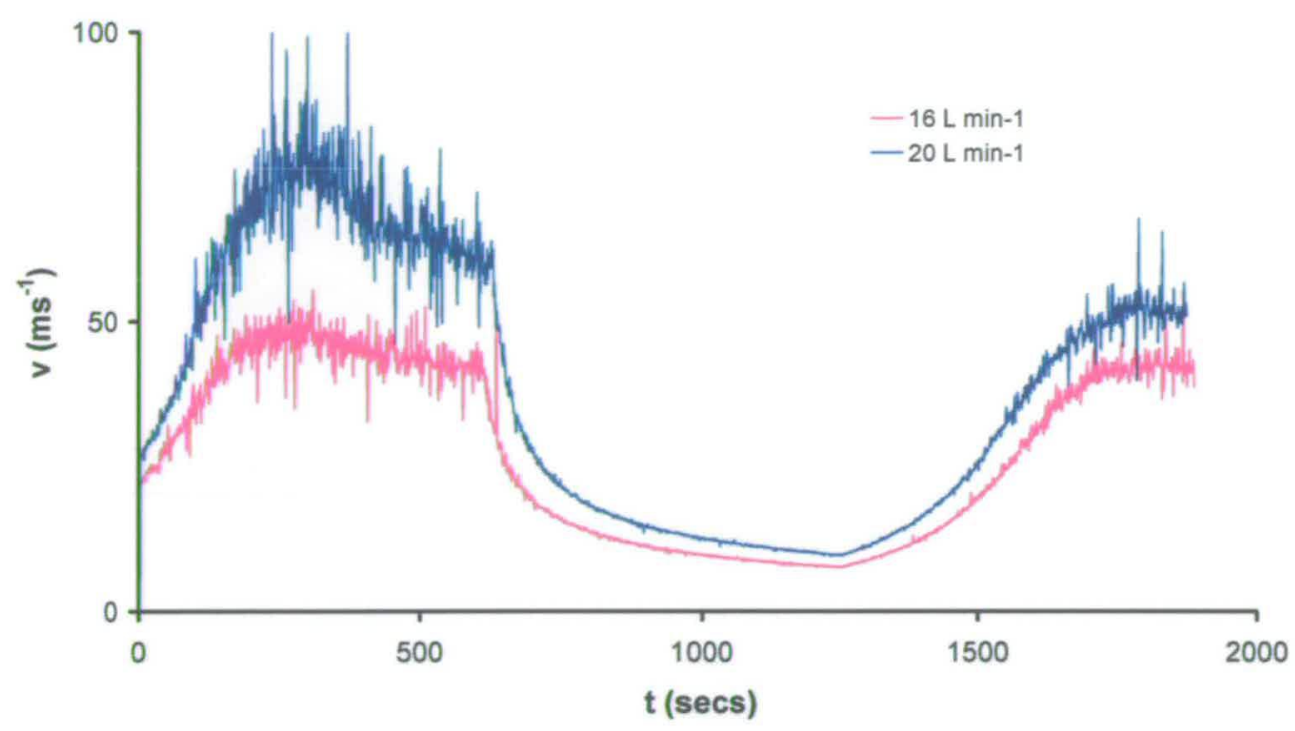

Figure 9.17: Comparison of the time-resolved flow velocity data for In718 machined in 15.0 $\% \mathrm{NaCl}$ electrolyte with $\mathrm{V}=13.0 \mathrm{~V}$ and with flow rates of $\mathrm{U}=16.0 \mathrm{~L} \mathrm{~min}^{-1}$ and $\mathrm{U}=20.0 \mathrm{~L}$ $\min ^{-1}$.

In718 was machined in $15.0 \% \mathrm{NaCl}$ and $21.7 \% \mathrm{NaNO}_{3}$ at a flow rate of $\mathrm{U}=20.0 \mathrm{~L} \mathrm{~min}^{-1}$ and with applied voltages of $\mathrm{V}=13.0 \mathrm{~V}$ and $\mathrm{V}=16.0 \mathrm{~V}$ respectively. During these experiments the equilibrium interelectrode gap was found to be $\mathrm{z}_{\infty}=0.43 \pm 0.03 \mathrm{~mm}$ and $\mathrm{z}_{\infty}$ $=0.38 \pm 0.03 \mathrm{~mm}$ for $\mathrm{NaCl}$ and $\mathrm{NaNO}_{3}$ respectively, consistent with those measured for these electrolytes at $U=16.0 \mathrm{~L} \mathrm{~min}^{-1}$ (section 9.2). Figure 9.18 and Figure 9.19 show the time resolved dissolution valency measurements taken at $\mathrm{U}=20.0 \mathrm{~L} \mathrm{~min}^{-1}$ for $15.0 \% \mathrm{NaCl}$ and $21.7 \% \mathrm{NaNO}_{3}$ respectively. 


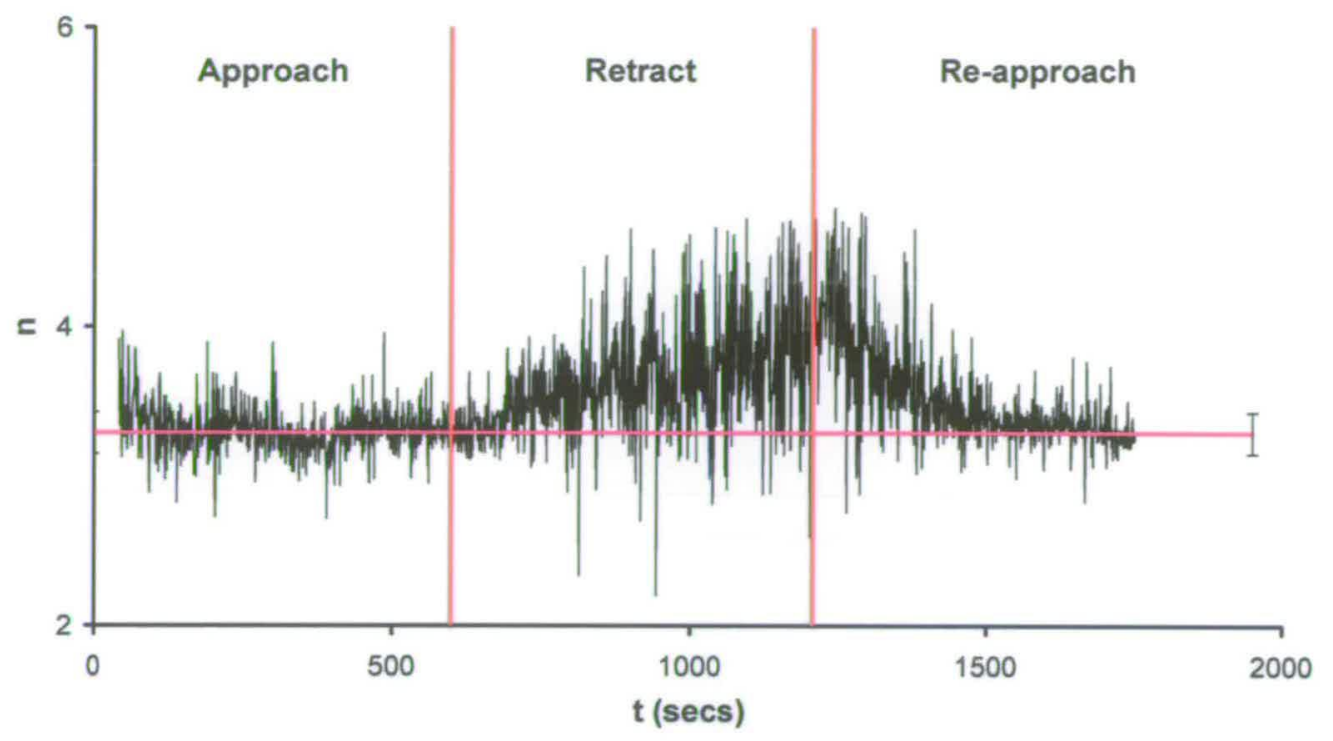

Figure 9.18: Experimental time-resolved $n$, with $\mathrm{p}=100$ from Equation 6.4, for $\operatorname{In} 718$ machined in $15.0 \% \mathrm{NaCl}$ at a flow rate of $\mathrm{U}=20.0 \mathrm{~L} \mathrm{~min}^{-1}$, the pink line depicts the average dissolution valency at equilibrium with an error shown.

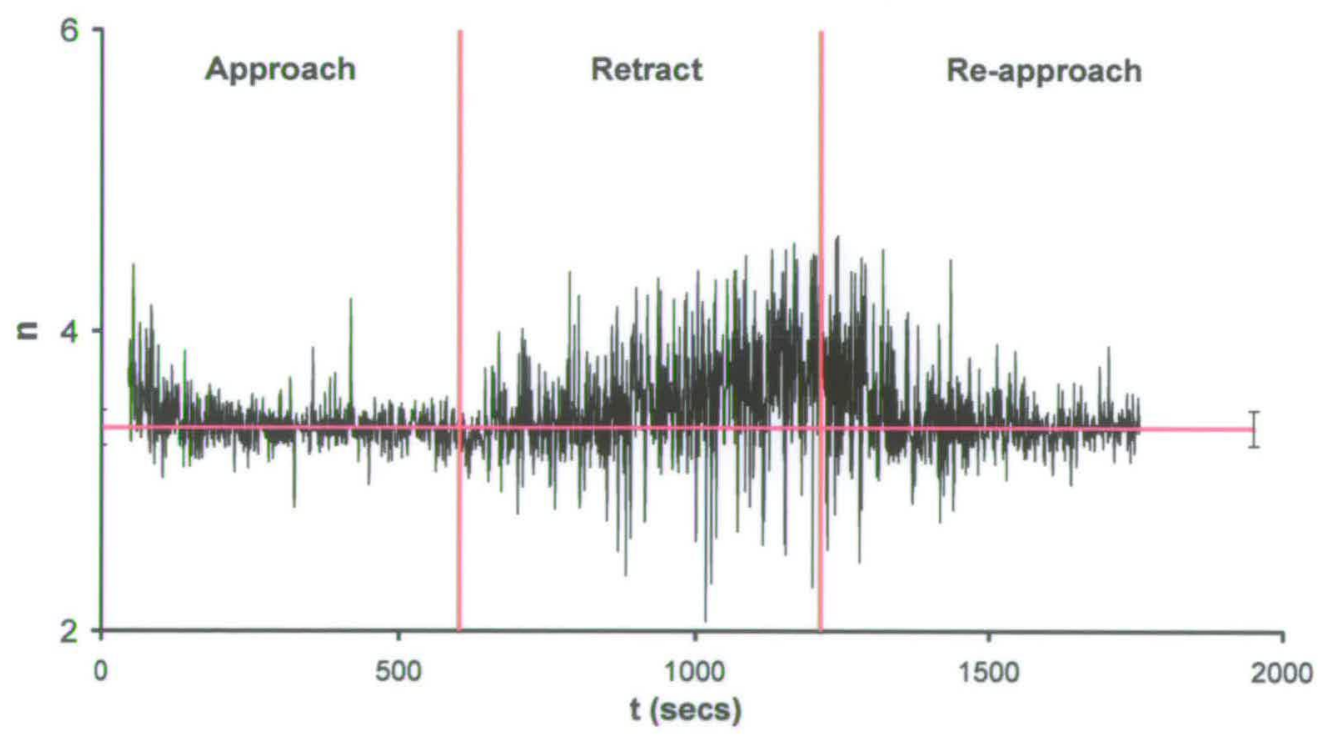

Figure 9.19: Experimental time-resolved $n$, with $p=100$ from Equation 6.4, for $\operatorname{In} 718$ machined in $21.7 \% \mathrm{NaNO}_{3}$ at a flow rate of $\mathrm{U}=20.0 \mathrm{~L} \mathrm{~min}^{-1}$, the pink line depicts the average dissolution valency at equilibrium with an error shown. 
These data show the equilibrium dissolution valency to be $n=3.3 \pm 0.2$ increasing to $n=3.8$ \pm 0.3 at low current densities for both electrolytes. These values are consistent with measurements made at $U=16.0 \mathrm{~L} \mathrm{~min}^{-1}$ (Figure 9.4 and Figure 9.5). Figure 9.20 and Figure 9.21 show comparisons of the $\mathrm{J}-\mathrm{V}_{0}$ characteristics for $\operatorname{In} 718$ machined at $\mathrm{U}=16.0 \mathrm{~L} \mathrm{~min}^{-1}$ (Figure 9.3) and $\mathrm{U}=20.0 \mathrm{~L} \mathrm{~min}^{-1}$ in $15.0 \% \mathrm{NaCl}$ and $21.7 \% \mathrm{NaNO}_{3}$ respectively.

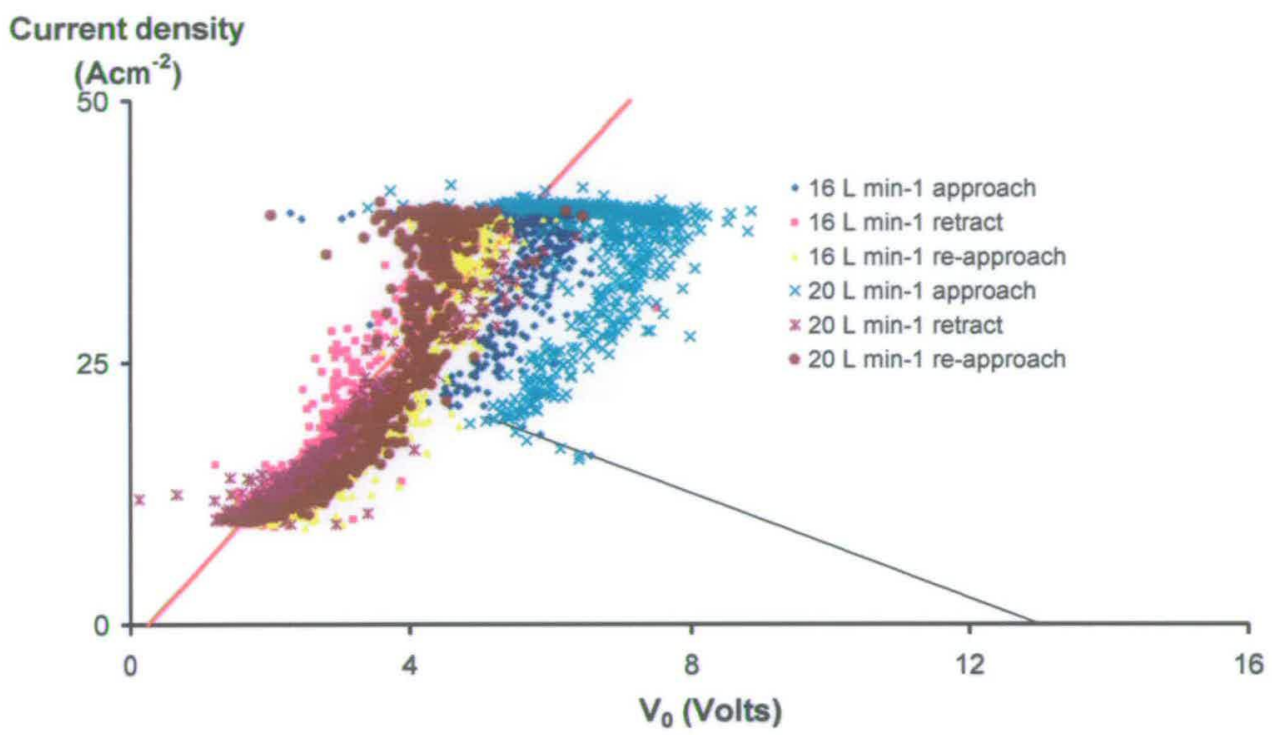

Figure 9.20: Comparison of the $\mathrm{J}-\mathrm{V}_{0}$ characteristics for In 718 machined $15.0 \% \mathrm{NaCl}$ with $\mathrm{V}=13.0 \mathrm{~V}$ and $\mathrm{U}=16.0 \mathrm{~L} \mathrm{~min}^{-1} 1$ and $20.0 \mathrm{~L} \mathrm{~min}^{-1}$. Also shown is the theoretical line calculated from Equation 7.2, and the linear least squares fit of the retract $\mathrm{J}-\mathrm{V}_{0}$ data for 15.0 $\% \mathrm{NaCl}$ (Figure 9.3) with an ohmic resistance of $0.13 \pm 0.01 \Omega \mathrm{cm}^{-2}$ and $\mathrm{V}_{0}$ axis intercept of $0.5 \pm 0.2 \mathrm{~V}$. 


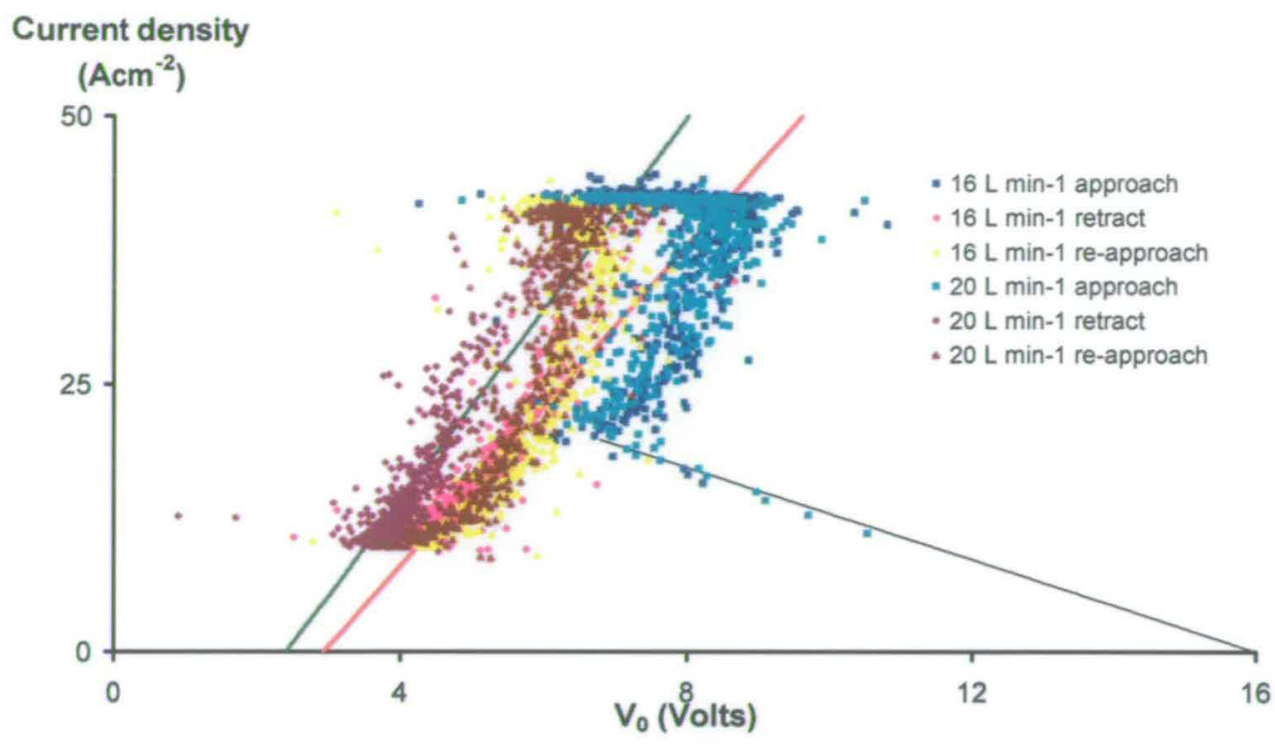

Figure 9.21: Comparison of the $\mathrm{J}-\mathrm{V}_{0}$ characteristics for $\mathrm{In} 718$ machined $21.7 \% \mathrm{NaNO}_{3}$ with $\mathrm{V}=16.0 \mathrm{~V}$ and $\mathrm{U}=16.0 \mathrm{~L} \mathrm{~min}^{-1}$ and $20.0 \mathrm{~L} \mathrm{~min}^{-1}$. Also shown is the theoretical line calculated from Equation 7.2. Finally, the linear least square fits of the retract $J-V_{0}$ data for $\mathrm{U}=16.0 \mathrm{~L} \mathrm{~min}^{-1}$ (Figure 9.3) with an ohmic resistance of $0.13 \pm 0.01 \Omega \mathrm{cm}^{-2}$ and $\mathrm{V}_{0}$ axis intercept of $2.9 \pm 0.2 \mathrm{~V}$ (red) and for the retract $\mathrm{J}-\mathrm{V}_{0}$ data for $\mathrm{U}=20.0 \mathrm{~L} \mathrm{~min}^{-1}$ with an ohmic resistance of $0.11 \pm 0.01 \Omega \mathrm{cm}^{-2}$ and $\mathrm{V}_{0}$ axis intercept of $2.4 \pm 0.2 \mathrm{~V}$ (green).

These data show flow velocity changes to have no appreciable effect on the dissolution valency; however a slight effect on the overall $\mathrm{J}-\mathrm{V}_{0}$ characteristics of In718 in the flow velocity range investigated was observed. Changes are observed in the $\mathrm{V}_{0}$ values in the approach phase of the $20.0 \mathrm{~L} \mathrm{~min}^{-1}$ data collected at $15.0 \% \mathrm{NaCl}$ (Figure 9.20) and in the retract phase of the $20.0 \mathrm{~L} \mathrm{~min}^{-1}$ data collected at $21.7 \% \mathrm{NaNO}_{3}$ (Figure 9.21). The reduction of both the ohmic $J-V_{0}$ resistance and the $V_{0}$ axis intercept, as seen for the $U=$ $20.0 \mathrm{~L} \mathrm{~min}^{-1}$ data in Figure 9.21, implies a reduction in ohmic resistance at the surface of the anode consistent with literature results for the reduction of the resistance of dehydrated surface films at increased flow rates [153]. It should, however, be noted that the linear least squares fit of the retract data collected at $20.0 \mathrm{~L} \mathrm{~min}{ }^{-1}$ in $21.7 \% \mathrm{NaNO}_{3}$ has the same gradient, to within experimental error, and a similar $\mathrm{V}_{0}$ axis intercept at $\mathrm{J}=0.0 \mathrm{~A} \mathrm{~cm}^{-2}$ to the retract data collected at $\mathrm{U}=16.0 \mathrm{~L} \mathrm{~min}^{-1}$. Since flow effects have not been observed during the retract phase in Figure 9.20 or for $\mathrm{Ti} / 4$ in $\mathrm{NaCl}$ or $\mathrm{NaNO}_{3}$ (Chapter 10) further experiments would be required to ascertain the validity of the results shown for $21.7 \%$ $\mathrm{NaNO}_{3}, \mathrm{U}=20.0 \mathrm{~L} \mathrm{~min}{ }^{-1}$ in Figure 9.21. 
$\mathrm{U}$ would also be expected to affect $\mathrm{J}-\mathrm{V}_{0}$ characteristics through significant product accumulation or reaction depletion, expected to occur at low $U$, resulting in changes in $\kappa$ along the flow path. This would be expected to produce trends in the segmented I-t characteristics with the currents at early times increasing or decreasing from segments 1 to 7 with increasing or decreasing $\kappa$ respectively, leading to the creation of a taper in the workpiece. No such effects were observed; this provides further evidence of the absence of any significant dependence of dissolution behaviour on $\mathrm{v}$ at these relatively high values of $\mathrm{U}$ and $\mathrm{v}$, appropriate to industrial ECM.

\subsection{Conclusions}

The data in this chapter show the ECM characteristics of In718 to have both similarities and differences to the $\mathrm{J}-\mathrm{V}_{0}$ characteristics observed for $\mathrm{Fe}$ and low chromium stainless steels (Chapter 7 and Chapter 8 respectively). The ECM dissolution valency for $\operatorname{In} 718$ has been shown to be independent of $z$, electrolyte type and concentration, electrolyte flow velocity and applied voltage. As with $\mathrm{Fe}$ and steels a low current density increase in observed dissolution valency has been observed for In718 under all the experimental conditions investigated in this chapter, consistent with an increase in dissolution valency or an alternative electro-oxidation reaction resulting in a low current density inefficiency in the dissolution process. As with $\mathrm{Fe}$ the low current density $\mathrm{J}-\mathrm{V}_{0}$ data are not observed to trend to lower $\mathrm{V}_{0}$ values, unlike SS410 and Jethete steels. In addition, the valency of $\mathrm{n}=3.8 \pm 0.3$ in this region is not consistent with $\mathrm{Cr}$ (III) dissolution. Therefore, unlike steel, preferential $\mathrm{Cr}(\mathrm{III})$ dissolution is not a likely explanation of this low current density, low voltage process.

As with both $\mathrm{Fe}$ and stainless steels, the $\mathrm{J}-\mathrm{V}_{0}$ characteristics for In718 show an initial linear decrease of $\mathrm{V}_{0}$ with increasing $\mathrm{J}$. As with $\mathrm{Fe}$ and steels these data are consistent with the initial breakdown of a passive layer and correspond closely to the theoretical gradient and intercept from Equation 7.2 for all the data shown in this chapter. This initial linear decrease of $\mathrm{V}_{0}$ with increasing $\mathrm{J}$ is followed by positive, essentially linear, $\mathrm{J}-\mathrm{V}_{0}$ data, as seen with $\mathrm{Fe}$ and steels. For In718 in both electrolytes (at concentrations greater than $5.0 \%$ ), except for $21.7 \% \mathrm{NaNO}_{3}$ at $\mathrm{U}=20.0 \mathrm{~L} \mathrm{~min}{ }^{-1}$, this positive, essentially linear $\mathrm{J}-\mathrm{V}_{0}$ data was found, during the retract phase, to have an ohmic surface resistance of $0.13 \pm 0.01 \Omega \mathrm{cm}^{-2}$, for 21.7 $\% \mathrm{NaNO}_{3}$ at $\mathrm{U}=20.0 \mathrm{~L} \mathrm{~min}{ }^{-1}$ an ohmic surface resistance of $0.11 \pm 0.01 \Omega \mathrm{cm}^{-2}$ was 
observed, which is consistent to within experimental error. The similar surface ohmic resistances observed for all the In718 data presented in this chapter imply similar surface dissolution processes at different interelectrode gaps, electrolyte type and concentration, electrolyte flow velocity and applied voltage which greatly simplifies ECM parameterisation. The $\mathrm{V}_{0}$ axis intercepts of the $\mathrm{J}-\mathrm{V}_{0}$ retract data at $\mathrm{J}=0.0 \mathrm{~A} \mathrm{~cm}$-2 were found to be $0.5 \pm 0.2 \mathrm{~V}$ for $\mathrm{NaCl}$ and $2.9 \pm 0.2 \mathrm{~V}$ for $\mathrm{NaNO}_{3}$ (with a value of $2.4 \pm 0.2 \mathrm{~V}$ for $21.7 \% \mathrm{NaNO}_{3}$ at $\mathrm{U}=$ $20.0 \mathrm{~L} \mathrm{~min}^{-1}$ ). This is consistent with data from Datta et al. which showed increased surface potentials when machining nickel in $\mathrm{NaNO}_{3}$ [167] compared to $\mathrm{NaCl}$ [136]. These potentials are consistent with the previously attributed active dissolution mechanism when machining in $\mathrm{NaCl}$ and a passive dissolution mechanism when machining in $\mathrm{NaNO}_{3}$.

Large temperature increases were observed when machining In718 in $5.0 \% \mathrm{NaNO}_{3}$ which have been attributed to appreciable Joule heating at the high applied potential, and relatively low electrolyte conductivity. These temperature increases have been shown to change the electrolyte conductivity resulting in large errors in the $\mathrm{J}-\mathrm{V}_{0}$ calculations if a constant $\kappa$ is assumed. Using thermocouple measurements and calibrations of the temperature dependence of $\kappa$ over the temperature range found during machining, the electrolyte conductivity changes can be corrected and the large apparent hysteresis in the data removed. However, even after these conductivity corrections significant differences in the $\mathrm{J}-\mathrm{V}_{0}$ characteristics are observed compared to measurements taken at lower temperatures. This is most likely due to the effects of temperature on the ECM dissolution processes.

In addition to providing insight into the $\mathrm{J}-\mathrm{V}_{0}$ characteristics of $\mathrm{Fe}, \mathrm{SS} 410$ and $\mathrm{J}$, the data in this chapter provide useful results for an ECM parameter base of this industrially useful material. The data presented have shown similar ECM characteristics for different $z$, electrolyte type and concentration, electrolyte flow velocity and applied voltage, greatly simplifying the ECM parameter base. 


\section{Chapter 10}

The ECM of Ti $6 / 4$ 


\subsection{Introduction and previous literature}

As with nickel based alloys, titanium alloys are used for applications within the aerospace and automotive industries (Chapter 9). This is because they have comparable high temperature strength, hardness and chemical wear resistance. However, as with nickel based alloys, these desirable material characteristics also make machining of these alloys by conventional methods problematic (section 1.3.1). For these reasons ECM is a valuable technique for machining titanium alloys, especially when making complex-shaped components with high surface integrity requirements, such as in engines.

In a previous study by Landolt et al. [58], using Electrochemical Micromachining with an electrolyte flow velocity of $10 \mathrm{~ms}^{-1}$, the dissolution valency of Ti in $5.0 \mathrm{M} \mathrm{NaNO}_{3}$ electrolyte was found to be 4.0 , while a lower valency of approximately 3.2 was observed for $5.0 \mathrm{M}$ $\mathrm{NaCl}$ electrolyte. In this study the current-cell voltage characteristics of the system were observed to intercept the potential axis (when $\mathrm{J}=0.0 \mathrm{~A} \mathrm{~cm}^{-2}$ ) at approximately $15 \mathrm{~V}$ and 28 $\mathrm{V}$ for $\mathrm{NaCl}$ and $\mathrm{NaNO}_{3}$ respectively. Landolt et al. attributes this initiation of anodic dissolution only at applied voltages well over $10 \mathrm{~V}$ to the predominance at low voltage of anodic film growth and oxygen evolution. ECMM, in this case, uses a channel cell configuration with microelectrodes defined by exposed photoresist. The channel cell configuration is consistent with the cell configuration in this work. However, Landolt et al. use stationary electrodes and, by virtue of the exposed photoresist definition of the workpiece, an increasing dissolution area. Therefore, differences may be expected between the characteristics found in this ECMM study and industrial ECM characterisation studies (section 7.1).

A study by Azumi and Seo [170], measuring cyclic voltammograms of Ti in a borate buffer at $\mathrm{pH}=9.84$ and $298 \mathrm{~K}$, showed potential values for the onset of dissolution of between 6 and $8 \mathrm{~V}$. This value was seen to increase with sweep number consistent with the growth of a passive layer. Current densities are typically below $3 \times 10^{-4} \mathrm{~A} \mathrm{~cm}^{-2}$, which is not appropriate to industrial ECM.

Initial characterisation of the ECM of $\mathrm{Ti} 6 / 4$ has been carried out previously at Edinburgh University using the chronoamperometric analysis detailed in section 3.2 [137]. In this work the time-averaged dissolution valencies and $\mathrm{V}_{0}$ values were found for $\mathrm{Ti} 6 / 4 \mathrm{in} \mathrm{NaCl}$ and 
$\mathrm{NaNO}_{3}$ electrolytes to be $\mathrm{V}_{0}=5.4 \pm 0.1 \mathrm{~V}$ and $\mathrm{V}_{0}=9.8 \pm 0.5 \mathrm{~V}$ respectively. The timeaveraged dissolution valencies at equilibrium were found to be $n=3.5 \pm 0.2$ for both electrolyte types, consistent with mixed valency $\mathrm{Ti}$ (III) / Ti (IV) dissolution.

As with $\ln 718$, studies into the time-resolved changes in $\mathrm{n}, \mathrm{J}$ and $\mathrm{V}_{0}$ for $\mathrm{Ti} 6 / 4$ would be a valuable contribution to the literature data already present for the dissolution of titanium and titanium-based alloys. All the work in this Chapter has been carried out on cell 2000 (section 4.6).

\section{2. $\mathrm{J}-\mathrm{V}_{0}$ characteristics of $\mathrm{Ti} 6 / 4$ in $\mathrm{NaCl}$ and $\mathrm{NaNO}_{3}$}

Figure 10.1 shows typical chronoamperometric data for segments 1-7, and typical z-t data, for an approach/retract and re-approach (section 4.8) experiment using Ti $6 / 4$ with $15.0 \%$ $\mathrm{NaCl}$ electrolyte and an applied voltage of $\mathrm{V}=15.0 \mathrm{~V}$.

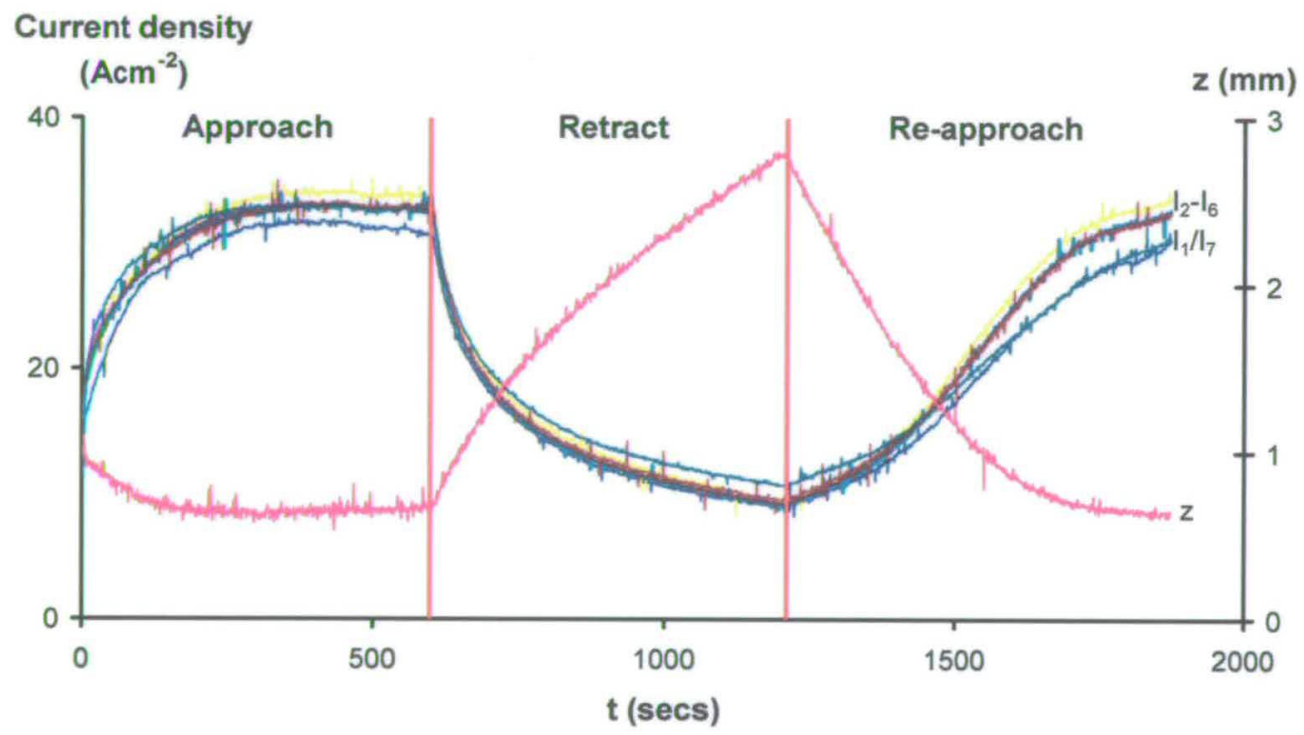

Figure 10.1: Experimental chronoamperometric transients for $\mathrm{Ti} 6 / 4$ machined in $15.0 \%$ $\mathrm{NaCl}$ with $\mathrm{V}=15.0 \mathrm{~V}$. Also shown is z-t data for the same experiment.

As with steels (Chapter 8) and In718 (Chapter 9) decreased values of $J_{1}$ and $J_{7}$ were seen during approach and re-approach phases with increased currents seen for these segments during the retract phase. This can be again attributed to the rounding effect described in 
section 8.3. From these data equilibrium values for segment 4 were found to be $J_{\infty}=33 \pm$ $0.7 \mathrm{Acm}^{-2}$ and $\mathrm{z}_{\infty}=0.63 \pm 0.03 \mathrm{~mm}$. Figure 10.2 shows typical chronoamperometric and $\mathrm{z}$-t data for the same material machined with $21.7 \% \mathrm{NaNO}_{3}$ electrolyte and an applied voltage of $\mathrm{V}=20.0 \mathrm{~V}$.

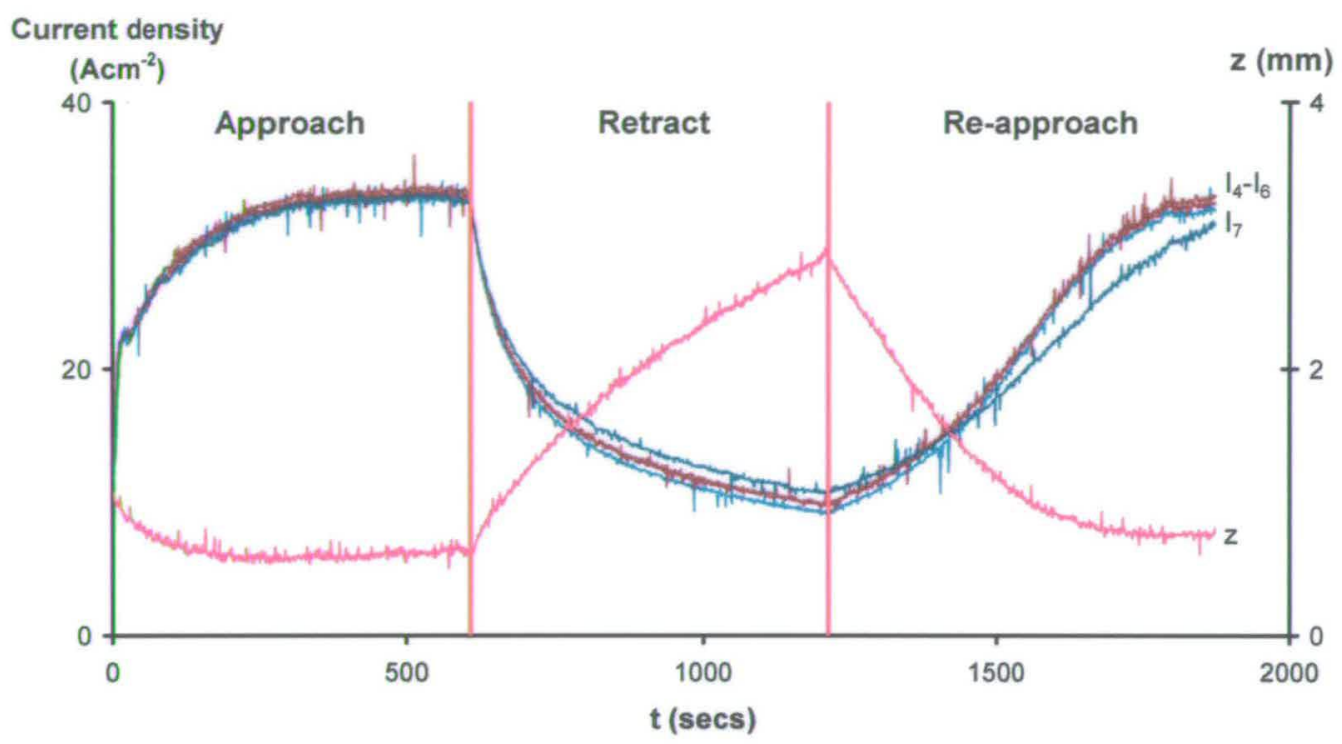

Figure 10.2: Experimental chronoamperometric transients for $\mathrm{Ti} 6 / 4$ machined in $21.7 \%$ $\mathrm{NaNO}_{3}$ with $\mathrm{V}=20.0 \mathrm{~V}$. Also shown is z-t data for the same experiment.

As seen for In718 machined in $21.7 \% \mathrm{NaNO}_{3}$, current data for segments 1-3 were unavailable for this experiment due to loose connections in the measuring system. However, since the $\mathrm{V}_{0}$ and $\mathrm{n}$ calculations require the use of the segment 4 current measurements, the subsequent analysis was unaffected. In the absence of these data, the segment 7 current density transient shows similar rounding effects to those observed for steels and In718 (section 8.3, Chapter 9) and for $\mathrm{Ti} 6 / 4$ in $\mathrm{NaCl}$ electrolyte. From these data equilibrium values for segment 4 were found to be $J_{\infty}=33 \pm 0.7 \mathrm{Acm}^{-2}$ and $\mathrm{z}_{\infty}=0.60 \pm 0.03 \mathrm{~mm}$, consistent, to within experimental error, with the values measured for $15.0 \% \mathrm{NaCl}$. As with In718, because of higher mean $\mathrm{V}_{0}$ values for $\mathrm{NaNO}_{3}$ produced by previous chronoamperometric measurements [137], the applied voltage was increased when machining in $\mathrm{NaNO}_{3}$ in an attempt to maintain a constant $\mathrm{J}_{\infty}$ and $\mathrm{z}_{\infty}$ between experiments. The percentage composition of the electrolytes was chosen to maintain a constant electrolyte concentration and a similar conductivity of $\kappa=0.20 \mathrm{~S} \mathrm{~cm}^{-1}$ [137]. The increased applied voltage could lead to increased temperature effects through Equation 8.1 (section 8.2). It is 
therefore possible that some temperature effects were present during this experiment (which could explain the curvature of the $\mathrm{NaNO}_{3}$ re-approach transient in Figure 10.3, section 10.3.1.2 vide infra); however, as these data were obtained prior to thermocouple measurement, thermocouple data were not available for $21.7 \% \mathrm{NaNO}_{3}$, which prohibited quantitative temperature correction.

Figure 10.3 shows the corresponding $\mathrm{J}-\mathrm{V}_{0}$ transients for $\mathrm{Ti} 6 / 4$ machined in $\mathrm{NaCl}$ and $\mathrm{NaNO}_{3}$.

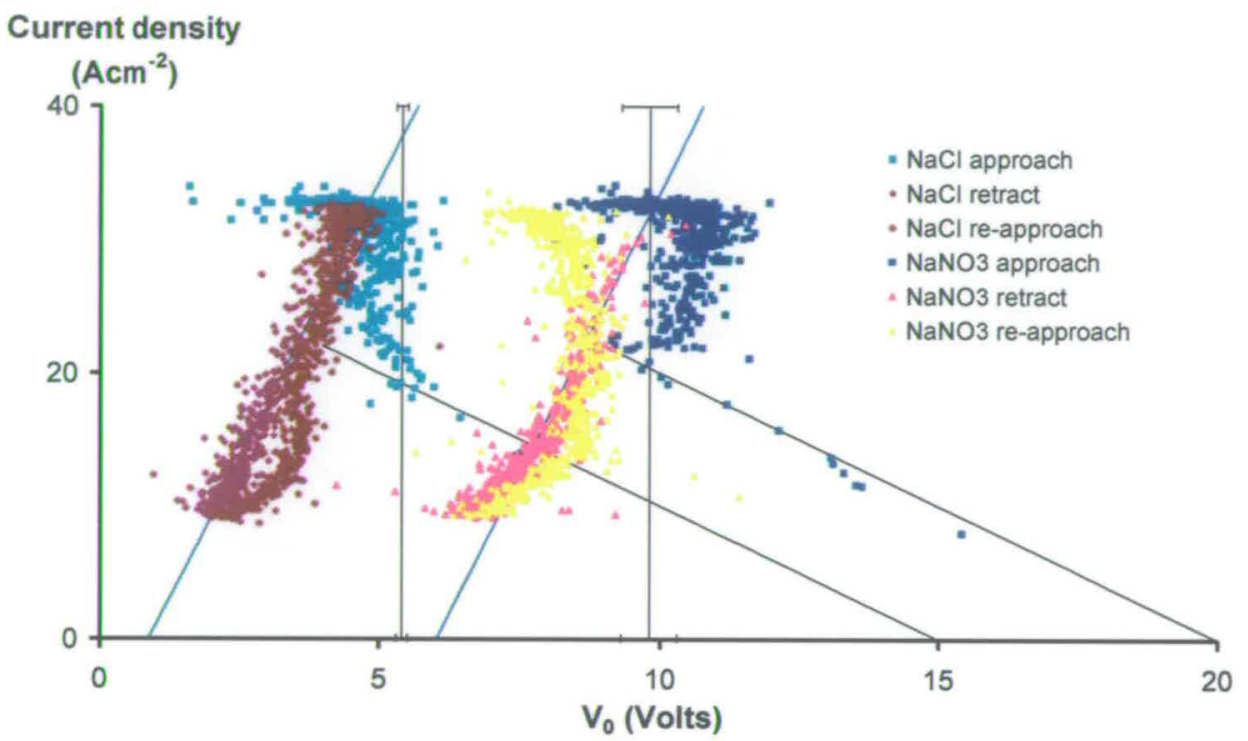

Figure 10.3: $\mathrm{J}-\mathrm{V}_{0}$ data for $\mathrm{Ti} 6 / 4$ machined in $15.0 \% \mathrm{NaCl}$ and $21.7 \% \mathrm{NaNO}_{3}$ at $\mathrm{V}=15.0$ $\mathrm{V}$ and $\mathrm{V}=20.0 \mathrm{~V}$ respectively. Time-averaged $\mathrm{V}_{0}$ values for Ti $6 / 4$ found through the chronoamperometric analysis in section 3.2 as $\mathrm{V}_{0}=5.4 \pm 0.1 \mathrm{~V}$ and $\mathrm{V}_{0}=9.8 \pm 0.5 \mathrm{~V}$ for $\mathrm{NaCl}$ and $\mathrm{NaNO}_{3}$ electrolytes respectively are given [137], with errors shown. Also shown are the theoretical lines calculated from Equation 7.2 with an experimental electrolyte conductivity of $\kappa=0.20 \mathrm{~S} \mathrm{~cm}^{-1}$ and initial interelectrode gaps $\mathrm{z}_{0}=1.00 \mathrm{~mm}$ for both $\mathrm{NaCl}$ and $\mathrm{NaNO}_{3}$. Finally, linear least square fits of the retract $\mathrm{J}-\mathrm{V}_{0}$ data are shown. These both give ohmic resistances of $0.12 \pm 0.01 \Omega \mathrm{cm}^{-2}$ and $\mathrm{V}_{0}$ axis intercepts of $0.9 \pm 0.2 \mathrm{~V}$ and $6.0 \pm$ $0.2 \mathrm{~V}$ for $\mathrm{NaCl}$ and $\mathrm{NaNO}_{3}$ (green and blue) respectively.

For both electrolytes, the close comparison of the mean values of these ultrasound approach $\mathrm{J}-\mathrm{V}_{0}$ data with those previously obtained using the chronoamperometric method [137] is reassuring and indicates the accuracy of this method. Figure 10.3 also shows that for 
machining in $\mathrm{NaCl}$ and $\mathrm{NaNO}_{3}$, the $\mathrm{J}-\mathrm{V}_{0}$ characteristics for Ti $6 / 4$ show some similarities with those observed for $\mathrm{Fe}$ (section 7.2.2), steels (section 8.5) and In718 (section 9.2). The initial linear decrease of $\mathrm{V}_{0}$ with increasing $\mathrm{J}$ for $\mathrm{Ti} 6 / 4$ shows the same close correspondence to the theoretically calculated lines found using Equation 7.2. As with $\mathrm{Fe}$ (section 7.2.2), Jethete (section 8.5) and In718 (section 9.2), the data on this line start at low $\mathrm{J}$ and high $\mathrm{V}_{0}$ and move to high $\mathrm{J}$ and low $\mathrm{V}_{0}$ consistent with breakdown of a passivating layer on the workpiece. Following this initial breakdown of passivity, the $\mathrm{J}-\mathrm{V}_{0}$ transients follow positive, essentially linear, ohmic characteristics as seen for all the previous materials studied in this work. The linear least squares fits of the $\mathrm{Ti} 6 / 4 \mathrm{~J}-\mathrm{V}_{0}$ retract data in both electrolytes shows an ohmic resistance of $0.12 \pm 0.01 \Omega \mathrm{cm}^{-2}$, the same, to within experimental error, as that measured for $\operatorname{In} 718$ (section 9.2). As with $\operatorname{In} 718$, the retract data were analysed to minimise the effects of the approach and re-approach hysteresis consistent with driving the ECM process with a feed rate of $0.5 \mathrm{~mm} \mathrm{~min}^{-1}$.

It is satisfying that, as with In718, both the chronoamperometric and the ultrasound method produce greater mean $\mathrm{V}_{0}$ values when machining Ti $6 / 4$ with $\mathrm{NaNO}_{3}$ compared to machining with $\mathrm{NaCl}$. This is consistent with literature evidence by Landolt et al. showing increased surface potentials when electrochemical micromachining of $\mathrm{Ti}$ in $\mathrm{NaNO}_{3}$ compared to $\mathrm{NaCl}$ [58]. However, the large difference between the $\mathrm{V}_{0}$ axis intercepts shown in Figure 10.3 are $0.9 \pm 0.2 \mathrm{~V}$ and $6.0 \pm 0.2 \mathrm{~V}$ in $\mathrm{NaCl}$ and $\mathrm{NaNO}_{3}$ respectively and those found by Landolt $e$ al. of $15 \mathrm{~V}$ and $28 \mathrm{~V}$ implies different conditions in the two systems. Indeed, the values found for $\mathrm{NaNO}_{3}$ in this work are far more consistent with those found by Azumi and Seo [170] in borate buffer, with a potential, at $\mathrm{J}=0.0 \mathrm{~A} \mathrm{~cm}^{-2}$, of between 6 and $8 \mathrm{~V}$. These voltages increased on cycling, consistent with the formation of a thicker passivating film in borate buffer. Therefore, as with steels, such positive $\mathrm{V}_{0}$ axis intercepts at $\mathrm{J}=0.0 \mathrm{~A} \mathrm{~cm}^{-2}$ seem consistent with a passive dissolution mechanism (section 9.2) [153]. As with steels and $\mathrm{In} 718$, a much lower $\mathrm{V}_{0}$ axis intercept was observed for $\mathrm{NaCl}$, consistent with an active dissolution process (section 8.5 and section 9.2).

Figure 10.4 and Figure 10.5 show the time-resolved dissolution valency data for $\mathrm{Ti} 6 / 4$ machined in $\mathrm{NaCl}$ and $\mathrm{NaNO}_{3}$ respectively. 
Parameterisation of Electrochemical Machining

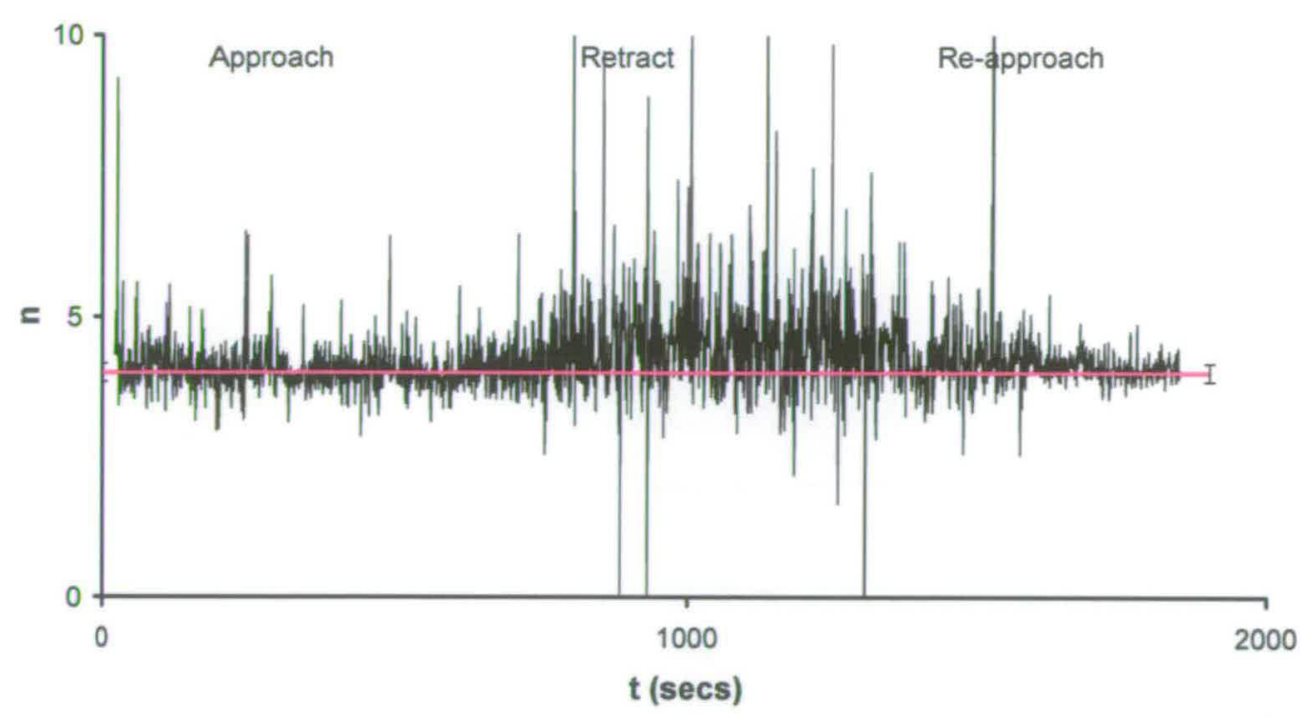

Figure 10.4: Experimental time-resolved $n$, with $p=100$ from Equation 6.4, for $\mathrm{Ti} 6 / 4$ machined in $\mathrm{NaCl}$, the pink line depicts the average dissolution valency at equilibrium with an error shown.

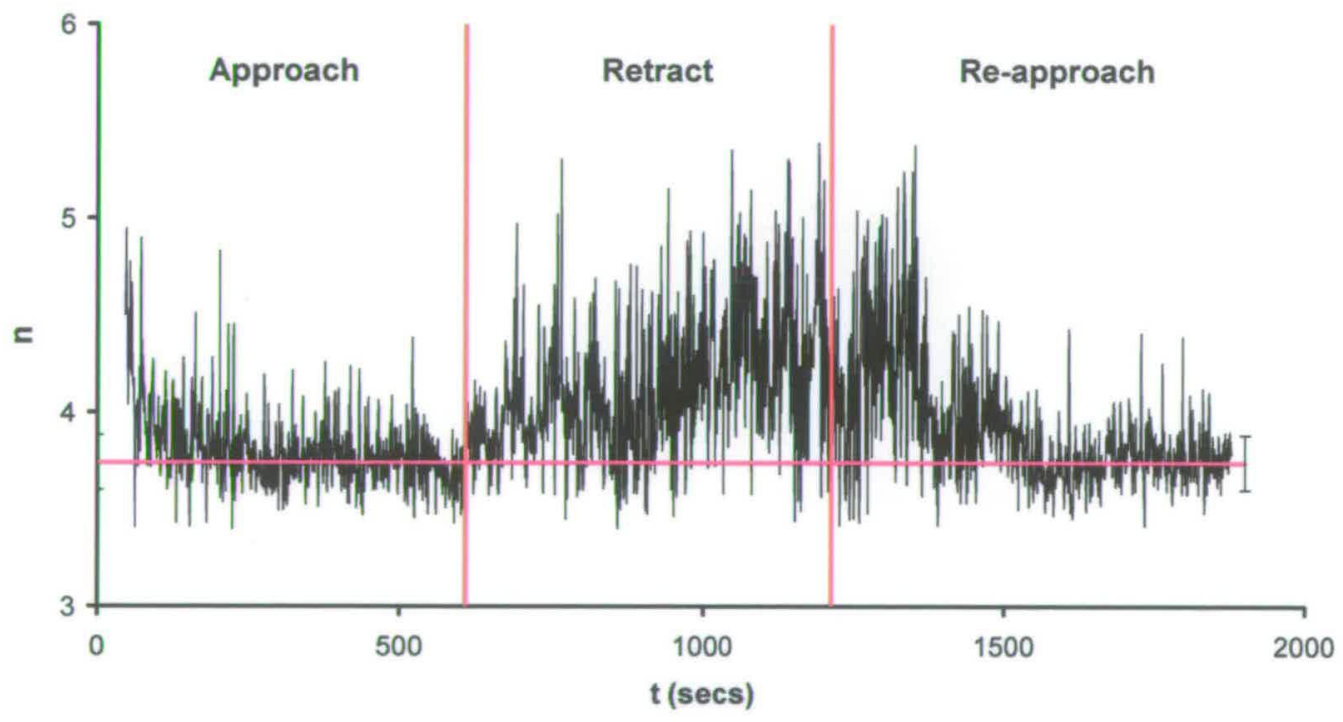

Figure 10.5: Experimental time-resolved $n$, with $p=100$ from Equation 6.4, for $\mathrm{Ti} 6 / 4$ machined in $\mathrm{NaNO}_{3}$, the pink line depicts the average dissolution valency at equilibrium with an error shown. 
These figures show the equilibrium dissolution valencies for Ti $6 / 4$ to be $n=4.0 \pm 0.2$ and $n$ $=3.7 \pm 0.2$ for $\mathrm{NaCl}$ and $\mathrm{NaNO}_{3}$ respectively. These $\mathrm{n}$ values are consistent with the chemically reasonable dissolution valencies of $\mathrm{Ti}$ (IV) in $\mathrm{NaCl}$ and either $\mathrm{Ti}(\mathrm{IV}$ ) or mixed valency $\mathrm{Ti}$ (III) / Ti(IV) in $\mathrm{NaNO}_{3}$. However, as with In718, care must be taken when assigning individual valencies since it is possible that the same mean valency could be achieved by balancing a lower valency for one element with a higher valency of another. These values are the same to within experimental error. The equilibrium dissolution valency observed for $\mathrm{NaNO}_{3}$ is consistent, to within experimental error, with the value of $\mathrm{n}=3.5 \pm$ 0.2 obtained in previous studies by Mount et al. [137]. These values are also broadly consistent with values measured by Landolt et al. [58], of $\mathrm{n}=4$ and $\mathrm{n}=3.8$ for ECMM of Ti in $\mathrm{NaNO}_{3}$ and $\mathrm{NaCl}$ respectively. As with $\mathrm{Fe}$ (section 7.2.2), stainless steels (section 8.5) and In718 (section 9.2) higher dissolution valencies were observed during low current density machining. Figure 10.4 and Figure 10.5 show $n$ to increase to $n=4.4 \pm 0.3$ for both electrolytes. This increase in $\mathrm{n}$ is consistent with either an increase in dissolution valency or a decrease in efficiency of the dissolution process (Equation 2.35) at low current densities. This is consistent with Landolt et al. [58] who attribute low voltage inefficiencies when machining $\mathrm{Ti}$ in $\mathrm{NaCl}$ and $\mathrm{NaNO}_{3}$ to oxygen evolution and anodic film growth in both systems. For Ti $6 / 4$, as with In718, the low current density increase in dissolution valency was found to be of the same magnitude in both electrolytes.

It is interesting that, as with $\operatorname{In} 718$ (section 9.2) and Fe (section 7.2.2), but unlike stainless steels (section 8.5), no low current density trend to lower $V_{0}$ values is observed for Ti 6/4 in Figure 10.3.

\subsection{Effects of changes in ECM conditions}

\subsubsection{Electrolyte concentration changes}

\subsubsection{1. $25.0 \%$ electrolyte concentration}

Ti $6 / 4$ was machined in $25.0 \% \mathrm{NaCl}$ and $25.0 \% \mathrm{NaNO}_{3}$ with applied voltages of $\mathrm{V}=15.0$ $\mathrm{V}$ and $\mathrm{V}=23.0 \mathrm{~V}$ respectively. During these experiments the equilibrium interelectrode gap 
was observed to have increased to $\mathrm{z}_{\infty}=0.71 \pm 0.03 \mathrm{~mm}$ for $25.0 \% \mathrm{NaCl}$ and $\mathrm{z}_{\infty}=0.85 \pm$ $0.03 \mathrm{~mm}$ for $25.0 \% \mathrm{NaNO}_{3}$ compared to $\mathrm{z}_{\infty}=0.63 \pm 0.03 \mathrm{~mm}$ and $\mathrm{z}_{\infty}=0.60 \pm 0.03 \mathrm{~mm}$ for $15.0 \% \mathrm{NaCl}$ and $21.7 \% \mathrm{NaNO}_{3}$ respectively (section 10.2). As with In718 (section 9.3.2.1), this increase in equilibrium interelectrode gap size was expected due to the increase in conductivities of $25.0 \% \mathrm{NaCl}$ and $25.0 \% \mathrm{NaNO}_{3}$ over $15.0 \% \mathrm{NaCl}$ and $21.7 \% \mathrm{NaNO}_{3}$ respectively. The equilibrium dissolution valency was found to be $n=4.0 \pm 0.3$ and $n=3.7$ \pm 0.2 for $25.0 \% \mathrm{NaCl}$ and $25.0 \% \mathrm{NaNO}_{3}$ respectively increasing to $\mathrm{n}=4.4 \pm 0.3$ at low current densities for both electrolytes. This is consistent with measurements made at lower electrolyte concentrations (Figure 10.4 and Figure 10.5).

Figure 10.6 and Figure 10.7 show comparisons of the $\mathrm{J}-\mathrm{V}_{0}$ characteristics for $15.0 \%$ (Figure 10.3 ) and $25.0 \% \mathrm{NaCl}$ and $21.7 \%$ (Figure 10.3 ) and $25.0 \% \mathrm{NaNO}_{3}$ respectively.

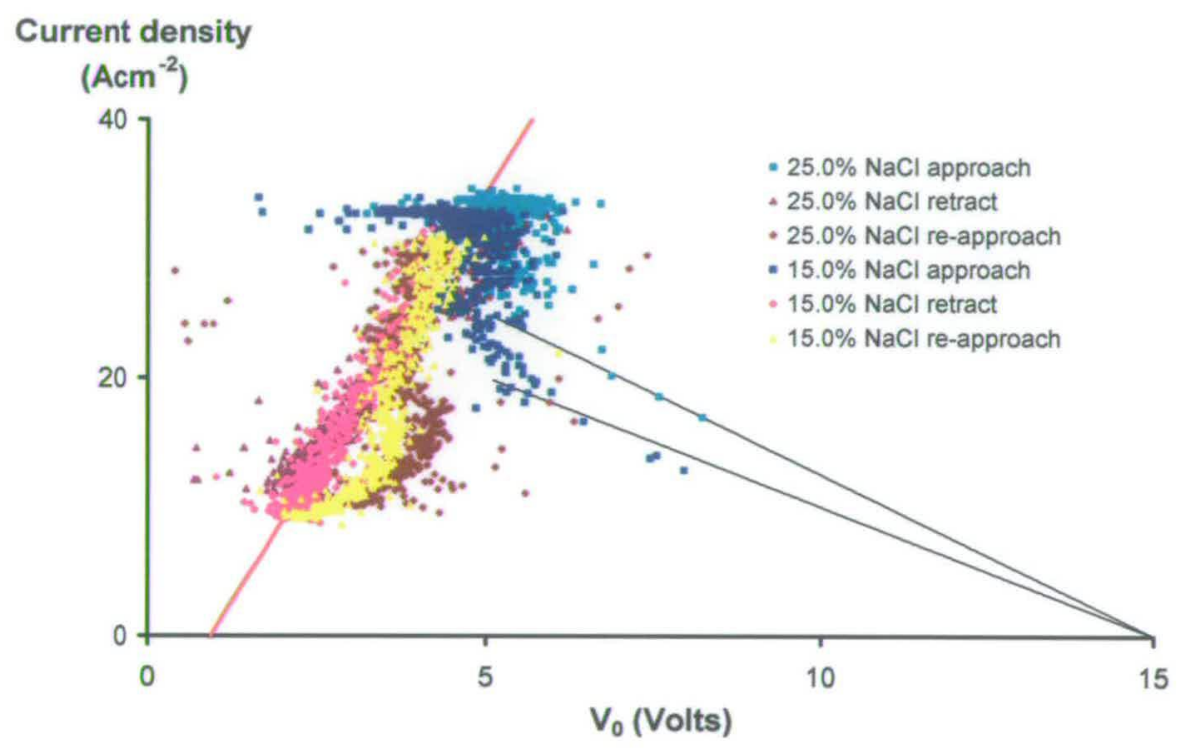

Figure 10.6: Comparison of the $\mathrm{J}-\mathrm{V}_{0}$ characteristics for $\mathrm{Ti} 6 / 4$ machined at $\mathrm{V}=15.0 \mathrm{~V}$ in $15.0 \%$ and $25.0 \% \mathrm{NaCl}$ electrolytes. Also shown are the theoretical lines calculated from Equation 7.2, with conductivities of $\kappa=0.20 \mathrm{~S} \mathrm{~cm}^{-1}$ and $0.25 \mathrm{~S} \mathrm{~cm}^{-1}$ for $15.0 \%$ and $25.0 \%$ respectively. Finally, the linear least squares fit of the retract $\mathrm{J}-\mathrm{V}_{0}$ data are shown with an ohmic resistances of $0.12 \pm 0.01 \Omega \mathrm{cm}^{-2}$ and $\mathrm{V}_{0}$ axis intercept of $0.9 \pm 0.2 \mathrm{~V}$. 


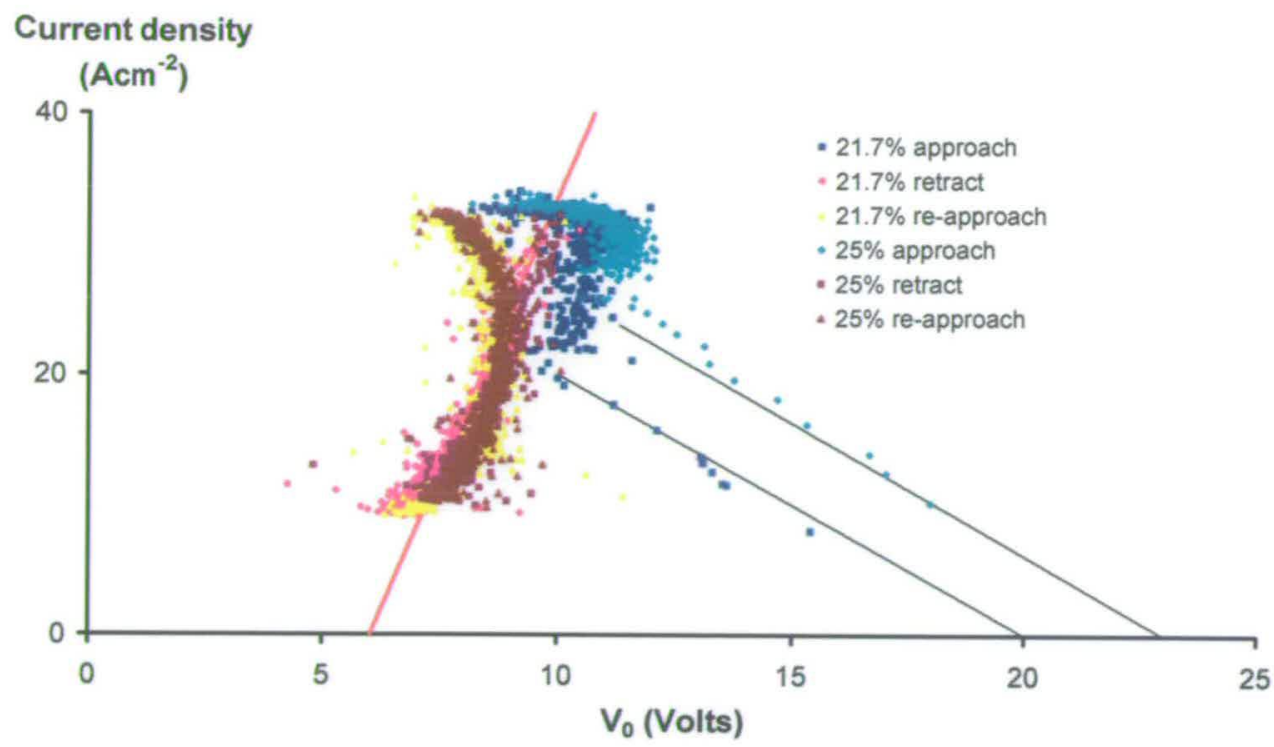

Figure 10.7: Comparison of the $\mathrm{J}-\mathrm{V}_{0}$ characteristics for $\mathrm{Ti} 6 / 4$ machined $21.7 \%$ and $25.0 \%$ $\mathrm{NaNO}_{3}$ electrolytes at $\mathrm{V}=20.0 \mathrm{~V}$ and $\mathrm{V}=23.0 \mathrm{~V}$ respectively. Also shown are the theoretical lines calculated from Equation 7.2, with conductivities of $\kappa=0.20 \mathrm{~S} \mathrm{~cm}^{-1}$ and $\kappa=$

$0.22 \mathrm{~S} \mathrm{~cm}^{-1}$ for $21.7 \% \mathrm{NaNO}_{3}$ and $25.0 \% \mathrm{NaNO}_{3}$ respectively. Finally, the linear least squares fit of the retract $\mathrm{J}-\mathrm{V}_{0}$ data are shown with an ohmic resistances of $0.12 \pm 0.01 \Omega \mathrm{cm}^{-2}$ and $\mathrm{V}_{0}$ axis intercept of $6.0 \pm 0.2 \mathrm{~V}$.

It is clear from these data that, as for steels and In718, an initial linear decrease of $\mathrm{V}_{0}$ with increasing $\mathrm{J}$ occurs in each case. The gradients and $\mathrm{V}_{0}$ axis intercepts are broadly consistent with the theoretical lines calculated using Equation 7.2 and the appropriate electrolyte conductivity, applied voltage and initial interelectrode gap parameters in all cases. The data on each line start at low $\mathrm{J}$ and high $\mathrm{V}_{0}$ and move to higher $\mathrm{J}$ and lower $\mathrm{V}_{0}$ consistent with a breakdown of passivity (as seen for Fe, Jethete and In718, section 7.2.2, section 8.5 and section 9.2). It is interesting to note that the $\mathrm{J}-\mathrm{V}_{0}$ data in this region for $25.0 \% \mathrm{NaNO}_{3}$ initially follow the theoretical lines calculated from Equation 7.2 but trend to higher current densities at lower $\mathrm{V}_{0}$ values. This has also been observed for $\operatorname{In} 718$ machined in $25.0 \%$ $\mathrm{NaCl}$ and $25.0 \% \mathrm{NaNO}_{3}$, and is consistent with the temporal mixing of the two terms in Equation 7.1.

Figure 10.6 and Figure 10.7 also show the subsequent positive, essentially linear, $\mathrm{J}-\mathrm{V}_{0}$ characteristics for $25.0 \% \mathrm{NaCl}$ and $25.0 \% \mathrm{NaNO}_{3}$ to have an ohmic resistance of $0.12 \pm$ $0.01 \Omega \mathrm{cm}^{-2}$ for the retract phase data, consistent with the $\mathrm{J}-\mathrm{V}_{0}$ data collected for $15.0 \%$ 
$\mathrm{NaCl}$ and $21.7 \% \mathrm{NaNO}_{3}$ and also consistent, to within experimental error, with the ohmic resistance measured for In718 (section 9.2). As with In718 (section 9.2), all these experiments show the same positive $\mathrm{J}-\mathrm{V}_{0}$ gradient despite changes in applied voltage, electrolyte concentration and type, and interelectrode gap. However, it should be noted that the conductivities of the electrolytes at a concentration of $25.0 \%$ could not be measured directly. Consequently a conductivity of $0.25 \mathrm{~S} \mathrm{~cm}^{-1}$ was assumed for $25.0 \% \mathrm{NaCl}$ in order to normalise this transient to the $15.0 \% \mathrm{NaCl}$ transient, while a conductivity of $0.22 \mathrm{~S} \mathrm{~cm}^{-1}$ was assumed for $25.0 \% \mathrm{NaNO}_{3}$ to normalise this transient to the $21.7 \% \mathrm{NaNO}_{3}$ transient. The differences in these normalised conductivities compared to those found when machining In718 in $25.0 \% \mathrm{NaCl}$ and $25.0 \% \mathrm{NaNO}_{3}\left(0.27 \mathrm{~S} \mathrm{~cm}^{-1}\right.$ and $0.21 \mathrm{~S} \mathrm{~cm}^{-1}$ respectively) imply an error of the order of $5-10 \%$ in making up the electrolyte solutions (section 4.8). This is reasonable, as the major error is defining the volume of the electrolyte, which can be measured to the order of $\pm 3.0 \mathrm{~L}$. Once normalised, all the data are the same to within experimental error. Such close correspondence of all the data with one common normalisation parameter further justifies the normalisation process, although it would be more accurate to independently measure the $25.0 \%$ concentration electrolyte conductivity. However, this was not possible due to the lack of a conductivity meter for these studies (section 9.3.2.1). As a result it can be concluded that the $\mathrm{J}-\mathrm{V}_{0}$ characteristics are essentially independent of the electrolyte concentration.

\subsubsection{2. $5.0 \% \mathrm{NaCl}$ electrolyte}

Figure 10.8 shows the time-resolved dissolution valency when machining $\mathrm{Ti} 6 / 4$ in $5.0 \%$ $\mathrm{NaCl}$ at an applied voltage of $\mathrm{V}=34.0 \mathrm{~V}$. This increased applied voltage is necessary to maintain reasonable values of $z_{\infty}$ in these relatively low conductivity electrolytes. 


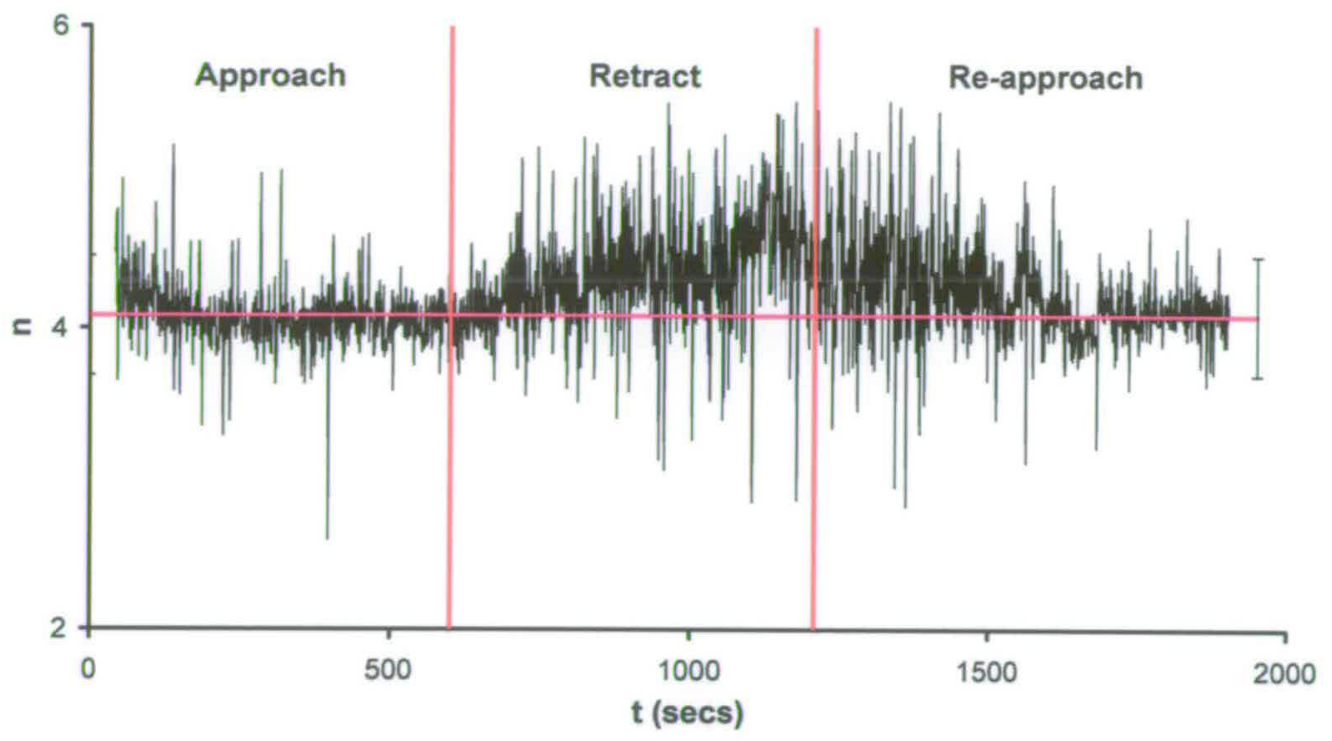

Figure 10.8: Experimental time-resolved $n$, with $\mathrm{p}=100$ from Equation 6.4, for Ti 6/4 machined in $5.0 \% \mathrm{NaCl}$ at an applied voltage of $\mathrm{V}=34.0 \mathrm{~V}$, the pink line depicts the average dissolution valency at equilibrium with an error shown.

This figure shows the equilibrium dissolution valencies for Ti $6 / 4$ in this electrolyte to be $\mathrm{n}=$ $4.0 \pm 0.3$ increasing to $n=4.7 \pm 0.4$ at low current density. These values are again consistent, to within experimental error, with previous measurements for Ti 6/4 in higher concentration $\mathrm{NaCl}$.

Figure 10.9 shows the time-resolved $\mathrm{z}$ data collected when machining $\mathrm{Ti} 6 / 4$ in $5.0 \% \mathrm{NaCl}$. 


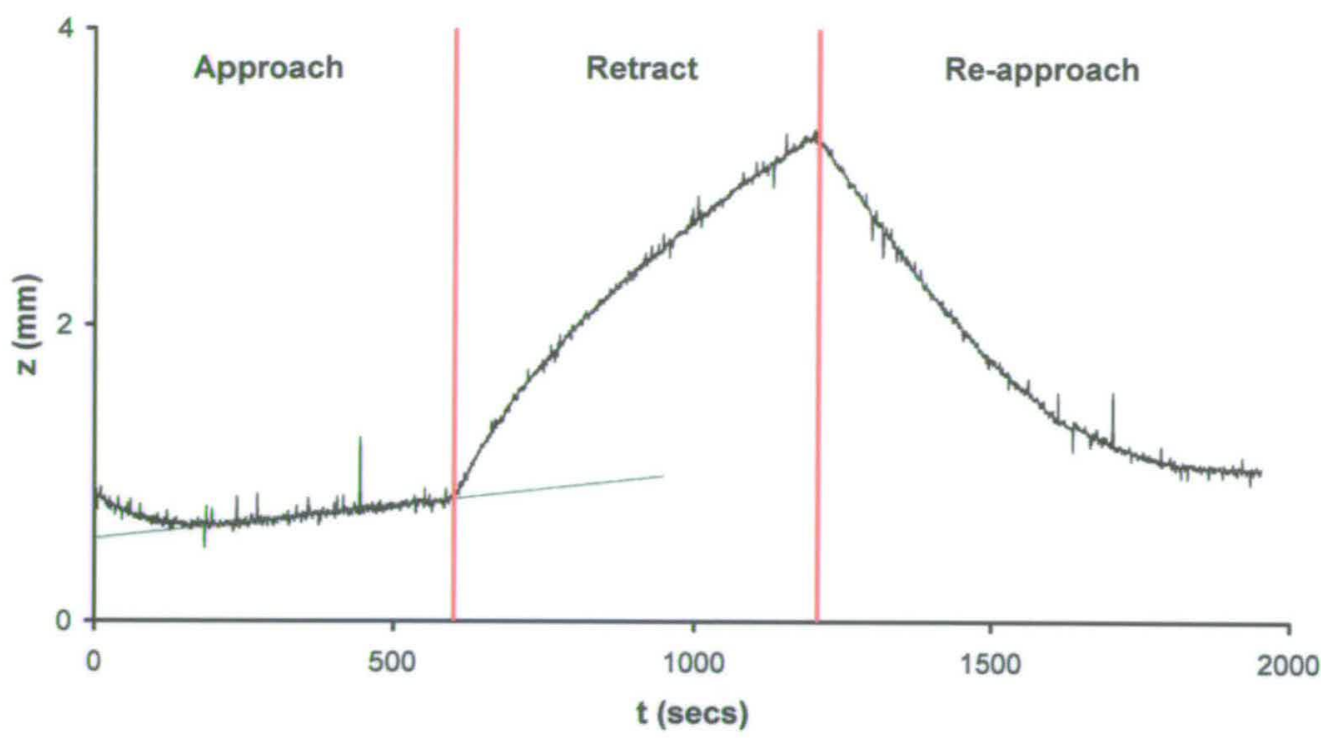

Figure 10.9: Plot of the time-resolved $\mathrm{z}$ data collected when machining $\mathrm{Ti} 6 / 4$ in $5.0 \% \mathrm{NaCl}$ at an applied voltage of $\mathrm{V}=34.0 \mathrm{~V}$. Also shown is the least squares fit through the approach phase $z_{\infty}$ data (green line).

Figure 10.9 clearly shows that $\mathrm{z}_{\infty}$ increases with time; this increase was found to occur at a rate of $4.5 \times 10^{-4} \mathrm{~mm} \mathrm{~s}^{-1}$ during the approach phase equilibrium period. At the start of the approach phase $\mathrm{z}_{\infty}=0.67 \pm 0.03 \mathrm{~mm}$ which was found to have increased by $22 \%$ to $\mathrm{z}_{\infty}=$ $0.83 \pm 0.03 \mathrm{~mm}$ at the end of the approach phase. This is consistent with increasing electrolyte temperature as seen for $\operatorname{In} 718$ (section 9.3.2.2).

Figure 10.10 shows the effects on the $\mathrm{J}-\mathrm{V}_{0}$ characteristics of an (incorrect) assumption of constant $\kappa$ when machining $\mathrm{Ti} 6 / 4$ in $5.0 \% \mathrm{NaCl}$ at an applied voltage of $\mathrm{V}=34.0 \mathrm{~V}$. 


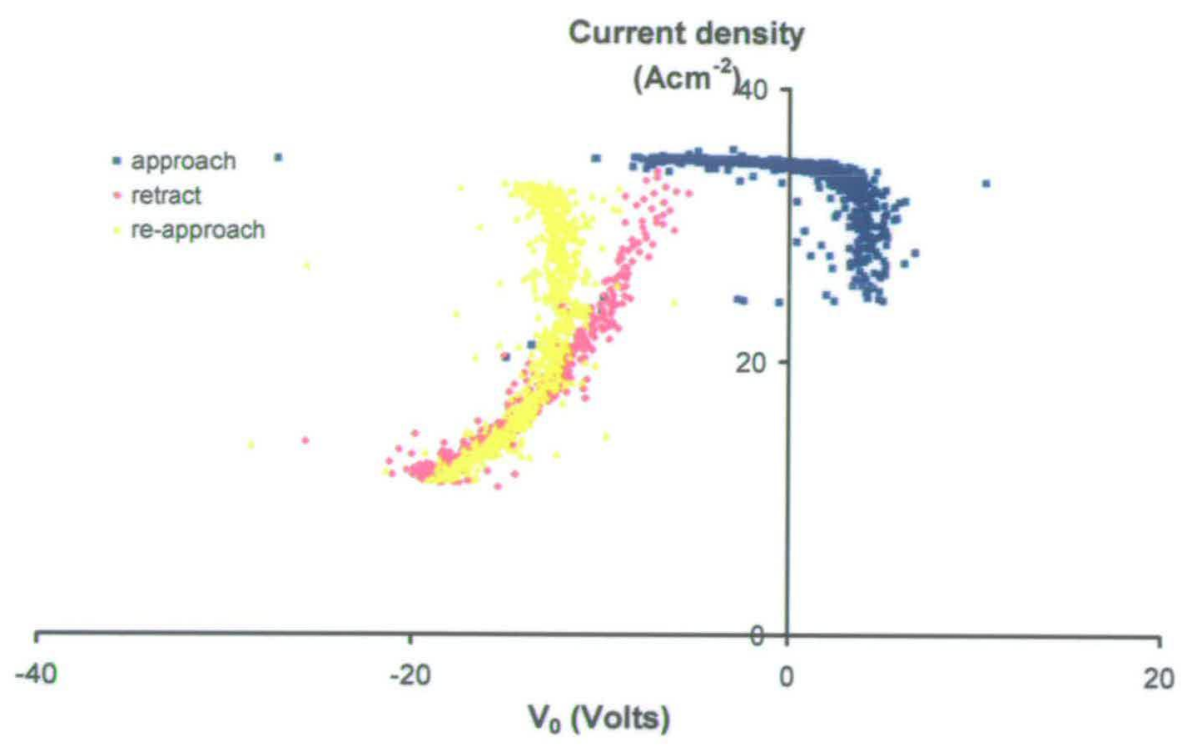

Figure 10.10: $\mathrm{J}-\mathrm{V}_{0}$ data for machining of $\mathrm{Ti} 6 / 4$ in $5.0 \% \mathrm{NaCl}$ with an assumed constant $\kappa$ equal to the initial bulk value of $\kappa=70.5 \mathrm{mS} \mathrm{cm}^{-1}$.

These data show $\mathrm{J}-\mathrm{V}_{0}$ characteristics consistent with significant Joule heating effects when machining in $5.0 \% \mathrm{NaCl}$ electrolyte (section 8.2 and section 9.3.2.2), namely large, negative and chemically unrealistic $\mathrm{V}_{0}$ values, a large hysteresis and curvature of the re-approach phase towards smaller $\mathrm{V}_{0}$ values.

Unfortunately no thermocouple data was available to correct the $\mathrm{J}-\mathrm{V}_{0}$ data shown in Figure 10.10 , however, an experiment carried out at a lower applied voltage $(\mathrm{V}=19.0 \mathrm{~V})$ showed ECM characteristics with smaller temperature effects. Figure 10.11 shows the time-resolved $\mathrm{z}$ data collected when machining Ti $6 / 4$ at this lower applied voltage. 


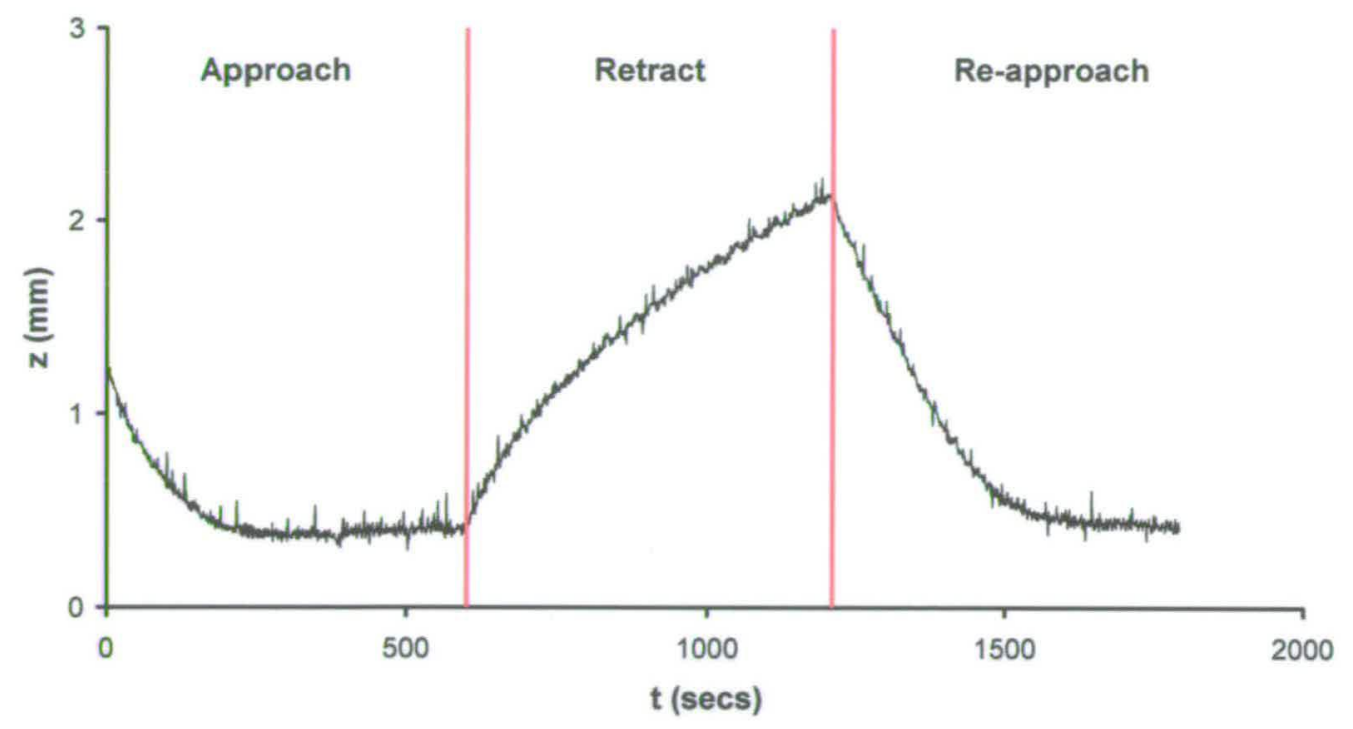

Figure 10.11: Plot of the time-resolved $\mathrm{z}$ data collected when machining Ti $6 / 4$ in $5.0 \%$ $\mathrm{NaCl}$ at an applied voltage of $\mathrm{V}=19.0 \mathrm{~V}$.

These data clearly show no temperature related increase in the equilibrium machining gap when machining at a lower applied voltage. For these data the equilibrium interelectrode gap was observed to be $\mathrm{z}_{\infty}=0.38 \pm 0.03 \mathrm{~mm}$. For this experiment the equilibrium dissolution valency was found to be $n=4.0 \pm 0.2$ increasing to $n=4.7 \pm 0.4$ at low current density, consistent with previous measurements for $\mathrm{Ti} 6 / 4$ in $\mathrm{NaCl}$. Figure 10.12 shows the corresponding $\mathrm{J}-\mathrm{V}_{0}$ data for $\mathrm{Ti} 6 / 4$ machined in $5.0 \% \mathrm{NaCl}$ at an applied voltage of $\mathrm{V}=19.0$ $\mathrm{V}$ compared with $\mathrm{J}-\mathrm{V}_{0}$ data collected at $15.0 \% \mathrm{NaCl}$ (Figure 10.3). 


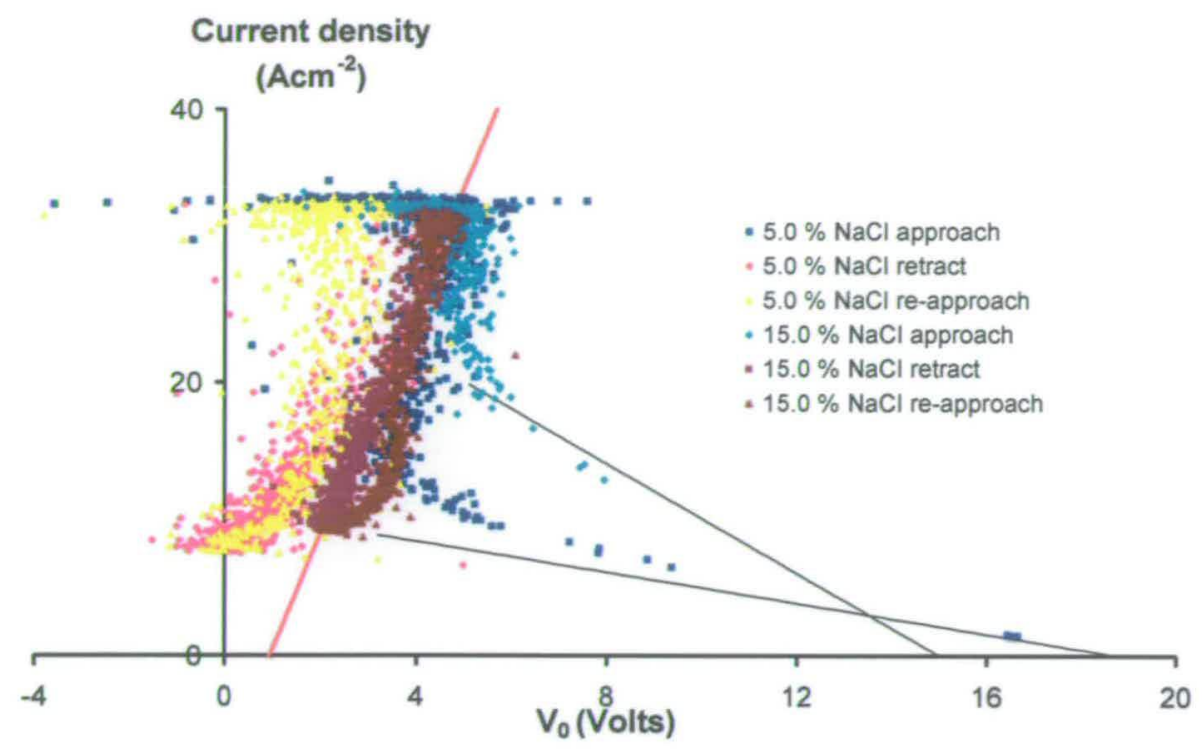

Figure 10.12: Comparison of the $\mathrm{J}-\mathrm{V}_{0}$ characteristics for $\mathrm{Ti} 6 / 4$ machined $5.0 \%$ and $15.0 \%$ $\mathrm{NaCl}$ electrolytes at $\mathrm{V}=19.0 \mathrm{~V}$ and $\mathrm{V}=15.0 \mathrm{~V}$ respectively. Also shown are the theoretical line calculated from Equation 7.2 with experimental electrolyte conductivities of $\kappa=70.5$ $\mathrm{mS} \mathrm{cm}^{-1}$ and $0.2 \mathrm{~S} \mathrm{~cm}^{-1}$ for $5.0 \%$ and $15.0 \% \mathrm{NaCl}$ respectively. Finally, the linear least squares fit of the $15.0 \% \mathrm{NaCl}$ retract $\mathrm{J}-\mathrm{V}_{0}$ data with an ohmic resistance of $0.12 \pm 0.01 \Omega$ $\mathrm{cm}^{-2}$ and $\mathrm{V}_{0}$ axis intercepts of $0.9 \pm 0.2 \mathrm{~V}$ is shown.

Figure 10.11 and Figure 10.12 clearly show reduced temperature effects when machining Ti $6 / 4$ in $5.0 \% \mathrm{NaCl}$ at a lower applied voltage, compared with the data in Figure 10.9 and Figure 10.10 respectively. This reduction in temperature effects is consistent with a reduction in applied potential, on which the Joule heating effect is linearly dependent (Equation 8.1), resulting in less significant electrolyte heating at this applied voltage. Indeed, the bulk electrolyte temperature difference when machining $\mathrm{Ti} 6 / 4$ at an applied voltage of $\mathrm{V}=19.0 \mathrm{~V}$ was found to be $\Delta \mathrm{T}=-1.0^{\circ} \mathrm{C}$ while that measured when machining at $\mathrm{V}=34.0 \mathrm{~V}$ (Figure 10.10) was found to be $\Delta \mathrm{T}=+6{ }^{\circ} \mathrm{C}$. It is clear from Figure 10.12 that for $5.0 \% \mathrm{NaCl}$ the initial linear decrease of $\mathrm{V}_{0}$ with increasing $\mathrm{J}$ occurs with a gradient and $\mathrm{V}_{0}$ axis intercept broadly consistent with the theoretical lines calculated using Equation 7.2 for the first data points. However, the $\mathrm{J}-\mathrm{V}_{0}$ data in this region trends to higher current densities at lower $\mathrm{V}_{0}$ values, consistent with the temporal mixing of the two terms in Equation 7.1 and seen previously for In718 machined in $25.0 \% \mathrm{NaCl}$ and $25.0 \% \mathrm{NaNO}_{3}$ (section 9.3.2.1) and for $\mathrm{Ti} 6 / 4$ machining in $25.0 \% \mathrm{NaNO}_{3}$ (section 10.3.1.1). It is satisfying that the approach transient gradient is consistent with an ohmic resistance of $0.12 \pm 0.01 \Omega \mathrm{cm}^{-2}$, the same 
value as that observed for all previous $\mathrm{Ti} 6 / 4$ and $\operatorname{In} 718$ experiments. However, as seen when machining In718 in $5.0 \% \mathrm{NaNO}_{3}$ (section 9.3.2.2), Figure 10.12 shows the retract and re-approach transients trending to smaller $\mathrm{V}_{0}$ values than those seen for $15.0 \% \mathrm{NaCl}$, with a small, but significant residual re-approach curvature towards smaller $\mathrm{V}_{0}$ values.

\subsubsection{Flow effects}

Ti $6 / 4$ was machined in $15.0 \% \mathrm{NaCl}$ and $21.7 \% \mathrm{NaNO}_{3}$ at a flow rate of $\mathrm{U}=20.0 \mathrm{~L} \mathrm{~min}^{-1}$ and with applied voltages of $\mathrm{V}=15.0 \mathrm{~V}$ and $\mathrm{V}=20.0 \mathrm{~V}$ respectively to investigate any flow effects on the $J-V_{0}$ characteristics (section 9.3.3). During these experiments the equilibrium interelectrode gap was found to be $\mathrm{z}_{\infty}=0.58 \pm 0.03 \mathrm{~mm}$ and $\mathrm{z}_{\infty}=0.60 \pm 0.03 \mathrm{~mm}$ for $\mathrm{NaCl}$ and $\mathrm{NaNO}_{3}$ respectively, consistent with those measured for these electrolytes at $\mathrm{U}=16.0 \mathrm{~L}$ $\min ^{-1}$, to within experimental error (section 10.2). Figure 10.13 and Figure 10.14 show the time-resolved dissolution valency measurements made at $\mathrm{U}=20.0 \mathrm{~L} \mathrm{~min}^{-1}$ for $15.0 \% \mathrm{NaCl}$ and $21.7 \% \mathrm{NaNO}_{3}$ respectively.

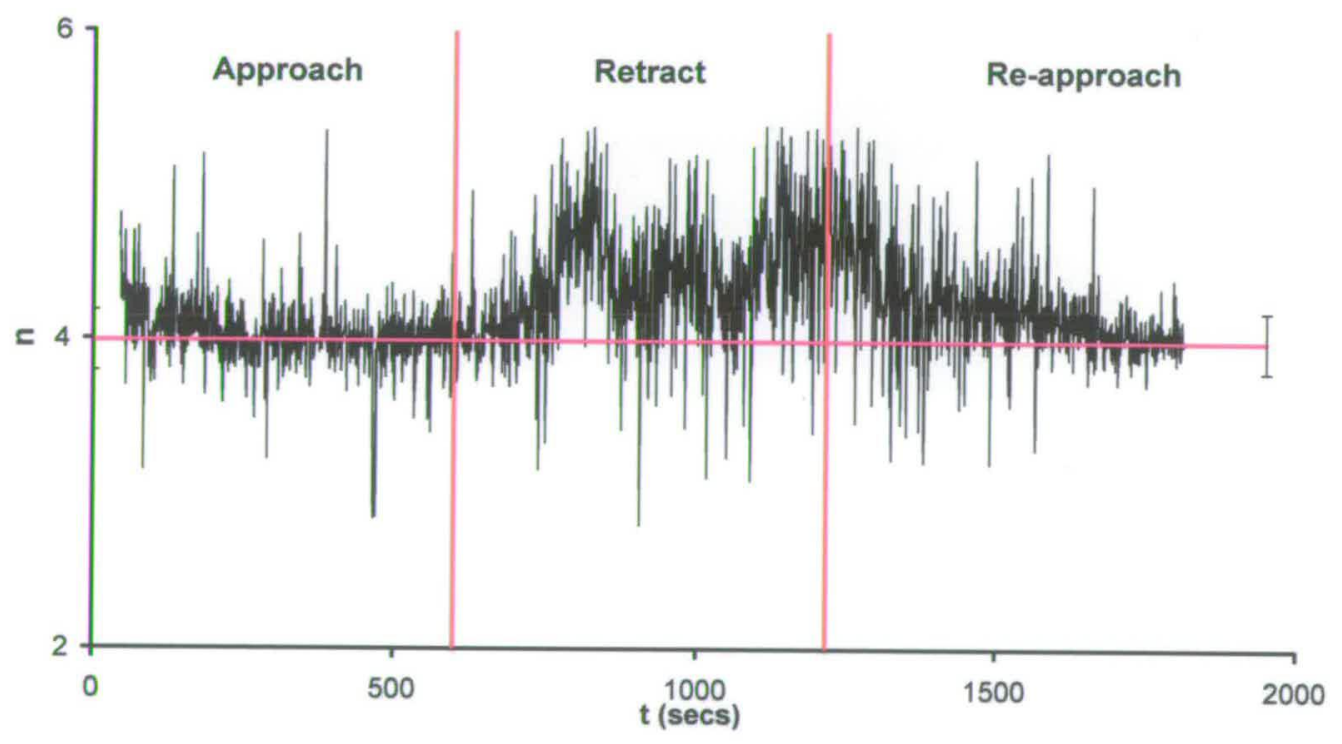

Figure 10.13: Experimental time-resolved n, with $\mathrm{p}=100$ from Equation 6.4, for Ti 6/4 machined in $15.0 \% \mathrm{NaCl}$ with an electrolyte flow rate of $\mathrm{U}=20.0 \mathrm{~L} \mathrm{~min}^{-1}$ and an applied voltage of $\mathrm{V}=15.0 \mathrm{~V}$, the pink line depicts the average dissolution valency at equilibrium with an error shown. 


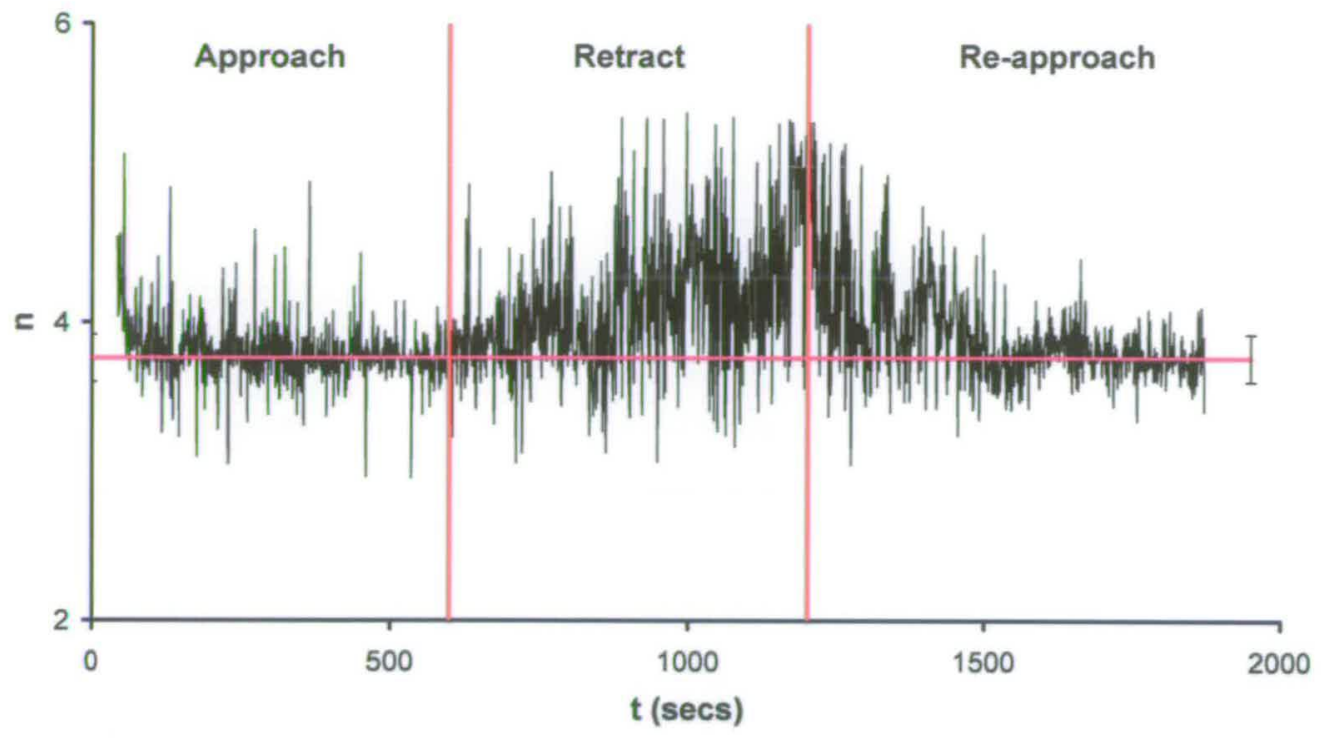

Figure 10.14: Experimental time-resolved n, with $p=100$ from Equation 6.4, for Ti 6/4 machined in $21.7 \% \mathrm{NaNO}_{3}$ with an electrolyte flow rate of $\mathrm{U}=20.0 \mathrm{~L} \mathrm{~min}^{-1}$ and an applied voltage of $\mathrm{V}=20.0 \mathrm{~V}$, the pink line depicts the average dissolution valency at equilibrium with an error shown.

These data show the equilibrium dissolution valency to be $n=4.0 \pm 0.2$ increasing to $n=4.8$ \pm 0.3 for $\mathrm{NaCl}$ and $\mathrm{n}=3.8 \pm 0.2$ increasing to $\mathrm{n}=4.6 \pm 0.3$ for $\mathrm{NaNO}_{3}$, consistent, to within experimental error, with measurements made at $\mathrm{U}=16.0 \mathrm{~L} \mathrm{~min}^{-1}$ (Figure 10.4 and Figure 10.5). Figure 10.15 and Figure 10.16 show comparisons of the $\mathrm{J}-\mathrm{V}_{0}$ characteristics for $\mathrm{Ti} 6 / 4$ machined at electrolyte flow rates of $U=16.0 \mathrm{~L} \mathrm{~min}^{-1}$ (Figure 10.3) and $U=20.0 \mathrm{~L} \mathrm{~min}^{-1}$ in $15.0 \% \mathrm{NaCl}$ and $21.7 \% \mathrm{NaNO}_{3}$ respectively. 


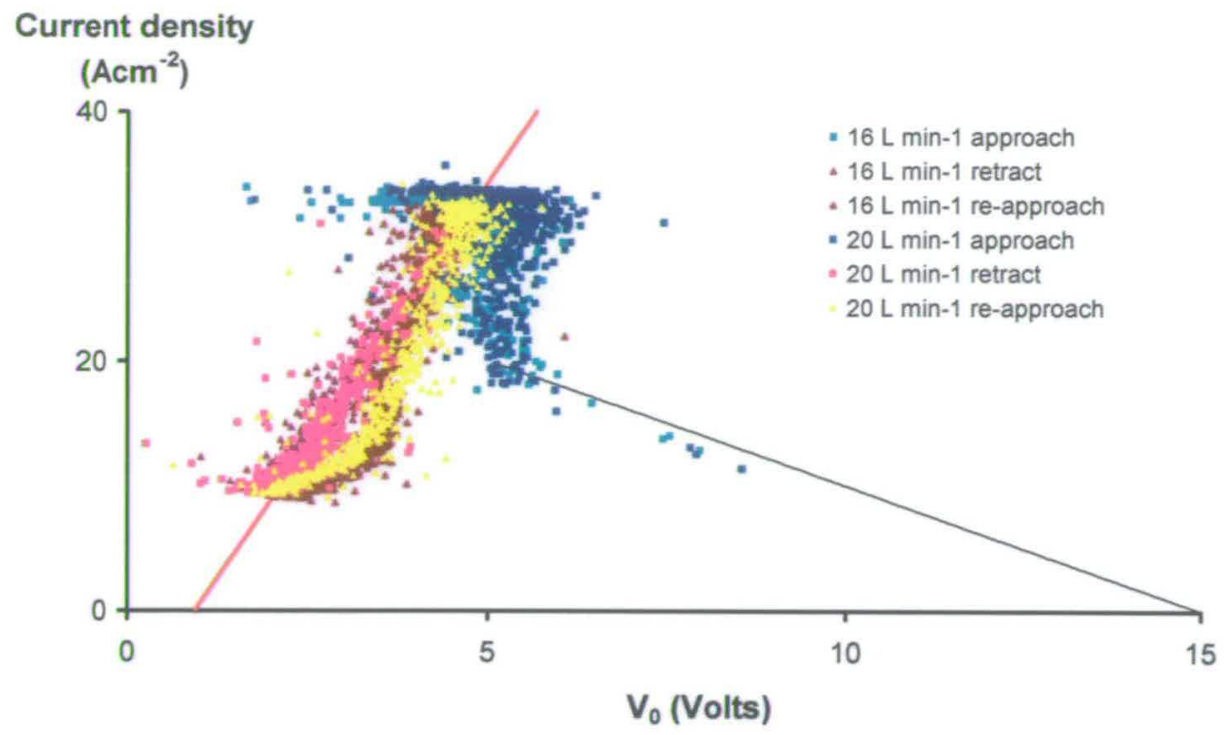

Figure 10.15: Comparison of the $\mathrm{J}-\mathrm{V}_{0}$ characteristics for Ti $6 / 4$ machined in $15.0 \% \mathrm{NaCl}$ with $\mathrm{V}=15.0 \mathrm{~V}$ and $\mathrm{U}=16.0 \mathrm{~L} \mathrm{~min}^{-1}$ and $20.0 \mathrm{~L} \mathrm{~min}^{-1}$. Also shown is the theoretical line calculated from Equation 7.2, with a conductivity of $\kappa=0.2 \mathrm{~S} \mathrm{~cm}^{-1}$. The linear least squares fit of the retract $\mathrm{J}-\mathrm{V}_{0}$ data with an ohmic resistance of $0.12 \pm 0.01 \Omega \mathrm{cm}^{-2}$ and $\mathrm{V}_{0}$ axis intercepts of $0.9 \pm 0.2 \mathrm{~V}$ is shown. 


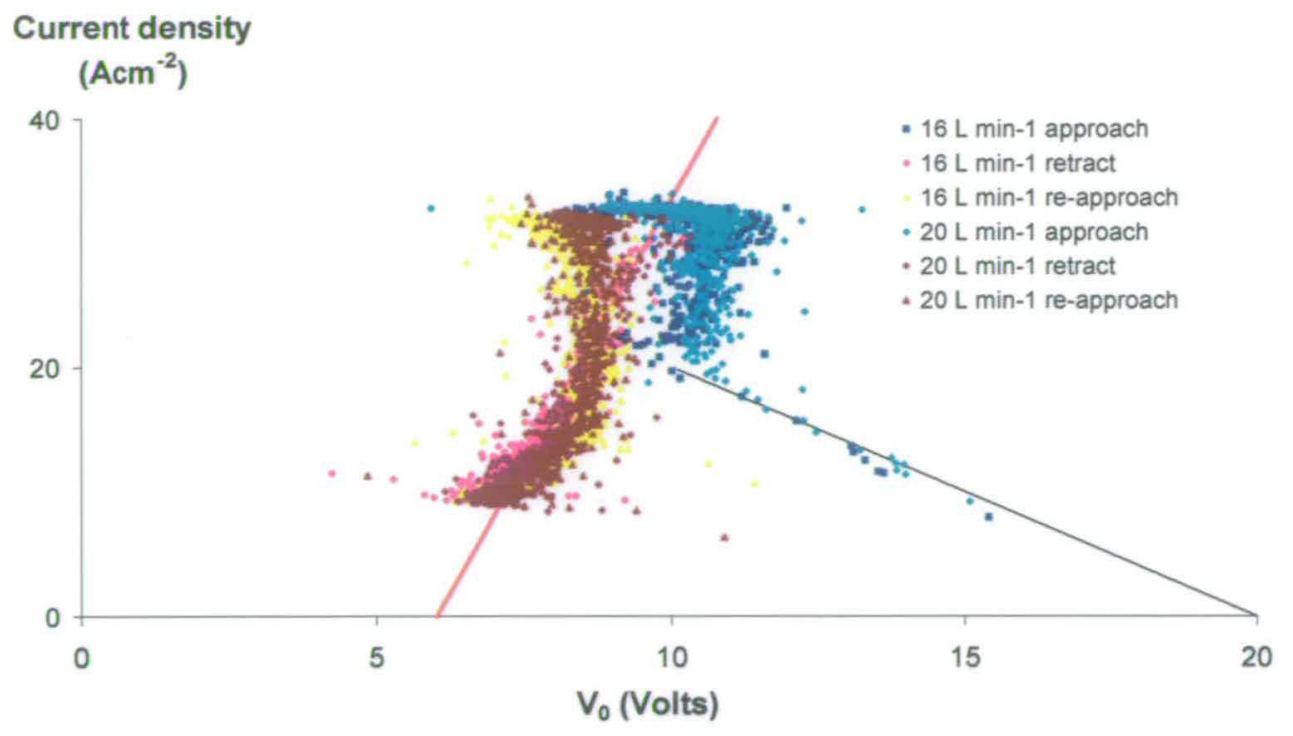

Figure 10.16: Comparison of the $\mathrm{J}-\mathrm{V}_{0}$ characteristics for Ti $6 / 4$ machined in $21.7 \% \mathrm{NaNO}_{3}$ with $\mathrm{V}=20.0 \mathrm{~V}$ and $\mathrm{U}=16.0 \mathrm{~L} \mathrm{~min}-1$ and $20.0 \mathrm{~L} \mathrm{~min}^{-1}$. Also shown is the theoretical line calculated from Equation 7.2, with a conductivity of $\kappa=0.2 \mathrm{~S} \mathrm{~cm}^{-1}$. The linear least squares

fit of the retract $J-V_{0}$ data with an ohmic resistance of $0.12 \pm 0.01 \Omega \mathrm{cm}^{-2}$ and $\mathrm{V}_{0}$ axis intercepts of $6.0 \pm 0.2 \mathrm{~V}$ is shown.

These data show flow velocity changes to have no appreciable effect on either the dissolution valency or the overall $\mathrm{J}-\mathrm{V}_{0}$ characteristics of $\mathrm{Ti} 6 / 4$ in the flow velocity range investigated. As with In718, no trends in the segmented I-t characteristics with the currents at early times increasing or decreasing from segments 1 to 7 with increasing or decreasing $\mathrm{k}$ respectively or taper in the final interelectrode gap were observed showing that there are no significant changes in $\kappa$ along the flow path and implying independence of the dissolution behaviour on flow velocity at the values investigated.

\subsection{Conclusions}

The data in this chapter show the ECM characteristics of Ti $6 / 4$ to have both similarities and differences to the $\mathrm{J}-\mathrm{V}_{0}$ characteristics observed for $\mathrm{Fe}$, low chromium stainless steels and In718 (Chapter 7, Chapter 8 and Chapter 9 respectively). As seen for all previous materials studied, the ECM dissolution valency for $\mathrm{Ti} 6 / 4$ has been shown to be independent of interelectrode gap, electrolyte type and concentration, electrolyte flow velocity and applied 
voltage. A low current density increase in observed dissolution valency has also been observed for $\mathrm{Ti} 6 / 4$ under all the experimental conditions investigated in this chapter, consistent with an increase in dissolution valency or an alternative electro-oxidation reaction resulting in a low current density inefficiency in the dissolution process. As with $\mathrm{Fe}$ (section 7.2.1) and In718 (section 9.2) the low current density J- $\mathrm{V}_{0}$ data are not observed to trend to lower $\mathrm{V}_{0}$ values, unlike SS410 and Jethete steels (section 8.5).

As with both $\mathrm{Fe}$, stainless steels and $\mathrm{In} 718$, the $\mathrm{J}-\mathrm{V}_{0}$ characteristics for $\mathrm{Ti} 6 / 4$ show an initial linear decrease of $\mathrm{V}_{0}$ with increasing $\mathrm{J}$, consistent with the initial breakdown of a passive layer, which corresponds closely to the theoretical gradient and intercept from Equation 7.2. This initial linear decrease of $\mathrm{V}_{0}$ with increasing $\mathrm{J}$ is followed by positive, essentially linear, $\mathrm{J}-\mathrm{V}_{0}$ data, as for all previous materials studied in this thesis. For Ti $6 / 4$ in both electrolytes (at concentrations greater than $5.0 \%$ ) this positive, essentially linear $\mathrm{J}-\mathrm{V}_{0}$ data was found, during the retract phase, to have an ohmic surface resistance of $0.12 \pm 0.01 \Omega \mathrm{cm}^{-2}$. The similar surface ohmic resistances observed for the In 718 data presented in Chapter 9 and the Ti 6/4 data presented in this chapter imply similar surface dissolution processes for both workpiece materials at different interelectrode gaps, electrolyte type and concentration, electrolyte flow velocity and applied voltage which greatly simplifies ECM parameterisation. The $\mathrm{V}_{0}$ axis intercepts of the $J-\mathrm{V}_{0}$ retract data at $\mathrm{J}=0.0 \mathrm{~A} \mathrm{~cm}^{-2}$ were found to be $0.9 \pm 0.2 \mathrm{~V}$ for $\mathrm{NaCl}$ and $6.0 \pm 0.2 \mathrm{~V}$ for $\mathrm{NaNO}_{3}$. These potentials are consistent with the previously attributed active dissolution mechanism when machining in $\mathrm{NaCl}$ and a passive dissolution mechanism when machining in $\mathrm{NaNO}_{3}$.

Large temperature increases were observed in the $\mathrm{J}-\mathrm{V}_{0}$ characteristics when machining Ti $6 / 4$ in $5.0 \% \mathrm{NaCl}$ which have been attributed to appreciable Joule heating at the high applied potential, and relatively low electrolyte conductivity. These temperature increases have previously been shown to change the electrolyte conductivity resulting in large errors in the $\mathrm{J}-\mathrm{V}_{0}$ calculations if a constant $\mathrm{\kappa}$ is assumed. A significant reduction in the Joule heating effect was observed when $\mathrm{Ti} 6 / 4$ was machined in $5.0 \% \mathrm{NaCl}$ at a lower applied voltage, however, some high temperature characteristics were still observed in the retract and reapproach phases in this $\mathrm{J}-\mathrm{V}_{0}$ data.

The data in Chapter 7, Chapter 8, Chapter 9 and this chapter have provided an insight into the $\mathrm{J}-\mathrm{V}_{0}$ characteristics of Fe, SS410, J, In718 and Ti 6/4. The similarities between the ECM characteristics for different materials, interelectrode gap, electrolyte type and concentration, 
electrolyte flow velocity and applied voltage, allow a great simplification of the ECM parameter base. This will be shown in Chapter 12 . 


\section{Chapter 11}

Comparison of $\mathbf{n}$ and $\mathrm{J}-\mathrm{V}_{\mathbf{0}}$ data for different workpiece materials in cell 2000 


\subsection{ECM parameter measurements}

The essential parameters for the data presented for Fe (Chapter 7), SS410 and Jethete (Chapter 8), In718 (Chapter 9) and Ti6/4 (Chapter 10) have been summarised in Table 11.1.

\begin{tabular}{|c|c|c|c|c|c|c|c|c|}
\hline & $\begin{array}{c}\text { Electrolyte } \\
\text { type }\end{array}$ & Conc $^{n} / \%$ & V / Volts & $\mathrm{U} / \mathrm{L} \min ^{-1}$ & $\begin{array}{c}\mathrm{z}_{\infty} \pm 0.03 / \\
\mathrm{mm}\end{array}$ & $\mathrm{n}_{\infty}$ & \begin{tabular}{|c|} 
Ohmic \\
resistance \pm \\
$0.01 / \Omega \mathrm{cm}$ \\
2
\end{tabular} & $\begin{array}{c}\mathrm{V}_{0} \text { at } \mathrm{J}= \\
0.0 \mathrm{~A} \mathrm{~cm}^{-2} \\
\mathrm{~V}\end{array}$ \\
\hline $\mathrm{Fe}$ & \multirow{3}{*}{$\mathrm{NaCl}$} & 15.0 & 8.0 & 16.0 & 0.18 & $2.1 \pm 0.1$ & \multirow{3}{*}{0.16} & \multirow{3}{*}{$0.0 \pm 0.1$} \\
\hline $\mathrm{SS} 410$ & & 12.0 & 10.0 & 16.0 & 0.56 & $2.1 \pm 0.2$ & & \\
\hline $\mathrm{J}$ & & 12.0 & 15.0 & 16.0 & 0.76 & $2.1 \pm 0.2$ & & \\
\hline \multirow{8}{*}{$\ln 718$} & \multirow{5}{*}{$\mathrm{NaCl}$} & 15.0 & 13.0 & 16.0 & 0.43 & $3.3 \pm 0.1$ & \multirow{7}{*}{0.13} & \multirow{5}{*}{$0.5 \pm 0.2$} \\
\hline & & 15.0 & 11.0 & 16.0 & 0.22 & $3.3 \pm 0.2$ & & \\
\hline & & 15.0 & 13.0 & 20.0 & 0.43 & $3.3 \pm 0.2$ & & \\
\hline & & 25.0 & 13.0 & 16.0 & 0.64 & $3.1 \pm 0.1$ & & \\
\hline & & 5.0 & 45.0 & 16.0 & 0.72 & $3.5 \pm 0.2$ & & \\
\hline & \multirow{3}{*}{$\mathrm{NaNO}_{3}$} & 21.7 & 16.0 & 16.0 & 0.38 & $3.3 \pm 0.1$ & & \multirow{2}{*}{$2.9 \pm 0.2$} \\
\hline & & 25.0 & 19.0 & 16.0 & 0.64 & $3.3 \pm 0.1$ & & \\
\hline & & 21.7 & 16.0 & 20.0 & 0.38 & $3.3 \pm 0.2$ & 0.11 & $2.4 \pm 0.2$ \\
\hline \multirow{8}{*}{ Ti6/4 } & \multirow{5}{*}{$\mathrm{NaCl}$} & 15.0 & 15.0 & 16.0 & 0.63 & $4.0 \pm 0.2$ & \multirow{8}{*}{0.12} & \multirow{5}{*}{$0.9 \pm 0.2$} \\
\hline & & 15.0 & 15.0 & 20.0 & 0.58 & $4.0 \pm 0.2$ & & \\
\hline & & 25.0 & 15.0 & 16.0 & 0.71 & $4.0 \pm 0.3$ & & \\
\hline & & 5.0 & 34.0 & 16.0 & 0.67 & $4.0 \pm 0.3$ & & \\
\hline & & 5.0 & 19.0 & 16.0 & 0.38 & $4.0 \pm 0.2$ & & \\
\hline & \multirow{3}{*}{$\mathrm{NaNO}_{3}$} & 21.7 & 20.0 & 16.0 & 0.60 & $3.7 \pm 0.2$ & & \multirow{3}{*}{$6.0 \pm 0.2$} \\
\hline & & 21.7 & 20.0 & 20.0 & 0.60 & $3.8 \pm 0.2$ & & \\
\hline & & 25.0 & 23.0 & 16.0 & 0.85 & $3.7 \pm 0.2$ & & \\
\hline
\end{tabular}

Table 11.1: Table summarising parameter measurements taken for Fe (Chapter 7), SS410 and Jethete (Chapter 8), In718 (Chapter 9) and Ti6/4 (Chapter 10) collected using cell 2000.

\subsubsection{Dissolution valency measurements}

Table 11.1 shows the equilibrium dissolution valencies for $\mathrm{Fe}$ and the stainless steels in $\mathrm{NaCl}$ have all been found to be $\mathrm{n}=2.1 \pm 0.2$, consistent with low valency dissolution of $\mathrm{Fe}$ as $\mathrm{Fe}$ (II) and, in SS410 and Jethete, $\mathrm{Cr}$ as $\mathrm{Cr}$ (III) (Chapter 7 and Chapter 8). When machining In718 under all the conditions studied in this thesis, the equilibrium dissolution valency was found to be within the range $\mathrm{n}=3.3 \pm 0.2$, consistent with $\mathrm{Ni}(\mathrm{III}), \mathrm{Fe}$ (III) and $\mathrm{Cr}$ (III) dissolution (Chapter 9). Ti6/4 showed dissolution valencies consistent with $n=4.0 \pm 0.3$ 
and $3.8 \pm 0.3$ for $\mathrm{NaCl}$ and $\mathrm{NaNO}_{3}$ respectively consistent with either complete $\mathrm{Ti}(\mathrm{IV}$ ) dissolution or some mixed valency $\mathrm{Ti}(\mathrm{III}) / \mathrm{Ti}(\mathrm{IV})$ dissolution (although it should be noted that the all dissolution valencies collected for Ti6/4 are the same to within experimental error) (Chapter 10). These data show that under the conditions investigated, applicable to industrial ECM, the equilibrium dissolution valency is independent of the applied voltage, electrolyte type and concentration and electrolyte flow velocity. In all the studies in these chapters, an increase in observed dissolution valency was found at low current densities (corresponding to low $\mathrm{V}_{0}$ values). This increase was found to be independent of electrolyte type and concentration, applied voltage, electrolyte flow rate and workpiece material and is consistent with an increase in dissolution valency or a decrease in dissolution current efficiency. Low voltage valency increases are unlikely in single component systems; however they may be observed through preferential low current density dissolution of a higher valency alloy constituent (as postulated for $\mathrm{Cr}(\mathrm{III})$ in stainless steels in Chapter 8). However, although this preferential dissolution may occur for stainless steels, it cannot explain the high valency measurements at low current density observed for single elements such as Fe and is unlikely to explain these effects for all the materials studied in this work. Consequently, a low current density inefficiency is also consistent with a competing electrooxidation reaction which does not result in metal ion dissolution. A possible low current density inefficiency electro-oxidation reaction is the evolution of oxygen at the workpiece; this has previously been postulated [58], and has been suggested that this reaction could be suppressed at high current density through increased dehydration of the electrolyte in the vicinity of the anode surface, due to formation of a viscous saturated surface layer.

\subsubsection{J-V $\mathrm{V}_{0}$ characteristics}

\subsubsection{1. $V_{0}$ axis intercept at $J=0.0 \mathrm{~A} \mathrm{~cm}^{-2}$}

Table 11.1 shows the $\mathrm{V}_{0}$ axis intercepts (at $\mathrm{J}=0.0 \mathrm{~A} \mathrm{~cm}^{-2}$ ) for the positive, essentially linear, $\mathrm{J}-\mathrm{V}_{0}$ data for all the workpiece materials studied in $\mathrm{NaCl}$ to be near $0 \mathrm{~V}$. It has been argued (section 7.2.2) that these results can be compared to similar results previously collected against a standard or normal hydrogen electrode (SHE or NHE). If this is the case, these previous results $[136,153]$ show similar intercepts, which have been attributed to an active dissolution mechanism, whereby metal dissolution takes place without the effects of a surface oxide film. This correspondence of the tool potential at $\mathrm{J}=0.0 \mathrm{~A} \mathrm{~cm}^{-2}$, to the NHE 
or SHE potential is most likely due to the same electrochemical reaction occurring (Reaction 7.1), and that in each case the changes in potential due to changes in metal ( $\mathrm{Pt}$ to copper chrome) and $\mathrm{H}^{+}$activity are small compared to the overall value of $\mathrm{V}_{0}$. Table 11.1 also shows $\mathrm{V}_{0}$ axis intercepts of appreciably more than $0 \mathrm{~V}$ for In718 and Ti6/4 in $\mathrm{NaNO}_{3}$. Similar positive potentials at $\mathrm{J}=0.0 \mathrm{~A} \mathrm{~cm}^{-2}$ w.r.t. SHE and NHE have previously been found $[167,170]$, and have been attributed to passive dissolution processes in nickel and titanium, where dissolution through a surface oxide layer is assumed.

\subsubsection{Initial linear decrease of J with $V_{0}$}

It is interesting that when $\mathrm{NaCl}$ is used, initially there are a relatively small number of data points, beginning at relatively high $\mathrm{J}$ and relatively low $\mathrm{V}_{0}$ values, present on the theoretical transient predicted by Equation 7.2 (Figure 9.3 and Figure 10.3). In contrast, for $\mathrm{NaNO}_{3}$, a relatively large number of data points, beginning at relatively high $\mathrm{V}_{0}$ values and relatively low $\mathrm{J}$ are present. The initial value of $\mathrm{V}_{0}$ is an indication of how much of the applied voltage is initially dropped at the workpiece surface. Therefore, these results support the presence of a thicker oxide film initially in $\mathrm{NaNO}_{3}$ compared to $\mathrm{NaCl}$. The fall in $\mathrm{V}_{0}$ and increase in $\mathrm{J}$ is consistent with voltage driven film breakdown to enable ECM. Together, this is consistent with a voltage driven initial breakdown of a relatively thin passive film in $\mathrm{NaCl}$ to give active dissolution, which and can be attributed to the oxide thinning properties of chloride ions [171]. In contrast, the data for $\mathrm{NaNO}_{3}$ are consistent with voltage driven initial breakdown of a relatively thick passive oxide film, to give a thinner oxide film which enables passive ECM dissolution.

\subsubsection{Positive ohmic J-V $V_{0}$ slope}

Table 11.1 shows that, subsequent to onset of ECM machining conditions, positive ohmic J$\mathrm{V}_{0}$ relationships are seen; the slopes of these lines appear to be independent of electrolyte concentration, applied voltage and interelectrode gap. The analysis carried out in this work (Chapter 3) calculates $\mathrm{V}_{0}$ through subtraction of the potential required for bulk ion migration in the electrolyte from the overall applied potential (Equation 3.1). Consequently $\mathrm{V}_{0}$ contains all other voltages required to drive the electrochemical processes at the tool and the workpiece. It is therefore clear that the rate determining process(es) at these surfaces appear ohmic in nature. These ohmic $\mathrm{J}-\mathrm{V}_{0}$ characteristics eliminate the possibility of Tafel rate 
determining steps at the electrode surfaces, which would be expected for a simple electrochemically controlled dissolution process at the workpiece or hydrogen evolution at the tool (Chapter 2). Consequently, there are three possible sources of this ohmic resistance. The first is the ohmic resistance of the passive film at the workpiece surface. However, although large variation in $\mathrm{V}_{0}$ at $\mathrm{J}=0.0 \mathrm{Acm}^{-2}$ has been seen for $\mathrm{NaCl}$ and $\mathrm{NaNO}_{3}$, and previously attributed to active and passive dissolution processes (the absence and presence of a surface oxide respectively, section 11.1.2.1), it is interesting that the same ohmic resistance is observed, within experimental error, in each case. This implies that dissolution through a passive surface oxide is not the origin of the ohmic dependence of the $\mathrm{J}-\mathrm{V}_{0}$ data. The second possible explanation of the ohmic $\mathrm{J}-\mathrm{V}_{0}$ characteristics is $\mathrm{H}_{2}$ formation at the tool. This has been previously postulated to lead to gas bubble formation and an excluded volume near the tool surface [80]. This effect may be expected to lead to a reduction in electrolyte conductivity near the tool surface which could result in an ohmic resistance near the tool. However, $\mathrm{H}_{2}$ bubble formation would be expected to be grossly affected by cell pressure and electrolyte flow rate, both of which change during the course of an approach/retract/reapproach experiment. Consequently, in this work, changes in the ohmic resistance would be expected during machining, which is inconsistent with the constant ohmic resistances measured in Figure 9.3 and Figure 10.3. Furthermore, the excluded volume due to $\mathrm{H}_{2}$ bubble formation would be expected to increase along the flow path length resulting in differences in electrolyte conductivity, segment current and gap, which are also not observed. As a result, $\mathrm{H}_{2}$ bubble formation is also an unlikely explanation of the ohmic $\mathrm{J}-\mathrm{V}_{0}$ dependence observed in this work. Therefore, the most likely explanation of this ohmic dependence is the formation of a surface salt film on the anode during machining. This explanation is consistent with a number of previous studies carried out which report the presence of surface salt films during high current density dissolution [58,71, 136]. Landolt et al. [58] postulate two different mass transport mechanisms in anodic metal dissolution; dissolution product limited transport (denoted the salt film mechanism) and acceptor limited transport (denoted the acceptor mechanism). In the salt film mechanism, the rate of transport of dissolving metal ions from the anode surface into the bulk electrolyte through a surface salt film is the rate determining step. Landolt et al. suggest that at the limiting current in this mechanism, a thin salt film is present on the anode surface and the concentration of the dissolving metal ions at the salt film/electrolyte interface corresponds to the saturation concentration of the metal-electrolyte anion salt. In the acceptor mechanism the rate determining step is diffusion of an acceptor species (the electrolyte anions or water) to the anode to complex with the dissolving metal. At the limiting current in this mechanism, the concentration of the 
acceptor species at the workpiece surface is zero. Previous observations by Kuo and Landolt [172] on the convective mass transfer processes during anodic dissolution of iron in $5 \mathrm{M}$ $\mathrm{NaCl}$ using a $\mathrm{RDE}$, found mass transport limited currents determined by the rate of convective diffusion of $\mathrm{Fe}^{2+}$ away from the anode. At the limiting current the concentration of $\mathrm{Fe}^{2+}$ at the anode surface was found to correspond to the experimentally determined saturation concentration, $\mathrm{c}_{\mathrm{s}}$, of $\mathrm{FeCl}_{2}$ under these conditions $\left(\mathrm{c}_{\mathrm{s}}=2.16 \mathrm{~mol} \mathrm{dm}^{-3}\right)$ suggesting the presence of a surface salt film. A subsequent channel cell study by Datta and Landolt [136], showed consistent saturation concentrations $\left(c_{\mathrm{s}}=2.1 \mathrm{~mol} \mathrm{dm}^{-3}\right)$ at the anode surface for both iron and nickel in $5 \mathrm{M} \mathrm{NaCl}$, supporting the existence of a similar surface salt film in a channel cell system as seen in the RDE system. In this study, Datta and Landolt also showed the limiting current density, $\mathrm{J}_{\mathrm{L}}$, corresponding to the formation of the surface salt film, is given by the expected equation:

$$
J_{L}=\frac{n F D c_{s}}{\delta_{d t}}
$$

\section{Equation 11.1}

for dissolution of $\mathrm{M}^{\mathrm{n}+}$ away from the salt film across the diffusion layer, of thickness $\delta_{\mathrm{dt}}$, into the bulk solution, where $c_{M}{ }^{n+}=0$, where $\mathrm{n}$ is the dissolution valency, $\mathrm{F}$ is Faraday's constant, $A$ is the electrode area and $D$ is the diffusion coefficient of $M^{n+}$. Therefore $\mathrm{J}_{\mathrm{L}}$ is inversely proportional to the diffusion layer thickness, $\delta_{\mathrm{dt}}$, for iron and nickel (Figure 11.1). 


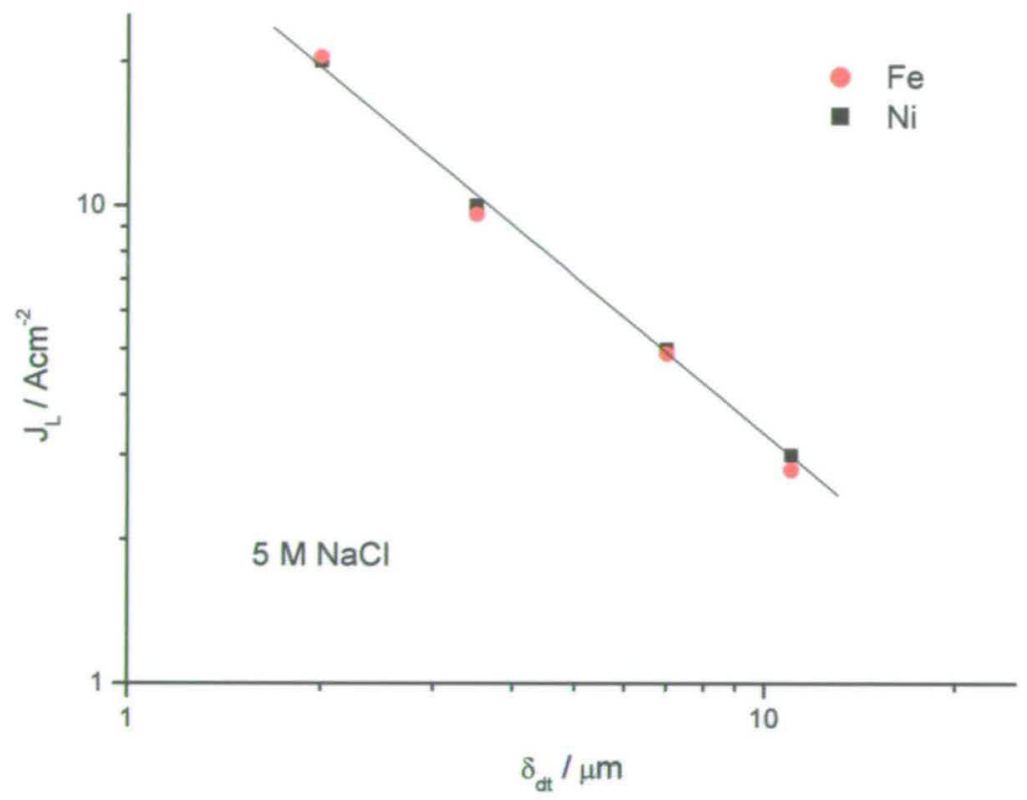

Figure 11.1: Limiting current density, $\mathrm{J}_{\mathrm{L}}$, plotted as a function of diffusion layer thickness, $\delta_{\mathrm{dt}}$, for high rate anodic dissolution of iron and nickel in $5 \mathrm{M} \mathrm{NaCl}(30 \% \mathrm{w} / \mathrm{w})$ in a flow channel cell [136]. The theoretical line is that expected for each metal from Equation 11.1.

The diffusion layer thickness under turbulent conditions in a channel cell is given by [146]:

$$
\delta_{d t}=\frac{50 b}{\mathrm{R}_{\mathrm{n}}^{7 / 8} \mathrm{P}_{\mathrm{r}}^{1 / 3}}
$$

\section{Equation 11.2}

Where $\mathrm{b}$ is half the height of the channel cell (for a typical initial interelectrode gap of $\mathrm{z}_{0}=$ $1.0 \mathrm{~mm}, \mathrm{~b}=5 \times 10^{-4} \mathrm{~m}$, see Figure 2.9), $R_{n}$ is the Reynolds number (calculated as $R_{n}=8900$ for an interelectrode gap of $\mathrm{z}_{0}=1.0 \mathrm{~mm}$, see section 2.3.2) and $\mathrm{P}_{\mathrm{r}}$ is the Prandtl number, found as the ratio of the kinematic viscosity, $v$, to the thermal diffusivity, $D_{T}$, of the electrolyte (Equation 11.3). 


$$
\mathrm{P}_{\mathrm{r}}=\frac{v}{\mathrm{D}_{\mathrm{T}}}
$$

\section{Equation 11.3}

At $298 \mathrm{~K}$, the kinematic viscosity of $5 \mathrm{M} \mathrm{NaCl}$ is $v=1.6 \times 10^{-6} \mathrm{~m}^{2} \mathrm{~s}^{-1}[136]$ and the thermal diffusivity of water $\mathrm{D}_{\mathrm{T}}=0.14 \times 10^{-6} \mathrm{~m}^{2} \mathrm{~s}^{-1}$ (the value for $5 \mathrm{M} \mathrm{NaCl}$ was not available) [173], from these values and Equation 11.3, the Prandtl number has been calculated to be $\mathrm{P}_{\mathrm{r}}=11.4$. Therefore, for this work, a diffusion layer thickness can be calculated, from Equation 11.2, of the order of $3.9 \mu \mathrm{m}$. From Equation 11.1, this corresponds to a limiting current density for salt layer formation of $\mathrm{J}_{\mathrm{L}}=9.2 \mathrm{~A} \mathrm{~cm}^{-2}$ for both nickel and iron in $5 \mathrm{M} \mathrm{NaCl}$ (Figure 11.1). This limiting value is only an estimation as the thermal diffusivity of pure water was used in Equation 11.2, and the value was calculated for an electrolyte concentration of $5 \mathrm{M}$ (compared to the $2.56 \mathrm{M} \mathrm{NaCl}$ solutions used in this work). However, despite this, the calculated value is of the same order as the lowest ECM current densities obtained during the ohmic $\mathrm{J}-\mathrm{V}_{0}$ behaviour for iron, iron based steels and the nickel based alloy In718 (Chapter 7, Chapter 8 and Chapter 9), implying the presence of a surface salt film for all the ohmic J-V data. It is interesting that this value for the limiting current density for the formation of a surface salt film is of the order of the current densities below which inefficiencies are noted in the dissolution valency measurements. It is therefore possible that these inefficiencies are connected to the incomplete formation of a surface salt film, e.g. increased hydration of the anode surface leading to oxygen evolution. It should be noted that the diffusion layer thickness will decrease linearly with the decreasing interelectrode gap during the approach phase (Equation 11.2). This will lead to an increase in the limiting current density for surface salt film formation with decreasing interelectrode gap. However, the observed current density, J, also increases. Repetition of the diffusion layer thickness calculation at equilibrium machining conditions $\left(\mathrm{z}_{\infty}=0.4 \mathrm{~mm}\right)$, with all other values remaining constant, gives a diffusion layer thickness of the order of $1.7 \mu \mathrm{m}$ (Equation 11.2) and hence a limiting current density for salt layer formation of $\mathrm{J}_{\mathrm{L}}=23.0 \mathrm{~A} \mathrm{~cm}^{-2}$ for both nickel and iron in $5 \mathrm{M}$ $\mathrm{NaCl}$ (Figure 11.1), which is lower than the equilibrium machining current densities measured for these materials. Therefore, it is reasonable to assume the presence of a surface salt film when machining iron, iron based steels and In718 under all the conditions used in this work. This is sensible as the overall current density is controlled by Equation 11.4. 


\section{Equation 11.4}

Given that $\mathrm{V} \gg \mathrm{V}_{0}$ and $\left(\mathrm{V}-\mathrm{V}_{0}\right)$ is therefore approximately constant, $\mathrm{J} \propto 1 / \mathrm{z}$. Consequently, since $\delta_{\mathrm{dt}} \propto \mathrm{z}$ (Equation 11.2), both $\mathrm{J}_{\mathrm{L}}$ (Equation 11.1) and $\mathrm{J}$ (Equation 11.4) will be proportional to $1 / z$ and $J$ will remain greater than $J_{L}$ at all times. Furthermore, the $J-V_{0}$ characteristics have been shown to be independent of electrolyte concentration, at and above $15.0 \%$ in this work, which is evidence against an acceptor dissolution mechanism. In conclusion, under the conditions used in this work, a salt film dissolution mechanism, as opposed to an acceptor mechanism [58], can be assumed.

It is interesting that in Figure 11.1, both iron and nickel show essentially the same $J_{\mathrm{L}}-\delta_{\mathrm{d} t}$ behaviour. From Equation 11.1, this indicates that the iron and nickel salts have essentially the same solubility. This most likely constituents of the salt films in $\mathrm{NaCl}$ and $\mathrm{NaNO}_{3}$ are $\mathrm{FeCl}_{2}, \mathrm{Fe}\left(\mathrm{NO}_{3}\right)_{2}, \mathrm{NiCl}_{3}$ and $\mathrm{Ni}\left(\mathrm{NO}_{3}\right)_{3}$. Table 11.2 shows the literature solubilities of iron and nickel salts in water.

\begin{tabular}{|c|c|}
\hline Salt & Solubility $/ \mathrm{mol} \mathrm{dm}^{-3}$ \\
\hline $\mathrm{FeCl}_{2}$ & 5.12 \\
\hline $\mathrm{Fe}\left(\mathrm{NO}_{3}\right)_{2}$ & 4.87 \\
\hline $\mathrm{NiCl}_{2}$ & 5.20 \\
\hline $\mathrm{Ni}\left(\mathrm{NO}_{3}\right)_{2}$ & 5.43 \\
\hline $\mathrm{FeCl}_{3}$ & 5.70 \\
\hline $\mathrm{Fe}\left(\mathrm{NO}_{3}\right)_{3}$ & 3.42 \\
\hline
\end{tabular}

Table 11.2: Solubilities of iron and nickel salts [146].

Although these molar solubilities are measured in water, and not in $\mathrm{NaCl}$ solutions, the solubility values are comparable to the saturation concentrations of $\mathrm{FeCl}_{2}$ and $\mathrm{NiCl}_{2}$ measured by Datta et al. in $5 \mathrm{M} \mathrm{NaCl}$ [136]. Table 11.2 also shows similar solubilities for $\mathrm{FeCl}_{2}$ and $\mathrm{Fe}\left(\mathrm{NO}_{3}\right)_{2}$ implying similar limiting currents for salt layer formation for iron in $\mathrm{NaNO}_{3}$ as found in $\mathrm{NaCl}$ (Equation 11.1). Also, interestingly, the solubilities of $\mathrm{FeCl}_{2}$ and 
$\mathrm{FeCl}_{3}$ are observed to be similar, showing little dependence of solubility on valency. Therefore, although $\mathrm{Ni}(\mathrm{III})$ solubilities were not readily available, the $\mathrm{NiCl}_{2}$ solubility is shown, which is also similar to $\mathrm{FeCl}_{2}$, supporting the observed similarity in solution concentration for salt film formation (Figure 11.1). Finally, the solubility of $\mathrm{Ni}\left(\mathrm{NO}_{3}\right)_{2}$, shown in Table 11.2, implies a similar limiting current for salt layer formation for nickel in $\mathrm{NaNO}_{3}$ as found in $\mathrm{NaCl}$ (Equation 11.1 and Figure 11.1).

Next onto the origin of the ohmic $\mathrm{J}-\mathrm{V}_{0}$ resistances observed in ECM. Figure 11.2 shows a schematic of the polarisation curves of titanium in $3 \mathrm{M} \mathrm{H}_{2} \mathrm{SO}_{4}$ at a $\mathrm{RDE}$ [58].

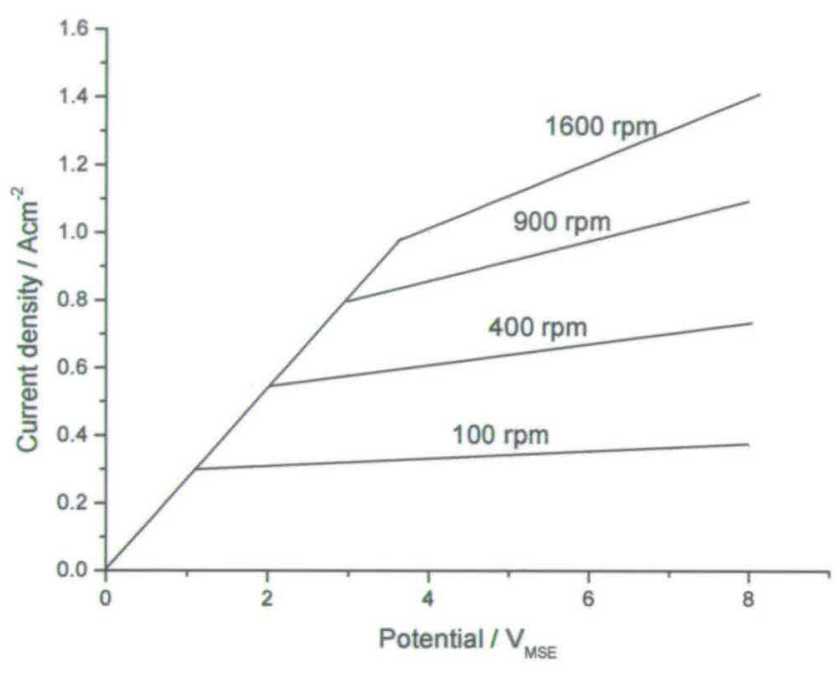

Figure 11.2: A schematic of the polarisation curves of titanium in $3 \mathrm{M} \mathrm{H}_{2} \mathrm{SO}_{4}$ measured using a Rotating disc electrode at different rotation rates [58].

The potential axis intercept at $\mathrm{J}=0.0 \mathrm{Acm}^{-2}$ of $\mathrm{V}=0 \mathrm{~V}$ is consistent with an active dissolution mechanism, which would be expected when machining titanium in an acid solution. This is followed by an ohmic resistance at low current densities, observed to be similar for all the rotation rates investigated, which is attributed to the $\mathrm{iR}$ drop between the reference electrode and the anode due to the electrolyte (this iR drop would not be expected in the results shown in this work since the ECM analysis removes the iR drop associated with ion migration in the electrolyte). At higher current densities, a "plateau" is seen, attributed to limiting current salt film formation [58]. However, this plateau is not completely flat (constant J w.r.t. V), but increases ohmically with a lower gradient. This is 
evidence for a migrational component of transport through the surface salt film, which would enhance the limiting current density. The resistances of the anodic salt films formed at different rotation rates were measured, using the gradients of the high current density ohmic regions in Figure 11.2, and the values are shown in Table 11.3 with the corresponding rotation rates and the diffusion layer thicknesses calculated using Equation 11.5.

$$
\delta_{d t}=0.642 D^{1 / 3} W^{-1 / 2} v^{1 / 6}
$$

\section{Equation 11.5}

Where $\mathrm{D}$ is the diffusion co-efficient of Ti $\left(\mathrm{D}=9 \times 10^{-10} \mathrm{~m}^{2} \mathrm{~s}^{-1}\right), \mathrm{W}$ is the electrode rotation rate in $\mathrm{Hz}$ and $v$ is the kinematic viscosity of $\mathrm{H}_{2} \mathrm{SO}_{4}\left(v=1.3 \times 10^{-6} \mathrm{~m}^{2} \mathrm{~s}^{-1}[174]\right)$.

\begin{tabular}{|c|c|c|}
\hline Rotation rate $/ \mathrm{rpm}$ & $\delta_{\mathrm{dt}} / \mu \mathrm{m}$ & Ohmic resistance $/ \Omega \mathrm{cm}^{-2}$ \\
\hline 100 & 50 & 80 \\
\hline 400 & 25 & 40 \\
\hline 900 & 17 & 16 \\
\hline 1600 & 13 & 13 \\
\hline
\end{tabular}

Table 11.3: Table to show the decreasing ohmic resistance with increasing rotation rate.

These data clearly show the resistance of the surface salt film decreasing with increasing electrode rotation rate, in line with decreasing diffusion layer thicknesses. This implies that the resistance of the surface salt film is controlled by the diffusion layer thickness. These iR characteristics of the high current density region of the polarisation curves in Figure 11.2 are consistent with the ohmic $\mathrm{J}-\mathrm{V}_{0}$ dependence observed in this thesis. The salt layer resistance found for titanium in this work $\left(0.12 \Omega \mathrm{cm}^{-2}\right.$, Table 11.1) is two orders of magnitude smaller than those found using a RDE (Table 11.3). However, the diffusion layer thickness measured at the start of the ohmic $\mathrm{J}-\mathrm{V}_{0}$ region in this work $(4.7 \mu \mathrm{m})$ is only half the value of the diffusion layer thickness at the highest rotation rate in Table 11.3, implying a significantly smaller ratio of the salt film resistance to the diffusion layer thickness in this work compared to the study by Landolt (Figure 11.2). This may be a consequence either of the turbulent flow regime using in this work, or the higher voltages applied to the workpiece surfaces compared to the RDE studies. 
The rate determining step of dissolution, in the presence of a surface salt film, would be expected to be either metal ion transport through the film, from the metal surface to the electrolyte solution, or complexing ion or water transport through the film to the metal surface. Landolt interprets his data as diffusional dissolution of species through the salt film [165]. However, the data shown in Figure 11.2, and the ECM data in this work, are consistent with a significant migrational transport component in these films. This eliminates water diffusion as the most likely rate determining step since water is a neutral species and therefore would not be expected to give such an ohmic $\mathrm{J}-\mathrm{V}_{0}$ dependence. It is interesting that similar ohmic $\mathrm{J}-\mathrm{V}_{0}$ slopes are seen across different workpiece types (Table 11.1), which suggests similar diffusion co-efficients for the diffusing ion species in each case.

It is also interesting that the data shown in Figure 11.2 shows a decrease in the ohmic resistance observed in the plateau region with increasing rotation speed. This is consistent with decreasing salt film thickness with increasing rotation speed, due to the enhanced mass transport of metal ions away from the salt film surface. However, in the ECM data measured in this work, constant ohmic resistances are observed during ECM experiments (Table 11.1) which suggests constant salt film thicknesses. However, it should be remembered that changes in the interelectrode gap during these experiments increase dissolution current (leading to increased salt film thicknesses) and decrease the diffusion layer thickness (leading to decreased salt film thicknesses) by the same magnitude (I $\propto 1 / z$, Equation 11.4, and $\mathrm{z} \propto \delta_{\mathrm{dt}}$, Equation 11.2), which could lead to essentially constant salt film thicknesses.

It is worth pointing out that the analysis thus far has assumed a flat anode surface. It is possible that, under these ECM conditions, eddies and pockets of near stationary electrolyte flow are present adjacent to the rough anode surface which may be expected to have some effects on the surface salt layer formation and thickness. Profilometry, using the Talysurf method, was used to measure a typical surface roughness average for Ti 6/4 machined in $\mathrm{NaCl}$ as $\mathrm{R}_{\mathrm{a}}=0.35 \mu \mathrm{m}$. Since this roughness average comprises approximately of the order of $1 / 10^{\text {th }}$ of the diffusion layer thicknesses calculated for this work, this is unlikely to affect the properties of the diffusion layer significantly. 


\subsection{Conclusions}

In summary, the results presented in this chapter are consistent with the following model for ECM dissolution at the workpiece surface, for all the materials investigated.
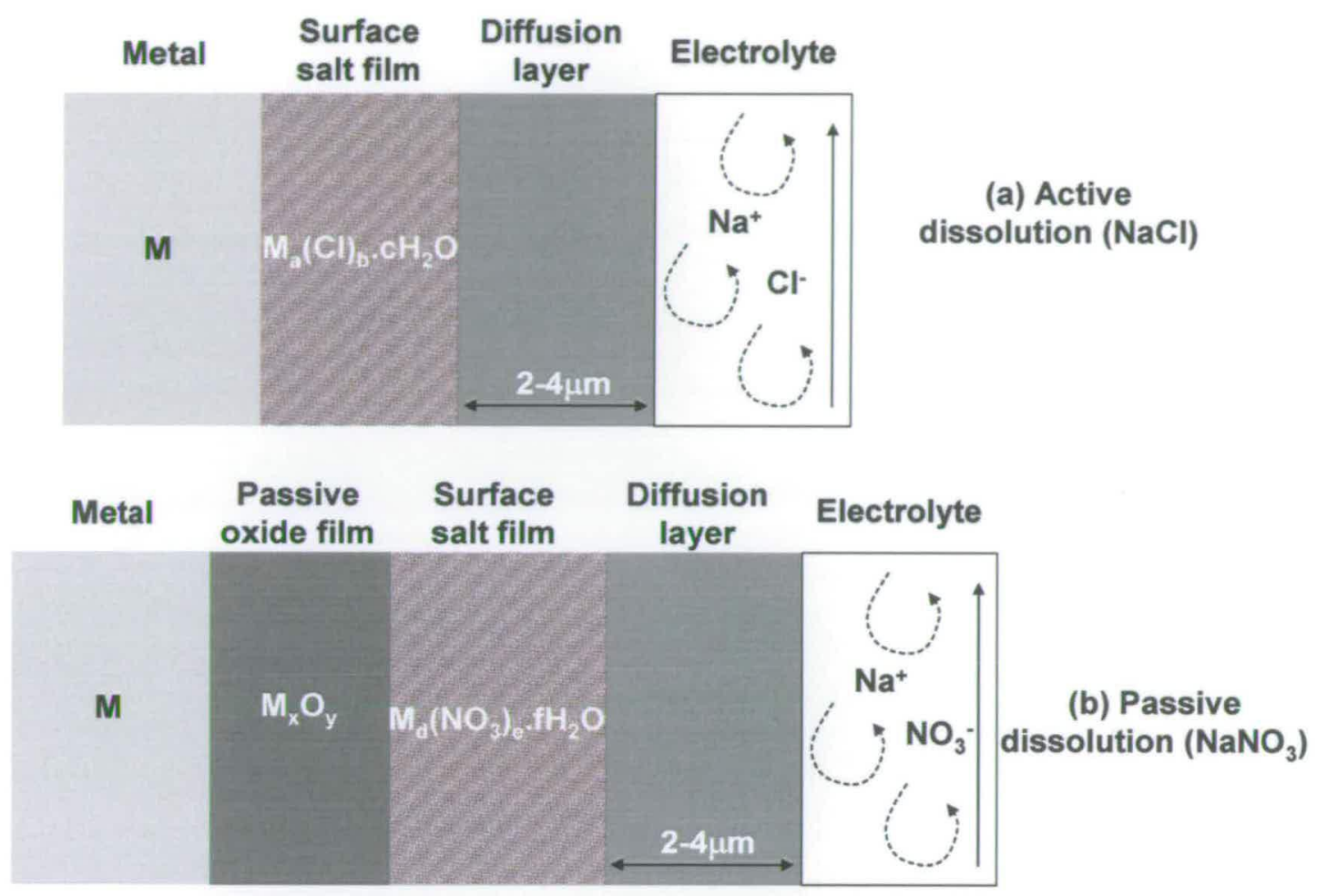

Figure 11.3: Schematic showing the proposed surface composition for the active and passive dissolution mechanisms discussed in this thesis, the thicknesses shown are not to scale.

In $\mathrm{NaCl}$, the results are consistent with Figure 11.3(a). The $\mathrm{V}_{0}$ axis intercepts at $\mathrm{J}=0.0 \mathrm{~A}$ $\mathrm{cm}^{-2}$ found were consistent with active metal dissolution from the workpiece material. The current densities were found to be high enough for the formation of a surface salt film on the workpiece. Subsequent to this a diffusion layer separates the surface salt layer from the turbulent electrolyte region. In $\mathrm{NaNO}_{3}$, the results are consistent with Figure 11.3(b). In this case the $\mathrm{V}_{0}$ axis intercepts at $\mathrm{J}=0.0 \mathrm{~A} \mathrm{~cm}^{-2}$ were found to be consistent with a passive dissolution process through a surface oxide film. The increase in the $\mathrm{V}_{0}$ axis intercept for passive dissolution in $\mathrm{NaNO}_{3}$ is consistent with the extra voltage required to enable dissolution through a surface oxide film. As with $\mathrm{NaCl}$, the current densities were found to be high enough for the formation of a surface salt film on the workpiece which was 
separated from the turbulent electrolyte region by a diffusion layer. In both electrolytes the ohmic J- $\mathrm{V}_{0}$ dissolution process has been attributed to the presence of a rate determining surface salt film. No appreciable extra ohmic resistance was observed during passive dissolution, in $\mathrm{NaNO}_{3}$, consistent with there being no appreciable extra resistance, to that of the salt film, due to the oxide film. Similar ohmic resistances were observed for different workpieces consistent with similar saturation concentrations and thicknesses of the salt film and similar diffusion co-efficients for the rate determining ion species. 


\section{Chapter 12}

Enhanced computer simulations of Ti 6/4 ECM using experimental $\mathrm{J}-\mathrm{V}_{0}$ parameters. 


\subsection{Introduction}

Experimental measurements of $\mathrm{J}-\mathrm{V}_{0}$ characteristics (the data in Chapter 7, Chapter 8, Chapter 9 and Chapter 10), in addition to providing insight into the surface dissolution processes (Chapter 11), also enable a more accurate ECM parameter base to be produced to enable enhanced ECM simulation of these materials for a given flow path length. The results in Chapter 7, Chapter 8, Chapter 9 and Chapter 10 have shown that, under a variety of experimental conditions, some or all of these parameters cannot be assumed to be constant throughout the experiment. In this chapter, a model calculation for $\mathrm{Ti} 6 / 4$ in a $\mathrm{NaCl}$ electrolyte using both ideal experimental conditions (constant $\mathrm{n}, \mathrm{V}_{0}$ and $\kappa$ ) and experimentally collected, non-ideal $\mathrm{J}-\mathrm{V}_{0}$ data (with constant values of $\mathrm{n}$ and $\mathrm{\kappa}$ ) is used to compute I-t and z-t data which is compared to experimentally collected data for these parameters. The work presented in this chapter has been carried out in collaboration with David Curry, who performed the simulations using the ECM parameter base provided by experimental work presented in this thesis.

\subsection{Simulation procedure}

The software used was MATLAB used in conjunction with the finite-element package FEMLAB. The geometry used for both the ideal and non-ideal simulations represents the electrolyte gap with the upper and lower boundaries defining the profile of the planer tool and workpiece. The workpiece boundary is broken down into a number of points, evenly distributed along its length. Figure 12.1 shows the boundary conditions applied in the model.

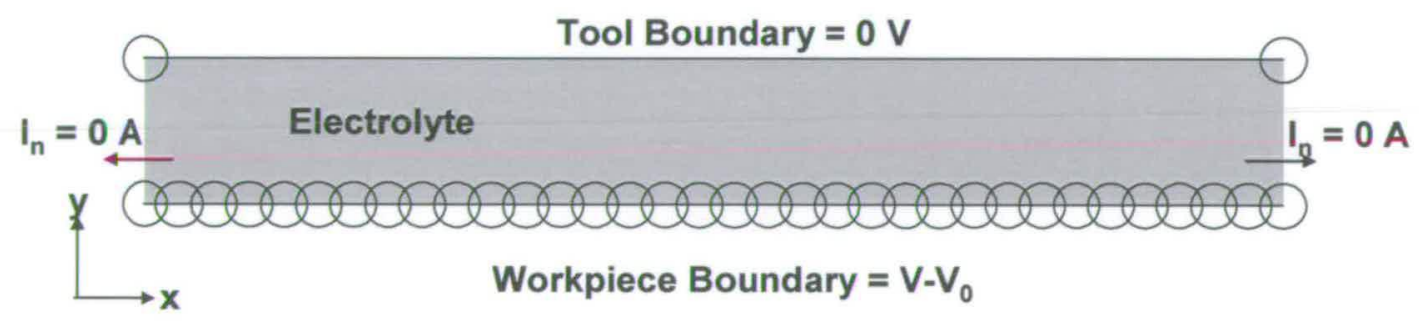

Figure 12.1: Geometry and boundary conditions used in simulation of I-t and z-t parameters. 
The voltage at the tool boundary was set equal to zero while the voltage at the workpiece boundary was assigned as $\mathrm{V}-\mathrm{V}_{0}$, in order to ensure that the migrational voltage across the electrolyte is of the correct magnitude (i.e. $\mathrm{V}-\mathrm{V}_{0}$ ). The side boundaries were constrained so that the normal of the current $\left(I_{n}\right)$ was equal to zero (Figure 12.1). In contrast to ideal simulations, $\mathrm{V}-\mathrm{V}_{0}$, applied as the workpiece boundary condition, was allowed to vary in magnitude throughout the simulation. The constant experimental conditions used for both the ideal and the non-ideal simulation were the experimentally measured dissolution valency $(\mathrm{n}=4)$, applied voltage $(\mathrm{V}=16 \mathrm{~V})$, feed rate $\left(\mathrm{f}=0.5 \mathrm{~mm} \mathrm{~min}^{-1}\right)$, electrolyte flow rate $(\mathrm{U}=$ $16.0 \mathrm{~L} \mathrm{~min}^{-1}$ ) and electrolyte conductivity (for $15.0 \% \mathrm{NaCl}, \kappa=0.2 \mathrm{~S} \mathrm{~cm}^{-1}$ ). The initial interelectrode gap was set to be $\mathrm{z}_{0}=0.8 \mathrm{~mm}$. Figure 12.2 shows typical approach data for the experimental ECM dissolution of Ti 6/4 (Chapter 10). These conform to a linear least square fit with an ohmic resistance of $0.11 \pm 0.01 \Omega \mathrm{cm}^{-2}$, consistent with the ohmic measurements made in Chapter 10, and a $\mathrm{V}_{0}$ axis intercept of $2.0 \pm 0.2 \mathrm{~V}$, consistent with the approach phase hysteresis resulting from driving the ECM process with a feed rate of 0.5 $\mathrm{mm} \min ^{-1}$ (section 7.2.2). This $\mathrm{J}_{-} \mathrm{V}_{0}$ ohmic data was used to calculate $\mathrm{V}_{0}$ at all $\mathrm{J}$ for the nonideal simulation.

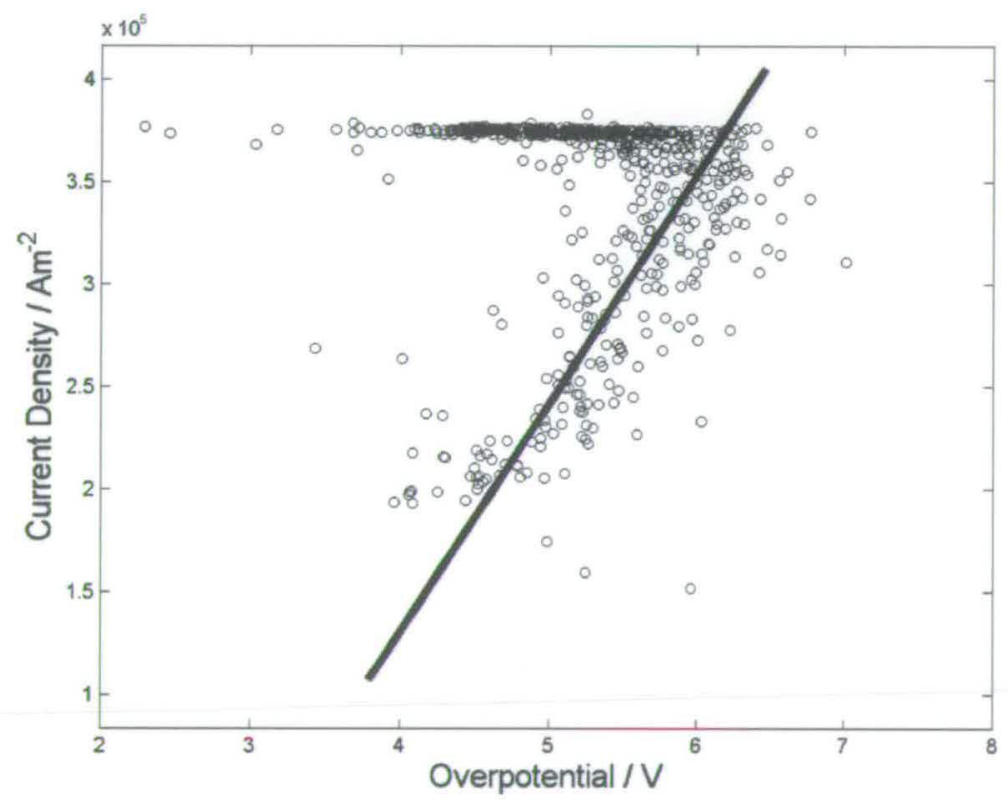

Figure 12.2: Linear least square fit (-) to the experimental ECM approach data for Ti 6/4 used to calculate $\mathrm{V}_{0}$ for non-ideal simulation. 
An ideal simulation was also performed, using the same experimental conditions, but with $\mathrm{V}_{0}$ set at $5.3 \mathrm{~V}$ at all times, the mean of the measured experimental $\mathrm{V}_{0}$ (Figure 12.2). For all simulations, the electric field, E, at all cell points, was evaluated through solution of the Laplace field equation:

$$
\frac{d^{2} E}{d x^{2}}+\frac{d^{2} E}{d y^{2}}=0
$$

\section{Equation 12.1}

where $\mathrm{x}$ and $\mathrm{y}$ are the co-ordinate axis shown in Figure 12.2. This enables the current to be calculated at all points using the electrolyte conductivity, $\kappa$, and the electrode area, A, through:

$$
I=E \kappa A
$$

\section{Equation 12.2}

Using this current, the material removal, normal to the workpiece, at each of these points, dh, is calculated for a small time step, dt, using (Equation 3.14). A spline representation is used to calculate the normals at each of the points distributed along the boundary. The change in workpiece thickness at each point, dh, is combined with the tool feed rate and the points are displaced, producing an interelectrode gap measurement after the small time step, dt. A spline is interpolated through the new coordinates, and a new set of evenly distributed points are defined. The new geometry, after $\mathrm{dt}$, is calculated, $\mathrm{V}_{0}$ is re-evaluated using the current measurement from the previous step, the new boundary conditions are applied (with the updated $\mathrm{V}_{0}$ for non-ideal simulations and constant $\mathrm{V}_{0}$ for ideal simulations) and the solution of the Laplace equation (Equation 12.1) for the electric field is repeated for the next time step.

The initial value of $\mathrm{V}_{0}$ was chosen by estimation at time $\mathrm{t}=0 \mathrm{~s}$ for the non-ideal simulation. The simulation can be terminated automatically based on either user defined conditions such 
as elapsed time, or experimental process parameters for example current values, or depth of material removed.

\subsection{Results and discussion}

Figure 12.3 shows the comparison between the current data collected experimentally and the ideal and non-ideal computer simulations carried out for $\mathrm{Ti} 6 / 4$. For the purposes of analysis, the data is split into two regions, region $\mathrm{A}$ (before equilibrium machining conditions are reached) and region B (during equilibrium machining).

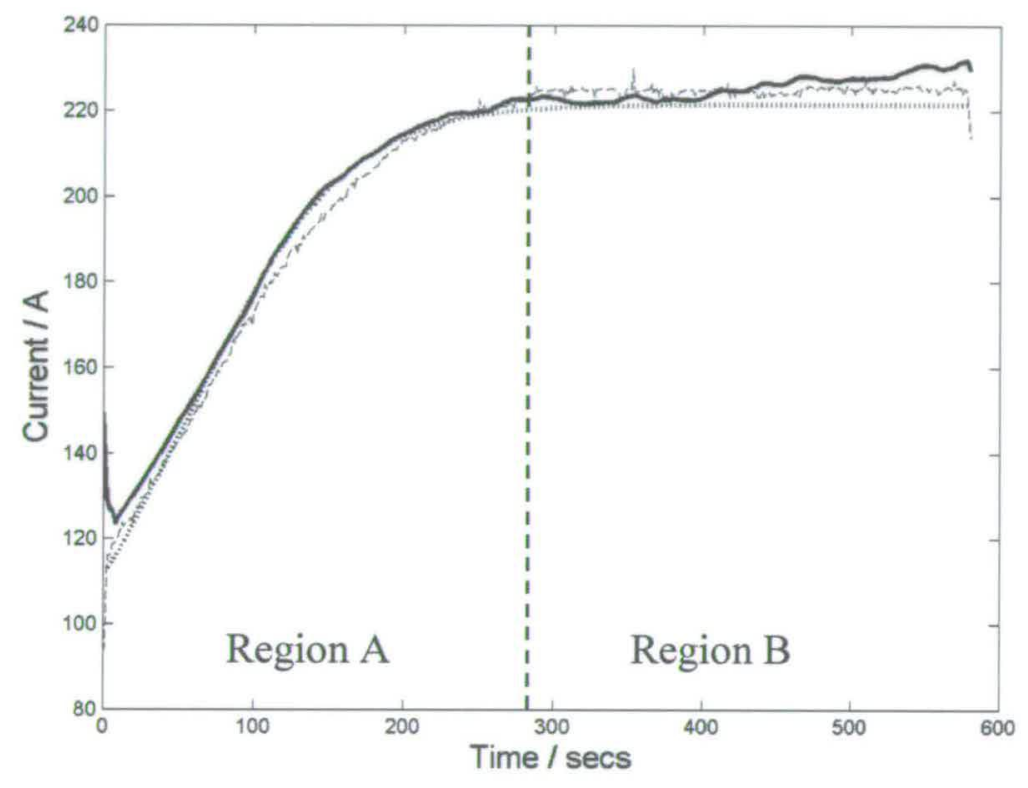

Figure 12.3: Comparison of I against $t$ for experimental (---), non-ideal simulation (-) and ideal simulation ("').

The initial decrease of I in the non-ideal simulation was a simulation artefact caused by an incorrect estimation of the initial $\mathrm{V}_{0}$ value. After this, both ideal and non-ideal simulations show comparable fits with the experimental data whilst machining in region A. Figure 12.4 shows the comparison between the interelectrode gap data collected experimentally and the ideal and non-ideal computer simulations carried out for this parameter. 


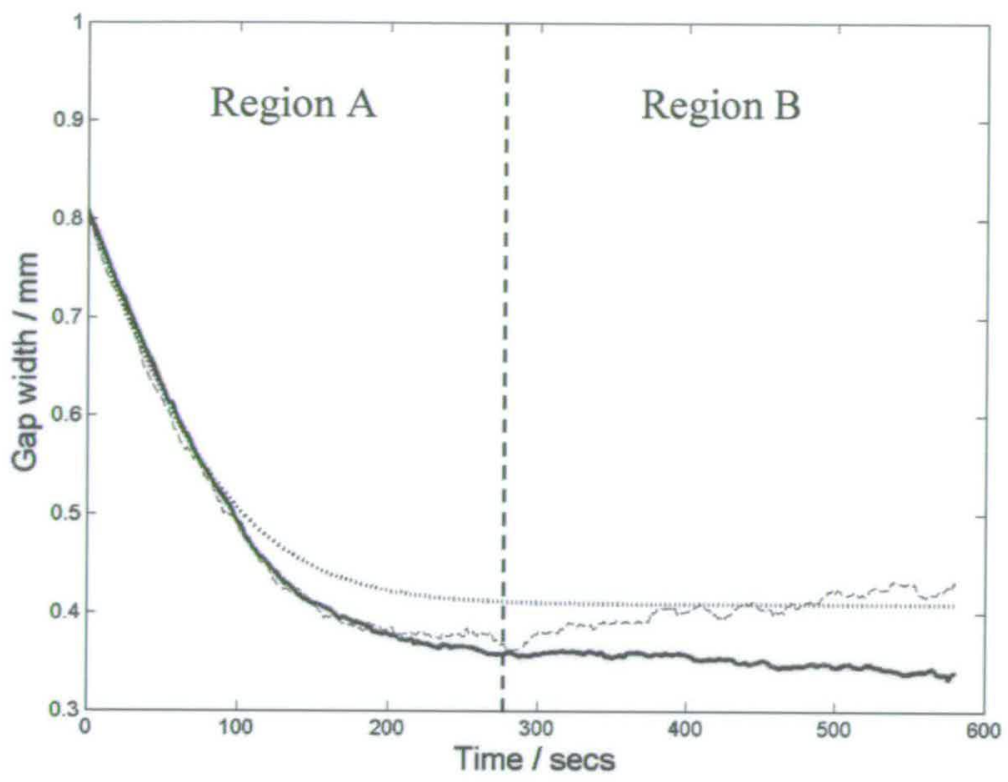

Figure 12.4: Comparison of the interelectrode gap for experimental (---), non-ideal simulation (-) and ideal simulation ('").

Figure 12.4 clearly shows a much closer correspondence between the experimental gap data and the non-ideal simulation than for the experimental data and the ideal simulation. This shows that, under these conditions, simulation using a mean $\mathrm{V}_{0}$ value is reasonably accurate between $\mathrm{z}=0.8 \mathrm{~mm}$ and $\mathrm{z}=0.5 \mathrm{~mm}$, but at smaller gaps in region $\mathrm{A}$ the error in $\mathrm{V}_{0}$ in the ideal case becomes appreciable (with the actual $\mathrm{V}_{0}$ value being greater than that used for the ideal simulation leading to smaller experimental $\mathrm{z}$ values). These data show the improvement of simulations incorporating experimental fitting of the $\mathrm{J}-\mathrm{V}_{0}$ data.

However, while the data in Figure 12.4 show that a linear least square parameterisation of J$\mathrm{V}_{0}$ significantly improves the simulation of $\mathrm{z}$ in region $\mathrm{A}$ (during electrode approach), significant errors are observed in the non-ideal simulations of $\mathrm{I}$ and $\mathrm{z}$ in region $\mathrm{B}$ (equilibrium machining, Figure 12.3 and Figure 12.4). The discrepancy between the experimental I data and the non-ideal simulation in region B (Figure 12.3) is most likely due to changes in the dissolution valency (Equation 2.5). To improve the accuracy of the nonideal I data simulation in region $\mathrm{B}$, and to further improve the accuracy in region A, the parameterisation of the variation in valency during ECM is also required. The increase in experimental gap measurements with time in region B (Figure 12.4) is most likely predominantly due to an electrolyte temperature increase. This can also be seen in the 
equilibrium data in Figure 12.2 where the experimentally determined $\mathrm{V}_{0}$ values are seen to apparently deviate to lower $\mathrm{V}_{0}$ than those predicted by the linear calibration. An electrolyte temperature increase leads to an increase in electrolyte conductivity (section 8.2) and a corresponding increase in the interelectrode gap, as seen for the experimental data in region $\mathrm{B}$ in Figure 12.4. To improve the accuracy of the non-ideal $\mathrm{z}$ data simulation, the calculation of the electrolyte temperature at all times and the parameterisation of the electrolyte conductivity as a function of temperature is required. The data for both the valency and temperature parameterisations can be found in Chapter 10 and section 8.2 respectively.

\subsection{Conclusions}

From this simulation the advantages of parameterisation for the computer simulation of ECM under non-ideal conditions is evident. Furthermore, the independence of the $\mathrm{J}-\mathrm{V}_{0}$ transients measured in this thesis on applied voltage, interelectrode gap, electrolyte flow rate and electrolyte concentration greatly reduce the parameter base required for simulation under different machining conditions. However, further improvements of the parameter simulations could be made through incorporation of dissolution valency and electrolyte conductivity parameterisation. 


\section{Chapter 13}

ECM in cell 2004 


\subsection{Introduction}

Part of the work done for this thesis included parameterisation of In718 and Ti6/4 in a newly developed ECM cell 2004 (section 4.7). The major differences between cell 2004 and cell 2000 , which may be expected to influence the ECM parameters, are the flow path length, the workpiece and tool dimensions and the electrolyte flow rate. In cell 2004 the flow path length was doubled in order to investigate $\mathrm{J}-\mathrm{V}_{0}$ characteristics at longer flow path lengths, with ECM conditions more appropriate to the industrial sponsor. However, in order to maintain similar measurable currents across a tool and workpiece for the same current densities the electrode width was halved. Consequently, while the area of the electrode surfaces was the same in both cells, the total dimensions of the cell 2004 electrodes was 7.5 $\mathrm{mm} \times 80.0 \mathrm{~mm}$ compared to $15.0 \mathrm{~mm} \times 40.0 \mathrm{~mm}$ for cell 2000 . Under comparable ECM conditions of applied voltage, feed rate and electrolyte conductivity, at the same current density the interelectrode gap is the same (Equation 3.1). The resulting halving of the cross sectional area of the interelectrode gap and the doubling of the flow path length therefore causes a corresponding pressure increase in the cell with respect to cell 2000 at the same current density. In order to reduce the cell pressure to prevent cell leakage, and to maintain the same electrolyte flow velocities as seen in cell 2000 , the electrolyte flow rate was halved. However, this would still lead to a greater pressure in cell 2004 compared to cell 2000 under comparable conditions of current density. The final difference was the use of four segments along the flow path length compared to the seven used in cell 2000. These segment dimensions were chosen to allow the ultrasound probe to fit into one of the segments in the tool for the direct $\mathrm{z}$ measurement tests discussed in Chapter 5. This area difference is allowed for in comparison plots through the use of current density measurements. For the measurements taken in this chapter, given the issues with direct $\mathrm{z}$ measurements in Chapter 5 , the ultrasound probe was positioned behind the workpiece measuring the workpiece thickness, as with cell 2000 (Figure 3.1), and analysis carried out as detailed in section 3.3. Consequently the data used for the ultrasound analysis was collected from segment 3 of the cell 2004 segmented tool. The object of the work presented in this chapter is to measure the performance of cell 2004 with respect to cell 2000 with a view to providing cell-independent ECM parameterisation. 


\subsection{Thermocouple measurements}

Figure 13.1 and Figure 13.2 show typical thermocouple measurements when machining In718 with cell 2004 in $\mathrm{NaCl}$ and $\mathrm{NaNO}_{3}$ respectively.

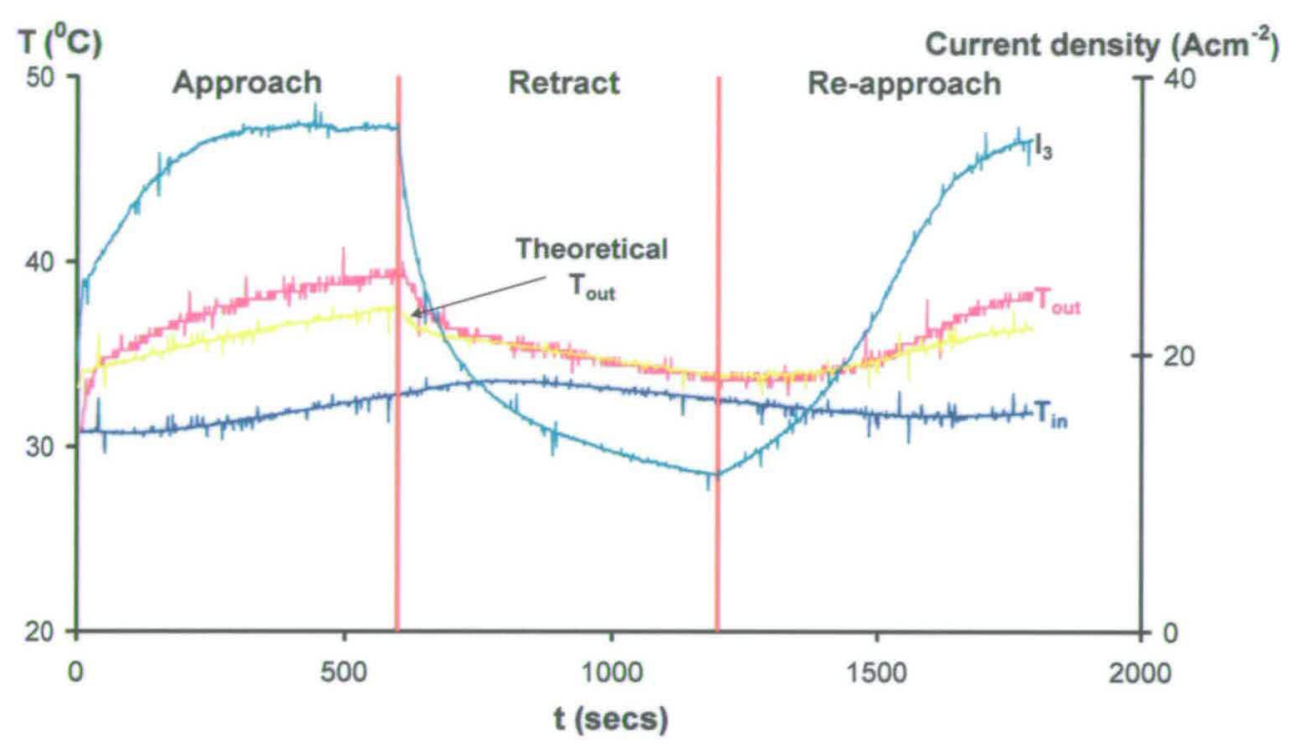

Figure 13.1: Inlet and outlet temperature profiles for In718 machined in $15.0 \% \mathrm{NaCl}$ at $\mathrm{V}=$ $13.0 \mathrm{~V}$. Also show is the theoretical outlet temperature calculated using Equation 8.1. Also shown is the segment 3 current density during machining. 


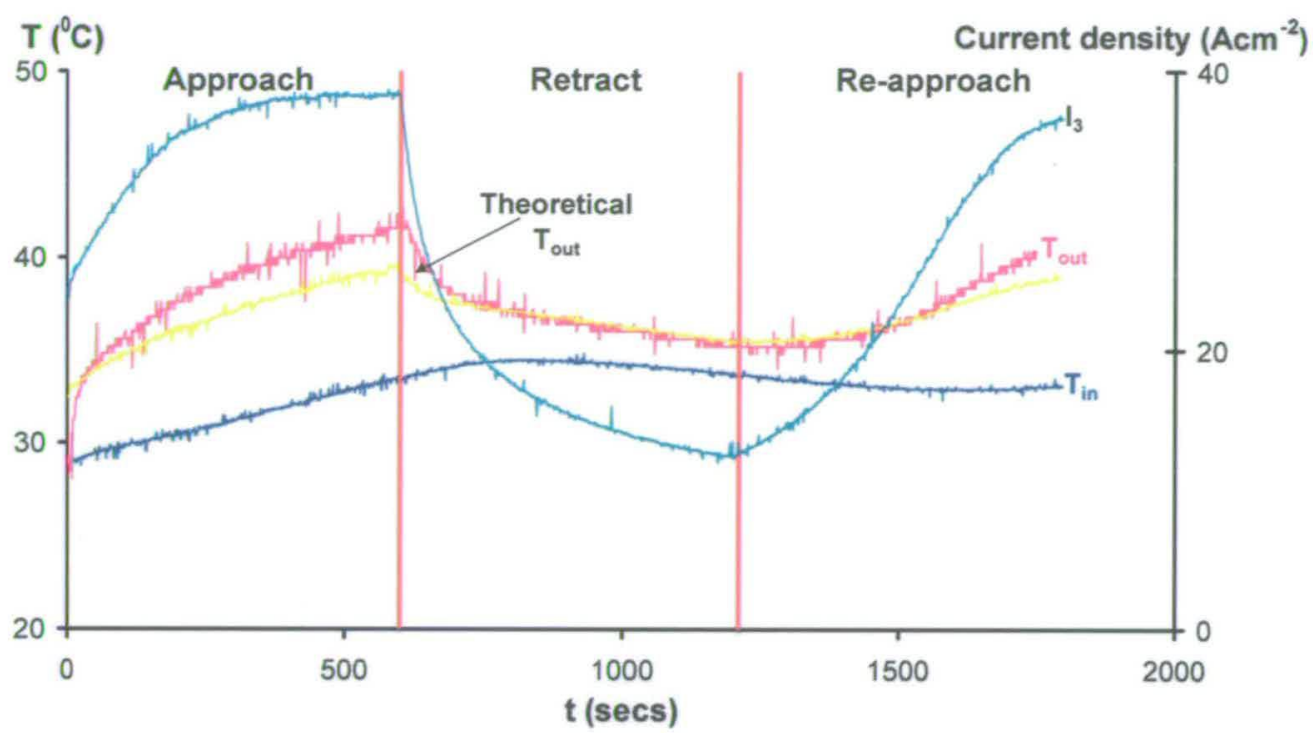

Figure 13.2: Inlet and outlet temperature profiles for In 718 machined in $21.7 \% \mathrm{NaNO}_{3}$ at $\mathrm{V}$

$=16.0 \mathrm{~V}$. Also show is the theoretical outlet temperature calculated using Equation 8.1.

Also shown is the segment 3 current density during machining.

These experiments were carried out using ECM parameters which ensure similar current densities, and hence comparable ECM dissolution, to those observed with cell 2000 (section 8.2). From these data it is clear that the electrolyte temperatures in cell 2004 are greater than those for cell 2000 (section 8.2). For $\mathrm{NaCl}$, Figure 13.1 shows a maximum temperature difference between the inlet and the outlet thermocouple of $7{ }^{\circ} \mathrm{C}$, with an overall temperature increase of the bulk electrolyte temperature during machining of $2{ }^{\circ} \mathrm{C}$ (from the inlet thermocouple). For $\mathrm{NaNO}_{3}$, Figure 13.2 shows a maximum temperature difference between the inlet and the outlet thermocouple of $8{ }^{\circ} \mathrm{C}$ with bulk electrolyte temperature increase during machining of $2{ }^{\circ} \mathrm{C}$ (from the inlet thermocouple). The origin of this temperature rise is a simple Joule heating effect in cell 2000 (section 8.2). Therefore, as with cell 2000, Equation 8.2 was used to calculate the theoretical temperature rise in this case, these are shown in Figure 13.1 and Figure 13.2. As with cell 2000, an initial steep increase in the outlet thermocouple temperature is observed. This is a consequence of the finite time required for the electrolyte from the cell to reach the outlet thermocouple position. Figure 13.1 and Figure 13.2 also show that at small z, i.e. the end of the approach and re-approach phases, the Joule heating relationship predicts outlet temperatures approximately $2.5{ }^{\circ} \mathrm{C}$ smaller than those measured. This effect has been seen previously in cell 2000 , with a difference of $0.4{ }^{\circ} \mathrm{C}$ observed at small $\mathrm{z}$ for stainless steels (section 8.2). Indeed, this small 
difference was noted when machining all materials in cell 2000. The larger temperature for cell 2004 is consistent with an additional electrolyte heating component, most likely from additional exothermic reactions in the gap. This effect would be expected to be greater for cell 2004, as observed, due to the reduced flow rate compared to cell 2000 , which would result in increased electrolyte heating and electrolyte transit time for the same amount of heat produced (Equation 8.2). However, Figure 13.1 and Figure 13.2 do show that the outlet temperature is predicted simply by the Joule heating at current densities of $I_{3}<22 \mathrm{~A} \mathrm{~cm}^{-2}$ during the approach, retract and re-approach phases. This confirms that the additional heating component is related to the magnitude of $\mathrm{J}$ (and hence the ECM dissolution) and is consistent with the exothermic reactions involving ECM products.

\subsection{Basic $\ln 718$ parameter measurements}

Figure 13.3 shows typical chronoamperometric data for segments $1-4$, and typical z-t data, for an approach/retract and re-approach (section 4.8) experiment using In718 with $15.0 \%$ $\mathrm{NaCl}$ electrolyte and an applied voltage of $\mathrm{V}=13.0 \mathrm{~V}$.

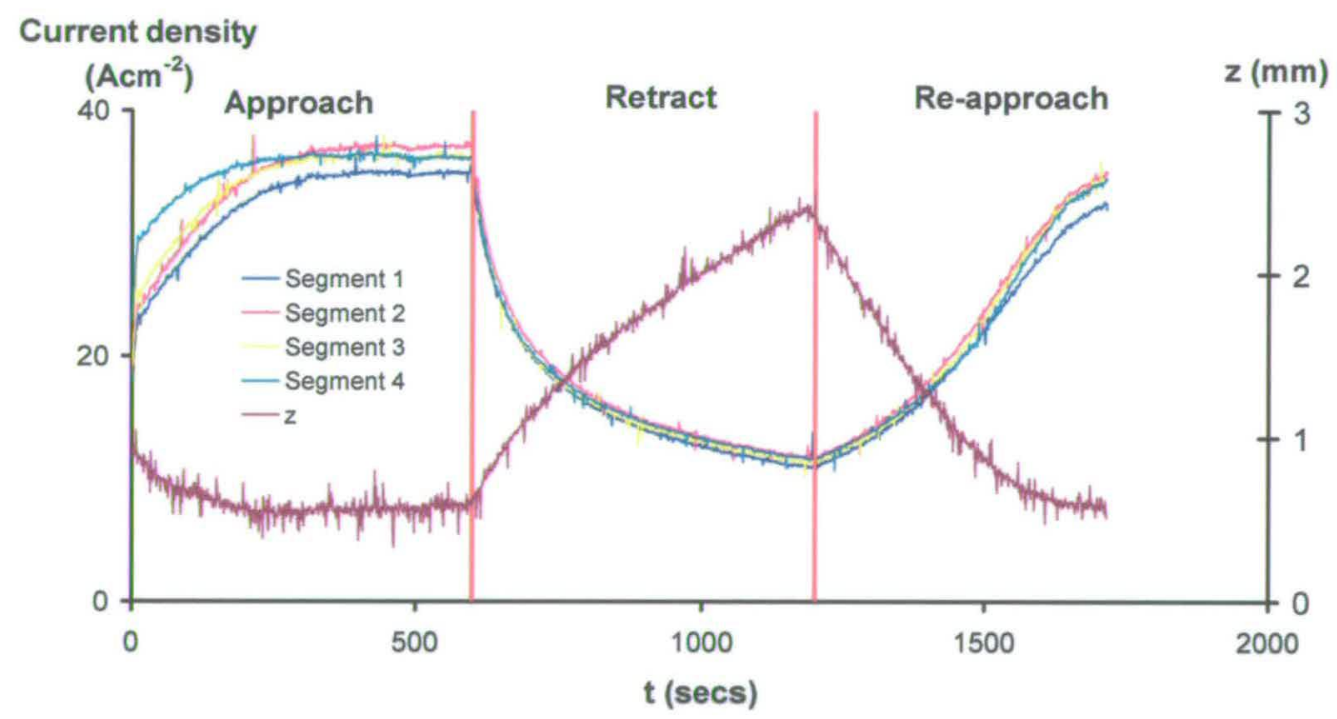

Figure 13.3: Experimental chronoamperometric transients for In718 machined in $15.0 \%$ $\mathrm{NaCl}$ with $\mathrm{V}=13.0 \mathrm{~V}$. Also shown is z-t data for the same experiment. 
These data show an increase in the initial segment 4 current density. This effect has been generally observed with cell 2004 and can be attributed to a taper in the initial interelectrode gap through difficulties in workpiece alignment due to the long workpiece length. The rounding effect seen with data collected in cell 2000 (section 8.3), in which the end segments show lower currents during the approach and re-approach phases and higher currents during the retract phase, is not as marked for cell 2004, this can be attributed to a smaller percentage contribution of the rounding current to the segmented current due to the increase in segment area. However, subsequent to machining, rounding of the workpieces was still observed visually for cell 2004 . These data suggest that smaller area tool segments, such as those used in cell 2000 , are beneficial to the study of the rounding effect. From these data equilibrium values for segment 4 were found to be $\mathrm{J}_{\infty}=36 \pm 0.7 \mathrm{Acm}^{-2}$ and $\mathrm{z}_{\infty}=0.57 \pm 0.03 \mathrm{~mm}$. Figure 13.4 shows typical chronoamperometric and z-t data for the same material machined with $21.7 \% \mathrm{NaNO}_{3}$ electrolyte and an applied voltage of $\mathrm{V}=16.0 \mathrm{~V}$.

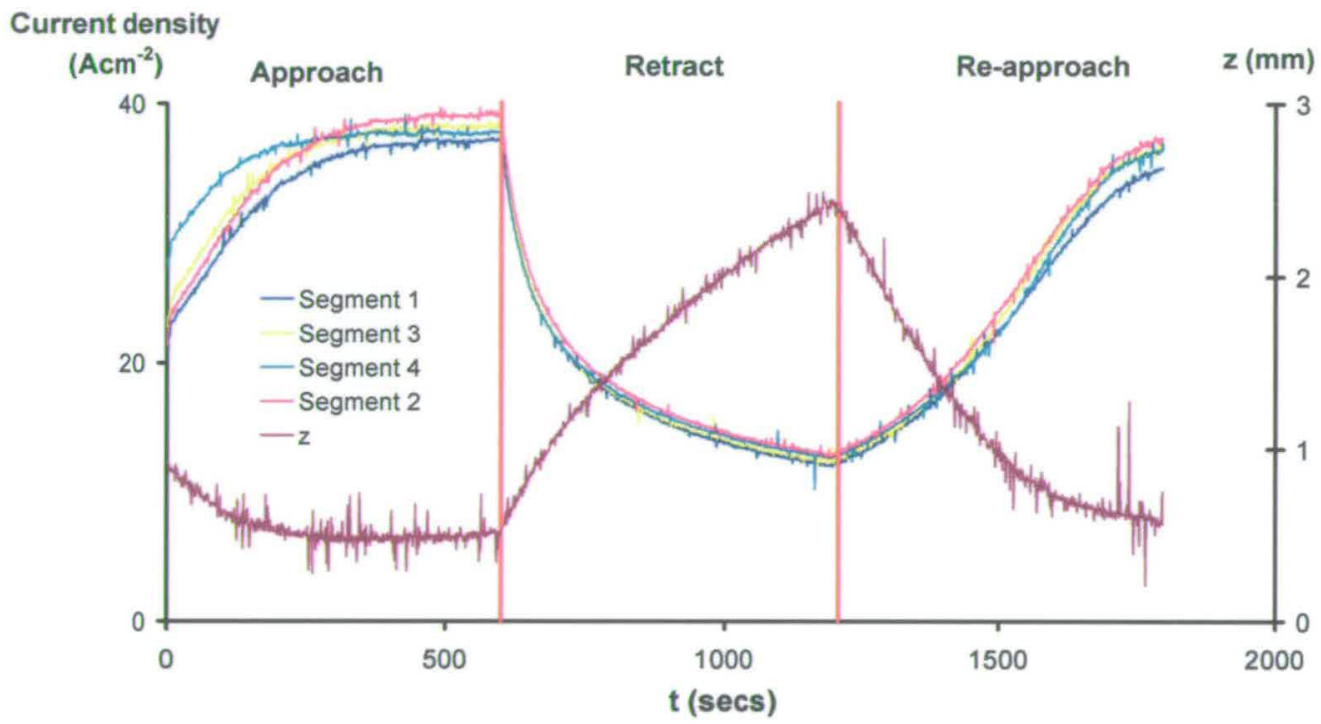

Figure 13.4: Experimental chronoamperometric transients for In 718 machined in $21.70 \%$ $\mathrm{NaNO}_{3}$ with $\mathrm{V}=16.0 \mathrm{~V}$. Also shown is z-t data for the same experiment.

From these data equilibrium values for segment 4 were found to be $\mathrm{J}_{\infty}=36 \pm 0.7 \mathrm{Acm}^{-2}$ and $\mathrm{z}_{\infty}=0.57 \pm 0.03 \mathrm{~mm}$. As for cell 2000 , the applied voltage was increased when machining in $\mathrm{NaNO}_{3}$, in line with higher mean $\mathrm{V}_{0}$ values for $\mathrm{NaNO}_{3}$ previously found by chronoamperometric measurements [137], in an attempt to maintain a constant $\mathrm{J}_{\infty}$ and $\mathrm{z}_{\infty}$ between experiments. The percentage composition of the electrolytes was chosen to 
maintain a constant molarity and hence a similar electrolyte conductivity of $\kappa=0.20 \mathrm{~S} \mathrm{~cm}^{-1}$ [137].

Figure 13.5 shows the corresponding $\mathrm{J}-\mathrm{V}_{0}$ transients for In718 machined in $\mathrm{NaCl}$ and $\mathrm{NaNO}_{3}$ with cell 2004.

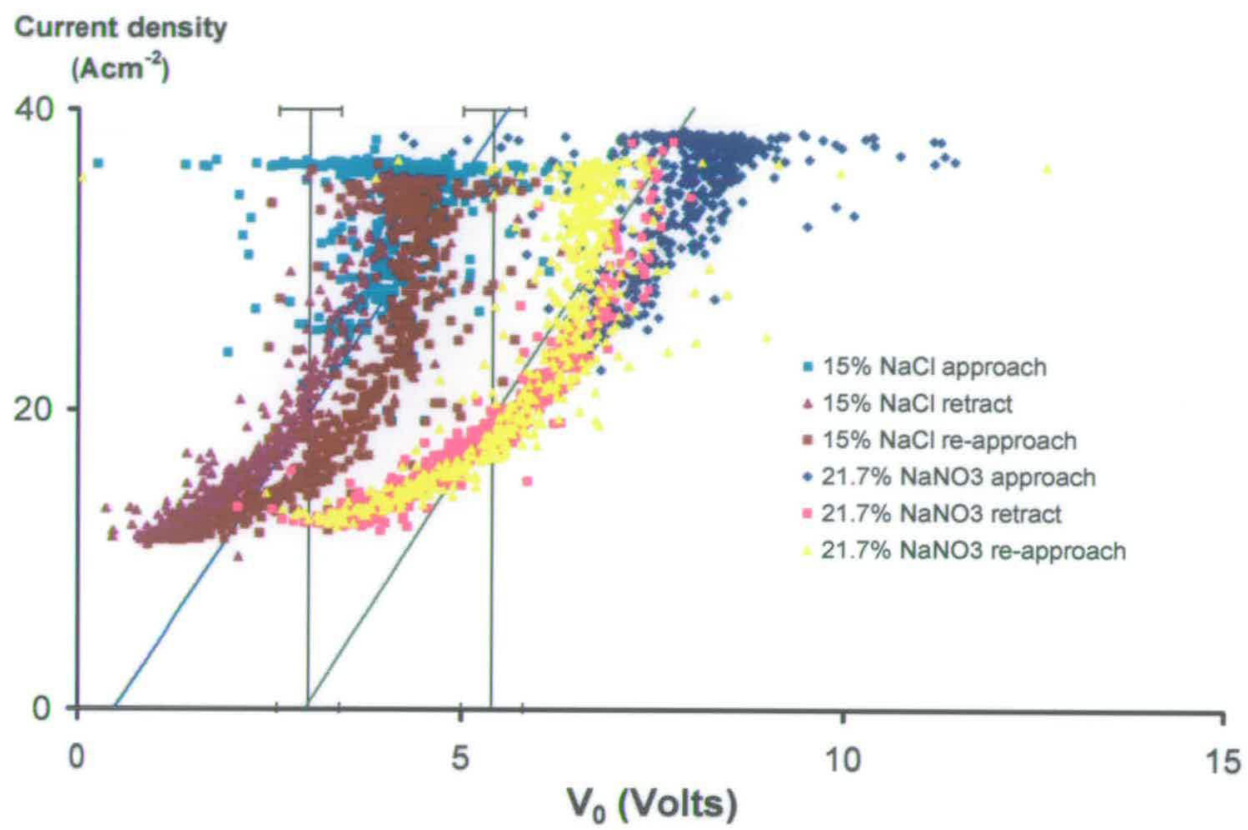

Figure 13.5: $\mathrm{J}-\mathrm{V}_{0}$ data for $\mathrm{In} 718$ machined in $15.0 \% \mathrm{NaCl}$ and $21.7 \% \mathrm{NaNO}_{3}$ at $\mathrm{V}=13.0$

$\mathrm{V}$ and $\mathrm{V}=16.0 \mathrm{~V}$ respectively in cell 2004 . Time-averaged $\mathrm{V}_{0}$ values for $\operatorname{In} 718$ found through the chronoamperometric analysis in section 3.2 as $\mathrm{V}_{0}=3.0 \pm 0.4$ and $\mathrm{V}_{0}=5.4 \pm 0.4$

$\mathrm{V}$ for $\mathrm{NaCl}$ and $\mathrm{NaNO}_{3}$ electrolytes respectively are given [137]. Also shown, for comparison, are the linear least square fits of the retract $\mathrm{J}-\mathrm{V}_{0}$ data for the same conditions in cell 2000, with ohmic resistances of $0.13 \pm 0.01 \Omega \mathrm{cm}^{-2}$ and $\mathrm{V}_{0}$ axis intercepts of $0.5 \pm 0.2 \mathrm{~V}$ and $2.9 \pm 0.2 \mathrm{~V}$ for $\mathrm{NaCl}$ and $\mathrm{NaNO}_{3}$ (green and blue) respectively.

These data clearly show similar J-V $\mathrm{V}_{0}$ characteristics for cell 2000 and cell 2004 (Figure 9.3 and Figure 13.5). As with cell 2000, machining In718 in cell 2004 also gives mean $\mathrm{J}-\mathrm{V}_{0}$ characteristics closely corresponding to those previously obtained using a chronoamperometric method. For closer examination of the $\mathrm{J}-\mathrm{V}_{0}$ differences between the two cells, Figure 13.6 and Figure 13.7 show the comparisons between $\mathrm{J}-\mathrm{V}_{0}$ transients collected in cell 2000 and 2004 for $15.0 \% \mathrm{NaCl}$ and $21.7 \% \mathrm{NaNO}_{3}$ respectively. 


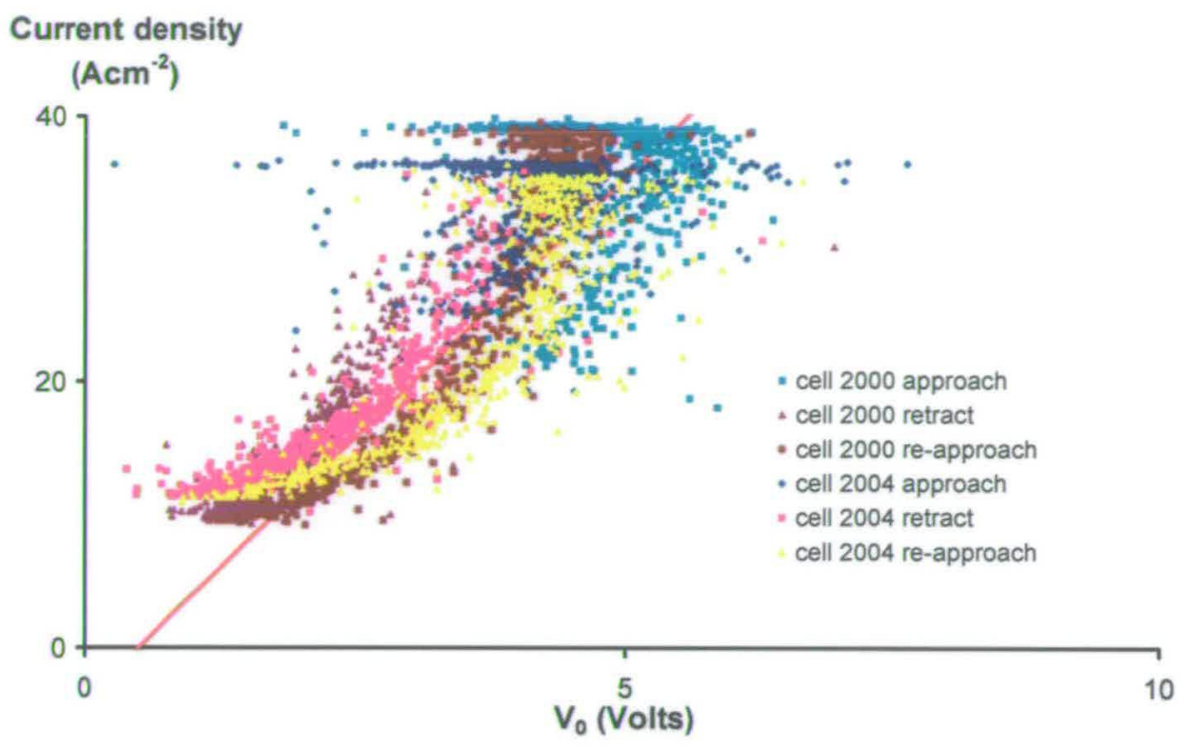

Figure 13.6: Comparison of the $\mathrm{J}-\mathrm{V}_{0}$ characteristics for In 718 machined in $15.0 \% \mathrm{NaCl}$ at $\mathrm{V}=13.0 \mathrm{~V}$ in cell 2000 and cell 2004 . For comparison, the linear least squares fit of the retract $\mathrm{J}-\mathrm{V}_{0}$ data for cell 2000 , with ohmic resistances of $0.13 \pm 0.01 \Omega \mathrm{cm}^{-2}$ and $\mathrm{V}_{0}$ axis intercepts of $0.5 \pm 0.2 \mathrm{~V}$ is given.

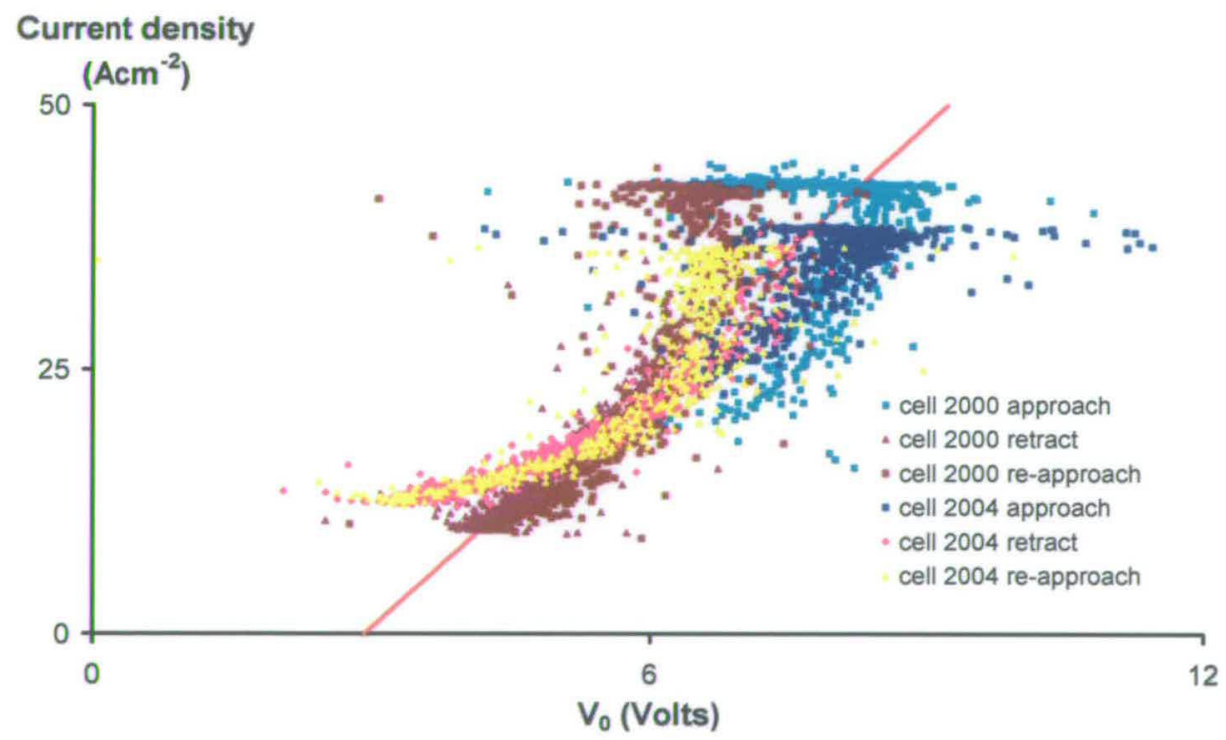

Figure 13.7: Comparison of the $\mathrm{J}-\mathrm{V}_{0}$ characteristics for $\operatorname{In} 718$ machined in $21.7 \% \mathrm{NaNO}_{3}$ at $\mathrm{V}=16.0 \mathrm{~V}$ in cell 2000 and cell 2004 . For comparison, the linear least squares fit of the 
retract $\mathrm{J}-\mathrm{V}_{0}$ data for cell 2000 , with ohmic resistances of $0.13 \pm 0.01 \Omega \mathrm{cm}^{-2}$ and $\mathrm{V}_{0}$ axis intercepts of $2.9 \pm 0.2 \mathrm{~V}$ is given.

The curvature of the $\mathrm{J}-\mathrm{V}_{0}$ transients measured in cell 2004 makes fitting the data with the linear least squares method problematic. This curvature can be explained, in part, through the increased temperatures measured in cell 2004 (section 13.2). Consequently, the cell 2004 data is compared to the retract fits calculated using the cell 2000 data. It is clear from Figure 13.6 and Figure 13.7 that the high current density $\mathrm{J}-\mathrm{V}_{0}$ data collected with cell 2004 is broadly similar to the data collected with cell 2000 . However, the low current density data is observed to trend to lower $\mathrm{V}_{0}$ values, a trend also seen for the stainless steels investigated in Chapter 8. A similar increase in observed dissolution valency has also been seen at low current densities (Figure 13.8 and Figure 13.9).

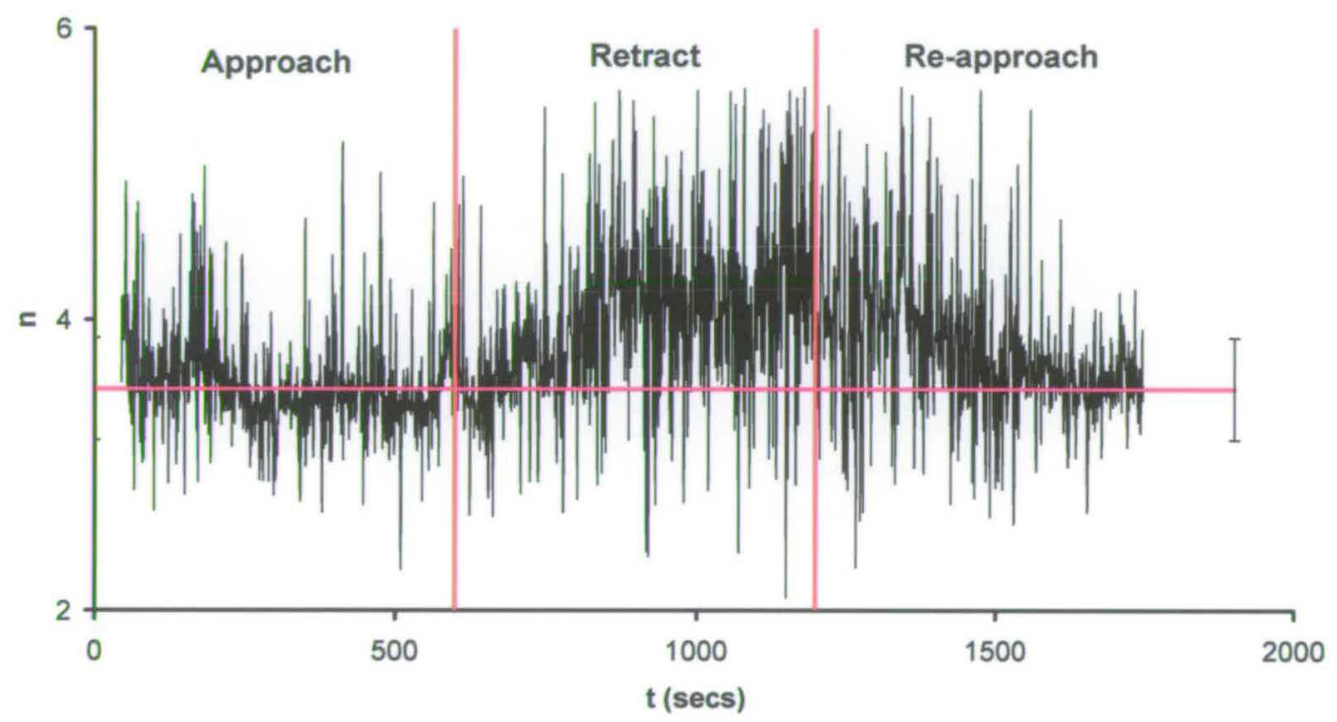

Figure 13.8: Experimental time-resolved $n$, with $\mathrm{p}=100$ from Equation 6.4, for $\operatorname{In} 718$ machined in $15.0 \% \mathrm{NaCl}$, the pink line depicts the average dissolution valency at equilibrium with an error shown. 


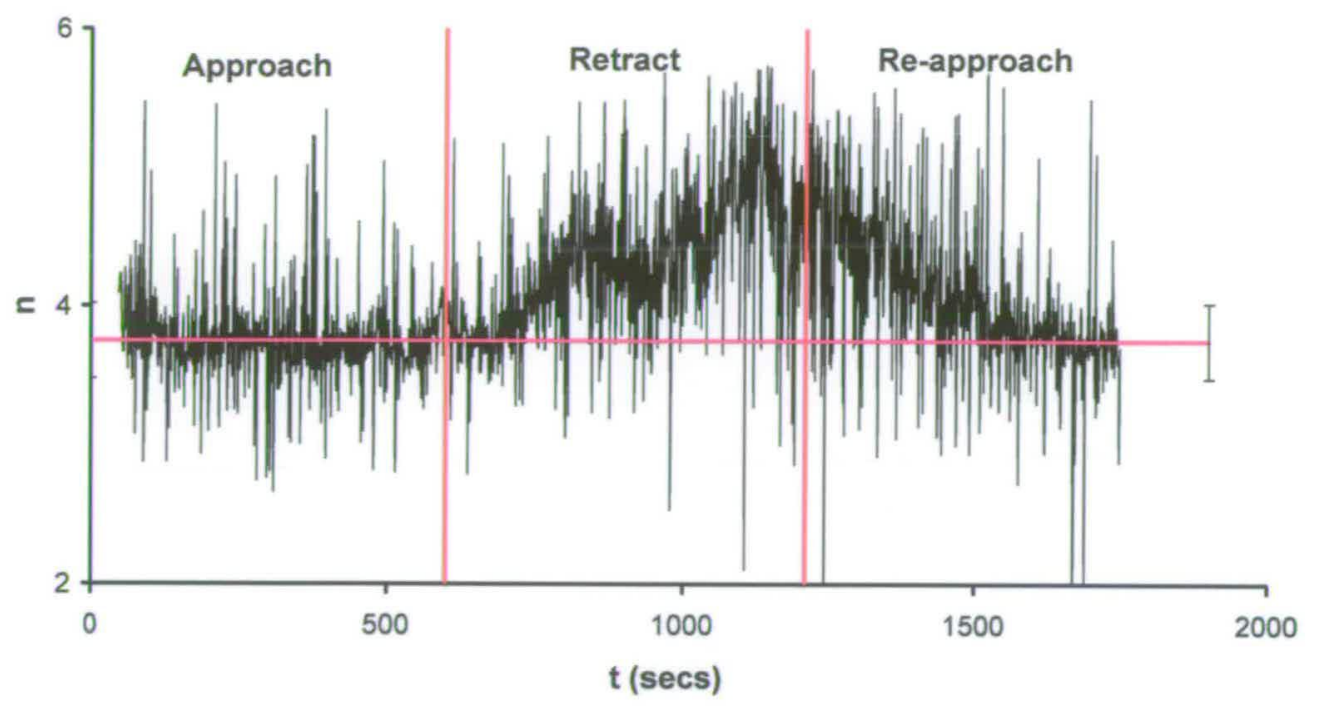

Figure 13.9: Experimental time-resolved $n$, with $p=100$ from Equation 6.4, for $\operatorname{In} 718$ machined in $21.7 \% \mathrm{NaNO}_{3}$, the pink line depicts the average dissolution valency at equilibrium with an error shown.

Figure 13.8 and Figure 13.9 clearly show similar valency trends, with increased valency at low current densities, to those seen when machining In718 in cell 2000. The equilibrium dissolution valencies for $\operatorname{In} 718$ was found to be $n=3.5 \pm 0.4$ and $n=3.7 \pm 0.3$ for $15.0 \%$ $\mathrm{NaCl}$ and $21.7 \% \mathrm{NaNO}_{3}$ respectively. The dissolution valency was observed to increase to $\mathrm{n}=4.6 \pm 0.4$ and $\mathrm{n}=4.8 \pm 0.3$ for $\mathrm{NaCl}$ and $\mathrm{NaNO}_{3}$ respectively. Figure 13.10 shows a comparison of these average dissolution valencies to those collected for In718 machined in $15.0 \% \mathrm{NaCl}$ and $21.7 \% \mathrm{NaNO}_{3}$ with cell 2000 . 


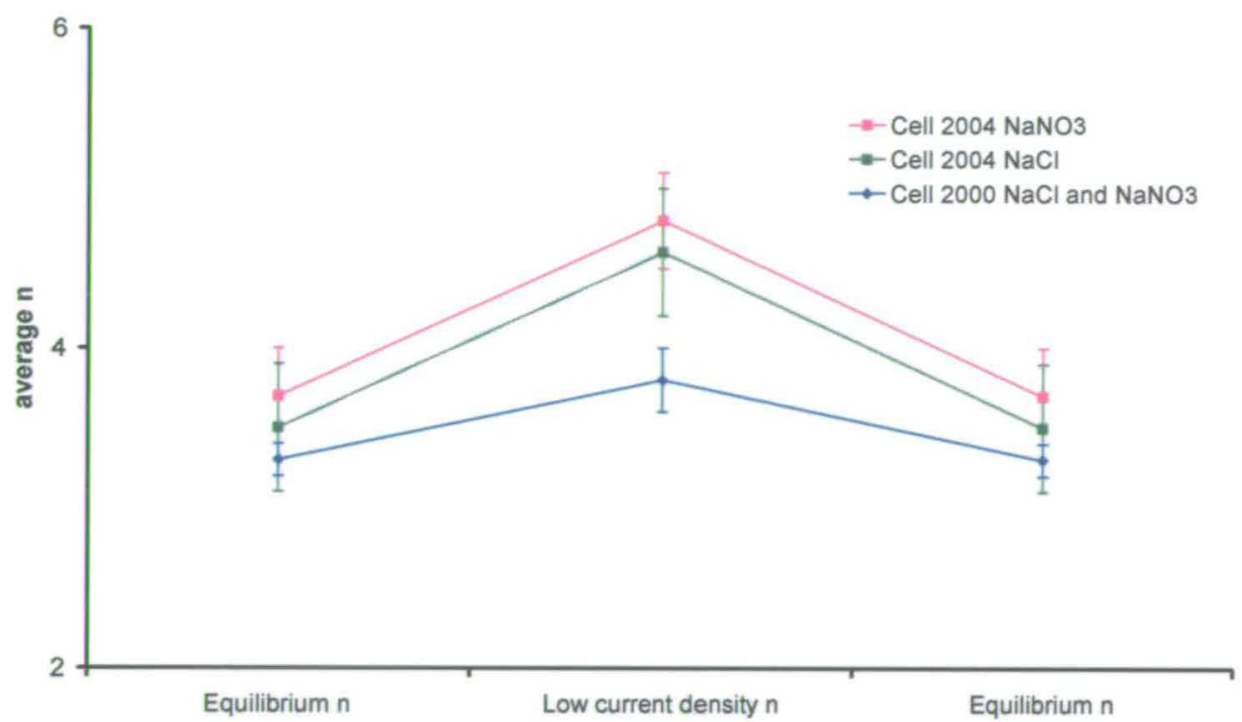

Figure 13.10: Comparison of the average equilibrium and low current density dissolution valencies for In 718 machined in $15.0 \% \mathrm{NaCl}$ and $21.7 \% \mathrm{NaNO}_{3}$ in cell 2000 and cell 2004 .

These data show that although all the measured equilibrium dissolution valencies are the same, to within experimental error, the low current dissolution valencies measured with cell 2004 are greater than those measured in cell 2000. This is consistent with either an increase in the dissolution valency or an increase in the alternative, low current density electrooxidation reaction in cell 2004. This could also explain the increased deviation of the low current density $\mathrm{J}-\mathrm{V}_{0}$ data to low $\mathrm{V}_{0}$ values for cell 2004 .

\subsection{Basic Ti6/4 parameter measurements}

Figure 13.11 shows typical chronoamperometric data for segments 1-4, and typical z-t data, for an approach/retract and re-approach (section 4.8) experiment using Ti6/4 with $15.0 \%$ $\mathrm{NaCl}$ electrolyte and an applied voltage of $\mathrm{V}=15.0 \mathrm{~V}$. 


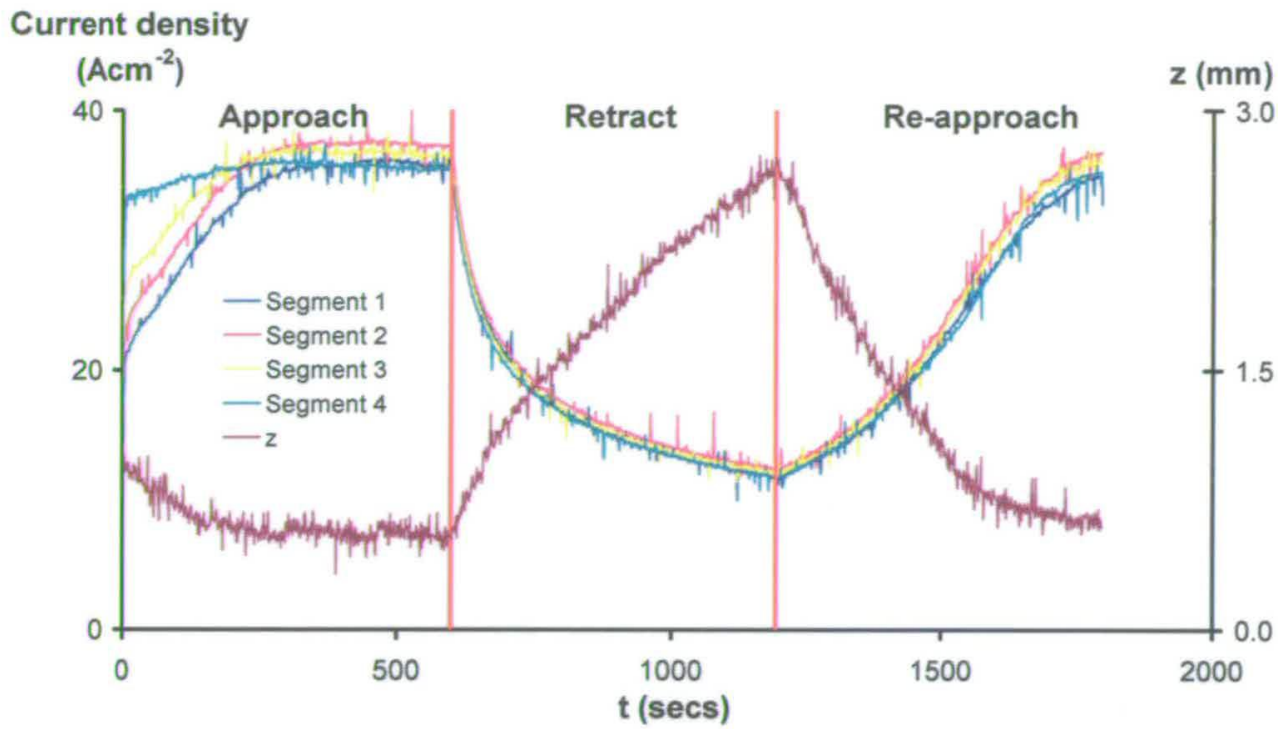

Figure 13.11: Experimental chronoamperometric transients for Ti6/4 machined in $15.0 \%$ $\mathrm{NaCl}$ with $\mathrm{V}=15.0 \mathrm{~V}$. Also shown is z-t data for the same experiment.

These data show typical $\mathrm{J}_{\infty}$ and $\mathrm{z}_{\infty}$ data for segment 3 in an approach/retract and re-approach (section 4.8) experiment of $\mathrm{J}_{\infty}=37 \pm 0.7 \mathrm{Acm}^{-2}$ and $\mathrm{z}_{\infty}=0.56 \pm 0.03 \mathrm{~mm}$. Figure 13.12 shows typical chronoamperometric and z-t data for the same material machined with $15.0 \%$ $\mathrm{NaNO}_{3}$ electrolyte and an applied voltage of $\mathrm{V}=23.0 \mathrm{~V}$. 


\section{Current}

density $\left(\mathrm{Acm}^{-2}\right)$

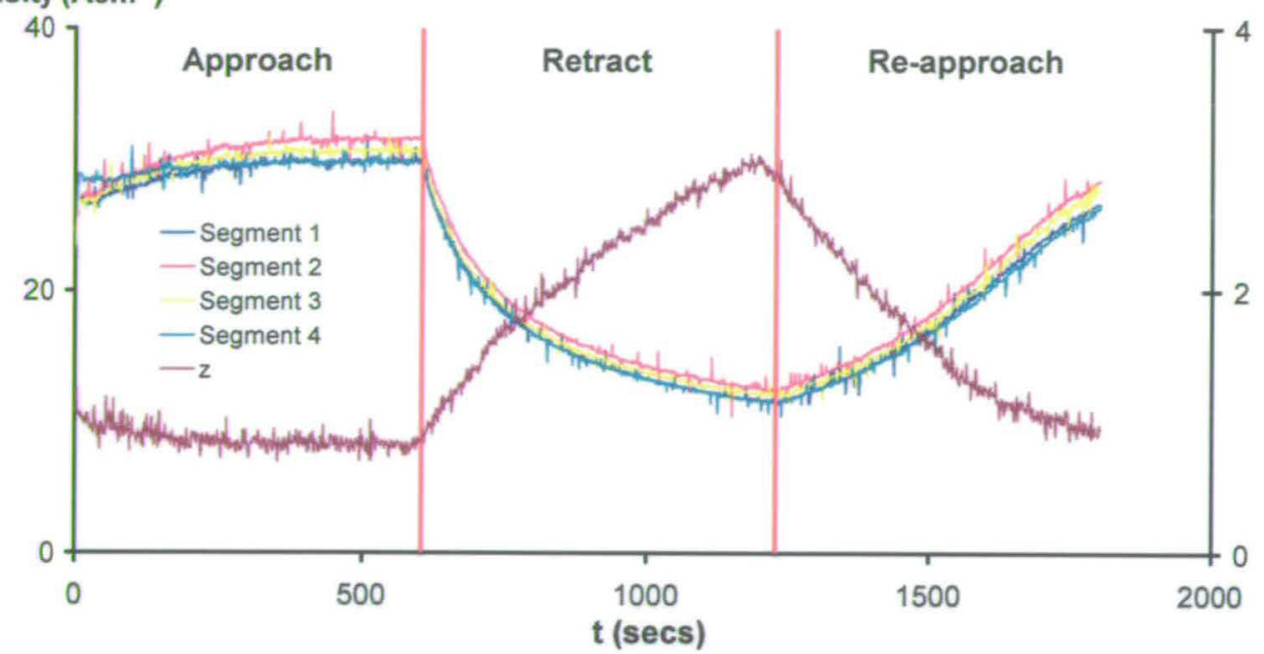

Figure 13.12: Experimental chronoamperometric transients for Ti6/4 machined in $15.0 \%$ $\mathrm{NaNO}_{3}$ with $\mathrm{V}=23.0 \mathrm{~V}$. Also shown is z-t data for the same experiment.

The equilibrium machining current and interelectrode gap for these data were found to be $J_{\infty}$ $=31 \pm 0.7 \mathrm{Acm}^{-2}$ and $\mathrm{z}_{\infty}=0.84 \pm 0.03 \mathrm{~mm}$. Figure 13.5 shows the corresponding $\mathrm{J}-\mathrm{V}_{0}$ transients for Ti6/4 machined in $\mathrm{NaCl}$ and $\mathrm{NaNO}_{3}$ with cell 2004 . 


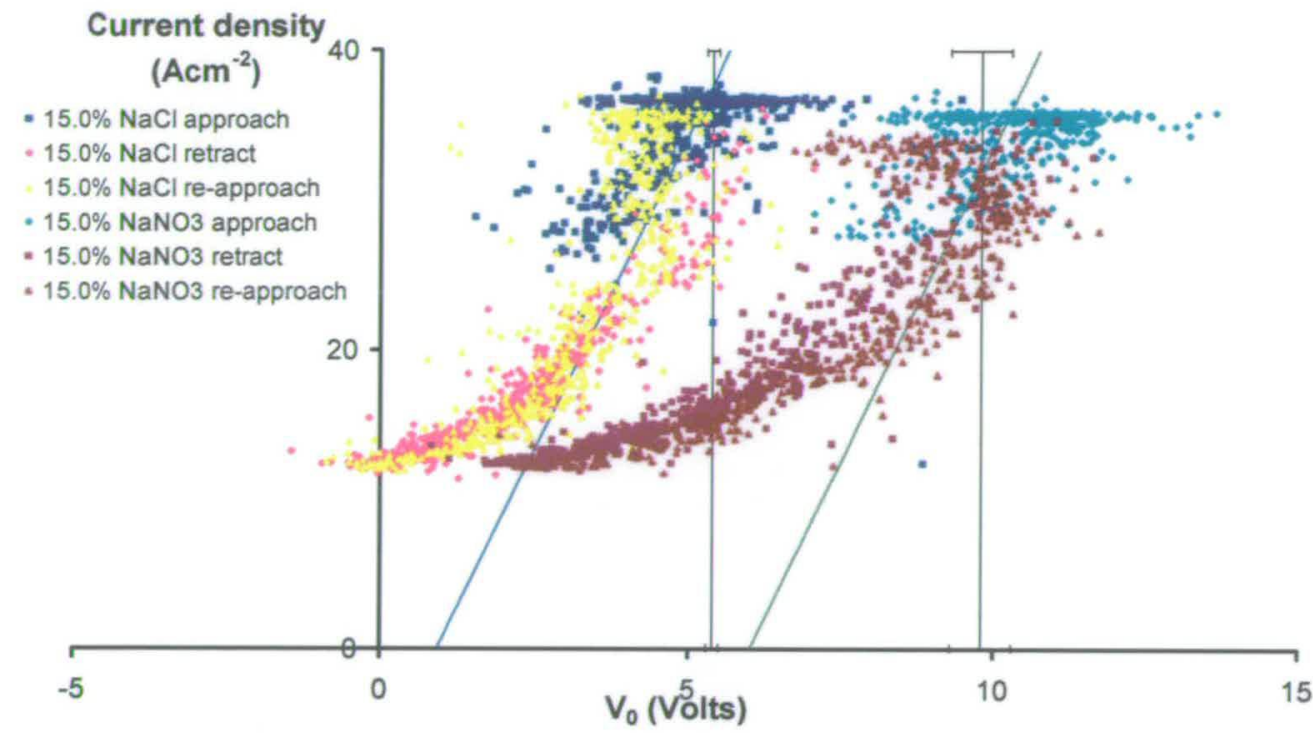

Figure 13.13: $\mathrm{J}-\mathrm{V}_{0}$ data for Ti6/4 machined in $15.0 \% \mathrm{NaCl}$ and $15.0 \% \mathrm{NaNO}_{3}$ at $\mathrm{V}=15.0$ $\mathrm{V}$ and $\mathrm{V}=23.0 \mathrm{~V}$ respectively. Time-averaged $\mathrm{V}_{0}$ values for Ti6/4 found through the chronoamperometric analysis in section 3.2 as $\mathrm{V}_{0}=5.4 \pm 0.1 \mathrm{~V}$ and $\mathrm{V}_{0}=9.8 \pm 0.5 \mathrm{~V}$ for $\mathrm{NaCl}$ and $\mathrm{NaNO}_{3}$ electrolytes respectively are given [137]. For comparison, the linear least square fits of the retract $\mathrm{J}-\mathrm{V}_{0}$ data for the same conditions in cell 2000 , with ohmic resistances of $0.12 \pm 0.01 \Omega \mathrm{cm}^{-2}$ and $\mathrm{V}_{0}$ axis intercepts of $0.9 \pm 0.2 \mathrm{~V}$ and $6.0 \pm 0.2 \mathrm{~V}$ for $\mathrm{NaCl}$ and $\mathrm{NaNO}_{3}$ (green and blue) respectively are given.

As with In718 (section 13.3), these data clearly show the similarities between cell 2000 and cell 2004 when machining Ti6/4 (Figure 10.3 and Figure 13.13). Machining Ti6/4 in cell 2004 gives mean $\mathrm{J}-\mathrm{V}_{0}$ characteristics closely corresponding to those previously obtained using a chronoamperometric method, consistent with the machining of Ti6/4 in cell 2000. For closer examination of the $\mathrm{J}-\mathrm{V}_{0}$ differences between the two cells, Figure 13.14 and Figure 13.15 show the comparisons between $\mathrm{J}-\mathrm{V}_{0}$ transients collected in cell 2000 and 2004 for $15.0 \% \mathrm{NaCl}$ and $15.0 \%$ and $21.7 \% \mathrm{NaNO}_{3}$ respectively. 


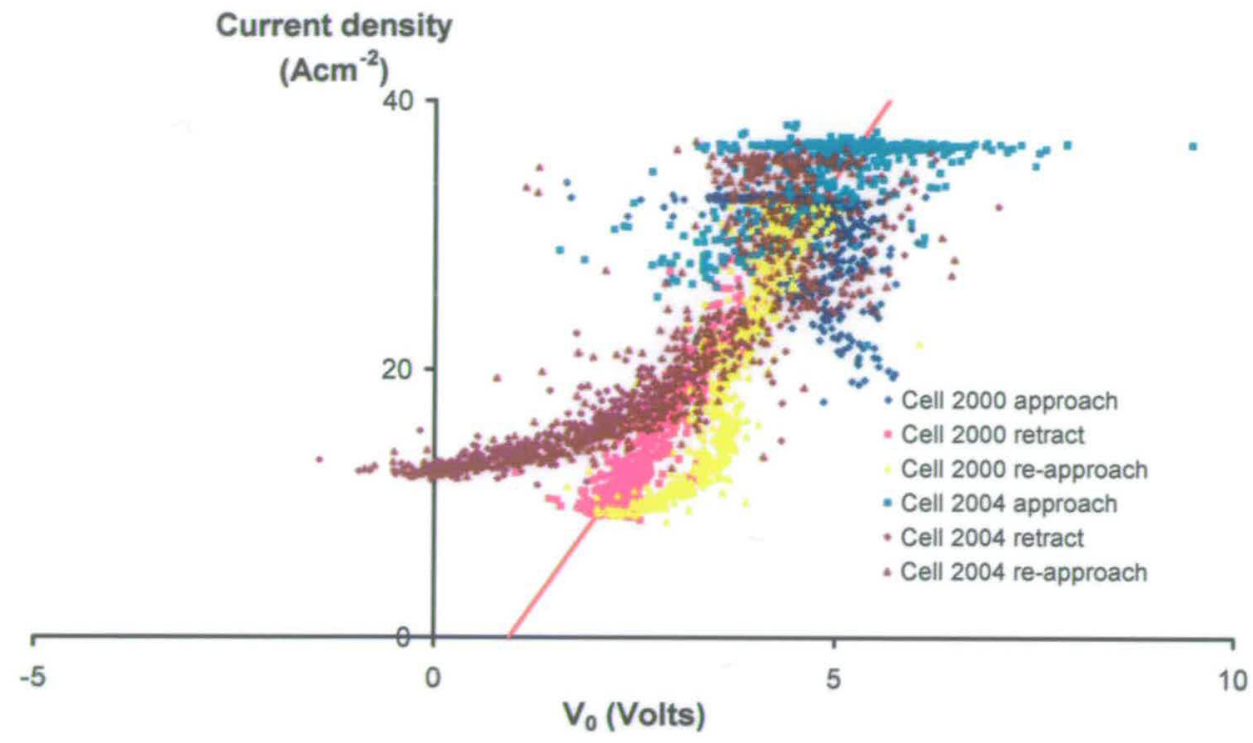

Figure 13.14: Comparison of the $\mathrm{J}-\mathrm{V}_{0}$ characteristics for Ti6/4 machined in $15.0 \% \mathrm{NaCl}$ at $\mathrm{V}=15.0 \mathrm{~V}$ in cell 2000 and cell 2004. Also shown is the theoretical line for cell 2000, calculated from Equation 7.2. For comparison, the linear least squares fit of the retract $J-V_{0}$ data for cell 2000, with ohmic resistances of $0.12 \pm 0.01 \Omega \mathrm{cm}^{-2}$ and $\mathrm{V}_{0}$ axis intercepts of 0.5 $\pm 0.2 \mathrm{~V}$ are given. 


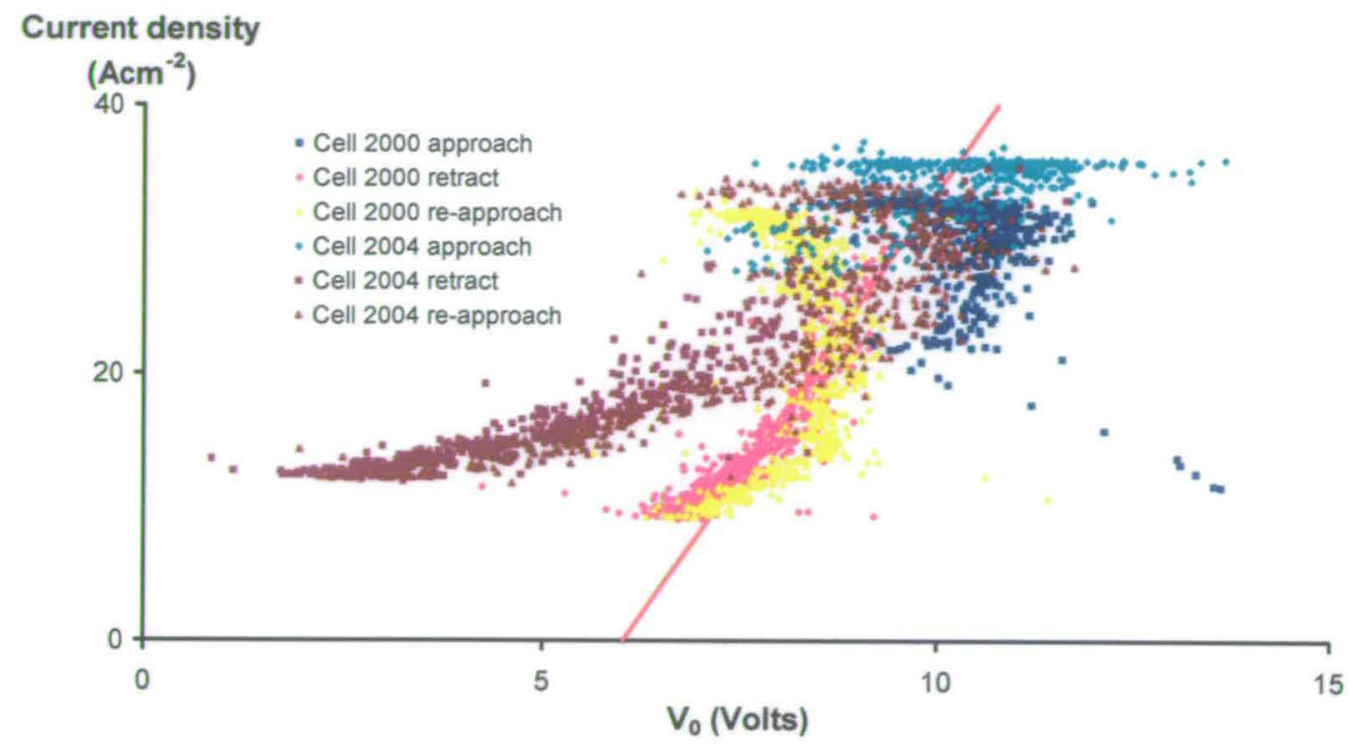

Figure 13.15: Comparison of the $\mathrm{J}-\mathrm{V}_{0}$ characteristics for Ti6/4 machined in $21.7 \% \mathrm{NaNO}_{3}$ at $\mathrm{V}=20.0 \mathrm{~V}$ in cell 2000 and $15.0 \% \mathrm{NaNO}_{3}$ at $\mathrm{V}=23.0 \mathrm{~V}$ in cell 2004 . Also given is the theoretical line for cell 2000, calculated from Equation 7.2. Finally, for comparison, the linear least squares fit of the retract $\mathrm{J}-\mathrm{V}_{0}$ data for cell 2000 , with ohmic resistances of $0.12 \pm$ $0.01 \Omega \mathrm{cm}^{-2}$ and $\mathrm{V}_{0}$ axis intercepts of $6.0 \pm 0.2 \mathrm{~V}$ are shown.

As with $\operatorname{In} 718$ (section 13.3), the curvature of the $\mathrm{J}-\mathrm{V}_{0}$ transients measured in cell 2004 makes fitting the data with the linear least squares method problematic. Consequently, the cell 2004 data is compared to the retract fits calculated using the cell 2000 data. It is clear from Figure 13.14 and Figure 13.15 that the high current density $\mathrm{J}-\mathrm{V}_{0}$ data collected with cell 2004 is broadly similar to the data collected with cell 2000 . The low current density data, however, is observed to trend to lower $\mathrm{V}_{0}$ values, consistent with the low current density $\mathrm{J}$ $\mathrm{V}_{0}$ data collected for In718 in cell 2004. An increase in observed dissolution valency has also been seen at low current densities when machining Ti6/4 in cell 2004 (Figure 13.16 and Figure 13.17). 


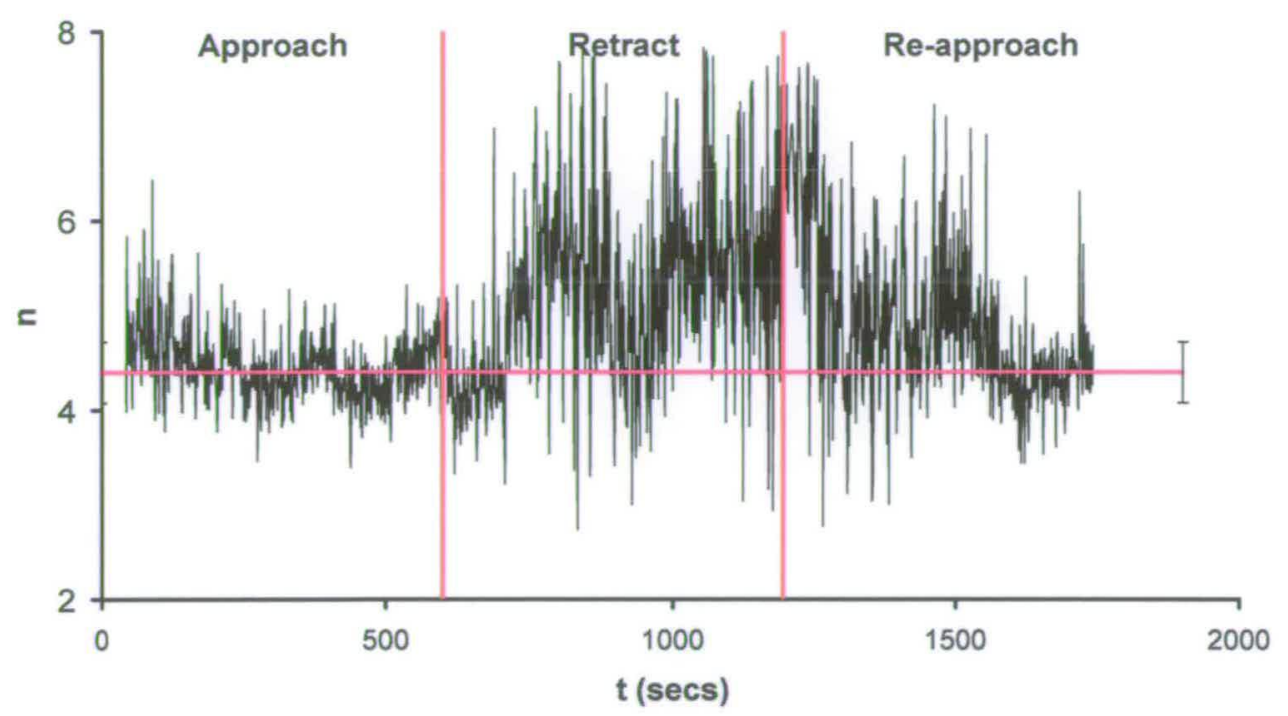

Figure 13.16: Experimental time-resolved $n$, with $p=100$ from Equation 6.4, for Ti6/4 machined in $15.0 \% \mathrm{NaCl}$, the pink line depicts the average dissolution valency at equilibrium with an error shown.

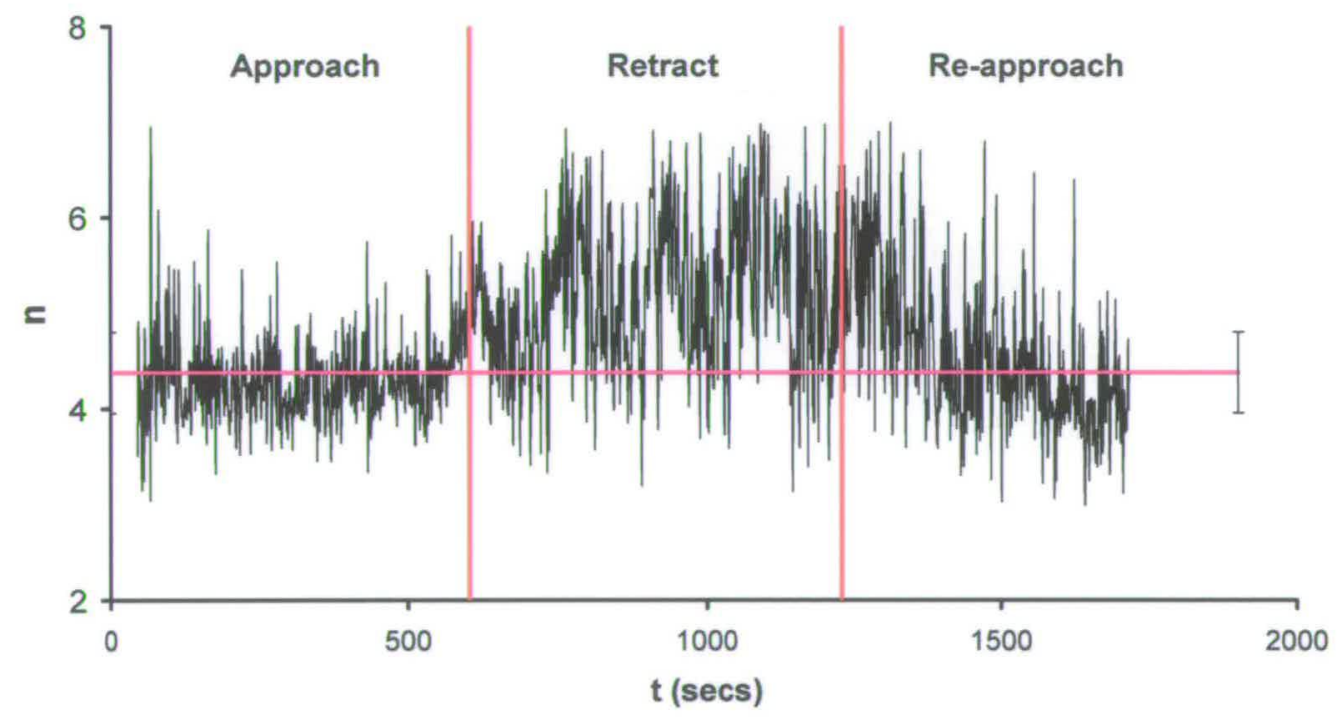

Figure 13.17: Experimental time-resolved $n$, with $p=100$ from Equation 6.4, for Ti6/4 machined in $15.0 \% \mathrm{NaNO}_{3}$, the pink line depicts the average dissolution valency at equilibrium with an error shown. 
Figure 13.16 and Figure 13.17 clearly show similar valency trends, with increased valency at low current densities, to those seen when machining Ti6/4 in cell 2000. The equilibrium dissolution valencies for Ti6/4 was found to be $\mathrm{n}=4.4 \pm 0.3$ and $\mathrm{n}=4.2 \pm 0.3$ for $15.0 \%$ $\mathrm{NaCl}$ and $15.0 \% \mathrm{NaNO}_{3}$ increasing to $\mathrm{n}=5.8 \pm 0.5$ and $\mathrm{n}=5.6 \pm 0.4$ for $15.0 \% \mathrm{NaCl}$ and $15.0 \% \mathrm{NaNO}_{3}$ respectively. Figure 13.10 shows a comparison of these average dissolution valencies to those collected for Ti6/4 machined in $15.0 \% \mathrm{NaCl}$ and $21.7 \% \mathrm{NaNO}_{3}$ with cell 2000 .

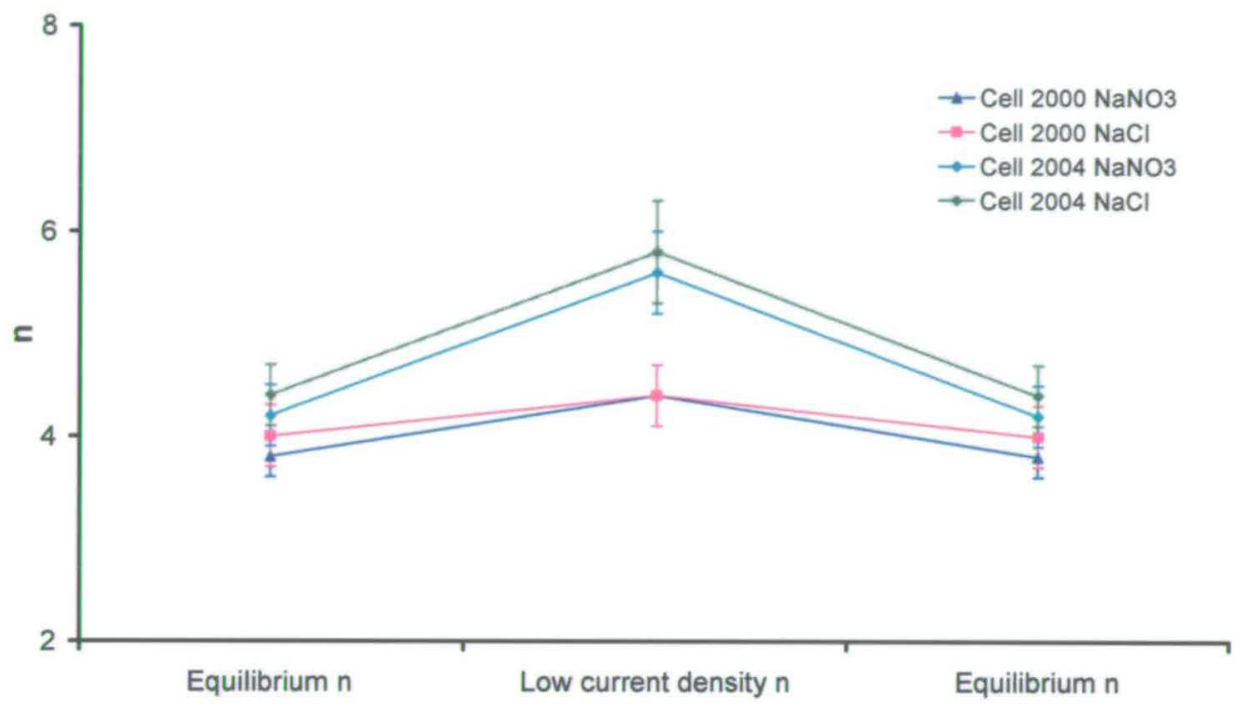

Figure 13.18: Comparison of the average equilibrium and low current density dissolution valencies for Ti6/4 machined in $15.0 \% \mathrm{NaCl}$ and $21.7 \% \mathrm{NaNO}_{3}$ with cell 2000 and $15.0 \%$ $\mathrm{NaCl}$ and $15.0 \% \mathrm{NaNO}_{3}$ with cell 2004.

These data show that although the equilibrium dissolution valencies are the same, to within experimental error, the low current dissolution valencies measured with cell 2004 are greater than those measured in cell 2000. As with In718 (section 13.3), this is consistent either with an increase in dissolution valency or an increase in the alternative, low current density electro-oxidation reaction in cell 2004. This could also explain the increased deviation of the low current density $\mathrm{J}-\mathrm{V}_{0}$ data to low $\mathrm{V}_{0}$ values for cell 2004 for Ti6/4, in the same way as for In718. 


\subsection{Cell 2004 parameterisation conclusions}

It has been established in section 13.2 that increased outlet temperatures have been observed consistent with additional heating through exothermic reactions in the interelectrode gap. The exothermic energy requirement for a temperature increase of $2.5^{\circ} \mathrm{C}$ above that expected from a simple Joule heating effect (seen in Figure 13.1 and Figure 13.2) with an electrolyte flow rate of $\mathrm{U}=8 \mathrm{~L} \mathrm{~min}^{-1}$, assuming a specific heat capacity of $\mathrm{C}=4.186 \mathrm{~J} \mathrm{~cm}^{-3} \mathrm{~K}^{-1}$, with a feed rate of $\mathrm{f}=0.50 \mathrm{~mm} \mathrm{~min}^{-1}$ for $\operatorname{In} 718$ is $\mathrm{E}=2000 \mathrm{~kJ} \mathrm{~mol}^{-1}$. Possible exothermic reactions occurring in the electrolyte when machining In718 are the formation of nickel, iron or chromium oxides or hydroxides (e.g. $\mathrm{NiO}, \mathrm{Ni}(\mathrm{OH})_{2}, \mathrm{Fe}_{2} \mathrm{O}_{3}, \mathrm{Fe}_{3} \mathrm{O}_{4}, \mathrm{Fe}(\mathrm{OH})_{2}, \mathrm{Fe}(\mathrm{OH})_{3}, \mathrm{CrO}_{3}$ or $\mathrm{Cr}_{2} \mathrm{O}_{3}$ ) from the dissolution products. The enthalpies of formation for these possible products are found in Table 13.1.

\begin{tabular}{|c|c|}
\hline Possible product & Enthalpy of formation $/ \mathbf{k J ~ m o l}^{-1}$ \\
\hline $\mathrm{NiO}$ & -244.3 \\
\hline $\mathrm{Ni}(\mathrm{OH})_{2}$ & -678.2 \\
\hline $\mathrm{Fe}_{2} \mathrm{O}_{3}$ & -822.2 \\
\hline $\mathrm{Fe}_{3} \mathrm{O}_{4}$ & -1117.1 \\
\hline $\mathrm{Fe}(\mathrm{OH})_{2}$ & -568.2 \\
\hline $\mathrm{Fe}(\mathrm{OH})_{3}$ & -824.2 \\
\hline $\mathrm{CrO}_{3}$ & -589.5 \\
\hline $\mathrm{Cr}_{2} \mathrm{O}_{3}$ & -1128.4 \\
\hline
\end{tabular}

Table 13.1: Enthalpies of formation of possible ECM products formed in electrolyte [146].

It is clear that these enthalpies of formation do not totally explain the temperature increases, above those expected for the Joule heating effect, observed in Figure 13.1 and Figure 13.2. This may be explained through further exothermic reactions such as the reduction of metal dissolution ions by hydrogen gas produced at the tool.

The comparisons between machining In718 and Ti6/4 in cell 2000 and cell 2004 have been summarised in Table 13.2. 


\begin{tabular}{|c|c|c|c|c|c|c|c|c|c|}
\hline & $\begin{array}{c}\text { Electrolyte } \\
\text { type }\end{array}$ & Cell & $\operatorname{Conc}^{n} / \%$ & V / Volts & $\begin{array}{c}\mathrm{z}_{\infty} \pm 0.03 / \\
\mathrm{mm}\end{array}$ & $\mathrm{n}_{\infty}$ & $\begin{array}{c}\text { Low } \\
\text { current } \\
\text { density n }\end{array}$ & \begin{tabular}{|c|} 
Ohmic \\
resistance \pm \\
$0.01 / \Omega \mathrm{cm}$ \\
2
\end{tabular} & $\begin{array}{c}\mathrm{V}_{0} \text { at } \mathrm{J}= \\
0.0 \mathrm{~A} \mathrm{~cm}^{-2} \\
\pm 0.1 / \mathrm{V}\end{array}$ \\
\hline \multirow{4}{*}{ In718 } & \multirow{2}{*}{$\mathrm{NaCl}$} & 2000 & 15.0 & 13.0 & 0.43 & $3.3 \pm 0.1$ & $3.8 \pm 0.2$ & \multirow{4}{*}{0.12} & \multirow{2}{*}{$0.5 \pm 0.2$} \\
\hline & & 2004 & 15.0 & 13.0 & 0.57 & $3.5 \pm 0.4$ & $4.6 \pm 0.4$ & & \\
\hline & \multirow{2}{*}{$\mathrm{NaNO}_{3}$} & 2000 & 21.7 & 16.0 & 0.38 & $3.3 \pm 0.1$ & $3.8 \pm 0.2$ & & \multirow{2}{*}{$2.9 \pm 0.2$} \\
\hline & & 2004 & 21.7 & 16.0 & 0.50 & $3.7 \pm 0.3$ & $4.8 \pm 0.3$ & & \\
\hline \multirow{4}{*}{ Ti6/4 } & \multirow{2}{*}{$\mathrm{NaCl}$} & 2000 & 15.0 & 15.0 & 0.63 & $4.0 \pm 0.2$ & $4.4 \pm 0.3$ & \multirow{4}{*}{0.11} & \multirow{2}{*}{$0.9 \pm 0.2$} \\
\hline & & 2004 & 15.0 & 15.0 & 0.56 & $4.4 \pm 0.3$ & $5.8 \pm 0.5$ & & \\
\hline & \multirow{2}{*}{$\mathrm{NaNO}_{3}$} & 2000 & 21.7 & 20.0 & 0.60 & $3.7 \pm 0.2$ & $4.4 \pm 0.3$ & & \multirow{2}{*}{$6.0 \pm 0.2$} \\
\hline & & 2004 & 15.0 & 23.0 & 0.84 & $4.2 \pm 0.3$ & $5.6 \pm 0.4$ & & \\
\hline
\end{tabular}

Table 13.2: Table summarising parameter measurements taken for In 718 and Ti6/4 using Cell 2004.

It is satisfying that both cell 2000 and cell 2004 show broadly consistent high current density $\mathrm{J}-\mathrm{V}_{0}$ characteristics in both electrolytes for In718 and Ti6/4 with broadly consistent ohmic dependence and $\mathrm{V}_{0}$ axis intercepts to within experimental error. This implies active and passive dissolution mechanisms for $\mathrm{NaCl}$ and $\mathrm{NaNO}_{3}$ respectively in both electrolytes and the presence of a high current density, rate determining surface salt film, as seen with cell 2000 (Chapter 11). The results in this chapter show that the flow path length and flow rate decrease in cell 2004 has had little effect on the $\mathrm{J}-\mathrm{V}_{0}$ characteristics for In 718 and Ti6/4 for high current densities. This is re-assuring as this lack of dependence on cell characteristics greatly simplifies the ECM parameter base for these two materials when machining at high current densities.

However, Table 13.2 shows that while the equilibrium dissolution valencies between the two cells are the same to within experimental error, the dissolution valencies at low current densities for cell 2004 are larger than those measured with cell 2000. It is interesting that this increase in dissolution valency occurs for all materials studied in both cell set-ups in this work and occurs to a greater extent in cell 2004. This is consistent with an increase in the low current density oxygen evolution suppressed at high current densities by dehydration of the electrolyte in the vicinity of the anode, i.e. the formation of a surface salt film (Chapter 11) [58]. The increased low current density effects observed for cell 2004 imply an increase in the limiting current density for the formation of the surface salt film, which would be expected to result from a decrease in diffusion layer thickness (Figure 11.1). However, from theoretical calculations (Equation 11.2) the diffusion layer thickness would be expected to 
remain the same. This indicates that the diffusion layer thickness may differ from the theoretical predictions for cell 2004; further experiments are required to quantify this effect. 


\section{Chapter 14}

Final Conclusions 


\subsection{Conclusions}

The following conclusions can be made from the work in this thesis:

1. This experimental system can be used to collect electrochemical data under ECM conditions relevant to industry.

While the literature shows a number of high current density studies of the ECM of nickel and titanium, the high flow rates, common in industrial ECM, are rarely used in these studies. This thesis has shown significant differences between these parameter studies and those relating to industrial ECM due to conductivity variations along the flow path length resulting from electrolyte temperature changes (section 9.3.2.2). Furthermore, the absolute anode potentials are rarely measured accurately due to large iR drops present in the electrolyte at the high current densities used for ECM studies. The system developed in this thesis has been shown to be capable of ECM studies at high flow rates with the ability for timeresolved surface anode potential measurements throughout machining (Chapter 7, Chapter 8, Chapter 9, Chapter 10 and Chapter 13).

2. The addition of thermocouples to the ECM system at the cell inlet and outlet have allowed the correction of the electrolyte conductivity data for Joule heating and allowed observation of exothermic reactions in the interelectrode gap.

Joule heating of the electrolyte causes changes in the electrolyte conductivity during machining. These changes were corrected for through the addition of thermocouples at the cell inlet and outlet allowing electrolyte temperature measurements (section 8.2). In cell 2004 the experimental outlet temperature was greater than that predicted by Joule heating, due to exothermic reactions in the interelectrode gap (Chapter 13). This observation shows the possibility of monitoring exothermic reactions in this way. Further studies are required to establish the exact exothermic reactions occurring during ECM.

3. Cell 2000 has been used to analyse $z, n$ and $V_{0}$ characteristics during the ECM of $\mathrm{Fe}$, SS410, Jethete, In 718 and Ti6/4 under a variety of machining conditions. 
Parameterisation of Electrochemical Machining

A critical comparison between the ultrasound analysis used in this thesis and a previous chronoamperometric analysis for this cell has proved the efficacy and enhanced accuracy of the ultrasound analysis, along with its potential for time-resolved measurement. Consequently cell 2000 has been used to demonstrate variations in $\mathrm{n}$ and $\mathrm{V}_{0}$ during ECM with a planar segmented tool for the materials Fe (Chapter 7), SS410, Jethete in $\mathrm{NaCl}$ (Chapter 8) and In718 (Chapter 9) and Ti6/4 (Chapter 10) in $\mathrm{NaCl}$ and $\mathrm{NaNO}_{3}$. The machining characteristics at various electrolyte concentrations and flow rates have been investigated.

4. Cell 2004 has been used to assess the feasibility of direct gap measurements using an ultrasound probe positioned behind the tool.

An initial assessment of the feasibility of direct interelectrode gap measurements from behind the tool was made (Chapter 5). Although this was not possible using the current system, measurements could be made in the future with an adapted ECM system.

5. Cell 2004 has been used with the ultrasound probe positioned behind the workpiece to characterise $\mathbf{z}, \mathbf{n}$ and $\mathbf{V}_{0}$ characteristics during ECM in a longer flow path length cell.

The ultrasound set-up with the probe measuring the thickness of the workpiece was used with a longer flow path length cell (cell 2004) to determine the ECM characteristics for In718 and $\mathrm{Ti} 6 / 4$ in $\mathrm{NaCl}$ and $\mathrm{NaNO}_{3}$ electrolytes (Chapter 13). These were shown to be broadly consistent with the shorted flow path length (cell 2000) at high current densities, but to show significant variations at low current density. The data provide an insight into the surface salt film formation and characteristics in different ECM systems. Further studies are required with this cell configuration to probe the exact mechanism of formation and characteristics of the surface salt film in cell 2004.

6. The ECM dissolution characteristics of the materials studied have been shown to be consistent with either active or passive dissolution processes in $\mathrm{NaCl}$ and $\mathrm{NaNO}_{3}$ electrolytes.

When machining in $\mathrm{NaCl}$, the $\mathrm{I}-\mathrm{V}_{0}$ data for all the materials studied were consistent with an active dissolution mechanism. In contrast, when machining in $\mathrm{NaNO}_{3}$, the $\mathrm{I}-\mathrm{V}_{0}$ data for all the materials studied were consistent with a passive dissolution process (Chapter 7, Chapter 
Parameterisation of Electrochemical Machining

8, Chapter 9, Chapter 10 and Chapter 13). These observations are consistent with previous literature studies into the dissolution properties of pure iron, stainless steels, nickel and titanium.

\section{The I- $\mathrm{V}_{0}$ characteristics were shown to have an ohmic dependence.}

For all the materials studied the I- $\mathrm{V}_{0}$ data showed an ohmic dependence (Chapter 7, Chapter 8, Chapter 9, Chapter 10 and Chapter 13). In both electrolytes the ohmic I- $\mathrm{V}_{0}$ dissolution process has been attributed to the presence of a rate determining surface salt film. The resistance of this salt film was found to be independent of electrolyte type and broadly independent of workpiece materials type, consistent with similar salt saturation concentrations expected in all these cases. Additional studies into materials for which the surface salt films have different saturation concentrations would provide further insight into surface salt film formation during ECM.

\section{High observed dissolution valencies were seen at low current densities in all the experiments conducted in this work.}

The increased dissolution valency observed for stainless steels at low current density has been attributed to either preferential low current density dissolution of $\mathrm{Cr}$ (III) or an alternative electro-oxidation reaction occurring in the cell at low current densities (Chapter 8). Since all the materials studied in this work displayed similar low current density increases in observed dissolution valency (Chapter 7, Chapter 9, Chapter 10 and Chapter 13), which cannot be explained at preferential low current density $\mathrm{Cr}$ (III) dissolution, it is most likely that in all cases this low current density behaviour can be attributed to an alternative electro-oxidation reaction. The data presented in this thesis are all consistent with studies by Landolt showing this inefficiency most likely due to oxygen evolution at low current density suppressed at high current densities by dehydration of the electrolyte in the vicinity of the anode and the formation of a surface salt film (see conclusion 7). 


\section{Appendix 1}

Derivation of chronoamperometric theory

$$
\begin{aligned}
& \frac{d z}{d t}=k\left(\frac{1}{z}-\frac{1}{z_{\infty}}\right) \\
& \text { as }\left(\frac{1}{z}-\frac{1}{z_{\infty}}\right)=\frac{z_{\infty}-z}{z z_{\infty}} \\
& \frac{d z}{d t}=k\left(\frac{z_{\infty}-z}{z z_{\infty}}\right) \\
& \therefore \int_{z_{0}}^{c} \frac{z z_{\infty}}{z_{\infty}-z} d z=\int_{0} k d t \\
& \Rightarrow \int_{z_{0}}^{e} \frac{z z_{\infty}}{z_{\infty}-z} d z=[k t]_{t_{0}}^{t} \\
& \Rightarrow \int_{z_{0}}^{e} \frac{z}{z_{\infty}-z} d z=\frac{k}{z_{\infty}}\left(t-t_{0}\right) \\
& \text { as } \mathrm{t}_{0}=0 \Rightarrow \frac{k}{z_{\infty}}\left(t-t_{0}\right)=\frac{k t}{z_{\infty}}
\end{aligned}
$$

substituting in $\int_{z_{0}}^{e} \frac{z}{z_{\infty}-z} d z$

let $u=\mathrm{z}_{\infty}-z$

$\therefore \frac{d u}{d z}=-1$ and $d z=-d u$

$\therefore \int_{z_{0}}^{e} \frac{z}{z_{\infty}-z} d z=-\int \frac{-u+z_{\infty}}{u} d u=\int 1-\frac{z_{\infty}}{u} d u$

$=u-z_{\infty} \ln u$

re - substituing for $u=\mathrm{z}_{\infty}-z$

$$
\int_{z_{0}}^{e} \frac{z}{z_{\infty}-z} d z=\left[\left(\mathrm{z}_{\infty}-z\right)-z_{\infty} \ln \left(\mathrm{z}_{\infty}-z\right)\right]_{z_{0}}^{z}
$$

applying limits

$$
\int_{z_{0}}^{e} \frac{z}{z_{\infty}-z} d z=z_{0}-z+z_{\infty} \ln \frac{\left(z_{\infty}-z_{0}\right)}{\left(z_{\infty}-z\right)}
$$

$\therefore$ overall

$$
\begin{aligned}
& \int_{z_{0}}^{e} \frac{z z_{\infty}}{z_{\infty}-z} d z=\int_{0}^{k} k d t \\
& \text { solves to give } \frac{z_{0}-z}{z_{\infty}}+\ln \frac{\left(\mathrm{z}_{\infty}-z_{0}\right)}{\left(z_{\infty}-z\right)}=\frac{\mathrm{k}}{z_{\infty}^{2}}\left(\mathrm{t}-\mathrm{t}_{0}\right)
\end{aligned}
$$




\section{References}

\section{Chapter 1}

1. Rolt L. T. C., Tools for the Job, Charles Churchill and Company Ltd, 1965, p12

2. De Barr, A. E., Oliver, D. A., Electrochemical Machining, MacDonald and Co, 1968

3. Ezugwu E. O., Wang Z. M., Journal of Materials Processing Technology, 68, 1997, 262

4. Metalcutting, American Machinist, McGraw Hill Publications Company, 1979, p 182

5. Ezugwu E. O., Bonney J., Yamane Y., Journal of Materials Processing Technology 134, 2003,233

6. Arunachalam R. M., Mannan M. A., Spowage A. C., International Journal of Machine Tools and Manufacture, 44, 2004, 1481-1491

7. Miller S., Interdisciplinary Sci. Rev. 21(2), 1996, 117-129

8. Dexter S. C., Aquacultural Engineering, 5(2-4), 1986, 333-346

9. Davies P. A., Dunstan G. R., Howells R. I. L., Hayward A. C., Metal Powder Report, 59(10), 2004, 14-19

10. Choudhury I. A., El-Baradie M. A., Journal of Materials Processing Technology, 77, $1998,278-284$

11. Kalpakjian S., Manufacturing Processes for Engineering Materials, $2^{\text {nd }}$ Edition, AddisonWesley, Reading, MA, 1992

12. Dimiduk D. M., Materials Science and Engineering A 263, 1999, 281-288

13. Honnarat Y., Materials Science and Engineering A 213, 1996, 115

14. Clifton D., Mount A. R., Jardine D. J., Roth R., Journal of Materials Processing Technology, 108, 2001, 338-348

15. Fergus W. J., Materials Science and Engineering A, 338, 2002, 108

16. Yoshihara M., Miura K., Intermetallics, 3, 1995, 357-363

17. Chen G., Sun Z., Zhou X., Materials Science and Engineering A 152, 1992, 597

18. Mantle A. L., Aspinwall D. K., Journal of Materials Processing Technology, 118, 2001, $143-150$

19. Bentley S. A., Mantle A. L., Aspinwall D. K., Intermetallics, 7, 1999, 967

20. Kadolkar P., Dahotre N. B., Applied Surface Science, 199, 2002, 222-233

21. Lin T-R., Journal of Materials Processing Technology, 127, 2002, 8-16

22. König U., Surface and Coatings Technology, 33, 1987, 91-103

23. Grzesik W., Nieslony P., Wear, 256, 2004, 108-117 
The Parameterisation of Electrochemical Machining

24. Constable C. P., Lewis D. B., Yarwood J., Münz W. -D., Surface Coatings and Technology, 184, 2004, 291-297

25. Kelly P. J., Arnell R. D., Ahmed W., Afzal A., Materials and Design, 17, 1997, 215-219

26. Choy K. L., Progress in Materials Science, 48, 2003, 57-170

27. Hanyu H., Kamiya S., Odagi H., Murakami Y., Saka M., Thin Solid Films, 413, 2002, 139

28. Hanyu H., Murakami Y., Kamiya S., Saka M., Surface and Coatings Technology, 169$170,2003,258-261$

29. Sun F. H., Zhang Z. M., Chen M., Shen H. S., Journal of Materials Processing Technology, 129, 2002, 435

30. Saijo K., Surface Coating Technology, 43-44(1), 1990, 30-40

31. Nesladek M., Vandierendonck K., Thin Solid Films, 270(1-2), 1995, 184-188

32. www.azom.com/details.asp?ArticleID=1264\#_High_Speed_Steels

33. Paul S., Chattopadhyay A. B., International Journal of Machine Tools and Manufacture, 36(1), 1996, 63-72

34. Hong S. Y., Markus I., Joeng W. -C., International Journal of Machine Tools and Manufacture, 41, 2001, 2245-2260

35. Paul S., Dhar N. R., Chattopadhyay A. B., Journal of Materials Processing Technology, $116,2001,44-48$

36. Liu Z. Q., Ai. X., Zhang H., Wang Z. T., Wan Y., Journal of Materials Processing Technology, 129, 2002, 222-226

37. Dudzinski D., Devillez A., Moufki A., Larrouquère D., Zerrouki V., Vigneau J., International Journal of Machine Tools and Manufacture, 44, 2004, 439

38. www.metals.about.com/library/bldef-Work_Hardening.htm

39. Del Valle J. A., Picasso A. C., Romero R., Acta Metallurgica, 46, 1998, 1981-1988

40. Sharman A., Dewes R. C., Aspinwall D. K., Journal of Materials Processing Technology, $118,2001,29-35$

41. Reichelt K., Jiang X., Thin Solid Films, 191, 1990, 91-126

42. Jindal P. C., Santhanam A. T., Schleinkofer U., Shuster A. F., International Journal of Refractory Metals and Hard Materials, 17, 1999, 163-170

43. Kramer B. M., J. Eng. Ind. 109, 1987, 87-91

44. Kertesz J., Pryor R. J., Richerson D. W., Cutler R. A., J. Met., 40(5), 1988, 50-51

45. Weller E. J., Nontraditional Machining Processes, second edition, Society of Manufacturing Engineers Publications, 1984 
The Parameterisation of Electrochemical Machining

46. Wakuda M., Yamauchi Y., Kanzaki S., Journal of Materials Processing Technology, 132, $2003,177-183$

47. McGeough J. A., Advanced Methods of Machining, Chapman and Hall, 1988

48. Lindberg, R. A., Processes and Materials of Manufacture, 1983

49. www.menke-funkenerosion.de/english/Geschichte.html

50. Ho K. H., Newman S. T., International Journal of Machine Tools and Manufacture, 43, 2003,1287

51. www.answers.com/topic/laser-acronym-1\&method $=6$

52. Riva R., Christ B., Rodrigues N. A., Trava-Airoldi V. J., Corat E. J., Proc. SPIE, 2789, 345-351

53. Borini S., Amato G., Rocchia M., Boarino L., Journal of Applied Physics, 93(8), 2003, 4439-4441

54. Pittaway L. G., British Journal of Applied Physics, 15, 1964, 967-982

55. Abate K., BMC Industries, Cortland, N. Y.

56. www.corrosion-doctors.org/Biographies/FaradayBio.htm

57. Figoux H., Jacquet P. A., French Patent 707526, 1930

58. Landolt D., Chauvy P.-F., Zinger O., Electrochimica Acta, 48, 3185, 2003

59. Wilson, J. F., Practice and Theory of Electrochemical Machining, Robert E Krieger Publishing Company, 1982

60. Howarth P., The Electrochemistry of Electrochemical Machining, PhD Thesis, University of Edinburgh, 2003

61. U.S. Environmental Protection Agency, Toxological Review of Hexavalent Chromium, CAS No. 18540-29-9, 1998

62. http://www.epa.gov/iris/toxreviews/0144-tr.pdf

63. http://www.ohcow.on.ca/resources/handbooks/chromium/Chromium.pdf

64. Directive 2000/53/EC of the European Parliament and of the Council of 18 September 2000 on end-of-life vehicles [Official Journal L269, 21.10.2000]

65. http://www.europa.eu.int/scadplus/leg/en/lvb/121225.htm

66. Standard Note SN/SC/1239, last updated 12 June 2003, End-of-Life Vehicles Directive, House of Commons Library

67. Clifton D., Process Characterisation for ECM, PhD thesis, University of Edinburgh, 2001.

68. Hewidy M. S., Jain V. K., Int. J. Prod. Res. 25, 1987, 433

69. Kozak J., Rajurkar K. P., Wei B., Trans. ASME, J. Eng. Ind., 116, 1994, 316

70. Zhu D., Xu H. Y., Journal of Materials Processing Technology, 129, 2002, 15 
The Parameterisation of Electrochemical Machining

71. Datta M., IBM Journal of Research and Development, 42(5), 1998, 655

72. Baltic Offshore Pipeline, Environmental Impact Assessment, October 2001, Recommendations for Design. Pipe Materials, Girth Welds and Non-destructive Testing.

73. Offshore Technology Report: Fatigue Performance of Repaired Tubular Joints (OTH 89 307) HMSO, ISBN 0014130965

74. Clifton D., Mill F., Esanola J., Kare R., Dover W. D., Journal of the Society for Underwater Technology, 24(4), 2000, 131

75. Disegi J. A., Injury, International Journal of the Care of the Injured, 31, 2000, S-D14-17

76. Bejar M. A., Gutiérrez F., Journal of Materials Processing Technology, 37, 1993, 691

77. Kozak J., Journal of Materials Processing Technology, 76, 1998, 170-175

78. van Osenbruggen C., de Regt C., Philips Tech. Rev., 42(1), 1985, 22-32

79. Jain V. K., Murugan S., Int. J. Prod. Res., 24(2), 1986, 439

80. Hopenfield J., Cole R. R., Transactions of the ASME, Journal of Engineering for Industry, 1966, 455

81. Pramanik D. K., Agarwal R. A., De A. K., J. of the Inst. of Engineers, 57(5), 1977, 250

82. Zhou Y., Derby J. J., Chem. Eng. Sci., 50, 1995, 2679

83. Butt R., J. Comput. Appl. Math., 47, 1993, 151

84. Landolt D., J Electrochem Soc., 119(6), 1972, 708

85. Datta M., Landolt D., Electrochimica Acta, 25, 1980, 1263

86. Hopenfield J., Cole R. R., Trans. of ASME J. Eng. Ind. B8, 1969, 755-765.

87. www.sppusa.com/reference/white_paper/wp_ss.pdf

88. Cox P. A., The Electronic Structure and Chemistry of Solids, Oxford Science Publications, 1995

89. www.azom.com/details.asp?ArticleID $=470$

90. www.jjjtrain.com/vms/engineering_metal_stds.html

91. www.angelfire.com/biz/BuildingPathology/stainless.html

92. www.normas.com/ASTM/BOOKS/DS56I.html

93. www.azom.com/details.asp?ArticleID $=1774$

94. www.worldstainless.org/what_basic.php

95. www.stainless-steel-world.net/basicfacts/app_food.asp

96. www.stainless-steel-world.net/basicfacts/app_cpog.asp

97. www.stainless-steel-world.net/basicfacts/app_abc.asp

98. www.stainless-steel-world.net/basicfacts/app_medical.asp

99. www.stainless-steel-world.net/basicfacts/app_auto.asp

100. Olsson C. -O. A., Landolt D., Electrochimica Acta, 48, 2003, 1093 
The Parameterisation of Electrochemical Machining

101. Marshall P., Austenitic Stainless Steels, Microstructure and Mechanical Properties, Elsevier Applied Science Publishers, 1984

102. Castro R., de Cadenet J. J., Welding Metallurgy of Stainless and Heat-Resistant Steels, Cambridge University Press, 1975

103. Honeycombe R. W. K., Bhadeshia H. K. D. H., Steels, Microstructure and Properties, Edward Arnold, 1995

104. www.weldingengineer.com/Stainless $\% 20$ Steel.htm

105. 2nd Edition Handbook of Corrosion Data. Edited by B.D. Craig, D.S. Anderson, ASM International, 1995

106. Keir J., Phil. Trans. Royal. Soc., 80, 1790, 359

107. Müller W. J., Trans. Faraday Soc., 27, 1931, 737

108. www.tf.uni-kiel.de/matwis/amat/def_en/kap_2/basics/b2_1_6.html

109. Smart L., Moore E., Solid State Chemistry, An Introduction, Second Edition, Stanley Thornes Ltd, 1995

110. Cornell R. M., Schwertmann U., The Iron Oxides, Structure, Properties, Reactions, Occurrence and Uses, VCH, 1996

111. Gallagher K. J., Feitknecht W, Mannweiler U, Nature, 217, 1968, 1118

112. Revie R. W., Baker B. G., Bockris J. O'M., J. Electrochem. Soc., 122, 1975, 1460

113. Olefjord I., Elfström B., -O, Corrosion 38, 1982, 46

114. Tjong S. C., Yeager E., J. Electrochem. Soc., 128, 1981, 2251

115. Schroeder V., Devine T. M., J. Electrochem. Soc., 146, 1999, 4061

116. Seo M., Sato M., Lumsden J. B., Staehle R. W., Corro. Sci., 17, 1977209

117. Murphy O. J., Bockris J. O’M., Pou T. E., Cocke D. L., Sparrow G., J. Electrochem. Soc., 129, 1982, 2149

118. Davenport A. J., Oblonsky L. J., Ryan M. P., Toney M. F., J. Electrochem. Soc., 147, 2000,2162

119. Toney M. F., Davenport A. J., Oblonsky L. J., Ryan M. P., Vitus C. M., Physical Review Letters, 79(21), 1997, 4282

120. Vesel A., Mozetič M., Zalar A., Applied Surface Science, 200, 2002, 94-103

121. Grosvenor A. P., Kobe B. A., McIntyre N. S., Surface Science, 574, 2005, 317-321

122. Montemor M. F., Ferreira M. G. S., Hakiki N. E., Da Cunha Belo M., Corrosion Science. 42, 2000, 1635-1650

123. Büchler M., Schmuki P., Böhni H., J. Electrochem. Soc., 145, 1998, 609

124. Bardwell J. A., Sproule G. I., Graham M. J., Journal of the Electrochemical Society $140,1993,50$ 
The Parameterisation of Electrochemical Machining

125. http://education.jlab.org/itselemental/ele022.html

126. Tang H., Prasad K., Sanjinès R., Schmid P. E., Lévy F., J. Appl. Phys., 75, 2042, 1994

127. Sul Y. T., Johansson C. B., Jeong Y., Albrektsson T., Medical Engineering and Physics, $23,329,2001$

128. Ohtsuka T., Guo J., Sato N., J. Electrochem. Soc., 133, 2473 (1986)

129. Blackwood D. J., Electrochimica Acta, 46, 563 (2000)

Chapter 2

130. www.psigate.ac.uk/newsite/reference/plambeck/chem2/p02081.htm

131. Fisher A. C., Electrode Dynamics, Oxford University Press, Chapter 1

132. Aitkins P. W., Physical Chemistry Fifth Edition, Oxford University Press, 1997, p834843

133. New Techniques for the Study of Electrodes and their Reaction, edited: Compton R. G, Elsevier, Chapter 6

134. www.engineeringtoolbox.com/reynolds-number-21_237.html

135. Von Kárman T., Angew Z., Math. Mech., 1, 1921, 244

136. Datta M., Landolt D., Electrochimica Acta, 25, 1980, 1255

Chapter 3

137. Mount, A. R., Eley, K. L., Clifton, D., J. of App. Electrochem., 2000, 30, 447-455

138. Jain V.K., Rajurkur, K.P., Precision Engineering, 1991, 13, 111

139. Clifton D., Mount A. R., Alder G. M., Jardine D., Int. J. Mach. Tools Manufact., 2002, $42,1259-1267$.

Chapter 4

140. Massey B. S., Mechanics of Fluids, $6^{\text {th }}$ Edition Chapman and Hall, London, 1995.

141. Douglas J. F., Gasiorek J. M., Swaffield J. A., Fluid Mechanics, $3^{\text {rd }}$ Edition, Logman, London, 1995.

142. www.flow-control.globalspec.com/LearnMore/Flow_Control_Flow_Transfer/Pumps/Po sitive_Displacement_Pumps

143. Mount A. R., Howarth P. S., Clifton D., J Electrochem Soc., 2003, 150(3), D63 
Chapter 5

144. www.van.hep.uiuc.edu/van/qa/section/States_of_Matter_and_Energy/Dissolving/ 20030404181819.htm

145. Grenthe I., Spahiu K., Plyasunov A. V., Modelling in Aquatic Chemistry, OECD Publications, 1997, Chapter 9

146. Handbook of Chemistry and Physics, $84^{\text {th }}$ Edition, $2003-2004$, CRC Press, $14-42$

147. www.panametrics-ndt.com/ndt/ndt-technology/ultrasound_flaw_detection.html

148. Lynnworth L. C., IEEE Transactions on Sonics and Ultrasonics, Vol. SU=22 (2), 1975

Chapter 6

149. Hopenfield J., Cole R. R., Trans. Of ASME J. Eng. Ind. B8, 1969, 755-765.

150. Bejar M. A., Gutierrez F., J. Mater. Processing Tech. 37, 1993, 691-699.

\section{Chapter 7}

151. Lohrengel M. M., Klüppel I., Rosenkranz C., Bettermann H., Schultze J. W., Electrochimica Acta., 48, 3203 (2003).

152. Lohrengel M. M., Rosenkranz C., Corrosion Science, 47, 2005, 785

153. Haisch T., Mittemeijer E., Schultze J. W., Electrochimica Acta, 47, 2001, 235

154. Hoare J. P., LaBoda M. A., McMillan M. L., Wallace A. J., J. Electrochem. Soc., 116, 1969,199

155. Harb J. N., J. Electrochem. Soc., 133, 1986, 439C

Chapter 8

156. http://biomed.tamu.edu/biomaterials/Metals.htm

157. Yong L., Yunfei Z., Guang Y., Liangqiang P., Sensors and Actuators A, 108, 2003, 144

158. Kirchner V., Cagnon L., Schuster R., Ertl G., Applied Physics Letters, 79(11), 2001, 1721

159. Mao K. -W., J. Electrochem. Soc., 118, 1971, 1876

160. Chin D. T., Wallace A. J., J. Electrochem. Soc., 120, 1973, 1487

161. Mileham A. R., Jones R. M., Harvey S. J., Precision Engineering, 4(3), 1982, 168 
The Parameterisation of Electrochemical Machining

162. Mount A. R., Clifton D., Howarth P., Sherlock A., Journal of Materials Processing Technology, 138, 2003, 449

163. http://hyperphysics.phy-astr.gsu.edu/hbase/thermo/spht.html

164. Mount A. R., Howarth P., Clifton D., Journal of Applied Electrochemistry, 31, 2001, 1213

165. Landolt D., Electrochimica Acta, 32, 1987, 1

166. Shih C. -C., Shih C. -M., Su Y. -Y., Su L. H. J., Chang M. -S., Lin S. -J., Corrosion Science, 46, 2004, 427

\section{Chapter 9}

167. Datta M., Landolt D., J. Electrochem. Soc., 122(11), 1975, 1466

168. Datta M., Mathieu H. J., Landolt D., Electrochimica Acta, 24, 1979, 843

169. Datta M., Landolt D., Electrochimica Acta, 25, 1980, 1263

Chapter 10

170. Azumi K., Seo M., Corrosion Sci., 2001, 43, 533

Chapter 11

171. Graham M. J., Corrosion Science, 37(9), 1995, 1377

172. Kuo H. C., Landolt D, Electrochimica Acta, 20, 1975, 393

173. www.coolingzone.com/Content/Library/Tutorials/Tutorial\%201/DNHT.html

174. www.kayelaby.npl.co.uk/general_physics/2_2/2_2_3.html 